UCRL-50021-79

Distribution Category

UC-21,22

\title{
Laser Program Annual Report - 1979
}

\section{Volume 2}

\author{
Scientific Editor: Lamar W. Coleman \\ Publication Editor: John R. Strack \\ MS date: March 1980
}

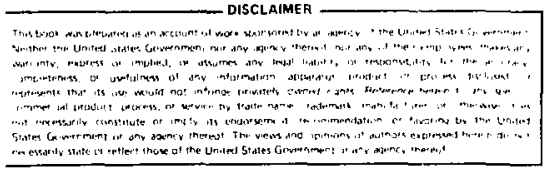

\section{Lawrence Livermore National Laboratory} University of California $\bullet$ Livermore, California $\bullet 94550$ 


\section{Contents}

\section{Volume 1}

Section 1-Laser Program Overview

Introduction

Major Activities

Program Resources

Program Facilities

Section 2-Solid-State Laser Systems and Technology

Introduction

Argus and Shiva Laser Facility Operations . . . . . . . . . . . . . . . 2-3

Nova . . . . . . . . . . . . . . . . . . . . . . . . . . . . . . 2-58

Research and Development . . . . . . . . . . . . . . . 2-138

Basic Research . . . . . . . . . . . . . . . . . . . . . . . 2-237

\section{Volume 2}

\section{Section 3-Target Design}

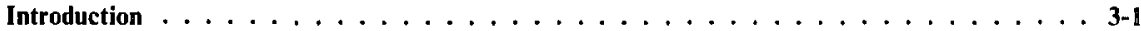

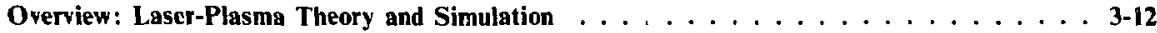

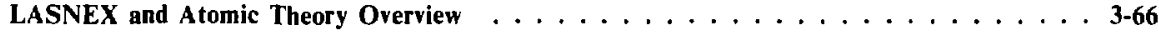

Section 4-Target Fabrication

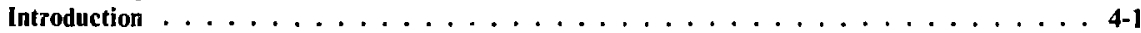

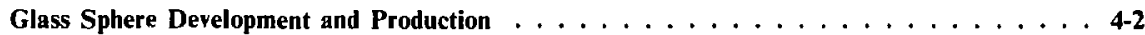

Coatings and Layers $\ldots \ldots \ldots \ldots \ldots \ldots \ldots \ldots$

Double-Shell Targets . . . . . . . . . . . . . . . . . . . . 4-29

Analysis and Characterization $\ldots \ldots \ldots \ldots \ldots \ldots \ldots \ldots \ldots$

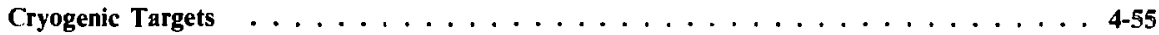

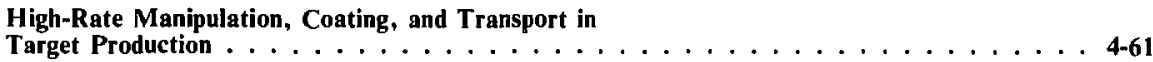

Section 5-Diagnostics Technology

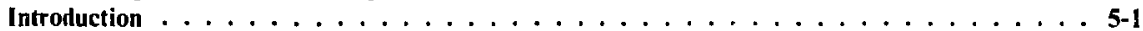

Temporal Response of the XRD-31 X-Ray Diode $\ldots \ldots \ldots \ldots \ldots \ldots$

Filtered-Mirror Sub-keV X-Ray Measurement System . . . . . . . . . . 5-5

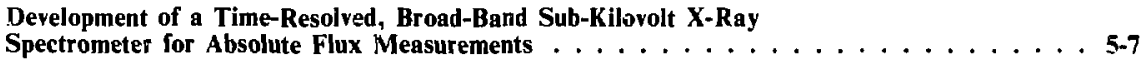

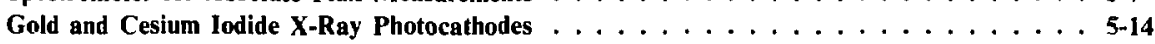

CCD Applications for Transient Digitizers . . . . . . . . . . . . . . 5-17

Reflector-Diffractor Spectrograph Measurements on Shiv a . . . . . . . . . 5-21

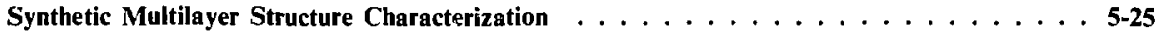

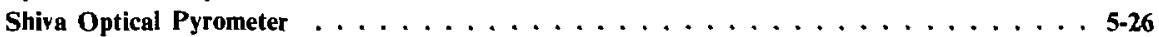

Shiva Raman Light Spectrograph . . . . . . . . . . . . . . . . 5-27

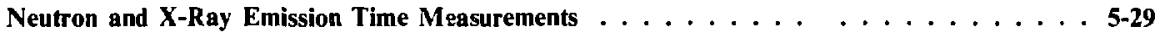

D-D Neutron Measurements . . . . . . . . . . . . . . . . . . 5-32

Compression Diagnostics for High-Dersity,

Low-Temperature Targets . . . . . . . . . . . . . . . . . . . . . 5 544

X-Ray Imaging of Laser Fusion Targets $\ldots \ldots \ldots \ldots \ldots \ldots \ldots$ 
Implosion Measurements with Neutron Activatio rechniques . . . . . . . . . 5-39

Plasma Diagnostics Using X-Ray Spectroscopy . . . . . . . . . . . . . . . . 5-42

X-Ray Backlighting with a Wolter Microscope and X-Ray

Streak Camera . . . . . . . . . . . . . . . . . . . . . . . . 5-54

Micro-Fresnel Zone Plate Developments . . . . . . . . . . . . . . 5-57

Management and Analysis of Experimental Data . . . . . . . . . . . . . 5-67

Section 6-Laser Fusion Experiments and Analysis

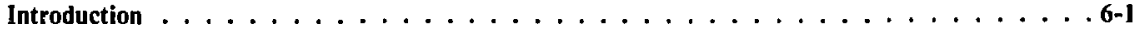

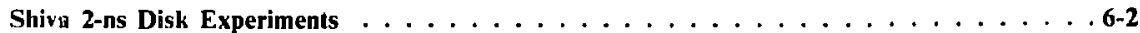

Sidescatter in Laser-Irradiated High-Z Targets . . . . . . . . . . . . . . . . 6-7

Electron Transport Analysis Using Layered Slab Targets . . . . . . . . . . . . . . 6-12

Z-Dependence of Sub-keV X-Ray Emission and Laser Intensity

Threshold for Inhibited Electron Thermal Conduction . . . . . . . . . . . . . 6-19

High-Energs $\ddot{z}$-Kay Measurements from Disks of Different Z . . . . . . . . . 6-26

Angular Distribution of Suprathermal X Rays . . . . . . . . . . . . . . 6-28

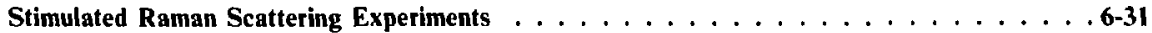

Experimental Configuration of the $2 \omega_{\mathbf{0}}$ Experiments $\ldots \ldots \ldots \ldots \ldots \ldots$.41

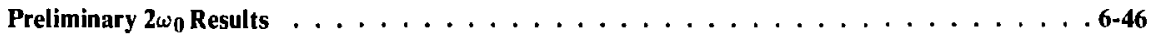

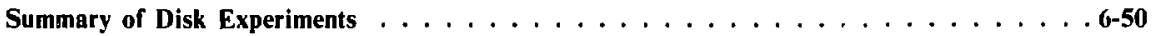

$10 \times$ Liquid Density Target Experiments . . . . . . . . . . . . . . 6-52

D-T Fuel Density Determination from Measurements of Pusher

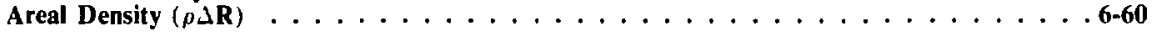

\section{Volume 3}

\section{Section 7-Advanced Quantum Electronics}

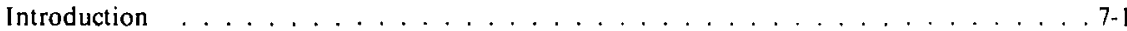

Pulse Compressor Laser Systems . . . . . . . . . . . . . . . . . . . . . . . . . 7-2

Advanced Concepts and Supporting Research . . . . . . . . . . . . . . . . 7-45

Laser Theory and Design . . . . . . . . . . . . . . . . . . . . . 7-67

Advances in Atomic and Molecular Theory . . . . . . . . . . . . . . . . 7-98

Section 8-Energy and Military Applications

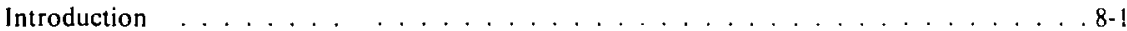

A Conceptual Design Strategy for Liquid-Metal-Wall Inertial

Fusion Reactors . . . . . . . . . . . . . . . . . . . . . . . . 8-3

New or Revised Calculations of the High-Yield Lithium-Injection

Fusion-Energy (HYLIFE) System . . . . . . . . . . . . . . . 8-13

Evolution of the HYLIFE Fusion-to-Electricity Converter . . . . . . . . . . . 8-70

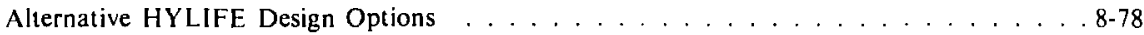

Material Considerations for Liquid-Wall ICF Energy CLaverters . . . . . . . . . . . . 8-87

Cost-Performance Studies of an Inertial-Fusion Power Plant . . . . . . . . . . . 8-107

Conceptual Design of a KrF-Fusion-Laser System . . . . . . . . . . . . . 8-119

Section 9-Advanced Isotope Separation

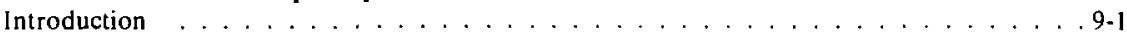

Laser Development . . . . . . . . . . . . . . . . . . . .9-4

Atomic Vapor Spectroscopy f. . . . . . . . . . . . . . . . . 9-15

Advanced Laser Research . . . . . . . . . . . . . . . . . . . . . . . . .9-24

Photon-Atom Dynamics . . . . . . . . . . . . . . . . . . . . . . . . . . . . . . .

Collision Physics and Chemistry . . . . . . . . . . . . . . . . . . . . .9-43

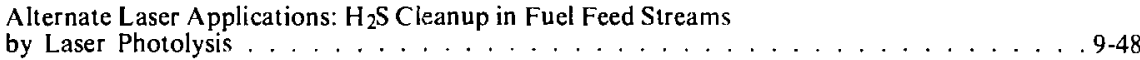




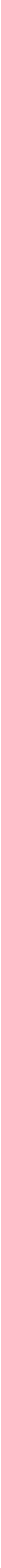

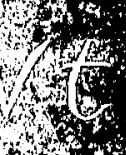
$+2$

ros $3 \times 5$

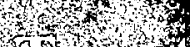

$x, 70$ - 


\section{Target Design}

\section{Contents}

Introduction

High-Z Disk Modeling

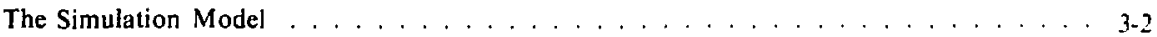

Simulation Results . . . . . . . . . . . . . . . . . . . . . . . . . . . . . . $3-3$

Scaling Laws . . . . . . . . . . . . . . . . . . . . . . . . . . . . . . . . . . .

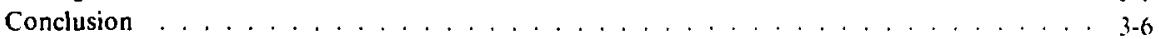

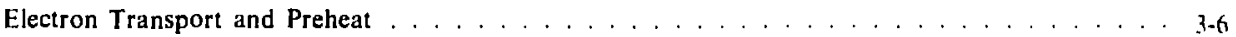

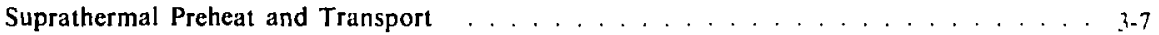

Shock Propagation . . . . . . . . . . . . . . . . . . . . . . . . . . . . . . . 3.9

Nuclear Preheat Calculations for Heavy-Ion Fusion . . . . . . . . . . . . . . . . . . 3-10

Overview: Laser-Plasma Theory and Simulation . . . . . . . . . . . . . . . . . . . . . . . . .

Nonlinear Inverse Bremsstrahlung and Heated-Electron Distributions . . . . . . . . . . . . . . . 3-13

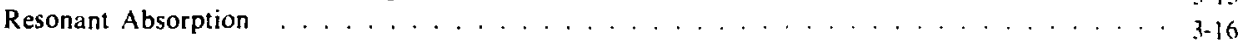

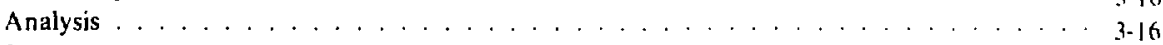

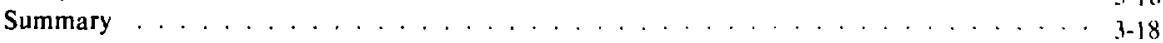

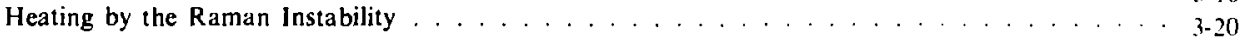

Theoretical Estimates of Raman Scattering . . . . . . . . . . . . . . 3-20

Hot Electrons . . . . . . . . . . . . . . . . . . . . . . . . . 3-21

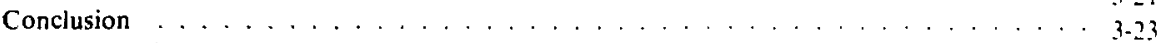

Two-Dimensional Simulations of Quarter-Critical-Density Heating

in Large Plasmas . . . . . . . . . . . . . . . . . . . . . . . . . . . . . 3-23

The Effect of Reheating on the Hot-Electron Temperature . . . . . . . . . . . . . . 3-25

Effects of Driver Bandwidth Upon Resonantly Heated Electrons . . . . . . . . . . . . . . . . . 3-27

Nonlinear Behavior Of Stimulated Scattering In Large Underdense Plasnias . . . . . . . . . . . . 3-28

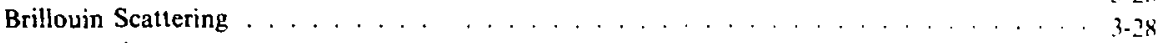

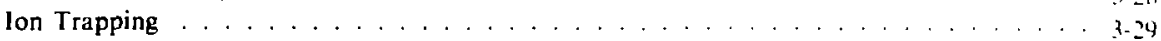

Harmonic Generation . . . . . . . . . . . . . . . . . . . . . . . . . . . . . .

Ion Tail Formation . . . . . . . . . . . . . . . . . . . . . . . . . . . . . . . . . . .

The Effects of Gradients . . . . . . . . . . . . . . . . . . . . . . $3-33$

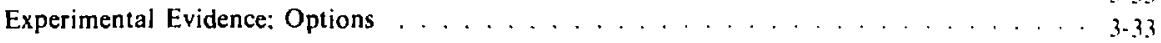

Summary . . . . . . . . . . . . . . . . . . . . . . . . . . . . . . . . . .

Physical Processes Affecting Stimulated Brillnuin Scattering at Different

Enhancement of Stimulated Brillouin Scattering in the Presence of a

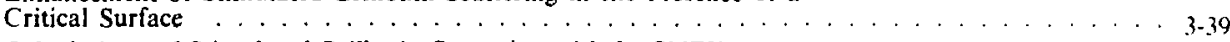

Calculations of Simulated Brillouin Scattering with LASNEX . . . . . . . . . . . . . . . .

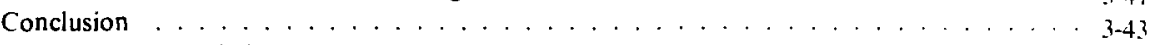

Steepening of Ion Acoustic Waves . . . . . . . . . . . . . . $3-43$

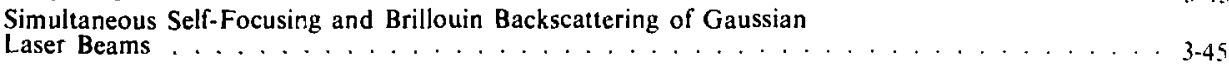

Spatial Structure of Filamented Light . . . . . . . . . . . . . . . . . . 3-47 
Update of Model for Ablative Liser Fusion Implosions

The Ablative Flow

Scaling Laws

Hydrodynamic Calculations of Steady-Flow Spherical Profiles . . . . . . . . . . . . . 3-55

The Computational Model . . . . . . . . . . . . . . . . 3-55

Calculational Methods . . . . . . . . . . . . . . . . . . . 3-57

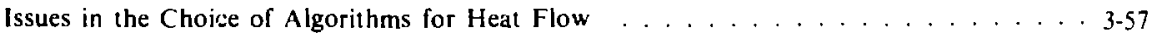

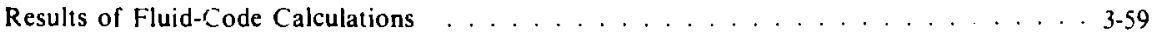

Examples of Validity Checks . . . . . . . . . . . . . . . . . . . 3-60

Conclusion . . . . . . . . . . . . . . . . . . . . . . . . . . . . . . . . . .

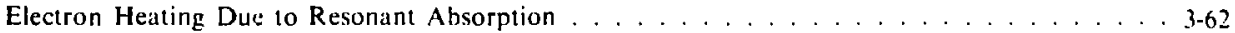

Electron-Heat Flux inhibition Due to Ion Acoustic Turbulence . . . . . . . . . . . . . 3-64

Plasma Code Development . . . . . . . . . . . . . . . . . . . . . . 3-65

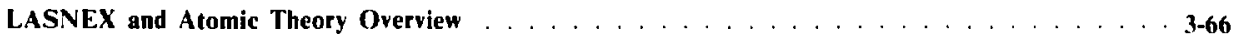

Improvements in the LASNEX Atomic Physics Package . . . . . . . . . . . . . . 3-66

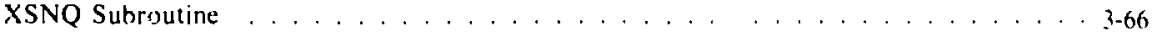

Screening Coefficients . . . . . . . . . . . . . . . . . . . . 3-67

Pressure Jonization Model . . . . . . . . . . . . . . . . . . . . . . . . . 3-68

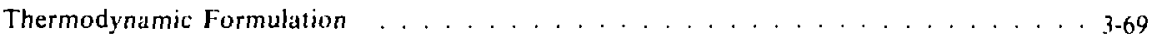

Energy Deposition by Fast Jons . . . . . . . . . . . . . . . . . . 3-72

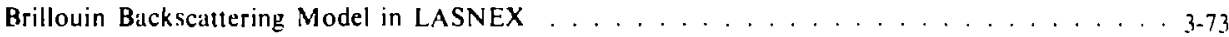

New Features of the SBS Model . . . . . . . . . . . . . . . . . . . . 3-74

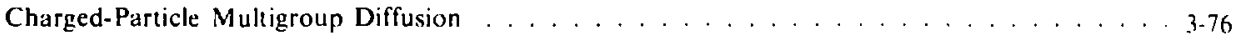

Solving Tridiagonal Linear Systems on the Cray-I Computer

at Vector Speeds . . . . . . . . . . . . . . . . . . . . . . . . 3-77

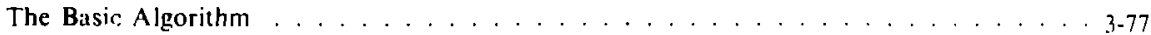

Implementation on the Cray-1 . . . . . . . . . . . . . . . . . . . . .

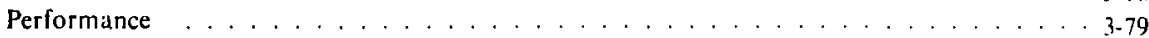

A Vectorizable Incomplete Cholesky-Conjugate Gradient (ICCG) Algorithm

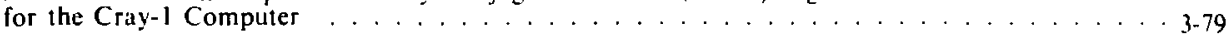

Hot-Electron Momentum Deposition in LASNEX . . . . . . . . . . . . . . 3-82 


\section{Target Design}

Introduction

The Target Design program at LLL combines the efforts of the plasma, code development, and design groups, in

- Developing theories of beam-plasma interaction, implosions, and thermonuclear microexplosions.

- Building plasma and implosion-burn computer codes.

- Using these theoretical and computational tools to design targets and simulate experiments.

In late 1979 a prototype of our high-density target design was irradiated at Shiva. and achieved sufficiently high neutron yield to enable an experimental determination of density. Preliminary measurements indicated that this target reached our prediction of approximately 100 to 200 times liquid density-a several-fold increase over the 50 to 100 times density achieved earlier in the year. We have thus increased achieved density by two of the three orders of magnitude (from normal density to 1000 times liquid density) required for highperformance ICF-reactor targets. Inferred thermonuclear parameters associated with this record density are $\mathrm{n} \tau \approx 5 \times 10^{14} \mathrm{~cm}^{-3}$-s and an ion temperature of $0.5 \mathrm{keV}$.

During the last year we attained a record thermonuclear ion temperature of $13 \mathrm{keV}$ with an unusual type of exploding-pusher target irradiated at Shiva: with another type of exploding pusher we equaled our record exploding-pusher neutron yield of $3 \times 10^{10}$. achieved in 1978. The discrepancy between this neutron yield and our 1977-78 prediction of $10^{12}$ is due primarily tc our anticipation of a higher implosion symmetry and laser-light absorption efficiency than actually occurred, and secondarily to the effects of less laser power and a longer pulse length that we assumed.

Advances in our modeling of energy transport and suprathermal electrons have greatly improved the agreernent between LASNEX calculations and our high-density experimental data ( $x$-ray microscope images, argon line images, and radiochemical measurements). Several of our laser-plasma theoretical predictions have been confirmed experimentally, including the behavior of Raman scattering in 2-ns disk experiments, improved coupling in short-wavelength disk experiments, and delayed suprathermal electron generation in highdensity experiments, all of which play a key role in many of our ICF targets. On the other hand, analysis of our gold disk data has turneri up small discrepancies which might be due to neglect of significant physical processes: experiments with more sophisticated diagnostics (for measuring the jensity profile, for example) are required to explore these discrepancies. Our plasma theorists have also formulated important nonlinear corrections to inverse bremsstrahlung, and improved our understanding of resonant absorption, filamentation. and Brillouin scattering.

The newly developed Cray-1 version of our primary target design code LASNEX has twice the computing capability of the old CDC 7600 version. LASNEX atomic physics. Brillouin scattering, and multigroup diffusion physics have all been improved, and a Monte Carlo photon-transport option has been added.

We have made advances in the design of targets for achieving 100 to 1000 times liquid density with Shiva, for ignition with Nova, and for high gain with reactor drivers. Scaling laws and analytic approximations for a number of critical phenomena are in good agreement with the detailed experimental results. 


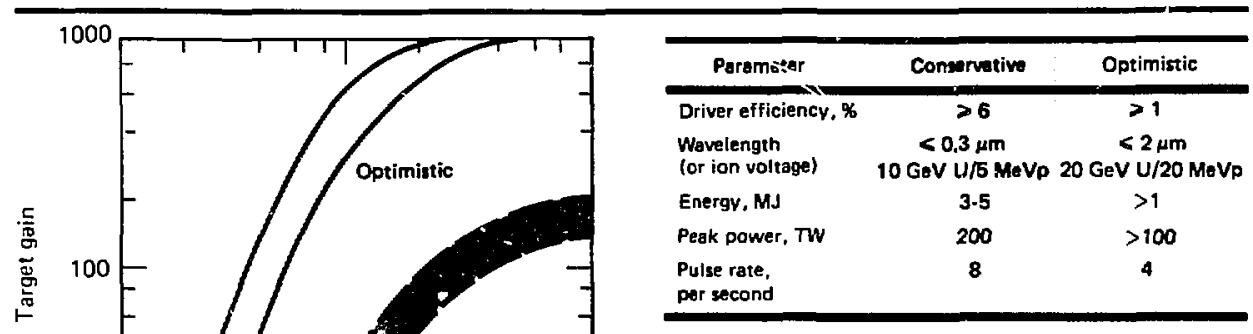

Our predictions of fusion-reactor driver requirements have not changed since last year, nor did our calculated target gain curves change in 1979 (Fig. 3-1). We expect to achieve the conservative levels initially and then follow a learning curve leading toward the more optimistic levels. The Fig. 3-1 Input energv (J) "conservative" estimate in the figure is based on conservative assumptions about implosion instabilities and thermonuclear ignition and burn, and assumes efficient beam-target coup!ing (with ions or short-wavelength lasers). Target designs based on these conservative assumptions will achieve ignition at the LLL. Nova laser facility (including frequency doubling) and at the Sandia Proton Beam Fusion Accelerator (PBFA II). Ignition is defined as energy deposited in D-T by thermonuclear heating. which equals thermal energy in D.T due to implosive heating.

\section{Author: J. H. Nuckolls}

\section{High-Z. Disk Modeling}

In the past year we have continued our efforts at achieving classical (inverse hremsstrahlung) ahsorption of laser light incident on disk targets. "Shiva 2-ns Disk Experiments" in Section 6 of this annual report describes in detail the highly successful resulis of this eflort, which has achieved $80 \%$ absorptions with gold disks hoth at Argus (uing $0.53-\mu \mathrm{m}$ light. 600-ps pulses, and mid- $10^{14} \mathrm{~W} \mathrm{~cm}^{2}$ intensities) and at Shiva (using $1.06-\mu \mathrm{m}$ light, 2-ns pulses, and mid-10 $13 \mathrm{~W} / \mathrm{cm}^{-}$intensities). Thus we have moved deep into the efficient inversebremsstrahlung absorption regime.

These experimental data supplement basic theoretical progress made in the past year on the process of inverse bremsstrahlung and on its competition with stimulated Brillouin scattering. (The theory is described in detail in several articles in this section that deal with laser-plasma theory and simulation.) Some aspects of this theoretical work were incorporated into our L.ASNEX modelingl of the disk esperiments, and the improved model has heen tested by comparing its predictions with previous disk experiments and with the abosementioned Argus and Shisat dala. The data present a formidable test for the model. als the experimental parameters involve factors of 2 in laser wavelength. 10 in pulse length and spot sise. 20 in target $Z$. and 100 in intensil?.

In this article we deseribe the effects of our various additions to the modeling and show comparisons with our experimental data. We find the model's absorption predictions to be belou the experimental results, implying less severe inhibited electron transport or additional absorption mechanisms such as ion acoustic turbulence. In addition. we consider some simple models of inverse bremsstrahlung absorption and compare their scaling predictions with the LASNEX simulations and experimental data.

The Simulation Model. Before describing additions to the model. we briefly review the elements of the standard disk calculational model, which has 
been shown to give good agreement with previous data.'

- A portion of the incident energy is completely discarded from the model's calculations, to account for stimulated scattering. The amount discarded is determined from previous experiments: theoretical arguments are used to scale from one $x$ periment to another.

- The light is absorbed by inverse bremsstrahlung on its way to the critical-density surface. Where some $20 \%$ of what remains is at sorbed into a hot-electron spectrum to simulate resonalnce absorption.

- The thermal-electron heal conduction from eriticat and belou (the regions where the light is atwrbed) wo the werdense plasmat is inhibited by the 2-strean mechanism: the inhibition factor varies from 1 10 0.02 as $Z T_{\text {. }} T_{1}$ varies from 310.30 above 30 the factor remains at 0,02 .

- Since conduction is inhibited, steep lemnerature and density gradients form al eritical. An alom moving rapidly across these stcep gradients is out of equilibrium, so the model must use non-LTE calculations of the mean atomic population levels and of the equation of state.

One of the major improvements in this model is the "langdon factor" that reduces the classical absorption due to nonlinear effect..- In inverse bremsstrahiung the electrons oscillating coherently in the laser's f-field collide with an ion and become isotropic. Since collision frequency scales as $v^{-3}$. the slowest electrons absorb most efficiently. thus heating up and speeding up, depleting the distribution of the slow. efficient absorbers. Usually electron-electron collisions will restore a Maxwellian distribution. thus repopulating the depleted lowenergy portions of the field. For high- $Z$ targets. however, electron-ion collisions (inverse bremsstrahlung) occur $Z$ times faster than the electron-electron collisions, so the depletion of the slow. efficient absorbers continues, thus reducing the net absorption. The figure of merit for this process is $\alpha \equiv Z\left(v_{0 \mathrm{sc}^{\prime}}^{2} v_{\mathrm{i}}^{2}\right)$. Calculations by Langdon show that for $\alpha \geqslant 10$. the inverse bremsstrahlung absorption opacity should be reduced by half: For $\alpha \leqslant 0.1$ there is no correction (the nonlinear effect is negligible) and fo: intermediate values of $\alpha x$ the multiplier varies smoothly from I to 0.5 .

We have also changed the way a onedimensional problem is set up. Instead of a pure disk (rectangular LASNEX zoning) we now use a portion of a sphere whose radius of curvature equals the illuminated spot-size diameter. The spherical divergence of the underdense plasma then mocks up, in one dimens: $n$, the two-dimensional effect of large plasma blow-off. Comparisons with two-dimensional runs show that this onedimensional method of running the code indeed minics major (mo-dimensional features

Although considerable progres; has been made in understanding the competition between inverse bremsstrathlung and timulated scaltering in large underdense platsmats, the need for more sophislicalled rescalroh remains all areis for major improvement in the model. Man! physical effects determine the amount al scatter, including reinforcing effects (such ats light reflecting back off the critical surface) and weakening eflect (such as concective damping ol the scallering in the presence of a density gradienth. These effects and others are stil being studied theoreticilly before thes can be incorporated into the LASNEX corde. The Brillouin scattering model currentl! incorporated into LASNEX rdiscussed later in this section in "Briliouin Backscaltering Model in LASNEX") has shown great promise. Since this addition to the basic model is still undergoing igorous development, however, the work refered to in the remainder of this article does not include this addition.

Simulation Results. On our lirst set of runs we reinvestigaled results for Au disks irradiated at Argus for 1 is with 1.06- $\mu$ m light. Figure 3-2 shows absorption predictions is intensity for these shots. for two different inhibition models: classical conduction, and 2-stream inhitition of thermal electrons. Recill that these results are not corrected for cattering losses. As intensity increases the background temperature rises, lowering collision frequencies and thereby reducing inverse bremsstrahlung. The classical conduction model transports more energy into the overdense plasma and blows out roore plasma into a shallow gradient, thus increasing the absorntion as compared to the inhibited model. In addition, the inbihited model bottles up the energy in the absorption regioti, increasing its temperature. which reduces the inverse brem.strablung as compared to the classical model.

Figure 3-2 also shows the absorption predictions with a correction for stimulated scattering, 
Fig. 3-2. Absorption predictions (for either ciasical of instited condaction models) vs laser intensity. Dotted liaes are the same curves

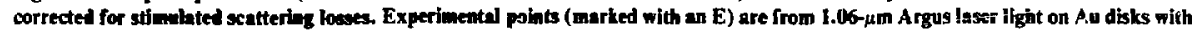
I-ns palses.

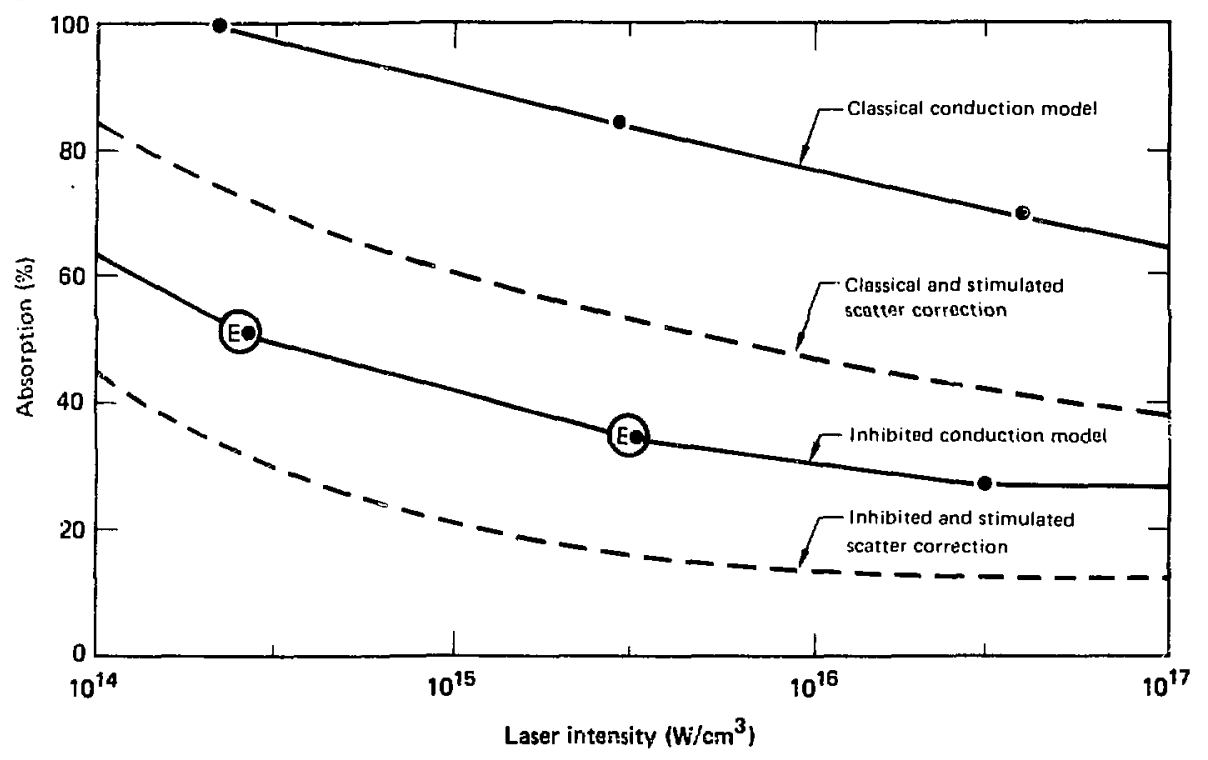

deducus from experiments. Note that the experimental points lie between the two corrected predictions: since previous modeling did not include the Langdon factor, absorption predictions were higher, thus making the inhibited model match the experiment. The possible implications of these new results are either that the 0.02 inhibition factor is too severe. or that we have not modeled other absorption processes (such as ion turbulence) which would increase the absorption prediction and bring the inhibited model back into agreement with experimental data. Despite the new absorption resulss, though. the inhibited model does predict the correct amount of energy that is radiated (in the range from 100 to $1500 \mathrm{eV}$ ) from the disk, whereas the classical conduction model predicts too much. This effect is due to the fact that the radiation is produced most efficiently in denser material, which is heated more efficientiy under classical conduction conditions.

As mentioned earlier, we now have a broader data base with which to compare the model. Figure 3-3 also shows absorption predictions vs intensity
Fig. 3-3. Same as Fig. 3-2, but for 1.86- $\mu \mathrm{m}$ Shiva laser light at 2 ns. No corrections were made for stimulated Brillouin scattering due to uncertainties in the actual amount of scatter.

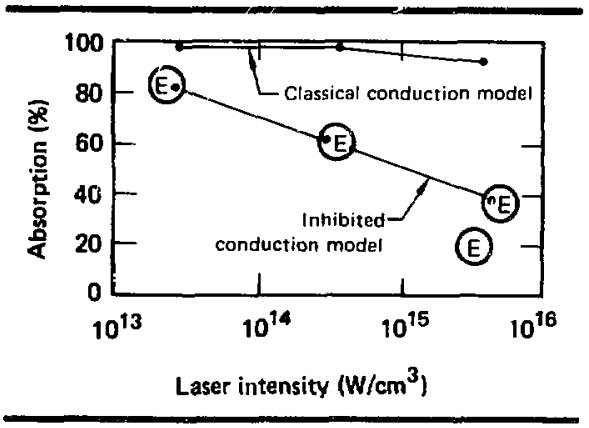

for the classical and inhibited model, but for 2-ns experiments at Shiva. Corrections for scattering have not been made and cannot be experimentally estimated at this time. It appears, as before, that the uncorrected inhibited model matches the experi- 
Fig. 3-4. Same as Fig. 3-3, but for CH disk targets.

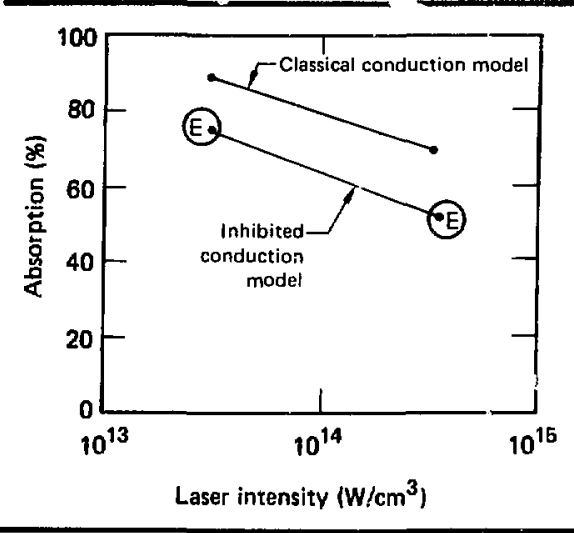

Fig. 3-5. Absorption vs material, for Argus 600-ps laster light at $0.53 \mu \mathrm{m}$ incident on Au or $\mathrm{CH} ; \mathrm{I}=3 \times 10^{14} \mathrm{~W} / \mathrm{cm}^{2}$.

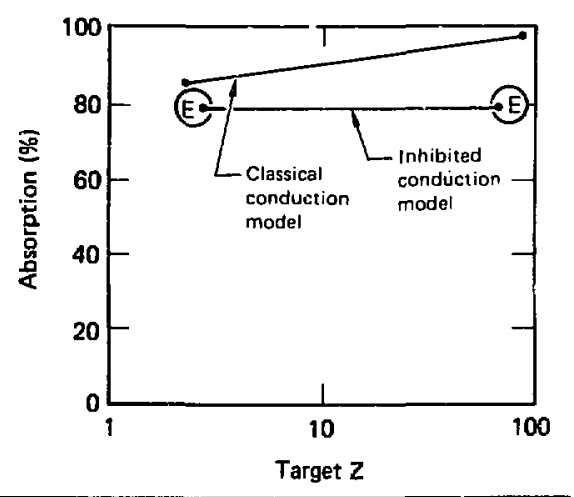

ments quite well, with the probable outcome that a stimulated scattering correction would result in the experimental point's lying squarely between the two models' predictions. Figure 3-4 shows precisely the same behavior for $\mathrm{CH}$.

Figure 3-5 shows green-light results with $\mathrm{CH}$ and $A u$ at $3 \times 10^{14} \mathrm{~W} / \mathrm{cm}^{2}$ for $600 \mathrm{ps}$. The LASNEX curves are uncorrected for stimulated scattering. However, in this case there was expeimental evidence that such scattering was negligible. Thus, the inhibited model fits these data better.

There are two surprises in these 2-ns data. First, the longer pulse and scale length (compared to the $1-n s$ data) slightly increased inverse bremsstrahlung at lower intensities (as expected) but apparently did not increase the stimulated scattering. Secondly, the parylene with mucil lower $\mathrm{Z}$ absorbed nearly as well as the gold These questions will be dealt with shortly.

Scaling Laws. As we sweep from low to high intensity we pass from the very strong inverse bremsstrahlung regime, is the weak one (with Brillouin scattering increasing all the way along). In this section we present results of scaling laws for inverse bremsstrahlung (and stimulated scaltering) in hoth limits, by setting $\left(f_{a h}\right.$ b) equal on $^{\text {? }}$ ?

The rak absorption linut, in which $f_{\text {ath }}=1$ $\exp (-t) \approx \epsilon \sim \|_{\mathrm{ci}}\left(n_{\mathrm{c}}\right)\left(\mathrm{n} / \|_{\mathrm{c}}\right)^{2} \mathrm{~L}$, yiclds

$$
f_{\text {abs }}-(2 \tau)^{0.6} J^{-0.4} \lambda^{-2.0}
$$

and่

$$
T_{e}-(1+2)^{0.4}
$$

These equations (and thos: that follow) were derived assuming that the density scale length. $L$. scales as $C_{、} T \sim T_{c}^{!}$?. This assumption is somewhat questionable for at least two reasons. First, for very long pulses spherical divergence distl.rbs this reiation. Second, for high intensity there is a steepening of the critical surface and a shoriening of scale lengths.

Since the quality factor $Q$ (a measure of the amount of stimulated scattering) scales as ( $\left.v_{\text {anc }} / v_{c}\right)^{\text {? }}$ $(\mathrm{L} / \lambda)$, using Eqs. (1) and (2) we ?ind

$$
\mathrm{O}_{\mathrm{SBS}} \cdot \lambda(\mathrm{I} \tau)^{0.8} Z^{-0.2}
$$

In the strong absorption limit ${ }^{3} f_{\mathrm{a} / \mathrm{s}}=\mathrm{I}$ and the absorption occurs at lower density $\left[n_{a}=n_{d} L_{-a} / L_{i}\right)$, where $l_{. a}$ is defined as $\left.c^{-1} !_{0}^{l_{i i}} u_{e i}\left(n_{c}\right)\left(n: n_{c}\right)^{2} d x=1\right]$. We find

$$
\mathrm{I}_{\mathrm{e}}-\left(Z_{-\mathrm{I}} \mathrm{I}_{\lambda}^{4}\right)^{0.2}
$$

and

$$
\mathrm{Q}_{\mathrm{SHS}}-1 \tau^{0.3} \lambda^{2.5} \mathrm{z}^{-0.6}
$$

We now consider the scaling with each parameter individually. Shorter wavelengths lead to greatly increased inverse bremsstrahlung [Eq. (1)] and decreased scattering [Eqs. (3) or (5)]. These 
scalings were reflected in the high absorption results in the green light experiments. Lower intensity leads to higher inverse bremsstrahlung [Eq. (1)] and decreased scattering [Eqs. (3) or (5)]. This was also seen in the low-intensity 2-ns Shiva data. In the low intensity-high absorption regime, scattering depends only weakly on pulse length [Eq. (5)]. In the high intensity-low ahsorption regime, however, both inverse bremsstrahlung [Eq. (1)] and stimuhated scaltering [Eq. (3)] are strongly dependent on pulse jength, with stimulated scattering actually scaling slightly stronger with $r$. Indeed, there are experimental indications of this: for mid- $10^{14} \mathrm{~W} / \mathrm{cm}^{2}$ irradiations, the $2-n s$ result on All $(60 \%$ absorption) is barely above the $1-n$ s result $150 \%$ absorption), while for mid-10 $15 \mathrm{~W} / \mathrm{cm}^{2}$ irradiations, the 2-ns result (25\% absorption) is actually below the 1-ns result (36\% ahsorption). The lesson to be learned is that if w" wish to absorb effiejently at long pulse angths by avoiding stimulated scattering, we must be sure to be in the strong inverse bremsstrahlung regime (by having low I and/or short $\lambda$ ) where $Q_{S B S}$ scales weakly with $x$.

The only mystery ihat remains is in the $\widetilde{Z}$ scaling. Equations (1) through (5) show that inverse bremsstrahlung should increase with $Z$, wisie SBS should decrease with $Z$, resulting in a higher- $Z$ target absorbing better. The experiments, however, showed comparable $\mathrm{CH}$ and $\mathrm{Au}$ absorptions, as did the LASNEX code model. Study of this prohlem is underway, including the possibility that

- The Langdon factor acts more strongly on Au $\left(\alpha x \equiv Z v_{0 c_{c}^{2}}^{2} v_{e}^{2}\right)$ than on $\mathrm{CH}$, thus reducing the inverse bremsstrablung for Au.

- Two-stream inhibition is less effective for low $Z$ (figure of merit $Z \mathrm{~T}_{\mathrm{c}} / \mathrm{T}_{\mathrm{i}}$ ), which would render the $\mathrm{CH}$ "uninhibited"; it would then absorb more efficiently than in the inhibited situation (which holds for Au).

Since hoth the experiments and simulations run the full range from low to high inverse bremsstrahlung, we take as a global scaling law the geometric mean of the two limits, resulting in

$$
\mathrm{T}_{\mathrm{e}}-\lambda^{0.4} \mathrm{I}^{0.5} \mathrm{z}^{0.3} \tau^{0.3}
$$

This scaling has been compared to LASNEX runs for which we find

$$
\mathrm{T}_{\mathrm{e}}\left(\text { LASNEX) } \sim \lambda^{0.4} \mathrm{I}^{0.4} \mathrm{Z}^{0.3} \tau^{0.4}\right.
$$

in reasonable agreement with $\mathrm{Eq}$. (6).

Conclusion. Our improved LASNEX model tracks many of the experimental trands which now cover a wide parameter range. An improved stimulated scattering package will help determine how well the model really stands up quantitatively. The nonlinear inverse bremsstrahlung correction lowers absorption which under many conditions implies the need for somewhat less inhibition or for invoking additional absorption mechunisms such as ion turhulence. On the other hand, LASNEX may be underestimating the absorption due to its including the average temperature (suprathermals and thermats in the busically nonlinear inverse bremsstrahlung phenomenon. $\bar{i}_{A V}$ is thus too high, and reduces the cillculated absorption. An experimental determination of density and $1 \mathrm{em}$ perature proliles would be extremely useful in secifying the correct physics to apply to the model. Finally, our simple scaling rules help to elucidate the experimental and code results.

\section{Author: M. D. Posen}

\section{References}

1. M. D. Rosen, 1). W. Phillion. V. C. Rupert, W C. Mciod, W. L. Kruer, J. J. Thomsisn, H. N. Kornblum, V. W. Slivinsky, G. J. Caporaso, M, J. Boyle, and K, G. Tirsell, "The Interaction of $1.06 \mu \mathrm{m}$ Laser Radiation with High Z Disk Turget," Phyr, Fluide 22, 2020 (1979).

2. A. B. Langdon, Nonlimear Incerse Bremsstrahliang and Heated Electrom Distributiom, Lawrerice Livermore Laboratory, l.ivermore, Callil., UCRL-82353 (1979).

3. C. E. Max and K. G. Estabrook, Wavelength Scaling in Laser Fusion from a Plasma Phisics Poim of View, Lawrence Livermore Laboratory, Livermore, Calif., UCRL-8267! (1979).

\section{Electron Transport anu Preheat}

One of the key requirements in the design of a high-gain target is keeping the fuel at a low adiabat in order io facilitate very high compressions. When laser light impinges on the outside of a pusher two physical processes (diagramed in Fig. 3-6) may occur that could raise the adiabat and significantly degrade the implosion:

- Laser-generated suprathermal electrons penetrating deep into the pusher may cause the preheated inside surface of the pusher to explode inward, sending a shock wave ahead that preheats the fuel.

The large ablation pressure set up near the outside of the pusher (which will ultimately implode 


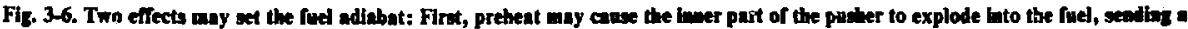
shock wave ahead of it that beatu the fuel. Secondly, large ablation driven shock may arrive lin the fuel too early and rabe the adiabat.

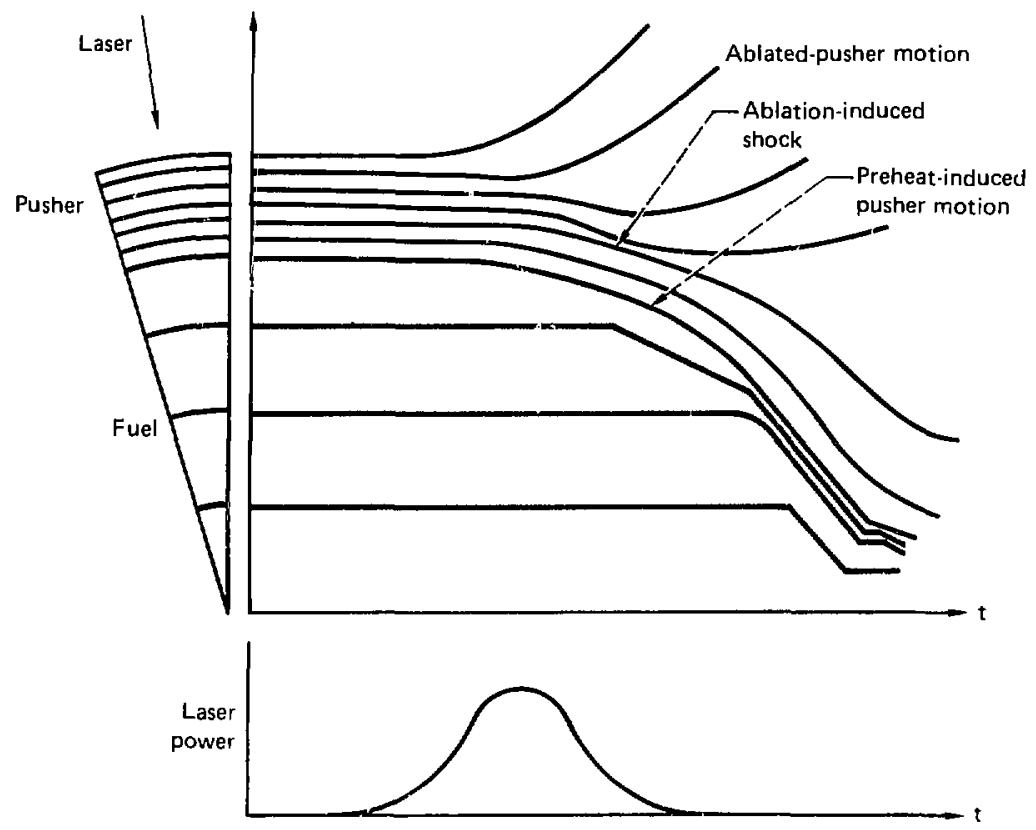

the capsule) sends a shock wave through the pusher that propagates through the fuel as well. If the timing of this shock is premature, the fuel temperature will be raised too soon, thus making high compression more difficult.

While our LASNEX code models these two phenomena (both temporally and spatially) quite accurately, it would nevertheless be useful to have simple analytic expressions for these effects. Such expressions would enable the target designer to pick the right place in parameter space without spending computer resources in a trial-and-error search for a low-adiabat implosion. In this article we develop such simple formulas, both for suprathermal preheat temperatures and for adiabat settings duc to shocks generated by the ablation process.

Suprathermal Preheat and Transport. In lasertarget experiments we usually monitor the highenergy (20 to $100 \mathrm{keV}$ ) $x$ rays produced when hot electrons slow down in dense material and emit bremsstrahlung. From the slope of that distribution we deduce $T_{11}$, the suprathermal electron temperature. and from its magnitude we find $E_{H}$, the amount of energy in the hot electrons. Along with actual measurements we can appeal to theoretical predictions, since $T_{H}$ can be related to laser intensity through particle codes $^{4}$ and simple heories. ${ }^{5}$ For conditions in which inverse bremsstrahlung absorpton plays only a minor role (high intensity, a short pulse, and a low- $Z$ pusher), $E_{H}$ has been measured $^{6}$ (and theoretically predicted ${ }^{7}$ ) to be in the range of 25 to $30 \%$ of the incident energy (100\% of the absorbed energy). For longer-pulse, lowerintensity irradiations we must rely on past experiments $^{8}$ and LASNEX simulations which show. $E_{H}$ to be only a few percent of the incident energy.

How does a suprathermal distribution (characterized by $T_{H}$ and $E_{H}$ ) transport energy through and 
deposit energy in a slab of material? What temperature, $T_{e v}$, does that material reach? Assuming an ideal gas and applying the simplification $A \approx 10 \rho$ (vilid lor glass and gold), we find that

$$
\Gamma_{\mathrm{cv}}(Z+1) \approx 7 \times 10^{5} \mathrm{e}\left(\mathrm{J} / \mathrm{cm}^{3}\right) \text {. }
$$

where $\mathrm{e}$ is the internal energy per unit volume. Tak:ng ionization energy into account would slightly winer $T$ : the ionization state $Z$ can be approximated fir gold as $Z \approx 1.6 \mathrm{~T}^{1 / 2}$ and for glass as $Z \approx 0.8$ $1:=$ ro find $T$ we need to know

$$
c(x)=\left.\frac{i}{A} \frac{d f}{d x}\right|_{x}
$$

Where 1 is the irradiated area. Thus, finding the preheill lemperature at a distance $x$ within a suprathermally bombarded slab requires an expression fur $d^{*}: d x$ as a function of $x$.

We begin by hypothesizing an expression for the average $d \mathrm{E} / \mathrm{dx}$.

$$
\frac{\mathrm{d} U}{\mathrm{dx}}=\frac{-\mathrm{E}:}{\lambda(\mathrm{F})},
$$

where $\lambda$ is the range ${ }^{y}$ of an electron. (This equation is analogous to a conventional energy loss, where $\lambda$ would be a mean free paih along the direction of motion.) For energies between 10 and $100 \mathrm{keV}$, for solid material.

$$
\lambda(\mathrm{H}) \approx \lambda_{\mathrm{H}}\left(\frac{\mathrm{E}}{\mathrm{T}_{\mathrm{H}}}\right)^{2} \approx 0.4\left(\frac{\mathrm{T}_{\mathrm{H}}}{\mathrm{Z}_{\mathrm{N}}}\right)^{2} \sqrt{\mathrm{Z}_{\mathrm{N}}}\left(\frac{\mathrm{E}}{\mathrm{T}_{\mathrm{H}}}\right)^{2}(\mu \mathrm{m}) .
$$

This expression fits the energy scaling and coefficient presented for aluminum data in Ref. 9. The sca ir. $\mathrm{E}$ of the expression with nuclear charge, $Z_{N}$, includes the transport effect of multiple-pitch-angle scattering in high- $Z$ material, which changes the direction of the electron without changing its energy. Thus the electron is taking a random walk, scattering randomly $Z$ times before losing its energy to a collision with another electron. This is the origin of $\sqrt{Z_{N}}$ in Eq. (11), since in a random walk the distance moved is the random step size times the square root of the number of steps.

Substituting Eq. (11) into Eq. (10) yields the Thomson-Whiddington law,

$$
E(x)=E_{0}\left[1-\left(\frac{2 x}{\lambda\left(E_{0}\right)}\right)\right]^{1 / 2},
$$

which gives the energy of a sample electron (which had energy $E_{0}$ at $x=0$ ) as it proceeds through the siab. In order to consider an entire distribution of electrons, we define the following quantities:

$$
\begin{gathered}
\tau_{H}=\frac{1}{2}\left(m v_{H}\right)^{2}, \\
f_{M}(v) \equiv N_{H}\left(\sqrt{\pi v_{H}}\right)^{-3} \exp \left[-\left(\frac{v}{v_{H}}\right)^{2}\right],
\end{gathered}
$$

and

$$
(g(v))=\int_{0}^{\infty}\left(4 \pi v_{0}^{2}\right) r_{M}\left(v_{0}\right) g\left(u_{0}\right) d\left(u_{0}\right),
$$

giving, for' $1=\mathrm{N}_{H}$,

$$
\mathrm{E}_{\mathrm{H}}=\left\langle\mathrm{I}_{\mathrm{TUt}}\right\rangle=\left\langle\frac{m v_{0}^{2}}{2}\right\rangle=\left(\frac{3}{2}\right) \mathrm{N}_{\mathrm{H}} \mathrm{T}_{\mathrm{H}} \text {; }
$$

thus, from Eq. (12),

$\langle F(x)\rangle=\int_{0}^{\infty} 4 \pi v_{0}^{2} f_{M^{\prime}}\left(v_{0}\right)\left(\frac{m v_{0}^{2}}{2}\right)\left[1-\left(\frac{2 \times v_{H}^{4}}{\lambda_{H} v_{0}^{4}}\right)\right]^{1 / 2} d v_{0}$.

This gives an expression for the energy at some distance $x$ within the slau when a suprathermal distribution has impinged on the slab at $x=0$. For convenience we define $Y \equiv v / v_{H}$ and $L \equiv 2 x / \lambda_{H}$. Recall from Eq. (9) that we need to know $\mathrm{dE} / \mathrm{dx}$, so performing the differentiation of Eq. (14) yields

$$
\left(\frac{\mathrm{dE}}{d x}\right)=\frac{3 \mathrm{E}_{H}}{2 \lambda_{H}} \int_{L / 4}^{\infty} y^{2} e^{-y}\left(y^{4}-L\right)^{-1 / 2} e^{-y^{2}} .
$$

We have evaluated the integral analytically (using the method of steepest descent) and checked the results numerically. For $\mathrm{l} \leqslant \mathrm{L} \leqslant 100$, a simple fit to the integral yields the elegant result, 


$$
\frac{d E}{d x}=\frac{E_{H}}{\left(3 \lambda_{H}\right) L^{\log _{10} L}} .
$$

For thin samples $(\mathrm{L} \ll 1)$ the right-hand side should be $E_{H} / 2 \lambda_{H}$. Substituting Eq. (16) into Eq. (9) and then into $\mathrm{Eq} .(8)$ yields the final formula

$$
T_{e v}(Z+1) \approx \frac{2 \times 10^{-5} E_{H}(J)}{A\left(\mathrm{~cm}^{2}\right) \lambda_{H} H^{(\mathrm{cm})} L^{\log _{10} L}},
$$

where we recall the definitions

$$
\begin{gathered}
L=\frac{2 x}{\lambda_{I I}}, \\
Z_{\text {glass }} \approx 0.8 \mathrm{~T}_{\mathrm{ev}}^{1 / 2},
\end{gathered}
$$

and

$$
\lambda_{\mathrm{H}}=0.4\left(\frac{\mathrm{T}}{\mathrm{z}_{\mathrm{N}}}\right)^{2} \sqrt{\mathrm{z}_{\mathrm{N}}} 10^{-4}(\mathrm{~cm})
$$

Thus, given $T_{H}$ and $E_{H}$ we can find $T_{e x}$. And, as the pressure within th: preheated material is. in the jdeal gas approximation,

$$
P=(0.1) T_{e v}(Z+1) M B .
$$

the pressure can be found as well.

Several refinements are required for this theory:

- Suprathermals may be affected by electric fields set up by resistive cold-electron return currents and hence would not transport "classically" as is assumed here.

- The temperature may be increased if suprathermals exiting the cold end of the slab are turned around by sheath potentials and redeposited. On the other hand, the temperature may be decreased since once the material is heated it expands, so that internal energy is converted into hydrodynamic energy, lowering $T$.

- Theoretical predictions for $T_{H}$ and $E_{H}$ must take into account the losses of suprathermals as they reflect of: the large corona in the underdense plasma. This effect ieduces the original soarce $T_{H}$ and $E_{H}$ to that distribution $E_{H}, T_{H}$ that actually deposits in the slab. The experimental me asurement, on the other hand, is the actual $T_{H}$ and $E_{H}$ depositing in the slab and emitting bremsstrahlung. so that is exactly the relevant $E_{H}$ and $T_{H}$ for this calculation.

While the treatment presented here is based on the expression hypothesized in Eq. (10), a more careful treatment has been carried out by Caporaso and Wilson, ${ }^{10}$ where the full diffusion equation is solved. The final result there is within $20 \%$ of Eq. (17) for $1 \leqslant L \leqslant 100$.

Thus far we have calculated how the inside of a pusher is preheated. As far as preheating the fuel, the reader is referred to Ref. 11, which relates specific internal eriergy deposited in the pusher to the shock it sends into the fuet as it explodes. Also calculated is the useful fraction $f$ of the absorbed energy [in our case $\left(\mathrm{nE}_{\mathrm{H}}\right.$ ] that contributes to the shock heating of the fuel, since as mentioned above the hydrometion (explosion) begins as soon as the material hegins to he:tt up.

Shock Propagation. The other contributor affecting the fuel adiabat is the main shock from the laser-driven ablation process. Thore are many papers in the literature that relate the ablation pressire. $P_{A}$. to the laser and target parameters; the reader may choose his favorite. Given a pressure wave of strength $P_{\Lambda}$ propagating through the pusher, how will the fuel be shocked? Standard hydrodynamic theory ${ }^{12}$ predicts that the shock speed effectively doubles as it ieaves the heavy pusher and propagates into the cold gas fuel. Strong shock theory relates $P$ to $; 0 v_{5}^{2}$ so that

$$
P_{f}=4 \rho_{f} \frac{P_{A}}{\rho_{p}},
$$

where $\rho_{f}$ and $\rho_{\mathrm{p}}$ are the initial fuel and pusher densities, respectively. Typically $\rho_{\mathrm{f}}=0.01$ and $\rho_{\mathrm{p}} \approx 2$; thus, the fuel is shocked with a pressure that is $1 / 50$ the pusher pressure. The timing of the shock can be approximated through the strong shock relations

$$
\begin{gathered}
v_{\mathrm{S}}^{2}=\frac{(\gamma+1) \mathrm{P}_{\mathrm{A}}}{2 \rho_{\mathrm{p}}}, \\
\Delta t=\frac{\Delta \mathrm{R}}{v_{\mathrm{s}}},
\end{gathered}
$$


where $\Delta R$ is the pusher thickness. The temperature of the post-shock-heated pusher is roughly

$$
\mathrm{T}_{\mathrm{ev}}(\mathrm{Z}+1)=2.5 \mathrm{P}_{\mathrm{A}}(\mathrm{MB}):
$$

this is the same ideal equation as Eq. (18) except that the density is assumed to be shocked to 4 limes its initial density [strong shock theory states that $\rho$. $=(\gamma+1) /(\gamma-i) \rho_{0}=4 \rho_{0}$ for $\left.\gamma=5 / 3\right\}$.

F:quations (21) and ( 17$)$ should be compared in practice to get a feel for what sets the adiahat-does the preheat dominate or does the shock?

We have done experiments ${ }^{13}$ to measure both preheat and shock at the backs of samples: $T_{11}$ and ${ }_{n}$ were also measured in the experinetsts. Fquation (17) successfully predicted the ohserved preheat temperatures: l:qs. (20) and (21), along with a reasonable model for $P_{1}$, successfully predicted the strength and time of arrivil of the shock signais. Comparisons with the IASNIX code were also quite favorable. These successes give us confidence in the valiatity of our simple theories.

Our ability to calculate the fuel adiahat from given laser and target paraneters gives us the lirst ingredient of a simple implosion model: work on completing the model is under way.

\section{Author: M. D. Rosen}

\section{References}

4 K. (i. Hestabrouk and W. I Kruer. "Properties of Ress-

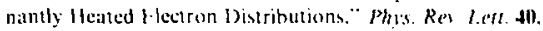
42 (1978)

5. W. 1. Kruer, H- Breaking amplitude in Harm. Inhomogeneous Plasmo wrence I ivermore I aboratory. l.ivermore, Calif.. I IC a $16014(1977)$

6. K. R. Manes, V. ( Rupert. I. M. Suerbacti. P. H. I.ee. and l. I: Siain. "Polarization and angular Dependence of 1.06 $\mu$ n 1 aser I ight Abserption by Planer Plasmas," Phrs Rer t.ett. 39. 28] (1977).

7. J. J. Tbomson, H. I. Kruer, A. H. Langdon, C. I: Max, and W. C. Mead. Theoretical Interpretation of Angle. and Pslarization-fependent laser Light Abserption Measuremonts. lawrence livermore laboratory. Livermore. Calif". L'CR1.-79628 (1977).

8. M. D. Rosen, I) V. Phillion, V. C. Rupert. W. C. Mead. W. L. Kruter, J. I Thonison, H. N. Kornblum, V. W. Sljvinsky. (i. J. Caruraso. M. J. Boylt. and K. G. Tirsell. "The interation $111.06 \mu \mathrm{m}$ Laser Radiation with Higb $Z$ Dist. Targets." Phys. Finds 22. 2020 (1979).

9. L. Kats and A, 5. Penfold, "Range-Fnergy Relations for Electrons and the Determination of Beta-Ray End-Point Energies by Absorption.' Rev. Mod. Phys. 24. 28 (1952).
10. (;. J. Caporaso and S. S. Wilson. A Simple Model for Suprathermal Fiectrm Deposititn in High-Z Plasmas. lawrence livermore laboratory. Livcrmore. Calif.. I.C. $-83308(1978)$.

11. M. D. Rosen and J. H. Nuckolls, "Ixploding Pusher PerFormance-A Theoretical Model." Phis. Fluids 22. 1393 $11977\}$.

12. R. I.elevier, lecures on IIydrodynaics and Shock Waves. I awrenes livermore laboralory. Livermore. Calif.. ( ' ( RI, $-4333(1965)$

13. 11, 1) Rosen, thalysis of R.ong l'ulse Physics Experiments ot 1.I l. Late 1977-Larly 1979, I awrence Livernore I aboratory. l.jvermore, (alif. l:( Rl.-83022 (1979).

\section{Nuclear Preheat Calculations for Heavy-Ion Fusion}

Some of the energetic heavy ions incident on a fusion target will produce nuclear reactions. Typically the velocities of the reaction products are comparable to the velocity of the beam. Thus the energy of a reaction product is roughly proportional to its mass: its charge, $Z$, is also roughly proportional to its mass and to its energy.

At a given velocity, io a energy loss per unit length is proportional to $Z$. The energy of the beam and reaction-product ions, though, is proportional to $Z$. Thus the reaction fragments can penetrate more desply into the target than the beam ions. In some cases these frugments may penetrate into and pretheat the fuel: it is therefore important to determine if this preheat is large enough to have a deleterious effect on target performance.

A high-gain, ion-driven larget of the type shown in Fig. 3-7 can tolerate a fuel preheat of about $10^{-4} \mathrm{~J} / \mathrm{g}$ (Ref. 14). The beam energy required to drive such a turget is about $1 \mathrm{MJ}$ per $\mathrm{mg}$ of fuel. Thus the fuel can tolerate preheat corresponding to about 1 part in $10^{5}$ of the beam energy. Fortunately, only 1 to $1 \%$ of the beam energy arrives early enough to contribute to preheat, so that I part in $10^{3}$ to $10^{4}$ of the beam energy can be deposited in the fuel without serious consequences. For more complicated multishell target designs one must also consider preheat in materials other than fuel. For the designs we have considered, the allowable levels are also on order of $10^{\circ} \mathrm{J} / \mathrm{g}$. but in some cases this figure represents the total energy input and not just that arriving in the first 1 to $10 \%$ of the pulse.

In order to investigate the nuclear preheat level quantitatively we have written a computer code which calculates the energy deposition of beam ions and nuclear reaction products as a function of 
Fig. 3-7. Section of a spherical ion-bean fusion target. Additional details about this type of target are given in the 1976 Annual Report. ${ }^{14}$

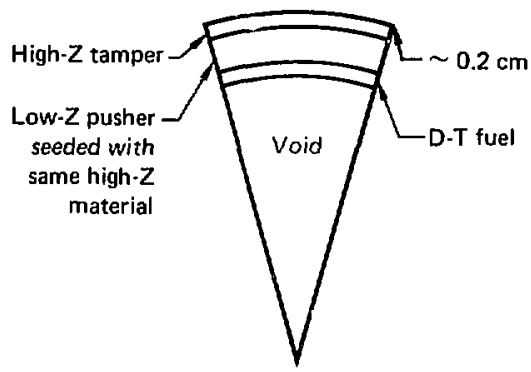

penetration into a planar slab. consisting of layers of different materials to simulate a target. The angular distribution of the incident ions with respect to the normal is chosen to approximate the distribution expected with respect to the sphere radii under plausible assumptions about target radii and beam focusability. Since detailed differential nuclear-reaction cross sections for the multitude of possible fragments are not available at energies of interest for heavy-ion fusion $(\sim 1$ to $20 \mathrm{GeV})$, we assume that

- The tcial cross section is geometrir.

- About $20 \%$ of the nucleons are emitted as neutrons or isotopes of light elements $(H$. He. or Li).

- i he muitiplicity of other nuclear frugments increases slowly with increasing atomic number. with the multiplicity values constrained by baryon conservation.

Thus according to our simple model about 20 light nuclei are emitted per interaction. and the probability of emission of any particular heavier nucleus is on the order of $1 \%$. All fragments are assumed to have the same velocity as the projectile. The use of geometric cross sections is adequate for our purposes. ${ }^{15}$ as long as we use an incident ion beam that does not have a large cross section for electromagnetically induced fission.

Figure 3-8 shows the results of a typical calculation for $10-\mathrm{GeV}$ heavy ions incident on a typical high-gain, ion-driven target. Note the jump in deposition at the interface between the high- $Z$ layer and the low- $Z$ layer behind it: the interface between the low- $Z$ layer and the D-T fuel occurs at
Fig. 3-8. Energy deposition as a funution of peretration. The contribution of muclear debris to energy deposition in the fuel has been multiplied by $10^{3}$ to make it visible. The fuel region extends to $0.2 \mathrm{~g} / \mathrm{cm}^{2}$ in this calculation although it would be much thinner in an actugl target.

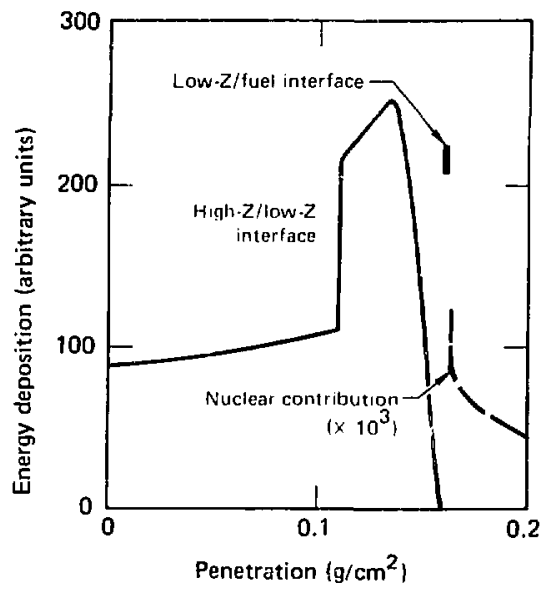

a penetration of $0.16 \mathrm{~g} . \mathrm{cm}^{2}$. We have ignored statistical range straggling in this calculation because it is not important for calculating nuclear preheat. (He do include straggling in our larget design codes.) Because straggling has been ignored. all incident ion bean deposition stops at 0.158 $\mathrm{g} / \mathrm{cm}^{2}$ : thus the energy deposition in the fuel represents only the nuclear preheat from the reaction products. The product of density and thickness of the fuel is about $10^{-3} \mathrm{~g} / \mathrm{cm}^{2}$. so we can eas:ly estimate from Fig. 3-8 that the energy deposited in the fuel by nuclear debris is less than 1 part in $10^{6}$ of the total beam energy and is thus too smail (by 1 or 2 orders of magnitude) to be significant. It is not possible to construet a multiplicity model that increases the calculated preheal by an order of magnitude without violating baryon conservation.

We conclude that the preheat produced by nuclear reactions of $10-\mathrm{GeV}$ heavy ions is not significant for the target design considered. Preliminary calculations show that this conclusion is also true for multishell targets. and seems likely to be true for nedily all types of targets. To verify this, we plan to extend detailed calculations to a wider 
range of ion kinetic energies and targets. The accuracy of our energy-deposition code will be improved by more realistic modeling and by com. pitrison with experimental nuclear data as they become available. Note, however, that while such dit.l are useful for precise target design work, they at init needed to establish the feasibility of heavy111 tasion

Suthor: R. O. Bangerter

References

i iaver fremeram temaal Raport-1476. L.a urence Livermore

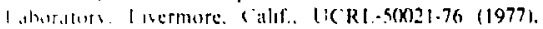
1.4 .4

$h$ V Mel ris and I ( Peng. "Nucleus-Nucleus Total

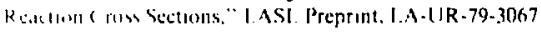

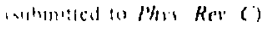

\section{Overview: Laser-Plasma Theory and Simulation}

in 1979 we continued to address critical laserplissmat coupling issues which impact target design. Particular attention was given to processes which ife operattive in the large underdense plasmas that charactere reactor targets: hot-electron generation Wis the Raman and 2-plasmon-decay instabilities. timultied Brillouin scattering, and self-focusing and filamentation of the laser beam. Based on an examination of uptions for improving the coupling we chose to 'undertake experiments with shorterwavelength laser light. and measurements to date confirm our predictions of improved absorption and reduced hot-electron temperature.

Progress in advancing our understanding of laser-plasma coupling was made in many different areas. The theory of inverse bremsstrahlung was extended to include the self-consistent modification of the velocity distribution of the heated electrons. The classical absorption rate was found to be reduced by a factor of approximately two, by self-consistent inodification of the velocity distribution. for many Lases of practical interest in high- $Z$ plasmas. Our calculations of electron heating by Raman and 2 plasmon-decay instabilities were extended to include large regions of underdense plasma; we subsequently found a sizable absorption into electrons with a temperst?re of -40 to $\$ 00 \mathrm{keV}$, provided there is a large region of plasma with a density of about one-fourth the critical density $\left(0.25 \mathrm{n}_{\mathrm{c}}\right)$. An experimental search for the Raman instability was initiated. and this instability was indeed observed. Firther investigations of electron heating via resonance absorption were conducted with simulations of a capacitor model: these simulations allowed us to better assess the effect of the background electron temperature on the temperature of the resonantly-heated electrons, especially in the regime of a severe steepening of the density profile.

Calculations of stimulated Brillouin scattering were given a high priority:

- We developed an improved model for the effect of self-consistent ion heating on the scattering. This model, which focuses on ion-tail formation, compares well with our computer simulations.

- We discovered a possible enhancement of Brillouin scattering by light classically reflected from the critical density surface.

- OUr I.ASNEX model of Brillouin scattering was extended to take into account both ion tail formation and stabilization of the scattering by gradients in the plasma density and expansion velocity. Calculations using this model of the scattering have been very promising. and are currently in at least semi-quantitative agreement with the experiment.

- In addition to calculating intensity profiles for filamenied light, we developed a simple modcl for the effect of filamentation on stimulated Brillouin scattering.

Uncertainties in the plasma conditions and in electron transport continued to receive attention. Hydrodynamic calculations of steady-flow spherical profiles in the presence of electron transport inhibition were found to be in good agreement with a theory we had developed. We examined coronal energy losses into fasi ions, and developed an improved description of these losses. The effect of ion turbulence on enhancing the resistivity and reducing the heat transport by electrons was further examined both theoretically and in microwave experiments. Finally, all these developments strongly recommend precise measurements of the plasma conditions in laser-irradiated targets.

Author: W, L. Kruer 


\section{Nonlinear Inverse Bremsstrahlung and Heated-Electron Distributions}

We have found that when $Z v_{0}^{2} / v^{2}>1$ (where $v_{0}$ is the peak velocity of oscillation of the electrons in the high-frequency electric field of the laser. $v_{\mathrm{c}} \equiv$ $\left(T_{0}, m_{c}\right)^{1} 2$ is the electron thermal velocity. and $Z$ is the ionization state) laser-light absorption in a plasma via inverse bremsstrahlung results in a nonMaxwellian velocity distribution for which the absorption is reduced by up to a factor of two compared to the absorption in a Maxwellian plasna as usually assumed. Transport and atomic proeesses are also altered. Lespecially in materials with 7. but also for $7 .=1$, this is significant at intensities lower than those for which another absorption linearity (analyeed many times before $e^{(6-19}$ ) is importunt. for which the measure is $v_{i}^{2} v_{c}^{2}$. This ratio ma! be expressed as $v_{0}^{2} v_{\mathrm{e}}^{2}=4 \times 10^{-16} \mathrm{~A}^{2} \mathrm{~T}_{\mathrm{c}}$. where $I$ is the intensity in $W . \mathrm{cm}^{2} . \lambda$ is the vacuun wavelength in $\mu \mathrm{m}$. and $T_{c}$ is the electron $1 \mathrm{~cm}$ perature in keV.

Vonlinear inverse bremsstrahlung arises when $7 v_{0}^{2}, v^{2}>1$. as electron-electron (e-e) collisions are not rapid enough to evolve a Maxwellian distribution from the hat-topped velocity distribution produced by inverse bremsstrahlung. Consider the ratio of the e-folding time for electron heating to the e-e equilibration time $\tau_{\text {ec }}$ required to reestablish a Muxwellian distribution: the ratio is (thermal energy/ $J \cdot F=3 r_{0} v_{e}^{2} v_{0}^{2}$. We have expressed the ahsorption ${ }^{17.20}$ in terms of $v_{0}$ and $\tau_{e}{ }^{\prime}=$ $\left[4(2 \pi)^{\mid}=3\right] n_{\mathrm{e}} Z \mathrm{e}^{+} \ln .1 \mathrm{miv} \mathrm{e}^{3}$ the same as the standard Maxwell-neighted $e-i$ scattering rate $e^{21.22}$ (except for a small modification ${ }^{20} 10 \ln .1$ ). The heating time is shorter than $\tau_{\mathrm{ee}}\left(\cong \tau_{\mathrm{c}} Z\right.$ ) when $Z v_{0}^{2} \cdot v_{\mathrm{e}}^{2}>3$. in which case non-Maxwellian distributions are possible.

in computer modeling of experiments in which inverse bremsstrahiung is thought to be the dominant absorption mechanism, agreement with experimental data often requires that we invoke mechanisms which reduce absorption. Perhaps the effect described here has such a role in experiments on high-7.-doped glass disks. ${ }^{23}$ In these experiments. with intensities of $\sim 10^{14} \mathrm{~W} / \mathrm{cm}^{2}$ at wavelength $1.06 \mu \mathrm{m}$. electron temperatures of $\sim 400 \mathrm{eV}$ and $Z \sim$ 10 ("a conservative choice tending not to overestimate absorption ${ }^{\cdot 23}$ ), we find $v_{0}^{2} / v_{e}^{2} \cong 0.1$. so the conventional nonlinearity ${ }^{16-19}$ makes only a $1.5 \%$ reduction in opacity. Since $Z v_{0}^{2} / v_{e}^{2} \cong 1$. however, the nonlinearity described here results in a $40 \%$ reduction. comparable to other refinements ${ }^{23}$ invoked to improve agreement with experiment. The reduction in opacity found here has also been helpful in recent modeling of high- $Z$ disks (see "High-Z Disk Modeling" earlier in this sectiun).

We derive the equation of evolution of the electron distrihution function. 5 . due to e-i scattering in the presence of an oscillating electric field. For ho ${ }^{\prime} \ll$

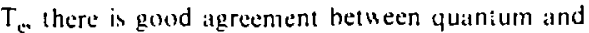
classical deseriptions 17.19 except for modificitions (1) In A. so for clarity we use a simple classical model. ${ }^{1 \times .22}$ Assuming uniform density and ficld, the kinclic equation is

$$
\frac{\partial f}{\partial t} \frac{c}{m e}+\frac{\partial f}{\partial v}=A \frac{\partial}{a v} \cdot\left[\frac{v^{2} \frac{1}{s} \cdot \underline{v}}{v^{3}} \cdot \frac{\partial f}{\partial v}\right]+C_{c t}(f) .
$$

where $A=\left(2 n_{c} Z e^{4}\right.$ mo $)$ ln 1 in the ustal notation: $C_{e x}$ is the e-e collision operator. Expansion in Legendre functions, $f(\Sigma, l)=\mathbf{E}_{1}(v, 1) \mathrm{P}_{1}(\mu)$, smplifies the e-i collision operatur. The lirst iso equations in the expansion of Ey. (22) are

$$
\frac{\partial f_{0}}{\partial t} \frac{e t}{m e} \frac{1}{3 r} \frac{\partial}{\partial t}\left(r^{2} f_{1}\right)=c_{0}
$$

and

$$
\frac{\partial f_{1}}{\partial t} \frac{\mathrm{el}}{m_{\mathrm{e}}}\left[\frac{\partial f_{0}}{\partial v}+\frac{2}{5 v^{3}} \frac{\partial}{\mathrm{in}}\left(v^{3} f_{2}\right)\right]-\frac{2 \mathrm{~A}}{v^{3}} f_{1}+c_{1}
$$

where el:(t) $m_{w}=$ s 1 cos wit. We have truncated the expansion by neglecting la: this implies $v_{0}^{2}, v_{2}^{2} \ll 1$. But the eflects of $1 \frac{7}{1}>1 \frac{2}{6}$ for a Maxwellian distribution have been discussed extensively, an $\rfloor$ since our purpose is to demonstrate modifications that are possible even when $v_{0}^{2} \ll v^{2}$. We are justified in making this simplification.

At intensities of $\sim 10^{1+4}$ the time-dependence of $f_{1}$ is predominantly at the high frequency e: it is the slow variation of $f_{0}$ which is relevant. $C_{0}$ is evaluated using only $f_{0}$ (Ref. 22, Eq. 7-7lb). As e-e collisions do not much affect the oscillating flux $f_{1}$, except indirectly through the slow variation of $f_{0}$. we drop $C_{1}$. We thus obtain 


$$
\begin{aligned}
& L \cdot I=\frac{s}{3} A \pi_{c} m_{c} v^{2} \int_{0}^{\infty} d v g \frac{\partial t 0}{\partial v} \\
& \frac{4 \pi}{3} x_{0} m_{e} v_{0}^{2} \int_{0}^{\infty} d v \frac{\partial y}{a v} f 0
\end{aligned}
$$

and

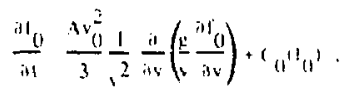

111h

$$
u v|=| 1+\left.w^{2} z_{v i}^{2}(x)\right|^{\prime}\left(1+\frac{v^{6}}{v^{6}}\right)^{1}
$$

where ids $\equiv 21$ s"is the electron sciltere. ratc

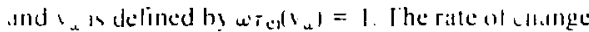
(1) knetic theres calculated from 1 4. (26) in consis-

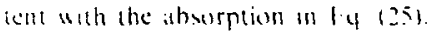

The functum gacounts for the changeover trom the primarity reiative response of the faster chectoms th the resistise response of the slou, trongly xillered stectrom. Genally. collisions are reilled as a perturbation on the excillation of the

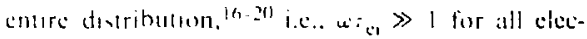
Iroms: $"$ g! $\equiv 1$ and. from F.q. (26), absorption is

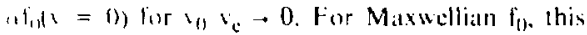
kerds th the standard linear absorption re-

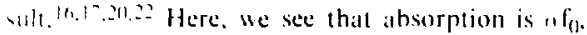
c.lluatcd where $\mathrm{g}$ increases rapidly from $\cong 0$ to $\cong 1$. I L.. It the velocily $\gamma_{u}$ for which scatlering and light treyuences are matched, wt $\left(v_{w}\right) \cong 1$. Since $v_{.} \ll v_{c}$ in cales ot interest, the absorption is little changed by this correction. ${ }^{-2}$

Numericil solutions illustrate the evolution of $f_{0}$ and its effect on absorption and other macroscopic properties. We use a numerical scheme similar to one in Ref. 25, in which the Fokker-Planck coefficients are cialculated from $f_{0}$ without linearization, except that the energy consistency between the finite difference forms of Eqs. (25) and (26) is preserved, and the rapidly decreasing "tail" of the distribution is correctly represented (att least in tilermal equilibrium): hoth properties are independent of the spacing of the velocity rones. which are larger at higher velocities.

He consider first the effect of inverse bremsstrahlung alone. corresponding to the limit $\left\langle\frac{2}{4} \gg 1\right.$. An initially monoenergetic distribution diffuses and slows in balance so that no net gain in kinetic energy results /curve (a) in Fig. 3-9). When electrons reach fow velocities [such that wir eilv) ? 1) their loss ol energy is slowed while upward diflusion of fiater particles continues. so net absorption hegins. This is the meaning of the result that the absorption rate depends on $f_{0}$ at $1 \cong v_{x}$. B! the time the electom have gaimed only $10 \%$ in energy leurve

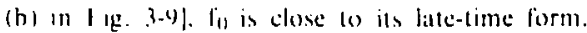
described by at similarity solution of the form $\mathrm{u}^{-3}$ $\exp \left(-s^{5}\right.$ su $)$. with $u^{5}=5$ s from 1 y. (20) with $\mathrm{g} \equiv \mathrm{I}$. For this distribution. the

Fig. 3-9. Evolution of an initially monoenergetic distribution $f_{0}$ due to e-i collisions in the presence of an oscillating Feld; $t$ e collisions are negiected correspending to targe $Z v_{0}^{2} / v_{c}^{2}$. The initial velecity, indicated by the dashed line, is $5.8 \mathrm{v}$. At first, electrons diffuse both up and down in balance so that $f_{0}$ evolves greatly before it gains only $1 \%$ in energy (a). Therester, the slowest clectrons cannot lose more energy, while ofhers continue to diffuse upward, resulting in net absorption. When the energy has increased by $10 \%(\mathrm{~b}), \mathrm{f}_{0}$ is close to the self-simitar solution (c), which is normalized to the seme cnergy as (b). Both axes are linear. $A$ change in initia] velocity or in the constant $A$ scales the times corresponding to curves (a) $\mathrm{H}(\mathrm{c}$ ), but does not alter their shape.

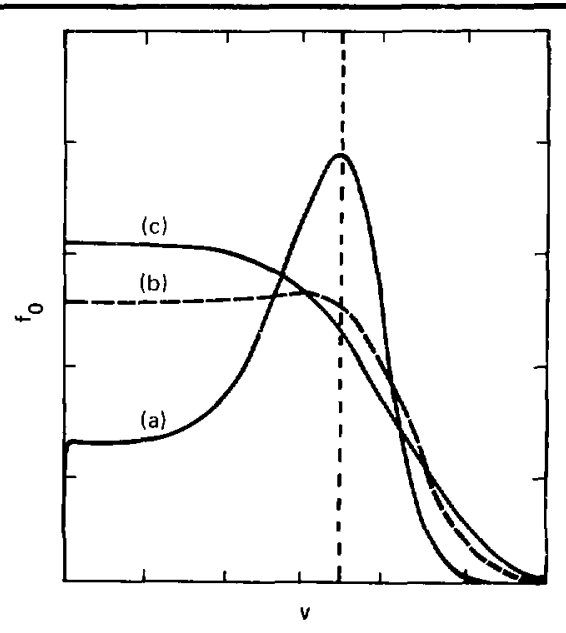


absorption is only $45 \%$ of what it would be if electron-electron collisions enforced a Maxucilian distribution.

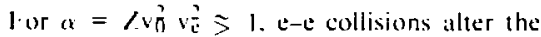
results only sligbtly. For example. with $a=6$ the absorption is still only 49", of its Maxweltian value. and inverse tremsstrahlung contributes equally with te- collisions to diffusion of suprathermats into the "tail" af the distribution. With a $=0.5$. the distribution is still depressed and hattened near $=0(1 \mathrm{ig} .3-10)$. and absorption is reduced $1067^{\prime \prime}$ i $10^{\circ}$ normal. 11 hen a is only 0.05 the reduction is 12 " for ans a. the reduction factor is $1-0.553 \mathrm{ll}+$ $(027 \text { a } 1)^{109} \mid$ within \pm 0.005 sec atso fig, 3-11.

Fall quantitative waluation of this effect reyuires incorporation of spatial gradients and transport. In turn. the transport itself, and the degree of ionialtion. Z, are themselves affected bs these distributions. Heat conduclitits and collisional ioriation are determined mainly by the tail of the cisiribution, which is Iruncaled here. On the other hand, alomic recombination is alfected b: the slow electrons "here our distributions are also deficient compared to a Maxuellian. Enhanced ion fluctuation (above ther:nal level) ate similarly (o) the high- $Z$ condition in increasing the absorption

Fig. 3-10. Distributions corrs sponding to various values of $Z v_{0}^{2} / r_{e}^{2}$, where $\left.v_{c}^{2}=/ v^{2}\right) / 3$. These are of course not steadystate, but are distributions plotted as $Z v_{0}^{2} / v_{e}^{2}$ decreases through specified values while the pissma heats up from a mech lower temperature. $Z v_{0}^{2} / v_{s}^{2} \gg 1$ corresponds to the selfsimilar solution, and $Z v_{G}^{2} \cdot r_{e}$; $\$$ ! is Maxwellian.

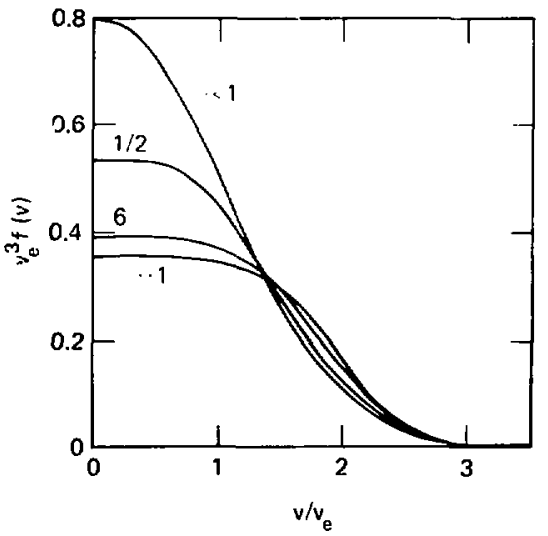

Fig. 3-11. Comparison of reduction in opacity by the nonlinear mechanisms of (a) this article, and (b) of Refs. 16-19. The absciss of (b) is mormalized to correspond to ( $(n)$ for $Z$ $=1$ and eyual light intensity in circular polarization. If $Z>$ 1 , (b) would remain closer to 1 . Thus, for any $Z$ and $v_{u}^{2} / v_{e}^{2} \leq$ I, the mechanism of this article dominates.

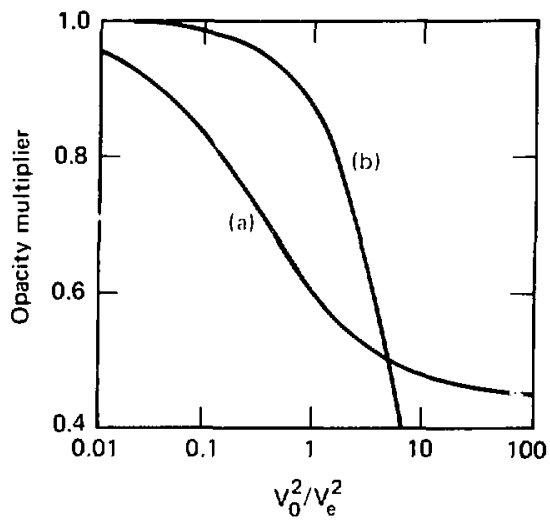

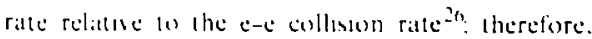
non-Vlaswellat" distributums again result. atthough whth a dolferemt dependence than discussed here. ! inall!. we note that the nonlinear results in Refis. 1610 19 appear 10 be mapplacable to amy in-

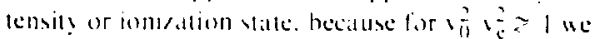
lind a much larger effect than thes do. white for $\frac{2}{1} \frac{2}{1}=1$ the encillat ang electron distribution is not en isotropes. much las Vitucllian as the! assume. I correct treatment of the latter situation remains 10 be donce.

Author: A. B. Langdon

\section{References}

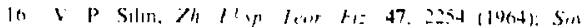

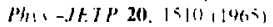

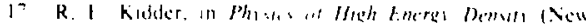

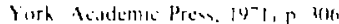

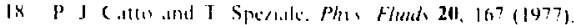

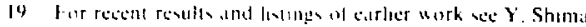
and H. Yaltom. $P / m, R_{a}$ \& 12. 2lote (1975): H. Brusk. J Pha 4 8. 1260(1975): I. Sihlewsinger and J. Wright. Phrs $R_{0,1}+20,19.34(1474)$

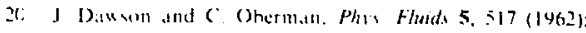

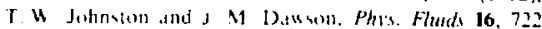
(1973) 
2l S l. Bragensku, in Reviews of Plasma Phrsics. M. A. I.rontowh. Ed. (Neu York: Consultints Bureilu, 1265). al I. p 205

$\because \quad$ I P Shkatrofik!. T. W. Johnston, and M. P. Bachynski. the Partic le Kmethe of Plasmas (Reiding. Mass: Addison.

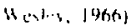

If I) Shat el al, Phat Fluids 21, 1634 (1978).

$\because$ It I Irutonkox. In Revews of Plosma Phisics, p. 174

1 killen. I A Mirin. and M. E. Rensenk, in Mrthods $m$

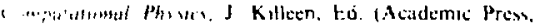

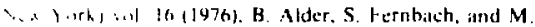

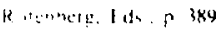

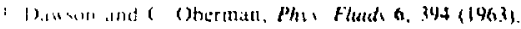

\section{Resonant Absorption}

Betiune the duminant latser-plasma coupling $\because$ H.1511mm are enhanced in the relative'y thin "is d-dellill layer, it is essential to devel ip a self. m14:trl description of the dynamies thes. Here se has that wen weak resonant absorption ${ }^{27}$ acts iongly upon the hydrodynamic evolution of this rgun. W w whance resonant absorption and wipprew wher mechanisms. The coronal rarefacs., Luhbul a sep in density including critical and firl plasmat helow the suberitical density at which the Hou emerees from the ste,

Driven resonant electron oscillations transfer .hworbed energy to the plasma via wavebreaking. ciecting particles toward low density. ${ }^{27}$ These hot collisimnies electrons are confined by the elecirostatic potential, and subsequently pose two challenges to laser (inertial confinement) fusion:

- To prevent them from preheating the larget.

- To use their associated energy to drive the Implosion.

Recent theoretical work ${ }^{2 \times .29}$ describes the scaling of hot-electron temperature in the high-intensity regime: briefly. the laser beam or driven wave steepens the density profile from estimates based on the usual coronal rarefaction and thereby slows the increase of the hot-electron temperature with increasing laser intensity. Here we present new and improved scating laws for the temperature of hot electrons produced by resonant absorption in the modest-intensity regime: we define the modestintensity regime as that in which the driven wave pressure dominates the laser radiation pressure. We can thus justifiably neglect electromagnetism, as the laser's primary contribution to the system is energy, while in the high-intensity regime it contributes both energy and momentum.

We have derived our results from a study performed with the one-dimensional electrostatic particle simulation code ESI. ${ }^{30}$ with which we investigated the scaling of energy, momentum. and charge balance of resonant electron oscillations in driven expanding plasmas. Our simulatıon code evolves the dynamics of an initially uniform slab of electrons and ions which expand into the vacuum under the influence of a high-frequency pump: the pump strength is independent of position in the critical region. This is the familiar infinitewavelength or capacitor model. ${ }^{27}$ The pump frequency is chosen to be well below the plasma frequency of the initial slab. By mapping the hotelectron heating rate onto absorbed laser intensity we can cast our results into a form useful for laboratory application and comparison to electromagnetic simblations.

Analysis. The critical-density region and driven resonant wave are illustrated in Fig. 3-12. Momentum balance on the underdense side of critical is established by the wave pressure and the reaction pressure of electrons ejected toward the vacuum. In other words, the saturated energy density of the wave is just that which balances the momentum flux of the ejected hot electrons. such that $E^{2} 8 \pi$ $(\pi / 2)^{1}=\pi / v_{11}$. Here $f$ is the absorption fraction of laser intensity $l$ into hot electrons of velocity $v_{H}$ $\left(T_{H} / \mathrm{m}\right)^{\prime} ?$, and the factor $(\pi \cdot 2)^{\prime} 2$ results from $\mathrm{cm}$ ploying a half-Maxwellian distribution in computing the hot-electron momentum flux in terms of its energy flux. The momentum flux of the hot electrons exceeds that of the laser radiation whenever $(\pi / 2)^{1}{ }^{2} \mathrm{fl} / \mathrm{v}_{11}>(2-\cap \mathrm{I} / \mathrm{C}$. or

$$
\left(\mathrm{T}_{11}^{\prime} \text { modest }<\left(511 \frac{\pi}{2}\right) \frac{\mathrm{r}^{2}}{(2-r)^{2}}(\mathrm{kev})\right. \text {. }
$$

Experience indicates that 0.3 is a useful estimate for f: Eq. (28) then becomes $T_{H}<25 \mathrm{keV}$. Evidently the modest-intensity regime in the laboratory ${ }^{31}$ is $\left.\mathrm{II}\left(\mathrm{W} / \mathrm{cm}^{2}\right) \lambda^{2}(\mu)\right|_{\text {modest }}<10^{16}$.

Momentum balance on the overdense side of critical is established by the background coldelectron pressure and the wave pressure. Thus $\left.-\nabla\left(n T_{C}\right) \sim-r\left(E^{2} / 8 \pi\right) \sim-\nabla\left[(\pi / 2)^{1.2} \mathrm{f}\right] / v_{H}\right]$, so that by crude integration we can estimate the upper density associated with resonant absorption, $n_{\mu}^{*}$, to be 


$$
\frac{n_{u}^{*}}{n_{c}} \sim 1+\left(\frac{\pi}{2}\right)^{1 / 2} \frac{\mathrm{Cl}}{n_{G} \Gamma^{2} C^{2} H}
$$

For the experimental parameters of Ref. 32 this steepening is more than three times that associals with the radiation pressure.

In general the radiation pressure contribution should be included in estimating the upper density, An upper densit! grtalter than that given in Eq. (29). however. should not be expected to change the d! namics of the driven resonant wale qualitalicals from w hat is described here. The longer seite length $\left(\sim c e_{p}\right)$ over which the radiation pressure acts ugevests a doubly stepped profile in which the resonant wave dominales locally but for which the max. imum densit! in given b! radiation pressure. In this situation war results maly be extended to the highintensily rę̧ ime.

Driven ststems are chatracterised by the sungle dinensionless parameter, bo ve. This is the rallus of the jitter selocity of an electron sxcillating irecls in the pump lietd, $v_{0}=\mathrm{eE}_{0} \mathrm{~m}_{\mathrm{i}}$ ), and the inilial (colds) electron thermal velocily, $v_{c}=\left(T_{c} m\right)^{l}=$ The spate and time scalles of the rarefaction and driecn resonant wave arte determined selloconsistently

Our principal results are the arration with $b_{0}$ 's of the hot-electron temperature, the heating ratte. and the densities delimiting the step. In general the stepped densit! profile dominates our resuls, and $w$ hile a description of the system in terms of a single seate lenglh is not a fatlure lor cerlan purposes, It is not appropriale. Feven for bo 'c less than im $M_{1}^{1}=$ - 130 we find that "ascebreaking governs the system: convection, soliton formation. etc. have never been seen.

In Fig. 3-13, the nurmalised hol-electron temperature. $T_{1} T_{C}$, and the normatised hot-electron heating rate. $Q_{1} n_{6} T_{C}(c$, are plotted against of ' $t$. vote the break in the dita alt $s_{0} v_{c}-1$. Coldplasma modeling is appropriate for $v_{0} v_{C}>1$. While $v_{0} v_{c}<1$ is the warm-plasma regime.

To identify the hot-electron heating ratte with ahsorbed laser intensity, we begin with $Q_{H} n_{c} T_{C^{\prime}} \mathrm{C}$ and $T_{H} T_{C}$ as functions of vo $v_{C}:$ we identif! $Q_{H}=$ fI. and eliminate ${ }^{\circ}{ }^{\prime}{ }^{C}$ to obtain the data displayed in Fig. 3-14. Note the role played by the background temperature as $T_{H} T_{C}$ varios against $\mathrm{n}\left(\mathrm{W} \cdot \mathrm{cm}^{2}\right) \lambda^{2}(\mu) \mathrm{T}_{C}^{3}{ }^{2}(\mathrm{eV})$. The break in the scaling is clearly evident. In the warm-plasma limit, $\mathrm{Cid}^{2} \mathrm{~T}_{\mathrm{C}}^{3} \geq 10^{11}$. curves 1 and 2 have slopes of 25
Fig. 3-12. In the critical-density region the driven resonant wave supports itself against the background plosma pressure on the overdense side by the reaction pressure associated with the momentun! flux of hut electrons ejected toward the underdense side. The curves are simulation data for $v_{0} / v_{c}=1$ at $t$ $=1200 / w_{0} ; w_{0}=\therefore p_{\text {ondx }} / 5$.
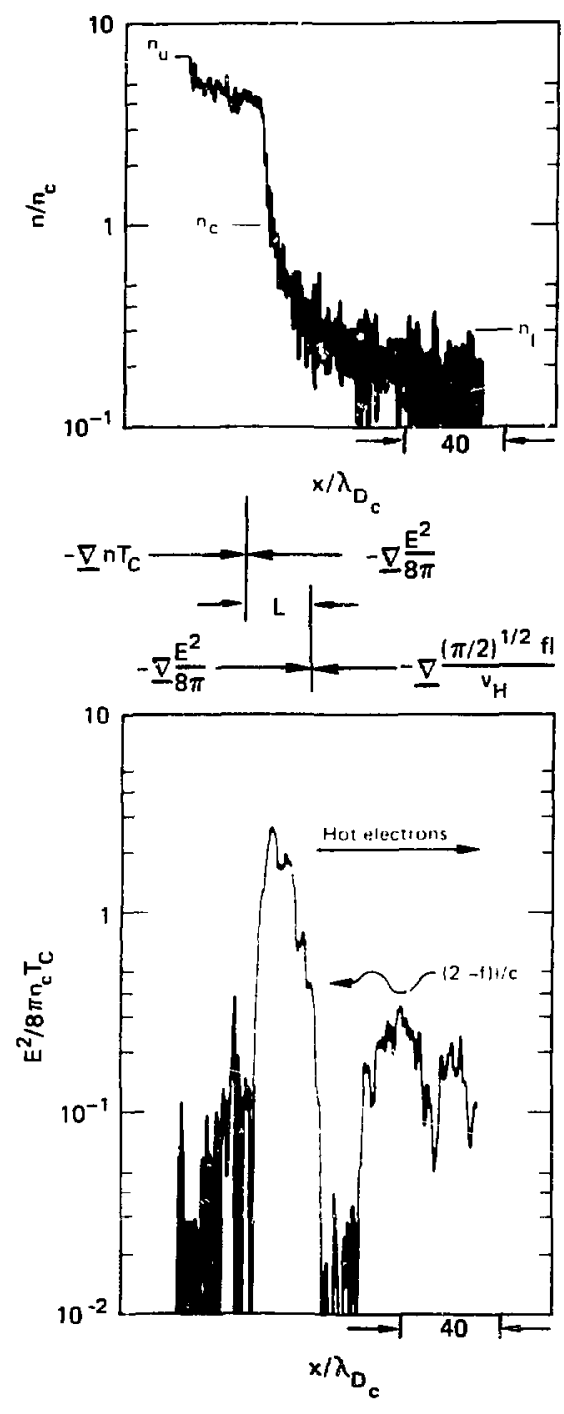
Fig. 3-13. The hot-lectron temperature, $T_{H}$, and the hotclectron heating rate, $Q_{H}$, increase with insreasing pump strength. $v_{0}$. Note the break in the scaling at $v_{0} / v_{c} \approx 1$ which separates the regimes of warm-plasma dynamies, $v_{0} / v_{c}<1$, and cold-plasma dynamics, $v_{0} / v_{c}>1$. The points plosted at $v_{0} / v_{c}=I$ are runs of the same physical system with different pump frequencies. The difference in results there represents the uncertainty of our results throughout.

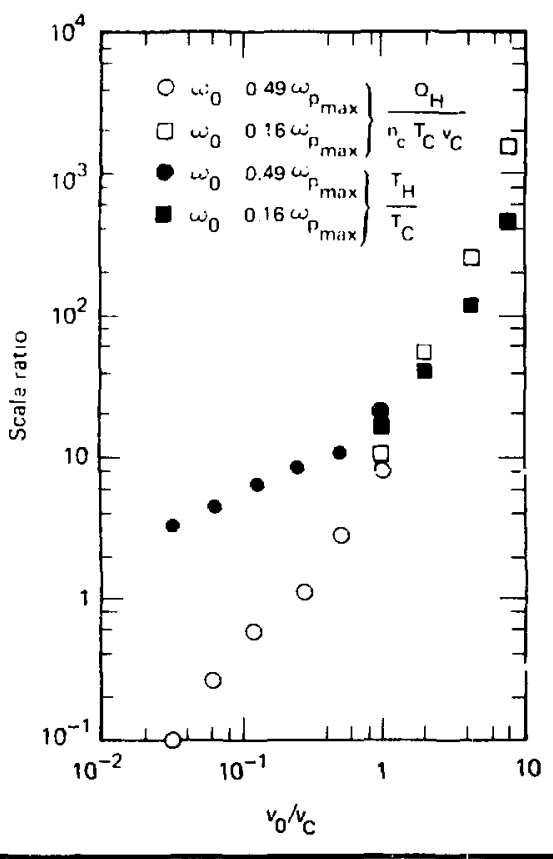

and 13 respectively. Curve $\mathrm{i}$ yields the prelerred

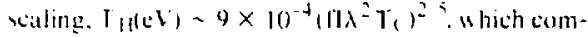
pares brorably with that given in Ref. 29: still within confidence limils. curve 2 yield $\mathrm{TH}_{\mathrm{H}}-4 \mathrm{x}$ $\left.10^{3}, 1 \lambda^{2}\right)^{1} 3 T_{1}{ }^{2}$ ? which compares lavorably with the scaling given in Ref. 28 . Note that the scaling with cold hackground temperature is as strong ats that with intensity. The cold-plasma limit given in Ref. 33 is recovered for $10 \lambda^{2} \mathrm{~T}_{i}{ }^{2}>10^{11}$. where $\mathrm{T}_{\mathrm{H}}$ $\sim 9 \times 10^{-7}\left(11 \lambda^{2}\right)^{2}$ ? independent of $T_{C}$.

Comparison of our results with the data of Giovanelli ${ }^{31}$ suggests that the interaction in the laboratory is governed by cold-plasma dynamics for
$1 x^{2}<10^{15}$ where the 2,3 power obtains, and by "arm-plasmal dymanics for $1 \lambda^{2}>10^{15}$ where the 2.5 or 1.3 power obtains.

The 2,3-power scaling of hot-electron tenperalture "Its suggested previously by flux-linit and stochatis-beating estimates. ${ }^{34}$ We have confirmed the 2 3-power sciling here viat cold-plasma estimales for resonant absorption:

- Wavebreaking accurs when electrons oscillate in the walce with a velocity, $v_{c}$ - eE; mu. which seales with the effective phase velocily of the wave so $v_{p} \sim 1 .(2 \pi w)$. The hot-clectron temperalure seales with the energy an elecoron san gain in riding the wave across the resonant region, $\mathrm{T}_{11} \propto$ ct:I.

- Combining these ue find $E^{2} \cdot 8 \pi \sim n_{6} T_{11}$

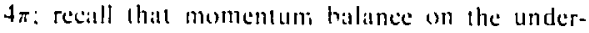
dense side of the wave requires $\mathrm{H}^{2} 8 \pi-(\pi) ! 1=$ n il

- Combining agilin yelds $\left.T_{11} \propto(1 \mathrm{l})^{2}\right)^{2} ?$ exatclly the disired result.

Find ly lig. 3-15 shows the relative upper and lower densities hounding the step al critical densils. $n_{4} n_{6}$ and $n_{1} n_{c}$ respectively as functions of

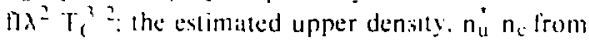
F. decreases from some 25 locial (cold) Debye lengths at $f\left(\lambda^{2} T_{1}^{3}=-1\right)^{3 /}$ to about $I$ as $f T \lambda^{2} T_{i}^{3}=$ exceeds $10^{11}$. Typical values of the lower densily are seen to he less than hall crilical. The agreement of we estimated upper densily is yuile good. except for the strongest pumps: neglect of the lhow contribution to momentum talance in the step region ${ }^{27}$ may be the mijor source of error.

Summary. We have shown that the dependence of hot-electron temperature upon background cold. electron temperature is as strong as the dependence upon laser intensity. We have also shown that the self-consistent coronal rarefaction, including resonant absorption. exhibits a stepped density profile. We have demonstrated an equilibrium in which the driven resonant wave supports itself against the background plasma pressure by the momentum flux of ejected hot electrons. This demonstration pruvides a first-principles model of profile modification in the modest-intensity regime, as the local reaction of the flow to the momentum deposited at the critical density surface by hot-electron production. The upper density of the stepped density profile may be much greater than critical density. 
Fig. 3-14. Identi combined to obta with slope $2 / 5, c$
. . a heating by trot electron production, $Q_{H}$, with absorhed laser intensity, $n$ *ie two curves in Fig. R-1 13 are itng of hot-electron temperature with the laboratory observables $f, I\left(W / \mathrm{cm}^{2}\right), \lambda^{2}(\mu)$, and $T_{C}$ ( $(\mathrm{V})$. Curve $I$ with slope $1 / 3$, and curve 3 with slope $2 / 3$ are discussed in the text.

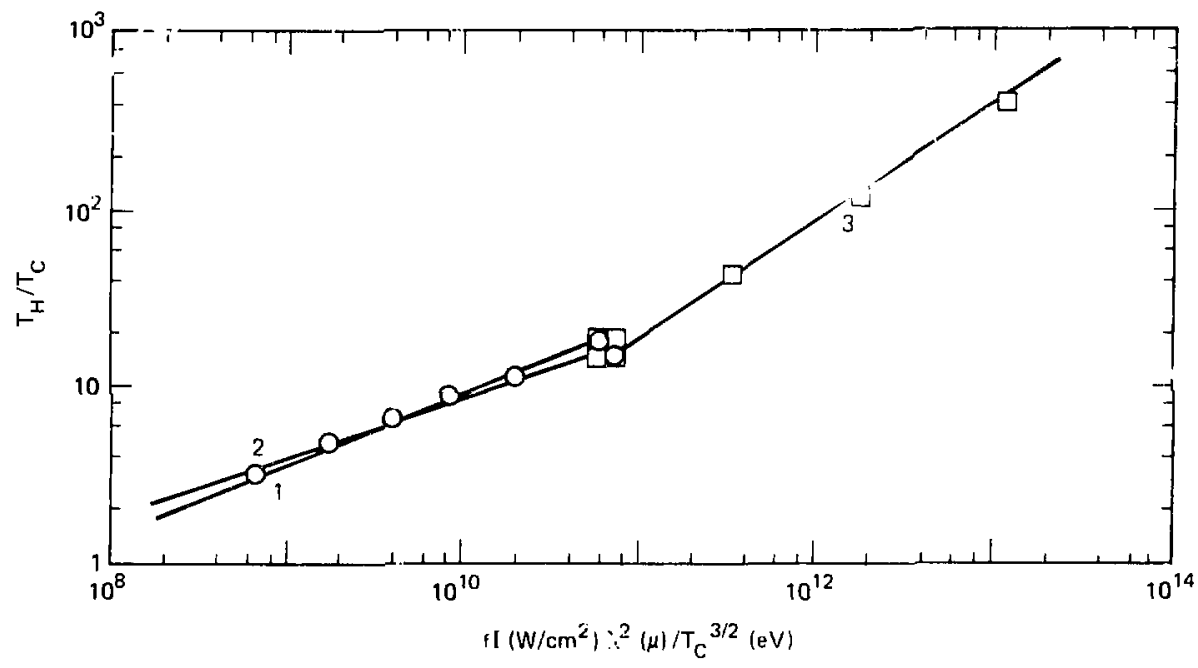

Fig. 3-15. The upper, $n_{u}$, and lower, $n_{1}$, densitics delimiting the step st critucal density, $n_{c}$, increasc and decrense respectively svith increasing pump strength. The estimated upper density, $m_{u}^{*}$ of Eq. $(29)$, is in apreement with ohservations except in the most strongly driven systems.

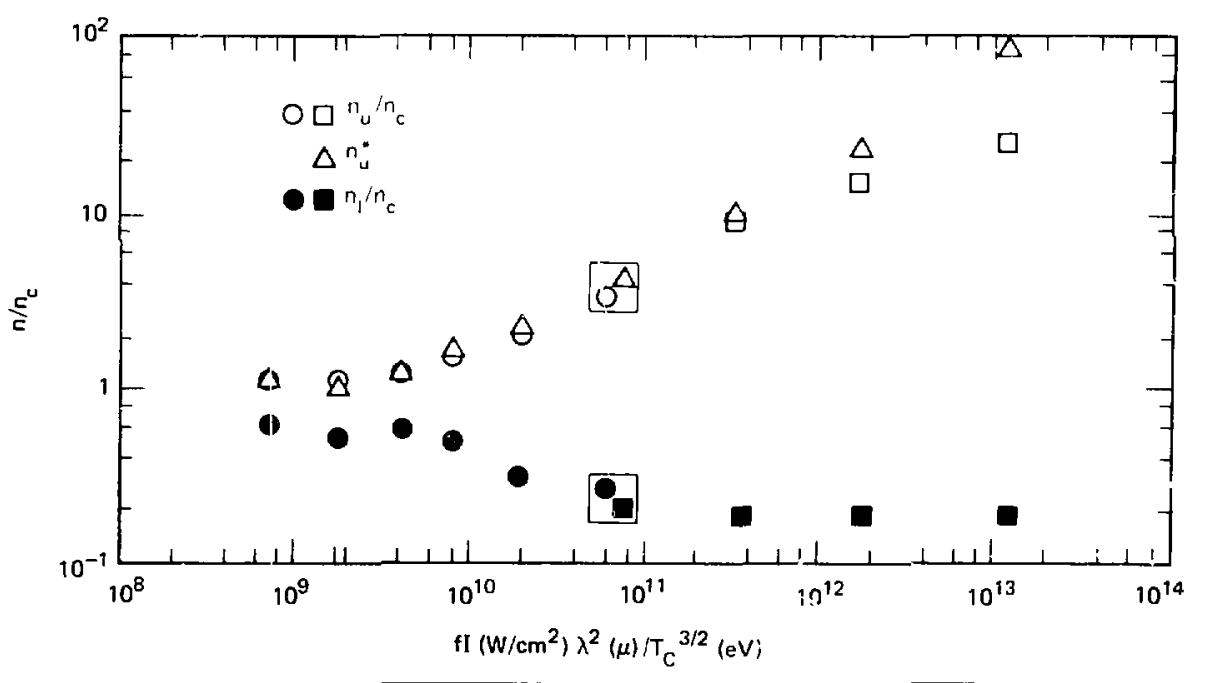


and much greater than that associated with radiatiisn pressure steepening; experimentai observations of such structures have been reported recently. ${ }^{32}$ The associated lower density and the absence of a :urmang region with $L / \lambda_{0}>1$ act to reduce the effects of parametric instabilities, classical collisions. and entunced turbulence. The jump in electrostatic rotential atssociated with the density jump contrinules to the formation of a hot corona.

\section{Buthora: J. R. Albritton and A. B. Langdon}

\section{Rererences}

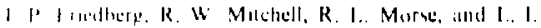

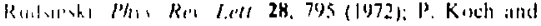

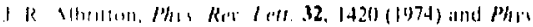

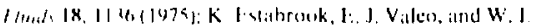

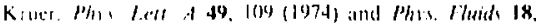
11 111975

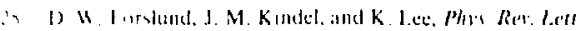
39. $2 x+1(1977)$.

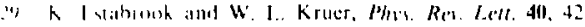
(19?

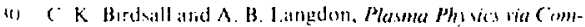
pader Sumdation (Universtly of California Press. Berkeles irrid bon Angeles, 1975).

'I R. I edenceps, M. D. J. Burpess, (j. D). Enright, and M. C.

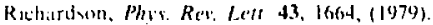

32. D. V. Cistranclli, LA-UR 76-2242 (1976): K. R. Manen et il., J. Opt. Sac Am., 67, 717 (1977).

33. J. R. Albriaton, E. I. Thorsos, and I. A. Williams, t.shoralusy for L.aser Finergeties, Rochester, N.Y.. Li.F: Rept. No. 85 $\{197 x)$.

34. R. L, Murse and C: W. Nielsom, Phyx. Fhods 16, 9(19) (197.3).

35 . D. W. liomlund, J. M. Kindel. K. I.ce, and I.. 1.. l.indman, Phas. Rer Le'dl. 36. 35 (1976).

\section{Heating by the Raman Instability}

Stimulated Raman scattering is the parametric decay of an incident photon into a scattered photon plus a longtitudinal electron-plasma wave (epw). The net gain scales with $I\left(\lambda_{0} / 1.06 \mu \mathrm{m}\right)^{2} l / \lambda_{0}$, where I is the laser intensity $\left(W / \mathrm{cm}^{2}\right) . r$ is the length of the underdense plasma in the decay region, ano $\lambda_{0}$ is the laser wavelength: thus the process can be quite significant for the long scale lengths expected in reactor targets. The frequency matching conditions are $\omega_{0} \rightarrow \omega_{\mathrm{r}}+\omega_{\mathrm{e}}$, where $\omega_{0}$ and $\omega_{\mathrm{r}}$ are the angular frequencies of the incide.tt and reflected light waves, respectively, and $\omega_{e}$ is the frequency of the electronplasma wave. As can be seen from the frequency matching condition, this process can occur for densities $\leqslant 0.25 n_{c}$ where $n_{c}$ is critical density.

Raman scattering is a resonant, absolute instability for $n \approx 0.25 n_{\mathrm{c}}$, and occurs most efficiently at that density (with reflection and absorption up to $50 \%$ ). As the density decreases, the epw wavelength $\lambda_{e}$ becomes shorter with respect to the laser wavelength and in particular with respect to the Debye length $\lambda_{D}:=\left[\mathrm{T}_{\mathrm{c}} /\left(4 \pi \mathrm{n}_{\mathrm{e}}^{2}\right)\right]^{1 / 2}$. The electron landau damping becomes considerable for $\lambda_{e} ₹$ $10 \lambda_{I}$, which occurs for high electron temperatures and low densities $\mid n_{\mathrm{c}} / n T_{\mathrm{c}}(\mathrm{keV}) \widetilde{>} 30$, where $T_{\mathrm{c}}$ is the electron temperature]. Here the instahility is a variation of Raman scattering called stimulated Compton scattering. There i's also an intermediate regime for densities between about 0.1 and 0.25 (depending on $T_{1}$ ) for which the instability is convective. moderaluly damped, and can still absorb and seatter a significant amouni of light.

Since $\omega_{\mathrm{c}}$ is much greater than the frequency of an ion sound wave, the Raman instability absorbs much more than Brillouin scattering, but that absorption is typically into fast electrons which can preheat a pellet core.

Theoretical Estimates of Raman Scattering. Kinetic simulations show a rapid evolution of the electron distribution function into the nonlinear state in which the electron distribution is composed of a cold thermal Maxwellian plus a self-consistent tail of heated electrons ${ }^{36-38}$, which in turn damps the plasma wave. The characteristic temperature $T_{\text {Ilot }}$ of this tail is found from the simulations to be about $\left(\mathrm{m}_{\mathrm{c}} / 2\right) \mathrm{v}_{\mathrm{p}}^{2}$, where $\mathrm{v}_{\mathrm{p}}$ is the phase velocity of the epw. The electron density of the tail is determined by balancing the energy absorbed by eiectrons via the damped plasma wave with the heat flux carried off by the electron tail. The epw damping is then estimated from the rate of Landau damping by the heated electrons. This simple model is completed by computing the reflection from the damped olasma wave. The analysis is just like that for the reflection due to a damped ion wave, ${ }^{39}$ and results in an analogous expression for the reflectivity:

$$
\Lambda(t-\lambda)=B\{\exp \{x(1-\Lambda)\}-\Lambda\},
$$

where

$$
\Lambda=\frac{\omega_{0}}{\omega_{\mathbf{T}}} \mathbf{r}
$$


Fig. 3-16. Reflecticn duc to Ramen instability as a function (a) of the density and (b) background temperature. The solid lines are the theoretical estimnte for noise level $B=3 \times 10^{-4}$ and the circles are the simulation results for $I\left(\lambda_{0} / 1.06 \mu \mathrm{m}\right)^{2}$ $=2.5 \times 10^{15}, M_{1} / m_{e}=100, \bar{z} T_{e} / T_{i}=5, c / \lambda_{0}=127$.

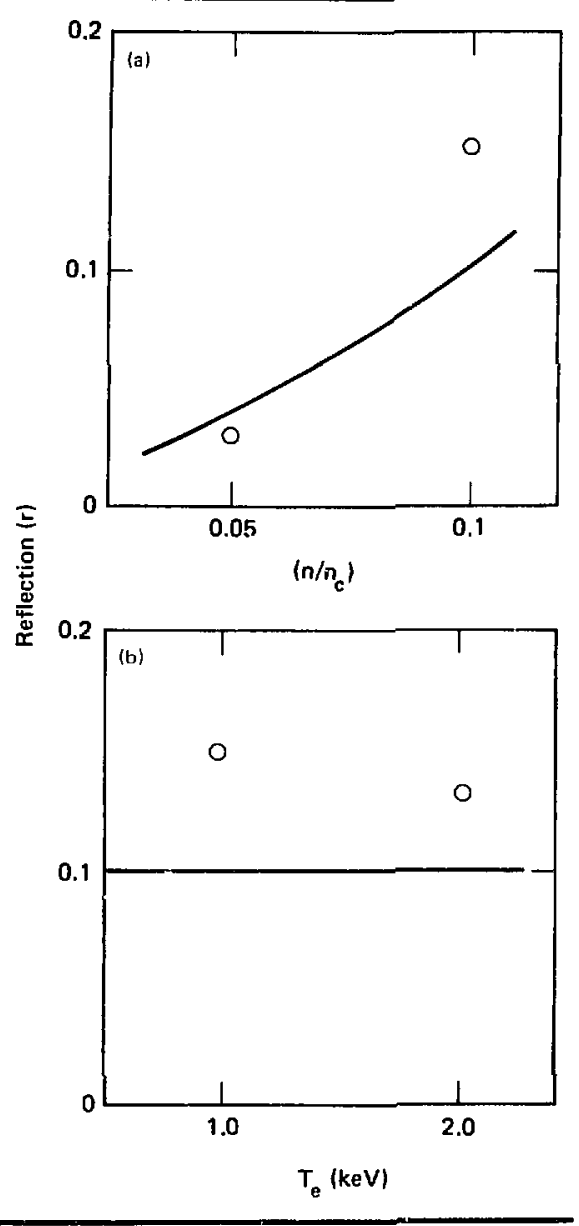

and

$$
x=\frac{k_{p}^{2} \mathrm{p}_{\mathrm{r}}}{8 \mathrm{k}_{\mathrm{r}}}\left(\frac{\mathrm{osc}_{\mathrm{c}}}{{ }^{2}}\right)^{2}\left(\frac{\omega_{\mathrm{pc}}}{\gamma}\right) .
$$

Here $r$ is the fraction of light reflected, $k_{p}$ and $k_{r}$ are the wave numbers of the plasma wave and reflected light wave, respectively, $\gamma$ is the Landau damping rate, $c$ is the velocity of light, $v_{o s c}$ is the oscillation velocity of an electron in the electric field of the incident light wave $\alpha \lambda_{0} \sqrt{1}$. and $B$ is the initial noise level of the reflected transverse wave.

Our theoretical estimates of the Raman reflectivity are compared with sample simulation results in Fig. 3-16. In these simulations a particle code was used to propagate the laser light through a uniform region of low-density plasma. Note that the variation with background density and temperalure of the computed Raman reflectivity of order $10 \%$ is in reasonable agreement with the simple model. Note also that rather large regions of plasma are needed to give a significant refiectivity, even neglecting the effect of gradients which are especially significant for Raman scattering. Hence it is not surprising the Raman scatlering has only recently been observed in our experiments.

Hot Electrons. Figure 3-17 shows the hotelectron distribution observed in a sample computer simulation. Note the high-energv electron tail which is generated by the electron-piasma wave as it damps. This tail is roughly Maxweltian in shape and has a characteristic temperature of $1: \mathrm{keV}$. As a first approximation. this temperature is independent of intensity. and depends mainly on the den.ity and background temperature which determine the phase velocity of the Raman-generated plasma wave. This hot temperature is approximalely

Fig. 3-17. Particle number vs energy, showing the Maxwellian nature of the Kaman-heated elactrons.

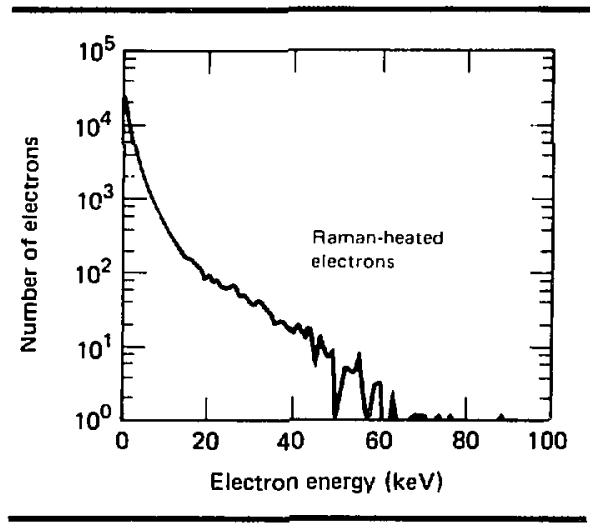


Fig. 3-18. Raman-heated electron temperature, and scattered light frequency vs plasma density. The boxes are simulation resuIts and the fraction of the light absorbed is next to the box.

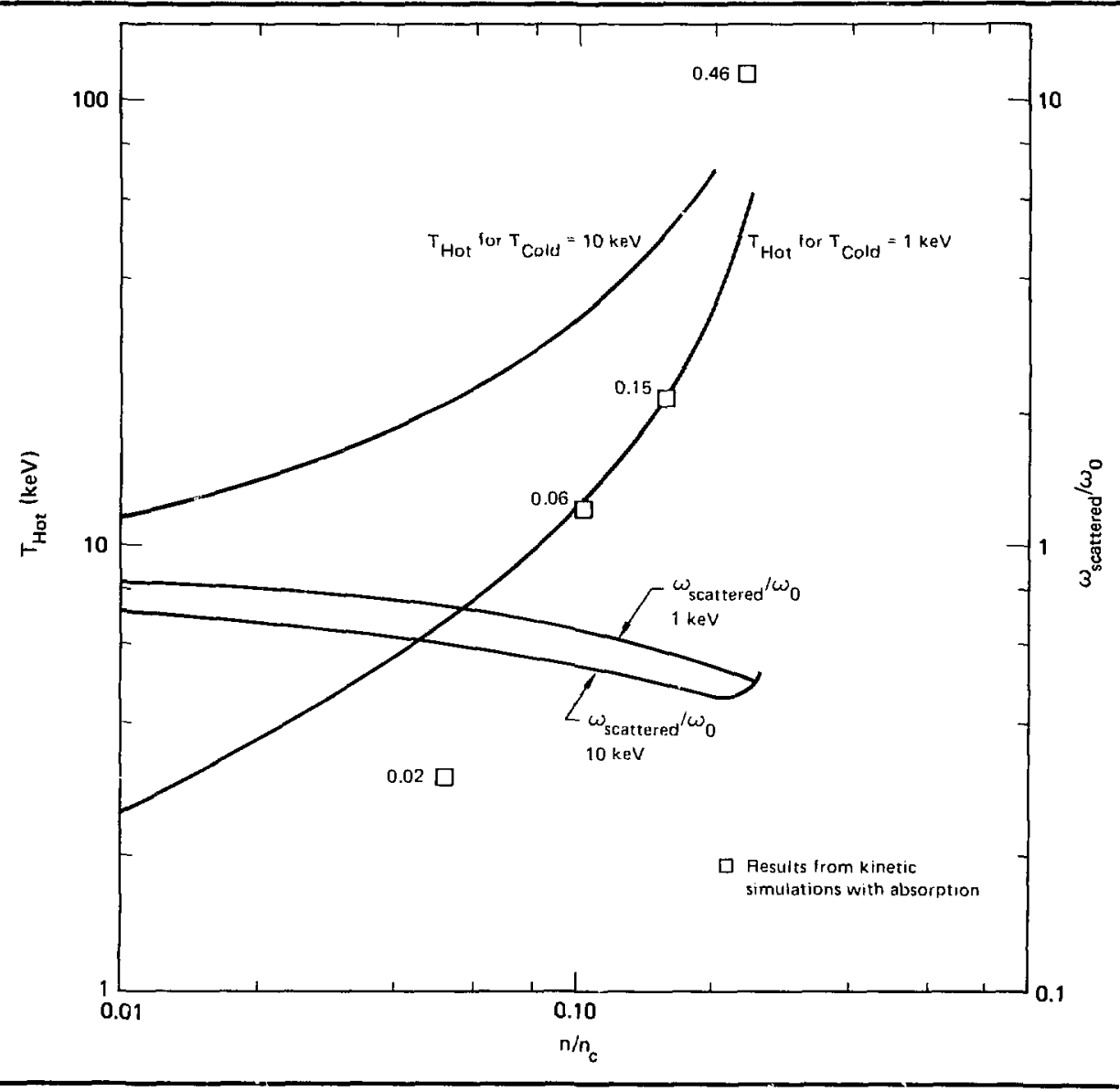

$\left(m_{\mathrm{e}} / 2\right) v_{\mathrm{p}}^{2}$. Figure $3-18$ plots the hot-electron temperature vs background density for two background temperatures: the theory is described in Ref. 37.

The density gradient threshold of Ranan scattering is determined by balancing the growth rate and the convective loss of the electron-plasma wave with the scattered electromagnetic wave across a mismatch length. ${ }^{40}$ The threshold is given at $0.25 \mathrm{n}_{\mathrm{c}}$ by $1\left(\lambda_{0} / 1.06 \mu \mathrm{m}\right)^{2} / 10^{16}>35\left(\lambda_{0} / L\right)^{4 / 3} \alpha \lambda_{0}^{0.7}$, where
$L=(1 / n d n / d x)^{-1}$. Raman is in natural competition with inverse bremsstrahlung and it is interesting to calculate the inverse bremsstrahlung absorption before the light reaches $0.25 \mathrm{n}_{\mathrm{c}}$. In a iinear density gradient, the e-folding absorption length from $n / n_{\mathfrak{c}}=0$ to $1 / 4$ is $\sim 1.5 \times 10^{4} \lambda^{2} \mu^{2} \mathrm{~T}_{\mathrm{e}}^{3 / 2}(\mathrm{keV}) / Z$ where $\lambda_{0 \mu}$ is the vacuum wavelength in micrometers. In the free-streaming flux limit. $T_{e}(\mathrm{keV})=\left(1 \lambda_{0}^{2} \mu / 5\right.$ $\left.\times 10^{13}\right)^{2 / 3}:$ this is faster than the $\lambda_{0 \mu}^{0 .{ }^{2}-0.7}$ scaling from LASNEX (see "High- $Z$ Disk Modeling" earlier in this section). We can now put the absorption length into the density gradient threshold with 
the temperature scaling to estimate the intensity for which Raman marginally occurs: $1>7 \times 10^{12}$ $\bar{Z}^{4 / 7} / \lambda_{0 \mu}^{18 ;} ;$ for $\bar{Z}=40$ and $\lambda_{0 \mu}=1$, the intensity is $5 \times 10^{13}$

Raman absorption decreases with a large bandwidth, as shown in Fig. 3-19. The bandwidth was chosen to be the same as is projected for an experiment on Cycleps: seven lines of equal intensity separuted by $6 \%$, for a total width of $36 \%$. When the growth rate is less than the line separation. we expect each line to behave independently. The linear growth rate at $0.25 \mathrm{n}_{\mathrm{c}}$ was $0.06 \omega_{0}$ which is about equal to the line width for the higher intensity shown. At early times the bandwidth does not help. but later, when the heating provides some damping. there is a decrease in Raman absorption. loor lower intensity. where the line width is greater than the growth rate. the bandwidth mostly drops the Ramar below threshold. Ranian and Brillouin act to reduce each other somewhat. since Raman heating partially reduces Brillouin and Brillouin reflection partially reduces Raman.

Some processes self-consiste tly decrease the instability. Raman scattering occurs mosi strongly and with the hottest electrons at quarler-critical density. where the $2 \omega_{p e}$ instabitity ${ }^{4 l}$ and Raman sidescattering also occur. $A l l$ three instabilities act to steepen the density profile and hence drive it

Fig. 3-19. Percentage of the light absorbed by Raman vs bandwidth of the laser for several total laser intensities. The simulation plasma had $130 \lambda_{0}$ from densities 0.14 to $0.37 n_{c}$.

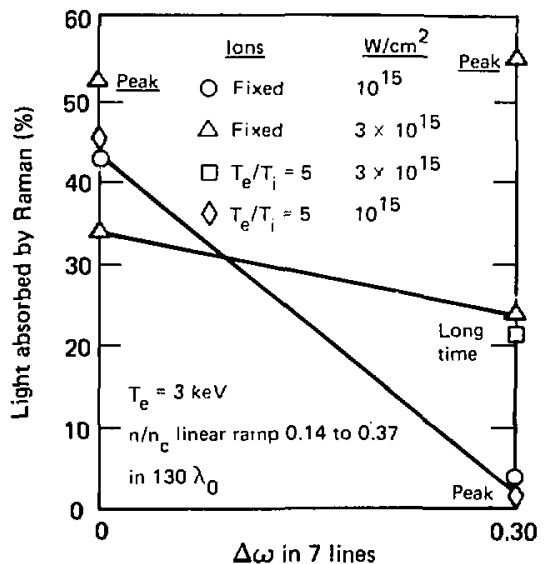

below threshold. The hot electrons generated by all these processes as well as by the other absorption processes also help to damp Raman.

Conclusion. The heated-electron temperature depends primarily on the phase velocity of the electron-plasma wave and is roughly independent of the light intensity. The heated-electron number does depend on the $\mathrm{I}\left(\lambda_{0} / 1.06 \mu \mathrm{m}\right)^{2}$, howcver. For every joule of energy absorbed. 1 to 2 or more joules are scattered (see "Stimulated Raman Scattering Experiments" in Section 6).

The characteristic dimensions of reactor targets are in millimeters, which means that Raman has thousands of light wavelengths in which to grow. lurthermore, a laser hot spot, focus. or filament could raise the light intensity sufficiently to drive Raman scattering vigorously. One problen with a lilament is that if it pushes the density below quarter-critical there will inherently be a region where the density will always be at $0.25 n_{0}:$ this issue is discussed in "Spatial Structure of Filamented light" later in this section.

futhors: K. C. Fstabrook and W. I. Kruer

\section{References}

36. K. (i. I stibrook. 1). W. Phillion. and V. C. Rupert. Li.l. laser fusion lfonthl. I aurence l ivermore laboralors. I ivermore. (allil.. M1: 79-6r (June 1979).

37. W. I Kruer K. (i. Istahrouk, and K. It, Sind. "Inslability- iencraled laser Reflection in Plasmas." Sucl fiveion 13. 952 (1973)

38. W. I. Kruer K. (i. lstubrook, A. H. I.angdon and B. F. I asinski. "Raman Bilckscalter in High Temperature. Inhomogeneous Plasmas." to be puhlished in Phis. Fluids.

39. IJ. M. Pbillion. IH. I. Krues, and I'. (". Rupera, "Hrillouin

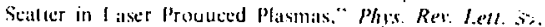
$1529(1977)$

40. N $>$ Rosenhluth. "Parametric Instabilities in Inhomogeneous Media." Phys. Reve Lat 29. 565 (1972).

41. A. B. Ian, don. B. I. lasinski. and W. 1. Kruer, "Vimlinear Salluratien and weurrence of the Two-

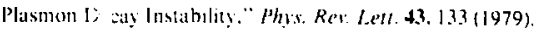

\section{Two-Dimensional Simulations of Quarter-Critical-Density Heating in Large Plasmas}

Witin a view toward planning and interpreting long-pulse-length experiments. we have begun a new ZOHAR ${ }^{42}$ simulation study of the highfrequency parametric instabilities which occur in 
the neighborheod of quarter-critical density. These instabilities, which have been discussed previously ${ }^{73+4}$ in the context of the steep density gradicnts dssociated with short-pulse-length experimients. are

- The $2 w_{p e}$ instability in which the laser light at frequency wo decays into two electron-plasma anven

- The Raman instability in which the decay s.ie are an electron-plasnia wave and an elecintiagnelic walve at $\sim w_{0} / 2$.

In l.1rge regions of relatively flat underdense pis mat, these instabilities may ahsorb a significant moun of the laser light into an unwelcome highenerge clectrun component. The Raman instahility maly also scitter the light at frequency $\sim \omega_{0} / 2$ before 11 can be absorbed at higher densities.

To find upper bounds in this parameter regime. we hegin with a simulation in which the ion motion is fromen. The saturation mechanism which we idenlified in our previous two-dimensional simulaimons. ${ }^{13}$ however, cannot then eccur; that is, we umit the process by which ion fluctuations driven un by the beating of the instability decay waves counle these decay waves into shorter-wavelength plasma waves which are then damped. The results we present here, then. confirm our understanding of the linear theory and provide upper limits or a worst-case scenario on the absorption, the electron distribution. and half-harmonic emission.

We model in two dimensions $(x, y)$ a plasma slab $21 \lambda_{0}$ long in the $x$ (laser) direction, at density $023 n_{\llcorner}: n_{c}$ is the critical density, at which the plasma frequency equals the laser frequency. The oscillatory velocity is $0.03 \mathrm{c}$ and the thermal velocity is $0.077 \mathrm{c}$ (where $\mathrm{c}$ is the speed of !ight), corresponding to $10^{15} \mathrm{~W} / \mathrm{cm}^{2}$ for an Nd:glass laser and a $3-\mathrm{keV}$ background. At this high eleciron temperature, $\mathrm{k}_{0} \lambda_{\mathrm{D}} \sim 0.13$ at $0.25 \mathrm{n}_{\mathrm{c}}\left(\lambda_{D}\right.$ is the electron Debye length) and the decay plasma waves are longwavelength. Mach of our work ${ }^{42.44}$ on the linear theory of the $2 \omega_{n e}$ instability in hot plasmas, when $k_{0} \sim k_{\text {plasma. }}$ is then applicable. The expected modes grow with $k_{y} / k_{0} \sim 0.8$, the most vigorous. The analytically calculated growth rate ${ }^{44}$ for that mode,

$$
\frac{\gamma}{\omega_{0}}=\frac{k_{0} v_{0}}{4}\left(1-3 \sqrt{3} \frac{k_{y} v_{e}^{2}}{v_{0} \omega_{0}}\right)=0.002 .
$$

is in remarkably good agreement with the growth rate in the model as determined from the electrostatic energy.

The absorption fraction is 50 to $60 \%$ into a hotelectron distribution which cuts off at $500 \mathrm{keV}$. This generated distribution is not a simple Maxwellian with a well-defined temperature. It is tempting to characterize it as a sum of Maxwellians with the temperature of each one corresponding to the phase velocity of a plasma decay wave. Such a description, with temperatures ranging from 40 to $130 \mathrm{keV}$ and more, is not unique. An alternative parametrization is as a $\sim 50-\mathrm{keV}$ Maxwellian with a very high-energy tail.

About $3 \%$ of the incident light is re-emitted at frequency $\omega_{0} / 2$. If we assume that half of the $\sim \omega_{0} / 2$ light produced is reabsorbed at its critical surface. then the Raman instability accounts for $\sim 10 \%$ of the absorption at most. Therefore, under the conditions of this simulation, the $2 \omega_{\mathrm{pc}}$ instability is the dominant absorption mechanism.

We stress again that the ion fluctuations and their associated saturation mechanism are not included in these results. Note also that the angular spectrum of decay waves is too limited in onedimensional simulations of the Raman instability to drive up ion fluctuations. In applying our results to experimental conditions, we then expect less absorption in a given length into a softer electron distribution than is reported here. More definitive answers await the two-dimensional mobile-ion simulations which are next on our agenda

In applying our simulation results to experiments in which high Z-disks are irradiated by moderate-intensity laser light, collisional damping of Raman and $2 \omega_{p e}$ instability must be taken into account. We find that

$$
\gamma_{\max } \gamma_{e \mathrm{i}}=\frac{14 \sqrt{1_{14}} \lambda_{\mu}^{2} \mathrm{~T}_{\mathrm{e}}^{3 / 2}}{Z}
$$

at $0.25 \mathrm{n}_{\mathrm{c}}$. Here, $\gamma_{\max }=\mathrm{k}_{0} v_{0} / 4, \mathrm{I}_{14}$ is the intensity in units of $10^{14} \mathrm{~W} / \mathrm{cm}^{2}, \lambda_{\mu}$ is the laser wavelength in micrometers, and $\mathrm{T}_{\mathrm{e}}$ is the background e!cctron temperature in $\mathrm{keV}$. The growth rate found in the simulation described above for $10^{15} \mathrm{~W} / \mathrm{cm}^{2}$ and $3 \mathrm{keV}$ was one-third the maximum, and if we assume that $Z=50$, then

$$
\gamma \tau_{\mathbf{e j}} \sim 1.7
$$


In this parameter regime, collisional damping would therefore play a central role.

Inverse bremsstrahlung is the dominant absorption mechanism in this parameter regime, and we need to check whether the laser intensity left at quarter-critical satisfies the $2 \omega_{p e}$ threshold condition. To make a preliminary estimate, assume a linear density gradient and note that the threshold condition in Ref. 44 may be expressed as

$$
\mathrm{I}_{1 / 4}>\frac{\mathrm{Te}}{\lambda_{\mu} \cdot \mathrm{L}_{\mu}} 1.77 \times 10^{16} \mathrm{~W} / \mathrm{cm}^{2}
$$

where $l_{1}+$ is the intensity at $0.25 \mathrm{n}_{\mathrm{g}}$. For short scale lengths, as in exploding-pusher experineats. there is litte absorption but the intensity must be high enough to sitisfy the gradient threshold. On the other hand. for long density gradients, there may be too nuch absorption below quarter-eritical density for this inequality to hold. Thus. there is some minimun intensity as a function of scale length below which $2 \omega_{\text {pe }}$ will nol occur. For lixed hackground temperature. this minimun incident intensity occurs when the gradient scale length is approximately equal to an absorption length and is $\sim 5$ $\times 10^{13} \mathrm{w} / \mathrm{cm}^{2} / \lambda_{\mu}^{3}$. Therefore prior absorption by inverse hrensstrahlung may reduce the potency of the high-frequency instabilities at quarter-eritical density.

\section{Authors: B. F. l.asinski and A. B. Langdon}

\section{References}

42. A. B. l.angdon and B. F. L.asinkki. "I:kelromagnetic and Relativistic Plasma Simulation Models." in Methods in Compuational Phrsies. I. Killeen. Fod. (Academic l'ress. Nex York vol. 16 (1976), B. Nlder, S. Iermbach. and $M$. Rotunberg. 1:ds.. p. 327

43. A. B. Langdon. B. F. Lasinski, and W. B. Kruer. "Nonlinear Saturation and Recurrence of the TwoPlusmon Decay Instubility." Phis. Rev Lest 43. 133 (1979).

44. Laser Program Anmal Report-1477, Law rence l.ivermore l.aboratory. Livermore, (alif., LCRI.-5002)-77 (1978). p. 4.49 .

\section{The Effect of Reheating on Hot-Electron Temperature}

Theory and simulations of resonant absorption have been described in the past few annual reports and in the literature ${ }^{45-47}$ Briefly, resonant absorption is the direct conversion of the transverse laser light to longitudinal electron-plasma waves (epw) at the critical density $\left[10^{21}\left(1.06 \mu \mathrm{m} / \lambda_{0}\right)^{2} \mathrm{~cm}^{-3}\right]$. The oscillating longitudinal electric field of the epw heats the electrons by accelerating them down the density gradient to a temperature of approximately $\left.21 \mathrm{~T}_{\mathrm{E}}^{0.25} \| \mathrm{I}\left(\mathrm{W} / \mathrm{cm}^{2}\right) / 10^{1 \mathrm{f}}\right]\left(\lambda_{0} / 1.06 \mu \mathrm{m}^{2}\right\}$ (Ref. 48). This article extends the previous work by studying the effects of magnetic fields and collisions (albedo) which return the healled electrons for further heating. We find that a magnetic field increases their temperature and collisions do not (see also "Resonant Absorption" earlier in this section).

We studied the nonlinear processes of resonant absorption with ZOHAR, our two-dimensional relativistic, electromagnetic, kinetic simulation code. ${ }^{+5.46 .45}$ We examined processes for forming macroscopic magnetic fields, ${ }^{+9}$ modeling macroscopic. slowly (time) varying magnetic fields by assuming the field to be an initial condition uniform in space over the simulation region. The time scale of the simulation is less than a picosecond of real time but is about 90 luser cycles, which is adequate if the magnetic field is large enough for the gyroradius of the suprathermal electrons not to run into the boundary condition and for there to be enough time for several gyro cycles $\left[2 \pi /\left(\mathrm{eB} / \mathrm{m}_{\mathrm{c}} \mathrm{c}\right)\right]$. Consequently, we chose it magnetic field of $10 \mathrm{MG}$ for laser intensity $10^{1 \mathrm{th}}$. The magnetic lield is a factor of 3 to 10 times larger than that determined by twodimensional LASNIX calculations but this is necessary to fit the problem into the computer in a reasonable amount of computer time. We checked the scaling by also running smaller simulated fields.

Figure 3-20 graphs the heated-electron temperature vs time and sbows aboul a $20 \%$ increase in temperature per electron gyro period. The $20^{\circ}$ figure is uncertain by a factor of two because the plasma is diamagnetic. reducing the external magnetic field (Fig. 3-2l), and because the anbipolar electric field expands with the hydro motion. Note that the heating nears saturation at late time: this is caused by the reduced magnetic field. and by an effect described by Morales and Lee ${ }^{50}$ in which very fast electrons pass though the short region of the epw too quickly to be affected by the electric field.

Short-pulse experiments ${ }^{5 t}$ showed that $\mathbf{T}_{\mathrm{Ho}}$ deduced from $x$-ray diagnostics increased with the 
Fig. 3-20. Resonant absorption $T_{\text {Hot }}$ vs time in laser cycles for the case of a 10-MG magnetic field imposed upon the simulation region us an initial condition $\left(\omega_{c} / \omega_{0}=0.1\right.$; laser intensity $=9 \times 10^{15} \mathrm{~W} / \mathrm{cm}^{2} ; \mathrm{T}_{\mathrm{Cou}}=4 \mathrm{keV}$; saturation at (a) may be due to diagmagnetic effect that reduced $\psi_{\varepsilon} / \omega_{0}$ to 0.05: heat rate $\sim 20 \%$ per pass-or about $7 \mathrm{keV}$ per pass; $n_{\text {tiog }}$ does not increase, and the "hots" become hotter).

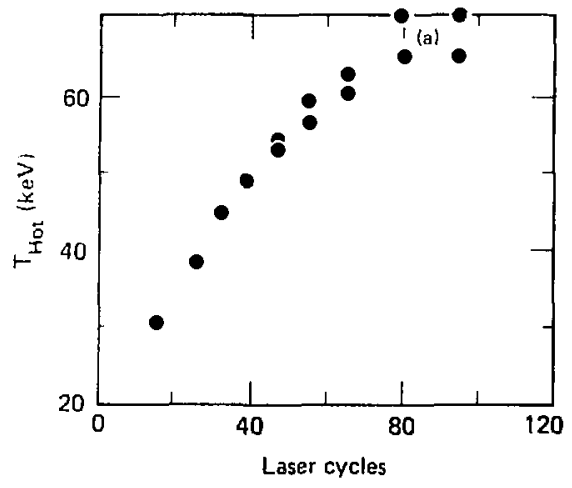

Fig. 3-21. The density profile and modified magnetic field as a function of $x / \lambda_{0}$.

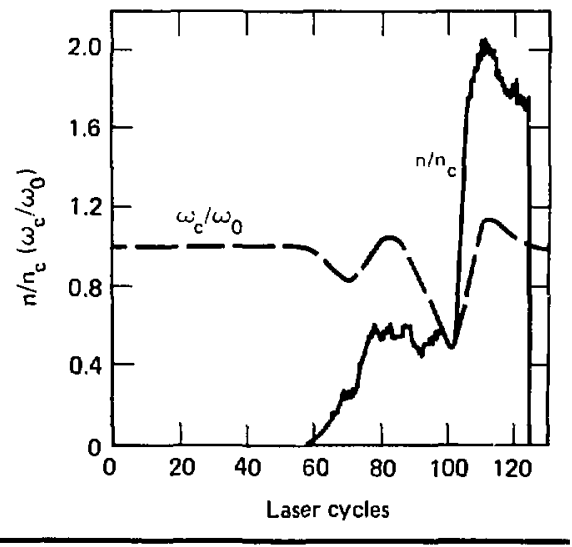

target $\bar{Z}^{0.25}$; it was suggested that perhaps the $\bar{Z}$ dependence might be due to collisions in the highdensity region which returned some of the hot electrons to be heated again. 52 The collision frequency is dominantly "ej, which is proportional to $\bar{Z}$. This "albedo" effect has an extensive literature in secondary emission of electrons from vacuum tube anodes. Typically, a beam of electrors lose about half their number and 0.3 of their energy in the high-collisional medium. We mocked this up in ZOHAR with a boundary condition that analyzed the dynamic electron distribution and returned half the heated electrons with 0.7 of their energy. The hot density increased but the hot temperature remained about the same. since the electrons lost about as much energy in the mock high-density region as they gained from the heating. Rosen ${ }^{53}$ analyzed the asymptotic limit of $n_{\text {Hot }}$ and $T_{\text {Hot }}$ given the scatlering material's number albedo $\beta$ and energy albedo $\kappa$, and assuming the electrons receive $\alpha$ of the uriginal energy per reheating pass through the resonance absorption region:

$$
T_{H \omega t}=T_{H o t}[] \quad(1 \cdot \alpha) \beta \mid /(1-\beta K)
$$

and

$$
\mathrm{n}_{\text {Hot }}=\mathrm{n}_{\text {Hot }} /(1-\beta) \text {. }
$$

where $T_{\text {fot }}$ and $n_{H_{1}(n)}$ are the ero-alhedo hotelectron temperature and density. In the ZOHAR simulation just described. $\beta=0.5, \kappa=0.7$, and $\alpha \approx$ 0.3. which explains why $T_{l l o l}$ remained the same and $n_{l l o t}$ increased. Since the number of electrons returned $v i$ energy is not a simple multiplier. perhaps a more careful treatment of the alhedo nodel might vield results closer to the experiment.

The $\overline{7}$ effect may be due to the fact that the drift velocity threshold for electron-ion instahilities decreases with increasing $Z$. and the resulting transport inhihition increases both the density and temperature gradient which can drive macroscopic magnetic fields. Transport inhibition increases $T_{\text {cold }}$ which in turn increases $T_{\text {Hol }}$, but not enough to explain the experiments. Valeo and Bernstein ${ }^{54}$ proposed that overdense collisions among the cold electrons provide an increase in $T_{\text {cold }}$ at critical density, since there would not be as much cooling from the cold electrons from the overdense region. Mason ${ }^{55}$ suggested that the density dip from a supersonic expanding plasma ${ }^{56}$ with spherical geometry could inhibit cold-electron transport by its electrostatic field.

Long-pulse-length experiments show much more scattering in $T_{\text {Hot }}$ 's $Z$ and no clear $Z$ dependance, indicating there may also be a 
geometric effect. For example, a low-Z̄ target will expand more into the laser. causing somewhat less irradiance than a high- $\bar{Z}$ target. This effect has also been investigated, but preliminary results show that the effect is not strong enough.

In conclusion, we have found that $\mathbf{T}_{\text {Hot }}$ clearly increases with macroscopic magnetic fields. since the heated electrons are recycled by the magnetic field to be reheated again. The experimentally observed $\mathrm{T}_{\mathrm{Hol}} \sim \bar{Z}^{0.25}$ for short pulse lengths has not been clearly explained by the albedo effect because the hot electrons cool as much by overdense colli. sions as they heat by resonance absorption. The $\bar{Z}$. dependence may be due to transport inhibition increasing $T_{\text {cold }}$ or by increasing magnetic fields from $\nabla n X r T$, or trom geometric effects.

\section{Authors: K. G;. Fstabrook and M. D. Kosen \\ Major (ontributors: W. I. Kruer and J. H. Nuckolls}

\section{References}

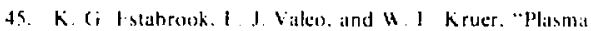
Heating and Ciradient Modificatuons hy Resonant Shoreptoon." Phis lett i 49. $109(1974)$

46. $K$ (i. I stabrosk, I I. Valeo, and W. I. Kruer, "I wo Dimensional Relalivistic Simulations of Revonance 1 h. iorpluen." Phis Fluids 18.1151 (1975).

47. D. W Forslund. I. $M$ Kindel, and $k$ I ee, "1 heors of Hor-

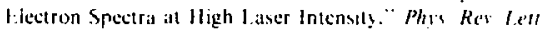
39. $264(1977)$.

4X. A. B. langdon and B. F. lasinskı. "I.leciromagneluc and Relatuvistic Plasma Simulation Models." in Metheds in Compuational fhysics. J. Killeen. Id. Acadentc Press. ven York, 1976), wol. 16. B ilder, S. Iernbalch, and 11 Rutenherg. Fods

49 ( . I Max. \& V. Manheimer. and I. I. Thom won, "I.nhaneed Transport seross laser (ieneraled Magnetic l. relds," Phis Flude $21,128(1978)$.

50. (i. J. Morales and Y. C. l.ee. "Iffecl of I ocallased blectrke fields on the t-volution of the Velocity Distribution 1 -unctuon." Phys Rev leet 33. 1534 (1974).

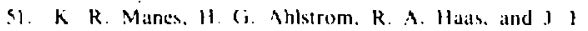
Holurichter. "light-Plasma Interaction Studies with 1 high power (;lase l.aser." I. Opl Soc Am. 67. $717(1977)$

52 I. H. Tuekols. Laurence l.ivermore l.ahoratory, I.juermore, (alif.. private communication (1979).

53. W. D. Rosen and $K$. (i. Fstahrook. "Analysis of the $\zeta$ Dependence of laser Generaled Suprathermal t.lectron Temperalure," lawerence livermore lahoratory. I ivermore. Calif.. LCRL.-K2280. 1979.

54. 1. 1. Valeo and I. B. Bernstein. "Fust-ion (jeneration in I.user-Plasma Interactions." Phys. Fluids 19. 1348 (1976).

55. R. J. Mason, "Nonte Carlo (Hyhrid) Suprathermal Electron Transport," Phis Rev. Lett 43. 1795 (1979).

56. C. F. Max and (. . . Mckec. "Effects of Flow on Densily Profiles in Laser-Irradiated Plasmas." Phis Rev. Lent 19. 1336 (1977).

\section{Effects of Driver Bandwidth Upon Resenantly Heated Elections}

It is predicted that broadband or multiple-line drivers can significantly lower the hot-electron temperature due to resonant absorption in a plasma. ${ }^{57.58}$ To test the bandwidth-dependence of the hot-electron temperature due to resonant absorption. we constructed an experiment which we then performed at microwave frequencies using band widths of $\lrcorner \omega \omega \leqslant 0.01$. We were able to determine. for the range of bandwidths available to us. a sealing las for the botelectron temperature as a function of bandwidth.

The work was done on the PROMETHEUS I low-density, pulsed-discharge plasma device at UC Davis. Microuaves $\left(f_{0}=1.2\right.$ (iH/) traveled down a cylindrical waveguide in the $T M_{01}$ mode and were incident upon an inhomogeneous, uninagnetized pulsed-discharge plasma $\left(n_{\mathrm{e}}<3 \times 10^{10} \mathrm{~cm}^{-3}\right)$. The plasma was essentially collisioniess so collisional absorption was negligible. The electromagnetic field extended 10 critical density $\left(n_{6}=1.8 \times 10^{10} \mathrm{~cm}^{-3}\right)$ where the electric field ( $\mathrm{b}$, parallel to $\mathrm{rn}_{\mathrm{e}}$ ) resonant1) drove electrostatic waves. resulting in resonant absorption of the microwaves. The broadtiand signal uas generaled by mixing a (jaussian noise source with the 1.2-(ill/ center frequency.

The heated-electron data were collected using planalr-disk I.angmuir probes placed in the overdense region to avoid rf interlerence. Over one hundred samples of the probe signal were ateraged by a computerised datat acquisition system.

The result ol driver bandwidth upon electron heating is given in 1:ig. 3-22 (all datal are taken at a peak power ol $200 \mathrm{~W}$. where vo, $v_{p} \approx 0.13$ ). We see that increasing the microwave bandwidth decreases the hotelectron temperature. We find that $T_{11} \propto$ $\left.\left(S u^{*} \cdot\right)^{6}\right)^{35}$ for the range of handwidths studied. The temperature of the heated electrons changes temporally as the density profite is modified by the ponderomotive force. Heated electrons appear on the same time scale as the rise time of the $r f$ signal ( - $50 \mathrm{~ns})$. The temperatures show' in Fig. 3-22 are the asymptotic values obtained after the density profile has reached a steady state $\left(\omega_{\text {pit }} \geqslant 100\right)$. We 
Fig. 3-22. Hot-electron temperature and density as a function of bandwidth.

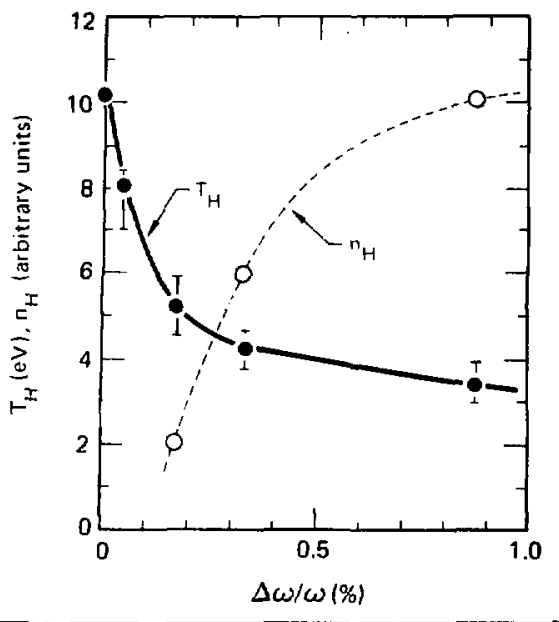

we that the mereitsing microw ave bandwidth lowers I 11 whike increasing the hot-clectron density $n_{11}$.

B. detining at heat tlux proportional to the climsiail free-streaming balue. $\left.Q^{H} \propto \mathrm{n}_{41} \Gamma_{1}\right\}^{2}$. ue find that the heat flux observed is nearly constant iner the range of handwidths used. as is the absorpthen la cxpected . Our results suggest that a hroadband fater driver ean reduce the hot-electron temperallure produced by resonant absorption while not salcrifucing absorption

Authors: R. B. Spielman, W. MI. Bollen, K. Mizuno, and J. S. Detiroot (All I niversity of (atifornia at Davis)

\section{References}

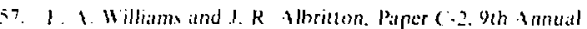
conference on Inomakous absorption of libclromatgnetic Waves. I niversty of Rochester. Rochester. N.Y.. May 15 10 18. 19:9.

58. I. Speriale, Paper $(2$, 9th annual conference on anomatous thorptom of teled ronagnetic Waves, Universils of Rochester, Rochevter, N.Y.. May is to 18.1979.

\section{Nonlinear Behavior Of Stimulated Scattering In Large Underdense Plasmas}

As lasers increase in energy and targets become larger, longer-pulse-length laser light is used to irradiate them. Much larger underdense plasmas are then created. and the size. $L$, of this plasma as seen by the light with wavelength $\lambda_{0}$ becomes an important parameter. This size parameter can be crudely estimated as

$$
\frac{L}{\lambda_{0}}=\min \frac{\left(v_{c \times p}{ }^{\top}, R\right)}{\lambda_{0}}
$$

where $v_{\text {exp }}$ is a typical plasma expansion speed. $\tau$ is the pulse length of the light, and $R$ is either the radius of the arget or the focal spot radius. Note that the characteristic size parameter seales ats $\tau, \lambda_{0}$ and that it is sometimes set by geometrical considerations.

The mix of laser-plasma coupling processes is expected to be sensitive to the size of the underdense plasma. When $1 . \lambda_{0} \geq 10$, the coupling is primarily determined by processes which occur at or near the steepened. rippled eritical-density surface. When I. $\lambda_{11}>10^{-2}$. processes such as inverse bremsstrahlung. stimulated Brillouin and Raman scattering. and filamentation can begin to play a sizable role: for example, a signilicant friction of the incidene light may be scattered by the Brillouin instability helore it reaches the critical-density surlace.

We consider here several nonlinear effects which limit Brillouin backscattering of intense ligbt in large underdense plasmats. After brieny considering ion trapping and harmonic generation. we focus on the self-consistent ion heating which occurs as an integral part of the Brillouin scattering process. In the long-term nonlinear state. the ion wave amplitude is determined by damping on the selfconsislently formed heated ion tail. $A$ simple model of the scattering is presented and compared with particle simulations.

Brillouin Scattering. Brillouir scattering ${ }^{59.60}$ can be most simply described as the resonant decay of an incident photon into a scattered photon plus an ion sound wave. Hence

$$
\omega_{0}=\omega_{t}+\omega_{j 3} \text {. }
$$

where $\omega_{0}$ and $\omega_{1}$ are the frequencies of the incident and reflected light waves and $\omega_{\mathrm{ia}}$ is the frequency of the ion sound vave. As is apparent from this frequency matching condition. this process occurs throughout the underdense plasma. In addition, since $\omega_{0} \gg \omega_{\text {ial }}$, nearly all of the energy of the incident light wave is transferred to the scattered wave. 
$\omega_{\mathrm{i}}$. Hence Brillouin scattering is a particularly dangerous energy-loss mechanism.

It is easy to show that Brillouin scattering is potentially an important effect in terms of decreasing laser absorption as well. Consider a light wave with electric vector $E_{i}$ incident on a uniform underdense plasma with density $n_{p}$. and let there be an ion sound wave of amplitude $\delta$, with frequency and wave number appropriate to scatter the incident wave into a backscattered light wave with electric vector $F_{r}$. Using Maxwell's equations and separating out the fast tine- and space-scale dependences, we readily obtain coupled equations for the slowly-varying amplitudes:

$$
\frac{\partial I}{\partial r}=-a \frac{\delta n}{n_{p}} r_{j}
$$

and

$$
\frac{\partial \mathrm{F}_{i}}{\partial \mathrm{x}}=\alpha \frac{\delta \pi}{\pi_{p}} \mathrm{l}_{\mathrm{r}} .
$$

Here

$$
a=\frac{\pi}{2}\left(\frac{1}{\lambda_{0}}\right) \frac{n_{p}}{n_{c}} /\left(1 \frac{n_{p}}{n_{c}}\right)^{1 / 2}
$$

where $\lambda_{0}$ is the wavelength of the incident light and $n_{c}$ is the critical density. These equations are readily integrated to determine the reflectivity. $r$. If we consider a plasma with size $L$. and neglect $F_{\text {. }}(I$.$) com-$ pared to F.l L). we obtain

$$
r=\tan H^{2}\left(\alpha \frac{\delta n}{n_{p}}\right)
$$

A simple example is very instructive. Consider an underdense plasma with $n_{p}=0.1 n_{0} . L=10^{3} \lambda_{(1)}$. and an ion wave amplitude of only $1 \%$ of the plasma density. Equation (41) then predicts that $r \simeq 80 \%$. showing that even a small density fluctuation can lead to sizable scattering in a large underdense plasma.

Given that Brillouin scattering can be a significant effect, it is very imporiant to understand what nonlinear effects serve to limit the amplitude of the ion sound wave. We will first estimate the effects of ion trapping and harmonic generation and then concentrate on the important effect of selfconsistent ion heating. which produces a tail which damps the ion wave. We will emphasize the nonlinear regime in which the light pressure is less than or comparable to the pressure of the underdense plasma. In the opposite regime. which obtains at very high intensity. enhanced profile steepening will clearly act to significantly reduce the scattering. ${ }^{61}$ In order to focus on the nonlinear behavior, we will neglect the effect of gradients and simply treat the underdense plasma as an equivalent region of plasma with uniform density.

Ion Trapping. Ion trapping is one effect commonly invoked to limit the ion wave amplitude. As the ion wave amplitude increases. its potential becomes large enough to nonlinearly bring ions into resonance with the waves Since such ions are efficleally accelerated by the "ave. a strong damping results, which serves to restrict the ion wave amplitude from further increase. If the ions are cold. the trapping condition is simply $\mathrm{Ze} \phi=\mathrm{Mv}_{\mathrm{p}}^{\frac{2}{2}} / 2$. where $\phi$ is the potential, $M$ and $Z$ are the ion mass and charge, and $v_{p}$ is the phase velocity of the wave. Neglecting Debye length corrections. the trapping condition corresponds to $\delta \mathrm{n}: \mathrm{n}_{\mathrm{p}} \simeq \mathrm{e} \phi / \theta_{\mathrm{c}} \simeq 1 / 2$. which is a large anplitude.

It is important to realise that even a snutl ion temperature significantly reduces the trapping amplitude. ${ }^{2}$ This temperature aflect is readily estimated if one assumes a so-called "waterbag" velocity distribution for the ions. In one dimension such a distribution is constant with a velocity beween $\pm \sqrt{3},(x$, is the ion thermal velocity) and zero for olher velocities. Sinee the majority of the ions in a Maxuellian distribution have velocities $\geq 2 v_{1}$. the waterhag distribution gives a reasonable first approsimation for the onset of strong trapping. which nou ocsurs when the lastest ion is nonlinearly brought into resonance with the vare: that is.

$$
\begin{aligned}
& 200=\frac{y}{2}\left(v_{p}, \sqrt{3} r_{i}\right)^{2} . \\
& \frac{\delta n}{n_{p}}-1: 2\left(\sqrt{1+\frac{3 \theta_{i}}{Z \theta_{c}}}-\sqrt{\frac{3 \theta_{i}}{Z \theta_{c}}}\right)^{2} .
\end{aligned}
$$

Where $\|_{1}$ is the ion temperature, $Z$ is the ion charge and $\theta_{\mathrm{c}}$ is the electron temperature. For $\theta_{\mathrm{i}} / Z \theta_{\mathrm{e}}=0.1$. 1.q. (42) predicts a fluctuation amplitude of $\delta n_{/} / n_{p} \simeq$ 0.13 . Clearly the ion temperature serves to signilicantly reduce the amplitude, but note that the trapping amplitude is still of order $10 \%$, unless the 
wns are quite hot $\left.\mid a_{1}, Z_{c} A_{c} \sim O(\}\right) \mid$. Strong trapping dise not on general limil the nuctuation amplitude (1) a imatl value.

Harmonic Generation. Harmonic generution is Anther effect which acts to limit the ion walve amNitude. provided the wave number limes the elecfor bebye kength $\left(\mathrm{k} \lambda_{\mid \mathrm{J}}\right)$ is small. If we neglect 4. ne elfe-s. the frequency of an ion sound wave is - pris proportsonal to its wave number. Such a 1.. all then steepen. sance harmonics are resind druen If we consider an ion sound valve

1. implutude ón. watve number k, and frequency and compule the growth of las second harmonic

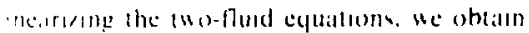

$$
\frac{i_{n} 2 k 1}{n_{i}}+2\left[\frac{n n k z}{n_{p}}\right]^{2} \omega_{i k}{ }^{\prime}
$$

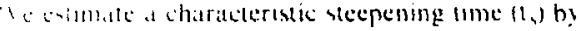

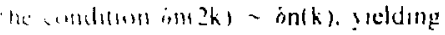

$$
w_{i k}, 2 / \frac{\operatorname{sn(k)}}{n_{p}} .
$$

II hen kilde correctuon are included. the rese-

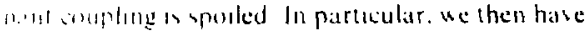

$$
w_{i k} \cdot \frac{k_{s}}{\sqrt{1+k^{2} x^{2}}} \cdot k v_{c}+2 u
$$

where w whe ion sound velocilv and $J u=\varepsilon_{i k} k^{2}$ $v_{j}=$ for $k \lambda_{1}$ e $\ll 1$. Significant harmonic generais $m$ then at least reyures that $6 \geq 1$ Ju. where $3 w$ as (w) he evaluated for the second harsonic. This cerresponds to the condition

$$
\frac{\delta \pi(k)}{\pi_{\mathrm{p}}} \cdot 4 \mathrm{k}^{2} \lambda_{\mathrm{BC}}^{2}
$$

The estimate in Fq. (46) suflices to shou that harmonis generation is not very effective in the hot underdense plasmas which are typical of recent laser-irradiated targets. For example, let us consider the jon wave rroduced by Brillouin backscattering in an anderd w ise plasmat with $n_{p} / n_{c}=0.33$ and an electron tempc ature of $3 \mathrm{keV}$. Then $\mathrm{k}^{2} \lambda_{\mathrm{D}_{\mathrm{c}}}^{2} \simeq 0.07$ :
In this case. significant harmonic generation would require that the ion wave reach a very sizable amplitude $\left\{\delta n(k) / n_{p}>0.3\right.$ for this example].

Ion Tail Formation. Let us now take a somewhat different approach and focus on the important changes in the ion velocity distribution which are a natural consequence of the Brillouin reflection. As shown by computer simulations, the long-term nonlinear state ${ }^{6.3}$ is one in which the Brillouin-generated ion wave is damped by a heated ion tail. which indeed is necessary to carry off the energy deposition into the con wave. The greater the reflectivily. the larger the ion tail. and so the larger is the con wave damping. Hence the reflectivity is at least partially self-correcting.

I very simple model can be used to estimate the reflectivity in the nonlinear state. Since the ion tail is produced by an ion wave with phase velocity $x_{\text {. }}$ its characteristic lemperature is approximately $\|_{11}=M v_{5}^{2}$ an estimate within a factor of about two of agreenient with our simulation. The magnitude of the ion heating (the tail density. $n_{11}$ ) is then readily delermined by balancing the energy flux deposited in the damped ion wave with the flux carried off the tail.

$$
f \frac{\omega_{i k}}{\omega_{01}} 0.8 n_{11} 01 \sqrt{\frac{\theta_{11}}{M}} \text {. }
$$

where $r$ is the reflectivity. I and $\boldsymbol{\omega}_{11}$ are the intensity and frequency of the incidene light wave. and the heal flux catried off by the ion tail has been eslimated in the free-streaming limit. Having determined $n_{1}$. We can now describe the ion wave damping simply as l.andau damping on the heated ion tail. i.e.. $v_{1} w_{1} \simeq 0.8 n_{1} \cdot \pi_{p}$. more sophisticated estimates. including for example resonance broadening. just enhance the damping.

He complete our simple model by computing the reflection from the damped ion wave. The amplitude to which the wave is driven is essentially the ponderomotive force due to the beat between the incident and reflected waves divided by the damping rate:

$$
\frac{\delta n}{n_{p}}=\frac{Z k^{2} e^{2} E_{i} E_{I}}{2 m M \omega_{0}^{2} \nu_{i} \omega_{i}}
$$

where $Z$ is the churge of the jons. $\omega_{j}$ and $\nu_{i}$ are the ion wave frequency and energy damping rate. $M$ 
and $m$ are the ion tnd electron mass, and $t_{\text {, }}$ and $t_{\text {. }}$ are the electric vectors of the incident and reflected light wases. Substitutung 14 ( 18 ) anto 14.499$)$ and integrating ther gives the well-known result ${ }^{\text {th }}$ for the reflectivity. r

$$
\begin{aligned}
& \text { rl] } n=B\{\operatorname{explan} \text { nl } r\} \text {. }
\end{aligned}
$$

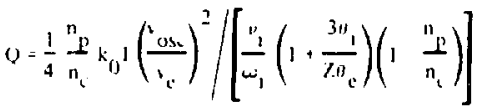

Here I w algan the wa of the platsma. np, whe

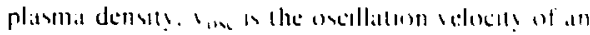

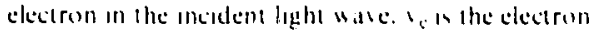
thermal weloits. and $B$ a the nome level of the rellected wate at $=1$, mormalued to the intensts at the incident light wate at $=$ )

I he reflectum is now determuned as a functwon (1) planma comdanom is an example. comsder

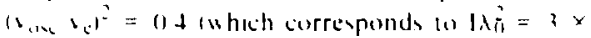
$111^{1} 11-\mu^{2} \mathrm{~cm}^{2}$ and $\mu_{c}=?$ kell and $n_{r} m_{0}=113$

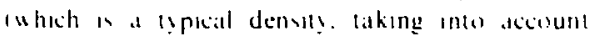
modest protile veepenong near the critkeal density.

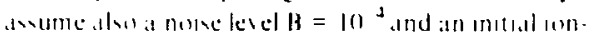
eiectron lemperalure ratlo of o ? Our model prede.

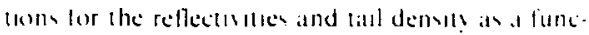

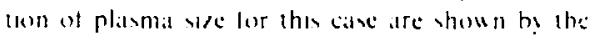
solid lines in 1 ig 3.23 these predicturn ate at reasonatle agrement wath imulation results for comparson. the open circles denote the hong-term Brolloum rellectlon! calculated in a ins domensional smulation code wheh used particte wons and huld electrons Vote that the reflectus! rather small $\left(-10 \%, 1\right.$ when I $\lambda_{1}$ - 10 , and graduatl increases 60 a value of $-50 \%$; when $1 \quad \lambda_{11}$ - 100$)$ vole also the substantial ion tall whoch sellconsistently forms The mean un temperature yuickl! becomes of order unily. Self-comsistent ion healung is a vers potent effect.

1 igure 3-2t compares the model predietom (solid line) with simulation results for the refleclivits as a function of plasma densils, when the scale length and intensily are fixed: $1 . \lambda_{1}=20$ and (bax $b)^{2}=13$. The circles denote simulation results using a code which treats both the electrons and the ions as particles. For these runs. the initial ion-electron temperature ratio was 0.2 and the ionelectron mass ratio was 100 . The result at $n_{p} n_{c}=$ 0.3 .3 prosides a point of a comparison with the numerical results using the hybrid code discussed in
Fig. 3-23. The reffection due to the Brillowin hastability and the ion tail density as a function of the sire of the underdense plasens. The paraiacters are $\left(v_{\text {onc }} / v_{e}\right)^{2}=0.4, n_{p} / a_{c}=0.33$, and $\theta_{i} / Z n_{e}=0.2$ initially. The solid lines denote the model predictions $\left(\mathrm{B}=10^{-4}\right)$. The circles denote simulation results using * kybrid code $(m / M=1 / 400)$.
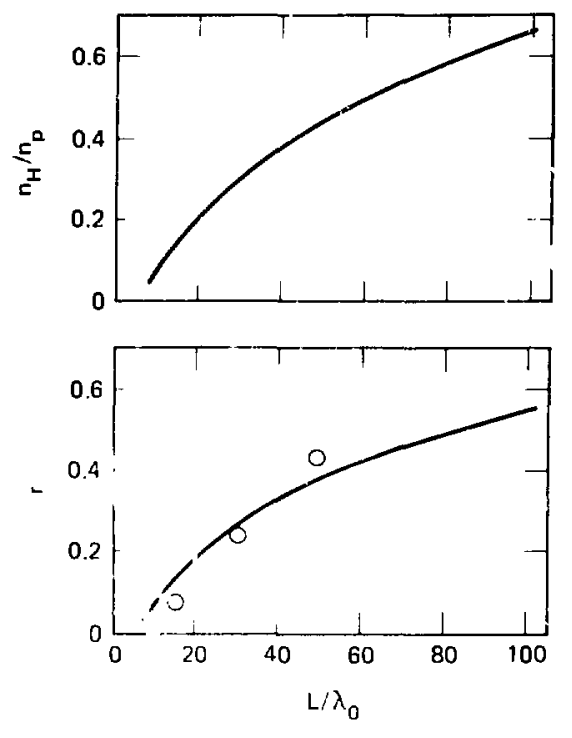

the presum tegese. and the agreement a yuste

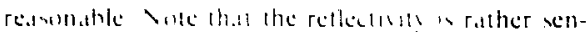

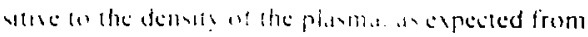

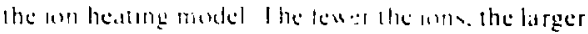

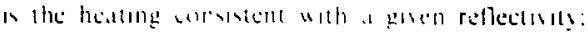
hence proble steepening neat the ritcal densily san plat an mportant role an reductug the saltering. by

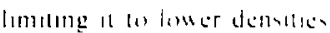

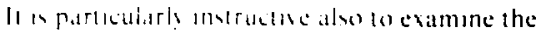

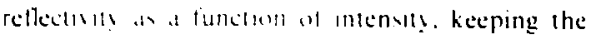

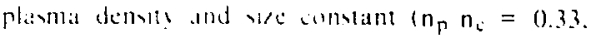
I $\lambda_{1}=8$ in 1 geare 325 shows the simple model predictun kir the retlestult as a lunction of

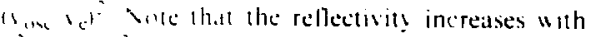

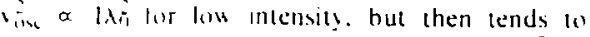

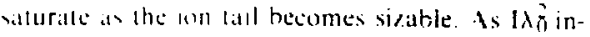
cratses. the self-combsent ion heating increases. "hict, ack w compe" s.lic for further increases in rellectubl! 
Fig. 3-24. The reflection due to the Brillouin instability as a function of the backgrcund plasma density. Here $\left(v_{\text {axd }} / v_{e}\right)^{2}=$ $0.4, \mathrm{~L} / \lambda_{0}=20$, and $\theta_{i} / Z t_{c}=0.2$ initially. The solid tine is the simple model prediction $\left(B=10^{-4}\right.$ ). The open circles dente simulation results using a particle code $(\mathrm{m} / \mathrm{M}=$ $1 / 100)$.

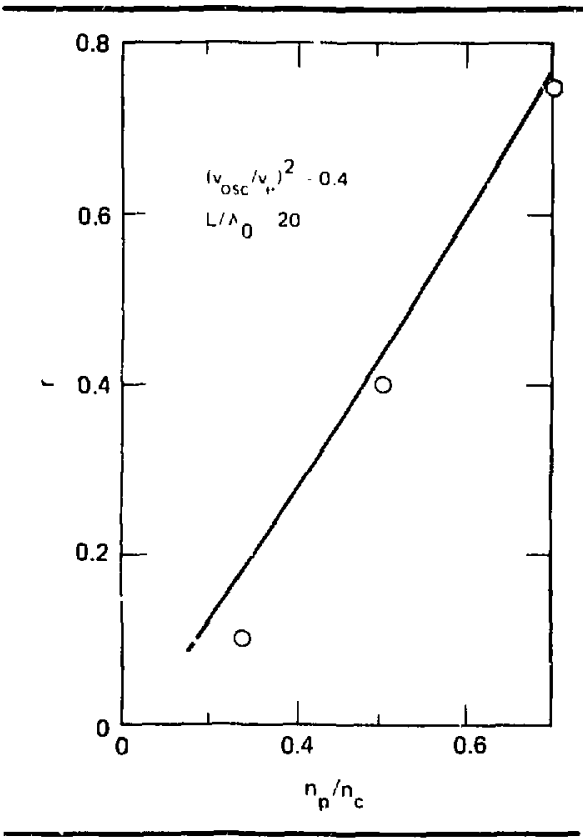

Fin. 3-25. The simple model prediction for the reflection due to the Brillouin instability as a futh fon of $\left(v_{\mathrm{med}} / \mathrm{v}_{\mathrm{e}}\right)^{2}$. Here $n_{\mathrm{p}} n_{\mathrm{c}}=1 / 3, L / \lambda_{0}=\$ n, B=10^{-4}$ and $\left\|_{\mathrm{i}} / Z\right\|_{\mathrm{e}}=0.2$ initially.

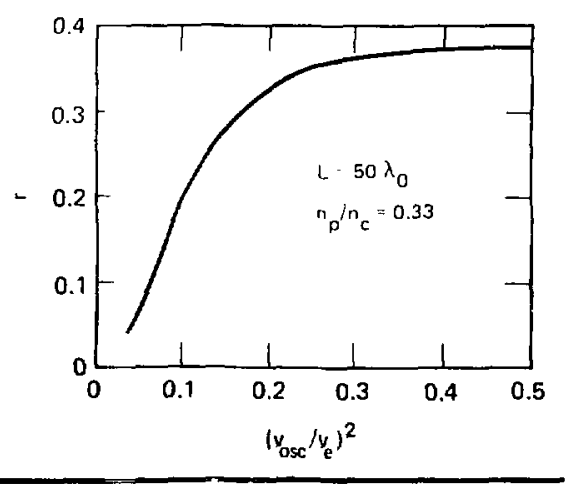

A crude aralycic estimate of the reflectivity on line salurated state is

$$
r \quad 1 \int\left[1+n_{B} 1 / \frac{\pi}{2} \frac{L}{\lambda_{0}}\left(\frac{n_{j}}{n_{i}}\right)^{2}\right] .
$$

Vote the dependerece of the rethection on the sise parameler $\left(1 . \lambda_{0}\right)$ and the plasma density $\left(n_{p} n_{c}\right)$ : this result indicales that. lise a given $n_{\Gamma} n_{a}$ and $l$.. long-wavelength lisers are less sensitive to Brillouin scatlering at high incensity. simply hecatuse there are fewer wavelengltos of underdense plasma avaliable (1) reflect the light, of course. for a given $n_{p}$ alnd 1 . short-walelength light tradergoes less scattering orcasuse the plasma in more underdense to the light.

We note that simpler ion heating models bist. give imilar results as long as the reflectivity and ion heating are sirable. for example, since the fratum of the ione in the heated tail is siable. it is conconient (1) approximitte the ion heallng als a main body heating. In the case. we simply balance the energes llus deposited into ion waves with the energ! flua carred off by a heated Mixwellian distribution with densily nip. The effective temperature of this distrihution is then

$$
\frac{\theta_{i}}{Z \theta_{c}}-\left[r \frac{n_{c}}{n_{p}}\left(\frac{v_{0 s c}}{v_{c}}\right)^{2}\right]^{2 / 3} .
$$

and smilar rellectistlien are oblained.

The fat that the numbers are not greatly sensitive to the details of the ion heating is anocher mantestation of the partially self-correcting feature of the scaltering in this simple model. It should be noted. however. that details of the ion heating ma! be significanl for understanding the lirequency spectrum of the reflected light. The normal modes of a multitemperature distribution differ in detail from the normal modes of a single-temperature distribution. Simulations show that when a heated taii develops, that tail becomes heavily populated and another higher-energy t...I is formed. We also note that ion lails are less sensitive to expansion cooling. "at an effect which can compete with the ion heating in an expanding plasma.

finally we can crudely estimate the time required for the plasma to approach this nonlinear stalle. The time. 1 . . io produce the heated tail is 


$$
\mathrm{t}_{11}-\frac{1}{2 \mathrm{r}} \frac{\mathrm{n}_{\mathrm{H}}}{\mathrm{n}_{\mathrm{c}}} \frac{\mathrm{v}_{\mathrm{e}}^{2}}{\mathrm{v}_{\mathrm{OSc}}^{2}} \frac{\mathrm{L}}{\mathrm{c} s} .
$$

Assuming $n_{11}-0.2 n_{p} \cdot\left(n_{p} n_{c}\right) v_{c}^{2} v \dot{2}=1, r=12$. $\theta_{c}=+\mathrm{kel}$ and $l .=50 \lambda_{k}$. Fq. (52) gives $l_{f 1} \sim 20 \mathrm{ps}$. of course. in practice. it may be difficult to observe a transient state in which the ions are initiall! heated. since this heating can gradually accumulate an the laser pulse increases from low to high inten. sits. In addition. other effects such ats shock heating can independenty contribute to the ion heating.

The liffects of firadients. It should be noted that gradients are not necessarnly an ellectove in reducing Brillouin scillering in the nonlineat regime as they are in the linealr regime. It is well known that gradients can greally increase the

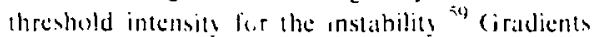
l,mii the region oner a hich any gise il three wases ciln resonanty merat. Voting that the wate numhers of the tl: rete nalles $\left(k_{1}\right.$, with $\left.i=1.2 .3\right)$ are now it functson al position. let us deline

$$
K=k_{1}(x) \quad k_{2}(x)-k_{3}(x)
$$

il ame point. $k=0$ (the waven ate resonantly coupledi. but awalg trom this point a mismalth derelons which sp̧onls the resonamt couplang. Hence the three waves can resonantly interact onl wer me interaction region which cian he estimated b?

$$
\int_{0}^{\operatorname{lin} t} x d x \quad 1,2
$$

Iay lor expandang about the point of resonance $\left.\right|_{h}=$ $A(1))+A^{1} x$, where $\left.A 10\right)=(1)$ then gives

$$
\operatorname{sint} \frac{1}{\sqrt{1}} \ll \mathrm{I}
$$

Propagation of wate energy out of this interaction region introduces an enhanced damping rate of approximately $s_{p 1}$ int. "here $v_{g}$ is the group velocily of the ith wave. Hence the threshold intensity is increased.

Well above the threshold set by gradients. however. the reflectivity from a given interaction region is determined nonlinearly. and one must further add up the contributions of the different interaction regions. Hence the bulk of the plasma still ontributes to the net reflection. Note that nonlineariy the refiectivity is not an exponential funetion of L (see Fig. 3-23). so the net contribution ${ }^{67}$ from a number of smallw regions need not be much less than the coisiribution from one larger region tthis assumes a distributed and or broadband noise source). (jrudients will no doubt play a quantitatively significont role. but probably not the erucial role which they often play in the inear theory. Perhaps the most important aspect of allowing lior plasma expansion and inhomogeneity will be in properly determining the various density regions available for swallering. since the rellectivity depend: aensilively on $n_{p} n_{c}$.

Experimental Evidence: ()ptions. Recent exper-

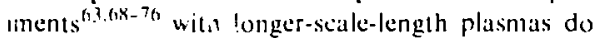
indicate that significant Brillouin scattering is possible. In these experiments. sicable underdense plasmas $\left.\left(1 . \lambda_{1}\right)>30\right)$ were formed in various ways: $\therefore$ using a prepulse plasma. a long-pulse-l.ngth plasma in the sense of $\tau \cdot \lambda_{1}$, $\omega r$ a pretormed plasma. Table 3-1 shows a brief summary of some of the experimental results for the back-reflection of $1.06-\mu \mathrm{m}$ and $10.6-\mu \mathrm{m}$ laser liznt. Note that a peak reflectivity of shout sor has been observed with either wavelength. Vote also that the pulse lengths (or prepulse delays) used in the experiments with I14.6$\mu \mathrm{m}$ light are much longer that those used with $1.06-$ $\mu_{\text {iii }}$ light. reflecting the expected $L \lambda_{\text {rrdependence. }}$

I idially, if Brillouin scattering does prove to be a erious pre' lem. there are some options for reducing the sca sring (although many more experiments are needed to determine the intensity and wavelength windows appropriate for laser fusion applicalionsi:

- Une option. of course. is lo operate at lower antensity $\left({ }^{\prime} \lambda \hat{)}\right)$. so that the plasma is more collisional and the Brillouin instability is more veakly diven. Indeed. recent experiments ${ }^{77} \cdot 78$ with several-ns pulses of 1.06- $\mu \mathrm{m}$ light have shown a high absorption $\left(-7(10\right.$ to $80 \%)$ at intensities of $\sim 10^{13}$ to $10^{14} \mathrm{Mem}$ :

- Increasing the bandwidth and or reducing the wavelengtb of the light are other effects ${ }^{74}$ in the same direction: the plasma absorbs more by inverse bremsstrahlung and the instability is more weakly driver.

- The complementary approach is to operate at high intensity and try io minimize the scattering by using its dependence on $n \cdot n_{p}$ and $L / \lambda_{0}$. 
Table 3-1. A brief summary of recent experimental results for the peak reflectivity $\left(r_{B}\right)$ of laser light (for more details see Refs. 63, 68-76).

\begin{tabular}{|c|c|c|c|c|c|}
\hline Inrcatution & $\lambda_{0}(\mu \mathrm{m})$ & Target & Pulse length & $\mathrm{I} \frac{\mathrm{W}-\mu^{2}}{\mathrm{~cm}^{2}}$ & Peak $x_{B}$ \\
\hline NRL & 1.06 & disk & $\underset{(\sim 2 \mathrm{~ns})}{\mathrm{pp}}+50 \mathrm{ps}$ & $10^{15}-10^{16}$ & $50 \%, f / 2$ \\
\hline L.LL & 1.06 & $\begin{array}{c}\text { disk, } \\
\text { spheres }\end{array}$ & $200 \mathrm{ps}-1 \mathrm{~ns}$ & $\begin{array}{l}3 \times 10^{14} \\
3 \times 10^{16}\end{array}$ & $\begin{array}{l}50 \%, f / 1 \\
15 \%, f / 2.6\end{array}$ \\
\hline$U$ of $R$ & 1.06 & spheres & $\underset{(\sim 2 \mathrm{~ns})}{\mathrm{pp}}+50 \mathrm{ps}$ & $10^{15}-10^{16}$ & $25 \%, \mathrm{f} / 2$ \\
\hline KMS & 1.06 & gas jet & $100 \mathrm{ps}$ & $10^{14}-10^{15}$ & $40 \%, f / 3.5$ \\
\hline I.ASL & 10.6 & sphere & $\underset{(10-35 \mathrm{~ns})}{\mathrm{pp}}+1 \mathrm{~ns}$ & $\sim 10^{17}$ & $10.50 \%, 8 / 2.5$ \\
\hline U of Alberta & 10.6 & $\begin{array}{c}\text { gas jet } \\
(0.1 \mathrm{~N})\end{array}$ & $35 \mathrm{~ns}$ & $\sim 10^{15}$ & $60 \%, 8 / 2$ \\
\hline LCLA & 10.6 & $\begin{array}{c}\operatorname{arc} \\
\left(0.015 \mathrm{~N}_{\mathrm{c}}\right)\end{array}$ & $50 \mathrm{~ns}$ & $-2 \times 10^{13}$ & $5 \%, 8 / 7.5$ \\
\hline U of Wash. & 10.6 & solenoid & $\sim 400 \mathrm{~ns}$ & $\sim 5 \times 10^{11}$ & $5 \%, f / 6.6$ \\
\hline
\end{tabular}

Summary. The size of the underdense plasma in units of the laser-light wavelength is an important paraneter aflecting the mix of coupling processes. Stimulated scattering of the light in large underdense plasmas is a very real concern. We have considered several nonlinear effects which limit the Brillouin scattering. Our calculations emphasize the importance of the self-consistent changes in the veiocity distribution which are produced when the electrostatic waves damp into the particles. Simple theoretical models. formulated and compared with computer simulations. have shown that the reflectivity is at least partially setf-correcting. As the reflectivity increases, the velocity distribution becomes nore distorted, enhancing the damping of the electrostatic wave and acting to reduce the reflectivity.

We emphasize that there are significant uncertainties in our attempts to quantitatively understand the level of stimulated scattering: more quantitative predictions will require a realistic treatment of the effects of gradients in the underdense plasma. The incorporation ${ }^{66.80}$ of a model for stimulated scattering into a hydrodynamic code is being actively pursued. In general, even the zero-order plasma condi:ions are rather poorly known, because of uncertainties in the electron transport. More experiments to diagnose the underdense plasma conditions are needed. and the angular distribution ${ }^{81}$ of the stimulated scattering also requires further investigation. Two-dimensional simulations suggest that the scattering occurs over a sizable range of angles; tius the back-reflection through a high-f-number lens can be a gross underestimate of the level of stimulated scattering, Finally, the competition of this scattering with other processes such as filamentation is not well understood either theoretically or experimentally.

\section{Authors: W. I. Kruer and K. G. Estabrook}

\section{References}

59. C. S. I.iu. M. V. Rosenhluth, and R. B. White, Phys. Fhids 17. $1211(1974)$

(10). [). W' Forslund, J. M. Kindel, and E. Lindman. Phis Findeds 18. 1002. 10) 7 (1975).

61. W. I. Kiruar, E. J. Valeo, and K. (;. Fstabrook. Phys. Rev. Lefl. 35. 1076 (1975).

62. J. M. Jiawson. M. I.. Kruer, and B. Rosen, in Dyamics of Iomized (iases. M. Lighthill. I. Inai, and H. Sato. Fds. (Cniversity of Tokyo Press. I'okyo. 1973). pp. 47 to 61

6.3. I). W. Pbillion W. 1. Kruer, and V. C. Rupert, Phys. Rev. l.ett. 39. 1529 (1977): Laser Program Amual Report-1976. I.awrence I.ivermore Laboratory, Livermore. Calif.. UCRL-50021-76 (1977), p. 4-63.

64. C. L. Tang. J. Appl. Ph.s. 37. 2945 (1966).

65. J. J. Thonison and K. Mima, NA607A. Japan, to be published.

66. K. (j, Istabrook and J. A. Farte, Lawrence Livermore I aboratory, Livermore. Cali'.. UCRL-82620 (1979): R. G. livans. Rutherford Laboratory RL.-79-06I (1979). 
67. C. J. Randall, Lawrence Livermore Laboratory. Livernore. (alif.. private communication (1979).

68. B. H. Ripin et al., Phys. Rev: Letf., 39, 611 (1977).

69. M. D. Rosen et al.. Phis. Finids 22. 2020 (1979).

70. R. R. Turner and L. M. Coldman. Phus. Rev. Letr. 44. 400 (1980).

71. I. J. Mayer et al. KMSF preprint U-904 11979

72. (3. H!. NeCall. LASI preprint IA-UR-79-1298 119791.

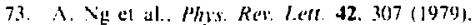

74. M. I. Herbat. C, 1. Clayton, and I. I. Chen. Phys. Rev I.eif. 43. 159) (1979).

75. R. Masses, K. Bergegren. and /. Pietryzk. Mhis. Re'r. Ie'll. 36. 96,3 (1976)

76. I here are numerous wher relerences to experiments, insluding I. M. (jorbunov et al.. .I:TP lett. 27. 226 (1978):

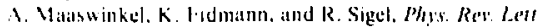
42. 1625 (1979): S. Viakai el al. Phis. Re't a 17. 1133 (1978).

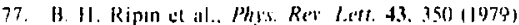

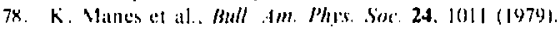

79. (. 1, Miax and K. (; 1 stabrook. "W'avelength Scalling in I aser I usion from a Plasma physics Proint of View," to be

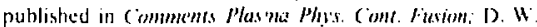
lorslund, Vinth Anomalous ibsorption Conlerence. Paper 1-2 (1979): I. Amiranoff wt ai.. Bull. Am. Phis Sisc 24. $1054(1979)$.

80. C. J. Randall. 1. J. Thomson, and K. (i. Pstabrook. Phys Rev Leell. 43. 92d (1979). D). (i. Colombant and $11 . M$. Manheimer, NRI. preprint (1979).

81. R. l.ehmberg. Phas Res. I.ett. 41. 86,3 (1978)

\section{Physical Processes Affecting Stimulated Brillouin Scattering at Different Laser Wavelengths}

The relatively large targets appropriate for an eventual laser fusion reactor are expected to produce substantial regions of underdense plasmat. Hence they are in a part of parameter space where stimulated Brillouin scattering (SBS) is of concern. due to its ability to cause premature reflection of the incident laser light. Wo have investigated physical processes which determine the level of SBS for lasers having a variety of wavelengths. in an effort to extend our understanding of wavelength sealing. ${ }^{2}$ In particular, it is expected that inverse bremsstrahlung absorption will limit $\mathrm{SBS}^{83}$ for short-wavelength lasers. whereas ion heating ${ }^{8-1}$ or ion trapping ${ }^{85}$ will be more important for longerwavelength lasers.

To investigate these effects quantitatively we have used a model describing SBS in the presence of inverse biemsstrahlung absorption and SBSinduced ion hcating. The incident. scattered. and reflected light waves are treated in the WKB limit, and the ion-wave amplitude is determined either by the ponderomotive force or by an ion-trapping limit. whichever is smaller. The ion waves are assumed to be at the strong damping limit. and ion energy equations determine the ion temperatures parallel and perpendicular to the direction of plasma expansion. $T_{\|}$and $T_{\perp}$. The background plasma is taken te be spherical. with given steadystate profiles of velocity and electron temperature $v(r)$ and $T_{s}(r)$. The background electron number density $n(r)$ is then determined from mass conservation. To account for profile-steepening at the critical-density surface, the electron density profile is assumed to rise steeply to critical as shown in Fig. 3-26. starting from an (adjustable) density $n_{p}<n_{c}$.

Within this model the equations describing the light waves may be written as follows. We define incident and Brillouin-scattered dimensionless normalized electric field amplitudes $\wedge_{0}$ and $\wedge_{\mathrm{s}}$ respectively, by.

$$
\text { I: } v_{0} \Lambda_{0,5} \exp \left(: \mathrm{i} \int \sqrt{\epsilon} \frac{\omega_{0}}{c} \mathrm{dr}\right)
$$

where $=\left(1-n \cdot n_{c^{\prime}}\right)^{\prime} ? F_{0}$ is the field amplitude incident at large $\mathrm{r}$ (in vacuum). and $\mathrm{E}_{+}\left(\mathrm{E}_{-}\right)$is the electric field of the incident (reflected) light. We also deline an inverse bremsstrahlung absorption factor by

$$
\mathrm{f}_{=}-\exp \left[\frac{p\left(\mathrm{n}_{\mathrm{c}}\right)}{c} \int\left(\frac{\mathrm{n}}{\mathrm{n}_{\mathrm{c}}}\right)^{2}\left(\frac{\mathrm{T}_{\mathrm{ec}}}{\mathrm{T}_{\mathrm{e}}}\right)^{3 / 2} \frac{\mathrm{dr}}{\sqrt{\epsilon}}\right] .
$$

Fig. 3-26. Schematic of electron density profile, $n(r)$, and of the three light waves: $A_{0}$ (incident), $A_{s}$ (Brillouin-scattere:"), and $A_{R}$ (reflected from the critical surface). Above a density $n<n_{e}$, the density profile is assumed to rise steeply to critical.

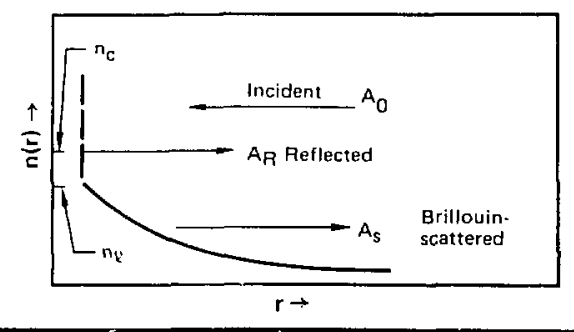


Then the incident. Brillouin-scattered, and reflected light waves (see Fig. 3-26) obey

$$
\begin{aligned}
& \frac{1}{2} \frac{\partial}{\partial r}\left(r^{2} \sqrt{\epsilon} A_{0}^{2} f_{-}\right)=\frac{k_{0}}{2}\left(\frac{n}{n_{c}}\right) A_{0} A_{s}{ }^{r}- \\
& x \min \left[\left(\frac{\mathrm{F}_{0}^{2}}{8 \pi n_{c} k T_{b}}\right) \frac{A_{0} \dot{A}_{s}}{\left(\frac{\nu_{i}}{\omega_{s}}\right)\left(1+\frac{3 T_{i}}{2 T_{c}}\right)}\left(\frac{\delta n_{j}}{n}\right)_{\max }\right] \text {, }
\end{aligned}
$$

$\frac{1}{2} \frac{\partial}{\partial r} r^{2} \sqrt{e} A_{s}^{2} r_{+}=$

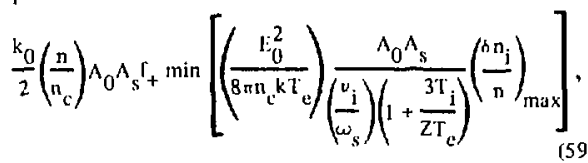

and

$$
\frac{1}{2} \frac{\partial}{\partial r}\left(r^{2} \sqrt{c} A_{R^{t}+}^{2}\right)=0
$$

where $u\left(\mathbf{n}_{\mathrm{d}}\right)$ is the classicul electron-ion collision frequency at the critical density, $v_{i}$ and $\omega_{3}$ are the damping and frequency of the ion waves. $Z$ is the ionic charge, $k_{1\}}=w_{0} / \mathrm{c}$ is the vacuum wave number of the incident light wave. and $\left(\delta \mathrm{n}_{\mathrm{i}} / \mathrm{n}\right)_{\text {mux }}$ is the maximum ion wave amplitude allowed by jon trapping or wavebreaking.

lon temperature is found from iwo ion-energy equations:

$$
\begin{aligned}
1 / 2 n_{i} v \frac{\partial T_{\|}}{\partial r}= & -\left(n_{i} T_{\|}\right) \frac{1}{2} \frac{\partial}{\partial r}\left(r^{2} v\right)-\frac{\nu_{i i}}{2}\left(T_{\|}-T_{\perp}\right) \\
& +\nu_{e i}^{L} n_{i}\left(T_{e}-T_{\|}\right)+\frac{\omega_{s}}{\omega_{0}}\left[\frac{1}{2} \frac{\partial}{\partial r}\left(r^{2} I\right)\right]_{S B S}
\end{aligned}
$$

and

$$
n_{i} v \frac{\partial T_{\perp}}{\partial r}=\frac{v_{i i}}{2}\left(T_{n}-T_{1}{ }^{\prime}+\nu_{e j}^{E_{e j}} n_{i}\left(T_{e}-T_{1}\right)\right.
$$

The terms on the right side of Eq. (61) represent, in turn, expansion cooling, coupling between the
Fig. 3-27. Profiles of background velocity and density to be used in parameter study of Brillouin scattering at various laser wavelengths. Other parameters: $r_{c}=0.24 \mathrm{~cm}, I_{L}=2 \times$ $10^{14} \mathrm{~W} / \mathrm{cm}^{2}, T_{e}=1.5 \mathrm{keV}, T_{1}\left(r_{c}\right)=1 \mathrm{keV}, Z=2$, and $A=$ 4.
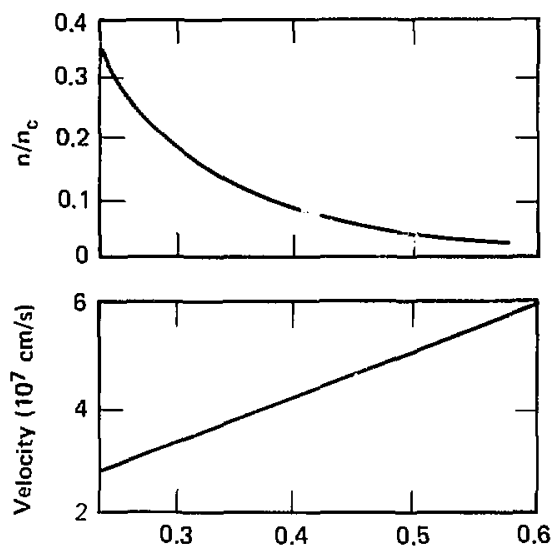

Radius (cm)

parallel and perpendicular ion temperatures at a rate $v_{\mathrm{ii}}$. coupling of energy from electrons al a rate "ti. and parallel ion heating due to Brillouin scallering itsell where I is the scattered light intensity. In the direction perpendicular to the flow velocity $v$ \{f:y. (62)\}, only coupling to ions and electrons contrihutes to $T_{\perp}$ directly.

We have wrillen a small computer code called SBS + IB, to numericaliy solve Eqs. (58) through (62) for fixed background profiles $n_{e}(r), v(r) T_{e}(r)$. and $T_{i}\left(r_{c}\right)$. W'e shall present below results of a preliminary parameter study using SBS + IB, which kept the background profiles constant and studied mechanisms determining the level of Brillouin for different laser wavelengths. For this parameter study we used profiles which might be typical of a large spherical reactor-sized target: $r_{\mathrm{c}}=0.24 \mathrm{~cm}, \mathrm{I}_{\mathrm{L}}$ $=2 \times 10^{14} \mathrm{~W} / \mathrm{cm}^{2} . T_{d}(r)=1.5 \mathrm{keV} . \mathrm{T}_{i}\left(r_{\mathrm{c}}\right)=1 \mathrm{keV}$, $Z=2$. and $A=4$. The velocity and density proliles are shown in Fig. 3-27. Electron density rose abruptly to critical above the density $n_{f}=0.33 \mathrm{n}_{\mathrm{c}}$.

We can use Eq. (61) to estimate when ion heating is expected to dominate over expansion cooling. A dimensionless parameter, $\mathrm{H}$, gives the ratio of the Brillouin heating to the expansion. cooling terms in Eq. (61): 
Fig. 3.28. Profiles from code SBS + IB of ion temperature and ion-wave amplitude $\delta n_{i} / n$, for two different laser wavelengths: (a) long wavelength, (b) short wavelength. Luser is incident from right; the noise level of the scattered wave at critical is $10^{-4}$. For $\lambda_{L}=2 \mu m$, ion heating is strong in the low-density plasma and is responsible for the decrease in $\delta n_{j} / n$ seen at large radii. For $\lambda_{\mathrm{L}}=1 / 3 \mu \mathrm{m}$, expansion cooling dominates over ion heating.
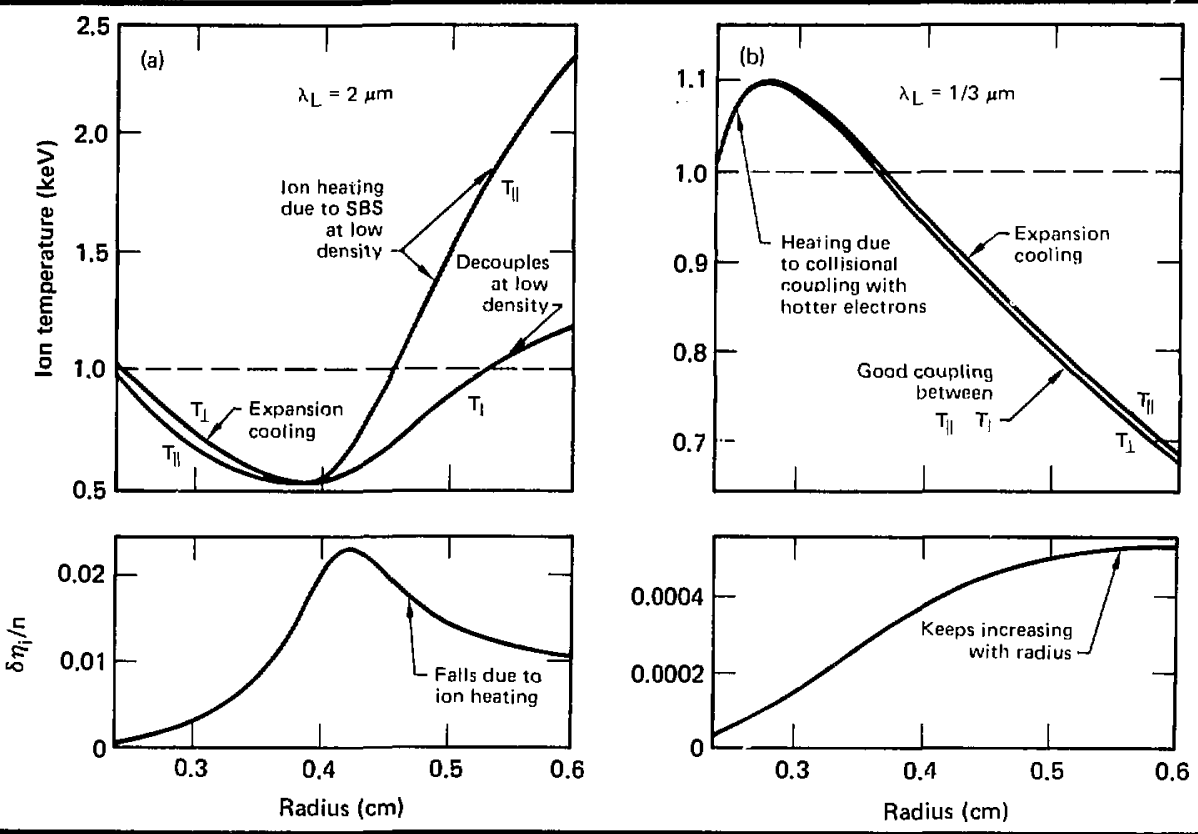

$H=\frac{\frac{\omega_{s}}{\omega_{0}}\left[\frac{1}{2} \frac{\partial}{\partial r}\left(I^{2} L\right)\right]_{S B S}}{(1 / 2) n_{i} v\left(\partial T_{\|} / \partial r\right)} \cong Q\left(\frac{E_{0}^{2}}{4 \pi n_{c} T_{e}}\right)\left(\frac{n_{c}}{n}\right)\left(\frac{Z T_{e}}{M T}\right)$

where $Q$ is the number of Brillouin gain lengths in at distance $r$ characterizing the hydrodynamic llow. $n_{c}$ is the critical electron density. and $M=v / c_{s}$ is the Mach number. A rough measure of the importance of ion heating in limiting the growth of Brillouin scattering is the ratio $\mathrm{H} / \mathrm{Q}$. because the heating rate is only relevant when Britlouin is substantial. This ratio of heating rate to SBS growth is, according to En. (63).

$\frac{\mathrm{H}}{\mathrm{Q}} \cong 4\left(\frac{\mathrm{I}_{\mathrm{L}}}{10^{16} \mathrm{~W} / \mathrm{cm}^{2}}\right)\left(\frac{{ }^{\lambda} \mathrm{L}}{1 \mu \mathrm{m}}\right)^{2}\left(\frac{1 \mathrm{keV}}{\mathrm{T}_{\mathrm{c}}}\right)\left(\frac{{ }_{\mathrm{c}}}{\mathrm{n}}\right)\left(\frac{\mathrm{ZT}_{\mathrm{e}}}{M \mathrm{~T}_{\mathrm{j}}}\right)$.

Ion heating is thus seen to be an important Brillouin limitation process for higher laser intensities $I_{L}$, longer laser wavelengths $\lambda_{L}$, lower electron densities $n / n_{c}$. cooler ions $\left(T_{i} \ll Z T_{e}\right)$, and smaller Mach numers, $M$.

Figure 3-28 illustrates this predicted scaling of ion heating with laser wavelength. showing strong ion heating for $\lambda_{\mathrm{I}}=2 \mu \mathrm{m}$. but only expansion cooling for $\lambda_{1}=1,3 \mu \mathrm{m}$. It is interesting that collisions are frequent enough in the $\lambda_{\mathrm{I}}=1 / 3 \mu \mathrm{m}$ case to maintain good coupling between the parallel and perpendicular components of ion temperature, and even to provide a hit of collisional heating from the hotter electrons near the critical surface. In contrast the two components of the ion temperature are decoupled when $\lambda_{\mathrm{I}}=2 \mu \mathrm{m}$. In the $\lambda_{\mathrm{L}}=2 \mu \mathrm{m}$ case, ion heating at low density causes the size of the ion density perturbation $\delta \mathrm{n}_{\mathrm{i}} / \mathrm{n}$ to decrease at large radii, whereas when $\lambda_{\mathrm{I}}=1 / 3 \mu \mathrm{m}$ the ion density perturbation amplitude is largest at large radii. Despite this ion heating. however, the actual magnitude of the ion perturbation is larger by about a factor of 50 
at $\lambda_{\mathrm{I}}=2 \mu \mathrm{m}$ than at $\lambda_{\mathrm{L}}=1 / 3 \mu \mathrm{m}$. Thus according io our model the total amount of Brillouin refleclivity at $\lambda_{l}=2 \mu \mathrm{m}$ exceeds that at $1 / 3 \mu \mathrm{m}$, despite lla presence of ion heating.

In contrast to ion heating, which is most impurtint at long laser wavelengths and high intenulas. inverse bremsstrahlur.g absorption can limit $\checkmark B Y$ all shorl wavelengths or low intensities. Under ihe latter conditions one can estimate the Brillouin sually lictor. ${ }^{\text {th }} Q$, by calculating the number of stis gain lengths in onc absorption length for insere hremsstrahlung:

$$
\ddots_{i l,} \quad \frac{n}{2}\left(\frac{1 \frac{2}{0}}{8 \pi n_{c} l_{c}}\right)\left(\frac{\omega_{s}}{\nu_{i}}\right) \int_{\infty}^{l} \frac{n(t)}{n_{c}} \frac{\left(d r / \lambda_{L}\right)}{\left(1-\frac{n(t)}{n_{c}}\right)} .
$$

"here $1_{\text {, wh }}$ is the distanec over which the incident thensity is decreased by a fuctor 1 ;e due to inverse bremsstrahlung absorption (10 be more exact one might want to use $2 \mathrm{~L}_{\text {ith }}$ or $3 \mathrm{~L}_{\text {abs }}$ as the upper limit for the integral). Reference 83 discusses this expresion for a llat density profile $n(r)=$ constant. For a nore general profile

$$
n(r)=n_{d}\left(\frac{r_{c}}{r}\right)^{\beta}, r>r_{c} \text { and } \beta>1 \text {. }
$$

one ohtuins. in the strong-absorption limit.

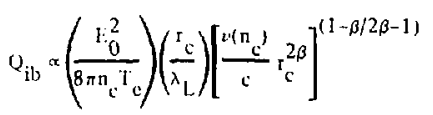

so that the wavelength scaling of the SBS quality factor is

$$
\mathrm{Q}_{\mathrm{ib}} \propto \lambda_{\mathrm{L}}^{(4 \beta-3 / 2 \beta-1)}, 1 \leqslant\left(\frac{4 \beta-3}{2 \beta-1}\right) \leqslant 2 .
$$

Thus when inverse bremsstruhlung is strong. Brillouin scattering is predicted to decrease strongly at short laser wavelengths. Figure 3-29 illustrates this decrease, showing the wavelength-dependence of electric field profiles for the incident and Brillouin-scattered waves; the results were obtained with the code SBS + IB. for the parameters deseribed in Fig. 3-27. The incident electric field
Fig. 3-29. Wavelength dependence of electric field profiles for the incident and Brillouin-scattered waves, for (a) $\lambda_{L}=$ $0.3 \mu \mathrm{m},(\mathrm{b}) \lambda_{\mathrm{l}}=0.5 \mu \mathrm{m}$, (c) $\lambda_{\mathrm{L}}=1.0 \mu \mathrm{m}$. Conditions of the laser and background plasma are described in Fig. 3-27; results are from code SBS + IB. Brillouin is predicted to be much stronger at $\lambda_{\mathrm{L}}=1 \mu \mathrm{m}$ than at $\lambda_{\mathrm{L}}=0.3 \mu \mathrm{m}$, according to this model.

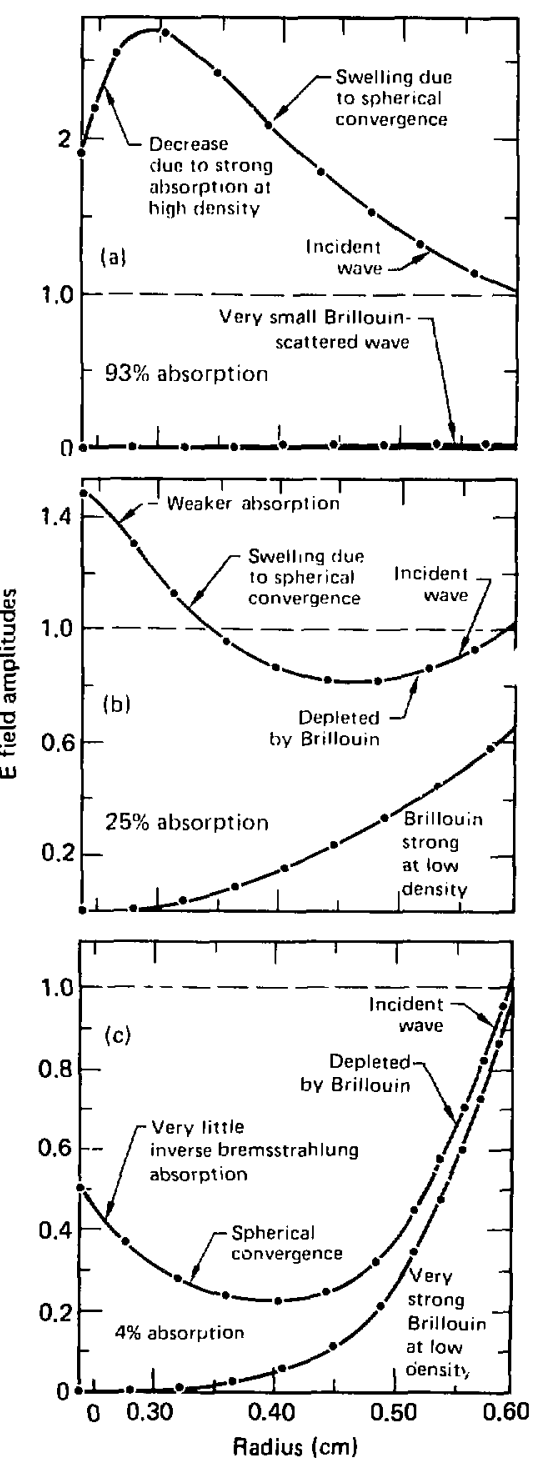


amplitude at $r=6 \mathrm{~mm}$ is normalized to unity. When $\lambda_{\mathrm{L}}=0.3 \mu \mathrm{m}$. there is virtua' no SBS: the Efield amplitude first swells due to spherical convergence and then decreases a bit near $r_{c}$ due to inverse bremsstrahlung absorption. When the wavelength is increased to $\lambda_{1}=0.5 \mu \mathrm{m}$. Brillowin becomes stronger as predicted by $\mathrm{Eq}$. (68). and absorption falls to $25 \%$. Near critical density inverse hremsstrahlung is present, but at a weaker leve] than for $\lambda_{1}=0.3 \mu \mathrm{m}$. When the laser wavelength is increased again to $1 \mu \mathrm{m}$. Brillouin becomes stronger still. and allows only $4 \%$ absorption to oecur. Figure 3-29(c) shows the incident wave so strongly depleted by SBS that litte amplification of E due to spherical convergence can oecur. Our calculations indicate that for these laser and targe parameters, ion heating does not begin to limit SBS until the laser wavelength has exceeded $\lambda_{\mathrm{I}}=1 \mu \mathrm{m}$.

There are several important ways in which the crude SBS model described here could be inproved. The Brillouin model developed recently ${ }^{86}$ for use in LASNFX simulations incorporates the selfconsistent reaction of background conditions into the presence of SBS. a feature crucial for quantitatively accurate modeling. A second important improvement to SBS + IB would be the inclusion of density and velocity-gradient effects ${ }^{87}$ on SBS, and a treatment which illowed some of the light reflected from critical to be a source for Brillouin backscattering. ${ }^{8 x}$ I inally, a parameter study incorporating wavelength variation (as in Fig. 3-29) should include the fact that the background profiles of density and temperature are different at each laser wavelength. We have examined the latter effect by using some L.ISNEX-generated profiles for the laser parameters of Fig. 3-29. More accurate plasmat profiles produced ever more severe Brillouin scattering at long- laser wavelengths. because the plasma profiles tended to be much more extended for long-wavelength lasers, thus providing more underdense plasma wavelengths for SBS to grow in.

\section{Authors: C. E. Max, C. J. Randall, and K. G. Estabrook}

\section{References}

82. C. F. Max and K. G. Estabrook. "Wavelength Scaling in Laser Fusion from a Plasma Physics Point of View." to be published in Comments Plasma Phys. Cont. Fusion.

83. Laser Program Annual Report-1977. Lawrence Livermore Laboratory, livermore, Calif.. UCRL-50021-77 (1978). p. 4-63.
84. D. W. Phillion, W. L. Kruse and V. C. Rupert, Phys. Ret. Letr. 39, 1529 (1977).

85. W. L. K ruer, "Nonlinear Estimates of Brilloun Scalter in Plasmas," Phys. Fluids 23. 1273. 1980: D. W. Forslund. J. M. Kindel. K. 1.ee and B. B. Godires. Phis. Fiuids 22. 462 (1979).

86. K. (i. Fslabroot: and J. A. Harte. ") () Reducing Brillouin Scatter." I.aw rence l.ivernore l.aburatory. I.vermore. Calif., LicRl.82620. Rev. 2. 1979). submitled to Phy Rev lintl.

87. K. Nishikalla and (. S. I.iu. in tdances in Plasma Phisic, vol. f. 1. Simon and w. Thompson. teds (Wiley de Som. Seu lork. 19761. p. 3 .

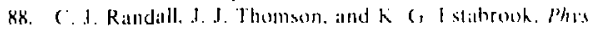
Re' Len 43, 924 (1979).

\section{Enhancement of Stimulated Brillouin Scattering in the Presence of a Critical Gurface}

Previous analyses of stimulated Brillouin scat tering (SBS) have not considered the effects of a partially reflecting boundary. such as the criticaldensity surlace in a realistic plasma expansion. Generally. these analyses have found that the instability exponentiates from some small noise level. $\alpha$, usually taken to be in the range of $10^{-4}$ lo $10^{-2}$ (relative to the ircident power). Thus for Sls to be appreciable. the effective interaction length. which is set by the finite length of the plasmal or by density or velocity gradients. must be much greater than the growth length. We discuss here a mechanism by which a reflecting surface can greatly increase the effective rioise level so that only a lew growth lengths are required for significant scattering. ${ }^{89}$

Consider a homogeneous slug of plasm. of length 1. (Fig. 3-30), moving with respect to a

Fig. 3-30. Schematic representation of sirmple model for SBS: a homogeneous slug of plasma moving with respect to a partially reflecting surface.

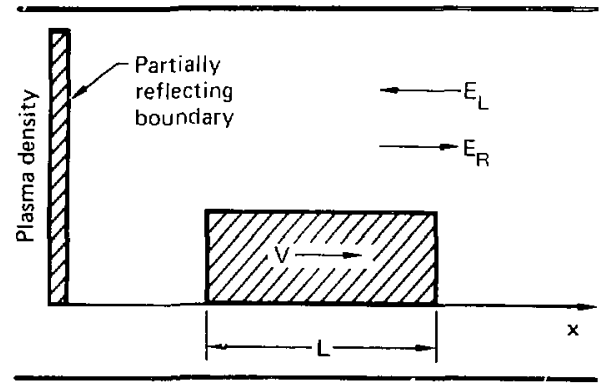


reflecting surface at a speed $v=M c_{s}$ toward the laser. Here, $M$ is the Mach number and $c_{s}$ is the sound speed. In the rest frame of the plasma, the left-going laser light. $E_{l}$, is Doppler-shifted up to $\omega_{1}$. $=\omega_{0}+k_{0} M c_{s}$, where $k_{0}$ is the free-space wave number and $\omega_{0}$ is the laser frequency, while the right-going (reflected) light, $E_{R}$, is Doppler-shifted down to $\left.w_{R}=w_{0}\right)-k_{n} c_{s}\left(M-2 M_{s}\right)$. where $M_{c}$ is the Mach number of the reflecting surface. The beating of these light waves drives an ion wave, $\tilde{n}$. with frequency $\omega_{1}=\omega_{1}-\omega_{R}=2 k_{0} c_{s}\left(M-M_{c}\right)$. The equations for the slowly varying amplitudes of these waves are

$$
\begin{aligned}
& c \sqrt{c} \frac{\partial \mathrm{l}_{\mathrm{L}}}{\mathrm{ix}}=\frac{\mathrm{i} \omega_{\mathrm{u}}}{4} \frac{\tilde{n}}{\mathrm{n}_{\mathrm{c}}} \mathrm{I}_{\mathrm{R}}, \\
& c \sqrt{c} \frac{\partial \mathrm{I} R}{\partial x}=\frac{-i \omega_{0}}{4} \frac{\tilde{n}^{*}}{n_{c}} E_{L}
\end{aligned}
$$

and

$$
\begin{gathered}
n\left[\left(\frac{M-M_{c}}{\sqrt{c}}\right)^{2}+i \frac{v}{2 k_{0} c \sqrt{c}}\left(\frac{M-M_{c}}{\sqrt{c}}\right)-1\right]= \\
\frac{e^{2} n_{0} E_{L} F_{R}^{*}}{2 m M c_{s}^{2} \omega_{0}^{2}} .
\end{gathered}
$$

Here $n_{0}$ and $n_{\mathrm{C}}$ are the background density and critical density, $\epsilon$ is the plasma dielectric constant. $v$ is the ion wave damping rate, and $m$ and $M$ are the electron and ion mass. We assume that the ion waves are heavily damped and so have neglected csañ $3 x$ compar d to vñ in Eq. (71). Substituting for $\tilde{n}$. we find equations for $\left|E_{L}\right|^{2}$ and $\left|E_{R}\right|^{2}$ :

$$
\begin{gathered}
\frac{\partial\left|E_{L}\right|^{2}}{\partial x}=\frac{\partial \mid E_{L} L^{2}}{\partial x}=\frac{n_{0} e^{2} k_{0}}{4 n_{c} \omega_{0}^{2} m_{M c}^{2} \sqrt{\varepsilon}}\left|E_{L}\right|^{2}\left|E_{R}\right|^{2} \\
\\
x \frac{\frac{\nu}{\omega_{s}} \eta}{\left(1-\eta^{2}\right)^{2}+\left(\frac{\nu}{\omega_{s}} \eta\right)^{2}}
\end{gathered}
$$

where $\eta=\left(M-M_{c}\right) / \sqrt{\epsilon}$ and $\omega_{s}=2 k_{0} c_{s} \sqrt{\epsilon}$. The boundary conditions are $\left|E_{L}\right|^{2}=E \delta / \sqrt{\epsilon}$ at the right end of the slab. where $E_{0}$ is the incident vacuum field, and $\left|E_{R}\right|^{2}=\Gamma\left|E_{l}\right|^{2}$ at the left end of the slab. where $\int$ is the reflectivity of the boundary. Solving Eq. (72), we find that the fractional transmissivity. $T$, of the slab is given by

$$
1-T(1-f)=f \exp [T(1-D Q Q(\eta)]
$$

where

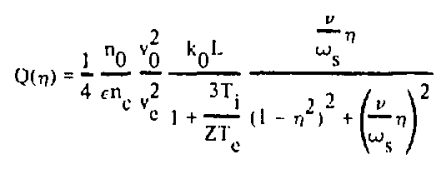

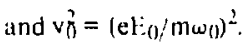

In deriving Eq. (73) we have neglected the light scattered by plasma fluctuations (noise) relative to the light reflected by the boundary, $\alpha \ll \mathrm{fT}$. If there is no reflecting surface ( $f=0)$ then this noise level determines the transmissivity as shown previously. ${ }^{90}$

$$
\tau(1-T)=c \exp [T Q(\eta=1)] .
$$

There are two important new features in Eqs. (73) and (74). First, the quality factor, $2(\eta)$, has at resonance function behavior, reaching a maximum at the resonant Mach number $M=M_{c}+\sqrt{\epsilon}$, and falling off with characteristic width "w, lor other Mach numbers. This behsvior is demonstrated in Fig. 3-31, wheh shows the calculated transmissivity for AURUS'1 simulations of the moving-slug prob'sm. Sccondly, the transmissivity in the presence of a reflecting surface [Eq. (73)] is almost aways much less than that obtained without the

Fig. 3-31. AURLS results for transmissivity, $T$, vs relative Mach number $M-M_{c}$, for the case $Q(\eta=1)=3.6, f=0.7$. Curve (a), $\gamma / \omega_{s}=0.25$; curve (b), $\nu / \omega_{s}=1$. The transmissivities are slightly lower than predicted by Eq. (73), because of transient elfects.

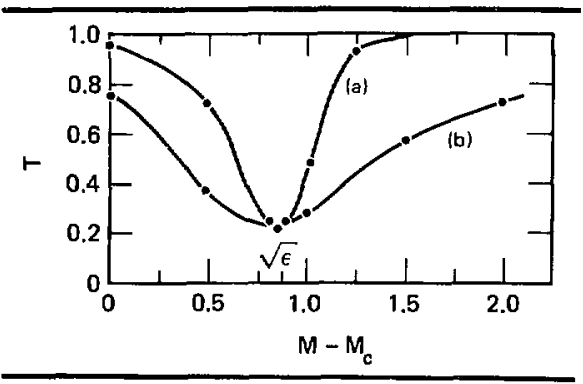


Fig. 3-32. Transmissivity, T, vs $Q$ for exact resonance, $M$ $M_{c}=\sqrt{\epsilon}$. Curve (a) is Eq. (75) with $\alpha=10^{-4}$. Curves (b) and (c) are Eq. (73) with $f=1,0.27$, respectively. The solid circies represent AURUS simulations; squares represents particle simulations. Also shown are AURUS simulations of partially overdense lsothermal expansions (triangles).

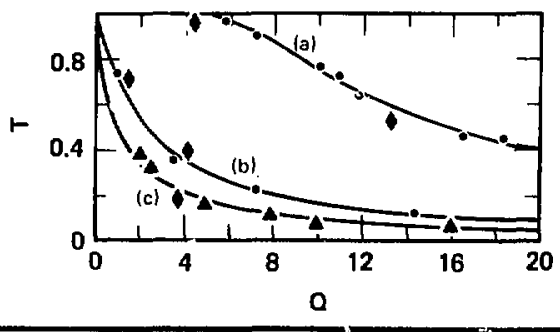

surface $\mid$ Eq. (75)]. Only if at $T \approx 1$ or $Q(\eta) \ll Q(\eta=$ 1) are these transmissivities comparable. In fact, for the ease of a perfectly reflecting boundary $(f=1)$ Eq. (73) becomes particulatly simple: $\mathbf{T}=11+$ $Q(\eta))^{-1}$ (Ref. 89). Figure 3-32 demonstrales this strong enhancement of SBS for various boundary reflectivities.

Out simple moving-slug model (Fig. 3-30) is a good approximation of the case where the critical surface is steepened, either due to the ponderomotive force or to localized heating in a strongly flux-limited plasnia. ${ }^{92}$ We now consider simulations of SBS in an isothermal expansion. which is appropriate for plasma heating with classical heat transport. Part of the plasma is overdense, so that total reflection takes place at the critical surface $(f=1)$. We allow various damping rates and scalc lengths. Even though the resonance condition. $M-M_{c}=\sqrt{\epsilon}$, is satisfied only at a single point, the width of the resonance is rather large, as can be seen from Fig. 3-31; essentially, a scale length of plasma participates in the scattering. Figure 3-32 shows the transmissivity points from the AURUS simulations. It can be seen that they fit the theoretical curve $\mathbf{T}=(1+Q)^{-1}$ very well.

Finally, we consider a simulation that includes ablation from solid density. The target was a glass disk, irradiated with $1-\mu \mathrm{m}$ light at an intensity $10^{15}$ $W / \mathrm{cm}^{2}$. Of the light reaching the critical suriace, $30 \%$ was absorbed ( $f=0.7$ ); this corresponds to absorption due to collective plasma effects (parametric decay, resonance absorption, etc.), and agrees with experimental measurements. ${ }^{93}$ We allowed classical heat transport, but self-consistent ion heating was
Fig. 3-33. Plot of electron density for a fluid simulation including ablation from solid density. Density fluctuations maximize nest the resonance point $M-M_{c}=\sqrt{\epsilon}$.

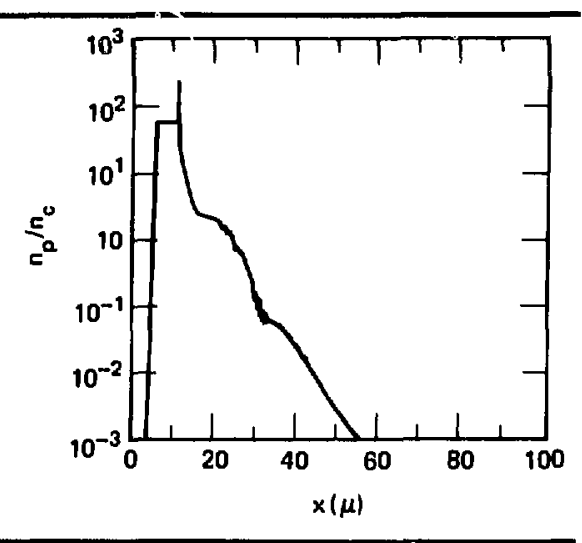

not allowed. Rather, "as set $"=\omega_{\mathrm{s}}$. Figure 3-33 shows the density profile $n_{n} / n_{c}$ all a lime $100 \mathrm{ps}$ into the calculation. The SBS-attive region is $0.1 \leqslant$ $n_{p} / n_{c} \leqslant 1$. The position of maximum scattering is at the point predicted by the resonance eondition. Evaluating $Q$ at this time we find $Q=1.25$. From Eq. (75), for $f=0.7$ the predicted transmissivity is $T$ $=0.51$, whereas the simulation gives $\mathrm{T}=0.48$-certainly adequate agreement.

Authors: C. J. Randall, J. J. Thomson, and K. G. Fstabrook

\section{References}

89. ('. J. Rar Jall, J. J. Thomson. and k, (i. I:slabrook, Phys Ro'v. l.e't1. 43, 924 (1979)

99. D, I. Phillion. W. I. Kruer, and V. C. Rupert. Phys Rev lostt. 39. 1529 (1977).

91. Laser Program innual Report-1978. Lawrence l.ivermore 1.aboratory, I.ivermore. (ulif, UCRL-50021-78 (1979). pp. $3-53$

92. M. D. Rosen et al., Phys. Fluids 22. 2020 (1979).

93. K. Mines. V. C. Rupert. J. M Auerbach, P. H. Y. Lee, and J. F. Swain. Phys. Rev I.elf. 39. 281 (1977).

\section{Calculations of Stimulated Brillouin Scattering with LASNFX}

Last year's annual report outlined the fundamentals of our Brillouin model in LASNEX, and results of the laser wavelength scaling of an 
Fig. 3-34. Absorption and Brillowin reflection vs Jaser wavelength for several intensities. The manber above the libe is the intesaity in $W / \mathrm{cm}^{2}$. (Becaise light that has been reffected by Brillouh may be subsequently absorbed, the fractions of Brilloain scatter and absorption may total more than 1.) The experinental triangles correspond to the absorption of the LASNEX result nearest to it at the aame laser wavelength. Note that the density and velocity gradients have essentially pushed Brillouin below threshold for the $3 \times 10^{14}$ greenlight runs.

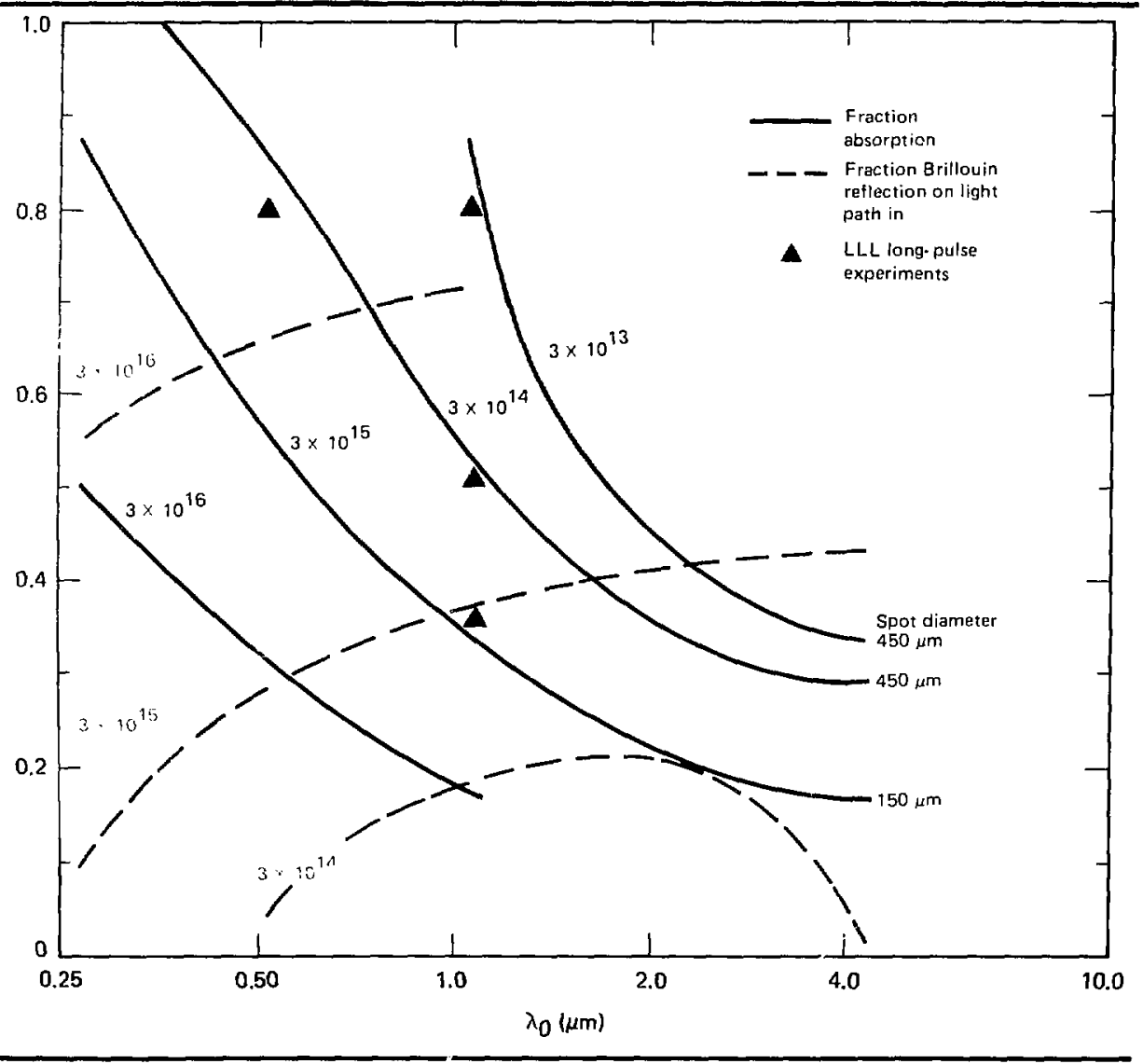

exploding-pusher model. ${ }^{9 \downarrow}$ In the last year, we have incorporaled and done test runs of a number of improvements (described in detail in "Brillouin Backscattering Model in LASNEX" later in this section).

All simulations were run on a gold disk, with laser plise length of 1 ns FWHM and spot diameters corresponding to experiments where available (shown in triangles). LASNEX has multipliers on the $\mathrm{rn}, \mathrm{rv}$, the heated ion density. and the saturated ion density amplitude; all these were set at $l$ in thesc runs. For consistenry, transport inhibition was used in all runs, such that heat was transported no faster than the ion sound speed for $Z T_{e} / T_{i} \gg 1$, which is the case of interest. For $I \lambda_{2}<$ $3 \times 10^{14}$, however, there is experimental evidence that perhaps this is not necessary, as less transport inhibition would mean more absorption.

The generators for these runs were modifications of the one discussed above (in "High-Z Disk Modeling") and were adapted to a treadmill rezoner 
between 0.1 and 1 critical density, giving 10 zones in all. The absorption and Brillouin scattering changed less than $2 \%$ from the coarse-zoned examples.

Figure 3-34 shows the absorption due to inverse bremsstrahlung and resonant absorption (solid lines), and reflection due to Brillouin scattering for light on the way to the target. After the light is reflected by Brillouin. it may

- Be absorbed by inverse bremsstrahlung.

- Be lost due to Brillouin again.

- Escape the target without being affected by either Brillouin or any absorption mechanism.

Conclusion. We have developed a model which predicts the fration of light absorbed $( \pm 10 \%)$ hy gold disks over a wide range of intensities and a factor of two in laser wavelength. We point out. however, that though the model is close to the short-pulse, green-light, French experiments, it underestinates (by 10 to $15 \%$ ) the absorption on $\mathrm{CH}$ disks as found by the long-pulse Shiva experiment:. We have self-consistently included a new inverse bremsstrahlung multiplier ${ }^{95}$ in LASNEX and found that the absorption is then too low. Absorption by ion acoustic turbulence is now being incorporated into LASNEX to find out if we can more clearls resolve the reason why $\mathrm{CH}$ and gold absorb about the same fraction and recover some of the absorption lost to inverse bremsstrahlung."

\section{Authors: K. G. Estabrook and J. A. Harte}

\section{Referenc's}

94. Laser Progrem Anmed Repurt-1978. L aurence I wermure

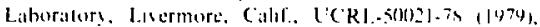
p. 3.6 .3 and p. $3-56$

95. A. B. i.tugdon. Phy. Ra Le't. 44. 575 (1980)

96. R. J. Fitehl ind W. 1. Kruter, Phit. Flusid 20, S: (1977).

\section{Steepening of Ion Acoustic Waves}

The nonlinear evolution of ion acoustic wal: $:$ s is a subject of both theoretical and practical importance. An interesting characteristic of a longwavelength ion acoustic wave is its tendency to steepen. i.c., to gencrate harmonics. Simple arguments ${ }^{97.98}$ indicate that this harmonic generaltion is efficient when the amplitude of the density fuctuation, in. associated with an ion wave with wave number. $k$. sutisfies the condition in $/ n_{n} ;$ $4 k^{2} \lambda_{D e}{ }^{2}$. where $n_{n}$ is the background plasma density and $\lambda_{\mathrm{De}}$ is the electron Debye length. This condition is easily satisfied, for example. by the ion waves generated via Brillouin scaltering in microwave simulation experiments, 99.100 or by the ion wates associated with the electron-ion decal instability in laser-produced plasmass.

We have examined harmonic generation by an ion acoustic wave in a one-dimensional approximation via numerical solutions of the istofluid description of a plasmil. The ion liuid is described by the continuily and lorece equations

$$
\frac{\partial n_{i}}{\partial 1}+\frac{\partial}{\partial x}\left(n_{i} u_{i}\right)=0
$$

and

$$
\frac{\partial u_{i}}{\partial t}+u_{i} \frac{\partial u_{i}}{\partial x}=\frac{\partial c l:}{M} u_{j} u_{i} .
$$

where $n_{i}$ and $u_{i}$ are the density and velocity of the jon fuid. $F$ is the electric field. $Z$ and $M$ are the ion charge and mass. and ${ }^{\prime} i$ is a collision frequency which models landau damping of the ion waves. If we neglect electron inertia and include a small ponderomotive force on the electrons. the analogous equations for the electron fluid reduce to

$$
n_{c}=n_{p} \operatorname{cop}\left(\frac{c c p}{0 c}+a\right)
$$

where $n_{e}$ and te are the electron density and $1 \mathrm{em}$ perature, $n_{p}$ is the unperturbed electron density, of is the electrical potential $(f:=-\lambda \phi$, ax). and $\alpha$ is the ponderomotive potential (normalized to "e, e) that drives the ion acoustic waves. We complete the model vith Poisson's equation:

$$
\frac{i^{2} G}{d x^{2}}=4 \pi n\left(n_{i} \quad n_{i}\right\} .
$$

To investigate the harmonic generation, we expand these equations to second order in the potential $\phi$. and then Fourier-transform: this results in a set of nonlinear coupled-mode equations. By appropriately choosing $\alpha$. we can excite an ion acoustic wave and compute the steady-state spec trum which results.

In the present example, an ion acoustic wave with wave number $k \lambda_{D C}=0.06$ is driven by a ponderomotive force. As the amplitude of this wave in. creases. energy is nonlinearly coupled with its har monics. Finally, the wave reaches the steady-state 
spectrum shown in Fig. 3-35, in which the energy Mousing into the driven wave is balanced by the encrys damped from the entire spectrum of waves. Vilc that a rich spectrum of harmonics is produced. Indeed. the rool-mean-square fluctuation amplitude len become nearly twice as large as the amplitude of the directly uriven ion wave.

Since the harmonics are supported by the dirculy drisen son wave. their generation represents a enh.med danning of this wave. This is shown by ine revilie in | Ig 3-36. where the steady-state am$i^{\prime}$ ade on the driven wave as plotted as a function of the implitude of the ponderomotive foree $(\alpha)$ that whe ihe wive the open cireles are results from the numercial culculations, and the solid line de114tc a 4 imple theoretical estimate that includes the ctleck on enhanced damping la calculation "Whusul the enbanced damping predicts larger icials-utate amplitudes). To obtain this estimate. "Le firs define an effective energy damping rate", as $\therefore 1$, where 1, a a uteepening time. is shown in Ref.

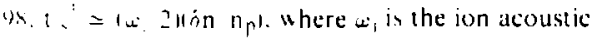
irequenc? The seady-ntate amplitude is then predicted w he

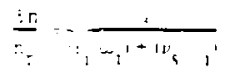

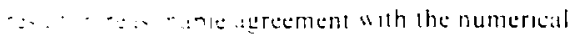

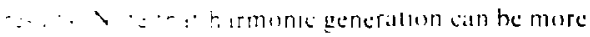

Fig. 3-35. The steady-state spectrum of ion acoustic waves found in numerical calculations in which one wave is driven by a ponderomotive force. In this example, the driven wave hac' 1 wave number $k_{i} \lambda_{1)_{e}}=0.06, v_{i} / \omega_{i}=0.05$ and $a=$ $5 \times 10^{-3}$.

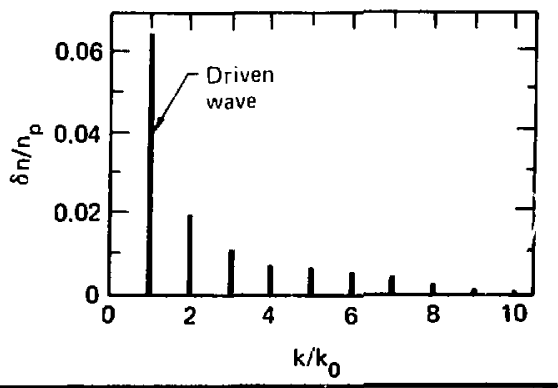

Fig. 3-36. The steady-state amplitude of a driven ion acoustic wave as a fanction of the ponderomotive potentisl that drives it. In these calculations, $v_{i} / \omega_{i}=\mathbf{0 . 0 5}$ and the wave number of the drivea wave is $k_{0} \lambda_{D_{e}}=0.06$.

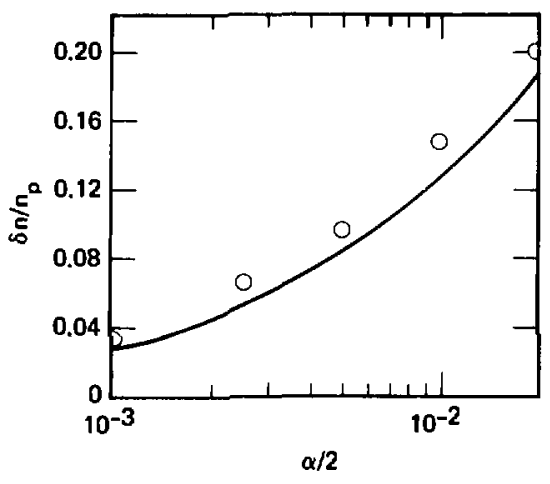

important than the damping of the driven walve when in $\mathrm{n}_{\Gamma}>r_{1} w_{1}$.

In summars, we have investigated harmonic generalion hy an ion acoustic wave. Our preliminars results show that this harmonic generation can atc like enhanced damping and ignificantly reduce the amplitude to which a long-waselength ion wale caln be driven. We need to extend our calculations we further explare the efficienty of the harmonic generation ats a runction of the salecength of the driven wate. and wallow for oher ion wase with wate numbers which are not an inlegral muliple of the wave number of the drisen walve. An important yuestion to he addressed in the role of nonlinear frequency shifts on the harmonic generation. We also plan to describe the ions as partisles, in order to explore the effect of harmonic generation on ion trapping.

\section{Author: W. I.. Kruer}

\section{References}

97. J. M. Dit wom, W. L. Kruter and B. Rosen. Dinamics af lomized Gotwes. M. Laghthill. I. Imill, and H. Satu, Eds. (Chnerstls of Tokson Press, Tohso, 1973). pp. 47 (w) 61.

98. U. L. Kruer and K. (i. Estabrocik. "Nonlintar Behabror af Stimulated Scalter in Large Underdense Plasmas." prepared for the Fifth Workshop on Laser Interaction with Mitter. Untversity of Rochester. Rochester, Nes York. Nowember s 10 9. 1979: also abailathle as Laurence Livermore Lahoratory. Livernore, Calil'., UCR L.8.374.3 $(1979)$. 
99. N. Luhmann, Linucraity of California at Los Angecte, L., Angeles, Calif. prosale communication. (1980).

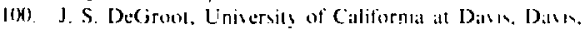
Cillo. protate conmunication (1980).

\section{Simultaneous Self-Focusing and Brillouin Backscattering of Gaussian Laser Beams}

Ion nealting is probably :he major saluration mechanism for stimulated Brillouin scattering (SBS) for long. moderate-intensits latser pulses. "Ii If the ions are heated of $T_{1}-Z T_{0 .}$ the spatial gromih length for elfi-fiocusing becomes comparable to that of SBS (Rel'. 102). so these lan procenses should the considered simultanesusly. Here we carry out an anilysiss of their intcratcion lor arimuthall! s!mmetrie laser be:ams in steady stalle. We assume that the beams are approximately Gausban. (A discussion of the salidity of the Ciasssian beam approsimation for pure self-focusing mat he lound in the next article.) He find that a small amount of SBS can caluse a self-lrapped laser heam lo diverge after undergoing a single focusing event.

He hegin with the equations for the towls varying (in $r$ and /) envelopen of the left-gonn ' and right-going light wases, $E_{-}$and $F_{+}$, the imsindependent plasme! densit!. $n$. and the SB: ion a are, $n$ :

$$
\begin{aligned}
& =2 \mathrm{ikz} \frac{\partial \mathrm{l}+\mathrm{d}}{\partial \mathrm{c}}+\frac{1}{\mathrm{I}} \frac{\partial}{\partial \mathrm{r}}\left(\mathrm{r} \frac{\partial \mathrm{l}}{\partial \mathrm{r}}\right)+\frac{\omega_{0}^{2}}{\mathrm{c}^{2}}\left(1-\frac{n}{n_{\mathrm{c}}}\right) 1 \cdot \frac{\omega_{0}^{2}}{\mathbf{2}^{2}} \\
& \times \frac{\overline{5}^{(*)}}{n_{i}} I_{7}=0 \text {. } \\
& n=n_{0} \exp \frac{-e^{2}\left(11+i^{2}+1 t^{2}\right)}{4 m \omega_{0}^{2}\left(T_{e}+3 T_{i} / 7\right)} .
\end{aligned}
$$

and

$$
2 i k \frac{\partial \bar{N}}{\partial z}+i \frac{\nu \omega_{s}}{r_{s}^{2}} \bar{N}+\frac{\bar{n}}{r} \frac{\partial}{\partial r}\left(\frac{r}{n} \frac{\partial \bar{N}}{\partial r}\right)=-\frac{2 Z e^{2} n k^{2}}{m M \omega_{0}^{2} c_{s}^{2}}+1 .
$$

In these equations $n_{0}$ and $n_{c}$ are the background and critical plasma density, "and "ase the ion-ware damping rate and frequency. $u_{0}$ and $k$ are the laser frequency and lo_al wave number. and $c$ and $c$, are the speeds of light and sound, respectively: in Eq. (80). (*) means "conjugate" for the + equation only. Appropriate to $T_{1}$ - $Z T_{2}$ we take the heal! damping limit of Ey. (82), neglecting the consection and diffration lerms:

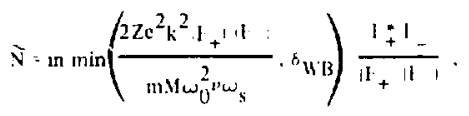

"here we hate included itn upper limit on the SBS ion wals amplitude. on 3 . (1) model tora trapping (ombunne lys. (81)), (81), and (83). we obtain

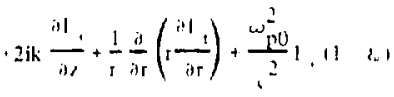

$$
\begin{aligned}
& =\frac{\omega_{p(j)}^{2}}{2 c^{2}} k_{n} \min \left(c_{1}+1+1, n s\right) \frac{1}{1} 1,=11 \text {. }
\end{aligned}
$$

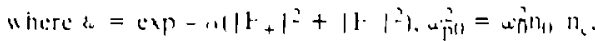
$\therefore=1 \pi+2(1)+i$. and $c=2,=211+r) \mid(1+$

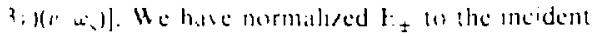
fickd $f_{n}$ with yules alecit! ${ }^{6}$. The electron ther-

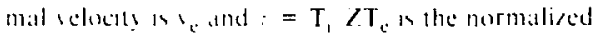
(1) lemperalure. Thu equatom can deserithe the simulansous SBS and self-focusng of laver heams

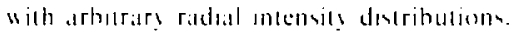

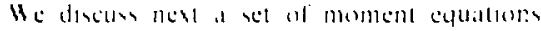

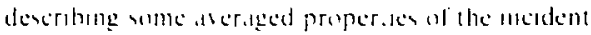
and eallered beam In dersing thene cyuations ne make the spprosm.tlont of replating the SBS xat-

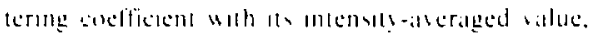

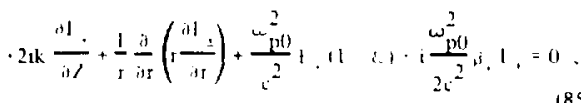

where

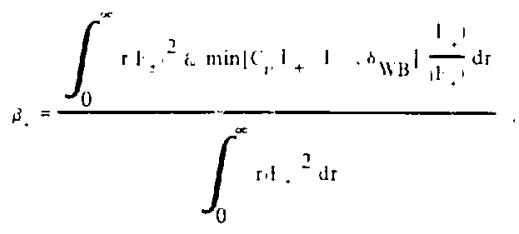

Taking the first wo radial moments of Ec. (8.5) sields equalions for power and mein square radius 


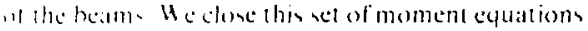
". makm! : cialusian heam approxumallon.

$$
1.11=2,120 \times\left[r^{2} \mathrm{a}^{2}(x)\right] .
$$

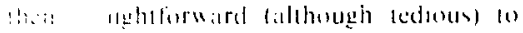

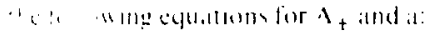

$$
\begin{aligned}
& \left(\frac{2}{2)}+\frac{w^{2}(0)}{2 n^{2} k} \cdot n^{2} \quad 0\right. \\
& \frac{d^{2}+1}{a^{2}} \quad \frac{11}{4} . \\
& \left.1-\frac{1}{2} \frac{2}{2^{2}}\left|1 \frac{1 \text { in }(p)}{p^{2}}\right|\right\} \text {. }
\end{aligned}
$$

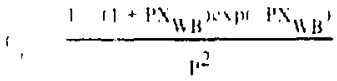

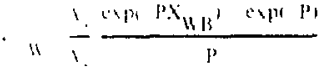

$$
\begin{aligned}
& \operatorname{lin} n \int_{0}^{x} \frac{1 \operatorname{sen}(1)}{1} d x .
\end{aligned}
$$

1901

1911

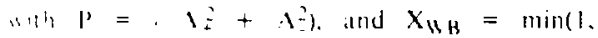
in is $(.1 .1$

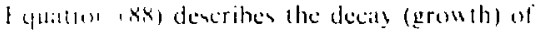
the ancolomt scallered bealm ats it propagites

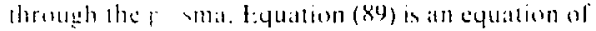
manom fire il roul mean square heam radius: this equalian hat an derived presiously, "os neglectung SBS. The mL ion of SBS hils adoed an explicil -

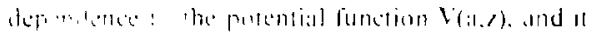
w the new follure which allows presiously trapped heams lo become untrapped in the presence of SBS.

This phenomenort in apparent in the first inlegral of Eq. (91):

$$
\frac{\mathrm{d}}{\mathrm{d} z}\left[\frac{\mathrm{l}}{2}\left(\frac{\mathrm{da}}{\mathrm{d} z}\right)^{2}+\mathrm{v}\right]=\frac{\partial \mathrm{V}}{\partial \mathrm{z}}=\frac{\omega_{\mathrm{po}}^{2}}{2 \mathrm{c}^{2} \mathrm{k}^{2}} \frac{\mathrm{d}}{\mathrm{dP}}\left[\frac{\mathrm{Lin}(\mathrm{P})}{\mathrm{P}}\right]_{\mathrm{a}^{2}} \frac{1}{\mathrm{~d} z}
$$

Fig. 3-37. Numerical solution of Eq. ( 103) (neglecting SBS), Plotted is $\mathrm{rms}$ beam radius, $a$, vs axial distence, $z$. Parameters are $V_{0}^{2} / Y_{c}^{2}=0.1, m_{0} / n_{c}=0.2, \tau=1, \operatorname{ka}(z=0)$ $=50, d u / d z(z=0)=0$.

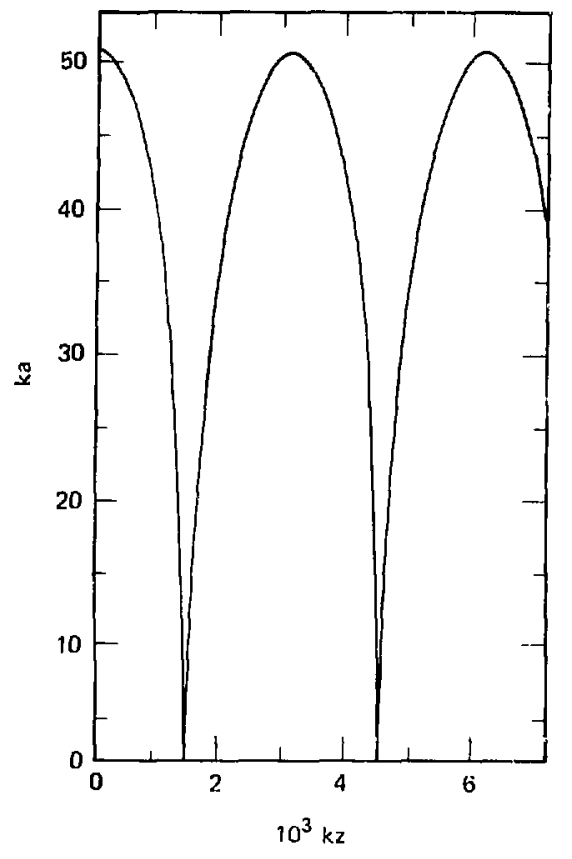

In the abserce of SBS, it $2 P$ is at constant. Thus the tual "energe!" of the heam $\mathrm{t}:=12(\mathrm{da} d /)^{2}+V$ is consersed. It a beam enlers the plasmat with $\mathrm{F}<0$ then $f$ will aluays be negallive and the heam will be trapped. with its rma radius oscillating nonlinearly at shown iri Fig. 3-37. In the presence of SBS, howerer, $\partial \mathrm{V}$ a $=\mathrm{dE} \cdot \mathrm{d} />0$, so that the beam must eventuall? escape, i.e. diverge indelinitely as $\mathrm{E}$ becomes positive.

An example of this behavior is shown in Fig. 3-38 Physically. the sciallered light beam helps to deplete the plasma density so that the incident beam acquires more inward radial momentum per unit intensity then it would in the absence of SBS. Then. as the incident light approaches the focus. scattering rises rapidly due to the increase in intensity. After passing through the focus the incident light intensity is decreased due to SBS. The incident beam does not deplete the plasma density enough for refraction to overcome the large radial momentum it acquired 
Fig. 3-38. Same parameters as Fig. 3-37, except that SBS is allowed; $\delta_{\mathrm{WB}}=0.1,1 / \omega_{\mathrm{s}}=1, \mathrm{~A}_{-}^{2}\left(\mathrm{kz}=\dot{\alpha}(00)=10^{-1} \mathrm{~A}_{+}(\mathrm{kz}\right.$

$=6000$ ).

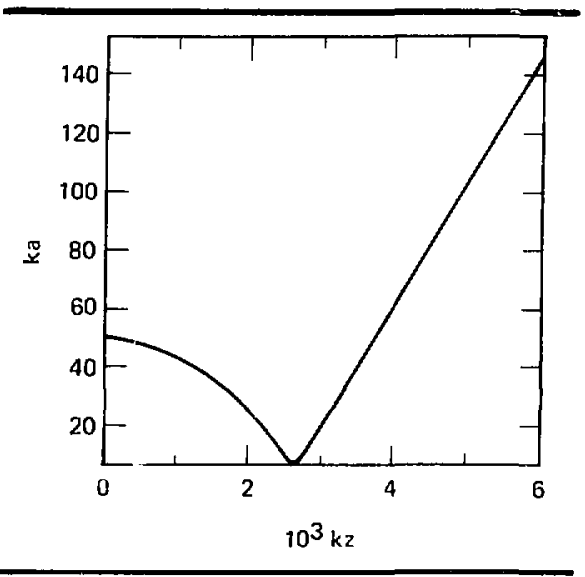

during its hight towards the focus, in a result the beam diverges inderinitely after a single focus.

The simple moment equations derived abose suggest a qualitatrely new feature of self-focusing when SBS is included. The equations, however. give only very approximate descriptions of the system s true behavior: more quantitative results require full numerical solution ol $l: q .(8+1)$.

\section{Author: (. J. Randall}

\section{References}

101. Laser Program lamsal Report-14

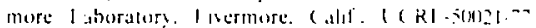

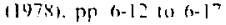

102 laser Program thmal Repert-14\%. I as rente I ber

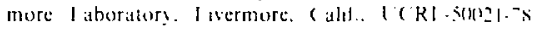
(1979). pp .3-36 10 3-42.

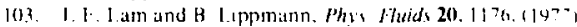

\section{Spatial Structure of Filamented Light}

Self-focusing. or filamentation. may become an increasingly dominant effect in present-day and future experiments. In contrast to modeling of earlier short-pulse experiments. we now envision beams traversing $i 0^{3}$ to $10^{-1}$ free-space wavelengths of underdense plasma. and reactor targets as large as a centimete in diameter

To investigate the spatial structure of selffocused light, we solve numerically a Schrödinger equation in cylindrical (r./) geometry with an exponential nonlinearil!, which deseribes the filamenlation of laser light in anderdenst plasmas due $w$ the ponderonotive loree. This work complements and extends presious andytic descriptions $114-106$ of selt-focusing in underdense plasman.

To derise the equation we want wo solve. we start whthe wate exuation

$$
r+2.11, \quad r^{3} \quad \frac{2}{2}, \quad \frac{1}{2} \cdot \frac{2}{2}
$$

for the laser-light electrof field acelor I propalgating

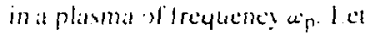

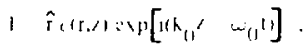

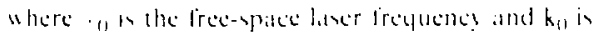

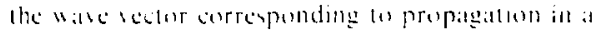

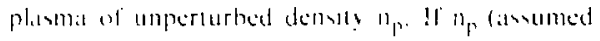

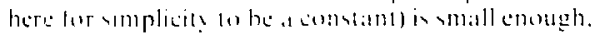

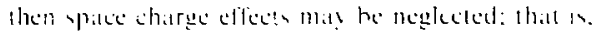

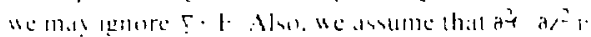

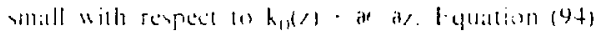
then hecomsen

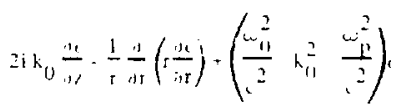

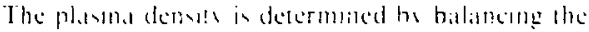

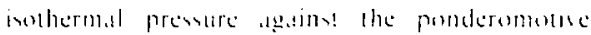
lorce: ihen

$$
u \frac{4 \pi c^{2}}{m} n_{n} \cdot v\left(\frac{1}{4} \frac{0^{2}}{n_{11}^{2}}\right) .
$$

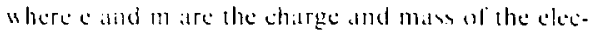

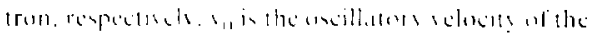
electron $1 ! 1$ the latser electere tield. with $\left|1_{11}\right|=$ c|e| musi: and $s_{\text {in }}$ is the clectron thermal velocis!

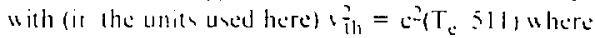
$\mathrm{T}_{\text {. }}$ is the elesten planmat comperature esprened in kiv.

The differencing setheme we use to selve tys. (96) and $(97)$ in chosen 10 preserse the consers ation properties of the Schrödinger equation. Variable 
zoning in both $\mathrm{r}$ and $\mathrm{z}$ leads to economy in code size and running time. The Crank-Nicholson scheme is used to advance $c(r, z)$ in $z$, and the nonlinearity is obtained by iteration. The flux is monitored as a check of the numerical scheme. The validity of the paraxial approximation as the beam focuses is checked by comparıng the magnitude of the second derivative, $a^{2} \epsilon / \partial z^{2}$, to the first derivative, $\left|\mathrm{k}_{0}\right| \cdot \mid \mathrm{at} / \mathrm{a} / \mathrm{s}$.

To compare to earlier work, ${ }^{104-106}$ we lirst discuss the self-focusing of an initial Gaussian pulse. In order to solve Eqs. (96) and (97) analyticully. the assumption is mide that the beam remains a Gaussian as it fo. uses. An advantage of the numerical solution is that no such assumption is necessiary.

Although difierent methods of solution are used in Refs. 104 to 106, the qualitative description is the sume. Above a rather low threshold power, $P_{c}$. there are self-trapped solutio is: for stationary selftrapped solutions the filament radius maty be as small as several free-space wavelengths. Numerically, we find that for a given Pcour work verifies the expression for the radius of the stationary selftrapped filanient given in Rel. 106. For powers above this critical power. houever, the numerical solution predicts very different behavior than that oblained in the anilytic solution. The beam does nol retalin its (iaussian sliape, and focuses in a shorter distance than predicted analytically.

As an example, we describe a numerical simulation for $P / P_{c} \sim 11$. Here, $v_{0} / v_{t h}=0.1$ and, if $b$ describes a lo-keV plasma, then the curresponding laser intensity is $2.4 \times 10^{14} \mathrm{~W} / \mathrm{cm}^{2}$ for 1.06- $\mu \mathrm{m}$ laser light. The initial shape is $\mathrm{e}(\mathrm{r}, \mathrm{r}=0)$ $=t(r=0,1=0)$ exp $\left(-r^{2}: \sigma^{2}\right)$ and $k_{1}{ }^{\pi}=700$. Also, $n=$ $0.1 n_{c}$. where $n_{c}$ is the critical density (the density at which the plasmal frequency equals the laser frequency).

Our results are illustrated by the flax contours of Fig. 3-39. These contours are chosen to give equal spitcing for a Gaussian beam. This shape is lost as the inner portion of the beam focuses down to a very narrow lilament. The power contained in this central inner portion is approximately the critical power-an intuitively pleasing result.

After this first focus, rings begin to appear beyond the central inner portion. The second max-
Fig. 3-39. Flux contours for an initial Gaussian profile with $\left|v_{0} / v_{t h}\right|=0.1$ and $k_{0} \sigma=700$. If the bean had retained its Gaussian shape, these contours would have remainied equally spaced.

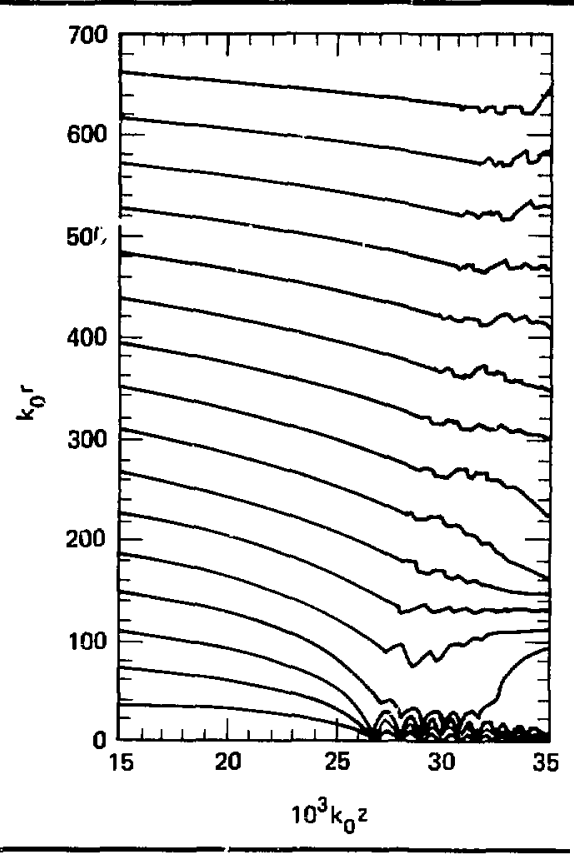

imat at $100 \leqslant k_{0}, r \leqslant 200$ is evident in these flux contours. The appearanee of rings beyond the first focus was first found in numerical work by Marburger and Dawes ${ }^{107}$ for a nonlinearily of the form $\left.|E|^{2} /\left(|+| E /\left.E_{s}\right|^{2}\right).\right]$

A snapshol of $\left|v_{0} / v_{t h}\right|^{2}$ vs $k_{0} r$ be jond tbe first focus is shown in Fig. 3-40. Note the evident nonGaussian nature: the maximum intensity is not on the beam axis at this point in $z$. Similar snapshots at any point in $z$ always show this well-defined central portion and a long tail: the Gaussian initial shape is not maintained during the beam evolution. Figure 3-41 shows $\left|v_{0} / v_{\mathrm{Lh}}\right|^{2}$ it $r=0$, vs $z$. Notice that after first focus at $k_{0} z=26700$, the intensity oscillates about a large value, never returning 10 its initial low value.

We compared our results with those of the analytical theory with the Gaussian shape ansatz by numerically integrating Eq. (26) of Ref. 106. The results for $\left|v_{0} / v_{\mathrm{th}}\right|^{2}$ as a function of $\mathrm{z}$ are indicated 
Fig. 3-40. Plot of $\left|v_{0} / r_{0}\right|^{2}$ vs $k_{0} r$ at $k_{0} z=29000$ for the initial Gaussian of Fig. 3-39. This snapshot is taken beyond the first intensity maximum.

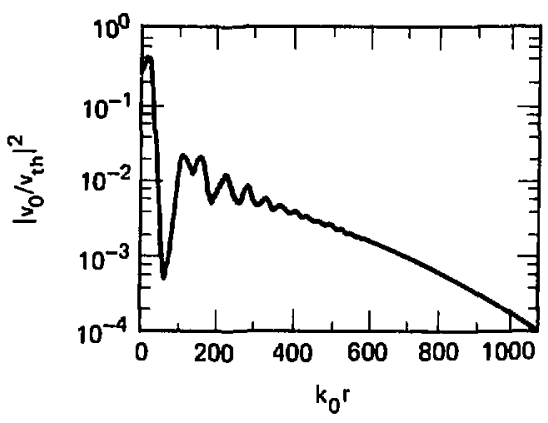

Fig. 3-41. Plot of $\left|v_{0} / v_{t h}\right|^{2}$ at $r=0$ as a function of $k_{0} z$ for the initial Gaussian of Fig. 3-39. The solid curve is the code result with first focus at $k_{0} z=26700$. The dotted line is the analytic result with the Gaussian shape assumption.

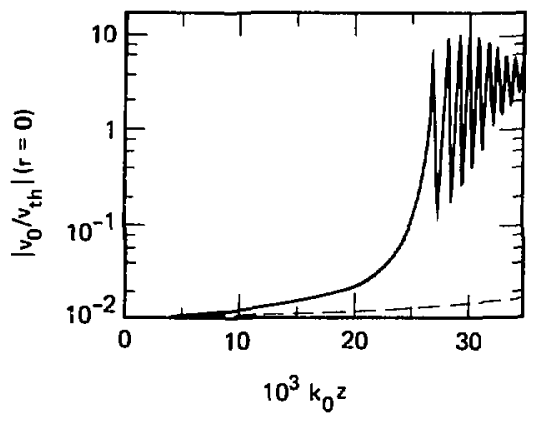

by the dotte line in 1:ig. 3-41. These analytic results are much too low and the resulting distance to first focus is more than iwice that found without the Gaussian shape assumption. At first focus. however. the analytic model has even sharper behavior than it numerical solution. The peak value of $\mid v_{0} / v_{t h}{ }^{2}$ is $1.5 \times 10^{2}$ and the resulting beam radius is less than the beam wavelength. According to the analytic model. the beam then oscillates by continually relaxing to its original state and refocusing. Thus the analytic model as developed in Ref. 106, with its Gaussian-shape ansatz. does not reproduce even the qualitative behavior found in the numerical solution, whether
Fig. 3-42. Plot of initial $\left|v_{0} / v_{\text {ti }}\right|^{2}$ vs $k_{0} r$ for the ring beam (solid curve) and a Gaussian of the same fux (dotted curve).

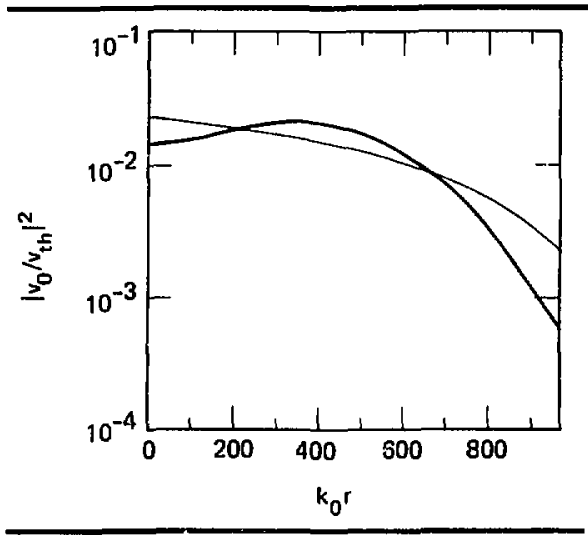

well before first focus, at lirst focus, or beyond the focus.

We should add a note of caution about the sharp behavior at first focus in our numerical results. There are several simplilications and assumptions in the derivation of Eqs. (96) and (97): steady state, cylindrical symmetry, slow variation with respect $10 \%$ scalar rather than vector optics, and no back-reflected light.

A : wovious advantage of the numerical solution is the ease with which we can study the propagation of initial pulse shapes other than Gaussian. To date we have tried "super-Gaussians" [ $\alpha^{-(r}$ " $\left.)^{4}\right]$ and "ring" beat.ns: we find rather similar behavior for both. For the initial profile of Fig. 3-42.

$$
\epsilon(\mathrm{r}, \mathrm{Z}=0)=\epsilon(\mathrm{r}=0)\left[1+2\left(\frac{\mathrm{I}}{\sigma}\right)^{2}\right] \exp \left[-\left(\frac{\mathrm{I}}{\sigma}\right)^{2}\right] .
$$

with $\mathrm{k}_{0}{ }^{\sigma}=500$ and the central intensity corresponding to $v_{0} / r_{t h}=0.12$, or $3.4 \times 10^{14}$ $\mathrm{W} / \mathrm{cm}^{2}$ at $10 \mathrm{keV}$ for Nd:glass. The flux contours are shown in Fig. 3-43. Again, these flux contours were chosen to give equal spacing for a Gaussian beam, but there is little evidence of Gaussian-like propigation.

The ring beam reaches first intensity maximum it $k_{0}{ }^{2} \sim 16000$. A Gaussian beam with the same 
Fis. 3-43. Flux confours for the ring beam described in Fig. 3-42.

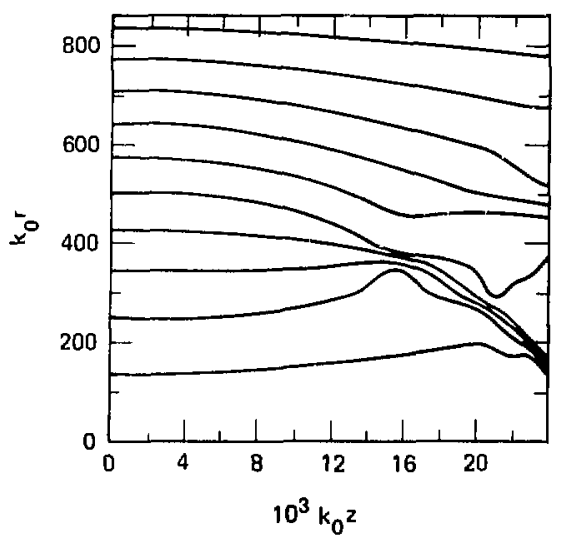

puser $\left(v_{0} / v_{11}=0.146, \mathrm{k}_{0} \sigma=922\right)$ reaches first focus further downstream, at $k_{0} z \sim 21000$. Also, the maximum intensity at first focus is a factor of five larger for the Gaussian bean.

After the "first focus" of the ring bean other rings appear, and these maxima slam into the center. This singularity at $r=0$ is a consequence of the cylindrical-symmetry assumption used in deriving Eq. (96). If aximuthally-dependent behavior were included, we would expect these rings to break off in'o sinall-scale filaments.

A central issue in the study of filamentation in i: $:$ an laser fusion applications is its competition with other processes in the underdense plasma. As a starl boward understanding this competition, we have included the effects of inverse bremsstrablung absorption with its densily-dependence in our code. The absorption length is assumed to be larger than the distance to first focus without absorption.

Nol surprisingly, this finite absorption length results in longer distances to first focus and lower maximum incensities. But note also that as the beam focuses, the density is lowered and the absorption rate decreases. Therefore, the behavior of the most intense part of the beam is qualitatively unaffected by the inclusion of an absorption length greater than the distance to first focus.

To apply these results to lasei-plasma experiments, we point out the scaling properties of Eq.
(95). If the density changes by a factor of $t$, then adjusting the radial size by $\mathrm{a}^{-2}$ and changing the propagation distance, $z$, by $k_{0}(n) / \alpha k_{0}(\alpha n) \sim 1 / \alpha$ regains the original equation. Thus one numerical example encompasses a family of results.

\section{A thors: B. F. Lasinski and A. B. Langdon}

\section{References}

104. C. E. Mix, "Strong Sell-focusing Dut to the Ponderomorive Force in Plasmas," Phys. Fiwids 19, 74 (1976).

I05. J. F. Lam, B. Lippman, and f: Tappert, "Self-Irapped Laster Beims in Plasmia," Phys. Fhideds 211, 1176 (1977).

106. 1). Anderson and M. Bonnedal, "Variational Approach tes Nonlinear Self-Foeusing of Gaussiau L_ister Beams," Phis. Fluids 22, 105 (1979).

117. J, H. Marburger and E. Datwes. "Dynamical Formation of a Small-scale Filamenl," Phys, Re1. LeIt. 21, 556 (1968).

\section{Update of Model for Ablative Laser Fusion Implosions}

A few years ago 108 we described our preliminary work on a model for ablative laser fusion implosions. Here we present an updated and expanded version of that model. which includes a more detailed description of the density jump at the critical surface.

There is now a rather complete literature describing scaling laws for laser fusion targets of the exploding-pusher variety, ${ }^{109}$ in which heat is carried dominantly by hot suprathermal electrons. In the present section we derive scaling laws for a different type of laser fusion target, called an "ablative" target. In ablative implosions hot electrons are not important to the overall energy flow. Rather, the inward flow of energy is via thermal conduction, and is roughly balanced by the outward flux of plasma energy and PdV work from the ablation surface. To conserve momentum the remaining cold material must be accelerated inward, or implode.

We shall present simple expressions describing the ablation pressure, critical radius, and ablation rate for spherical ablative targets. These quantities are functions of the laser intensity, laser wavelength, and target size. Effects of inhibited heat transport of a magnitude inferred from a large body of experimental data ${ }^{110}$ are explicitly included. Previous work on spherical laser-driven ablation was largely computational rather than analytic, "II exploring the regime where heat transport inhibition is not important. ${ }^{112.113}$ 
Although the present model has been developed for spherical geometry. Ref. 114 shows that it may be applied with some success to planar targets, by setting the effective ablation radius roughly equal to the spot diameter in a planar ir radiation.

The Ablative Flow. Classical expressions 115 for conductivity, $\kappa$, and hea! flux, q. are valid when the scale length for temperature variations, $L_{T}{ }_{T}$. is much longer than the electron-ion mean free path. When gradients are so steep that $L_{T}$ becomes less than at mean free path. the classical expression for $y$ implies that the characteristic speed for heat flow is much faster than the electron thermal speed. This seems physically unreasonable. at least for electron distribution functions close to Maxwellian. $A$ common remedy has been to postulate an upper lintit an the heat flux in this regime. Frequertly one expresses this "saturated" riagnitude of the heal flux, $4_{\text {ial }}$. in terms of the electron thermal speed $v_{t c} \equiv$ $\left(k T_{\mathrm{e} / \mathrm{m}}\right)^{\prime}$ ?

$$
u_{s a t}=f\left(n_{e} k T_{e}\right) v_{t e}=5 \phi \rho c^{3} .
$$

Here $n_{c}$ is ti:: number density of electrons. $f$ is the flux limit as usually defined, $\phi$ is the flux limit scaled to hydrodynamic variables, and $\mathrm{c}=(\mathrm{p} / \mathrm{p})^{12}$ is the isothermal sound speed. where $p$ and $p$ are the pressure and density.

At present the appropriate value for the flux limit. $f$, is uncertain. Interpretation of a variety of laser-plasma interaction experiments ${ }^{110}$ has been possible only when $f$ is assumed to be approximalaly' 0.03 . Work is in progress 10 understand the size of the flux limit $f$ and the mechanisms responsihle for inhibited energy transport.

In the work below, w'e will take the electronhest flux to be the minimum of the classical and saturated values. We leave $f$ as a parameter in our solutions: our only restrictions are that $f$ be constant in space, and, for the analytic work, that $\int \gtrless 0.4$ for a D-T plasma.

A typical temperature profile. $T(r)$. for a laser fusion target has a high temperature at the critical radius, and a low temperature at the ablation surface. As a result, one can show that conduction is typically classical near the cold pellet surface. $R_{i 3}$, whereas it may be saturated near the critical surface.

The overall flow is obtained by solving separately in the classical and saturated regimes, and then matching the solutions at the point where the classical and saturated heat flows are equal (details of the derivation not presented here are discussed in Refs. 111 and 116). The equations to be solved are mass conservation.

$$
\dot{\mathrm{m}} \equiv 4 \pi \mathrm{r}^{2} \rho \mathrm{v}=\text { constant }
$$

the equation of motion lor the gas.

$$
\rho v(d v / d r)=-d p / d r, p=\rho c^{2} .
$$

and the entrgy equation.

$$
\left.I^{2}(\mathrm{~d} / \mathrm{dr}) r^{2}\left[\rho v\left(v^{2} / 2+5 \mathrm{c}^{2} / 2\right)+\underline{\mathrm{q}} \cdot \hat{\mathrm{r}}\right]=\right] \mathrm{b}\left(\mathrm{r} \quad \mathrm{r}_{\mathrm{c}}\right) .
$$

$110^{n}$

In these equations $v$ is the radial thow velocity and I is the ahsorhed laser inlensity at the eritical surface. For this work we assume the electron and ion tem. peratures to be equal: extension to separate values of $T_{\mathfrak{e}}$ and $T_{i}$ is straightforward. $1 / 6$ The pressure is given by $\mathrm{p}=\mathrm{n}_{\mathrm{c}} \mathrm{T}_{\mathrm{c}}+\mathrm{n}_{\mathrm{j}} \mathrm{T}_{\mathrm{l}} \equiv \mathrm{nkT} / \mu$. The mean mass per parti le is $\mu \equiv A m_{p} /(7+1)$. where the ion mass and charge are $\mathrm{Am}_{\mathrm{P}}$ and $Z \mathrm{C}$. The critical surface for a laser of frequency $\omega$ lies at the radius $r_{0}$ where the electron density is $\mathrm{n}_{\mathrm{c}} \equiv \mathrm{m}_{\mathrm{c}} \mathrm{w}^{2} /\left(4 \pi \mathrm{c}^{2}\right)$. It is assumed that the laser deposits its energy at $r_{c}$. We are thus neglecting absorption in the underdense plasma ( $r>r_{c}$ ). Wi also assume that the latser light pressure is negligible. Finally, we assume a quasisteady state. We discuss the validity of these assumptions helow and in the following arlicle.

Inside the pellet surfice l:q. (102) may be integralted to ohtain

$$
(5 / 2) n i c^{2}\left(1+.1^{2} / 5\right)+4 \pi r^{2} q \cdot \hat{r}=A=\text { constant } .
$$

where $i=4 \pi r^{2} p$ is the mass loss rate and $M=v ; c$ is the isothermal Mach number. The constant of integration $\Lambda$ is the difference hetween the outward enthalpy flux and the inward conductive heat flux. For subsonic ahlation of an unpreheated puiet. both terms are very small at the pellet surface, so that $A=0$. Reference 111 shows that this approxinlation is an excellent one in the regine in question.

In the classical region close to the pelles surface. Eq. (103) can be solved analytically in the limit $\mathrm{M}^{2} / 5 \ll 1:$

$$
T / T_{c}=\left(\dot{n}_{1} / \dot{m}_{c}{ }^{2 / 5}\left(1-R_{i} / I\right)^{2 / 5},\right.
$$


Fig. 3-44. Dependence on $\cdots$ Jimit, $\phi$, of $M_{b}$, the Mach number in the saturated regin; and $r_{\text {, }}$, the radius where the saturated region begins. The ablation radius is $R_{n}$.

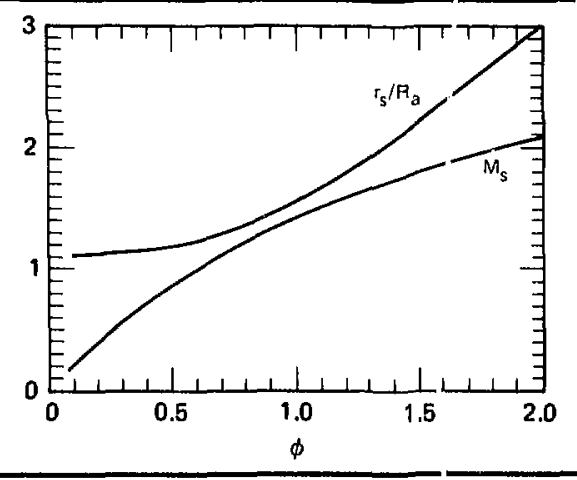

where $\dot{m}_{\mathrm{c}} \equiv 16 \pi \mu k\left(\mathrm{r}_{\mathrm{c}}\right) \mathrm{R}_{\mathrm{a}} / 25 \mathrm{k}$ is the ablation rat: that would obtain if the classial region extenr'ed out to the critical surface. In fact. the heat flux reaches the saturated value in 84 . (99) at some radius $r_{\mathrm{s}}<r_{\mathrm{L}}$ -

In the salturated region $\left(r_{s}<r<r_{c}\right)$, the energy equation is simply $M_{3}\left(1+M_{3}^{2} / 5\right)=2 \phi$ where $\phi$ is the renormalized flux limit delined by $4 . a \mathrm{l} \equiv 5 \phi p \mathrm{c}^{3}$. We conclude that the Mach number in th saturated rone, $M_{3}$ is constant and depends only on the flux limit $\phi$ (see r.ig. 3-44). The flow is super sonic in the saturated wone for $\$>0.6$. The monentum and continuity equations yield

$$
T=T_{c}\left(\frac{I}{r_{c}}\right)^{4 / 1+M_{s}^{2}}{ }_{, \rho=\rho_{1}}\left(\frac{I}{I_{c}}\right)^{2}\left[2+M_{s}^{2} / 1+M_{s}^{2}\right]
$$

in the saturated region, where $\rho 1 \equiv m\left(r_{-}-\delta r\right)$.

The dynamics in the classical zo te are discussed in Ref. 115. Briefly, the Mach number increases monotonically. reaching $M_{5}$ at the point $\tau_{s}$; hence the assumption that $M^{2} \ll 5$ made in deriving Eq. $(104)$ is approximately valid for $\phi ₹ 1\left(M^{2} ₹ 2\right)$. For larger values of $\phi$ the energy and momentum equations do not decouple, and they must be solved simultaneously 112: there is a family of subsonic solutions $\left(M_{s}<1, \phi \approx 0.6\right)$ and a unique transsonic solution.

The ablation rate $\dot{m}$ can be obtained if the above equations are supplemented by the jump conditions at the critical surface. Mass, nomentum. and energy conservation imply $[\rho v]=0,\left[\rho v^{2}+\rho c^{2}\right]$ $=0$, and

$$
\left(\dot{\mathrm{m}} / 4 \pi \mathrm{r}^{2}\right)\left[v^{2} / 2+5 \mathrm{c}^{2} / 2|+| q\right]=1
$$

where $\{y\} \equiv y\left(r_{c}+\delta r\right)-y\left(r_{c}-\delta r\right)$ for any variable $y$. Since the temperature is a maximum at $r_{c}$ where the laser energy is deposited, the jump in the heat flux at $r_{c}$ is $\left.[q] \quad 5 \phi(p)+\rho 2\right) c_{c}^{3}$ provided the heat flux is saturated on both sides of critical (the ease of classical heat flow for $r>r_{c}$ is analyzed in Ref. 111). llere $\rho_{1.2} \equiv \mu\left(r_{c} \pm \delta r\right), c_{c} \equiv c\left(r_{c}\right)$ and $T$ is assumed continurms. liquation (106) then gives

$$
k T\left(r_{c}\right)=\mu\left(1 / 10 t \rho_{c}\right)^{2 / 3} \mathrm{H}^{2 / 3}(\phi),
$$

where the function 11 is derived in Ref. 111 and is plotted in Fig. 3-45, and $I$ is the laser intensity at the sritical surface.

Scaling Laws. We have now described solutions in the classical zone near the ablation surface, and in the flux-limited region inside the critical surface. Next the global structure of the flow and its macroscopic properties are found by matching these separate regions together.

The classical and saturated solutions within $r_{c}$ must match onto each other at the point $r_{s}$ where the classical and saturated heat flows are first equal. ${ }^{111116}$ For subsonic flow $\left(\mathrm{M}_{\mathrm{s}}<1, \phi \geq 0.6\right)$ one finds $r_{s}=R_{i s}\left(11+M_{s}^{2}\right) / 10$. For $\phi>0.6 . r_{s}$ that radius where the trans-sonic solution for $M(r)$ passes through $M_{\mathrm{s}}: M\left(r_{\mathrm{c}}\right)=M_{\mathrm{s}}$. The resulting function $r_{s}(\phi) / R_{i a}$ is plotted in Fig. 3-44.

Fig. 3-45. Dependence on fitx limit, $\phi$, of $(a)$ the function $H(\phi)$ deflned in Eq. (107), ano of (b) the ratio $\rho_{1} / \rho_{c}$. which represents the size of the density jump at critical. Herz $p_{\mathbf{l}}=$ $\left(r_{c}-\delta r\right)$, and $\rho_{c}$ is the critical density.

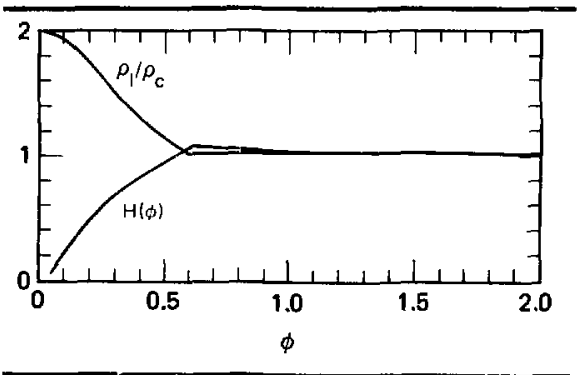


The ablation rate and pressure may be expressed ${ }^{\prime \prime \prime}$ in terms of a numerical parameter, $\hat{\sigma}$, that corresponds roughly to the ratio of the mean free path at $r_{c}$ to the ablation radius $R_{a}$ :

$\hat{o}=0.11\left[\frac{\mathrm{P}_{\mathrm{TW}}^{4 / 3} \mathrm{H}^{4 / 3} \lambda_{\mu \mathrm{m}}^{14 / 3} \mathrm{Z}^{4 / 3} \mathrm{~A}^{7 / 6}{ }_{\epsilon \delta_{\mathrm{T}}} \mathrm{T}}{\left(\mathrm{R}_{\mathrm{a}} / 0.1 \mathrm{~cm}\right)^{11 / 3} \phi^{7 / 3}(\mathrm{Z}+1)^{7 / 2}}\right] \times\left(\frac{10}{2 \mathrm{n} \Lambda}\right)$

(108)

Here $\epsilon \delta_{\mathrm{T}}$ is a function of $Z$ (defined in Ref. 115 ). P. $\mathrm{W}$ is the laser power in units of $10^{12} \mathrm{~W}, \lambda_{\mu \mathrm{m}}$ is the laser wavelength in micrometers, and $\ln 1$ is the Coulomb logarithm. ${ }^{115}$

The ablation rate $\dot{m}$ is then given by

$$
\begin{aligned}
& \dot{m}= 8.2 \times 10^{4} \mathrm{G}(\phi) \times\left[\left(\frac{\mathrm{R}_{\mathrm{u}}}{\lambda_{\mu \mathrm{m}}}\right)^{4 / 3}\left(\frac{\mathrm{A}}{\mathrm{z}}\right)^{2 / 3} \mathrm{P}_{\mathrm{TW}}^{1 / 3}\right] \\
& \times \hat{o}^{Z}\left[\left(1+\mathrm{M}_{\mathrm{s}}^{2}\right) /\left(22+7 \mathrm{M}_{\mathrm{s}}^{2}\right)\right]_{\mathrm{g} / \mathrm{s}},
\end{aligned}
$$

(109)

where $M_{s}(\phi)$ and $G(\phi)$ are shown in Figs. 3-44 and 3-46. and $A \mathrm{~m}_{\text {, }}$ and $Z$ are the mass and charge of the ions.

The ablation pressure $\mathrm{p}_{\mathrm{a}}$ (in cgs units) is

$$
\mathrm{p}_{\mathrm{a}}=\rho(\phi) \rho_{\mathrm{c}}^{1 / 3} \mathrm{l}_{0}^{2 / 3} \hat{\sigma}^{-}\left[\left(2-\mathrm{M}_{\mathrm{s}}^{2}\right) /\left(22+7 \mathrm{M}_{\mathrm{s}}^{2}\right)\right] .
$$

where $\beta(\phi)$ is shown in Fig. 3-45 and $I_{0}$ is the laser intensity (in $\mathrm{cgs}$ units) at the ablation surface $\mathrm{R}_{\mathrm{a}}$. Similarly, the temperature at the critical surface is

$k T_{c}=\mu\left(\frac{1_{0} I I}{10 \phi \alpha^{2} \rho_{c}}\right)^{2 / 3} \hat{\sigma}^{-2}\left[\left(1+M_{s}^{2}\right) /\left(22+7 M_{s}^{2}\right)\right]_{\text {ergs }}$.

For simplicity we now specialize to the flux limit value $\phi=0.3, f \simeq 0.03$, which to date seems most closely supported by $1.06-\mu \mathrm{m}$ experiments. We also assume $A \simeq 2 Z \gg 1$. Then the ablation rate $\dot{m}$ and pressure $\mathrm{p}_{\mathrm{a}}$ are given by 112

$$
\mathrm{n} \cong 2.3 \times 10^{4}\left[\frac{\mathrm{I}_{14}^{0.5}}{\lambda_{\mu \mathrm{m}}^{8.0}}\left(\frac{\mathrm{R}_{\mathrm{a}}}{0.1 \mathrm{~cm}}\right)^{1.9}\right] \mathrm{g} / \mathrm{s}
$$

and

$$
\mathrm{p}_{\mathrm{a}} \cong 7.2\left[\frac{\mathrm{I}_{14}^{0.6}}{\lambda_{\mu \mathrm{m}}}\left(\frac{\mathrm{ZR}_{\mathrm{a}}}{0.1 \mathrm{~cm}}\right)^{0.07}\right] \mathrm{Mbar}
$$

Fig. 3-46. The functions $\alpha(\phi), \beta(\phi)$, and $G(\phi)$ appearing in Eqs. $(109)-(111)$.
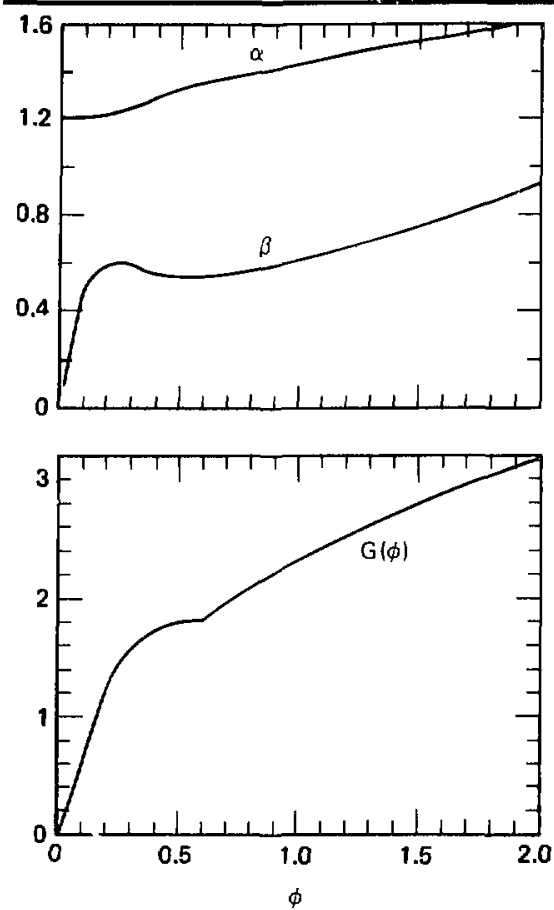

where $R_{a}$ is the ablation radius of the pellet, $I_{14}$ is the absorbed laser intensity at $R_{\mathrm{i}}$ in units of $10^{14}$ $W / \mathrm{cm}^{?} . A_{\mu m}$ is the laser wavelength in micrometers, and $Z$ is the ionic charge. Similarly. the radius and temperature of the critical-density surface are

$$
r_{c} \cong 1.6 R_{a}\left(l_{14}^{0.1} \lambda_{\mu m}^{0.4}\right)\left(0.1 \mathrm{~cm} / \mathrm{ZR}_{\mathrm{a}}\right)^{0.08}
$$

and

$$
\mathrm{T}_{\mathrm{c}}=1.7\left(\mathrm{l}_{14}^{0.5} \lambda_{\mu \mathrm{m}}^{0.8}\right)\left(2 \mathrm{R}_{\mathrm{a}} / 0.1 \mathrm{~cm}\right)^{0.1} \mathrm{keV} .
$$

Figure $3-47$ illustrates these scaling relations, and compares them with LASNEX simulation results (described in Ref. 111 and the next article). Figure 3-48 illustrates typical density and temperature 
Fig. 3-47. Parameter study showing (a) temperature at critical $T_{c}$, (b) critical radius $r_{c}$, and (c) ablation pressure $p_{1}$ as a function of absorbed laser power $P_{\mathrm{L}}$ in terawatts (1 TW $\left.=10^{12} \mathrm{~W}\right)$. Other parameters are $\lambda_{1}=2.65 \mu \mathrm{m}, \mathrm{R}_{\mathrm{q}}=0.1$ $\mathrm{cm}, t^{t}=0.3, Z=6, A=12$. The solid line is theoretical prediction; points are results of computer hydrodynamics calculations described in the following articles.
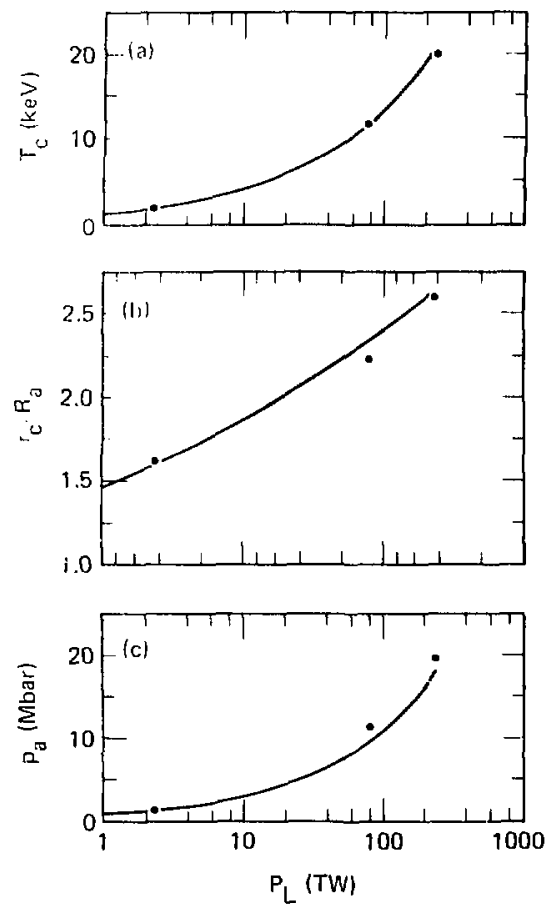

proliles from the present theory, for laser power $P_{1}$ $=78.5 \mathrm{TW}$. These compare quite well with the I. ASVi:X calculations.

These results of our model show that shorterwavelength lasers should produce higher ahlation rates and pressures for the same laser inlensity and target parameters. Niternatively, to produce a given ablation presetre. i.e. a given target implosion, a higher intensity laser is required at longer wavelengths. from $\mathrm{K} q$. $\{11,3\}$ with $p_{\mathrm{a}}$. Z. and $\mathrm{R}_{\mathrm{a}}$ lised. the sealing is $1 \propto \lambda_{1}^{1 .}$. $>1-\mu \mathrm{m}$ laser would thus have to be 3.3 times as intense as a $1 / 2-\mu \mathrm{m}$ laser to drive the same implosion. Collective effects
Fig. 3-48. Spatial profiles of density and temperature, for laser power $P_{L}=78.5 \mathrm{TW}$ und other parameters as in Fig. 3-47. Solid lines show theoretical predictions; dashed lines are numerical results described in the foltowing article from the hydrodynamics code LASNEX. The two agree quite welI.

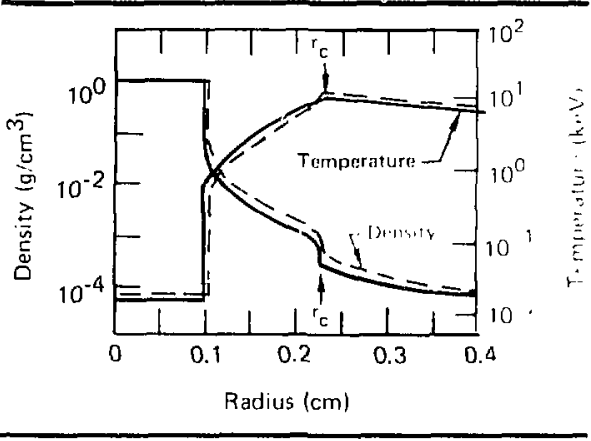

of hot-electron production and stimulated scattering not included in our model would tend to exacerhate this already strong wavelength scaling.

He conclude with a brief discussion of linitations on the validity of our theory. due to various assumptions we have made. These limits are considered in detail in the next article and Ref. 111

- Negleci ol inve:se bemsstrahlung absorption in the underdense plasma places a lower bound on the laser power or a lower bound on the laser wavelength.

- An upper limit on the allowable laser power arises from our assumption that an electron heated at the critical surface will not be able to penetrate to the elassical sone without suflering a collision.

- Our neglect of hol electrons is valid when the fraction of suprathermals is $₹ \mid 0$ " at the critical surf:ee, as is seen in long-pulse or short-wavelength experiments

- The steady-flow hypothesis means that our theory is valid only when the laser parameters vary slowly, compared to the time it lakes a fluid element to travel from the ablation surface to the eritical surface. For laser powers near al few hundred TW. wa: velenglhs near $1 \mu \mathrm{m}$. and target sizes near $1 \mathrm{~mm}$. this neans quantitatively ${ }^{11}$ that our theory should apply to portions of a laser pulse where the power is roughly constant over time scales of 1 to $2 \mathrm{ass}$.

Within these limitations. extensive comparisons with computational hydrodynamics 
calculations have shown the present theory to be accurate to better than 10 \%

Authors: C. F. Max, C. F. Mckee, and W: C. Mead

\section{References}

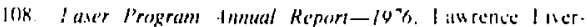
more I aboratory. I ivermbre. (alli. I ( RI-50021-7h (1977) p 4-9?

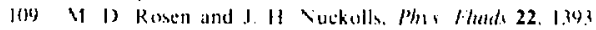
(1979): I k. Storm. J. I. I itsen. I H. Viucholls. II. \&;

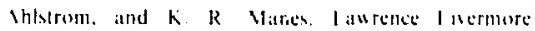

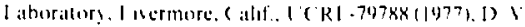

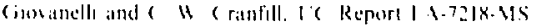

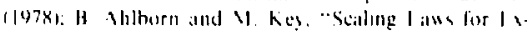
ploding l'usher Iarpels." Rutherford I ahorillurs Report RI - 74013.3 11975).

110 R. ( Malone. R I Mec rors, and R I Vorse. Phas

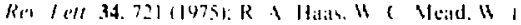

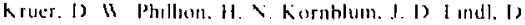

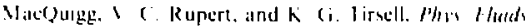
211. 22 (1977): B Yilakohi and I Brostom. Iha Ke's

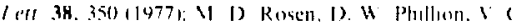

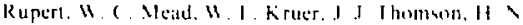

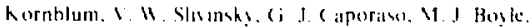

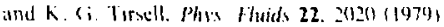

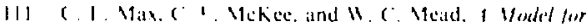

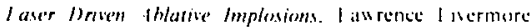
I aboralory. I wermors. (alll. I C RI-x354? llo be

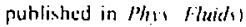

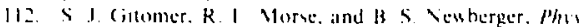
Heids 20. 234 (1977): I Ifontierth and R I Morsc. "Stationars I low Model of a hlatuels Impleded Sphericial

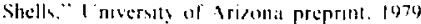

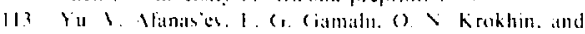

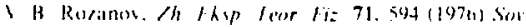

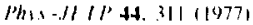

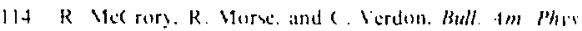
Sidi 23. $7 \times 7(197 k)$

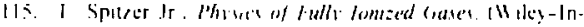
lerselence. ves rork, 1967). (hapler 5

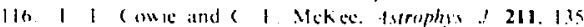
(1977)

\section{Hydrodynamic Calculations of Steady-Flow Spherical Profiles}

During the gestation of the theory discussed in the preceding article. we performed extensive numerical simulations using the fluid-dynamics computer code I. ASNLX ${ }^{117}$ We sought consistency and accuracy checks to evaluate important aspects of both the theory and the code. In the process we discovered and corrected several inconsistencies and inaccuracies in both. The theory benefitted from tests of many of the simplifying assumptions involved in oblaining the analytic solutions. The code benefitted from the use of theoretical profiles as a "test case," 10 evaluate the numerical art of solving simple fluid-dynamics and heat-flow problems.

In this section, we

- Present a description of the numerical modeling.

- Discuss istues involving the algorithm used to calculate heat flow.

- Comparc the results of parameter studies with theory.

- Show dy namical calculalions perforned to test the validity of two of the theoretical hypotheses.

Notation used throughoul correspond's to that used in the preceding article.

The ( omputational Model. The fluid-dynamics code I ASNitX has been used for the work presented here, but with simplified physics in order to be consistent with the assumptions of the theory.

llydrodynamies were modeled in onedimensional spherical coordinates using a standard l.agrangian explicil differencing scheme. $11^{7} .11 \mathrm{k}$ conal coordinales and velocities are interfacecentered. Density and cemperature are roneecentered. The artificial viscosily used had the form developed by White. "The equation of state of a fully ionired. ideall gas $(\alpha=5,3)$ was used. with pressure and specilic energy (in cas units) given bs

$$
\begin{gathered}
\Gamma_{\mathrm{tgs}}=9.61 \times 10^{14}\left(\frac{Z+1}{\mathrm{~A}}\right) \rho \mathrm{I}_{\mathrm{keV}} . \\
\mathrm{r}_{\mathrm{cLs}}=\frac{3}{2} \frac{\mathrm{P}_{\mathrm{cgs}}}{\rho} .
\end{gathered}
$$

The computer-code thermodynames were smplified to agree with the assumptions of the theors

- Al! radiation phesies and an conduction were turned oft.

- The electrons and ions were described as single-temperature Maxwellians with eidectron-ion collisional coupling increased by a later of $10^{6}$ above the Spiter ${ }^{20}$ salue. lo ensule $T_{c} \equiv T_{1}$.

We presen here our I.AS NI Y algorithms used to numerically solve the one-dimensional fluxlimited diflusion of thermal electrons. followed by at brief destription of an older version of the code w ith which we encountered some numerical difficulties. The notation used to represent plasma paraneters in the code is illustrated in Iig. 3-49. For the discussion 10 follow, temperatures are in kev, and the 
Fig. 3-49. Terminology for discussion of numerical hent-nux algorithm. Successive zones are indicated by index K. Density, temperature in $\mathrm{keV}$, pressure, and volume $\mathrm{V}$ are zonecentered, whereas radius and velocity are interface-centered.

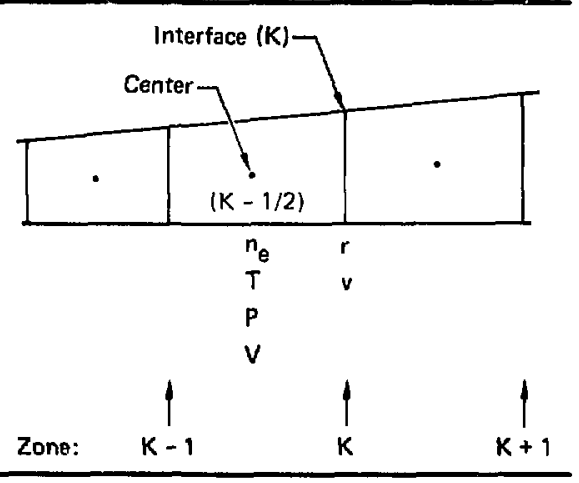

physical quantities will be tagged, as required for clarity, with

- The conal index $\mathrm{K}$.

- The time step index $n$. which indicates a quantity evaluated at the end of cycle $n$. In certain expressions mixcures of cycle-n and cycle-(n - 1) quantities are involved: quantities composed of variables from such mixed tinte-steps will be indicated by the cycle number "M."

- A superscript c or 1 as a reminder of cellor interface-centering.

The heat-flux crossing boundary $k$ during cycle $\mathrm{n}$ is obtained by solving

$$
\Delta \mathrm{O}^{\mathrm{I}}(\mathrm{K}, \mathrm{n})=\mathrm{K}_{\mathrm{uff}}^{\mathrm{l}}(K, \mathrm{M}) \cup \mathrm{K,n}^{\mathrm{T}} \mathrm{T}
$$

implicitly, with a ef (evaluated as described below) held fixed. The temperature gradient is defined as

$$
\nabla{ }_{K, n}^{1} T=\frac{T(K+1 / 2, n)-T(K-1 / 2, n)}{0.5 \times\left[r(K+1, n)-T\left(r^{2}-1, n\right) \mid\right.} .
$$

Since the conductivity depends strongly on temperature, the solution for $\triangle Q$ is actually explicit and the time-step is controlled by limiting the relative change in temperature, $\triangle T / T$, to a maximum value $\ll 1$ for every zone.

The effective conductivity is determined as

$$
\kappa_{\mathrm{eff}}^{I}=\operatorname{MAX}\left[\kappa_{\mathrm{MIN}}^{I}, \operatorname{MIN}\left(\kappa_{\mathrm{CLAS}}^{\mathrm{I}}, \kappa_{\mathrm{F}, L}^{\mathrm{I}}\right)\right] .
$$

The minimum cinductivity is normally set to ${ }^{\mathrm{M}} \mathrm{M} / \mathrm{N}$ $=10^{14} \mathrm{erg} / \mathrm{s}-\mathrm{cm}-\mathrm{keV}$, and has no impact on this work.

The classical conductivity at a cell center is well-determined in terms of cell-centered quantities at the end of the previous time-step:

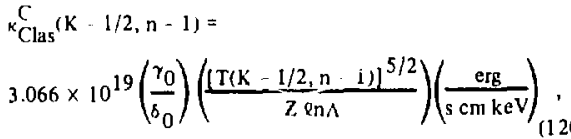

als in Braginskii. ${ }^{21}$ 1lere

$$
\begin{aligned}
& \gamma_{0}=\frac{0.9067}{z^{3}}+\frac{4.408}{z^{2}}+\frac{5.4053}{z}+1.20, \\
& \gamma_{0}=\frac{1.3008}{z^{3}}+\frac{1.5956}{z^{2}}+\frac{0.7778}{z}+0.0961,
\end{aligned}
$$

and we take $\ln .1=10$ to agree with the theoretical modei. In our parameter studies we have assumed a fully ionized plasma, $Z=6$, for which $\gamma_{0} / \delta_{0}=$ 8.068 . The averaging to obtain an interface-centered classical conductivity is perforned using

$$
\begin{aligned}
& { }^{\kappa} \operatorname{Clas}_{\text {an }}(K, M)=
\end{aligned}
$$

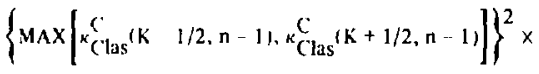

$$
\begin{aligned}
& \frac{\left(\Delta r_{K-1 / 2, n}+\Delta r_{K+1 / 2, n}\right)}{\left[\Delta r_{K} 1 / 2, n{ }^{K}{ }^{C}(\operatorname{las}(K-1 / 2, n-1)]+\right.}
\end{aligned}
$$

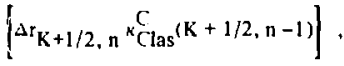

where

$$
\Delta r_{K-1 / 2, n}=r(K, n) \quad r(K-1, n)
$$

This averaging scheme was chosen historically to apply to $k$ elf. in order to satisfy the requirement that diagonal flux-limited transport in two-dimensional calculations not exceed transport along the mesh directions. In the code used for this work, however. averaging of this form was used for $\kappa_{C}$ las only. This thermal-conduction averaging scheme reported adequately for the calculations reported here.

The nux-limited "conductivity" is computed using 


$$
\begin{aligned}
\kappa_{\mathrm{FL}} & =\mathrm{f}_{\mathrm{e} \mathrm{m}_{\mathrm{e}} \mathrm{v}_{\mathrm{c}}^{3} \frac{1}{\nabla \mathrm{T}}} \\
& =2.120 \mathrm{fn_{ \textrm {c } }} \mathrm{T}^{3 i 2} \frac{1}{\nabla \mathrm{T}} \frac{\mathrm{erg}}{\mathrm{scm} \mathrm{kcV}} .
\end{aligned}
$$

In order to calculate an interface-centered quatntity. the three terms in Fq. (125) are "averaged" sepa, ately:

$$
\begin{aligned}
& \kappa_{I: L}^{I}(K, M)=2.120 f x \\
& {\left[\frac{V(K-1 / 2, n) n_{c}(K-1 / 2, n)+V(K+1 / 2, n) n_{c}(K+t / 2, n)}{V(K-1 / 2, n)+V(K+1 / 2, n)}\right]} \\
& \left.\times \operatorname{MAX}[T(K-1 / 2, n \quad 1), T(K+1 / 2, n \quad 1)]^{3 / 2}(\nabla)_{K, M}^{I}\right)^{-1} .
\end{aligned}
$$

Here, the mixed gradient is defined as in F:q. (1/8). but with coordinates from cycle $n$ and temperatures from cycle $(n-I)$.

We next briefly discuss a previous numerical scheme which presented some difficulties (discussed later in this article). The "old" heat-flow algorithm differed primarily in the evaluation of $k \mathrm{kl}$. and the sequence in which the averaging to obtain interfacecentered quantities occurred. First. the value of $k f_{t}$ at zone center $\mathrm{K}-1 / 2$ was computed from F.q. (125) by taking $n\left(T^{3}\right)_{K-1} z$ and $M A X(1 / T)$ between the interfaces $K$ and $K-1$. Next, a cell-centered effective conductivity was obtained using l:q. (119) for cell-centered quantities. Finally. a weighted averag: of cell-centered seffs of the form of liq. (123) was used to obtain interface-centered conductivities. As mentioned above. this algorithm had been chosen to prevent faster flux-limited heat-flow diagonal to the mesh in two dimensions. The effect in one dimension. however. was. in cases of strong gradients. to have $\nabla T$ in E.q. (117) and $(1 / \nabla T)$ in Eq. (125) computed on opposite sides of a cell. yielding a systematic error of significant magnitude.

Calculational Methods. As shown in the previous article, the profiles obtained theoretically have two regions where velocity, density, and temperature gradients are steep: the ablation surface. and the critical surface where the laser energy deposition is localized. The latter, particularly at low flux limits, is the most demanding for the code numerics.

In order to resolve the profiles around the critical surface. it treadmill rezoner was used. Relatively coarse zones were used above a density
10 to 50 times the laser critical density. As the matter ablated and flowed to lower density. it crossed a threshold density where the treadmill rezoner split the zones in half. as required to maintain a transition in zone mass down to a fine-zoned region in the outer part of the profile. Typically a ratio of coarse to fine zone mass of between 3 and 10 was found 10 be the best overall compromise in resolving the ablation and critical surfaces.

The initial conditions for the calculations were set up by numerically solving for the theoretical prolites, and then transferring them to the lagrangian mesh. The zones were chosen to have constant mass in the coarse- and line-roned regions. with a transition of a few zones, varying mass by a factor of about two. between the two regions. The initial velocity at each vertex was set to the theoretical value at that spatial location. The ane temperature was chosen to conserve internal energy. Typically. the transfer from theoretical profiles to at roughly 200-zone mesh conserved momentum io about $I \%$ and energy to about $0.1 \%$.

The calculations were run for 0.5 to 5 times the flow time (the time for a lluid element to flow from the ablation surface to the critical surface). The shorter runs could be coned more finely than the longer. since less matsis was ablated. The detailed comparisons presented in this and the preceding article were made after $\$ 2$ flow times. A duajtreadmill scheme could perhaps be employed to give improved resolution at hoth the ablation and critical surfaces for longer runs. within the constrants imposed by at reatsonable number of zones.

Issues in the (hoice of Algorithms for Heat Flow. One of the "artistic" features of numerical problem-solving is the development of suitable differencing algorithms. In the use of a fluid-code, it is frequently necessiry to perform repeated calcuiations, applying hoth quantitative criteria and "common sense" tests to assess the adequacy of a given algorithm. In the following diseussion. we present as an example a numerical algorithm which turned out 10 be inadequate for calculating strongly inhibited heat transport, yet which produced such sufficiently "reasonahle" results that its quantitative failures took a long time to isoiate. In fact, its shortcomings would have taken considerably longer to identify had we not been comparing our 
numerical results with the theoretical profiles presented in the preceding section. Our motivation in describing our experience with this algorithm is ii ilustrate the kind of subtle warning signs that “... appear when a numerical algorithm begins to $\therefore$ i

higure 3-50 shows fluid-code calculations of je: init and temperature profiles for flux limit $\phi=$ 1. computed using two different flux-limit buratum, as described above (for the relation be$\therefore$ and $f$, see $l: q$. (99) in the previous article). ' $i$. id algorithm, which uses a local maximum $\therefore \ldots i_{11} 11 \mathrm{tef}$ produced interesting density and temies.1!1: ytuctures near the critical surface. These

Fig. 3-50. Fluld-code-calculated deasity and temperature profiles using "old" (solid lines) and "new" (dashed lines) nux-limit algorithms for the $\phi=0.6$ case. Several calculations were required to show that the density and temperature structures seen using the "old" nux limit algorithm (see "The Computational Model," and "Issues in the Choice of Algorithms for Heat Flow") changed qualitatively with different time steps and zonings, suggesting numerical problems.
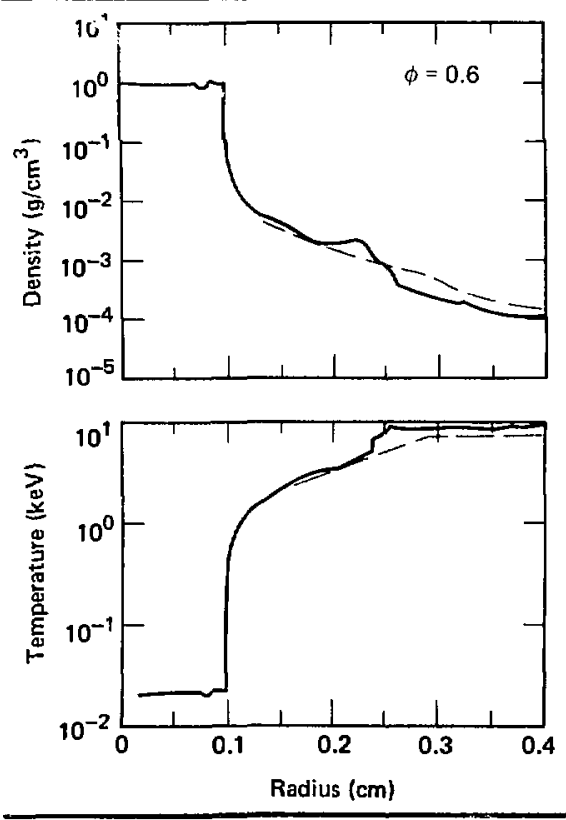

seemed physically reasonable. but calculations performed using different zoning patterns and timestep controllers showed that these structures were very sensitive to simple changes in the details of the calculations. This suggested numerical trouble. but the origin of the problem was very unclear.

Figure 3-51 shows similar profiles calculated for the case $\phi=0.3$. Here the "old" flux-limit algorithm, when zoned sufficiently finely near critical, exhibited a large temperature jump (a factor of two or three between adjacent zones). At this point, direct calculation of the code-generated heat Mux in ward from critical clearly showed a reduction by a factor of two to three from the expected value. This was traced to the old flux-limit algorithm which used the maximum value of $\mathrm{rT}$ from adjacent zones. and which could thus systematically un-

Fig. 3-51. Fluid-code calculations of density and temperature proftles for old and new Ilux-limit algorithms, for the $\phi=0.3$ cuse. Here the old algorithm produced a temperature jump at critical which behaved quite physically and systematically modified the calculated plasma parameters. The size of the temperature Jump was considerably reduced when an inproved hest-flow algorithm was employed.

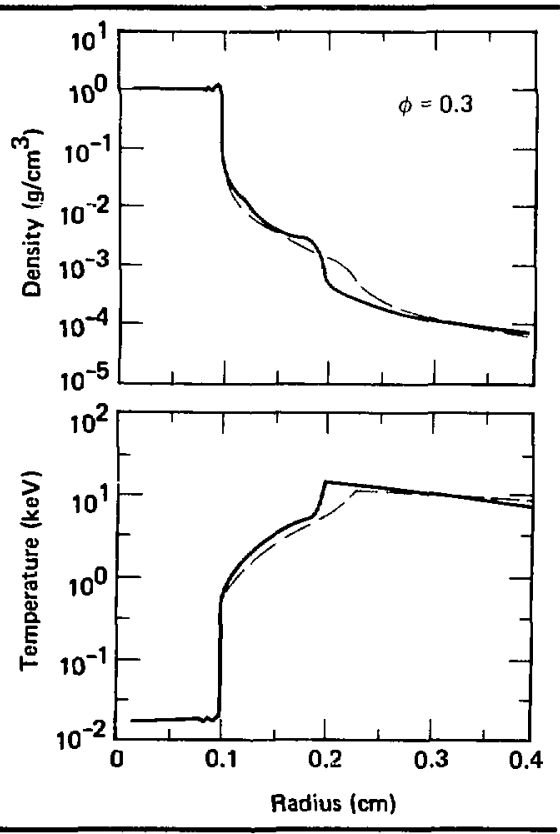


derestimate the heat flow through a given zone in certain steep-gradient situaitons.

This flux-limit algorithm was replaced by a simpler one which never displaced the temperature gradient used to compute the flux limit by one zone's width. The result was instantly gratifying: numerical behavior was vastly improved. sensitivity to zoning declined. and agreement between the fluid-code results and the theory was greatly enhanced.

Using the new algorithm. the temperature jump at the criticul-density surface, which had previously been significant, became quite small. This fict has led us to wonder about the physical reality of the large temperature jumps frequently seen in computer hydro-calculations of strongly inhibited heat flow. Our experience emphasizes that such temperature jumps can sometimes be artificts of the heal flow algorithm.

In reality. the existence and size of any temperature jump at critical density would be determined by microscopic physical processes occurring within the discontinuity. Neither our present theoretical model nor our hydrodynamies code includes such detailed physics. What our present hydro calculations do show is that it is at least dynamically self-consistent to have no temperature jump across the critical-density surface, for $\phi>0.3$.

In summary. strong transport inhibition place: considerable stress on numerical algorithms used to calculate flux-limited heat flow. Our experience suggests that extensive screening by numerical. analytical, or experimental cross-checks is necessary to establish the trustworthiness of a given heat-flow algorithm. The theoretical profiles developed in the present work may be useful for future investigators as a test-case for the adequacy of various heat-flow algorithms.

Results of Fluid-Code Calculations. Two parameter studies were performed using the improved algorithm: one varying the flux limit $\phi$ and the other varving the laser power $\mathrm{P}_{\mathrm{L}}$.

In the first parameter study, the flux limit $\phi$ was varied from 0.3 to 1.4. Laser and target conditions were: $\lambda_{\mathrm{L}}=2.65 \mu \mathrm{m}$. absorbed laser power $\mathrm{P}_{\mathrm{L}}$. $=78.5 \times 10^{12} \mathrm{~W}, A=12, Z=6$ (fully ionized carbon), and a solid pellet of initial radius $R_{a}(t=0)=$ $0.1 \mathrm{~cm}$. The results are depicted as circles or triangles in Figs. 3-52 to 3-55, for comparison with the solid lines which represent predictions of our
Fig. 3-52. Variation of the critical radius, $r_{c}$, and the radjus where heat flow becames saturated, $r_{s}$, with Dux limit. $\delta$. Solid lines are predictions of theory with absorbed laser power $P_{L}=7.85 \times 10^{13} \mathrm{~W}, \lambda_{1}=2.65 \mu \mathrm{m}, R_{k}=0.1 \mathrm{~cm}, \mathrm{~A}=$ $12, Z=6$. Points are results of computer hydrodynamics calculations described in the text. The saturated region becomes saaller and $r_{c}$ larger as $\phi$ increases.

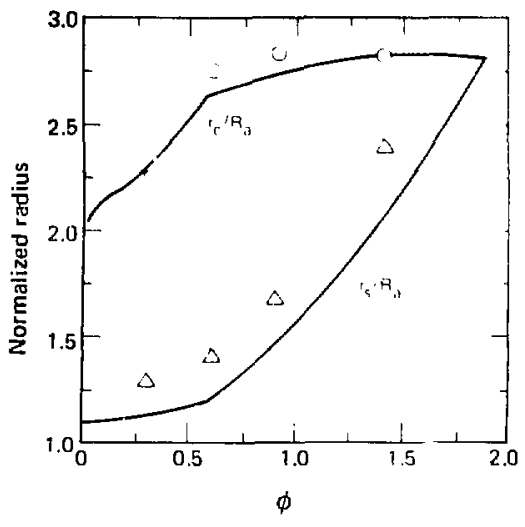

Fig. 3-53. Temperature at the critical surface as a function of flux limit, $\phi$, for absorbed power $P_{1}=7.85 \times 10^{13} \mathrm{~W}, \lambda_{1}=$ $2.65 \mu \mathrm{m}, R_{a}=0.1 \mathrm{~cm}, A=12, Z=6$. The solid line is theoretical prediction; paints are result of hydrodynamics calculations. To the right of the maximum in $\mathbf{T}_{c}(\phi)$, laser energy deposited at the critical radius is dominuntly carried away by inward heat flaw; to the left, by outward kineticenergy how.

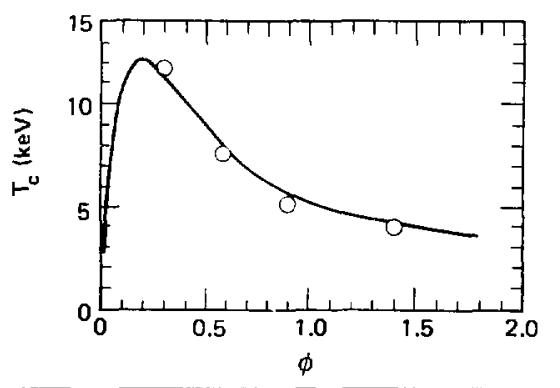

theory. The code values of $\dot{m}$ shown in Fig. 3-55 represent spatial averages over all $r>R_{a}$. In general the hydro calculations showed the Mach number in the saturated zone to be constant to a few percent 
Fig. 3-54. Ablation pressure, $P_{a}$, as a function of the flax limit, $\phi$. Solid line is prediction of theory with absorbed power $P_{L}=7.85 \times 10^{13} \mathrm{~W}, \lambda_{L}=2.65 \mu \mathrm{m}, R_{2}=0.1 \mathrm{~cm}, A=$ 12, $Z=6$. Points are results of computer hydrodynamies calculations described in the text. For fixed laser and target parameters, the ablation pressure falls strongly as transport becomes more inhibited.

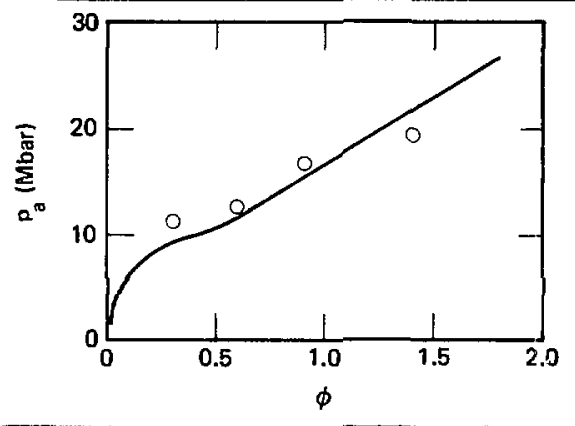

Fig. 3-55. Mass loss rate $\dot{m}$ as a function of Mux limit, $\phi$, for the same conditions as Fig. 3-54. Solid line is theoretical prediction. Points are results of hydrodynamics calculations; error bars represent $\pm 2 \pi$ at a given time in the computer run, and give a feel for the degree of spatial variation in $\stackrel{m}{\circ}$. (In a strict steady state, m would be constant in space.)

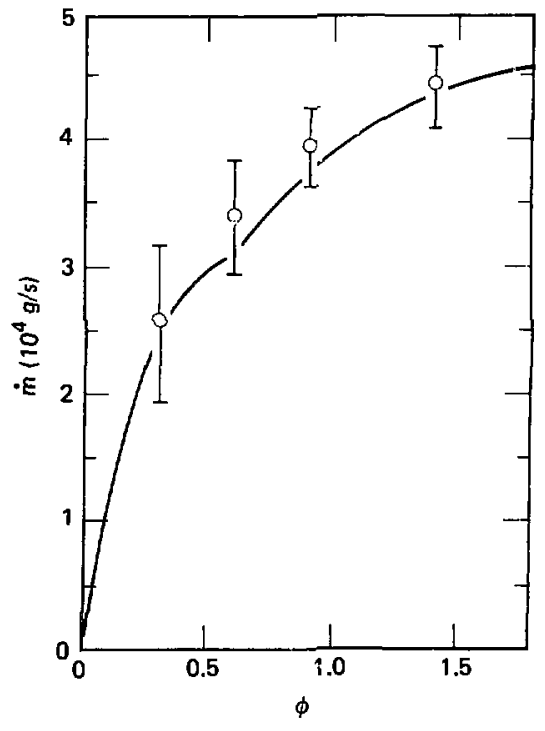

across the region. In contrast, $m$ showed more spatial variation at each given time. The error bars in Fig. 3-55. which represent $\pm 2 \sigma$, are an attempt to illustrate the magnitude of this spatial variation of $\dot{m}$ due to the lack of exact steady flow. The parameters $\dot{m} . r_{c}, T_{c}$, and $p_{a}$ show excellent agreement between theoretical and hydrodynamics results.

The second parameter study varied the absorbed laser power over a factor of 100 , from $2.35 \times$ $10^{12} \mathrm{~W}$ to $2.35 \times 10^{14} \mathrm{~W}$. Other conditions we e $\phi=$ $0.3, R_{a}(t=0)=0.1 \mathrm{~cm}, Z=6, A=12, \lambda_{1}=$ $2.65 \mu \mathrm{m}$. A bove the upper limit of $\mathrm{P}_{\mathrm{I}}=2.35 \times 10^{14}$ $W, \hat{\sigma}$ was too large to satisfy the validity conditions for the theoretical model. As in the previous study. theoretical profiles were used as initial conditions and the calculations wire compared with theory after at least one or two flow times. Figure 3-47 (in the previous article) shows the very good agreement between theory and dynamical calculations for $T_{C}$, $r_{c}$, and $p_{a}$ versus absorbed laser power $P_{1}$.

We conclude that when the laser and target parameters are within the range where our theory should apply. the steady-state model developed in this work agrees very well with numerical fluid code calculations.

Examples of Validity Checks. In this section we illustrate calculations which delineate the regions of parameter space where our theory should be applied. Predictions of the analytic model are compared with the numerical results. to explore what happens as the model is rushed to the edge of its region of validity. We consider here two examples: the steady-state hypothesis, and the neglect of radiation prcssure. More complete results are presented in Ref. 122.

A fundamental assumption of our steady-state ablation model is that characteristics such as the laser intensity and the density and velocity profiles do not change by large amounts in the time it takes a fiuid element to flow from the ablation surface to the critical surface. In reality of course, laser pulses often have rise times shurter than the hydrodynamic flow times. For reactor-scale targets, several classes of target designs use laser pulses which start at low. fairly constant power, then rise quickly compared to a flow time, and finally stay at relatively constant power for several flow times. We would like to be able to apply our quasi-steady state theory to such laser pulses during the constant-power intervals. 
Fig. 3-56. To test sensitivity to severe transients, the laser power in a fluid code calculation was given the temporal profile shown in ( 2 ). Target parameters are $R_{1}=0.1 \mathrm{~cm}, A=12, Z=6, \phi=0.6, \lambda_{2}=2,65 \mu \mathrm{m}$. Density profiles (b) are shown at four times during the evolution. At $t=0.16 \mathrm{~ns}$, the profile has not yet begun to repond to the higher incident laser power. The promles at $t=2.4$ and $4.0 \mathrm{~ns}$ show a density bulge around $\rho \cong 10^{-4} \mathrm{gm} / \mathrm{cm}^{3}$, which is due to the increased ablation of muterial in response to higher laser power. By $t=$ $6.7 \mathrm{~ns}$, the density bulge has propagated outward to the critical surface and the code's profile agrees quite well with the steady-state theory.
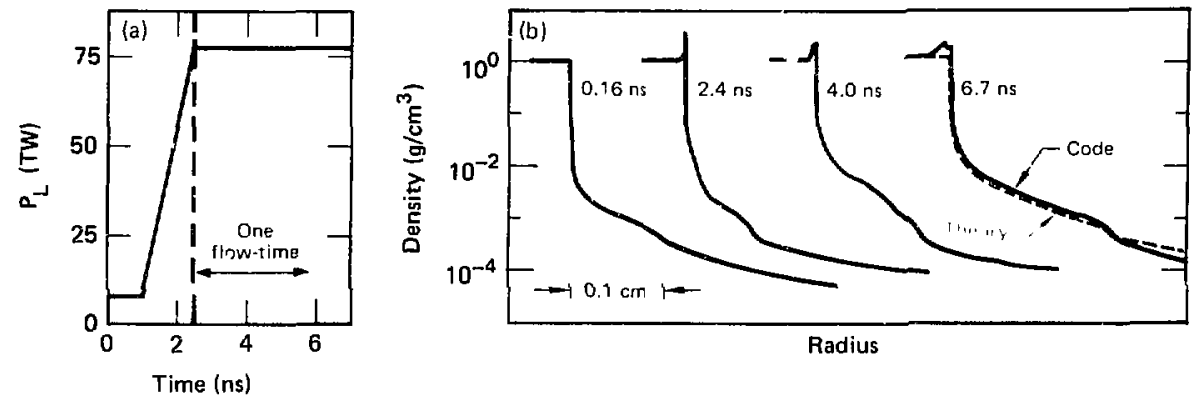

Radius

To test whether such it use of our model is likel: 10 be valid. we performed a computer calculiation using the laser power temporal profile shown in Fig. 3-56(a). The power was increased by a factor of 10 in less than 1 flow time, and was held coristant thereifter. We found that after 1.5 flow times had elapsed after the power transient, the dynamical code results converged to the steudy-state theory within better than $10 \%$ agreement.

ligure 3-56(b) illustrates in more detail how this convergence occurred. When the laser power was first increased, the critical radius was driver inward because the inner part of the target had not yet ablated enough material to maintain a larger critical surface. As the ablation pressure and ablation rate increased and the newly ablated material began to reash the critical surface, huwever, $r_{c}$ increased quite quickly. The evolving density profiles shown in Fig. 3-56(b) show this "bump" of newly ablated material moving outward. In the last profile shown. the computed density has relaxed to excellent agreement with the theory.

We conclude that the quasj-steady model can probably be reliably applied to situations where the laser power remains roughly steady for more than one or two flow times, independent of increases or decreases in transient power which may have preceded such a sleady-power regime

To test the validity of our assumption that $f^{2} 8_{\pi} \mathrm{n}_{\mathrm{c}} \mathrm{T}_{\mathrm{L}}$ is not large in the theoretical model, we ncrlormed a hydrodyzamics calculation in which the ponderomotive force was included in the hydro code in the WKB limit. ${ }^{117}$ We chose a value of $\phi$ large enough that $M_{s}$ would be greater than unity $(\phi$ $=0.9, M_{s}=1.331$. in the hopes of seeing a steadystate "shock plus D-Fronl" of the type predicted in Refs. 123 and 124. This structure arises when supersonic plasma approaching the critical density surlice encounters a now restriction produced by the laser rudiation pressure: the resulting shock raises the sound speed to permit a subsonic rarefaction wate (D-front) at the critical surface.

Our results are shown by the solid line in Fig. 3-57. illustrating the density profile. As expected. for these parameters the ponderomotive force makes only a small and localized perturbation to the overall plasma densily profile. With the exception of the immediate vicinity of the critical surface. the protile from the hydro code is in excellent agreement with the theory developed neglecting the ponderomotive force.

A second issue addressed in Fig. 3-57 is the existence of a steady shock plus D-front for $M I_{\mathrm{s}}>1$. The figare shows that a steady compressional structure did indeed lorm in the critical surface region. as predicted. An enlargement of this critical region appears it the inset (b). and numerical study of this structure showed it to be a shock plus D-front. This structure persisted in the same form for the entire duration of the computer run. We believe this is the 
Fig. 3-57. Fluid-cooe-generated density profiles incleded effects of 1 (solid line) and 10 (dotted line) times the WKB ponderomotive force. Lajer and target painmeters are alusorbed power $P_{l}=78.5 \mathrm{~W}, \lambda_{1}=2.65 \mu \mathrm{m}, \phi=0.9, \mathrm{R}_{\mathrm{a}}=0.1$ $\mathrm{cm}, Z=6, A=12$. This figure supports the result that (within the context of our model) the ponderomotive force does not cause any significant modification of the global densily profile (a). The inset (b) is a magnitication of the critical-surface region, and shows the supercritical density . ompression, or shock pius D-front, expected in this case, since $\mathbf{M}_{3}>1$.

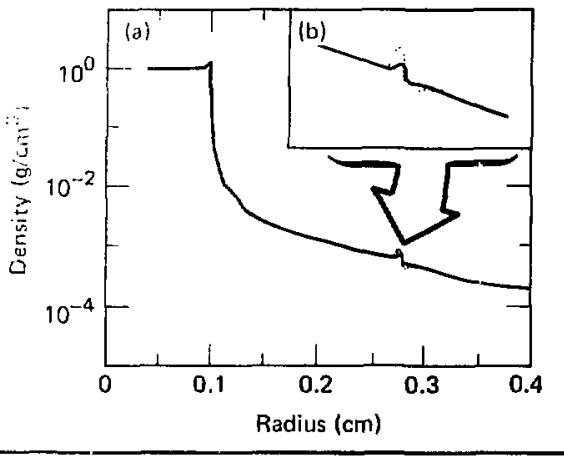

first computational confirmation that steady density structures above the critical density can persist for significant times. for laser pulses whose power is roughly constant in time.

Conclusion. We have presented salient methods and results from numerical simulation of steady. tiale spherical hydrodynamic nows. We find quantitative agreement to better than $\sim 10 \%$ between theory and simulation. over a wide parameter range for which the theoretical model assumptions hold. ite have also explored some examples showing the nature and extent of our model's applicability to situations near its limits of validity.

luthors: W. C. Mead, C. E. Max, and C. F. McKee Major Contributor: G. B. Zimmerman

\section{References}

117. C. B. /.mmermun and W. L. Kruer, "Numerical Simulation of l.sser Initiated Fusion." Comments Plasma Phys. Cont Fusion 2. 8.5 (19,5). (j. B. Zimmerman. Numerical Simularion of the High Density t tpproach to Laser Fusion. I.awrence Livermore l.uboratory. Livermore, Calif.. UCR 1.7481) (1973): J. A Harte. and (;. B. Zimmerman. Lawrence Livermore 1.aboratory. Livermore. Calir. U(II)-17517(1977).
118. W. D. Schulz, in Merhods in Compulational Physics. J. Killeen, L.d. (Academic Press, New York, 1964), vol. 3. B. Alder, S. Fernbach, and M. Rotenberg. Eds.. pp. I to 45.

119. J. White. "A New form of Artificial Viscosity." J. Comp. Phys. I1, 573 (1973): and J. White, "A New Form of Artificial Viscosity: Postscript." J. Comp. Phys. 12. 553 (1973).

120. 1.. Spitzer, Jr.. Physics of Fully lonized Goses. (Wiley - Interscience. New York. 1967). Chapter 5

121 S. I. Braginskii. "Transport Processes in a Plasma," in Reviews of Plasma Physics. Vol. 1. M. A. Leorlovich, L.d. (Consultants Bureau, New York, 1965), p. 205.

122. C. I. Max. C. I. McKes, and W. C. Mead. A Model for laser Driven thlative Implosions, Lawrence livermore 1 aboralory. I.ivermore, Calli., LCRL_-83542, submitted to Mys. Fluids

123. I. Mayer, R, Berger, and C. R. Max. KMS Jusion Report No. $1876(1979)$.

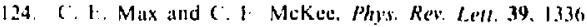
(1977).

\section{Electron Heating Due to Resonant Absorption}

Hot-electron production and electron-heat transport inhibition are studied experimentally with the PROMETHEUS I device at the University of California at Davis. This device models laser absorption due to resonant absorption and parametric instabilities. and electron-heat transport inhibition due to ion woustic turbulence and a dc magnetic field. Intense, p-polarized microwaves are incident onto an inhomogeneous. essentially collisionless plasma. The microwave field is mainly parallel to the density gradient so that electrostatic waves are driven near the critical surface, resulting in electron heating and resonant absorption of the microwaves.

The lime history of the electron energy distribution at high power ( $\left.v_{0 s c} / v_{\text {co }}=0.7\right)$ is illustrated in Fig. 3-58. The current vs voltage (I-V) characteristic of a one-sided Langmuir probe is shown in Fig. 3-59. This probe is in the overdense region near the critical surface. and pointed so that electrons moving up the density gradient are measured

- Just before the microwave pulse begins $(t=$ $0 \mu \mathrm{s})$, the electrons have a Maxwellian distribution (shown by the exponential shape of the currentvoltage curve) with a relatively cold temperature $\left(\mathrm{T}_{\mathrm{eo}} \approx 1.3 \mathrm{eV}\right)$.

After a short time $(0.1 \mu$ s, longer than the estimated wave-breaking time). high-energy electrons are observed $\left(E_{e} \geqslant 30 T_{e o}\right)$. These must be electrons accelerated by the resonantly driven electrostatic waves, because the ions have nol moved significantly. Aiso, the thermal electrons (those elec- 
Fig. 3-58. Time evolution of the electron current-voltage curve.

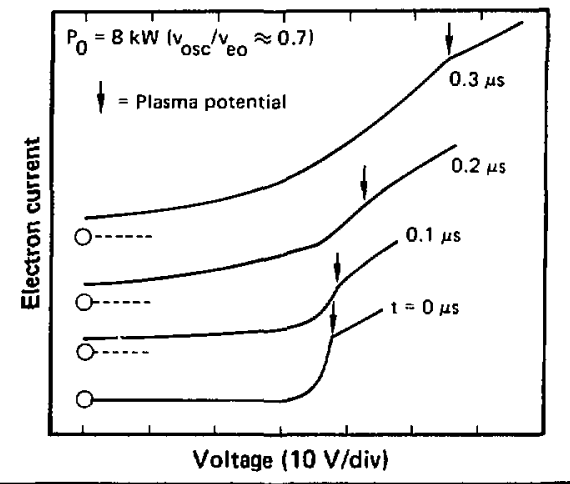

Fig. 3-59. Logarithm plot of typical I-V curves.

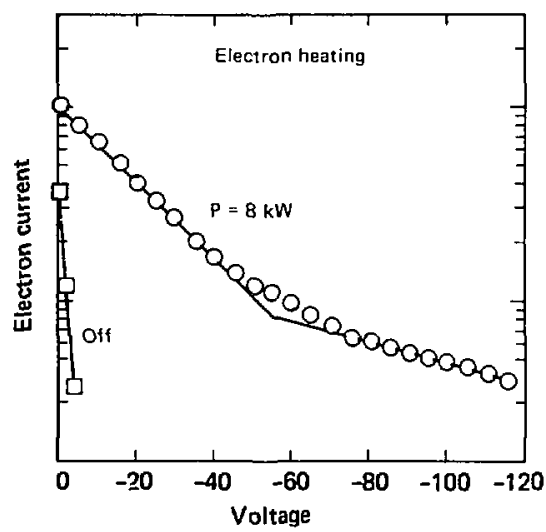

trons with energies near the plasma potential) $s . l$ have a relatively low temperature.

- A little later $(0.2 \mu \mathrm{s})$, however, significant thermal-electron heating (and a higher current of high-energy electrons) is observed. In addition, the plasma potential becomes more positive.

- Still later $(0.3 \mu \mathrm{s})$, the thermal electrons have been significantly heated. The electron energy distribution is bi-Maxwellian ${ }^{125}$ as shown in Fig. 3-59.

Notice that the thermal electrons are strongly heated. The hot electrons in a previous experiment, ${ }^{126}$ where s-polarized microwaves decayed to electron-plasma waves and ion acoustic waves, were hotter $\left[\mathrm{T}_{\mathrm{H}}\right.$ (s-polarization $) \approx 4 \mathrm{~T}_{\mathrm{H}}(\mathrm{p}$ polarization)]. The suprathermal election temperature, $T_{H}$, agrees well (within $40 \%$ ) with a scaling law ${ }^{125}$ which was derived from particle simulation calculations for laser parameters. This agreement is remarkable, because no normalization is used in the comparison: that is, the values of $v_{0 s c} / v_{\text {to }}, T_{0}$, angle of incidence, and absorption from the microwave experiments are directly substituted into the sculing law. In fact, the agreement would be within $10 \%$ in absolute value if the initial electron temperature were used.

The key difference between the simulation results and the results frum microwatve experiments is that the thermal electrons are strongly heated at high power in the microwave experiments. As shown in Fig. 3-60. the thermal electrons in the overdense region are heated as they approach the critical surluce (at $10 \mathrm{~cm}$ ). The estimated thermalelectron drift velocity at high power is much larger than the threshold value for the ion acoustic drift instability, $V_{c}\left(T_{c o} / T_{i o} \approx 10\right.$, so that $\left.V_{c} / v_{e o} \approx 0.05\right)$.

Strong ion acoustic turbulence $(\delta \mathrm{n} / \mathrm{n} \leqslant 20 \%)$ and a de electric field are observed in the overdense region where the thermal electrons are heated. The

Fig. 3-60. Temperature of thermal electrong traveling toward the critical surface in the overdense region. The critical surfoce is at $10 \mathrm{~cm}$.

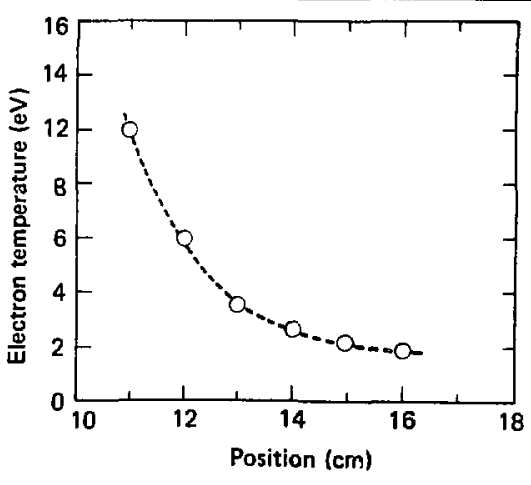


waves travel down the density gradient and have a broad spectrum in angle and frequency. Our measurements iniply that effective electron collision Ireyuencies due to the ion turbulence are as high as "all $\omega_{\mathrm{pu}} \approx 0.04(\approx 300$ times the electron collision frequency). This value agrees with theory $\left[u_{\mathrm{e}} \mathrm{er} / \omega_{\mathrm{pe}}\right.$ $\approx(\dot{m} / \mathrm{n})^{2}$. with the measured value $\left.\delta \mathrm{n} / \mathrm{n} \approx 0.2\right]$. We also observed large-amplitude ion waves $(\delta n / n \leqslant$ $20 \%$ ) in the underdense region which propagate up and down the density gradient. These waves are probably excited by parametric instabilities.

With such large levels of ion turbulence near the eritical surfice, thermal and even some suprathermal electrons should be seattered and confined neils the eritical surlace. We have verified that thermal electrons are confined near the eritical surfite. by measuring the decay of the thermal-electron cemperature alter the end of the microwave pulse. As predieted by diffusion theory, the thermalelectron temperature decays exponentially: the decay time gives "etripe $\approx 0.013$. in agreement with the above estimates. We also lind that the de electric field is increased by a locilized. weak magnetic field (wic $\left.\left.\omega^{\prime}\right) \leqslant f^{\prime}\right)$. The magnetic field is transverse to the electron flow and lacalised so that thermal (but not suprathermal) electrons are confined. The de electric field must then be increased so that the return current is maintained.

Authors: K. Mlizuno, R. B. Spiclman, J. S. DeGroot, and $\mathrm{W}$. M. Bollen (AII I niversity of California at Daris)

\section{References}

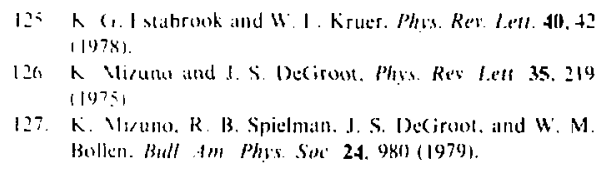

\section{Electron-Heat Flux Inhibition}

\section{Due to Ion Acoustic Turbulence}

Electron-heat conductivity in a plasma must be high enough so that the absorbed laser energy is transported to the ablation surface. There are strong indications that the electron-heat flux is severely inhibited in some laser experiments: mechanisms which could inhibit the electron-heat flux are thus under intense investigation.

One commonly invoked mechanism is driftdriven ion acoustic turbulence. Thermal electrons must drift towards the absorption surface to compensate for the flux of suprathermal electrons which carry the absorbed laser energy to the ablation surface. These drifting thermal electrons drive ion acoustic turbulence, if the electron drift velocity, $V_{d}$. is large enough (for example, $v_{d} \sim C_{s}$ if $Z T_{e} / T_{i} \gg$ I). The ion turbulence (in clumps, rather than individual jons) then scalters the electrons. Thus, the electron mean free path ean be shorter than the collisional value. and the heat flux is inhibited.

The key unsolved problem is the level of inhibition directly connected to the level of ion acoustic lurbulence (the electron mean free path due to the turbulence is $\lambda_{\mathrm{eff}} \approx \lambda_{\mathrm{De}} /(\delta \mathrm{n} / \mathrm{n})^{2}$, where $\lambda_{\mathrm{D}, \mathrm{e}}$ is the electron Debye length). We are performing modeling experiments ${ }^{128}$ and calculations ${ }^{129}$ with the objective of finding the level of electron-heat flux inhibition for laser-driven pellet parameters. Our previous results, reported in last year's annual report. ${ }^{130}$ showed large de electric fields, thermalelectron heating $\left(T_{c} \approx 10 T_{e o}\right)$, and large-amplitude ion acoustic turbulence $\left(\delta \mathrm{n} / \mathrm{n} \leqslant 0.2\right.$, or $\lambda_{\text {eff }} \geqslant 25$ $\lambda_{1}$ ) in the overdense region just above eritical density. Even at the highest power $\left(\mathrm{v}_{0 \mathrm{scc}} / \mathrm{v}_{\mathrm{eo}} \approx 0.7\right.$. where $v_{\text {osc }}=\mathrm{e} E_{0} / m_{0}$ and $E_{0}$ is the electric field of the incident microwaves), however, the dc electric field did not significantly inhibit the hot-electron heat flux: flux inhibition was $f_{H} \geqslant 0.5$, where $f_{H}$ is the factor of heat-flux reduction from free streaming. Nevertheless. our calculations indicate that thermal-electron heat flux is strongly inhibited $\left(f_{t} ;\right.$ $0.06, \lambda_{\text {eff }}>50 \lambda_{\left.D_{c}\right)},{ }^{129}$ and for some conditions the thermal-heat flux is reversed $\left(q_{t} \sim \nabla T_{c}\right.$ instead of $q_{t}$ $\sim-\nabla T_{\mathrm{e}}$ ). This strong inhibition is in apparent agreement with the inhibition factors which are required in the hydrodynamic calculations of some laser experiments.

The reason that the thermal electrons can be so strongly inhibited by ion acoustic turbulence is that the heat flux of the thermal electrons is made up of two terms (i.e., $q_{t} \sim \alpha V_{d}-\beta \nabla T_{e}$ ) which tend to cancel in laser-driven pellets. Thus, under conditions where $V_{d}$ is large enough to compensate for the hotelectron heat flux, $q_{t} \approx 0$ or even $-\nabla T_{e}$. The ion acoustic waves are driven by an effective drift $\left(v_{\mathrm{efr}} \simeq\right.$ $\gamma v_{d}+\nabla T_{e}$ ) however, so that $v_{\text {err }}$ can be large; the 
ion acoustic turbulence is strongly driven, but the heat flux is strongly inhibited.

We have made detailed measurements ${ }^{|3|}$ of the ion turbulence, and improved our computational model. ${ }^{129}$ Our measurements show that the spectra of ion acoustic turbulence peaks at a lower frequency than the frequency of the fastest-growing mode. This indicates that mode-coupling is shifting the lurbulence to lower frequencies. We have therefore incorporated a model of mode-coupling due to induced scattering from the ions 1.12 and electrons 1.33 in our calculations. We find good agreement between the calculated and measured ion acoustic spectra. Thus we can understand why the mean free path implied by the measurements is in fairly ciose agreenent with the Sagdeev formula, ielf $=10^{-2}\left(Z \mathrm{~T}_{\mathrm{c}} / \mathrm{T}_{\mathrm{i}}\right)\left(\mathrm{v}_{\mathrm{d}} / \mathrm{v}_{\mathrm{c}}\right) \omega_{\mathrm{zu}}\left(\lambda_{\mathrm{eff}} \approx \mathrm{v}_{\mathrm{c}} / \nu_{\mathrm{e}} \mathrm{fr}\right)$, which was derived 1.32 assuming that induced scattering is the dominant mode-coupling mechanism.

We have also constructed ${ }^{1.34}$ a computational model of the effect of the de electric field driven by the ion acoustic turbulence on the plasma density profile near the critical density. We have included hot ions in this model. since ion acoustic turbulence levels seen in the microwave experiments should result in strongly heated ions. Preliminary results indicate that the $\mathrm{dc}$ electric field in the overdense region can be large enough to reflect the ions before they get to the critical surface. The importance of this discovery is that the density in the eritical region must decrease so that the density profile is not in steady state. When the density has decreased to the point that the critical density is near the point of ion reflection, then a new cycle starts, with the ion reflection point moving farther in the plasma. This suggests that the density profile near the critical density would not have al steady state. but rather that a relaxation oscillation would be set up.

\section{Authors: ( . L. Yee, J. S. De(iroot, and W. Woo (All I niversity of (alifornia at Davis)}

\section{References}

IIK K. Wiruns, R. B. Spielman. J. S. Decirool. and $N$. M. Bullen. "Ilteds of the Return Current on Iilectron lleating and Thermal T ransport," abstract in hall. .Im. Phas Sid 24. $9 \times 0(1979)$. and submitted o Phys Re's. I ent

134. (. I Fee. W. Wou. and J. S. DeCjroot. "Thermal Heat I las and Anomalous $D$ ( Resistivity." ahsiratcl in Bull. Im th a Ser 24. 934 (1979). and submitted to Phys. Rer. lat
130. I aser Program tmual Report-1978. Lawrence I.ivermore 1.ahorators. l.ivermore. Calil.. UCRI,-50021-78 11979). np. 3-50 10 3-52.

1.31. W. M. Bollen. J. S. Decirool, K. Miruno, R. B. Spieiman, and R. L. Walriven. "lexperimental Investigation of Current Driven Jon scoustic Turbulence and Anomalous I) Resistivity." alsstrate in Rull. Am. Phys. Sod. 24.935 (1979). and submitted to Phiss. Finds.

132. R. /. Sagdeev and A. A. Cialeev, Rom-linear Plasma Theory. (Benjansin. Ness York. 1979) Pp. 68 1073.

13.3. Duk-ln (hoi and $W$. Horton. "Modilied Kadomtsev Spectrum from Renormalied Plasma Turbulence Iheory." Pher thath 17, 2(14h (1974).

13.4. W. Wuo and J. S. De(iroul. "] lliects af counter StreatmIng and Vortex Return Currents an Plasma Temperature

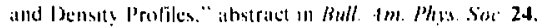
(9ko) (1979), and submitted to Phis Rev leet

\section{Plasma Code Development}

Much of the code development effort this year was spent converting codes to run on the Cray-1 computer. and tuning them to run elficiently. The vectorized (raly version of the 1 S 1 code ${ }^{13.5}$ wats used for the studies described in "Resonant Absorption" earlier in this section. The $Z O H A R$ code. 136 used in many plasmal studies, now runs about four times laster on the cray thatn on the (1) 7600 : much of this speed is duc to the reprogramming for the (ray of the 7600 machine-language particle integrators. In order lo achieve similar economies, new physics codes will be written only lor the (ray-1. bypassing the ( $D C$ 7600. I or example. two extensions of the onc-dimensional hadrodynamics code AURUS (Rel. 1.37) are being implenented on the cray. in one, a PIC model for the ions provides a fully kinetic description of their dynamies. In the second. a WKB treatment of the light allows efficient simulation of stimulated Brillouin scattering over very large plasmit regions.

Authors: A. B. langdon. B. F. Lasinski, and C. J. Randall

Major (untributor: ('. Barnes (Stanford (iniversity)

\section{References}

135. C. K. Birdaill and I B. I alled:m. Plasma Physics via Computer Simulanom 11 moversils of California Press. Herkeley and 1 os ingeles. 1975)

136. 3. B. I angdon and B. I I asmski. "kelectromagnetic and Relanivistic Platma Simulation Models." in Methods in Compatotomal phract. I. Killeen. lid. Academic Press. ven York. 1976), wol. 16. 13. Nlder. S. Fernbath, and M. Rotenberg. H.dh. P .3.27

137. T.aser Program famal Report-1978. 1.awrence l.ivermore lahorators. I isermore. Calif., (XCRL-50021-78 11979). pp. $3-53$ in 3.56 . 


\section{I.ASNEX and Atomic Theory Overview}

During 1979 we completely restructured and renrole the I. ASNl:X code for the Cray-l cominter several physics improvements were made in the corte as a ilatural consequence of this translation nowe and wher improvements were made in : . . re iceded to carry out current design work.

the allomic physics package, XSN, wals .... $+i_{1} / \ldots$ al 10 melude electron degeneracy and a for-

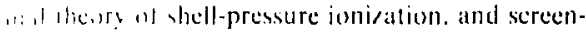

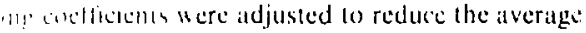
twa m mom/ation polentials. These improvements 111.t5: moly small changes in the cialculated Ireyenty-dependent opacities: however. pressures and energies, which are needed for nen-I.Tl: cotculalions. have improved significantly. and they are now themodynamically consislent. He halve begun (1) use XSY as well as at Thomas-lermiInate model to calculate ion-beam deposition in dense plasmiss

The Brillouin backscattering model that 'imits the amount of laser light available for atsorption hits been modilied to self-consistently cialculate situratton by densily gradients and ion damping. rcoordingly. calculated laser-light absorption on gold disks is now more in agreement with experiments.

Additional terms have been included in the multigroup diffusion equations used to transport charged particles from hermonuclear reactions. (ompared with standard diffusion models. the new metheds are less sensitive to done size and time step and provide better agreement with Monte carlo cilluulations.

In translerring I.ASNI:X to the (ray-I competer. we have made niany improvements to the structure, reliability. and speed of the code. By alutomiting the management of memory. we have elimialated many kinds of programming errors and made it possible for as many as cight people to work on the code simultaneously without interfering with one another. Nlso, we have found that it is now much easier to add new physics packages and user conveniences.

Most of LASNEX has been rewritten to take advantage of the fist vector instructions on the Cray-1. For some procedures, such as matrix inversion. this capability required us to develop totally new methods.

Currently, IASNLX is running about twice as fast on the Cray-1 as it does on the 7600. Compiler enhancements expected in the next lew months should allow us to realise vector speeds for mest of the coding. We anticipate further improvement in speed by a factor of two.

\section{Author: (i, B. Zimmerman}

\section{Improvements in the IASNEX Atomic-Physies Package}

The I ASNIX in-line atomic physics packalge XSNQ has been revised to improve its treatment of heavy atoms in dense plasmats. The changes give a signilicant improvenent in the physical deseription of atomic ionization. equation of state, and opacity. the revised code provides a unified atomic model uselul for the enlire range from low densities (including non-l Th: corditions) to very dense. degenerale malter.

SSVQ Subrouline. The I. ASNIX code uses XSNO. originally develoned by W. L.okke. for calculations of hot plasmats at low densities. ${ }^{3 k}$ The package is a convenient source of atomic data-including ionization state, energy levels, populations. tquation of slate, and optical-absorption coefficients ropacity-and it calculates both equilibrium and nonequilibrium problems. We are nuaking a continuing elfore to iniprove this package with respect to computational speed and physical acurati. $199 \cdot|4|$

Inertial confinement lusion research is uniquely interested in plasnlas of high density $(\mu>0.1$ g/ $/ \mathrm{cm}^{3}$ ): however, normal atomic-ionisation calculations encounter difficulties when itpplied to atoms in such a dense plasma. These difficulties, which occur for dense plasmas and are associated with continuam-lowering and pressure ionization. include

- I.arge regions where the ionization state $Z$ is constant and independent of aensity and temperature 
- Pressure ionization occurring as discontinuous jumps of ionization state.

- Slow computational convergence near these jumps.

- Disagreement with the statistical-atom (Thomas-Fermi. Thomas-Fermi-Dirac) calculations by as much as a factor of two.

Similar problems arise in other atomicjonization calculations for dense plasmas, including those based on the Saha equation. An additional difficulty specific to the XSNQ screened-hydrogenic model is produced by inaceurate ionization potentials for heavy elements: this problem is most evident at relatively low plasma lemperatures ti.e.. $<100 \mathrm{eV}$ ). Tuble $3-2$ gives a comparison that il. lust rates this problem for gold $(7 .=79)$.

Finally. there has been a continuing difficuly with thermodynamic consistency of $\mathrm{l}$.Tl: and nonI.Tl: equations of state.

We have developed practical solutions to these difficulties. Our changes retitin the XSNO energylevel scheme and preserve compatibility with the opacity calculation and non-1.Tl equations. The modified code is faster than the original hecause it achieves self-consistency in fewer iterations

Screening coefficients. The ionisation potentials are obtained from a sereening nodel originally developed by II. Mayer. ${ }^{1+2}$ For electrons of at!! shell. $\mathrm{n}(\mathrm{n}=$ principal yua ntum number $=1.2 .3 \ldots)$. a screened nuclear charge. $C_{n}$, is computed as

$$
z_{n}=7 \quad \sum_{i<n} \operatorname{S(n,i)p_{i}} \mathrm{I}_{n n^{3}} .
$$

where $t$ is the nuclear charge, $S(n, i)$ is the matrix of screening constants. $T_{n}=12\left|1-\left(12 n^{2}\right)\right| S(n, n)$ is the screening within the $m$ th shell, and $P_{n}$ is the num-

Table 3-2. A comparison of calculational models, for the first few ionization potentials of goid.

\begin{tabular}{cccc}
\hline $\begin{array}{c}\text { Ionization } \\
\text { stage }\end{array}$ & $\begin{array}{c}\text { Original XSNQ } \\
{[\text { Mayer S(n, i)] }} \\
(\mathrm{eV})\end{array}$ & $\begin{array}{c}\text { New XSNQ } \\
\text { [New S(n, i)] } \\
(\mathrm{eV})\end{array}$ & $\begin{array}{c}\text { Scofield } \\
\text { Hartree-Fock- } \\
\text { Stater (eV) }\end{array}$ \\
\hline 1 & 69.8 & 13.4 & 9.2 \\
2 & 82.6 & 23.0 & 20.5 \\
3 & 95.7 & 33.1 & 32.2 \\
4 & 109.3 & 43.6 & 46.2 \\
5 & 123.4 & 54.6 & 61.1 \\
\hline
\end{tabular}

ber of electrons in the mh shell for the iosi in question. (In thermodynamic equilibrium, $P_{n}$ is the average shell population.)

From the screened nuclear charges, a total fw: energy. $t_{\text {inoll }}$ is constructed by use of relatmin. hydrogenic energy levels, and this ion energes od! ferenced to produce the inniration potentials. I h: procedure predicts the ionsation polentials 1.1 ..: the elements in terms of a single fo-by-10 miltan sertening constants. Matyer calculated the wre...... constanls $S(n, i)$ by apply ing first-order perturt. $i_{1}, \ldots$ theory to hydrogenic wave fanction

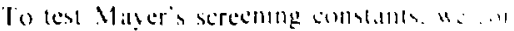
pared the predicted iombation porembith a...

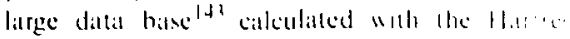
lack-slater theory. Ihe datal base colltam ve ionizittion polentials for 30 elemens

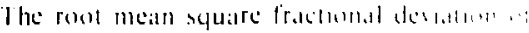

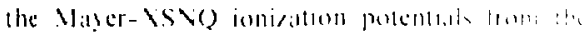

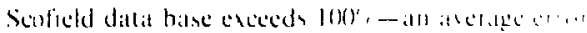

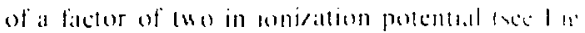

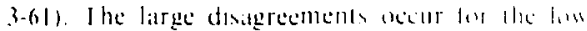

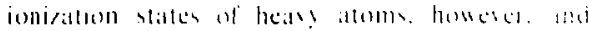
lherefore are important only for cold planmal I. (1)10 (1)

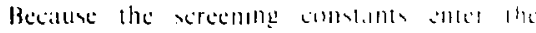

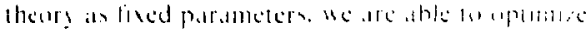

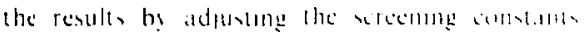

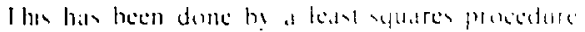

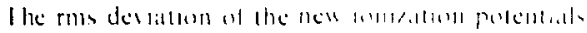

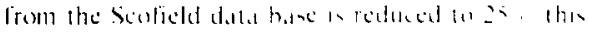

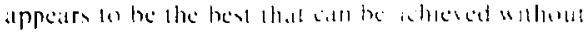

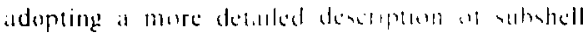

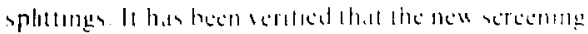

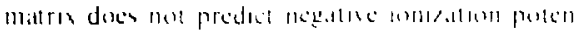

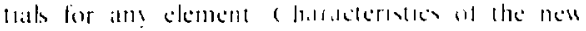

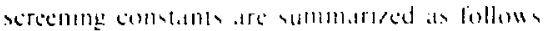

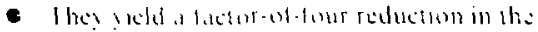

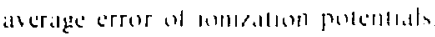

- Ihe new mitris retains the important "ym-

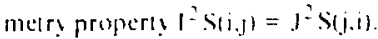

- Ihe matrix-elements are smoolh monotone functions of shell indices i.j (see l-ig. 3-62).

- Ihe largest alteration in any se, eening constint is kess than 10 ';

ligure $3-6,3$ compares iomsation potentials calculated from the new screnting constants with the Scofield data-base for aluminum, iron, barium. 
Fig. 3-61. Ionization potentials of four elements e re plotted as functions of the number of bound electrons. The solid line interpolates between the Hartree-Fock-Slater ${ }^{142}$ values; the points are produced by the XSNQ package with the original (Mayer) screening. Note the substantial disagreement for nearly neutral heary atoms.

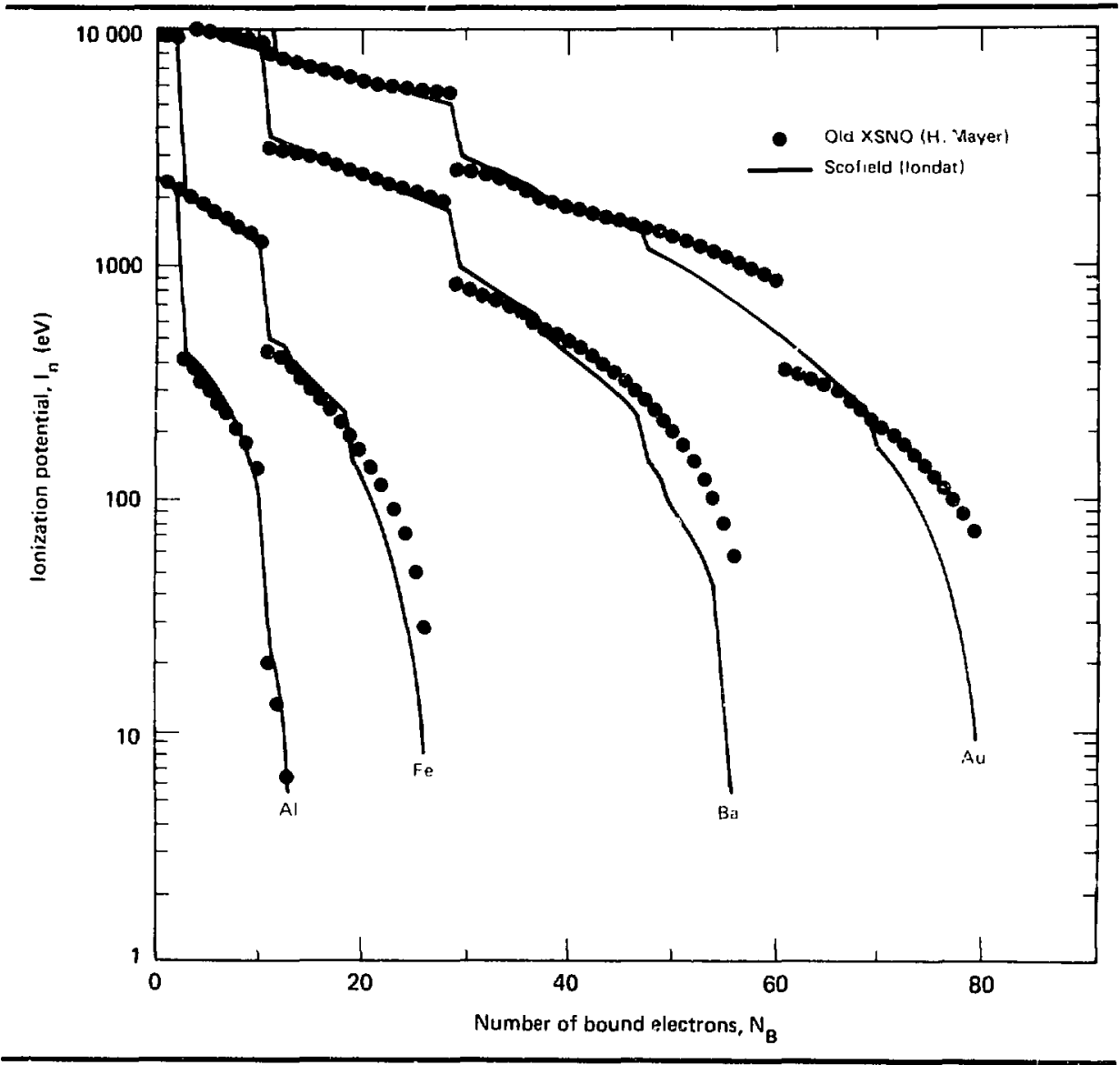

and gold. The first lew ionization potentials are signilicintly improved. (learly. these new screening constants will Improve the ability of L.ASNI:X to describe lou-lemperature material properties.

Pressure Ionization Vodel. When atoms press together at high density. bound electrons are released into the fres-electron continuum., a process called pressure ionization. In our approach, the pressure ionization is phenomenolugically described by introducing a shell degeneraty $D_{n}(p)$ that enforces the removal of the nth shell as the atum is compressed. The electrons of the $n$th shell are assigned an approximate orbit radius

$$
\left.I_{n}=0.529 \times 10^{-8} \mathrm{~cm}\right)\left(\frac{n^{2}}{Z_{n}}\right) \text {, }
$$

where $n$ is the principal quantum number and $Z_{n}$ is the screened nuclear charge in $m$ h shell: the shell merges smoothly into the continuum when the 
Fig. 3-62. The new screening constants $S(\mathbf{i}$,$) .$

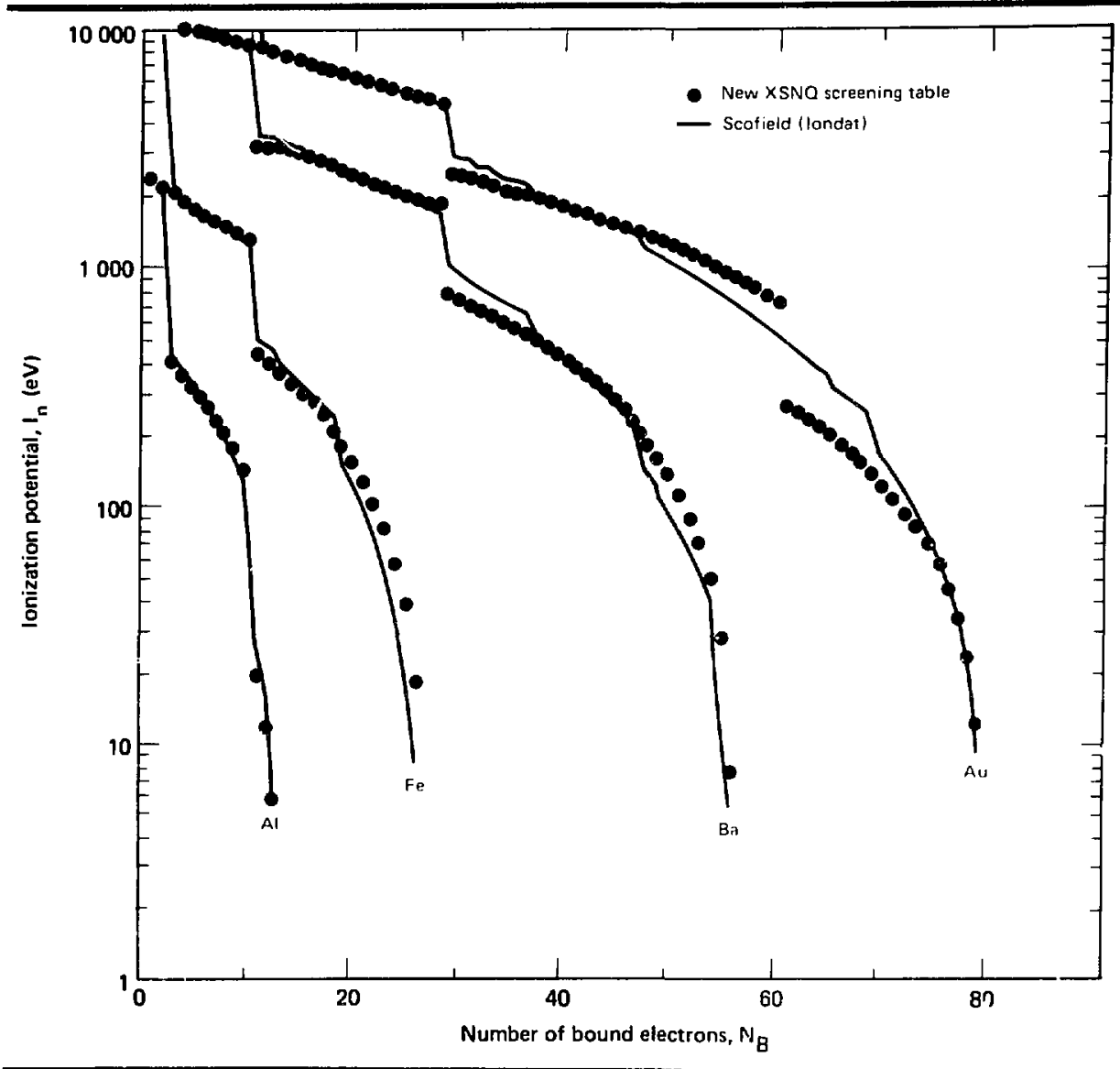

alerage atomic spacing drops belou $2 r_{n}$. In this model. the shell degeneraley is taken io be

$$
D_{n}=\frac{2 n^{2}}{1+\left(a \mathrm{ar}_{n}^{3}\right)^{\mathrm{b}}} \text {. }
$$

where $p$ is the material density. The coefficients a.b ars adjusted to produce approximate agreement with the Thomas-lermi-Dirat theory.

Thermodynamic Formulation. The Helmholy liee energy of the ion is constructed as

- Pree Energ:: $F_{\text {iọn }}=F_{i o n}-T S_{i o n}$.

- Fnergy: $F_{11 n}=\sum_{n} \in_{n} P_{n}$.
- Inturs $S_{\operatorname{mn}}=-h \mid P_{n} \log \left(f_{n}\right)+\left(D_{n}-P_{n}\right)$ log $\left(1 \quad i_{11}\right)$ where $\frac{1}{11}=$ anc-electron energs $11.3 .6 \mathrm{es}$

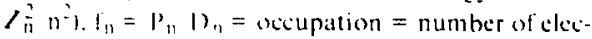
troms per quantum stale. and $\underline{k}=$ Bolumatsm onstant.

The cotal lree energy $1 \mathrm{f}$ is $\mathrm{f}$ iun plus comtribulions from fres clectrons and from continum lowering. The ire electrons are allowed artitrits degeneracy. $1 /$ thermodynamic averages $\left(f_{n} . l\right.$. pressure. energy) are derived from this free energ!. 


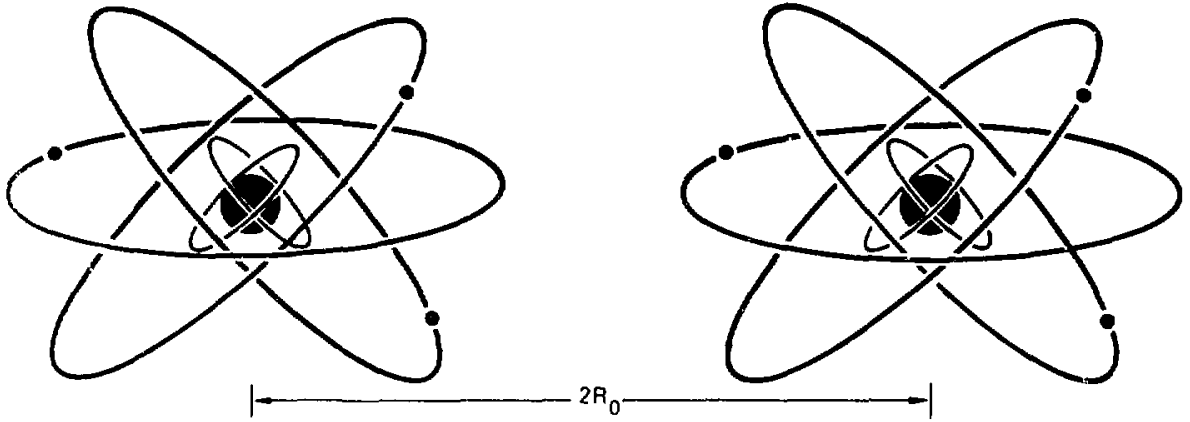

Fig. 3-64. Numerical test of thermodynamic consistency for the modified XSNQ. The quantity shown is $A=[T(\theta p / \partial T)-P] /[2 e / \partial V]$, which should be unity. The slight devintions from unity are caused by numerical differentintion on a finite $(50 \times 50)$ grid.

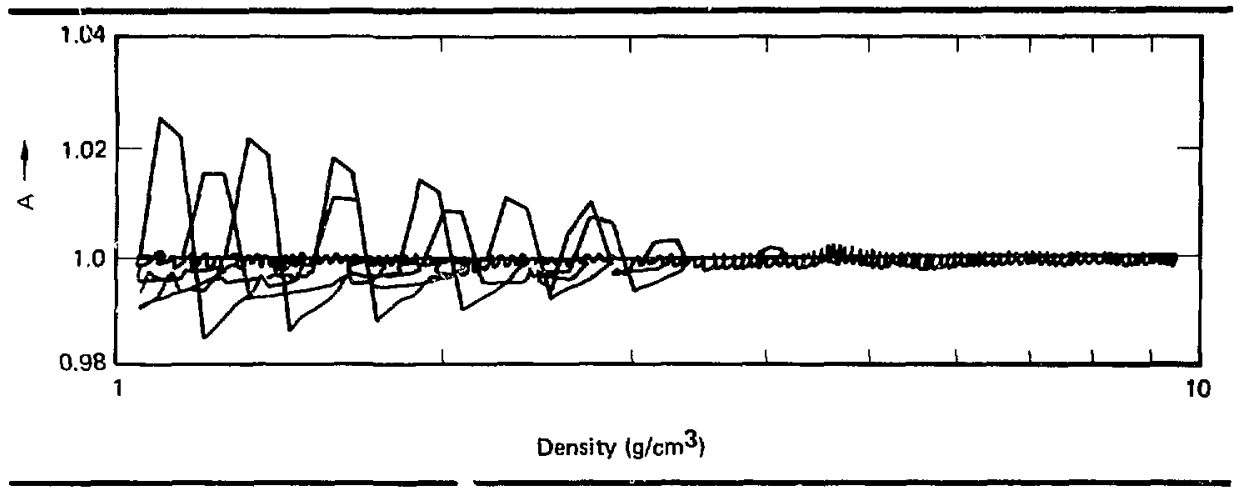

I The procedure gliarantees thermodynamic conwience. "hich a important lor hydrodynamic applications: the numerical results show excellent ther modinamic consulerin! isee fig. 3-64).

The pressure ohtained from this free-energy model contains three terms: free-electron pressure. including degeneracy effects: an electrostatic contribution obtatined from the continuum lowering: and a shell-compression pressure not considered by previous workers. The latter term is given by

$$
P_{\sin \| u}=k l \sum_{n} \frac{\partial D}{\partial v} \log \left(1-f_{n}\right)
$$

This term contributes when an occupied shell is squeezed into the continuum. The pressure and energy are ploted in Fig. 3-65 for aluminum. (This is the electronic pressure: solid-state bonding and ion kinetic contributions are omitted.) The ionization state. $Z$, is plotted in Fig. 3-66.

Addition of pressure ionization (in the form described here) and free-electron degeneracy has recuired several changes in the opacily and non-LTE portions of the XSNQ code. With these changes installed, the computed results appear to give a more realistic description of very dense plasmas.

The theory described here gives an immediate practical description of pressure ionization in dense plasmas. Efforts are underway to employ more 
Fig. 3-65. Modified ionization nodel results for aluminum: (a) electronic pressure, and (b) electronic energy.
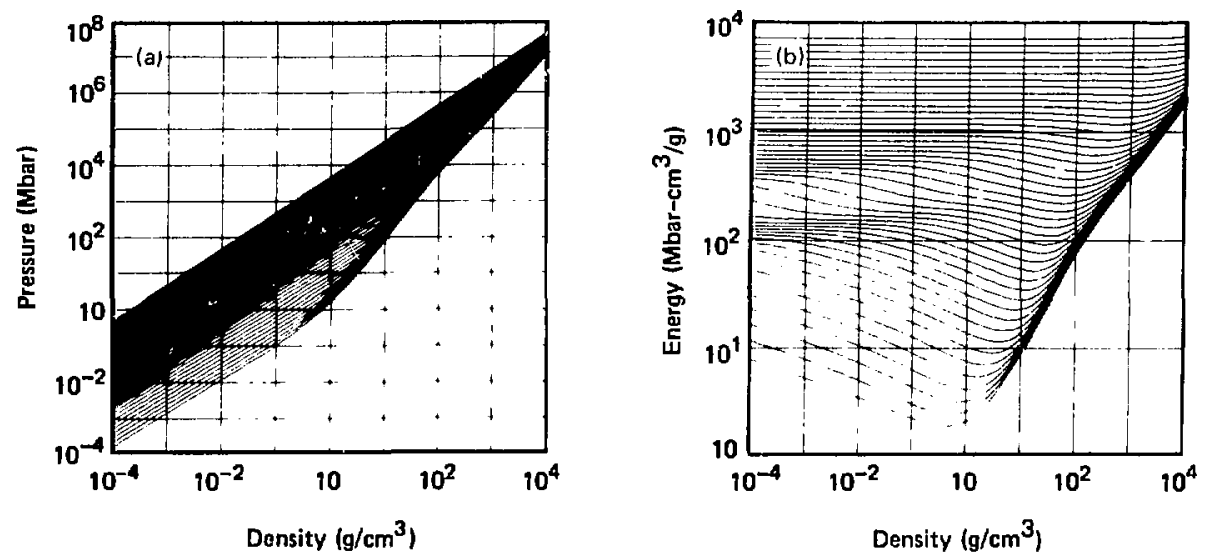

Fig. 3-66. The ionization state $Z(n, T)$ of aluminum: (a) as calculated by the original average atom model and (b) as calculated from the new model. In (a) the density range from $10^{-4}$ to $10^{4} \mathrm{~g} / \mathrm{cm}^{3}$; the temperature from 1 to $10 \mathrm{keV}$. Note the following unsatisfactury fentures: Пat shelves where $Z=$ constant; very abrupt jumps hetween shelves; wrinkied extensions of the jumps that continue up to high temperatures. All these features are associated with the pressure-ionization model. The calculation in (b) has been adjusted to give approximate agreement with the Thomas-Fermb-Dirac theory in the range of pressure ionization. The $Z(n, T)$ surface is considerably smoother.
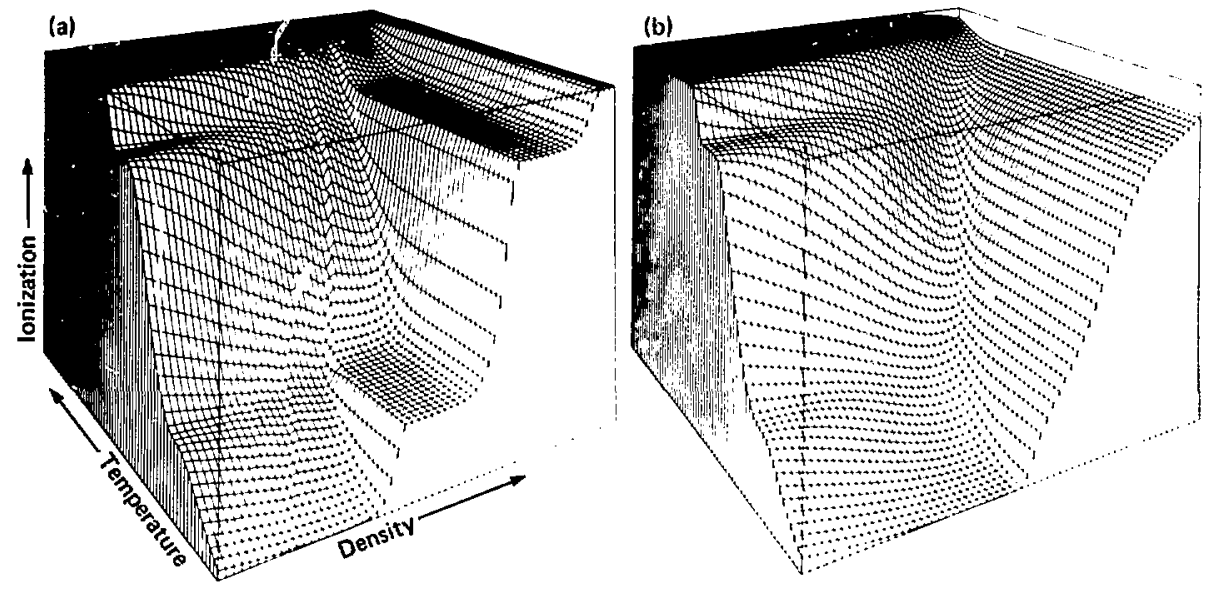
rigorous quantum-statistical calculations to supply more detailed information about spectroscopic phenomena encountered in pressure ionization.

\section{Authors: R. M. More and G. B. Zimmerman}

\section{References}

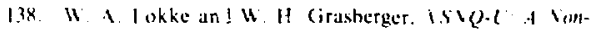
1.If Fmixum and absorplion Coefficiem Subroutine. 1 awrence I wermore 1 aboratory. I sermore. Caldf. I ( R I - 5227611977 )

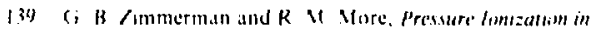
faserefusion larget simulatum. I anrence 1 is momore

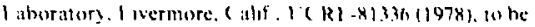

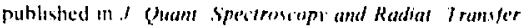

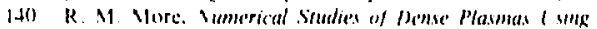
Sparistisal and tomization theortes, 1 alu rence I vermore

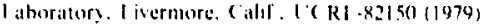

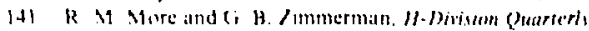
Report Juld through Seprember 19?, I a rence I nermore

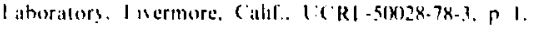

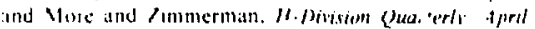
through fune 1070. ( ( RI - 50028-79-2. p I

14. M. Miger. "Metheds of Opilcty Cimleulation," Ios

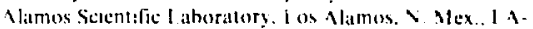
$6,4711947)$

14.4. I. H. Scofield. I au rence I wermore I ahoratory. prisale communicition (1974)

\section{Energy Deposition by Fast lons}

II idespread interest in heavy-ion fusion has prompted us to re-examine the I. ASNE: $X$ model for ion energy-deposition in hot dense plasmas. The existing l.ASvild deposition package follows textbook theory. ${ }^{\text {It }}$ separately calculating energy loss to bound and free electrons. The average ionization potential required for energy loss to bound electrons is calculated by a plausible scaling formula that is correct in the two limits of cold matter and fully iunired plasma. However. we believe that at initio energy-deposition calculations give a more convincing basis for heavy-ion target design. During 1979. we began development of two theoretical models that will be used to calculate heavy-ion energy loss. In the future. we hope to incorporate the results of our development effort into the I ASN LX code.

The practical problem concerning heavy-ion energy loss is that all existing experimental information refers to cold matter, but ICF target plasmus reach high temperatures and pre'sures. The actual rate of energy loss is expected to change dramiatically with the thermodynamic state of the target. 145146

To briefly describe our calculations, it is useful 10 w rite the energy lass per unit of path length in the general form

$$
-\frac{d r}{d x}=\frac{4 \pi Z^{2} e^{4}}{m v_{0}^{2}} N L
$$

"shere $/$ is the current charge of the fast ion. $v_{0}$ is its velocit! $m$ is the electron mass. and $N$ is the ion number densily in the target plasma. 1 . is the stopping number per larget atom. often calculated ats at logarithm of a ratio ol lengths fe.g. maximum and minimum impact paramelers). The inportant un. known quamitilies are $/$ and $\mathrm{l}$..

for fast ions, the charge stale $\Rightarrow$ depends mainly on the ion velocity $v_{0}$ and is believed to depend weakly on the plasma thermodynamic state. for this citse. we currently use the empirical formula given b! Betr. ${ }^{147}$ for slou ions, $Z$ cannol fall belou the thermal ionization state of an impurity ion in the plasma: this is calculated by ihomas-1ermi theory. The XSNQ code contains a uselul set of ratc equations and cross sections that can be modified to calculate the charge state of moving ions: this calculation is under way and will provide a hetler estimate of $/\left(v_{(i)}\right)$.

The stopping number, L, can be calculated by two methods. In one approach, the ions of the target plasma are described by ThomasFermi-Dirac theory. ${ }^{14}$ This theory includes many effects of high pressure and temperature. including thermal ionization, electron degeneracy, and strong ion-pair correlation. The theory is not completely quantum mechanical and omits effects of boundelectron energy quantization. To be certain that the omitted effects do not significantly alter the stopping power, we intend to verify our results by using the screened hydrogenic ionization model (XSNQ).

To calculate the stopping number per atom. L Atom, from the statistical model, we have adopted the local-density approximation of Lindhard and Winther ${ }^{149}$ :

$$
L_{A t o m}=\int n(r) L_{0}\left[n(r), T v_{0}\right]\left[1-\frac{Z e v_{1}(r)}{E}\right] d^{3} r \text {. }
$$

In this equation. $L_{0}\left[n(r), T . v_{0}\right]$ is the stopping number per electron in a uniform electron gas having 
density $\mathrm{n}$ and temperature $\mathrm{T}$ (for fast ions of velocity $v_{0}$ ). A similar theory is widely used to calculate energy loss in cold matter. ${ }^{150}$ We have modified the Lindhard-Winther approximation in the following ways:

- The electron density $n(r)$ is calculated from a finite-temperature theory (i.e.. TFD theory).

- The stopping number $L_{0}$ is obtained from the finite-temperature dielectric function.

- The factor in heavy brackets in Eq. (132) is a correction for curved orbits followed by heavy ions traversing the volume of a target atom. The term $v_{1}(r)$ is the ion-pair potential. ${ }^{151}$ and $E$ is the energy of relative notion of projectile and target ions.

- The classical Bohr minimum-impact parameter is used where appropriate (heavy-ion projectiles).

We have applied this formalism to calculate stopping ol helium ions (alpha particles) in gold at several densities: the results are shown in Fig. 3-67. The normal density results are (generally) within about 20\% of experimental data.

A second approach uses the XSNQ ionizationequilibrium model to provide detailed populations and energy levels for the target plasma. The average ionization potential of bound electrons is then formed directly by using hydrogenic oscillator strengths. The usual stopping theory ${ }^{144}$ is used to

Fig. 3-67. Energy loss per ntom for helium ions in gold at three densities calculated from the TFD model. The units of $S(E)$ are $\mathrm{eV}-\mathrm{cm}^{2}$ per $10^{15}$ atoms; $\mathrm{dE} / \mathrm{dx}$ is $\mathrm{S}(\mathrm{E})$ times the target ion number density.

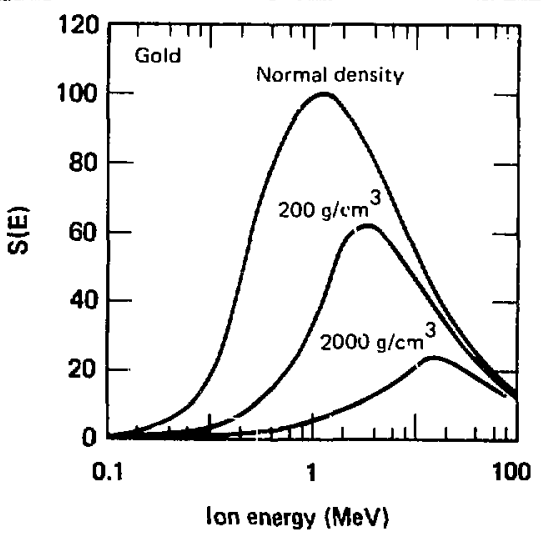

obtain the free-electron contribution. The advantage of this second approach is that it will provide an independent check on the validity of results obtained from the statistical model.

\section{Authors: R. M. More and D. S. Bailey}

\section{References}

144. J. D. Jackson, (Jassical Electrod)nomics (John Wiley \& Sons. $A$ w York. 1975), 2nd ed.

145. S. Skupsky, Phy. Rel A It, , 1977).

146. F. Nardi, t. Peleg, and 7 . Zinnsi un. Phy.r. Fluids 21, 574 (1978).

147. H. D. Bete. Rev. Mod, Phys. +13. 465 (1972).

148. R. D. Cowan and J. A. Ashkin Mhs. Ret. 105. 144 (1957).

149. J. Lindhord and A. Wint1 Danske Videnskabermes Selskab 34. $4(1964)$.

150. W. K. Chu and D. Powers. Phys. Rev. 187. 478. (1969): Phys. Ret. B 4. 10 (1971).

151. J. Lindhard, V. Nielse and M. Schurff. Danske ridenskabernes Selskab."., $10: 19081$.

\section{Brillouin Backscattering Model in LASNEX}

In the 1978 an 1 report ${ }^{152}$ we described our stimulated Brilloui scattering (SBS) model for LASNEX. In the $r$ "t year "re have made many improvements and additions, to more accurately model the Brillowan backscattering in underdense plasmas. We have changed the densi..--dependence of our model: included the effects of inhomogenenus media: added a self-consistent calculation of te hot-ion contribution to ion damping: improve the saturation calculation: improved the calculat in of backscattering when a very small fraction is reflected: and considerably expanded the treatment of backscattered light. Below, we describe these ch:' ges to our SBS model.

The fraction of light reflected in each zone by Brilloun backscattering is obtained by the following equation [compare with Eq. (54) on page 3-63 of Ref. 52]:

Reflection $=1-\exp$

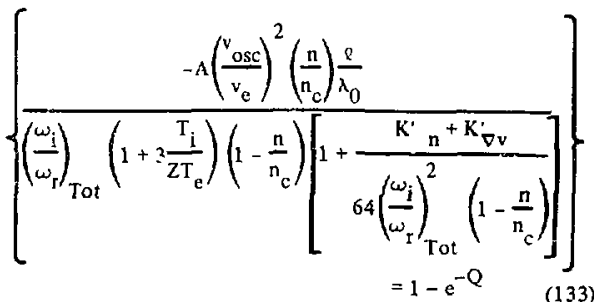


where

$$
K_{\Gamma_{\mathrm{n}}}=\frac{\lambda_{0}}{\Delta x}\left(\frac{\mathrm{n}}{n_{\mathrm{c}}}\right)\left[\left(\frac{C_{s}+v}{C_{s}}\right)^{2} /\left(1-\frac{n}{n_{c}}\right)^{1 / 2}\right]
$$

ind

$$
K_{\Gamma v}^{\prime}=2 \frac{\lambda_{0} \Delta v}{\Delta x}\left[\left(1-\frac{n}{n_{c}}\right)^{1 / 2}\left(\frac{c_{s}+v}{c_{s}^{2}}\right)^{2}\right] .
$$

The sradients are taken along the direction of the incident light and will be explained below. $A$ is a cinstim except in the case of very small backscattring. is discussed below: $v_{\text {osc }} / v_{\mathrm{c}}$ is the ratio of the uscillation velocity of an electron in the laser electric field to the electron thermal velocity: $n / n_{c}$ is the ratio of the electron number density to the electron number density at the critical surface (where $\omega_{0}=$ $\left.\omega_{p, c}\right): C: \lambda_{0}$ is the ratio of the pain length to the vacuum laser wavelength: $\omega_{i} / \omega_{r}$ is the ratio of the ion damping to the ion acoustic frequency; $T_{c}, T_{i}$, and $Z$ are the electron and ion temperatures and the ionization state. respectively: $C_{s}$ is the ion sound speed: $k_{0}=2 \pi / \lambda_{0}: v$ is the material expansion velocity, and $\lambda x$ is zone lergth.

Vew Features of the SBS Model. We have thoroughly investigated the density-dependence of the $Q$ factor, and we have now settled on the dependence shown in Eq. (133); this dependence gives the best fit to the sinulation data, and is theoretically justified. ${ }^{153}$

Both density and velocity gradients affect the stimulated Brillouin scattering instability. In the presence of inhomogeneities of the plasma properties, the resonance conditions are satisfied only in a finite region of space. Thus the instability is effectively damped if the waves propagate out of the resonant interaction region before significant growth occurs. In Eqs. (133) to (135), we show how the gradient terins are included in $Q$, the exponentiation length of SBS. The effects of inhomogeneous media on three-wave parametric instabilities were first derived by Rosenbluth. ${ }^{154}$ The form we have employed is derived by replacing Nishikawa's ${ }^{155}$ integration length in his Eq. $111-57$ by the mismatch length, ${ }^{154}$ as suggested by Kruer. ${ }^{156}$ As a diagnostic check, the code calculates the threshold conditions for density-gradient saturation,

$$
\left(\frac{v_{\text {osc }}}{v_{e}}\right)^{2}<\frac{4}{\pi} \frac{\left(\nabla \frac{n}{n_{c}}\right) \lambda_{0}}{\left(\frac{n}{n_{c}}\right)}\left(\frac{C_{s}+v}{C_{s}}\right)
$$

and velocity-gradient saturation,

$$
\left(\frac{v_{\text {osc }}}{v_{c}}\right)^{2}<2.55 \frac{(\nabla v) v_{0}\left(1-\frac{n}{n_{c}}\right)}{C_{s}\left(\frac{n}{n_{c}}\right)}
$$

and monitors the number of times that these conditions are satisifed.

In last year's annual report ${ }^{152}$ we mentioned $t$ iat the ions heated by SBS are optimally located in position and velocity space to do the most damping. and that the ion-ion equilibration time is usually large enough to make this nonequilibrium effect visible. Initially, we simply mocked up this phenomenon by a multiplier on the ion temperature in the $\left(\omega_{i} / \omega_{r}\right)$ calculation. Now the contribution of the hot ions to the damping is calculated selfconsistently. This improvement is indicated in Eq. (133) by the subscript "Tot" on $\left(\omega_{i} / \omega_{\Gamma}\right)$.

We now use the following equation to calculate ion damping:

$$
\left.\frac{\omega_{\mathrm{i}}}{\omega_{\mathrm{r}}}\right|_{\text {Tot }}=\left.\frac{\omega_{\mathrm{i}}}{\omega_{\mathrm{r}}}\right|_{\text {Hot }} \frac{n_{\mathrm{iHot}}}{n_{\mathrm{iTot}}}+\left.\frac{\omega_{\mathrm{i}}}{\omega_{\mathrm{r}}}\right|_{\text {Cold }} \frac{n_{\mathrm{iCol} \text { I }}}{n_{\mathrm{i} \text { Tot }}}
$$

where $n_{j H o t}$ and $n_{i C o l d}$ are the number densities of the hot and cold ions, and $n_{i}$ ot is the total ion density. The damping frequency of the cold ion is calculated as described in Ref. 152. The $\omega_{\mathrm{i}} / \omega_{\mathrm{r} H \text { or }}$ calculation is simplified by assuming $Z T_{\mathrm{e}} / \mathbf{T}_{\mathrm{H} \text { Hot }}=$ 2: i.e., the hot ions are accelerated by SBS to a temperat are $1 / 2 \mathrm{M}_{\mathrm{j}} \mathrm{C}_{\mathrm{s}}^{2}$

In our new self-consistent calculation, the density of the hot ions must be found to evaluate $\omega_{\mathrm{i}} / \omega_{\mathrm{r} T \mathrm{~T}}$. Ions are heated by the SBS; they remain hot for the ion-ion equilibration time, $\tau_{i j}$, which may be long compared to the other time scales of interest. Thus time histories of the fraction of hot ions as a function of electron density. $n_{\mathrm{e}}$ are required to

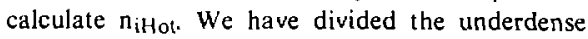


plasma into the following five sections so that the ratio of electron density to critical density, $n / n_{c}$. ranges from 0 to 0.0625 . from 0.0625 to 0.125 , from 0.125 to 0.25 , from 0.25 to 0.5 , and from 0.5 to 1.0 . The total run time is divided equally into 20 time blocks. For each of these 100 points in $\left(t . n_{\mathrm{e}}\right)$ space, the fraction of hot ions is stored and, of course. updated at each time step. Finally, we obtain the current $n_{\text {iHot }}\left(t, n_{\mathrm{e}}\right)$ where

$$
n_{i H o t}\left(t, n_{e}\right)=\int_{t-\tau_{i j}}^{t} n_{i H o t}\left(t^{\prime}, n_{e}\right) d t^{\prime}
$$

by the appropriate summation.

We found that our nonlinear saturation statement was not suitable for a probiem with zones that were greater in width than a few vacuum wavelengths. This is, of course, the usual situation in hydrodynamic calculations. In the old model, the maximum saturated backscattering occurred throughout the entire zone in which the saturation limit was reached, irrespective of the zone size, resulting in nonphysicaliy large amounts of backscattering. In the sal world, the maximum backscattering would not occur over many wavelengths: instead, SBS would be self-limiting through the intensity dependence, $\left(v_{\text {osc }} / v_{\mathrm{e}}\right)^{2}$. of the $\mathrm{Q}$ factor. The backscattering reduces the light intensity reaching $\lambda_{0}$ further into the plasmá.

io include this natural self-limitation in our SBS package, we wrote a saturation loop that is short and fast. Each zone in which saturation occurs is treated in pieces of length $\lambda_{0}$. The proper light in. tensity, with the backscattered part deleted, is used for each plasma slab of thickness $\lambda_{0}$. We have found that this technique works very well to correct the overestimate of scattering that occurred in the simpler model.

For quite small reflection. the total backscattering is smatler than the pump-depietion regime described by Eq. (133). The code monitors the space-integrated $Q$ from the previous time step and sets $A$ so that the total reflection agrees with the more compleie description of Ref. 157 for a noise level of $10^{-4}$.

Finally, we have expanded the options available fo ${ }^{-}$monitoring backscattered light. Last year, we reported that all of the backscattered light was assumed to be scattered out of the target and was lost from the problem. Now, ine user ruay select any of the following four options:

- All of the backscattered light is lost, as before.

- None of the backscatiered light is lost except that scattered into vacuum. Instead, the light backscattered on the way up the density gradient into the material is added back into the light ray at the scattering location, as the ray propagates back down the density gradient. Likewise, on the way back out of the material, the backscattered light will augment the ingoing ray on the next time step. This model is one-dimensional and allows only one laser.

- Some of the backscattered light is lost. but the rest is traced. This is a combination of the first two op:ions: the user defines a certain fraction of the backscattered light to be tracked, as in the second option: the rest of the light is then lost, as in the first option. This model may be thought of as allowing a fraction of the scattered light to be sidescattered and lost: the rest of the light is backscattered and trasked.

- The user may track the light that is backscattered as the laser goes up the density ramip. but not the light that is backscattered as the light propagates down the density ramp. Again, this is a combination of the first two options, using the second option on the way into the target and the first option on the way out.

In conclusion. the Brillouin backscalter model has been extensively expanded and improved. and this model now predicts 0 within $10 \%$ the absorptions seen in gold-disk experiments. The results of these predictions are discussed in "Calculations of Stimulated Brillouin Scattering with LASNEX." earlier on this sution.

\section{Authors: J. A. Harte and K. G. Estabrook}

\section{References}

152. laser Program Annual Repm-1978. Lawrence Livermore laboratory. Livermore, Calif. LCRL-50021-78 11979). PГ. 3-63 10 3-69.

153. H. I Kruer. Lawrence Livermore Laboratory. Livermore. (alif., privite communication (1979).

154. M. N. Rosenbluth. Phys. Rev. Leu. 29, 565 (1972)

155. $h$. Vishikawa. in Advances in Plasma Physics. vol 6. A. Simon and 4 . Thompson. Eds. (Wiley \& Sons. New York. 1976). p. 45.

156. W. 1.. kruer. Lawrence Livermore Iaboratory. Livermort. (alif., private communication (1979).

157. D. W. Phiilion, W. I. K ruer. and V. C. Rupert, Phys. Rev: loct. 39. $1529(1977)$. 


\section{( harged-Paiticle Multigroup i)iffusion}

During the past year we completely rewrote the ... rmonuclear-burn-product transport routines in ! s 1 . We undertook this prnject in order to $\therefore$ advantage of fast vector operations on the $\therefore: \because-1$ mputer. but many physics improvements atie ulcurporated at the same time. These improve:r:uten include:

- Consistent isotope production and depleW. for all reactions.

- The ability to transport any reaction iroduct of knock-on from elastic scattering.

- Inclusion of all important in-flight reactions and nuclear elastic scattering involving imopes of hydrogen and helium.

- The ability to use completely arhitrary energy-group structures for all charged particles.

- Inclusion of inertial terms in the flux equatoon for all charged particies.

The most important improvements have been the last two items of this list. In this article we discuss the importance of including the inertial terms.

Starting from the lokker-Planck equation. Ref. 158 takes the /ero and first moments to obtain

$$
\frac{\partial n_{l}}{\partial t}+v \cdot \vec{j}_{I}=\frac{1}{\tau_{e}} \frac{\partial}{\partial I:}\left\{\left[1+\left(I_{0} / l:\right)^{3 / 2}\right] \mathrm{I:n} \mathrm{n}_{\mathrm{l}}\right\}
$$

and

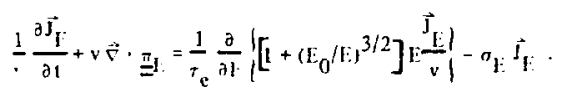

In these equations $n_{k}$ is the density of particles at energ! ..$~$ is their velocity. $\vec{J}_{k}$ is their flux. $\tau_{\mathrm{s}}$ is the slowing-down ume considering only electron collisions. $f_{0}$, is the energy at which energy losses to electrons and ions are equal. $\underline{\underline{\pi}} \mathrm{F}$ is the stress tensor. and $\sigma_{f}$ is the scattering cross section. The bracketed term in each equation represents the effects of energy loss: in F.q. (141) we can identify it as an inertial term. since it accounts for the fact that charged particles remember their direction as they lose energy. Previously. we had followed Ref. 158 and simplified : :q. $(1+1)$ by assuming

$$
\begin{aligned}
& \frac{\partial \overrightarrow{\mathrm{J}}_{\mathrm{L}}}{\partial \mathrm{t}}=0 \text { (steady-state), } \\
& \frac{\pi}{=} \mathrm{:}:=\frac{1}{3} \mathrm{n}_{\mathrm{E}} \text { (isotropic), } \\
& \frac{\partial}{\partial \mathrm{l}} \mid=G \text { (noninertial). }
\end{aligned}
$$

For comparison. the current I.ASNHX chargedparticle diffusion model can be characterised by

$$
\begin{aligned}
& \frac{\partial \vec{J}_{1}}{a t}=0 \text { (steady stats) } \\
& \underline{\underline{n}} \mathrm{~F}=1)(\omega) n_{1} \text { (osymptotic angular distribution), }
\end{aligned}
$$

To test these modifications. we ran a problem in which $3.5-\mathrm{MeV}$ a particles were emitted from the center of a uniform D-T sphere ineld at a temperature of $50 \mathrm{keV}$. Under these conditions the $\alpha$ particles deliver energy to thermal ions only near the end of their range, and thus the deposition to ions as a function of distance from the $\alpha$ source is a critical measure of the $x$-particle 1 ransport. In Fig. 3-68 we compare L.ASN!XX calculations with and without the inertial term to a Monte Carlo calculation of the same problem. We sec that the improve-

Fig. 3-68. Charged-particle inertial term produces a peaked deposition profile, in agreement with Monte Carlo calculations.

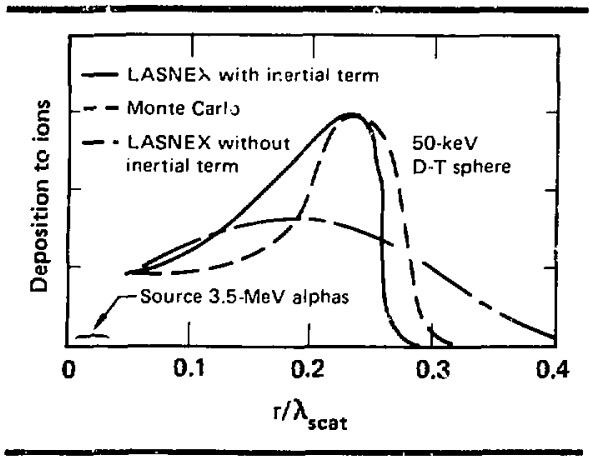


ments made to L.ASNEX's charged-particle diffusion method have greatly increased its ability to track the accurate Monte Carlo simulation.

\section{Author: (;. B. Zimmerman}

\section{Reference}

158. F. C. Corman. W. 1. Loewe. G. E. Cooper, and A. M. Winslow. "Multi-(iroup Diffusion of Energelic Charged Particles." Suct Fusion 15. 377 (1975).

\section{Solving Tridiagonal Linear Systems on the Cray-1 a mputer at Vector Speeds}

When we examine the aumerical algorithms used to solve the physics equations in LASNEX. wC find that a large number of subroutines require the solution of tridiagonal linear systems of equations. Radiation transport, thermal- and suprathermalelectron transport, ion thermal conduction. charged-particle and neutron transport. all require the solution of tridiagonal systems of equations. The standard algorithm that has been used in the past on CDC 7600's will not vectorize and so cannot take advantage of the large speed increases possible on the Cray-1 through vectorization. There is. however. an alternate algorithm for solving tridiagonal systems. called cyclic reduction, which allows for vectorization, and which is optimal for the Cray-1. Software based on this algorithm is now being used in LASNEX to solve tridiagonal linear systems in the subroutines mentioned above. The new algorithm runs as much as five times faster than the standard algorithm on the Cray-1.

The Basic Algorithm. Consider a tridiagonal linear system of equations

$$
b_{i-1} X_{i-1}+a_{i} X_{i}+c_{i} X_{j+1}=Y_{i} \quad i=1,2, \ldots, n,
$$

with $b_{0}=c_{n}=0$. The standard algorithm for solving these equations is:

$$
d_{i}=\left(a_{i}-k_{i-1} \dot{c}_{j-1}\right)^{-1} \quad \text { for } \mathrm{i}=1,2, \ldots, n
$$

and

$$
k_{i}=b_{j} d \quad \text { for } i=1,2, \ldots, n-1
$$

followed by

$$
W_{j}=Y_{i}-t_{i-1} W_{i-1} \quad i=1,2, \ldots, n
$$

and

$$
X_{i}=d_{i}\left(w_{i}-c_{i} X_{i+1}\right), \quad i=n,(n-1), \ldots 3,2,1 .
$$

This algorithm is recursive and cannot be vectorized. and therefore will only run abou' iwice as fast on the Cray-1 as it did on the CDC 7600 .

But note that the standard scalar algorithm is just I I lecomposition. ${ }^{159}$ If our original equation is written in matrix notation as $\mathrm{MX}=\mathrm{Y}$, with

$$
M=\left(\begin{array}{cccc}
a_{1} & c_{1} & 0 & 0 \\
b_{1} & a_{2} & c_{2} & 0 \\
0 & b_{2} & a_{3} & c_{3} \\
0 & 0 & b_{3} & a_{4}
\end{array}\right),
$$

then we may decompose $M$ as a lower-triangularmatrix I with unit diagonal clements and an uppertriangular-matrix 1 i. i.e.. $M=$ l.li. Here

$$
L=\left(\begin{array}{llll}
1 & 0 & 0 & 0 \\
v_{1} & 1 & 0 & 0 \\
0 & { }_{2} & 1 & 0 \\
0 & 0 & { }_{3} & 1
\end{array}\right)
$$

and

$$
L=\left(\begin{array}{cccc}
d_{1}^{-1} & c_{1} & 0 & 0 \\
0 & d_{2}^{-1} & c_{2} & 0 \\
0 & 0 & d_{3}^{-1} & c_{3} \\
0 & 0 & 0 & d_{4}^{1}
\end{array}\right) .
$$

Then ae solve $1.11=Y$ and $l X=W$ to find $X$.

To obtain an algorithm which allows some vectorization we reorder the rous and columins of our matrix so that first we take all the odd multiples of I. then all the odd multiples of 2. then all the odd multiples of $2^{2}$. etc. Thus we apply a permutation $P$ which takes the original ordering. $1.2 .3 \ldots . n$. into the new ordering

$$
\begin{gathered}
1,3,5,7, \ldots, 2,6,10,14, \ldots, 4,12,20,28, \ldots, 8,24,40,56, \ldots, \\
2^{\mathrm{q}}, 3 \cdot 2^{\mathrm{q}}, 5 \cdot 2^{\mathrm{q}}, 7 \cdot 2^{\mathrm{q}}, \ldots, 2^{\mathrm{p}} .
\end{gathered}
$$

Here $p$ is the tighest power of 2 with $2^{p} \leqslant n$.

Our original matrix equation now becomes 


$$
\left(P M P^{3}{ }^{\prime}()^{\prime} X\right)=(P Y) .
$$

If we perturm I I decumposilon on thes reordered matris uc oblatr a new algorithm called cyclic reduction that atgorthon is vecturbahie hecause we are edmomenong ali the odd variabley first (which are nes coupled to each other). Then all the odd mulaple-ist-2 barables (which are not coupled is cise othert. and wo un l urthermore. the fact that

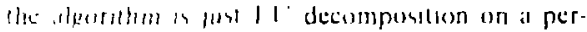

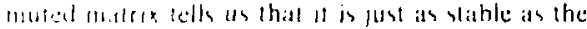

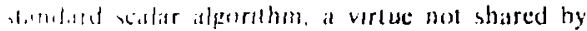

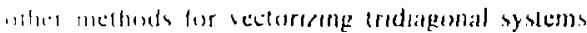

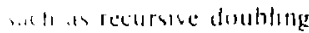

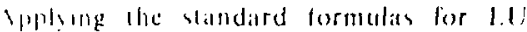

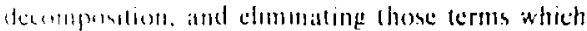

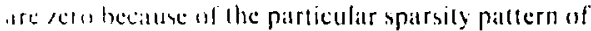
nur ferrdered mislrex, we oblain

$$
u^{\prime \prime}-b_{i}, u_{t}^{\prime \prime}-c_{i} \text { and } d_{i}^{\prime \prime}-a_{i}, j \cdot 1, \ldots, n \quad 11, n
$$

Iten for each $q=0,1,2,3, \ldots, p$ we take

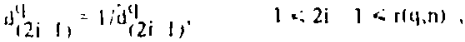

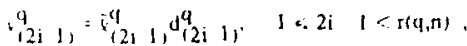

$$
\begin{aligned}
& \text { (12ii }=-12 i) \quad 2 \cdot 2 i<r(a, n) \text {, }
\end{aligned}
$$

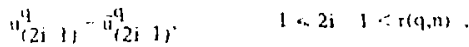

where $r(4, n)$ is $i$ h. largest integer. $r$. such that $r \cdot 24$ $\leqslant n$.

for the solve, we let

$$
\begin{aligned}
& u_{(2 i)}^{q}=\vec{u}_{(2 \mathrm{i})}^{\mathrm{q}} \mathrm{d}_{(2 \mathrm{i}-1)^{4}}^{\mathrm{q}}, \quad 2<2 \mathrm{i}<\mathrm{r}(\mathrm{q}, \mathrm{n}) . \\
& \left.j_{i}^{q+1}=\tilde{d}_{2 i}^{q} \quad(2 i-1)^{4} u_{(2 i-1)}^{u}-v_{(2 i)}^{4}\right)^{4}(2 i)^{4}
\end{aligned}
$$

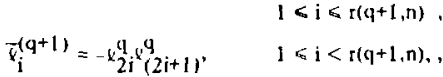

$$
\begin{aligned}
& \bar{u}_{j}^{(q+1)}=\cdot u_{2 i}^{q} u_{(2 i+1)^{q}}^{q} \quad l \leqslant j<r(0-r l, n) \\
& \mathrm{I} \cdot 2^{4} \leq \mathrm{n} \text {. } \\
& \mathrm{W}_{\mathrm{i}}^{\mathbf{o}}=\mathrm{Y}_{\mathrm{i}^{*}} \quad \mathbf{i}=1,2, \ldots, \mathrm{n} .
\end{aligned}
$$

Then for each $q=0,1,2, \ldots,(p-1)$ we take

$$
\begin{aligned}
& w_{i}^{(q+1)}=w_{(2 i)}^{q} \quad q_{(2 i}^{q}, w_{(2,1)}^{q} \quad u_{(21)}^{q} w_{(2 i+1)}^{q} \\
& \text { Ior } \mathrm{i}-1,2, \ldots, \mathrm{r}(\mathrm{q}+1, \mathrm{n})
\end{aligned}
$$

We set $x q=d f u q$. and then for each $q=\{p-1\}$. (p - 2),...4.3.2.1.0 we lake

$$
\left.x_{(2),}^{4}-x_{1}^{(4+1)}, \quad 2<2 i<n q, n\right)
$$

and

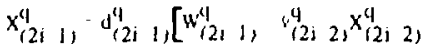

$$
\begin{aligned}
& \left.412 i 1, X_{(2 i)}^{(1)}\right], \quad 1<2 i \quad 1-r(y, n) .
\end{aligned}
$$

f.inally, $x_{1}=x_{1}^{0}$, for $i=1, \ldots, n$.

Clearly all the above operations at the q level of reduction are vectorizable with vector length $\mathrm{r}\left(\mathrm{q}_{+}+\right.$ $1, n)$. Thus we now have a vectorizable algorithm with vectorsation on lengths $n / 2, n / 4, n / 8, \ldots .1$.

Implementation on the (ray-1. The numbers $r(4, n)$ are easily calculated from $r(0, n)=n$ and

$$
r(q+1, n)=\sin 1+T R\{r(q, n), 1\}
$$

where $\operatorname{SIlll}: T R\left(n_{1}, n_{2}\right)$ is $n_{1}$ (in binary notation) shifted right $n_{2}$ positions with the rightmost $n_{2}$ bits of $n_{1}$ lost off the end.

it is important to give consideration to storage layout and possible memory bank conflicts. Consider for example the forward sweep of the solve. The "natural" storage layout is to store $w_{1}^{(y+1)}$ in the $\left[j \cdot 2^{(4+1)}\right]$ element of the $Y$ array and just keep overwriting $w_{(21)}^{4}$ with $w_{1}^{(4+1)}$. The problem with this is that vector reads from and writes te memory in increments of $2^{4}$ on the Cray-l cause bank connicts and loss of speed if $q>2$. lor $q=0.1 .2$ we can read or write one word per clock period. For $q=3$ this degrades to one word every two clock periods and for $q \geqslant 4$ the performance degrades to one word every four clock periods. To eliminate this problem we use the following storage scheme.

$W_{i}^{0}=Y_{i}$ is stored in the first $n=r(0, n)$ elements of the $Y$ array. $W_{1}^{l}$ is stored in the next $r(1, n)$ elements of the $Y$ array, and so on. Thus

$$
w_{j}^{q}=Y_{[s(q, n)+i]} \text {, }
$$

where $s(0, n)=0$ and $s(q+1, n)=s(q, n)+r(q, n)$. $X^{4}$ is stored in the same locations as $W_{1}^{a}$. The matrix elements $\ell_{1}^{\varphi}$ and $\mathcal{Y}_{1}$ are stored in $b_{[s(q, n)+i t} u_{i}$, and $\widetilde{\mu}^{q}$ are stored in $c_{[s(q, n)+i \mid,} d_{i}^{q}$ and $\widetilde{d}_{i}^{q}$ are stored in 


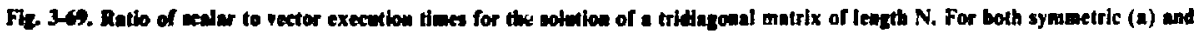
ary netric (b) matrices, the asymptolic rate of the vector cyclic reluction algorithm is more thes wix tibes the scalar rate, but this peedep can ine ackleved wily with relathely lone rectors.

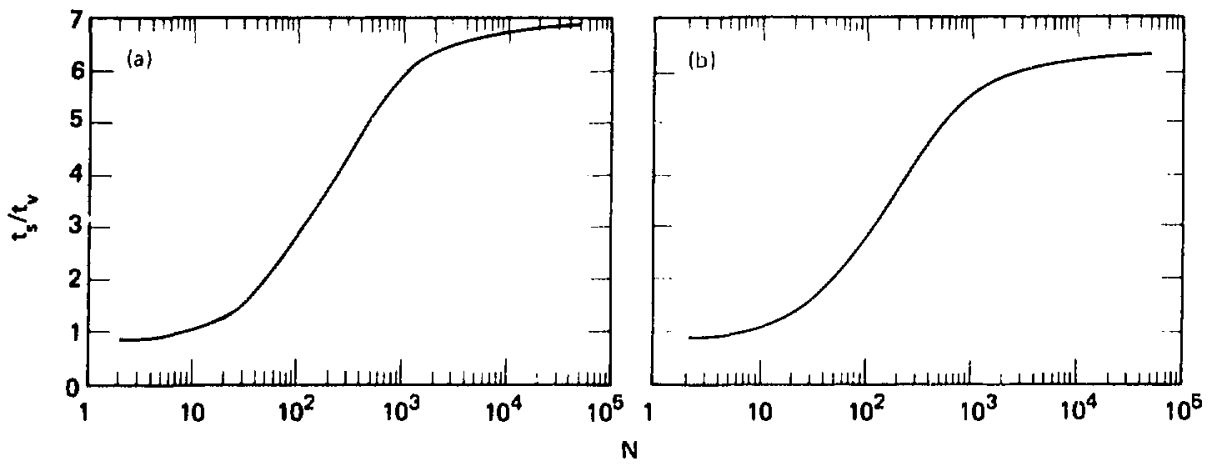

adsem + 1f. Whth this sorage scheme all memory reads and urites are done 13 increments of 1 and 2 . and all bank conllicts are eliminated. Since

$$
\sum_{q=0}^{n} r(q . n)<\sum_{q=0}^{p}\left(n / 2^{q}\right)<2 \pi \text {. }
$$

The arrays a.b.c. and y must now be $2 \mathrm{n}$ elemems long instead of $n$ elements long. and so he need iwice as much storage as is required for lite standard scialar aigorithm.

Performance. This algorithm was hand-coded for the (ray-l by R. I. von Holdt for both the symmetric and nonsymmetric matrix cases. In lige. 3-69 the hand-coded vector cyclic reduction algorithm is compared with the cray-l FORTRAN CITcompiled standard scalar algorithm. $N$ is the problem dimension and $\left(t_{s} / t_{v}\right)$ is the relative execution time of the scalar and vector algorithms. The cyclic reduction algorithm was also coded with C $\mathrm{FT}$ and it was asymptotically about 4.5 times faster than the CFT scalar algorithm: thus the hand coding made only a $50 \%$ improvement over $C \mathrm{FT}$.

\section{Author: D. S. Kershaw}

\section{Reference}

159. For a good discussion of L.U decomposition sec J. H. Wilkinson. The Algebraic Eigenvalue Prohlem (Oxford University Press. Oxford. 19651 chap. 4, sec. 36.
A V'ectorizahle Incomplete CholeskyConjugate Ciradient IC C $(i)$ Algorithm for the (ray-1 Computer

In I 4S\J the two-dimensional radiation transport. celectron thermal cond: ... ien thermal conduction and neutron transport atl use IC ( $($; 10 solve the tramsporl equations. We bave heen using

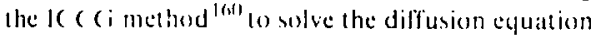
with a nine-point coupling lol schem: on the $C D C$ 7600 . In going from the $(2)(7600)$ to the cray-1. a large part of the algorithm consisth of solving tridiagonat linear sysems on eateh 1. line af the I agrangian mesh in a manner which is nol vectoriable. Therefore a direct translation from the $7600)$ to the crity would not give much increase in running speed because the vectorization potential of the ( ray cannot be used. We have developed an alternate IC $\mathrm{C}(\mathrm{i}$ algorithm for the Cray-l which utilizes the vertor tr: liagonal solver described in the previous article. This new algorithm allows lull vectorization and runs as much as seven times faster than the old algorithm on the Cray-l. It is now being used in (ray LASNEX to solve the twodimensional diffusion equation in all the physies subroutines mentioned above.

Thus we have an equation, $M X=Y$, where $M$ is positive definite and symmetric, and the sparsity 


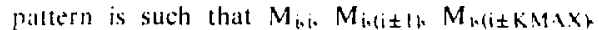
$\left.V_{1 \cdot 11 \pm k} \| 1 \times \pm 1\right)$ are the only nonero elements.

The IC C $\mathrm{s}$ method consists in finding an apFurnate (holesky decomposition for $M \approx 1$ LI. $?$ $a$ h then using the conjugate gradient algorithm. $i \because r_{11}=\left\{-11 K_{0} \text { and } p_{0}=(1 . D) I^{\mathrm{I}}\right)^{-1} \Gamma_{0}$, then

$$
\begin{aligned}
& \therefore \quad\left[r_{i}(1,1) 1{ }^{1},{ }^{1} r_{i}\right] /\left(y_{i}, M p_{j}\right) . \\
& \because: x_{i}+a_{i} p_{i} \text {. } \\
& i_{i, i} \quad T_{j} \quad i_{1} M_{P_{i}} \text {. }
\end{aligned}
$$

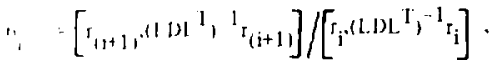

$$
\begin{aligned}
& i_{1+1}, 1[0]^{3}, I_{i j+1}+b_{j} p_{i}
\end{aligned}
$$

$1: 1, \quad 11.1 .2 .$.

llm algurlthm is all trivially vectori able exant for the exaluation of the approximate inverse

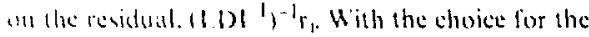
dpromumble (holesky decomposition given in Ret. low thas is a recursive operation which can only go it raliar speeds on the craly-1. To vectorise it we mus uxe a diflerent approximate decomposition whets altons for vectorization.

fo see how this is done we first wite the exalt (bulesk decomposition in block form, in which wur mattrix 11 cian be written

$$
Y=\left(\begin{array}{lllll}
A_{1} & B_{1} & 0 & 0 & \\
B_{1}^{T} & A_{2} & B_{2} & 0 & \\
a_{1} & B_{2}^{T} & A_{3} & B_{3} & \\
. & 0 & B_{3}^{T} & A_{4} & \ddots
\end{array}\right),
$$

where the $V_{1}$ and $B_{1}$ are tridiagonal niatrices of dumension KMIAK. and $i=1.2 .3 \ldots . \mathrm{MAX}$. The (holesky decomposition may now be writlen in block form. $M=$ L. D) ' . where

$$
L=\left(\begin{array}{lllll}
\mathrm{L}_{1} & 0 & 0 & 0 & \\
\mathrm{C}_{1} & \mathrm{~L}_{2} & 0 & 0 & \\
0 & \mathrm{C}_{2}^{\mathrm{I}} & \mathrm{I}_{3} & 0 & \\
0 & 0 & \mathrm{C}_{3}^{\mathrm{T}} & \mathrm{L}_{4} & \\
& & & \ddots & \ddots
\end{array}\right)
$$

and

$$
\mathrm{D}=\left(\begin{array}{lllll}
\mathrm{v}_{1} & 0 & 0 & 0 \\
0 & \mathrm{v}_{2} & 0 & 0 \\
0 & 0 & \mathrm{v}_{3} & 0 & \\
0 & 0 & 0 & \mathrm{v}_{4} & \\
& & & & \ddots
\end{array}\right)_{1}
$$

lere the $L_{1}$ are lower triangular matrices whose diagonal elements are all 1, the $D_{i}$ are diagonal matrices, and $\mathrm{I}_{\mathrm{i}}, \mathrm{C}_{\mathrm{N}}, \mathrm{D}_{\mathrm{j}}$ are given recursively by

$$
c_{i}=D_{i}{ }^{1} I_{i}{ }^{1} B_{i}
$$

and

$$
1_{i} b_{i} L_{i}^{T}=\Lambda_{i} \quad\left(C_{(i)}^{T}, D_{(i-1)}\left(C_{(i-1)}=\bar{\Lambda}_{j}\right.\right.
$$

for $\mathrm{i}=1.2 .3, \ldots, 1 . \mathrm{M} \wedge \mathrm{x}$.

If we write the unknown and right-hand-side vectors in block form,

$$
X=\left(\begin{array}{c}
x_{1} \\
x_{2} \\
x_{3} \\
\cdot \\
\cdot
\end{array}\right), \quad Y=\left(\begin{array}{c}
y_{1} \\
y_{2} \\
y_{3} \\
\cdot \\
\cdot
\end{array}\right)
$$

where $X_{i}, Y_{i}$ are vectors of length $K M A X$, then I.DI. ${ }^{\mathrm{T}} \mathrm{X}=Y$ may be solved by a forward sweep.

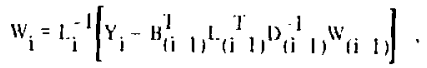

$|162|$

for $\mathrm{i}=1.2,3, \ldots 1 . \mathrm{M} \wedge \mathrm{X}$, and a backward sweep.

$$
x_{i}=L_{i}^{-T} D_{i}{ }^{\prime}\left[W_{i}-I_{-i}^{-1} H_{i} X_{(i+1)}\right] .
$$

for $\mathrm{i}=1 . \mathrm{MA}$. (1.M.1.X-1),...3.2.1.

Now let us examine the sparsity pattern of the $I_{1}$ and $\left(C_{1}\right.$ lor the case of exact Cholesky decomposition. $A_{1}$ is tridiagonal so $l_{1}$, will be lower tridiagonal. Since $B_{1}$ is tridiagonal. $\mathrm{Eq} .(160) \mathrm{im}-$ plies that $\left({ }_{1(1, j]}\right.$ will be nonzero for $j \leqslant j+1$ and zero for $j>i+1$. liquation (16I) thus implies that $A_{2}$ is at dense malrix (all elements nomsero) and so 
$L_{2}$ is dense lower triangular. Thus in general we find

$$
L_{i(j, k)}=\left\{\begin{array}{r}
0, k>j \\
\text { nonzero, } k \leqslant j
\end{array} .\right.
$$

and

$$
c_{i(j, k)}=\left\{\begin{array}{r}
0, k>j+1 \\
\text { nunzero, } k \leqslant j+1
\end{array} .\right.
$$

Thus we have extensive fill-in: that is, many elements which were ero in $A_{i}$ and $B_{i}$ become nonsero in $I_{1}$ and $C_{1}$. This greatly inereases both storage requirements and the amount of computation.

We seek an approximalle 1.DI. ' decomposition which has no fill-in. To accomplish this. in 1:4. (160) we throw away (i.e., neither compule nor .ure) al. but the eridiagonal part of $C_{i}$. Then in 1.4 .1161$)$ we throw away all but the tridiagonal part of $(" 11$, $D_{(i-1)} C_{(1-1)}$ thus malking $\tilde{A}_{i}$ and $L_{\text {. }}$ tridiagonal. We thus obtain an incomplete block decomposition. $M$ $\approx$ I.DI.'. where I., and $C_{1}$ have the same sparsity pattern as $A_{1}$ and $B_{1}$. Note that the solve [Fys. (I (62) and (I63)] does not use $C_{i}$ explicitly, and so the $C_{\text {, }}$ need nol be saved onee the $\widehat{A}_{(i+1)}$ have been calculated. Only the $I_{i}$ and $D_{j}$ need be stored. Now we have reduced sur incomplete decomposition and solve to a series of tridiagonal decompositions and solves.

In the previous article we have shown how to vectorize tridiagonal decompositions and solves on the Cray-1 using cyclic reduction. Here we simply apply this algorithm to the incomplete version of tigs. (160) through (I63) and we have a fully vectorizable ICCG algorithm.

In detail the algorithm is as follows:

- If $K: A A X<1$ MAX we transpose our grid (inierchange $K M A X$ and L.MAX) so that LMAX becomes the inner loop and KMAX the outer loop. Thus our block matrices will have the largest possible dimension and our vector tridiagonal solves will vectorize as well as possible.

- The incompleteness of the decomposition is accomplished in Eq. (160) by performing only the first two levels of cyclic reduction in evaluating $E_{1}=$ $D_{\mathrm{i}} \mathrm{C}_{\mathrm{i}}=\mathrm{L}_{\mathrm{i}}^{-1} \mathrm{~B}_{\mathrm{j}}$, and in noring elements outside the tridiagonal band. All higher levels of reduction couple only elements inside the tridiagonal band with (zero) elements outsicie the band and so have noeffect. If we use the notation of the previous article for the $L_{i}$ and $D_{i}$ matrices and suppress the block subseript " $i$ " for clarity. we then have explicitly:

$$
\left.\begin{array}{l}
E_{j,(j-1)}=B_{j(, 11,}, \\
\mathrm{r}_{j, j}=B_{j, j} \\
\mathrm{E}_{\mathrm{j},(\mathrm{j}+1)}=B_{\mathrm{j},(\mathrm{j}+1)}
\end{array}\right\}
$$

for all odd $j . \quad 1 \leqslant j \leqslant k M A X$.

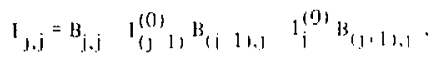

lor all even $1.2,1 \cdot k M A$.

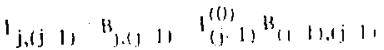

$$
\begin{aligned}
& \left.1_{j,(j+1)}=B_{1 .(3)+1)} 1_{j}^{(0)} B_{1}+1\right), 1+11
\end{aligned}
$$

for all $j=2 k$ with $k$ odd. $2 \leqslant 1 \leqslant k M 1 \times$. . I

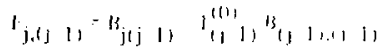

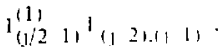

$$
\begin{aligned}
& 1_{j,(j+1)}=B_{j,(j+1)} \quad 1_{1}^{\prime \prime \prime \prime} B_{1}+1+1,1+11 \\
& 1_{(j, 2)}^{(1)}(0)+2,(1+1) \text {. }
\end{aligned}
$$

for all $\mathrm{j}:=2 \mathrm{k}$ with $k$ eren. $2 \leqslant 1 \leqslant k \backslash 111$

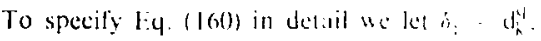
where $\mathrm{j}=\mathrm{k} \cdot 2 \mathrm{y}$ with $k$ odd. Them

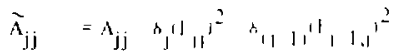

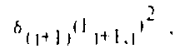

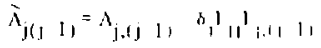

$$
\begin{aligned}
& \delta(1,1) 11,11111,1111
\end{aligned}
$$

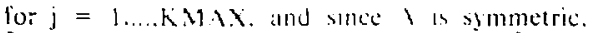

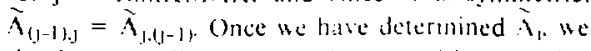
simply use cyclic reducton decomposition to solve $L_{i} \mid D_{i} I_{j}^{T}=\hat{n}_{1}$ for $L_{1}$ and ()$_{1}$.

We must make one modification of the decomposition alvorithm given in the previous arlicle. A negative pivot would destroy the positise definiteness of our approximate identuy. (1.DI $\left.{ }^{1}\right)^{-1} \mathrm{M} \approx 1$, so we must modify any negative pivot $t 0$ a positive 
value. We do this by changing $d_{(2 j-1)}^{q}=1 / \widetilde{d}_{(2 i-1)}^{\mathrm{q}}$ for $1 \leqslant(2 i-1) \leqslant r(q, n)$, from the previous article, to $\mathrm{d}^{4}(2 \mathrm{i}-1)=1 /\left|\widehat{\mathrm{d}} \mathrm{q}_{(2 \mathrm{i}-1)}\right|$

- We now solve for $X$ using Eqs. (160) and (161). If $Z$ is any vector (of length KMAX), then $B_{i} Z$ and $B_{i}^{T} Z$ are trivially vectorizable and $L_{i}^{-1} Z$ and $L_{i}^{-T} D_{1}^{-} Z$ are the forward sweep and the backward sweep of the cyclic reduction solve as described in the previous article.

We now have a completely vectorizable algarithm for linding an approximate Cholesky decomposition. L.DL ${ }^{\mathrm{T}} \approx \mathrm{M}$, of our matrix ind for calculating. (1.DL $\left.{ }^{T}\right)^{-1}$ r, the approximate inverse applied to the residuat. Thu; we have completely veclorided the ICC(i algorithm.

The new vector algorithm has been implemented on the Cray-l using Cray-I FORTRAN and is atout 10 to 15 times faster than the standard IC C $($; algorithm coded in FORT RAN on the CDC 7600 ).

luthor: D. S. Kershaw

\section{References}

tort 1) S kershaw. "The IC (i Vethod for the lecrative Solu. tim on Sivtems of l.inciar t quations." I camp. Phys. 26. i. $\left.1,(1)^{-}-x\right)$

:n1 1) 5 kershas, Differencing of the Difusion Fquation in l.aprangian If!drodynamies code's. L awrence tivermort

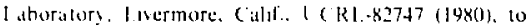
anpear in $\mathrm{f}$ comp rhas.

\section{Yot-Flectron Mome um Deposition in LASNEX}

Theory and simulations of resonant absorption have been described in past annual reports and in the literature. ${ }^{162-164}$ Briefly, resonant absorption is the direct conversion of the transverse laser light to longitudinal electron-plasma waves (epw) at the critical density $\left[10^{21}\left(1.06 \mu \mathrm{m} / \lambda_{0}\right)^{2} \mathrm{~cm}^{-3}\right]$. The oscillating longitudinal electric field of the epw heats the electrons by accelerating them down the density gradient to a temperature of approximately 21 $T_{\mathrm{d}}(\mathrm{keV})^{0.25}\left[\mathrm{I}\left(\mathrm{W}^{\prime} / \mathrm{cm}^{2}\right) / 10^{16}\left(\lambda_{0} / 1.06 \mu \mathrm{m}^{2}\right)\right]^{0.4}$. The momentum of the accelerated elcctrons acts with the ponderomotive pressure of the transverse and ingitudinal Electric field to steepen up the density gradient at critical. The consequences of this steepening are:

- Resonant absorption becomes accessible to a wide range of angles ${ }^{162.163}$ (LASNEX users can use an absorption-vs-angle mode).

- The $\left(T_{\text {Hot }} \mathrm{n}_{\mathrm{Hot}}\right)$ helps to push back critical density, $\mathrm{n}_{\mathrm{s}}$, which helps to explain why gold disk experiments have a red shift in the backscattered light. ${ }^{165}$

- The $\nabla$ steepening affects inverse bremsstrahlung absorption.

- Brillouin scattering.

I.ASNEX models resonant absorption in a very simple way. $A$ user-defined fraction. 1 $X I . R F L F$, of the laser energy reaching or exeeeding a given density, XLTHRESO* $n_{c}$, is deposited at the ray turning point. The energy dumped is deposited in the electron bins (if they are present) at a temperature specified (XLALPHA) or determined by a semi-empirical formuli (LTHOT). L $A$ SNEX has no knowledge of the resonant field nor of the dirceled motion of the heated clevisuis.

The momentum imparted by the resonant electrons may be calculated by energy flux conservation. Therefore the force exerted by the hot electrons at $\mathrm{n}_{\mathrm{c}}$ can be included in the "dump-all" (resonance absorption) model in LASNEX. A calculation of the time rate of change of momentum due to the hot electrons created by resonance absorption is given below with an equation appropriate for inclusion in I.ASNEX.

This model assumes that all of the laser energy deposited by resonance absorption is transferred to the hot electrons which are ejected from $n_{c}$ and stream down the density gradient. Thus, the conservation of energy densit: flux gives

$$
f\left(\frac{\dot{E} \times \mathrm{B}}{8 \pi}\right) \mathrm{c}=\frac{\mathrm{in}_{\mathrm{e}} \mathrm{n}_{\mathrm{Hot}}}{\sqrt{\pi v} \mathrm{Hot}_{\mathrm{Hot}}^{\infty}} \int_{0}^{\infty} \mathrm{dv}\left(\frac{1}{2}\right) v^{3} \exp \left(-\frac{v^{2}}{v_{\text {Hot }}^{2}}\right),
$$

which may be solved for $n_{\text {Hot, }}$ the number density of hot electrons. In this equation, $E$ and $B$ are the laser field quantities, $f$ is the fraction absorbed by resonance absorption, $m_{e}$ is the election mass and $v_{\mathrm{Hot}}=\left(2 \mathrm{~T}_{\mathrm{Hot}} / \mathrm{me}_{\mathrm{e}}\right)^{1 / 2}$ is the thermal velocity of the hot e'ectrons. Equation (164) gives 


$$
\frac{\mathrm{n}_{\mathrm{Hot}}}{\mathrm{n}_{\mathrm{c}}}=\left(\frac{\pi}{2}\right)^{1 / 2} \mathrm{f}\left(\frac{\mathrm{v}}{\mathrm{c}}\right)^{2}\left(\frac{\mathrm{m}_{\mathrm{e}} \mathrm{c}^{2}}{\mathrm{~T}_{\mathrm{Hot}}}\right)^{3 / 2} \text {, }
$$

where $\mathrm{c}$ is the speed of light and $v_{0}$ is the peak oscillation velocity of electrons in the laser electric field. The total flux of hot-electron momentum flowing down the density gradient is then

$$
G=\frac{n_{1} l o t}{\sqrt{\pi}} \frac{v_{1 I o t}}{d} \int_{0}^{\infty} d v m_{e} v^{2} \exp \left(-\frac{v^{2}}{v_{\text {Hol }}^{2}}\right) .
$$

Combining Eqs. (165) and (166), one finds

$$
G=\frac{V_{\pi} f 1}{v_{H o t}} .
$$

Thus the force due to the hot electrons is the rate of change of momentum as the hot electrons are created at $n_{c}$ and flow away down the density gradient. So, for a zone of cross section $A$.

$$
F_{\text {Hot }}=\frac{V_{\pi} \mathrm{flA}}{v_{\text {Hot }}} \text {. }
$$

or, in LASNEX units,

$$
\mathrm{F}_{\mathrm{Hot}}=0.095 \frac{\mathrm{ClA}}{\sqrt{\mathrm{T}_{\mathrm{Hot}}}{ }^{(\mathrm{keV})}}
$$

where $\mathrm{F}_{\mathrm{Hot}}$ is directed up the density gradient. In deriving Ec. (167) we have lised

$$
\begin{aligned}
& \left(\frac{v_{0}}{c}\right)^{2}=\left(\frac{21}{c}\right)\left(\frac{1}{m_{e} c^{2} n_{c}}\right)= \\
& 0.082\left[\mathrm{~L}\left(\text { jerks } / \mathrm{sh} / \mathrm{cm}^{2}\right)\left(\lambda_{0} / 1.06 \mu \mathrm{m}\right)^{2}\right] \text {, }
\end{aligned}
$$

where $I$ is the light intensity with vacuum wavelength $\lambda_{0}$.

Including Eq. (169) in LASNEX will improve the resonance absorption model. LASNEX does not know, however, that the hot electrons are all moving down the density gradient, so that the total problem momentum will not be conserved. But the local affect near critical density should be correct.

The importance of the hol-electron force may be estimated by comparing it with the ponderomotive force. The ratio of the two forces is

$$
\frac{\mathrm{l} \mathrm{ilor}_{\text {llor }}}{\mathrm{F}_{\text {Pon }}}=28.4 \frac{\mathrm{f}}{2-\mathrm{f}} \frac{1}{\sqrt{\mathrm{T}_{\text {1lot }}(\mathrm{keV})}} \text {. }
$$

so for a plasma with $f=0.3$ and $T_{110 t}=25 \mathrm{keV}$, the two forces are equal; whereas for a lower $\mathrm{T}_{\mathrm{Hol}}=$ $3 \mathrm{keV}, \bar{F}_{\mathrm{Hol}}$ is almost three times F Pon ( $\mathrm{T}_{\mathrm{Hot}}$ is also discussed in "Resonant Absorption" earlier in this section).

Authors: K. G. Estabrook and J. A. Harte

\section{References}

162. k. (i. tslabrook, I. I Valeo, and W. L. K ruer "Plasma Heating and (iradient Modifications by Resonant ab sorplon." Phis lent .4 49, 109 (1974).

iol K. (i. Fstabrook, H. J. Valeo, and H. L. Kruer. "Tro Dimenstonal Reldivistic Simulations of Resonance Absorption." Phys. Fluids 18. 1151 (1975)

lith 1) M' leorslund, J. M. Kindel, and $k$. Lee. Theory of Hot-tectron Spectra at High laser Intersity." Phys. Rev. Letl 39. $28+(1977)$.

16: M. D. Rosen. D. W. Phillion. V. C. Rupert. W. C. \$1: W. I. Kruer, I. T. Thomson. H. V. Kornblum. V. W. Siwinsky. (i. J. Caporaso. M. J. Boyle, and $k$, G. Tirsell. "The !nteraction of $1.06 \mu \mathrm{m}$ Laser Radiation With Higb Z Disk Taroets." Phis. Fluids 22. 2020 (1979). 
ariget Fabrication

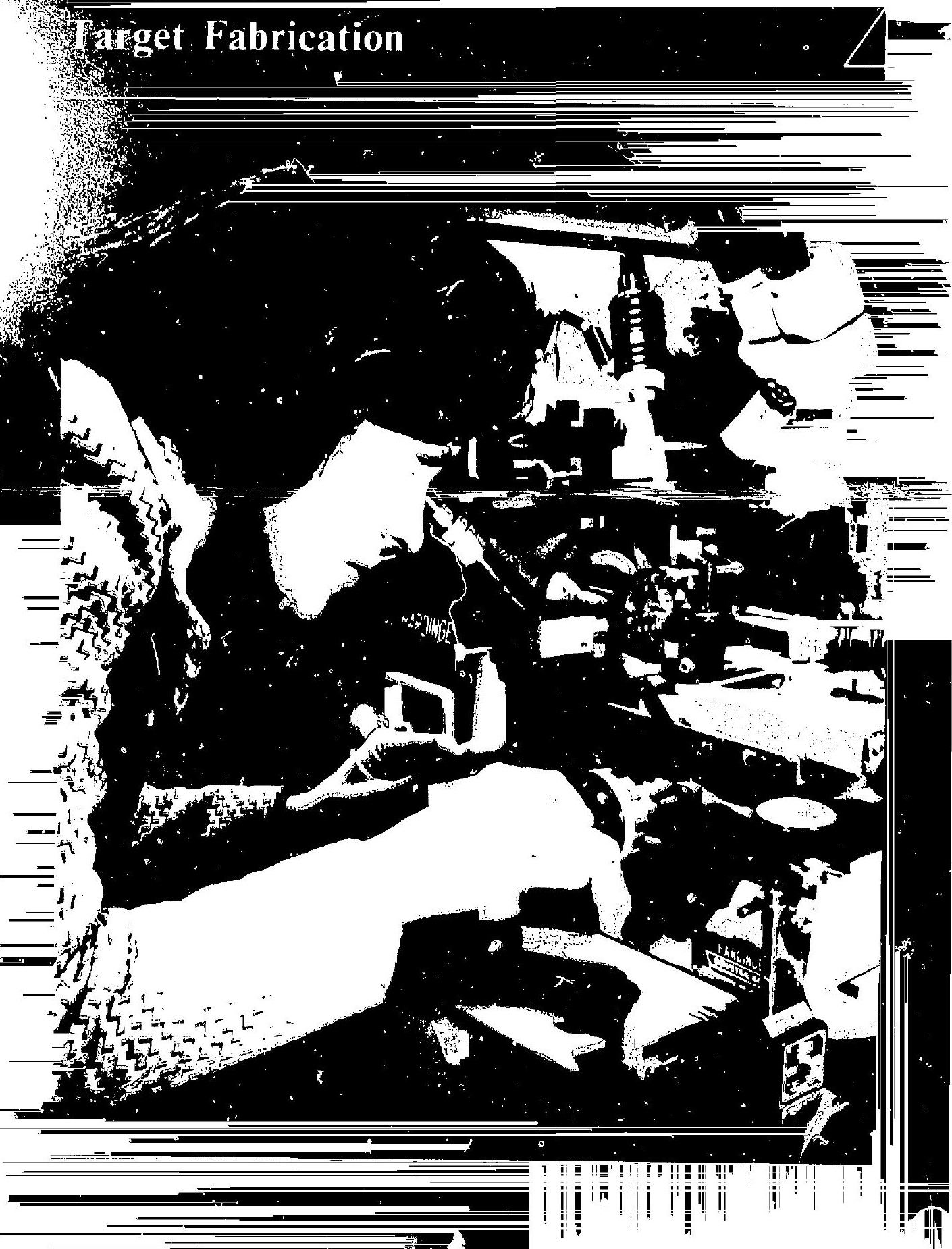




\section{Target Fabrication}

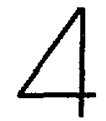

\section{Contents}

Introduction

Glass Sphere Development and Production . . . . . . . . . . . . . . . . . . . . . . . 4-2

Sphere Fabrication Processes . . . . . . . . . . . . . . . . . . . . . . . . . . . . 4-2

Diagnostic Gas Fills . . . . . . . . . . . . . . . . . . . . . . . . . . . 44

High-Z Glass Spheres . . . . . . . . . . . . . . . . . . . . . . . . $4-5$

D-T Fill Techniques . . . . . . . . . . . . . . . . . . . . . . . . . . 4-5

Coatings and layers $\ldots \ldots \ldots \ldots \ldots \ldots \ldots \ldots$

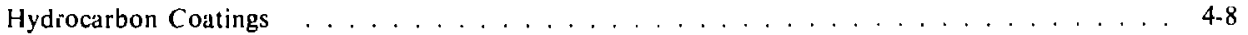

Plasma roating Process . . . . . . . . . . . . . . . . . . . 4-9

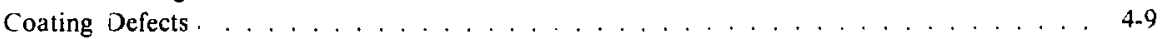

Production Results . . . . . . . . . . . . . . . . . . . . . . . 4-12

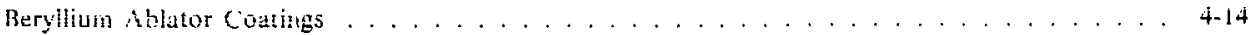

Sputtered High-Atomic-Number Coatings . . . . . . . . . . . . . . . . . . . 4-17

Screened Cage and Fixturing . . . . . . . . . . . . . . . . . . . 4-17

Microsphere Sticking Protlem ... . . . . . . . . . . . . . . . . . 4-19

Platinum Deposition Proress . . . . . . . . . . . . . . . . . . . 4-20

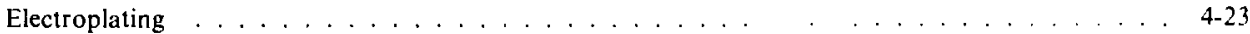

Molecular Beam Levitation During Coating . . . . . . . . . . . . . . . . . . . . 4-26

Double-Shell Targets . . . . . . . . . . . . . . . . . . . . . . . . . . . . . . . . . . . . 4-29

Machined Hemishells . . . . . . . . . . . . . . . . . . . . . . . . . . . . . . . 4-29

Assembly Procedure . . . . . . . . . . . . . . . . . . . . . . . . . . . 4-32

Alternate Approaches . . . . . . . . . . . . . . . . . . . . . . . . . . 4-32

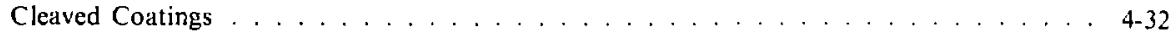

Molded Hemishells . . . . . . . . . . . . . . . . . . . . . . 4-32

Molecular Beam Levitation and Coating ................ . . . . . . . . . . .

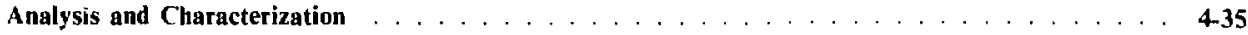

Production Characterization . . . . . . . . . . . . . . . . . . . . . . 4.35

Development of Automated Characterization Techniques . . . . . . . . . . . . . . . 4-38

Auıomatic X-ray Image Analysis and Sorting System . . . . . . . . . . . . . . . . 4-38

Automated Sphere-Mapping System . . . . . . . . . . . . . . . . . $4-42$

Automated Sorting linterferometer . . . . . . . . . . . . . . . . . 4-45

Materials Analysis Developments and Surface Studies . . . . . . . . . . . . . . . . . . 4-49

Optical Microscopy . . . . . . . . . . . . . . . . . . . . . . . . . . . . . . . . . . . . . . 449

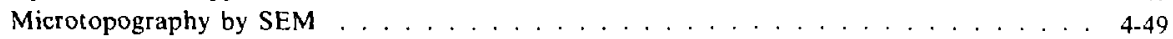

Surface Analysis . . . . . . . . . . . . . . . . . . 4-51 
Solid Hydrogen Spherical Pellets

Condensed D-T Layer Formation

Layers Condensed Through Fill Tubes

Liquid Layers on the Interior of Glass Spheres

Cryogenic Pylon

Iligh-Rate Manipulation, Coating, and

Iritrisport in Target P'roduction

lunet Shell Production

Second Shell Production 4-62

Iransporl L.evitator 4-6.3

Production Rate 4-64 


\section{Target Fabrication}

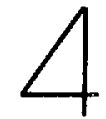

\section{Introduction}

Our primary task in target fabrication continues to be the development anciapplication of techniques for producing and characterizing targets used in current experiments. In addition, we must carry out the research and development that will make it possibie to produce the advanced targets required for future ICF studies.

The complexity of the targets, the diversity of materials used, and the variety of characterization methods required in our work all require expertise in a large number of technical fields. Materials science, plasma physics, cptics, electron microscopy, analytical chemistry, and cryogenics are only a few of the scientific fields in which the staff must be proficient in order to solve the problems of target fabrication.

The past year has seen several particularly noteworthy achievements in the area of target fabrication:

- The yield of target-quality glass shells from the high-temperature furnaces has increased to $99 \%$ in some batches.

- Special diagnostic gases have been put into the glass shells during theır formation.

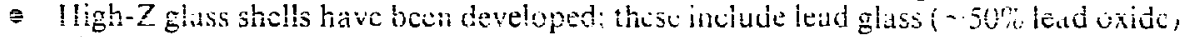
and tantalum glass $(\sim 50 \%$ tantalum oxide).

- Target-quality coatings of hydrocarbon $(\mathrm{CH})$ polymers more than $100 \mu \mathrm{m}$ thick have been deposited on glass shells.

- Layers of beryllium. platinum, and other metals have been applied to glass sinells.

- By using modern highly sophisticated micromachining techniques, we have produced hemishells of $\mathrm{CH}$ polvmers with microinch surface finishes (on the order of $250 A$ ).

- A powder or "sand" of frozen hydrogen spheres (10 $\mu \mathrm{m}$ diameter) has been produced as a potential method of filling cryogenic fusion targets to high densities.

- We have assembled multiple shell targets that have exceptionally good geometrical tolerances. (In Fig. 4-1, the components of a double-shell target oriented for assembly are shown suspended from a human hair.)

Detailed descriptions of these and many other advances in target fabrication are included in subsequent articles.

As target designs change and become more complex, both materials research and the development of characteriza-

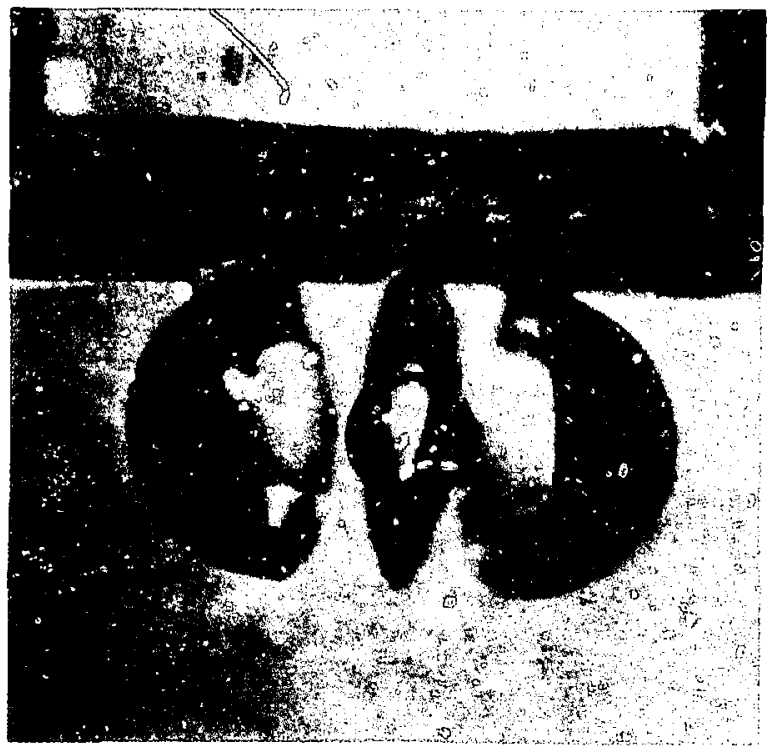


tion and assembly techniques must provide the means for producing these advanced targets. Creative, highly competent, and hardworking scientists, engineers, and technical support personnel will continue to be needed to achieve our goals.

The continued support of "outside" coistractors will also be a necessary and highly valued part of our program. The contributions of such organizations as the Rockwell International Rocky Flats Plant, the University of Illinois Charged Particle Research Laboratory, and the National Bureau of Standards Laboratory at Boulder nave been invaluable to the success of the target fabrication effort.

Author: $(\therefore$. D. Hendricks

\section{Glass Sphere Development and Production}

During the past year we continued both to meel the needs of the Laser fusion Program for quality glass microspheres and lo improve and extend the scope of our techniques. Our fat,rication processes are now refined to the point where we can tune our droplet system to produce microspheres with the desired dimmeters and wa!l thickneses our dried-gel capabilities are entranced by density-and diameler-sieve-cutting the resultant microspheres to narrou the mass distribution. We showed that filling the microspheres during fabrication with such highly reactive gases as bromine is feasible. We used our droplet systems to make lead oxide microspheres: we also began developing tantalum glass spheres using the dried-gel technique. In addition. we initiated a study of sol-gel and pelletizing techniques as possible improvements to our dried-gel processes. linally, based on studies of the working strengths and permeability of the glass microspheres, we developed improved techniques for filling the spheres with D-T. These advances are discussed in more detail in the articles that follow.

\section{Sphere Fabrication Processes}

We have continued to refine our understandin of the liquid-droplet process.' In this process, we use acoustic disintegration of a liquid jet to procice uniform drops of an aqueous solution of glassforming compounds. The size of the liquid drop is affected by the solids content in the glass-forming solution, the feed pressure of the solution. the orifice size, and the acoustic driving frequency. The uniformity of th drops is evidence that the resultant microspheres all have the same mass. In the pust. we obtained batches of spheres with uniform wall thick ness by sieve-cutting over a narrow diameter range.

We now obtain direcly from the furnace narrow-diametar distributions tunable to the desired sphere sioss. This advance is directly attributable to our understanding of the interplay among the size of the droplet. the lemperature

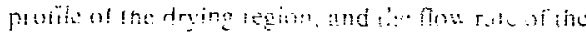
furnace atir. A key aid to this understanding is our use of a fast and accurate device for measuring sphere diamelers that enabled us to develop an exratustive statistical model of the liquid droplet precess. A diameler distribution of a batch from : furnace tuned to produce $140-\mu \mathrm{m}$-diam spheres appears in Fig. 4-2(a). The spheres continue to have narrow thickness distrihutions as well as excellent concentricity [see Fig. 4-2(b)]. The capability of tuning the dianteter of the sphere hals greatly enhanced our yield, so that only a couple of hours of running time are needed to produce a batch large enough to be processed through the fill and coating stages of target fabrication.

Wh also produce microspheres by using a dried-gel process.' Although more flexible in terms of available sizes, the dried-gel process has the disadvantage that the microspheres produced have a broad range of will thicknesses. which means that simple diameter-sieving will not result in a batch with a nairow wall-thick ness distribution.

To narrow the wall-thick ness distribution, we developed a density-sieve-cutting procedure to use in conjunction with diameter-sieve cuts. For spheres with densities between 0.6 and $1.5 \mathrm{~g} / \mathrm{cm}^{3}$ we succcsively sink and Mott the spheres in liquids such as pentane and chloroform to separate the desired wall 
Fig. 4-2. Broplet production: (a) diameter distribution of droplet batch and (i) interference picture of spheres showing excellest concentricity and narrow-thickness distributicn.

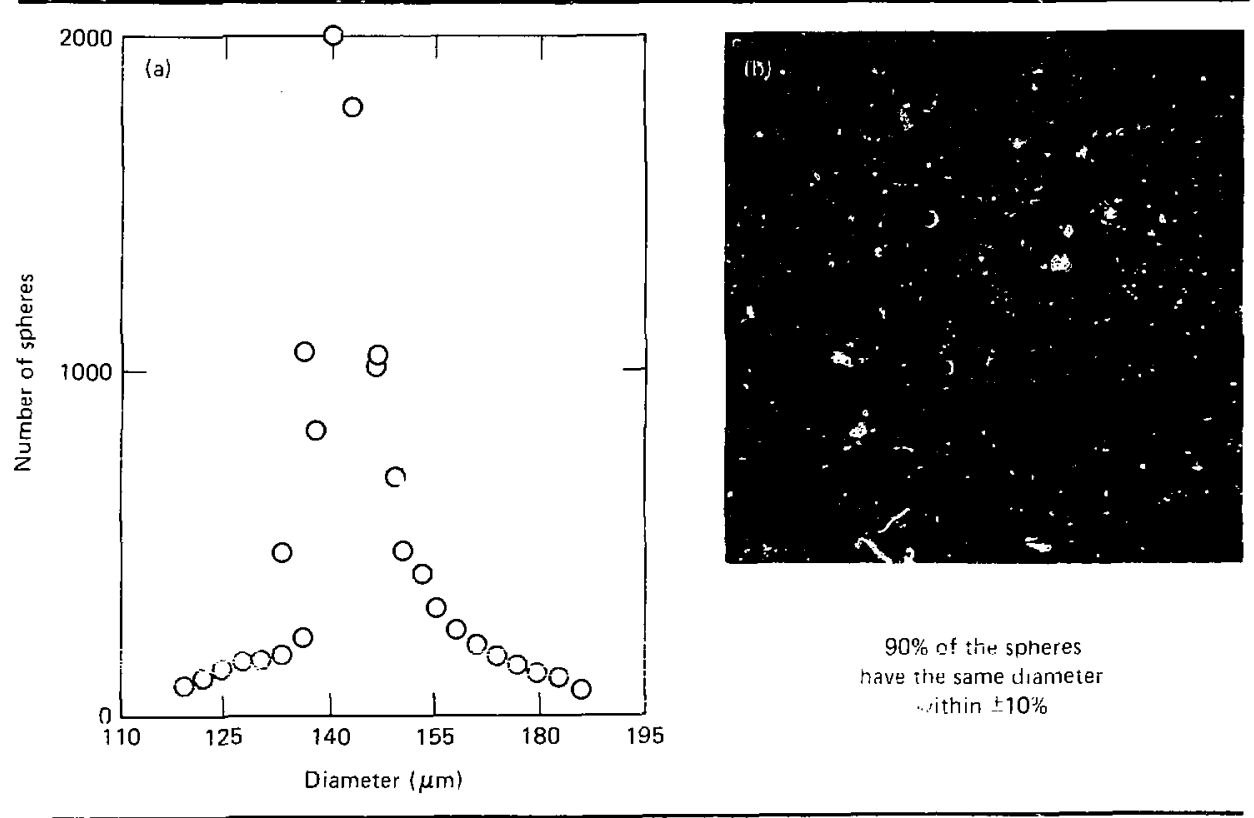

thicknesses for given diameter ranges. An example of a resulting dried-gel batch is shown in Fig. 4-3.

Such batches obtuined from the dried-gel process can be useful for coating. By combining spheres produced by the liquid-drcplet and driedgel techniques. we can toilor the thickress distribulion of sr: as in a coating batch such as shown in Fig. 4-4. 'ius gives us the flexibility to match target fabricati it resources to the target requirements. Consider the request for a $100 \mu \mathrm{m}$ i.d. $\times 10 \mu \mathrm{m}$ glass sphere coated with $100 \mu \mathrm{m}$ of $(H$. Nithough calculations indicate that $10 \mu \mathrm{m}$ is the optimum glass thickness. there is a reasonable likelihcod that spheres between 5 and $20 \mu \mathrm{m}$ thick will also be desired. Rather than coating batches with a variety of rarros thickness distributions (which would take months if done sequentially), it is more efficient to coat micruspheres having the tailored distribution shown in Fig. 4-4. The spike guarantes a reasonable probability of obtairing $10-\mu \mathrm{m}$ spheres and the hroad background covers the range of other polentially useful sizus.

Although a few more days of wrling lme in re-

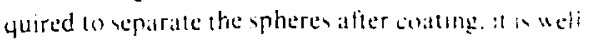

Fig. 43. Broad-thickness-distribution batch for coating spheres approximately $100 \mu \mathrm{m}$ in diameter with thicknesses ranging from 6 to $17 \mu \mathrm{m}$. This batch was obtained iy bưth density- and dianeter-sieve cutting a batch that was pruduced by the dried-gel method and that initially had a nuch broader distribution. Note the excellent concentricity.

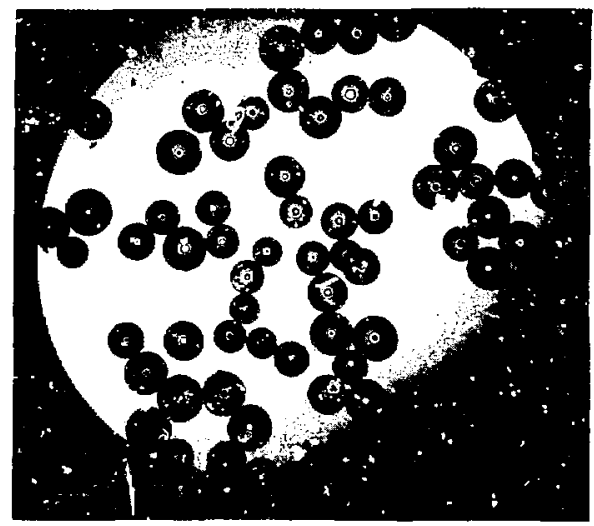


Fig. 4-4. Talioed thickeen ditrinution of gaberts for ef. ficlent costing with hydrocartien polywer.

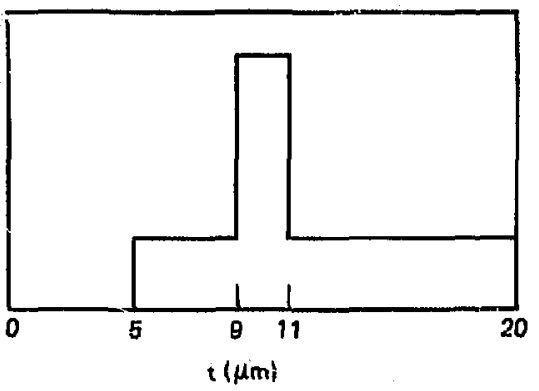

worth the effort in view of the time that would otherwise be spent making and coating separate balches of spheres. When target requirements are more well-defined, as in the past with $140-\mu \mathrm{m} \times 5$ $\mu \mathrm{m}$ spheres, we can use just the narrow distribution batch produced by the liquid-droplet process.

Authors: R. I. Woerner and V. F. Draper Major Contributors: J. E. Andrews, M. H. Corzette, W. E. Elsholz, G. A. Gleeson, C. L. McCaffrey, R. J. Moore, and S. I. Weinland

\section{Reference}

1. Laser Program inmual Report-1978. L.awrence Livermore I aboratory. I ivermore. Calif., UJCRL_-50021-78 (1979). pp. 4-2 to 4-7.

\section{Diagnostic Gas Fills}

Adding special gases to the D-T fuel in fusion targets provides the capability of making measurements to determine the D-T fuel density attained during implosions. ${ }^{2}$ At present, imaging and broadening of the $x$-ray lines emitted by argon seed gas is used. However, this technique is limited to relatively thin-walled targets because of the attenuation of the $x$-ray lines.

For the thicker-wall targets (i.e., ablative targets), we would like to extend the nes tronactivation techniques ${ }^{3}$ used on the glass pusher to find the $\rho \mathrm{R}$ of a diagnostic seed gas; ${ }^{79} \mathrm{Br}$ has teen proposed as the best choice for a gas from the standpoint of diagriostics.

Although we routinely use simple permeation to fill glass spheres with $\mathrm{D}-\mathrm{T}$, this method is not practical for the desirable diagnostic gases. By introducing argon into the furnace atmosphere, we have been able to trap up to 0.2 atmospheres (at room temperature) of argon in the micrespheres during the fabrication process. ${ }^{4}$ Bromine is highly reactive and therefore much more difficult to nandie. During the latter part of 1979, we began a series of experiment's io determine the feasibility of adding bromini: to the internal atmosphere of the microspheres.

Using a drued-gel system, we produced glass microspheres with up to 0.31 atmospheres of bromine trapped inside. The bromine is probably present as $\mathrm{HBr}$ and $\mathrm{Br}_{2}$. The small experimental furmace used for this experiment is capable of producing target-quality spheres 70 to $300 \mu \mathrm{m}$ in diameter with wells 1.5 io $3 \mu \mathrm{m}$ thick on a selection basis (i.e., by sorting through batches to find acceptable spheres). A major effort is still required to adapt this process to a liquid-droplet systen, for producing batches of acceptable spheres.

In our experiment, we used two furnaces, one located above the other. The upper furnice was operated at $1440^{\circ} \mathrm{C}$ and prodiced the spheres in a static air atmosphere. Below the upper furnace, separated by a cooled 12-in. zone, is a second furnace containing a bromine atmosphere. This furnace is operated at $1200^{\circ} \mathrm{C}$. The 12 -in. cooled zone prevents the bromine from rising to the upper furnace. If too much bromine is present in the upper furnace the glass material and bromine react violen1ly, preventing glass spheres from forming. However, if the spheres are first produced and then immediately passed through a bromine atmosphere, the bromine does not react with the glass but appears simply to permeate through it.

\section{Authors: R. L. Morrison and R. L. Woermer}

\section{References}

2. J. T. Larsen, V. W. Slivinsky, S. M. Lane. and N. M. Ceglio, Diagnosis of Laser Produced Implosions Using Argon X-Ray Lines, Lawrence Livermore Laboratory. Livermore, Calif., UCRL-83313 (1979).

3. E. M. Campbell, S. M. Lane, Y. L. Pan. J. T. Larsen, R. J. $W_{\mathrm{ahl}}$, and R. H. Price. Determination of Fuel $\rho R$ of $I C F$ Targess by Neutron Activation, Lawrence Livernore Laboratory, Livermore, Calif., UCRL-83073 (1979).

4. J. C. Koo. J. L. Dressier, and C. D. Hendricks. Low Pressure Gas Filling of Laser Fusion Microspheres, Lawrence Livermore Laboratory. Livermore, Calif., UCRL-81417-1 (1979). 


\section{High-Z Glass Spheres}

We have continued development of lead-glass spheres, which we began last year using the driedgel process. ${ }^{5}$ By extending the process to the liquiddroplet system, we produced several batches of thinwalled, lead-glass microspheres that contain about 30 mole \% of lead oxide. Since these microspheres were produced at an oven temperature below the critical evaporation temperature of lead oxide in the glass, the percentage of lead oxide in these microspheres is uniform and approximately equal to the oxide percentage in the aqueous solution. This was verified by secondary $\mathrm{x}$-ray emission data logether with index-of-refraction measurements. At $300^{\circ} \mathrm{C}$ the characteristic D-T fill time for these spheres was several hundred hours.

In an attempt to decrease the fill time constant and aid permeation studies, we reduced the lead content. Spheres with diameters of $\sim 150 \mu \mathrm{m}$ and walls $0.5 \mu \mathrm{m}$ thick and containing 25 mole \% lead were lilled at $400^{\circ} \mathrm{C}$. The measured permeability indicates that similar composition spheres $100 \mu \mathrm{m}$ in diameter and $10 \mu \mathrm{m}$ thick would take approximately 100 hours to fill. The concentricity of the thin. 25 mole \% spheres needs to be improved.

We also produced lead-oxide glass spheres with reasonably thick walls (5 to $13 \mu \mathrm{m}$ ). Some spheres were concentric, but problems remain in controlling the geometry and composition of the spheres.

The wash used for the alkali-silicate glasses ${ }^{6}$ is unsuitable for our high- $\mathrm{Pb}$ content glasses because of excessive surface deterioration. All the Pb-glass spheres washed with this technique showed some degree of cracking with subsequent uniform strip. ping of the surface.

We found that hydrogen peroxide was the best wash solution of those we examined. At all ranges of temperature and concentration, the surface appearance was enhanced, and we obtained the quality shown in Fig. 4-5. (An ethanol rinse follows the $\mathrm{H}_{2} \mathrm{O}_{2}$ wash in all cases.)

Additionally, we have developed a very promising new tantalum glass that may well provide all the characteristics required of high- $Z$ glasses. We have blown a glass containing $50 \mathrm{wt} \%$ tantalum into good quality microspheres by the dried-gel production method.

The dried gel is produced by using organometallic compounds in an ethanol solution. The gel forms very rapidly and can dry overnight because of
Fi. 45. Typical Ph-plass microephere suzfase after $\mathrm{H}_{2} \mathrm{O}_{2}$ naw.
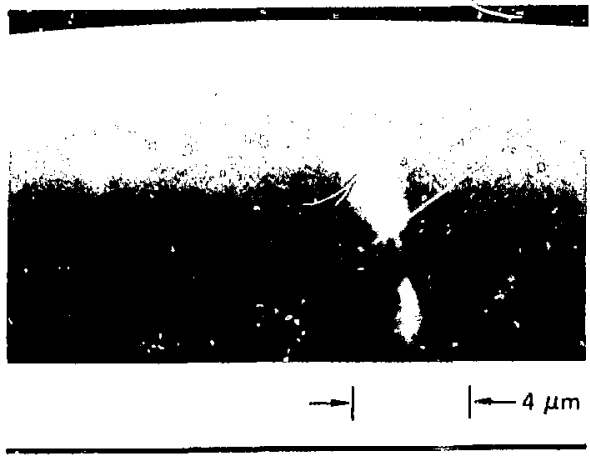

the high volatility of the alcohol. ine spheres produced from this gel show good sur e characteristics and can be filled with D-T by pre intly used methods. We have also demonstruted good concentricity in these spheres.

Further experiments with this glass will be devoted to controlling the diameter and wall thickness. If we can achieve this control, tantalum glass will very likely replace lead glass in satisfying our high- $Z$ glass requirements. In future experiments. we will develop production of tantalum glass by the li zuid-droplet technique. This method of production is important because it permits us to manufacture batches of very narrow diameter and wall-thickness distribution.

Authors: R. L. Morrison and R. L. Woerner

Major Contributors: J. C. Koo and J. E. Andrews

\section{References}

$\therefore$ Laker Prugram Anmal Repert-1978, Law rence Livermore Lithorators. Iivermore, Calif., UCR1-50021-78 (1979), p. 4.5 .

6. Laser Program Almual Report-1977. Lawrence Livermore Lahorators, I.ivermore, Calil.. UCRL-50021-77 (1978), pp. $5-13105.16$.

\section{D-T Fill Techniques}

During attempts to fill glass microspheres for botb the low-density thermonuclear-burn Shiva targets and the argon-diagnosed intermediatedensily experiments, a high proportion of the desired thin spheres broke. We reviewed the 
available data from past fills and precrush studies to determine whether the breakage could be correlated with the expected mechanical properties of the glass spheres. It appears that the spheres are failing under compression that is due to buckling.

We avoided this problem by combining our present unuerstanding of the mechanical properties of the spheres with the known permeation rates for D-T and $\mathrm{He}$ to prescribe the appropriate fillpressure steps and times for the various aspect-ratio spheres. ${ }^{7}$ More experiments are required to confirm and refine our knowledge of the mechanical strength and permeability of the spheses.

When a sphere is subjected to a uniform external pressure, the sphere wall undergoes a

Fig. 4-6. Strength limits of glass microspheres. Curve I (hoopstress) indlcates the compressive pressare llmit that applies to spheres with aspect ratios, $d / t<\sim 65$ and, since $\sigma_{e}$ $\sim \sigma_{1}(\sim 10$ kbar $)$, the extensive limit for all spheres. [Here, $P_{H}=4 \sigma /(t / /)$.) Curve II (elastic buckling) indicates the compressive limit for spheres with $d / t \sim 65$. The points represent the thinnest apheres known to have sarvived the varioess all prescures. During fill $(700 \mathrm{~K}), 20 \mathrm{mg} / \mathrm{cm}^{3}=233$ bar; after fll $(390 \mathrm{~K}), 20 \mathrm{mg} / \mathrm{cm}^{3}=100$ bar. $\left(P_{\mathrm{g}}=\right.$ $\left.\left[8 E / \sqrt{ } 3\left(1-\nu^{2}\right)\right]\left[1 /\left(0^{*} / t\right)^{2}\right].\right)$

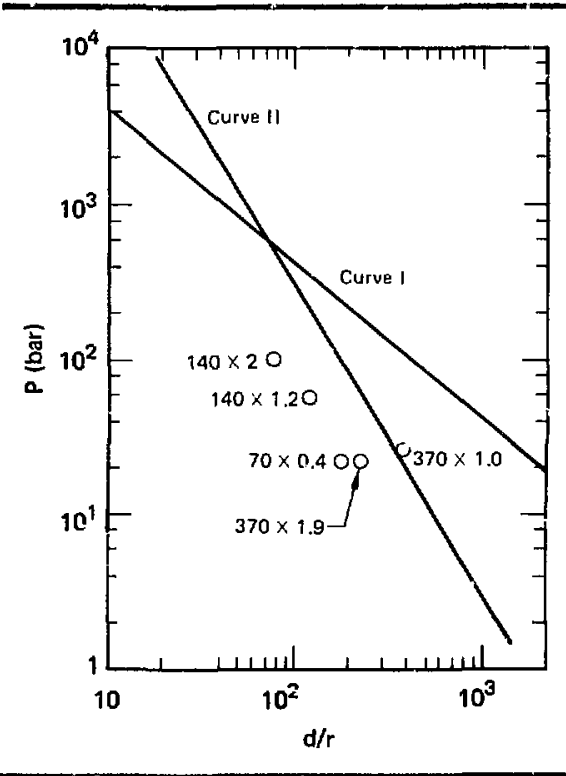

compressive-hoop stress. The failure limit for compressive-hoop stress is indicated by Curve $I$ in Fig. 4-6, where we have used a compressive stress limit of $10 \mathrm{kbar}\left(10^{9} \mathrm{~Pa}\right)$. This value is probably accurate to within $\pm 15 \%$; it was chosen on the basis of the measured value for bulk samples of mul. ticomponent glass systems that are reasonably close to the glass sphere composition.

Because of buckling. however, thin spheres will fail at much lower pressures than indicated by Curve I. In Fig. 4-6, Curve II is a plot of the elastic buckling pressure limit of the microspheres; it is also based on the measured values for bulk samples of similar multicomporsent glass.

The expression for the elastic buckling limit is valid as long as the stress remains less than the elastic limit or-since there is no well-defined elastic limit for glass-(at most) less than the yield point. As seen from Fig. 4-6, this is the case for microspheres with aspect ratios greater than about 65 . For smalier aspect ratics, inelastic buckling is the failure mode, and it occurs at pressure values lower than predictcd for elustic buckling. For spheres with aspect ratios less than 65, the compressive-hoopstress curve serves as an upper limit. The unsafe region above both limits is indicated in Fig. 4-6.

Along with the estimated curves for the strength limits of the microspheres, we have also plotted some rough data from various D-T fills. The points represent the thinnest sphere known to have survived the various fill pressures. The results are quite consistent with elastic buckling as the failure mode, even though the limit curves are approximations for ideal spheres, and concentricity and other structure defects greatly reduce the buckling pressure and provide scatter in the data. To be safe, the external overpiessure on the spheres should be kept a factor of 5 to 10 less than the failure limit indicated in Fig. 4-6.

How large a fill pressure the spheres can hold is governed by the tensile strength, $\sigma_{t}$, of the sphere material. Unfortunately, it is difficult to estimate accurately the tensile strengtî of glass. Unlike compressive strength, the tensile strength is unpredict. able and highly dependent on cross section. Measurements of $\sigma_{\mathrm{l}}$ for some glass compositions have varied from as low as $0.7 \mathrm{kbar}$ for bulk samples to as much as $100 \mathrm{kbar}$ for thin fibers. Additional factors, such as surface imperfections, also produce wide scatter in the data. Because the microspheres are relatively thin in cross section ( 1 to 
$10 \mu \mathrm{m}), 10 \mathrm{kbar}$ is a reasonable estimate for $\sigma_{1}$. Thus Curve $I$ in Fig. 4-6 can also represent the bursting limit of the spheres.

As seen from comparing Curves $I$ and Il in Fig. 4-6, the glass microspheres will buckle under compression at a lower pressure than they are capable of containing under exiension. If we wish to fill a sphere to a pressure higher than its buckling point, we can simply ramp-fill the sphere. This is done by applying the maximum allowable pressure difference and subsequently increasing the external pressure at the same rate as the pressure increases in the microspheres, thus maximizing the pressure differential and minimizing the fill time.

The time, $\lrcorner t$, needed to ramp-fill a sphere to a final pressure, $P_{F}$, is given by $\Delta t=\tau P_{F} / \Delta P_{\max }$. where $\lrcorner P_{\max }$ is the maximum overpressure that can be applied. Current f.ll tumes are typically 35 to
50 nours. The time constant, $\tau$, depends on the geometry of the sphere and the permeability of the sphere material. Based on preliminary experiments, we use $\tau_{D-T}=2 \times 10^{-2}\left(\ell r_{i} 2 / r_{o}\right)\left(h / \mu m^{2}\right)$ where $r_{i}$ and $r_{0}$ are the internal and external radii and $\ell$ the wall thickness of the sphere. A more accurate value for $\tau$ remains to be established, especially for spheres with walls less than $3 \mu \mathrm{nu}$ thick, many of which lose their fili at a faster rate than anticipated.

To supply the ramped pressure, we have developed a vanadium D-T hydride gas generator. $\checkmark$ anadium will form a hydride at room tempcrature, but will release the hydrogen at high pressure when heated. $A$ pressure of 10,000 psi can be obtained at less than $300^{\circ} \mathrm{C}$. The generator consists of a highpresiure chamber filled with vanadium granules that is connected to two transducers (one for control and the other connected to a recorder). These

Fig. 4-7. Schematic for vąnadlum hydride D-T ramp-filling system.

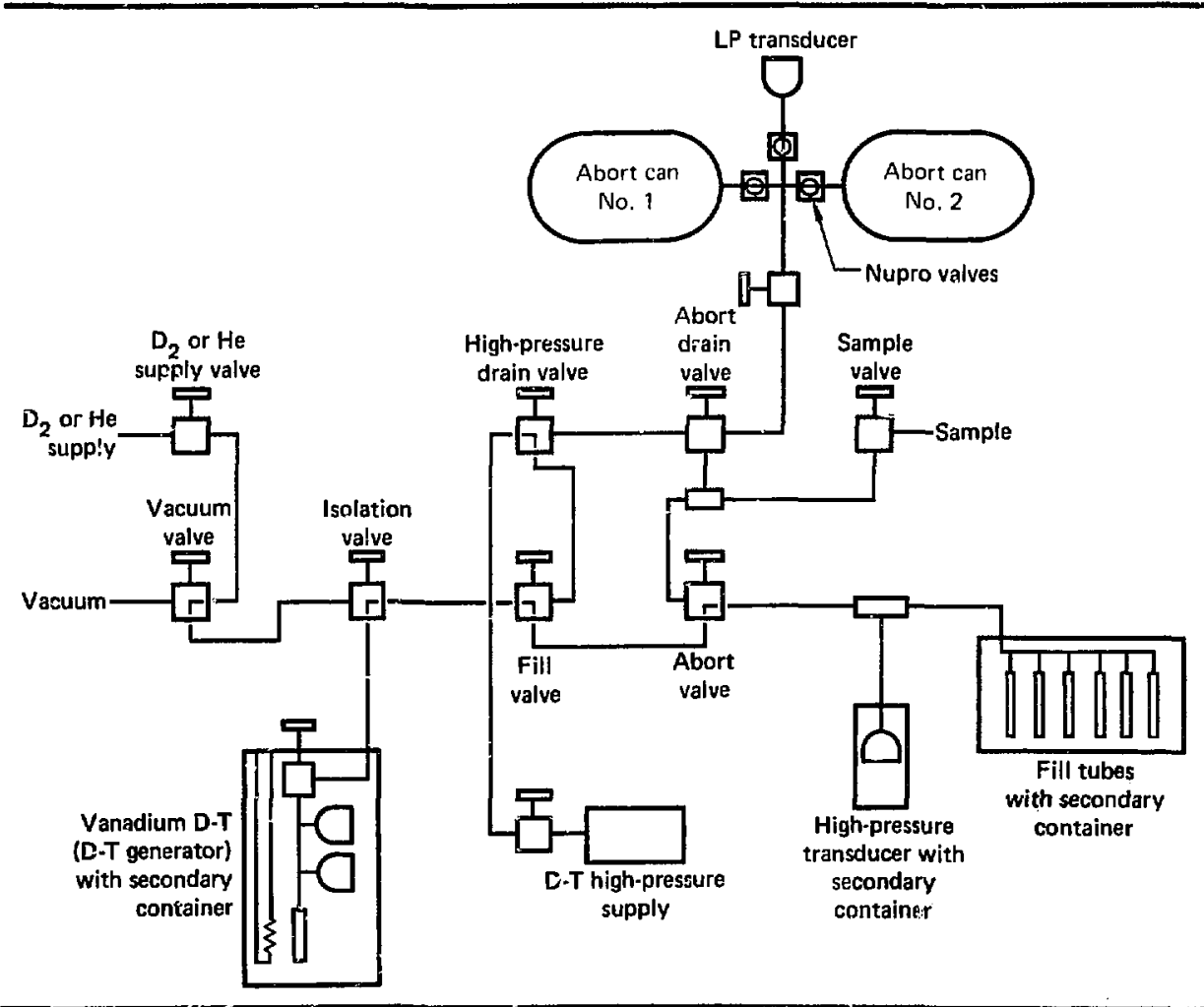


are connected through two high-pressure values to the sphere-loading system (Fig. 4-7). A heater surrounds the vanadium chamber, and two thermocouples are also connected to the chamber. (One conncets to a recorder and the other to an oveniemperature cutoff.) This entire unit is placed in a secundary container to prevent the loss of tritium in the sent the clamber. or a transducer, or other :quipment should rupture. Because there is no coninfell relationship between pressure and temineratture, we used the voltage signal from the messure transducer to control the heater power. l hie rignal is compared to a voltage that represents the uenired pressure at a given time. This powerrequured voltage signal notifies a power controller 1. hupply the power for the heaters. Oven temperalure and uverpressure safety cutoffs are connected to this unit.

\section{Authors: R. I.. Woerner and L. D. Christensen}

Major Contributors: I. M. Moen and B.W. Weinstein

\section{Reference}

7. R I. Muerner. B. W. Weinstein, I. M. Moen. and J, G Ritumann. "Working Strengths and D-T Fill Procedures for Cilass Microsphere laser Fusion Targets." prepared for $J$. Ippl Phys. I ebruary 1S. 1979 (a)so t.awrence Livermore Laboratory (1CRL-82728).

\section{Coatings and Layers}

The performance of many laser fusion targets can be improved by the addition of one or more layers around the microsphere fuel container. Coating these layers onto targets, whether the simple D-T filleo glass shells or the double-shell assemblies described in "Assembled Second Shells," is a difficult. two-step effort. We must first develop the coating processes to deposit materials with the desired properties, and then we must coat the microspheres and assemblies with extremely smooth and uniform layers of these materials.

Low- $Z$ coatings have been formed by plasmapolymerization of hydrocarbons as weil as by sputtering beryllium. High- $Z$ coatings have also been formed by sputtering platinum. For both plasma polymerization and the sputtering process, the exacting surface-finish requirements $(\leqslant 0.1 \mu \mathrm{m})$ constrain the choice of coating material, the deposition rate, and the energy deposition at the coating surface. The processes must virtually eliminate all defect-causing particles greater than $0.1 \mu \mathrm{m}$ (these particles originate from a number of sources). With D-T filled microspheres, the processes are further constrained: they must not heat the microspheres above $100^{\circ} \mathrm{C}$. a temperature that could cause loss of the fill gas by diffusion.

We must provide continuous, random motion of the microspheres during these processes to ensure a uniform coating. Strong electrostatic forces, however, cause these moving microspheres to adhere to one another, forming clumps. Further, in coating soft meials or coating with high fluxes, the shells additionally tend to clump or cold weld to each other or to the coating apparatus as a consequence of complex, poorly understood processes.

Microsphere adhesion and many of the problems with particulate contamination can be overcome by levitating single glass spheres in a coating process. One successful levitation scheme :ciles on a molecular beam support for the spheres.

a process that we describe in detail under "Molecular Beam Levitation During Coating." below.

An entirely different approach to depositing metallic layers is electroplating. Intrinsically a low temperature process, electroplating provides low mechanical stress for the shells during coating, and it is versatile in the materials it can deposit. Soft metals are the first coating that electroplating can add to our coating capability.

Our first article, "Hydrocarbon Coatings." describes advances in the plasma polymerization of hydrocarbons, which we initiated last year and which we now use in current production. Thereafter, "Beryllium Coatings" describes progress in sputtering beryllium. We discuss platinum sputtering used for a high-Z, high-density tamper layer on microspheres in "Sputtered High-Atomic Number Coatings." Finally, we review our progress toward electroplating metallic layers in the article "Electroplating."

Authors: S. F. Meyer and W. L. Johnson

\section{Hydrocarbon Coatings}

During 1979, we continued development of a plasma polymerization process to produce ultrasmooth hydrocarbon coatings in response to the 
need of the Laser Fusion Program for an intermediate-density target. The couting for this target must be far thicker than any ultrasmooth coating previously deposited on microshells by plasma polymerization. The specifications require a coating thickness greater than $100 \mu \mathrm{m}$ with a density of $1.0 \mathrm{~g} / \mathrm{cm}^{2}$ and a finish better than $0.2 \mu \mathrm{m}$ over the entire surface.

$A$ number of factors enabled us to provide hydrocarbon coatings that satisfy the specifications:

- Improved krowledge of and control over defect growth in the plasma polymerization process.

- Complation of an automated plasmia coater.

- Rapid and accurate characterization of the developed coatings provided by the Surface Analysis and Target Characterization (iroups.

- Improved glass microshell quality provided by the Glass Shell Group.

As a consequence. we are now able to produce hydrocarbon coatings up $10135 \mu \mathrm{m}$ thick. while maintaining a surface smoothness of better than $0.1 \mu \mathrm{m}$.

Plasma Coating Process. Plasma polymeri/ation of hydrocarbon gases has been studied extensively by others. ${ }^{x-10}$ Our own process is very similar to the process we used to form fluorocarbon coatings. We have described that process-as well as associated hardware-in the 1978 Annual Report." Briefly, the plasma-polymerization process activates a hydrocarbon monomer gas as it passes through an electrical discharge formed in ad helical resonator. The activated molecules then condense to form a polymer on a substrate. The plasma is formed inside a quart/ discharge tuhe covering a piezoelectrically driven vibrator pan that holds the glass microshells. Vibrating the microshelis into continuous random motion allows a uniform coating of polymer to be deposited.

The parameters that must be carefully controlled to give good reproducible contings with the desired surface finishes include the total gas pressure in the discharge tube. the flow rates of the monomer gas that forms the plasma. hydrogen, and a possible third gas that helps control polymerization. Coating rates are typically $1 \mu \mathrm{m}: \mathrm{h}$. and it is impractical to hand-control these parameters for the $100 \mathrm{~h}$ needed to produce a $100-\mu \mathrm{m}$ coating. We have overcome this difficulty by constructing an automated coater with prevision for self-protection in case of system failure. Gas-flow rates and total system pressure are independently controlled. and the coater is capable of unattended operation in excess of $100 \mathrm{~h}$.

Coating I)efects. Early efforts at coating microshells produced surfaces that were completely covered with dome-shaped defects. (When magnified. a target would resemble a caulifiower.) Such coatings have heen traced to the presence of small particles that can nucleate defects any time they setthe on the surface being woated. The sourees of these particles are atmosphere-borne dirt and particles formed during the polymeriation process itself.

$W_{2}$ minimise intrusion of atmosphere-borne dirt hy shiclding the apparatus hen it is open for loading. We also cleatn the quarle discharge tuhes for two hours in an argon discharge that further reduces particulates iiltroduced to the system. The (ilass Shell (iroup mainlains a high standard of surface cleanliness on the glass spheres thentselves hy using a series of $u$ ashes. 12

Polymer. of course, forms on any surface in contact with the plasma. The coating deposited on the interior walls of the quart/ discharge tube becomes a source of particulate contamination because tensile stress in the deposited coating causes the coating to crack and peel. The intrinse stress leading to the contamination is neutralised by controlling the systen gas composition and thus the coating properties We have found that a combinaltion of trans-2-hutene and hydrogen (flowing at 0.3 and $1.9 \mathrm{secm}$. respectively maintained at a total system pressure of $75 \mathrm{~m}$ Torr produces a minimum stress coating. In addition. it is necessary to surround the gas-discharge section of the plasma coater with a water jacket. This dielectric layer reduces the electric lield at the quarte reaction vessel "all and consequently decreases electron and ion bombardment that in part cause intrinsic stress in the film. Reduced discharge-power levels also reduces this sputtering effect. We have found that 20- $\mathrm{W}$ discharge power is optimal.

Finally. thick polymer coatings are typically deposited in two separate steps with a change of discharge tube between the steps. This prevents thick coatings from developing on the wall. Neutralization of intrinsic stress has increased coating thickness from a maximum of $2 \mu \mathrm{m}$ (the thickness at which the first flake contamination appears) to over 
$100 \mu \mathrm{m}$. which makes it possible to deposit thick coatirgs on targets in a single batch run. (As an aside, in contrast to tensile stress, a large compressive stress in the polymer coating on the discharge tube wall can cause the tube to explode after several hours of coating. This problem is also solved by the remedies discussed above.)

A second source of particulate contamination arising from the couting process itself is generation of particles in the gas phase. ${ }^{13}$ These particles, ranging in size from a few hundred angstroms to several micrometres. are created by gas-phase polymerization. The extent of gas-phase polymerization is limited by turning the discharge off for one second every ten seconds. This allows growing particles. which are levitated in the electrostatic fields of the discharge, to be swept out of the coating region.

t:ven after eliminating all these sources of particles. we found that defects were still appearing in the microsphere coatings. We suspected the cause of these defects to be irregularities present on the glass microshell itself.

The effect of a surface irregularity on coating quality is shown in Figs. 4-8 and 4-9. The surface quality over most of the microshell surface is better than $0.1 \mu \mathrm{m}$. The target quality of the coating. however. was destroyed by a substrate irregularity

Fis. 48, SEM photomicrograph of a defect in $\& 41-\mu \mathrm{m}$-thick conting that orighated at the mieroshell surface.
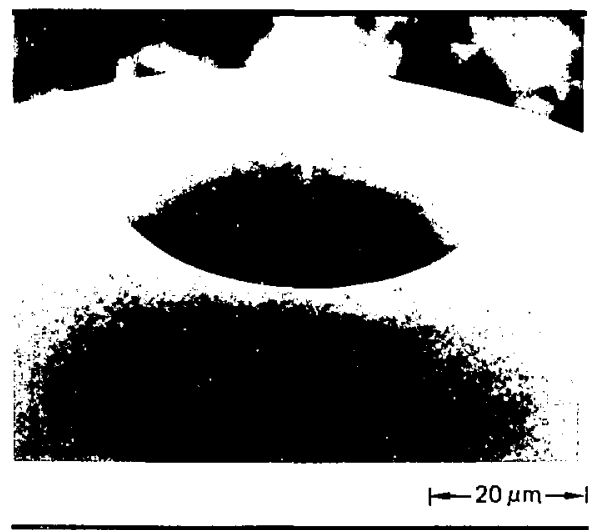

which. when covered by the growing coating, became a dome-shaped defect. We now have a clear understanding of the growth behavior of these defects and can predict the aspect ratio of defects based on a knowledge of substrate irregularities. We quantitatively studied the effect of coating substrate surface irregularities on coating defects and found that. to achieve target quality coatings, it is necessary to eliminate not only particles in the coater environment but also irregularities larger than $0.1 \mu \mathrm{m}$ on the glass microshell.

Known surface irregularities (polymer latex beads) were dispersed on a clean glass substrate. We masked the beads in a way that permitted us to produce a gradient of polymer coating. In the foreground of Fig. 4-10, the coating is less than $1 \mu \mathrm{m}$ thick. while further back the coating grows to $20 \mu \mathrm{m}$ thick. We found that the size of the defect expands laterally as the film thickness increases. However. the aspect ratio (the ratio of height to width) diminishes as the film thickness increases. Close examination of a thinly coated latex bead reveals that the coating has increased the width of the particle as well as its height. This can happen only with an omnidirectional coating flux.

\section{Fig. 49. Optical pwotomicrograph obtainet by oil immersion showing the profile of a defect growing from a mbatrate irrezwarity in a 135- $\mu$ m-thick coation.}

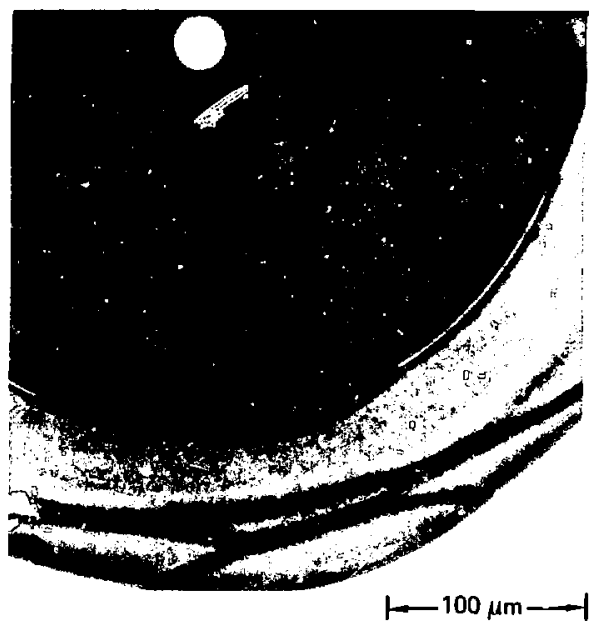


Fig. 4-10, Knom surface irrepdarities (1.1-p:m-dian latex sheres) conted with a grallent of plasens polyner op to $20 \mu \mathrm{r}$ thick.

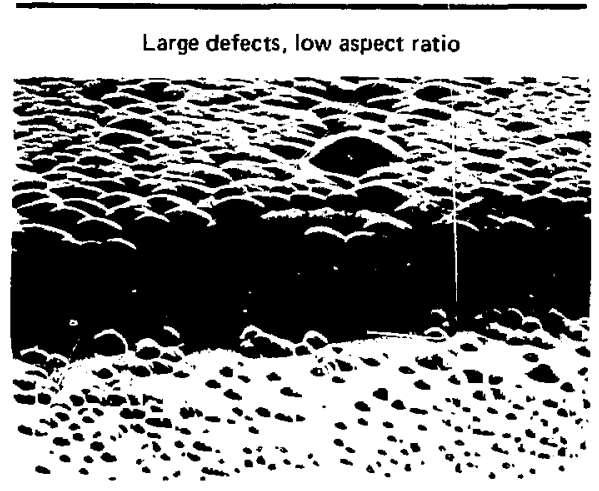

Small defects, high aspect ratio

$$
-1 \quad 10 \mu \mathrm{m}
$$

To further measure the spatial distribution of the coating flux, we mounted a single microshell on a stalk in the plasma discharge. Figure $1-11$ is a radiograph of the coated ball: the outside diameter of the glass ball is $144 \mu \mathrm{m}$, and a portion of the mounting stalk is still visible. The coating is neuriy uniform over the entire surface. indicating a uniform spatial distribution of the coatıng nux.

If an omnidirectional coating flux is assumed. the topology of defect growth becomes a simple geometric problem. For a hemispherical surface irregularity. successive layers of coating will replicate the contour of the original. forming a spherical defect that is concentric with the original (Fig. 4-12). The locus of the intersection of the defect with the smooth. unaffected surface can then be geometrically determined. Thus the defect size grows laterally as the film thickness increases: however. its height remains constant. Similar geometrical arguments for defect growth have also been made for spherical irregularities and defect growth on spherical substrates.

To investigate the growth rate of defects and to check the model, we plasma coated a range of sizes of latex beads. Figure 4-13 shows a normal view of the film defects resulting from coating over $1.1-\mu \mathrm{m}-$. $0.3-\mu \mathrm{m}-$, and $0.1-\mu \mathrm{m}$-diam latex spheres. The smallest sphere produced the smallest defects with the lowest aspect ratio. This shows that small defects produce disproportionately large defects
Fig. 411. Microsbell scomnted on a stalk coated with polymer. The wifonesty of the conting indicates an owwithrectional coat ba process. (Ball dianeter = $144 \mu \mathrm{m}$; contine thickess $=24.6 \mu \mathrm{E}$ top, $23.4 \mu \mathrm{m}$ sile.)

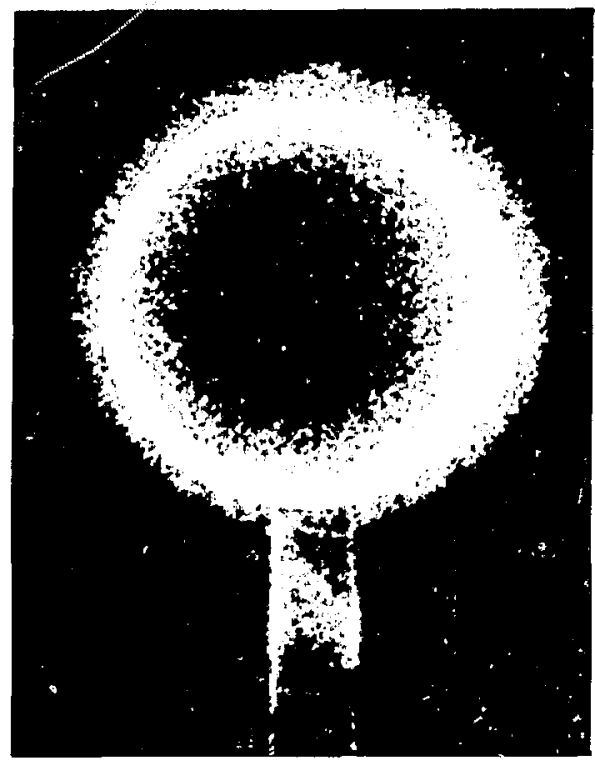

Fig. 412. Defects in plasma polymerized coatings grow by replication of the original irregularity. The defect radius is a fonction of the coating thickness and irregularity radius.

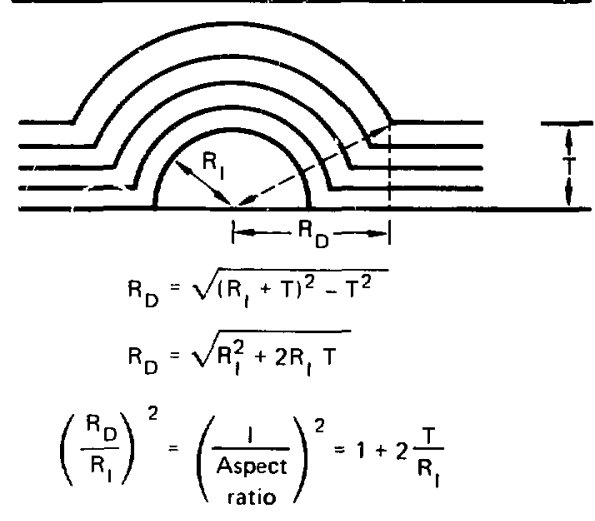




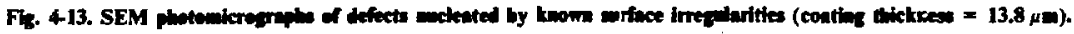
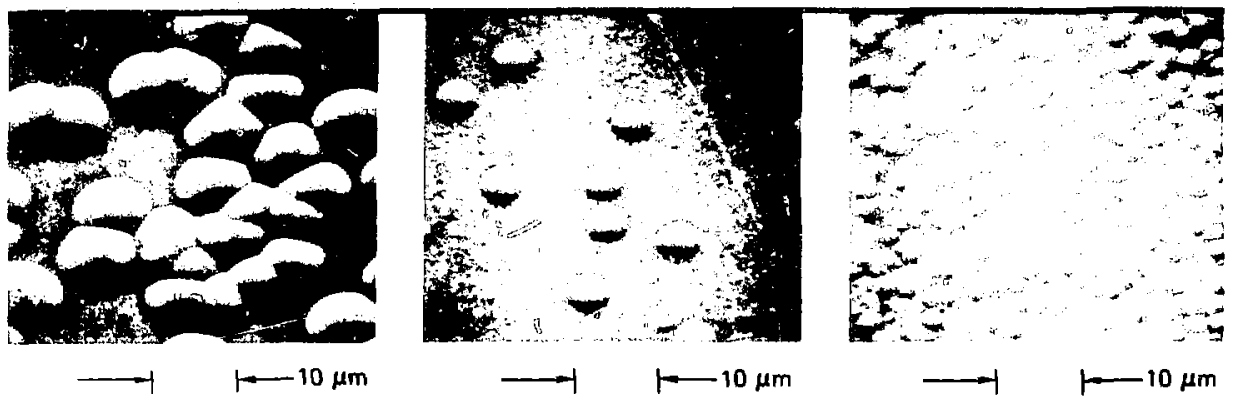

Fig. 414. Redeced defect dianeter as a fusction of reduced film thickness. Dats foints are incumust defects. The solid lincs show the growth belavior expected for spherical and hemispherical irregalarities as predieted by the geometrical growth model.

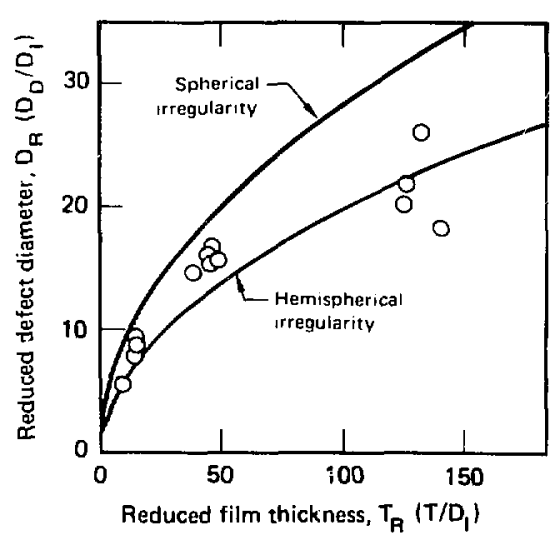

when the defect size is normalized to the size of the starting irregularity.

The data are summarized graphically in Fig. 4-14. where reduced defect diameter (normalized hy the latex sphere diameter) is plotted against reduced film thickness. The two solid lines show the behavior expected for the geometric growth models for films growing on hemispherical and spherical

Fix. 4-15. Hydrocarton conting (110 $\mu$ m thkck) on a 140-by-

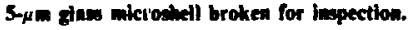

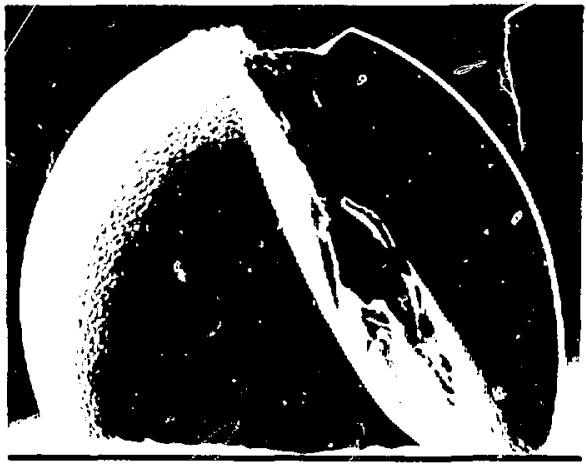

irregularities. Most of the experimental points lic between the expected curves. The close fit shows that the physical behavior is fairily well-modeled as an onınidirectional coating hux.

Production Results. Figure 4-15. a seanning electron microscope (SEM) photomicrograph of a target broken for examination. shows the coating to be concentric, homogeneous, and smooth. The coating thickness (measured from the photomicrograph) is $110 \mu \mathrm{m}$. and it look $106 \mathrm{~h}$ to deposit. The clean fracture displays the typically homogeneous. void-free coating. Inspection of the interlace between the glass and the hydrocarbon coating shows it to be also void-free. The surface finish shown in Fig. $4+15$ is better than $1 \mu \mathrm{m}$. which is still not adequate for target use. Further improvements in pir- 


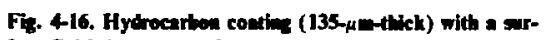
face fild letter tam $0.2 \mu \mathrm{m}$.
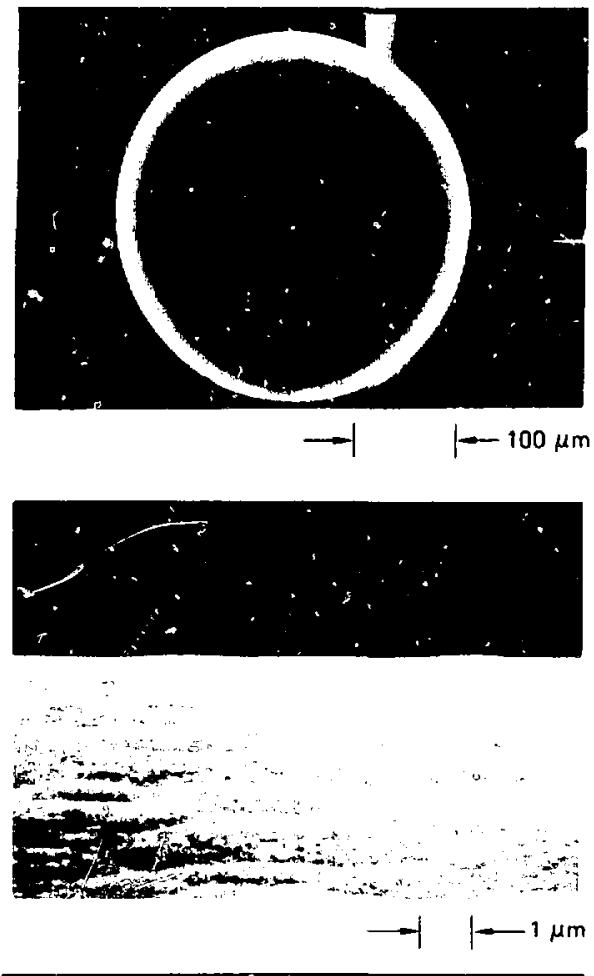

ticulate elimination and glass-shell quality resulted in surface finishes betler than $0.1 \mu \mathrm{m}$ on coatings $135 \mu \mathrm{m}$ thick (1.ig. $4-16)$.

Thick hydrocarbon coatings require different thickness charactermation techniques than are used for the thin fluorocarbon coatings. Coatings thicker than $40 \mu \mathrm{m}$ are heyond the range of the Zeiss interferometer. The Target characteriation (jroup in. stead applied $x$-ray microradiography wo the nondestructive measur:ment of the thick coated largets ligure 4-17. an $x$-ray meroradiograph of a hydrocarbon coated microshell. shows that the coating is conceritric with the substrate and measures $110 \mu \mathrm{m}$ thick. Volumes calculated from the measured thickness were combined with coating weights (determined on a quaru fiber balance) to yield coating densities of $1.00 \pm 0.05 \mathrm{~g} \mathrm{~cm}^{3}$.

Immersing the targets in an oil having nearly the sane index of refraction as the coating reduces
Fit. 417. X-ray radionicrograph of $=110-\mu$-thick hydrocartoe-coated target.

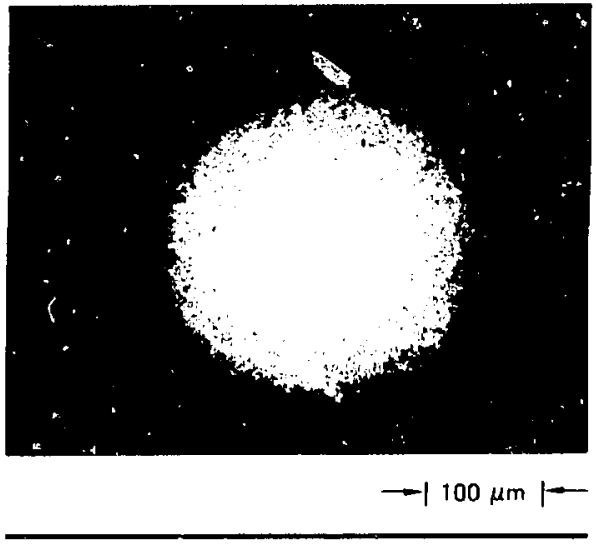

diffraction effects and allows a penetrating vieu of the internal structure of the coating. Figure $4-9$

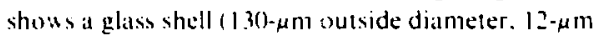
wall thickness) that is coated with hydrocarbon polymer lhydrogencarbon ratio averages 1.3:1: shorthand notation: $\left(1 I_{1}\right)$ lo a total thickness of $135 \mu \mathrm{m}$. I nondefected coillang is shown in tig. 4-65 (an "Materals inalysis Divelopments and Surface Studies." this section). Th: polymer coatung is lypeatly deposiled in las separate sleps to prerent intrinsic stress buildup in ine coathe that depessts on the discharge lube. The change ol dissharge tube lo present highly wressed thick colatugn causes a detinite color thange in the coating. (Jher rings are also visible withon the ecating that correspond lo slight ariattoms in process conditions.

Oil immersion alos allows us (1) exansine the glass inicroshell surlace after coating. Through this technique we have verilied the relationship belween coating defects and substrate irregularities. The result is that we have set quantitative quality standards on microsrell surface contumination.

Author: S. \&. I.etts

Najor Contributors: R. J. Hayes, C. W. Jordan. R. M. Krenick. S. F. Maye, D. W. Myers, and I. A. $W$ itt 


\section{References}

*. Il Kobayashi. M. Shen. and A. T. Bell. "Effects of Reacunn Conditions on the Plasma Polymerization of 1.thylene," Hacromol Sci-Chem. A8121. 373 (1974).

1) I T I owe and R. J. Fries. "Plasma Polymerized P-xylene an a l aser liusiun Target." Surface Sci. 76, 252 (1978).

(6) R I ipins. M. Cumbell. and R. J. Fried. "Plastic Coating: hor I iscr fuswon Targets." Prog. in Polymer Sci. (I979).

i user l'regram anmal Report-1978, L.awrence Livermore

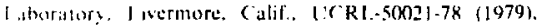
ipe $4.70+10+1]$

I. Laser Program tmmal Report-197k, L.an rence l.ivermore I aboritory. I wermore. Calif. I'C'RI -50021-78 (1979). P. + - 5

1: Y R Haren, K ( M Mthan, and W. I. James, "Plasma1)eposted Polymer films. II Transmission and Scinnung

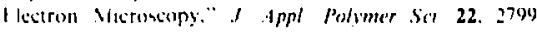
$11.17 \mathrm{x}$

\section{Beryllium Ablator Coatings}

In 1979 uc continued development work on coattng thick, dense, smooth Be on both micro. spheres and henispherical mandrels for secondshell applications. The targel shown in Fig. 4-43 ince "Douhle-Sheil Talrgets," this section) is a Itpical Be second-shell design. Hemispherical ceind-shell Be layers may be up $10200 \mu \mathrm{m}$ thick and should be dense, smocth, and have low inpurny content. We use a hemispherically shaped copper mandrel as the Be substrate. The Be coating hould also be machinathle because the critical concentricily $(<2 \%)$ and surface finish $(<1 \mu \mathrm{ml}) \mathrm{re}$. quirements of these shells may require precision machuning after deposition. The recentering ulerance $(1 \mu m)$ for remachining the coated mandrel whe required concentricity rules out an. highumperature $\left(5100^{\circ} \mathrm{C}\right)$ deposition process that $\therefore$ atald tleermally strain or deforn the mindrel. As ne whetion, we have developed roon-temperature deposilw iechniques using of sputtering with a slintrcid magnetron sputtergun to coilt thick. adhermi, dense Be onto water-cooled. Cu hentiuphersill mandrels.

The cyincirkal magnetron of sputtering system is capable of depositing $B e$ on a water-cooled matnJel all a rale of $5 \mu \mathrm{m}$ 'h-kW' rf using a 0.4-Pa $(\sim 3 \times$ $10^{-3}$ Torr) argon plasmia. A schematic of the cylindrical sputtergun and uater-cooled mandrel block is shown in Fig. 4-18. We have described our experimental procedures in detail eisewhere. ${ }^{14.15}$

The morphology of beryllium growth on copper mandrels held at $15^{\circ} \mathrm{C}$ during sputtering depends on the argon gas pressure, sputtering power (rate), and impurity-gas doping. A rough, open, low-density structure grows at high pressure and low power ( $3.3 \mathrm{~Pa}$ and $300 \mathrm{~W}$ ), as shown in Fig. 4-19(a). This film also hals an unusual $\{101\}$ texture orientation. Low-pressure and higher power $(0.49 \mathrm{Pal}$ and $1000 \mathrm{~W})$ sputtering results in thick $\mathrm{Be}$ coatings with a finc-grained, polycrystalline surface structure, bulk density, and $\{100\}$ orientation. An example is the 22- $\mu$ m-1hick film surface shown in Fig. 4-19(h). We can further improve the surface finish on thick Be coatings at $15^{\circ} \mathrm{C}$ hy gas-impurity doping during sputtering. With at 0.5 at. \% oxygen and $0.3 \mathrm{at} . \%$ nitrogen content in the Be coating, the surface finish is very fine-structured, as shown in Fig. 4-20. Analysis hy $x$-ray diffraction indicales only a very weak $\mathrm{Be}\{100\}$ line, suggesting the $\mathrm{Be}$ film is nearly amorphous. These low-lemperature, hinh-rate deposition techniques produce very-finegrained, dense. smooth Be coatings that we believe are suitahle for the precision machining operations necessi $y$ in constructing the Be second-shell layer of the proposed double-shell targets.

Thick $(>10 \mu \mathrm{m})$ Be coatings exhibit large crystal facets and unacceptably rough surfaces when deposited at high power onto uncooled. mechanically floating substrates. This is unfortunately the environment of a thermally isolated. moving-glass microsphere, during any physical vapor deposition (PVD) coating process. We have conducted additionai work with rf sputtering of Be onto floating planar glass substrates that are allowed to reach about $200^{\circ} \mathrm{C}$ during the sputtering process.

We have successfully suppressed the lightemperature grain growth such as the rough-faceted 002: crystallite surface shown in Fig. 4-21 to the much smoother. cone-free surfaces of Figs. 4-22 and $4-23$ by gas doping of oxygen or nitrogen. The surface structure in Fig. 4-21 is polycrystalline with a .110 orientation. presumably resulting from the 0.5 at. $\%$ of $\mathrm{N}_{2}$ and $\mathrm{O}_{2}$ contained in the Be. Heavier doping levels. up to 14 at.\% oxygen, produced the amorphous structure shown in Fig. 4-23. From these results. we are optimistic that low-pressure. high-energy if sputtering with controlled. low levels 
Fig. 4-18. Cylindrical if spittergun and mandrel substrate holder used for production of Be iemispherical shells.
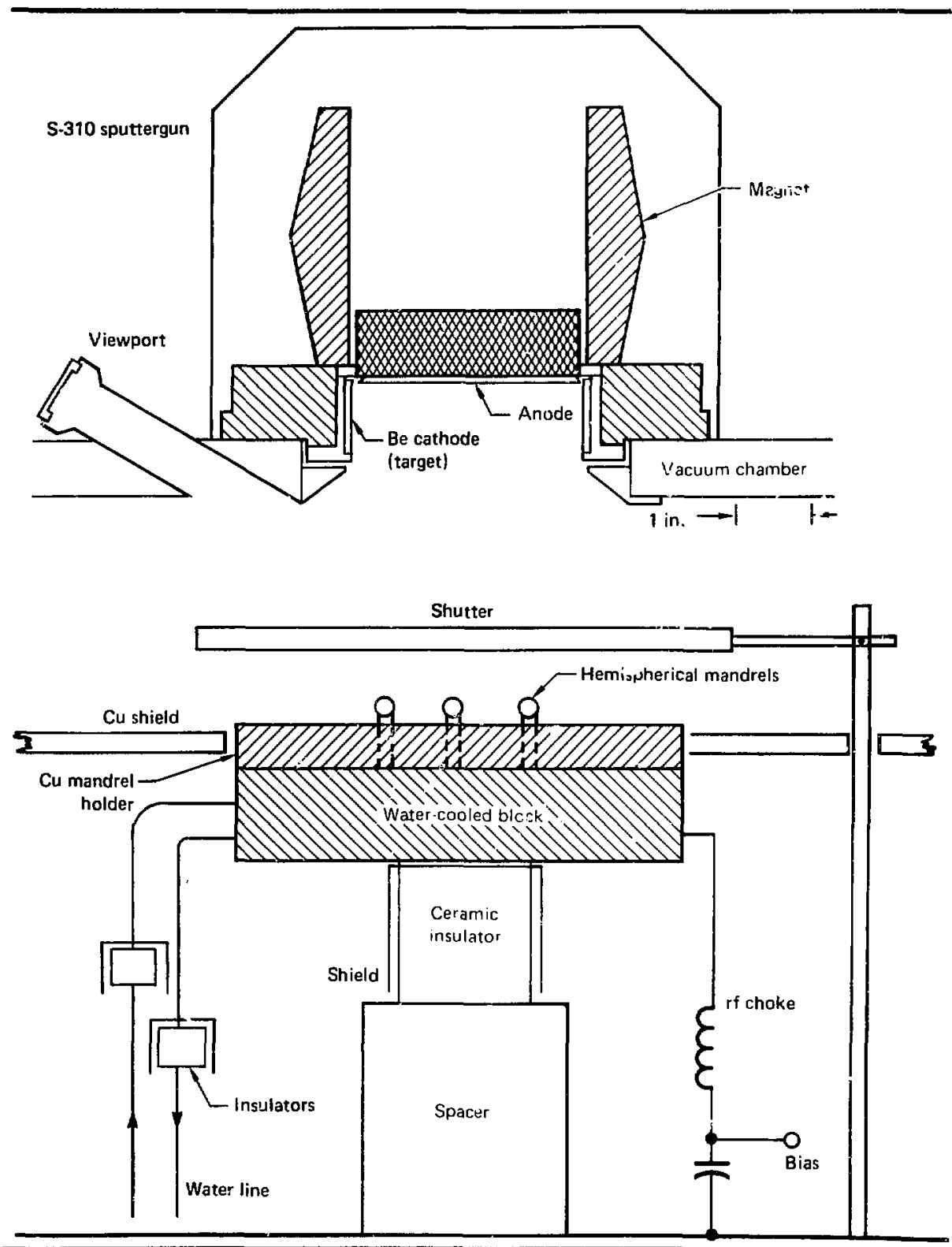


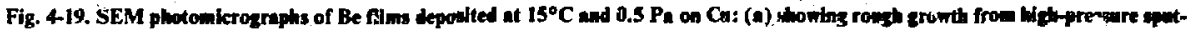

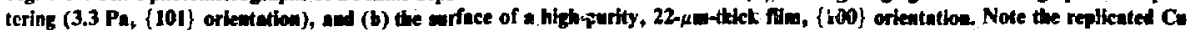
mandrel polbihiag contches in the ecenter right.

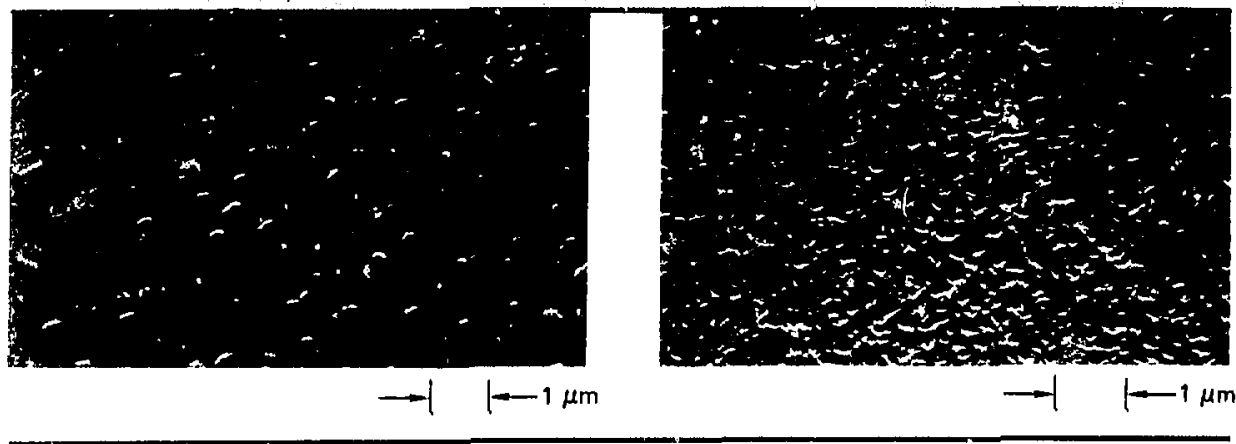

Fig. 4-20. SEM photomicrographs of $₫$ Be flim deposited at $15^{\circ} \mathrm{C}$, showing an improved surface finish with a impurity doping of 1.5 at. 9.

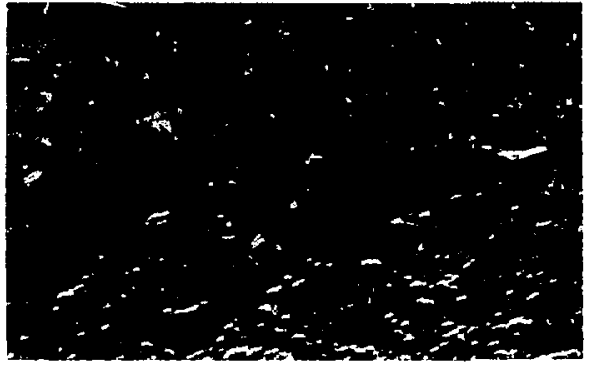

$$
\rightarrow 1 \quad 1 \mu \mathrm{rr}
$$

Fig. 4-22. SEM photomicrograph of the surface of a BE film deposited on a hot glass substrate with $\{110\}$ orientation and 2 at. $\%$ total impority.

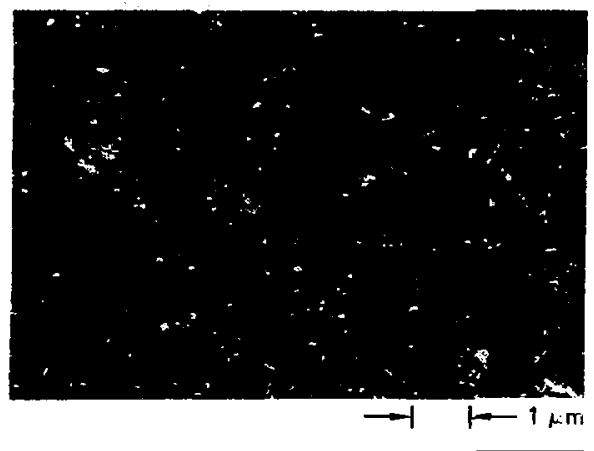

Fig. 421. SEM photomicrograph of a high-purity Be Fitm deposited on a hot glass whotrate stowing faceted $\{002\}$ crystallites growing from a subetrate area.

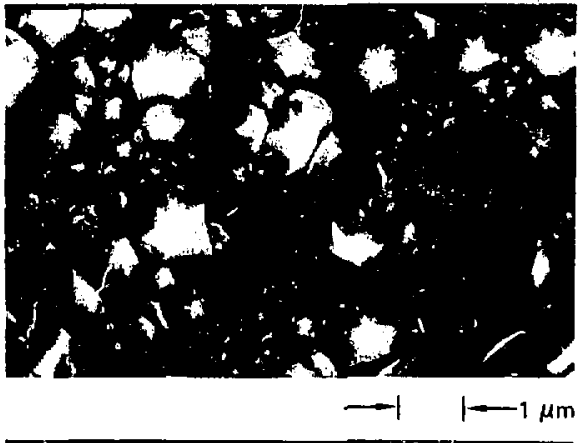

Fig. 423. SEM photomicrograph of an impurlty-stabilized, - morplows Be film deposited on hot glass ubstrates containing 14 at. $\%$ oxygen.

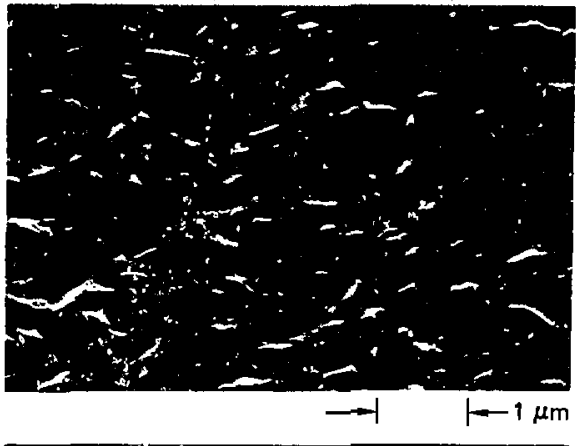


Fig. 424. SEM photomicrograph of the surface finish of a Be-couted glass microsphere after Be sputter deposition in a cooled vibrating cage: (a) 2- $\mu$ m-thick Be conting (b) 7- $\mu \mathrm{m}$ thick Be canting.
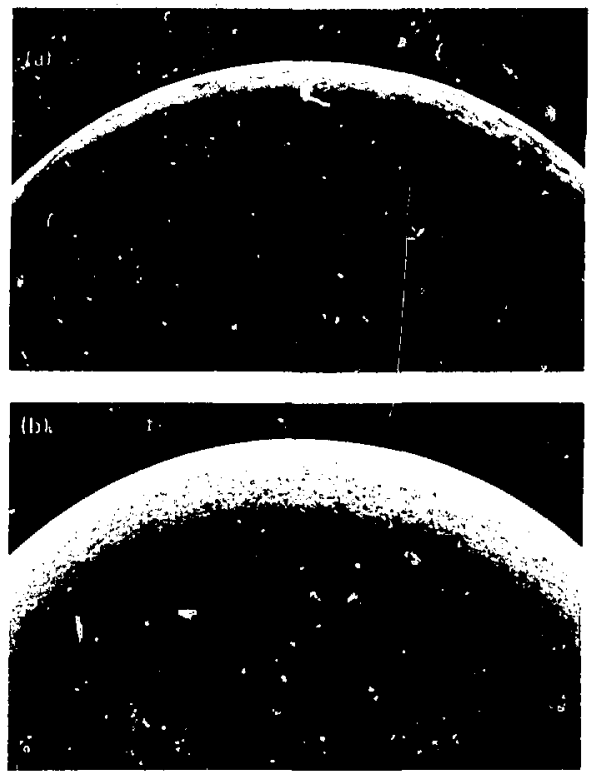

of gaseous impurities is a promising technique to refine the surface finish of thick Be coatings on hollow glass microspheres.

We have sputtered thin Be coatings on glass microspheres using the water-cooled vibrating cage techniques described in detail in the next article, "Spu:tered High-Atomic-N!umber Coatings." These Be coatings required no gas-impurity doping to produce smooth, cone-free surfaces. Two rfsputtered Be coatings on $140-\mu \mathrm{m}$ glass microspheres with thicknesses of $2 \mu \mathrm{m}$ and $7 \mu \mathrm{m}$ are shown in Figs. 4-24(a) and 4-24(b), respectively.

\section{Authors: R. J. Burt and S. F. Meyer}

Viajor Contributors: F. J. Wittmayer and K. L. Montgomery

\section{References}

14. Loser Program Annual Report-1978. Law rence livermore Laboratory. Livermore. Calif.. UCRL-50021-78 (1979). p. $4-17$.

15. R. J. Burt. S. F. Meyer and E. J. Hsieh. "RF Magnetron Sputtering of Thick Film Amorphous Beryllium." J. Vac Sii. Tech. 17, 407 (1980).

\section{Sputtered High-Atomic-Number Coatings}

High- $Z$, high-density coatings on glass microspheres are needed as the tamper layer in the proposed double-shell target shown in Fig. 4-43 (see "Double-Shell Targets," (his section). The tamper specifications are 30-nm surface smoothness, 100$\mathrm{nm}$ thickness uniformity, and thicknesses in the 5to $10-\mu \mathrm{m}$ range. The spherical nature of these substrates-coupled with the small dimensions $(\sim 100 \mu \mathrm{m} 0 . \mathrm{d}$.)-makes it difficult to achieve the required uniformity itnd surface finish by using con. ventional physical vapor deposition processes.

Previous attempts alt sputtering onto meving microspheres with a batch process at LLL, at Los Alamos. ${ }^{16}$ and at KMS Fusion. Inc.. ${ }^{17}$ have suffered from severe sticking problems, thereby preventing the continuous motion needed for reating uniformity. Only the Molecular Beam Levitator moving a single ball (described in "Molicular Beam Levitation During (oating," (his section) has been successful in a sputtering environment. ${ }^{16}$ Our introduction of the vibrating screened cage reported previously ${ }^{18}$ greatly reduced the sticking problems and allowed the first practical batch application of sputtering on microspheres.

We have successfully batch-coated microspheres with up to $6 \mu \mathrm{m}$ of $\mathrm{Pt}$, with a surface roughness of $100 \mathrm{~nm}$, thickness nonconcentricity of less than $300 \mathrm{~nm}$. and density greater than $98 \%$ of bulk $\mathrm{Pt}$. In the remainder of this article we outline the screened-cage batch process technique, the difficulties in microsphere coating, and our vari is solutions to these difficulties.

Screened (age and Fixturing. We conduct our microspbere couting experiments in a stainless steel. diffusion-pumped vacuum system equipped with a gas-flow controller and a Sloan S-310 Sputtergun with a custons matching network. Dataloggers and chart recorders monitor the deposition parameters during runs. The sputtergun and screened cage are illustrated schematically in Fig. 4-25.

The screened cage for bouncing and confining the microspheres consists of a piezoelectric crystal compressed between a massive steel base and a lowmass copper block. An axial bolt piov ; the compression and acts as the spring in the mechanical spring-and-mass system. Three copper rings bolted 
Fig. 4-25. Schematic illustration of sputtergun and screened cage showing their relative orientation and wajor components.
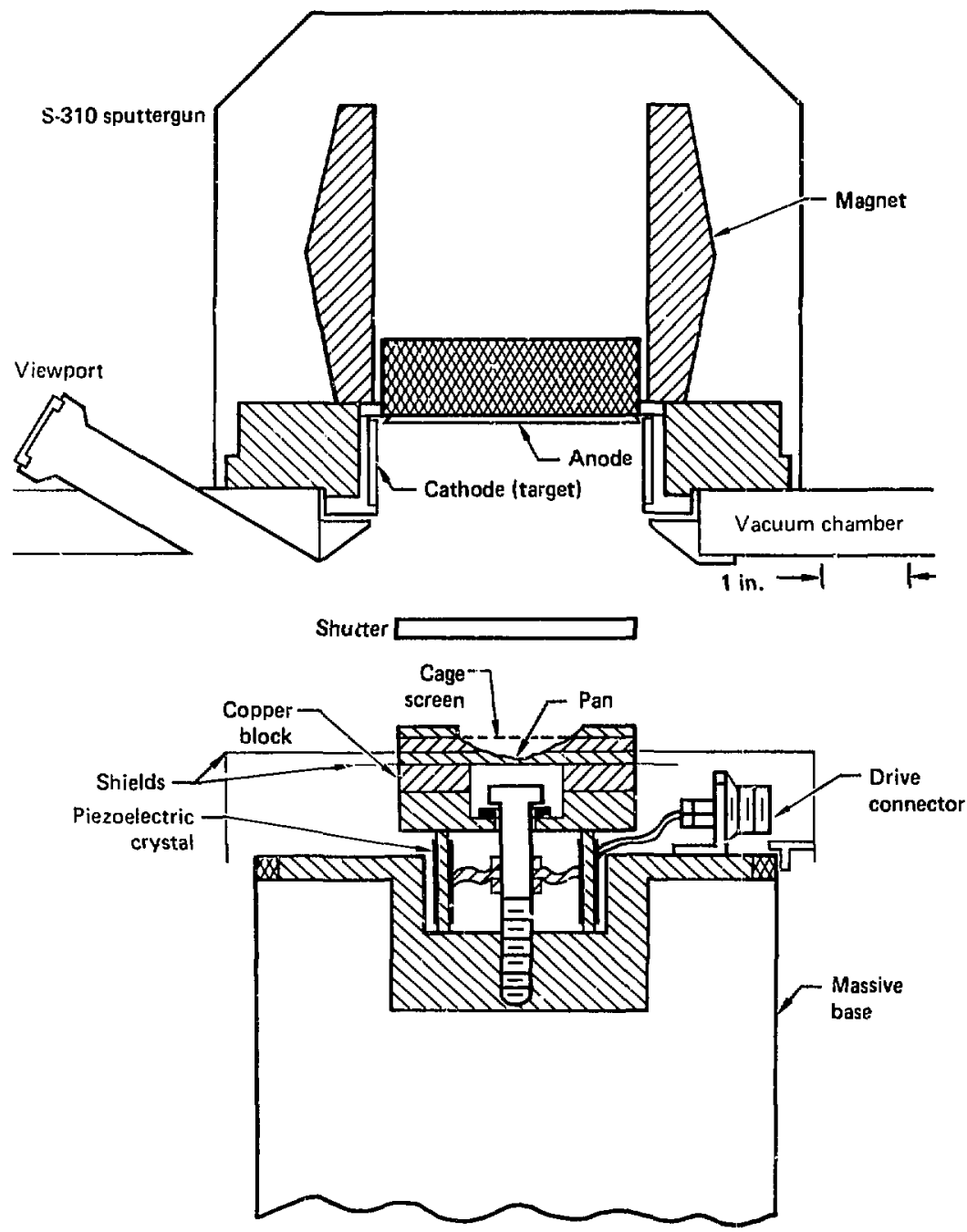

to the copper block clamp the screen and thin copper pan to form the cage. The pan acts like a mechanical amplifier to bounce the balls, since the pan amplitude is much larger than the displacement of the block and crystal. The block is electrically isolated from the crystal electrodes and the grounded base to allow independent biasing of the cage. Of course, the microspheres are not in continuous contact with the cage, so the biasing effect is rather indeterminate. Cooling for the cage is provided by 
channels in the copper block for circulating water or liquid nitrogen, and by conduction through the screen and copper jan.

Our initial me thod for driving the crystal used a frequency-modulated oscillator running at the crystal resonance and swept over a range of pain resonances. Unfortunately, as the cage temperature and mass changed during coating, the drive system required continual tuning to maintain optimum motion, and encountered problems with repeatability. To improve the reproducibility, we switched to a "white noise" drive. Since all frequency components are present, the cage tuning has little effect on the microsphere motion, and the drive can be calibrated with a simple rms voltage measurement.

To confine $70-\mu \mathrm{m}$ microspheres, we need to use a 300-mesh screen with an initial transmission of only $65 \%$, which is steadily reduced during coating. This small screen mesh restricts both the visibility and coating rate of the microspheres. The microsphere visibility is further restricted by the shallow angle of the side viewport shown in Fig. 4-25, and by the omnidirectional plasma glow. Improved iighting and better optics are highly desirable for reproducible results.

Microsphere Sticking Problem. Two clean metal surfaces, such as those produced in vacuum deposition, can cold-weld together by deforming slightly and bonding under impact. ${ }^{19}$ The coldwelding tendency depends directly on hardness and is worst for the soft metuls. such as $\mathrm{Cu}, \mathrm{Au}$, and $\mathrm{Al}$. The severity of the vactum welding problem with gold is graphically visible in the SEM micrograph in Fig. 4-26. The thin gold coatings on the two microspheres have sluck together to form a patch which has almost separated from the two spheres. A large number of roughly circular patches where the coating was completely torn away are visible here and on other spheres from the coating run. Suct welding either produces unacceptable defects or prevents a uniform coating.

The hard metals, such as Ta, Mo, and W, do not cold-weld easily: however, they present a nearly impossible stress-failure problem. Coatings of these metals flake off the system walls and rip the cage screen as a result of stress buildup, making a thick defect-free coating very difficult to deposit. By contrast, $\mathrm{Pt}$ is a nearly ideal metal because of its ductility, high $Z$ and high density, and relative resistance to vacuum welding.
Fig. 426. SEM photomicrograph of two gold-coated wherospheres showing cold-welding damage spots and torn flap of cold-wehded costing.

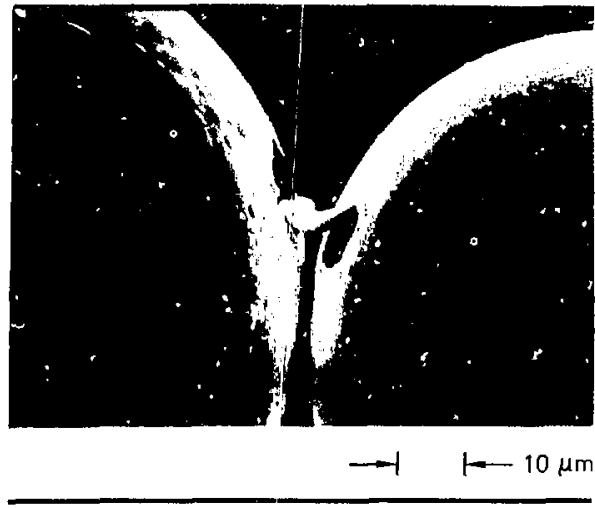

In addition to the rigid clumping of vacuum welding, a second type of sticking-believed to be electrostatic in origin-produces clumping of the microspheres to each other, to the pan. and to the screen. This sticking action is intermittent and usually reversible when the coating conditions are changed. This sticking usually takes the form of microspheres moving up to the underside of the screen and either adhering motionless to it. or moving intermittently around or it. Sometimes the microspheres c'ump together and stick to the pan without motio?. Increasing the bouncer drive or reducing the sputtering power releases the microspheres. which then return to normal bouscing in the pan. If the operator does not intervene during sticking. the microspheres will either be coated nonuniformly. or will become attached to the screen. pan. or other microspheres. Driving the cage too hard. on the other hand, can cause cracking and breakage in the Pt coating. Keeping the microspheres in constant motion without brcakage is a major problem.

We do not yet fully understand the exact mechanism of this sticking process. However, it seems to be caused by microsphere charging from the difference in electron and ion mobility in the sputtering plasma. The evidence for an electrostatic mechanism includes the following: 
- The screened cage reduces the sticking relative to an open pan.

- A bias voltage on the cage reduces the sticking.

- Rf sputtering has less sticking than de sputtering at similar power levels.

- Addition of an electronegative gas such as oxygen to the plasma atmosphere minimizes sticking.

- The degree of sticking is propertional to the plasma power density.

- Closing the shutter relieves the sticking (and stops the coating).

Adding oxygen as a dopant gas in our sputtering process is one of the most etfective methods for preventing sticking. We still do not fully understand why oxygen helps, hut its effect is clearly established. Once oxygen is introduced into the plasma during coating, the microspheres im- mediately become unstuck, and bounce freely. As the oxygen flow is gradually reduced, at a criticai level the microspheres clump back together again. The clumping and unclumping actions are reversibie and controlled strictly by the oxygen.

Platinum Deposition Process. As with beryllium coating reported in the previous article, we found that coating parameters such as operating pressure. cage bias, temperature, and impurity-gas doping have a substantial influence on the $P t$ coating that is in good agreement with Thornton's Structure Zone Mode ${ }^{20}$ shown in Fig. 4-27. The transition region, Zone $T$, produced by low deposition pressure and low substrate temperature, is the desired region for the very smooth. :niform coatings needed on laser fusion targets.

Our best coatings were achieved with a watercooled cage using 300-mesh screen. The deposition parameters included total pressure in the vicinity of

Fig. 4-27. Coating-growth structure zone model of Thornton ${ }^{20}$ showing how sputtered-coating structures change with temperature and inert gas pressure.

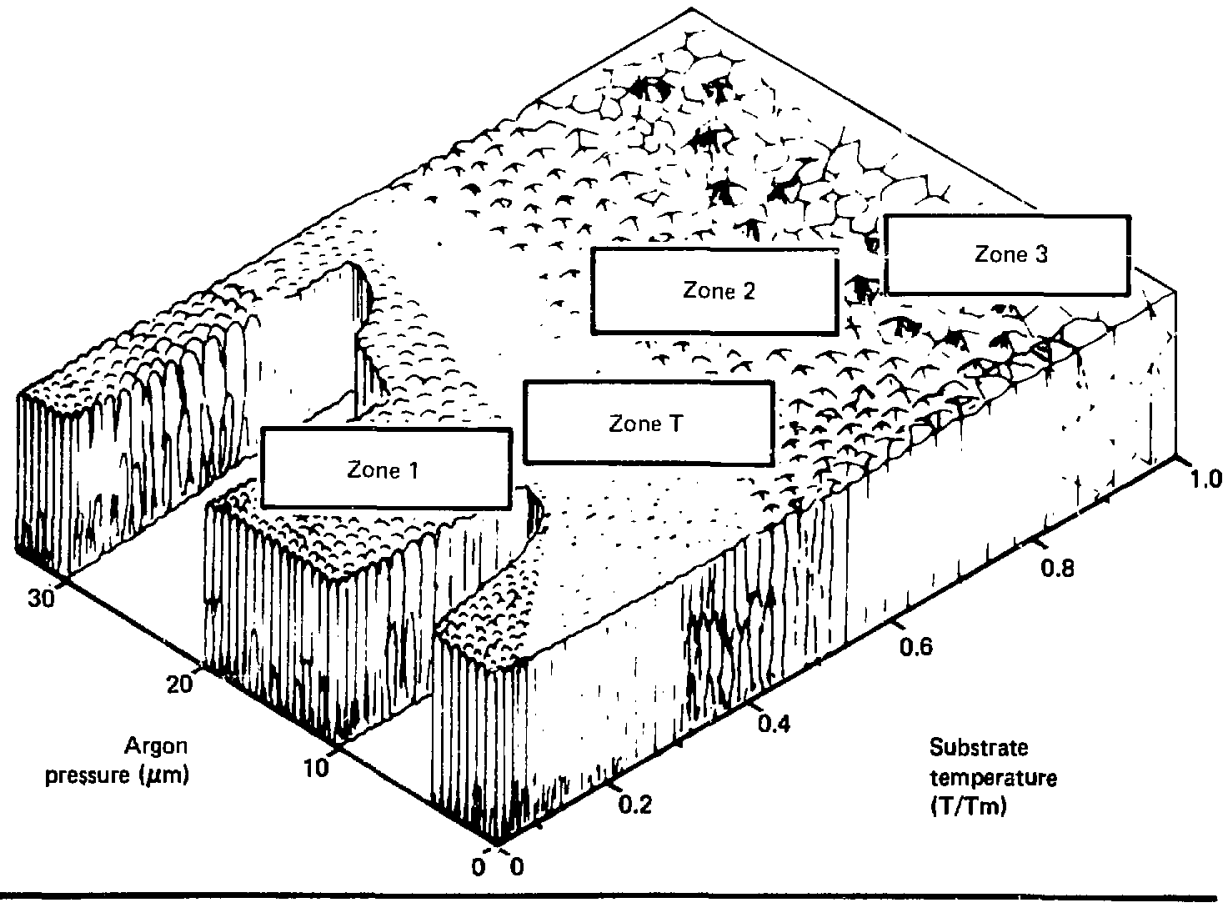


Fig. 4-28. Snooth platiram contine (100-nu surface flaish, bolk platimin denoity) grown on microsphere ginder best spattering conditiows. Cowe defects vidtle represent $300-\mathrm{mos}$ defects.

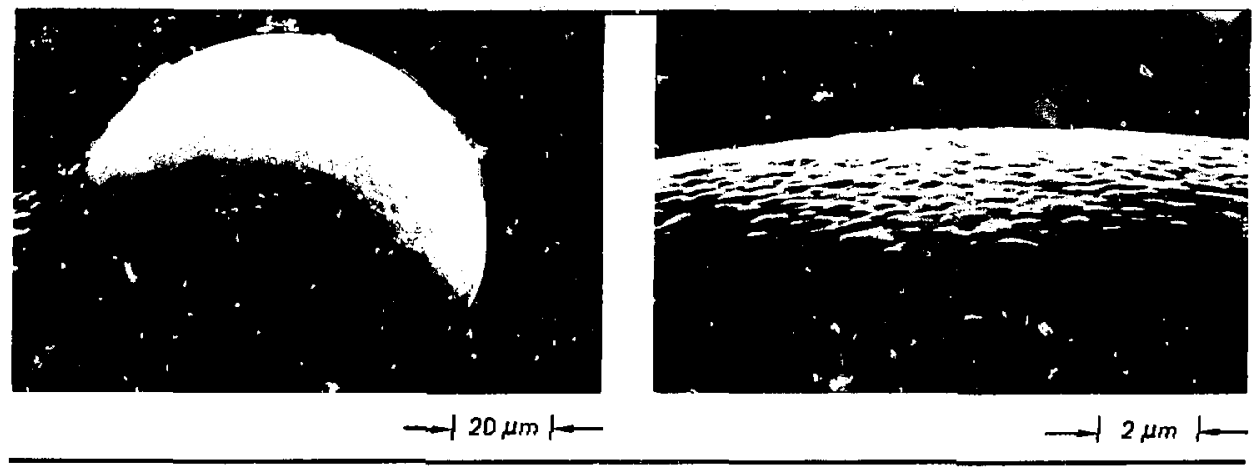

Fig. 4-29. Low-denolty, porows, Zone 1 siructure of Class I platinum coating on microsphere. Sptttering pressure above 1 Pa (7.5 mTorr) promotes this growth.

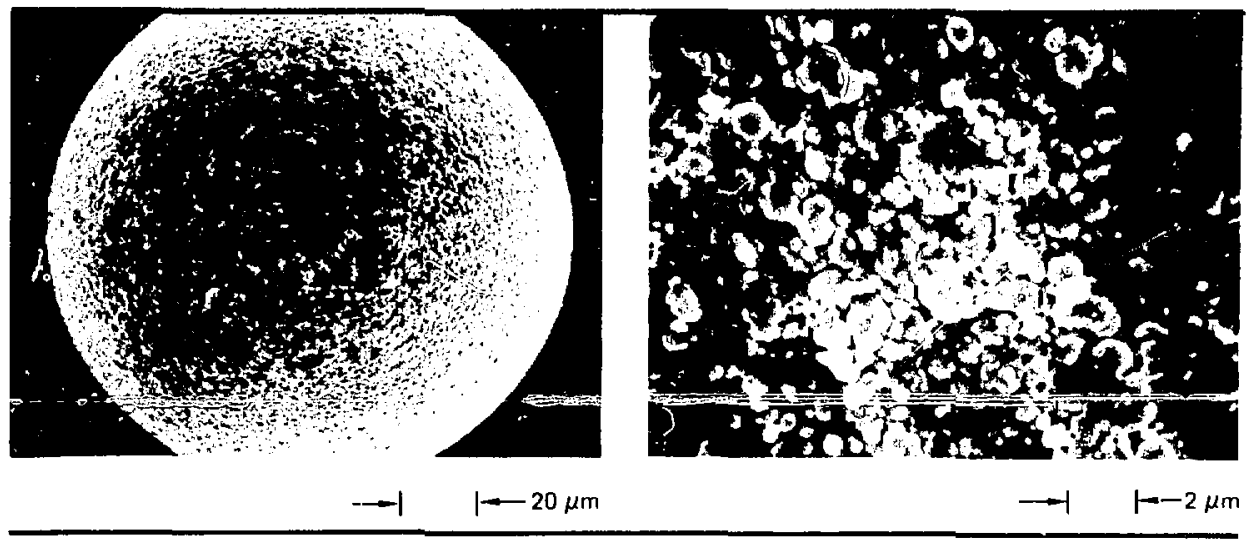

$0.8 \mathrm{~Pa}$ (6 mTorr), with oxygen flow of $0.5 \mathrm{secm}$. argon gas flow of $30 \mathrm{scem}$, bias of $-100 \mathrm{~V}$, and if sputtergun power of $300 \mathrm{~W}$. The white-noise-driven bouncing action was set for minimum motion without high-speed impact with the cage walls and screen. A typical Pt-coated microsphere that was coated with these parameters (shown in Fig. 4-28) is $\sim 98 \%$ bulk Pt density and has a $\sim 100$-n m surface finish. Deposition pressures of $0.8 \mathrm{~Pa}$ and lower typically produce dense, smooth coatings which are typical of Zone-T growth. However, the deposition rate of $1 \mu \mathrm{m} / \mathrm{h}$ appears to decrease monotonically with decreasing pressure. so operating at the lowest possible pressure is not necessarily desirable. Different deposition parameters produce coatings with characteristics that fall predominately into three other classes. Below, we discuss in $q$ reral terms how each coating parameter influences the coating structure.

The quality of the Pt coating decreases with increasing deposition pressure, in agreement with the Structure Zone Model. Pressures above about $1 \mathrm{~Pa}$ (7.5 mTorr) produce the Class 1 coating shown in Fig. 4-29, which is indicative of Zone I growth. 
These Zunc I coatings appedi grainy, porous, or loosely packed, and they are of low density. The inert gats pressure during sputtering and the degree of oblique incidence appear to be the dominant processes responsible for Zone I structure formaiion

The oxygen dopant also affects the Pt-coating characteristics. The addition of about $2 \%$ oxygen to the argon sputtering gas (which is the amount promlded by the 0.5 -sccm oxygen flow rate that produced the coating of lig. 4-28), relines the grain wructurs in the growing Pt coating. and hardens the metial against cold welding. With a large amount - It, ) of oxygen, the Pt coating becomes hard and bricle. With evidence of platinum oxide formation. lhe resulting class II structure shown in Fig. 4-30

Fig. 4-30. Class If platinum coating broken by impact with cage. Conting was hardened with excessive amounts of oxygen doping duringe sputtering.

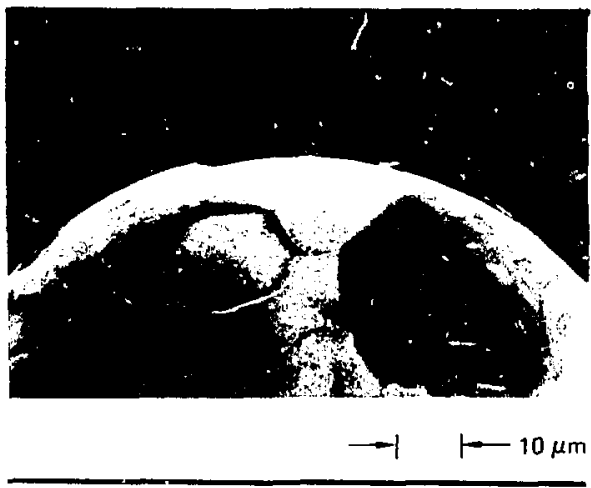

is brittie, extremely fine-grained, and characterized by cracks and broken layers from impact damage. On the other hand, when we use insufficient oxygen $(\ll 1 \%)$, the Pt coating is soft and forms the Class III structure shown in Fig. 4-31. Accretion of loose particles within the cage by cold welding probably accounts for the patchy surface. The impact of the bouncing action "hammered" the soft Pt particles into a semismooth surface. Tre desired oxygen dopant level falls between these two extremes and depends, of course, on both sputtering rate and system throughput.

The bouncing action is the third most important parameter (after total pressure and oxygen dopant) in controlling Pt-coating characteristics. The gross nontuniformity seen in some depositions is obviously the result of inadequate bouncing action. On the other hand, too vigorous a bouncing action causes cracking and breakage of the Pt coating. An effect of vigorous bouncing action that is not so obvious is that the impact of the microspheres onto the cage parts-the $\mathrm{Ni}$ screen in particular-can trigger the release of micrometre-sized particles. On impact, the small particles adhere to the $\mathrm{Pt}$ coating. producing "cone defect" nuclei. We achieved our cleanest coatings by reducing the bouncing action 10 to a level where the microspheres barely moved around, with minimum collisions with the screen. We need an improved optical observation system, however, to monitor this bouncing action adequately.

Traditionally, substrate bias voltage is used to increase the argon ion bombardment of the substrate to yield a cleaner and denser coating. With

Fig. 4-31. Piatinum costed microsphere showing "soft" Class III conting grown with insuficient oxygen dopigt.

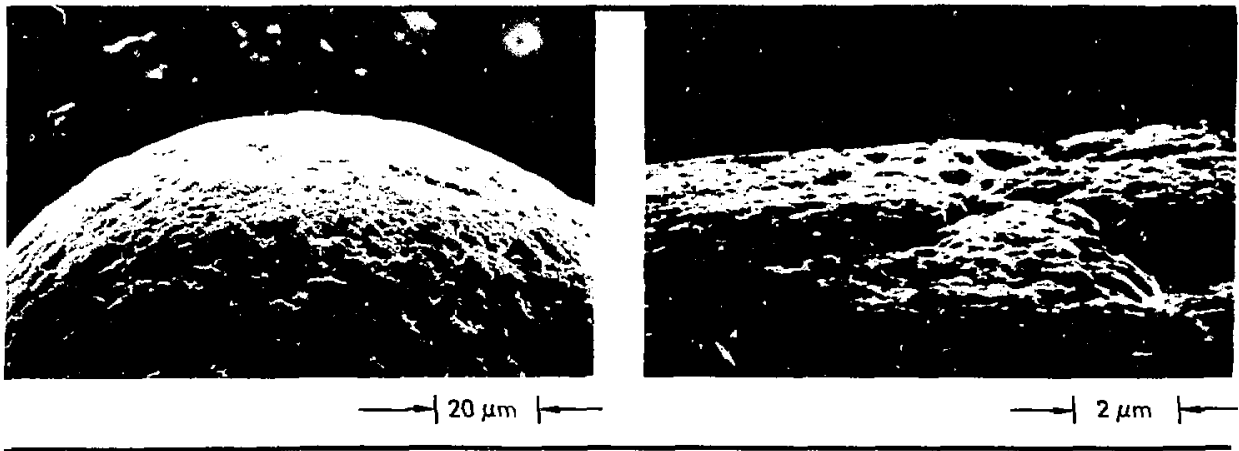


our cage configuration, it is not clear how well the ion flux penetrates the screen. In any case. the microspteres are not at bias potential most of the time because of the bouncing action. We do nol observe in the coating a pronounced diffurence between biased and unbiased runs. However, as noted above. the bias definitely helps prevent sticking.

We have been unable to direclly monitor the actual microsphere temperature during coating. We have inferred from measurements on isolated thermocouples that with a water-cooled cage the probable microsphere temperature is on the order of $150^{\circ} \mathrm{C}$, considerably lower than the onset tem. perature for diffusion-dominated grain growth in platinum (Zones 2 and 3 in Fig. 4-27). This coating temperature appears acceptable for all but very thin-walled microspheres, where some evidence indicates that the D-T fill can leak out during the coating process. Preliminary experiments with liquid-nitrogen cage cooling and with magnetic deflection of the plasma electrons have shown sume reduction in thermocouple temperature. We will investigate the magnetic deflection coating method further before reaching a conclusion. Efforts are continuing to fine-tune the set of coating parameters to achieve a smoother surface finish and to achieve a higher degree of repeatability.

Authors: S. F. Meyer and E. J. Hsieh

Major Contributors: R. J. Burt, G. T. Jameson. and A. L. Plake

\section{References}

16. 1. T. Towe and C, D. Hosford. "Magnetron Sputter couting of Microspherical Substrates." I. Vac Sci Tech 16. 197.1979.

17 W. J. Felmlee. KMS fusion. Inc private communication (19801).

18. Laser Program thinal Report-1978. L.aw rence Livermore laboratory. Livermore. Calif., LiCRI-50021-78 (19791. p. $4-14$.

19. D. I. Bourgette, D. V. Keller, and R. L. Stephenson, in Jacumm lfeiallurge. $O$. Winkler and $R$. Bakish eds (1) Isevier. Nen York, 1971), Pp, 825-8.38.

20. J. Thornton, "High Rate Thick Filn Growth." $1 \mathrm{~mm}$ Rev Maler. Sa 7, 239.260)(1977).

\section{Electroplating}

High-alomic-number elements required in advanced target designs can be deposited by electroplating under conditions that preserve fragile laser fusion targets. We have therefore begun a project to produce coatings by the use of electroplating, a method that offers unique advantages. It is a well established coating method that can be applied to deposit a wide variety of metals. Figure 4-32 shows the elements that can be electroplated from aqueous solutions. Other elements may be deposited from nonaqueous organic solvents at low temperatures or from fused salts at high temperatures. The solutionplating techniques (electroless. electroplating. and immersion plating) are also complementary to sputtering. It has been found very difficult to sputter smooth coatings of very soft metals such as gold or

Fig. 4-32. The highlighted eleunents can all be electrodeposited from aqueous solution. (Almost all elements can be electrodeposited with the proper choice of solvent.)

\begin{tabular}{|c|c|c|c|c|c|c|c|c|c|c|c|c|c|c|c|c|c|}
\hline $\mathrm{H}$ & & & & & & & & & & & & & & & & & $\mathrm{He}$ \\
\hline $\mathrm{Li}$ & $\mathrm{Be}$ & & & & & & & & & & & B & C & $N$ & 0 & $F$ & $\mathrm{Ne}$ \\
\hline $\mathrm{Na}$ & $\mathrm{Mg}$ & & & & & & & & & & & Al & $\mathrm{Si}$ & $P$ & $S$ & $\mathrm{Cl}$ & Ar \\
\hline$k$ & $\mathrm{Ca}$ & $\mathrm{Sc}$ & $\mathrm{Ti}$ & $v$ & $\mathrm{Cr}$ & $M n$ & $\mathrm{Fe}$ & Co & $\mathrm{Ni}$ & $\mathrm{Cu}$ & $\mathrm{Zn}$ & $\mathrm{Ga}$ & $\mathrm{Ge}$ & As & $\mathrm{Se}$ & $\mathrm{Br}$ & $\mathrm{Kr}$ \\
\hline $\mathrm{Rb}$ & $\mathrm{Sr}$ & $Y$ & $\mathrm{Zr}$ & $\mathrm{Nb}$ & Mo & Tc & Ru & $\mathbf{R h}$ & Pd & $\mathrm{Ag}$ & $\mathrm{Cd}$ & In & Sn & $\mathrm{Sb}$ & $\mathrm{Te}$ & 1 & $x e$ \\
\hline Cs & $\mathrm{Ba}$ & La & $\mathrm{Hf}$ & $\mathrm{Ta}$ & $w$ & $\mathbf{R e}$ & Os & Ir & $\mathrm{Pt}$ & Au & $\mathrm{Hg}$ & TI & $\mathrm{Pb}$ & $\mathrm{Bi}$ & $\mathrm{Pa}$ & At & $R_{n}$ \\
\hline $\mathrm{Fr}$ & $\mathrm{Ra}$ & $A c$ & & & & & & & & & & & & & & & \\
\hline
\end{tabular}


copper. ${ }^{21}$ Solution plating is well suited to deposition of these metals in a low-temperature. low-stress environment.

Two major problems arise in the plating of glass microsphere fusion targets: First the ultrasmooth nonconductive glass surface must be treated to achieve a very smooth. adherent and conductive surface (called a strike) for plating. Then. the conductive microspheres must be sustained in random motion while electrical contact with the cathode of the plating cell is retained. (The motion is essential to achieve a uniform coatiag trickness.)

Fig. 4-33. The Iypical silver strike surface deposited from an ammoniacal AgNO 3 solution.

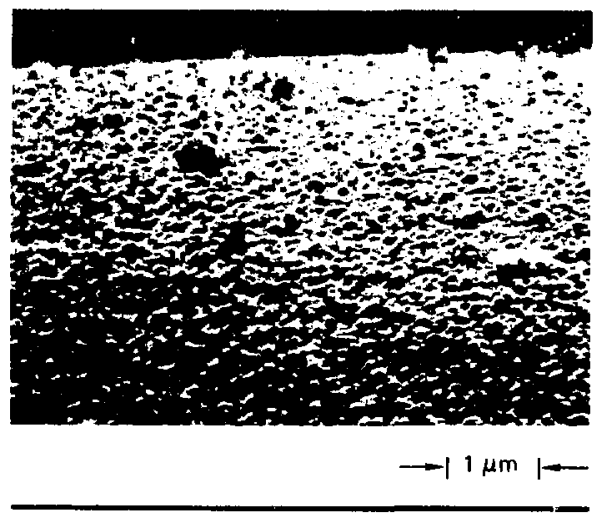

Most industrial processes for plating glass involve a chemical or mechanical etching of the glass to obtain a rough. high-surface-area substrate for plating. ${ }^{22.23}$ This is, however. unacceptable for use with fusion targets. Sputtering can produce smooth. relatively hard. thin metallic coatings on glass that accept overcoats of electrodeposited metal, as Fig. 4-33 shows. These sputtered coatings are often strained. however, and this can lead to plating difficulties. We have therefore developed an alternate method for the deposition of a strike layer.

Modifications to ancient mirror silvering techniques (Brashear) allowed us to produce a 0.14 $\mu \mathrm{m}$-thick silver strike with the surface shown in Fig. 4-34. The silver coating is adhesive and cohesive, and it readily accepts overplates of gold or copper. The nodular silver growths on the surface have formed at the solution-containe- interface. We can remove most nodules by washing and ultrasonic treatments. Other strikes we investigated--such as colloidal $\mathrm{PdCl}_{2}$ or precipitated metal sulfides-were completely unsatisfactory. Tin deposited by the thermal decomposition of $\mathrm{SnCl}_{4}$ coated unevenly. We expect further charges in the silver process to result in far better surface finishes. We may also find it advantugeous to follow the example of target builders at Los Alamos and make a nickel strike by the decomposition of $\mathrm{Ni}(\mathrm{CO})_{4}$ at the surface of glass micrespheres. ${ }^{24}$

The second major impediment to uniform electroplated surfaces is the plating tank or cell in which

Fig. 4-34. The surface finish of $\mathrm{a}$ thin $(\sim 1000 A)$ sputtered Pt strike. The utility of this smooth $P($ conting is anrred by teasile stress in the conting.

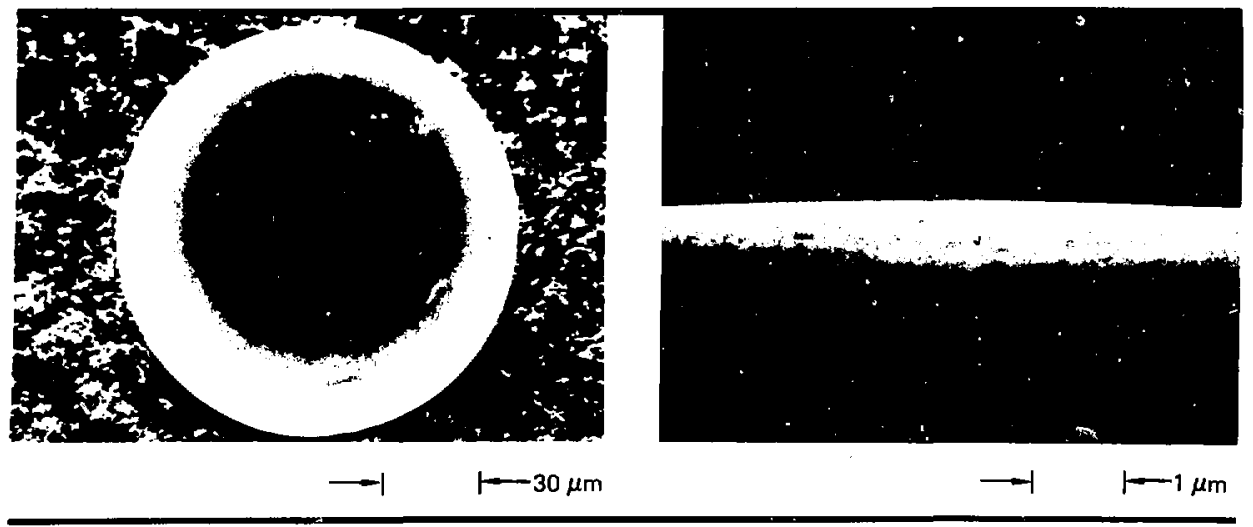


the plating occurs. To achieve the random contact of the cathode necessary for electroplating. we found the cells shown in Figs. 4-34 and 4-35 to be acceptable. The cells we have designed and developed also are capable of dispersing gas bubbles on the surface of the target (a problem that disrupts the plating of very small objects). ${ }^{23}$

The cell in Figure 4-35 has a cathode which is vibrated by an electromechanical transducer to impart motion to the miciospheres. Solution is also pumped through the screen to further aid microsphere motion and to replace depleted solution. An even more gentle motion can be achieved with the cell shown in Fig. 4-36. Mass flow, viscous forces. buoya $\mathrm{nt}$ and gravitational forces all can be balanced to prodace a rolling motion along the lapering walls of the cell. The result is uniform plating with very

Fig. 4-35. Vibrating cathode electrophating cell.

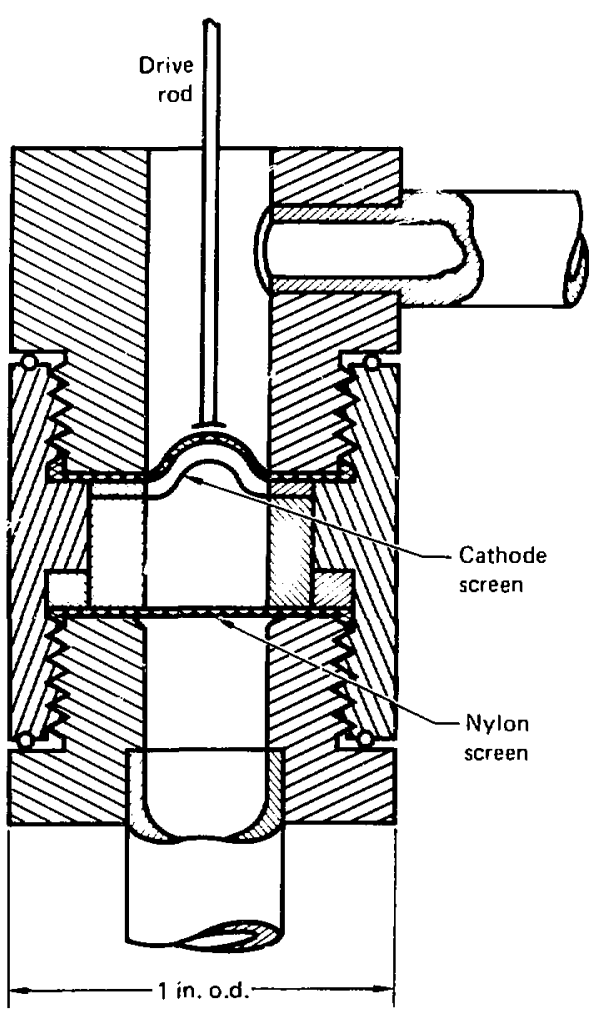

small loss of microspheres due to breakage or because they become plated to the cathode.

Although electroless and immersion plating would have allowed us to use simple plating cells. each of these processes was unsatisfactory. Immersion plating (also called displacement deposition) is the deposition of a metallic coating by chemical replacement of suhstrate atoms from a solution of a salt of the coating metal. The thickness of immersion coatings is limited. Once a continuous layer of coating is established. substrate atoms can no longer be replaced by the coating metal. Typically. gold can be immersion-plated only to about $0.025 \mu \mathrm{m}$ thick. ${ }^{23}$

H:lectroless plating uses the electrons provided by oxidation of a chemical reagent present in the solution to deposit metal atoms on a catalytic surfice. For the electroless process to be useful. it must be autocatalytic. Once the initial catalytic surface is completely covered with coating atoms. the newly produced coating must itself calalyze the deposition of more coating atoms. Ideally, the process should continue until all the coating atoms are removed or the supply of reducing agent is exhausted.

We found that the apparent benefits of the electroless system. simple containers, no external reducing potential. near-perlect throwing power and low

Fig. 436, Electroplating now cell.

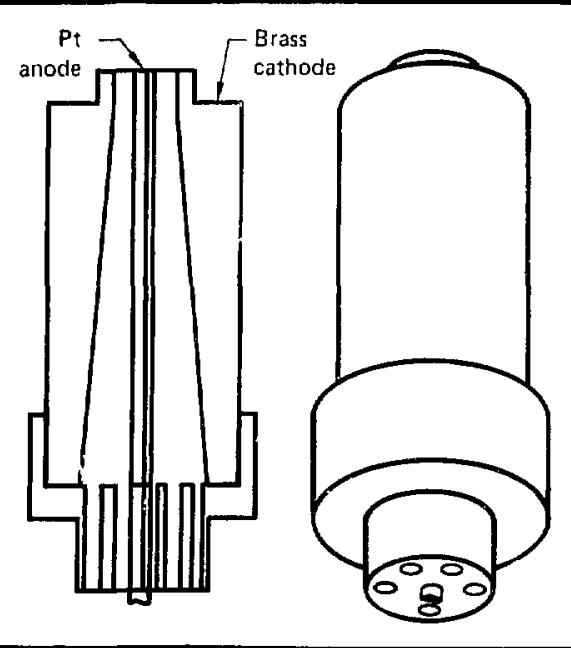


Fig. 4-37. Grain structure in an efectrolese An depodit.

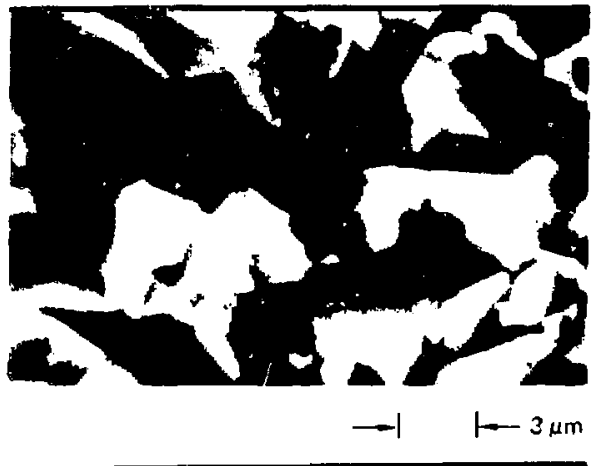

purants ware affset hy the need for the strike to serse at a catalyst and by the poor surface vilouthness caused by the relatively large grain sises $1.345 \mu \mathrm{m}$ shown in Fig. 4-37. In addition, in electroless plating-as in electroplating-the solution and macrosphere motion must always be maintained 1) replenish the couting species present in the active plating volume adjacent to the microsphere.

Although electroplating is well developed as an engincering lechniqus, only the gross feattures of the themistry af the solutions is understood. These detal hecume very important in the plating of tins laser lusion targets, whose uniformity and surface finish must he so extremely well controlled. The use of well-developed commercial plating haths has heen an aid to producing a consistent plated product. We have found that a commercially avaliable gold-plating solution, BDT-5/0, offers small grain sise (40 to $60 A)$. good leveling and high hrightness. Figure $4-38$ shows a cross section of a gold coating plated on a glass microsphere from BDT-510. We have also plated copper from a commercial pyrophosphate bath.

Author: J. D. Illige

Major Contributors: C. M. Yu, C. W. Jordan, G. R. Korbel, D. J. Lenz, and B. Krenick

\section{References}

21. Laser Program Ammal Report-1979, I.awrente Livermore Laboralory, Livermors, Calif., UCRL-50021-78 (1979). pp. 413 to 4-21. (See also "Sputtered High-AtomicNumber Coutings," this section.)
Fig. 438. Cross section of electroplated An $15 \mu \mathrm{m}$ thlek on 2 140-pratian dasa sphere.

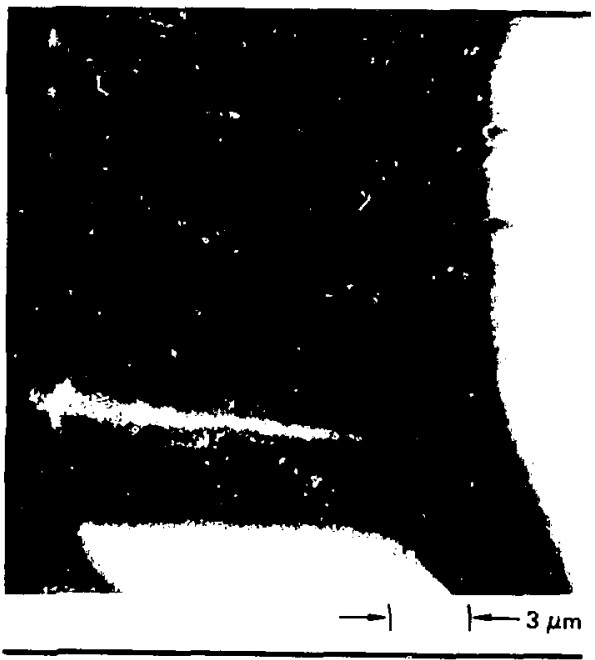

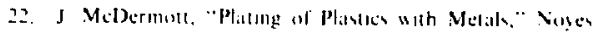
Dillas (ispersalim (1974).

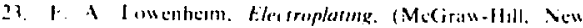
$\because$ irk. I978)

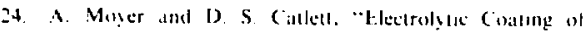

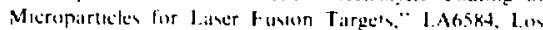

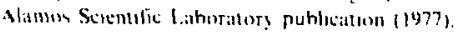

\section{Molecular Beam Levitation During Coating}

One attrattive possibility for producing the ahlative, outer shell of the double-shell targets (Fig. 4-43, next article) is to support them using molecular beam levitation during a coating process. This technique provides a unique means of support for these delicate turgets which allows them to rotate frecly in a contactless environment while being coated. This method of supporting individual microspheres is also advantageous because it minimizes particulate contamination, which provides nucleation sites for surface defects. ${ }^{25}$ of the various levitation schemes previously proposed (e.g., electrostatic, magnetic, optical, acoustic, etc.), molecular beam levitation appears to be the most easily adaptabie to discharge coating techniques. ${ }^{26,27}$

Beams of argon atoms can be formed by flowing argon gas through very small capillary tubes 
$\left(10.5 \mathrm{\mu m}\right.$ diameter) at low pressures. ${ }^{28}$ An individual microsphere can be supported on these berars and coaled in al plasmal.

To understand the physics of this katition device. we have made careful measurements of tho" and pressure of the levitation gas. It is import:, l to identify the gas flow regime in which levitation lakes place (viscous or free molecular), becaluse the associated forces are quile different.

The datil from one such measurement are shown in the graph in Fig. 4-39, "here the rise in chamber pressure, Py (1), was monitored an a function of different. lisert values al kevitator balck pressure, P, lor at 50-am capillary arras. The graph show that for bact pressures less thall I Torr. the dalis fall along the same straight line, correspending io a comstant. pressure-independent value ferr corle ductance. Al at back pressure of 10) Torr. the dallat deriate significantly. Indicating that the condecetance of the levititser has changed. Free meiccular now through colindrical tubes is describec hs

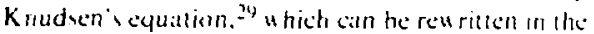
(i)rin:

$$
P_{2}(1)=P_{1}\left[1 \quad \exp \left(\frac{C_{\text {mol }}}{v}\right)\right] .
$$

where $V$ is the volume of the chamber, and $C_{\text {man }}$ is the coinductance. Conductance: in a colindrical tube is given b!

$$
C_{\mathrm{mol}}=\frac{8}{3} \frac{r^{3}}{\pi} \pi \sqrt{\frac{k t}{2 M \pi}} .
$$

and $r$ and $f$ are the whe dimensiens. In the calce of viscous 17un. Poiscuille' equation ${ }^{311}$ describes Hoss through eglindrical tubes, and it can be reuritten in the form:

$$
P_{2}(t)=P_{1} \tanh \frac{\phi P_{1} t}{2 V}
$$

where

$$
0=\frac{\pi r^{4}}{8 \pi 6}
$$

and $\eta$ is the constant of viscosit: for argon. On the basis of these two equations. we infer from the data in Fig. 4-39 that the levitator operates in the free
Fig. 4-39. Fraph shoring the rise in chamber pressure, $P_{2}(t)$, as a function of constant, levitatior back pressure, $P_{1}$.

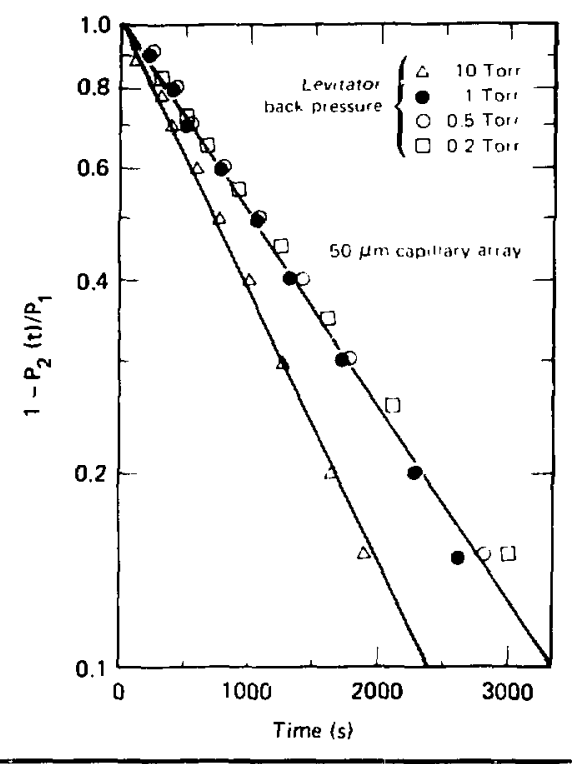

molecular llow regume lar hack prenares less than 1 Forr whth as 50 - $\mu$ m capllier arra! + 140-by $5-\mu m$ elam macomphere lestlate all a halck pressure of

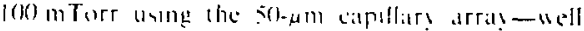
within the free-molecular-17um regume

Severai problem are anductuled wath molecular

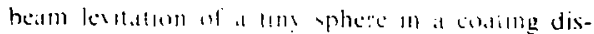

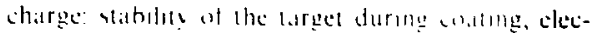

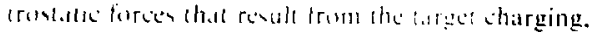

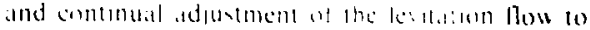
compensile for the acegh stded the sphere b! the coatung proses

He hate whed the nonblem of stabilits bs placing a pectelly beveled. comering ring over the capillary arras. The hesel ach a a circular dellece

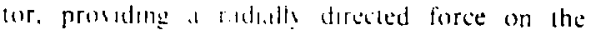
micresphere. Thw loscis a spong-like in nature. creatirig a potemtal mommum at the center of the ring. The levilded morosphere is therefore held very firmly in plice at the center oi the ring in Fig. 4to.

A second protilem, that of electrostatic force, is especially vesere whenever a metallic sphere is levitated ahove a melall $\ldots$ substrate in a plasma. The 
metal sptiere charges to the floating potential of the plisma and is consecpuently atlracted $w$ the sonduems nitane of the levitator by its oun image force. This inage forece altraction can be overcome by producing an opposing field using a d.c.-biatsed sereen placed athowe the levilated sphere.

In the course of placing thick plastic or other lou- $Z$ coatings un spheres, we ma! have to operate

\section{Fig. 4-40. A levituted microsphere is held firmly at the center of the ring by effasive llow from the lip.}

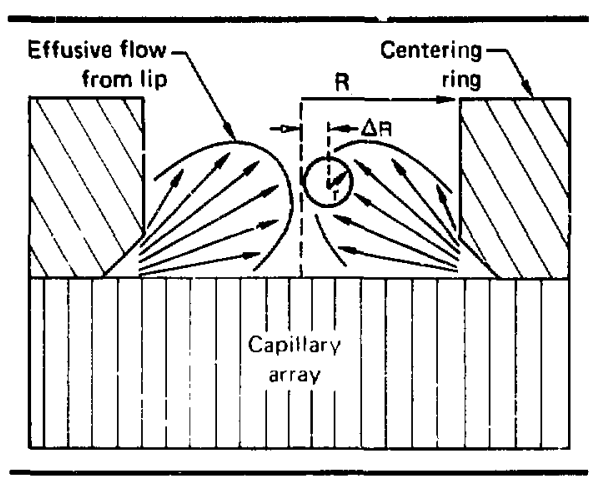

the levitation-coating system for several tens of hours. For this reason, we need a fully autonatic lesilator-coitting system. Schemes for such a system atre currently under developnent. One such scheme consists of imaging the levilated microsphere on a position-sensing detector. Then. its the microsphere louer hecatuse of the increased coating weight, change in height is decected, and a signal is fed back (1) the alve that controls the levitaltor gats flow. This whem is shown in Fig. 4-41.

We cursentls use a manually operated, moleculitr-heam, levitalor-coating system. The function of the system is to evalluate special problems such as coating double-shell largets or multiple-coatling targets. Figure 4-42 shom a microsphere that was coated with eopper by electroplating, then with $(-F$ plastic contling in the levilitter-coating ystem. Lesitation-coating may "ell be the on! alternative for fabricatting future gencration, multiple shell tilrgets, such an discussed in "Double-Shell Target," (the next artiele).

Author: J. K. (rane

Major (untrihurors: R. D), Smith, (i. R. Korbel, and C. W. Jordan

Fig. 4-41. A levilated sphere is imaged on a special detector, producing a signal based on the height of the ball. This signal can then be used to control the flow of levilation gas.

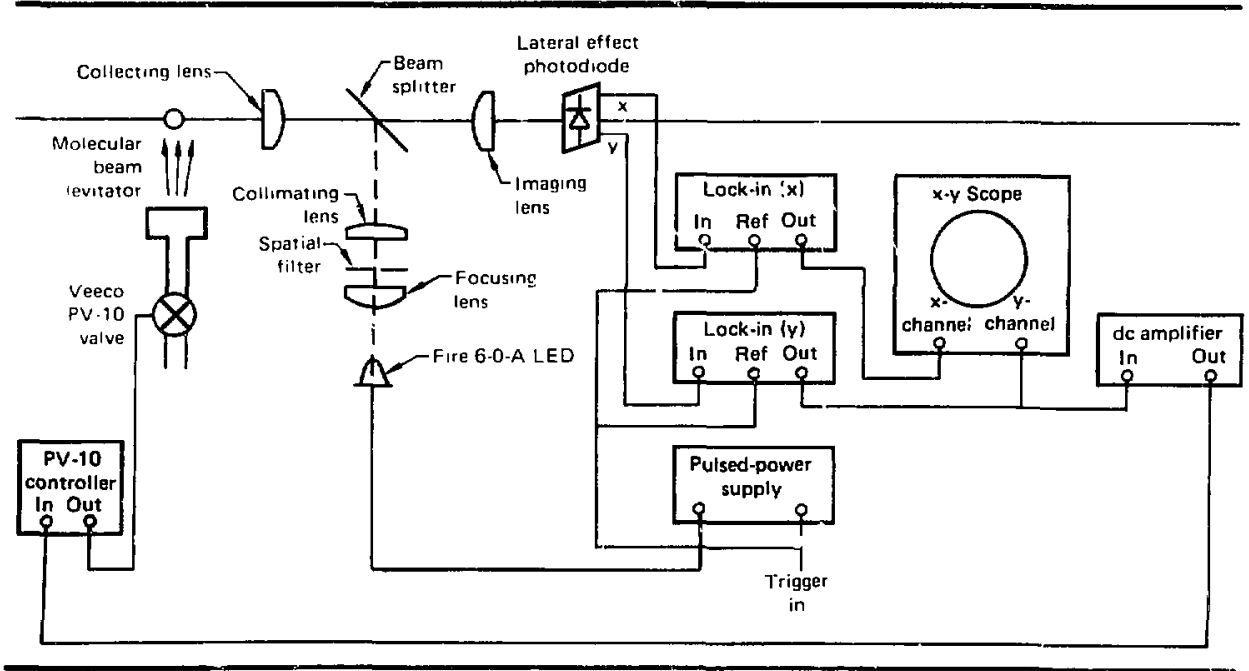




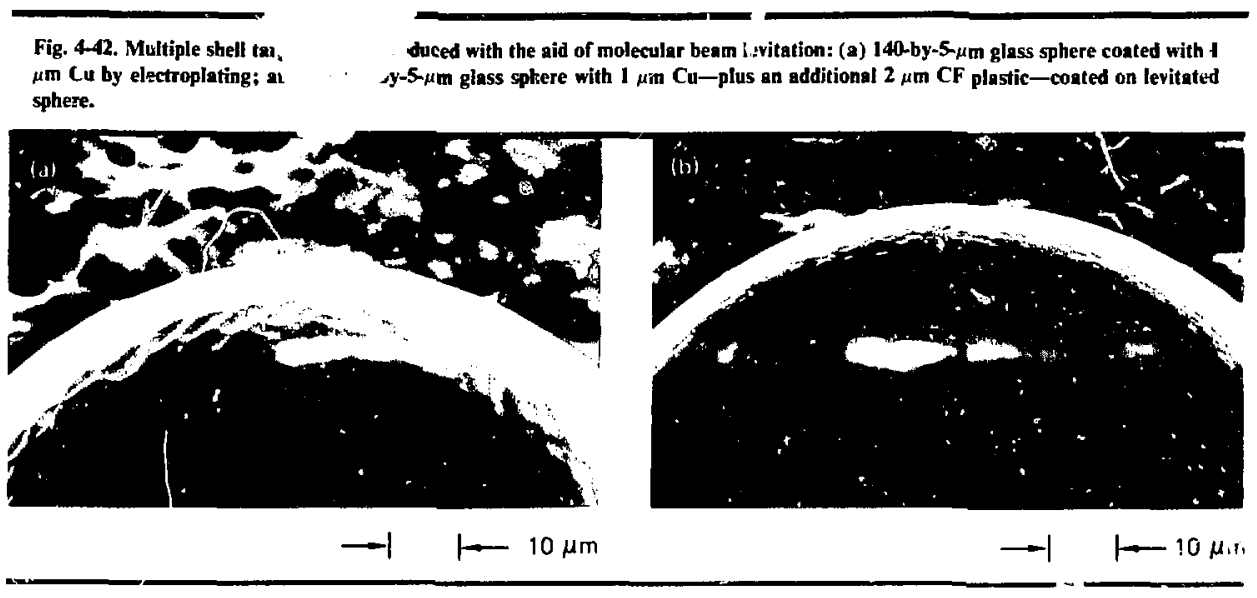

\section{Reference;}

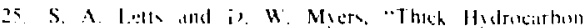

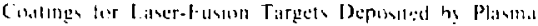

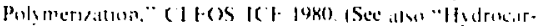
horl Coilthes," in the repurt.l

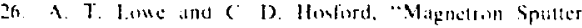

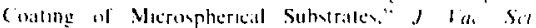
Tohthe 1h. 197 (1979).

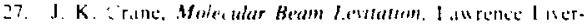

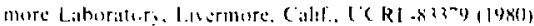

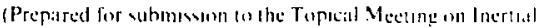

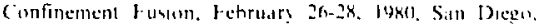
(alil)

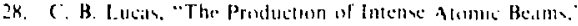
lar Imm 2.3. $195(1973)$.

29. 1. I. Matruel and R. (ilang. Hamdhosh of $\mathrm{Thm}$ thim

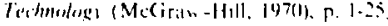

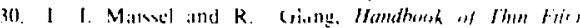

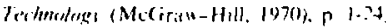

\section{Double-Shell Targets}

One method proposed for obtaining better performance in fusion implosion experiments is the use of a doutile-shell target cesign similar to that shoun schemationlly in Fig. 4-43. Double-shell largets make use of : he principie of "velocity nuitiplication": The outer shell i. imploded like an ordinary ahlatively driven target. accelerating the relatively massive outer shell pusher to a high velocity. When the outer shell pusher strikes the inner tamner, the less massive tamper recoils and implodes with a higher velocity.

In the first versions of these double-shell targets, the outer shell consists of a single layer of $\mathrm{CH}$ polymer. In "Macilined Henishells," we descrite the micromathining and asiembly lechnigues that have heen most successful for producing these lareats. In "Alternate Approalches." we describe alternalive processen that we hope will improse target qualits and reduce fabrication time.

\section{Machined Hemishells}

The simplest procedure for fabricalting doublewell targets is to anemble ( w o hemispherical shells ("hemishells") around the inner target sphere. So far the most aceesful melhod for producine $\mathrm{CH}$ hemishells has heen machinging with an ultraprecision single-point diatnond lathe. Two versions of - his process hase been dereloped, one at LLL and one at Resekwell International

The steps in the I.LL, process ate shown in Fig. 4-44: First a copper mandrel is cull with a hemispherical rip whose rasius is the desired inner redius if the hemishell. The mandrel is removed from the lathe and a hedrocarbon coating is applied. The mandrel is then replaced in the lathe, and the outer suriace of the hemisphere is cut.

In developing this process. we nere required to solve a number of difficult machining and materials problems. To ensure that the hemishell wali thickness will be untorm. the mandrel nust he repositioned in the lathe "ith an accuracy better than $0.1 \mu \mathrm{m}$. We accomplish this by holding the mandrel in a massive chuck assembly that hals been malchined to exalting tolerances. This chuck assembly can be replaced in the lathe rapidly wilh 
Fig. 4-43. General dowtile-sheil target design with a high-Z, high-density tamper conting on the glass microsphere, and a separate low-Z abla'or shell.

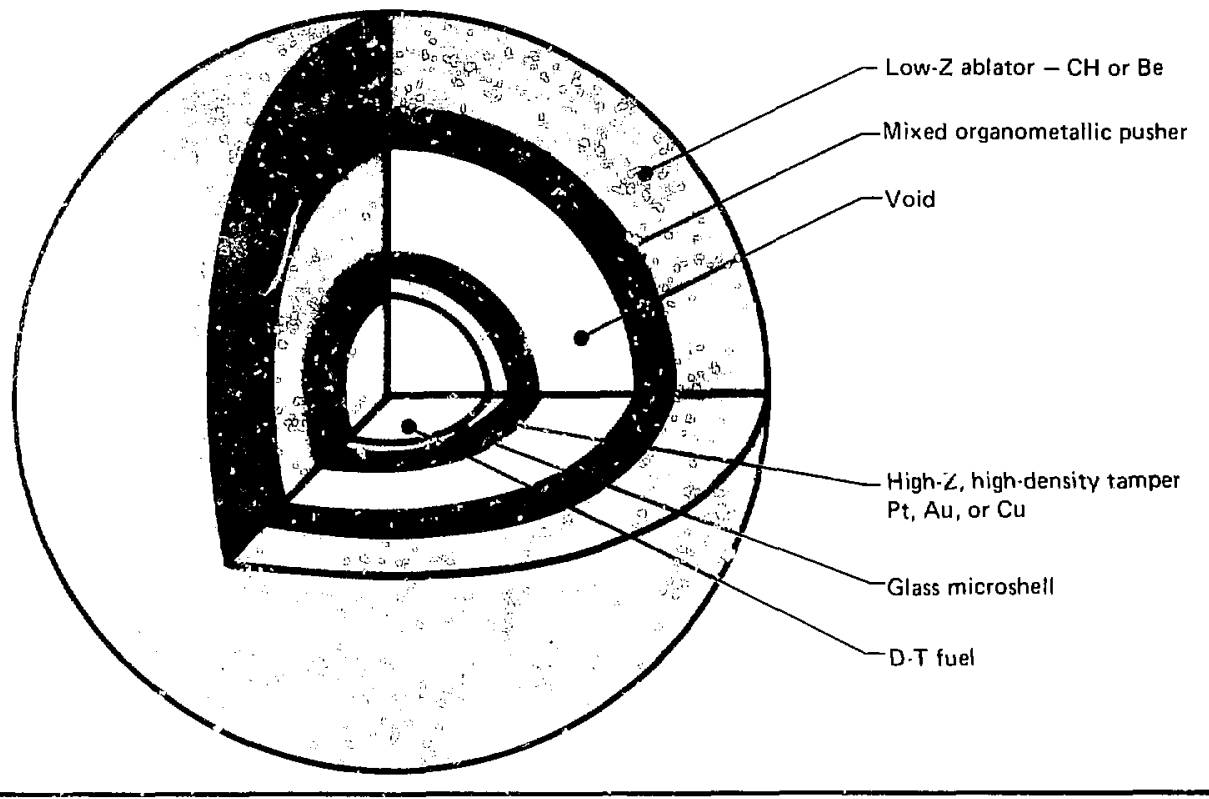

the required accuracy; in addition, it provides a convenient means for holding and transporting the mandrel.

Another problem we encountered was obtaining suilate mandrel material. Ordinury oxygenfree, high-conductivity (OFHC) copper has grain irregularities that cause imperfections in the matchined mandrels. To alieviate this problem, we ciectroplate a layer of high-quality copper onto sach mandrel before machiniig. The machining is done in the tine grained electroplated material, providing a smooth mandrel and, therefore, a smooth inner surface on the hemishells.

Onc of our most difficult problens was cutting a smooth, flat rim with sharp corners. Conventional diamond-cutting lools have a radius of about $25 \mu \mathrm{m}$. These tools tend to push the $\mathrm{CH}$ material in front of them, often forming a "lip" of material on the inner edge of the hemishell. This problem was solved by using an extremely sharp diamond tool with a ridius of ahout $0.02 \mu \mathrm{m}$.
The hydrocarbon material used for the hem sphere must satisfy several requirements:

- It must not contain any voids or other defects.

- Il musi he hard enough to be easily machinable hut elastic enough that it dies not develop cracks.

- The cure cycle must be at less than about $150^{\circ} \mathrm{C}$ to avoid any distortion of the mandrel and chuck assembly.

- The material must adhere to the mandrel sufficiently that the hemisphere does not come off during machining of the outer surface.

Our best results were obtained with a polybutadiene/vinyl toluene monomer mixture cured with 3\% Lupersol 101 peroxide. The polymer cures at $125^{\circ} \mathrm{C}$ for $16 \mathrm{~h}$. An altractive feature of this material is that by varying the amount of curing agent, we can control the hardness to suit machining demands. We found that a thin coating of polyvinyl alcohol (PVA) over the butadiene improves the curing process. The PVA reduces volatilization of monomi.. and peroxide and excludes oxygen, which would inhibit polymerization. 
Fig. 4-4\%. Sequence for producing a CH second shell by micromachining: (a) A copper mandrel is machined with a hemispherical tip. (b) The mandrel is coated with $\mathrm{CH}$ polymer and remachined to form the outer surface of the hemisphere. The copper mandrel is dissolved away, leaving the hemisphere shown in (c).
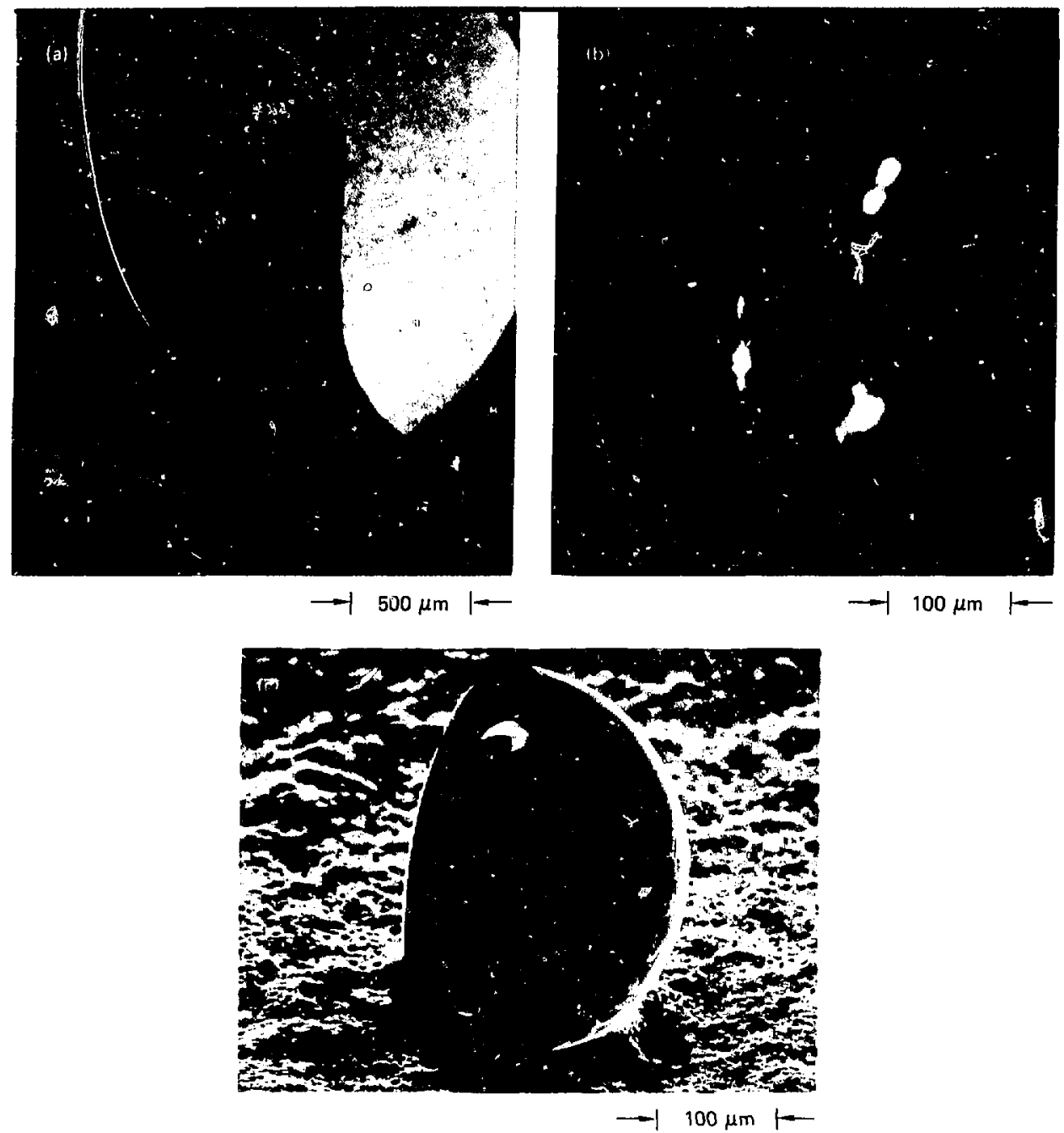

After the $\mathrm{CH}$ layer has been applied to the mandrel and the outer surface of the hemisphere has been cut, the machined hemisphere and mandrel are cleaned by ultrasonic agitation in a series of baths. The copper mandrel is dissolved away and the hemishell is cleaned in another sequence of baths.
The hemishell is then ready for final inspection and assembly.

Rockwell Internatıunal has developed an alte:nate process for making hemishells that provides more rapid processing. The copper mandrel is left in the lathe during the entire machining, coating, and 
remachining sequence. Because the mandrel remains in position throughout the process, the problems of repositioning are eliminated. However, the coating processes and materials that can be used are somewhat restricted. The mandrel cannot be placed in a special environment for curing, and the coating must be applied rapidly hecause the lathe is unavailable while coating is under way. The machining and materials problens encountered in the Rockwell process are similar to those described for the LLL method. The best ress: 'ts have been oblained with a molten polystyrene coating applied to a heated. slowly rotating mandrel. The composition of the polystyrene was carefully chosen 60 minimie stress catused by thermial slirinkage.

The two methods are complenentary. The LLL process allows nore flexibility becaluse the mandrel and chuck assembly can be readily moved shout for a complicated coating and curing procedure. Also, the lathe is not in use during the coating process. The Rockucll procedure provides more rapid processing and simpler fixluring. Handling is minimized, and there is no need for ultraprecise realignment after coating. Both processes have sllecessfully produced targel-quality hemispheres.

\section{Assembly Procedure}

A highly skilled operator assembles these double-shell targets under a high-power optical microscope using liny glass vacuum chucks and precision, hand-operated micromanipulators (sce Fig. 4-1).

The inner sphere is placed at the center of a plastic support film stretched over a wire loop. The sphere is covered with a second support film on a smaller wire loop. The two films adhere on contact, trapping the sphere. Severing the first film between the two concentric support rings leaves the sandwiched inner sphere supported by the smaller loop. We apply hydrostatic pressure 10 force the two films into (i sse contact with the sphere. The $\mathrm{CH}$ hemispheres are now honded around the inner sphere. One hemishell is held in position with a vacuum chuck. Through a fine glass capillary, held in a second manipulator, we extrude a minute bead of fast-curing adhesive on the edge of the hemishell. Then we center the sandwiched inner sphere over the hemisphere and press it onto the adhesivecoated rim. When the adhesive has cured, we repeat the process with the other hemisphere. This procedure requires a greal deal of skill. Precise alignment must be maintained, and each sequence must be completed in three to five minutes before the adhesive cures.

The targel ean be held in position for the laser by using the film sandwich on its wire loop for support. Alternatively, the completed target can he cut out of the support film with a laser machining facility.31 and mounted on al support stalk.

\section{Alternate Approaches}

Cleaved Coatings. One ahernate approach for making hemishells for multishell targets is to coat a spherical mandrel with hydrocarbon polymer, split the coating into two halves, and then remove the mandrcl. The advantages of this method are that many mandrels can be coalcd simultaneously with the same methods used to deposit $\mathrm{CH}$ ablator layers on single-shell targets, ${ }^{32}$ and that the two hemispheres will he an exalt malch for each other, even if the fracture is not smooth or located exactly on the equator. Figure 4.45 shows a coating fractured by this method.

The probiem with the method is that it is difficult to propagate a controlled fracture to consistently producc two nearly hemispherical parts with no pieces missing. Also, because neither of the two pieces is ever exactly a hemisphere, problems arise in atssemhling the target. The two nonhemispherical pieces distort the support films for the inner capsule, causing misalignment. Wc can overcome this, however, by using a small stalk or several small, hollow plastic spheres for supporting the inner sphere.

Molded Hemishells. Another approach being explored for making hemishells for multishell targets is molding. If successful, such a process would provide reproducible hemishells much faster and more cheaply than precision machining can.

Preparation of a mold cavity for fabrication of these tiny hemispheres is a challenging problem. With the newly developed "zero raoius" diamond machining tools, it is possible to cut the male mold section. However there are no tools small enough to allow direct machining of the female half of the mold. We solved this problem by machining a male mandrel from which the female mold section could 


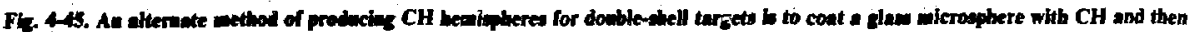

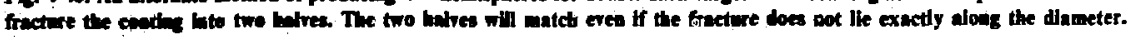

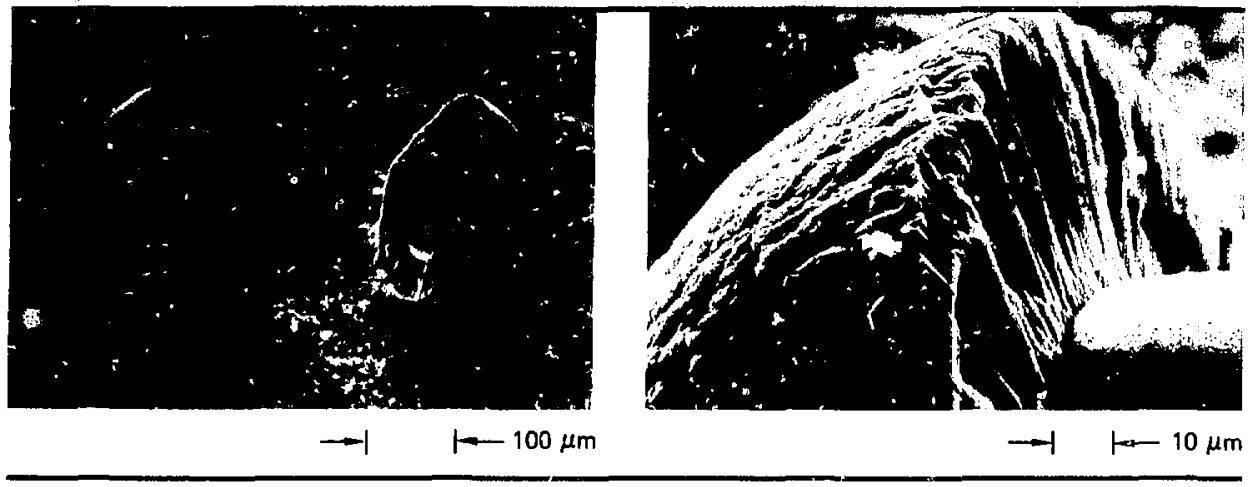

be replicated. By using nickel for the mandrel and electroplating it with copper, we were able to use the differential thermal expansion of the two materials to separate the female mold section from the mandrel. Figure 4-46 shows the mold confimuration.

Initial experiments used a room-temperaturecure silicone rubber to avoid damaging the delicate mold. Several hemishells were made with this material, demonstrating the feasibility of the concepr. However, when we attempted to polymerize a hydrocarbon monomer (styre!ne) in the mold, shrinkage and volatilization during cure resulted in voids.

We then tried several epoxies, wish are nonvolatile and cure at low temperatures with little shrinkage. Partial cure was achieved, but removing the hemispheres from ihe mold proved difficult. We found that mold release agents on both the male and female mold sections, along with differential cooling. were required to allow demolding. Scanning-electron microscope photograpliy revealed that great care had to be exercised to prevent surface irregularities that stem from buildup of the mold release agent. Mold cleaning has proved to be quite difficult.

We also aried thermoforming in the mold. Preserving the dimensional accuracy of the mold required us to observe an upper temperature limit of $75^{\circ} \mathrm{C}$. We formulated a special polystyrene with an unusually low glass-transition temperature, and it appeared to be quite moldable. We obtained improved surfaces by using a preformed, very smooth sheet of polymer, slightly thicker than the ultimate hemishell wall thickness. This sheet was pressed in the mold to form a hemisphere. The first hemispheres made by this process deformed to a pirtially flat'ened state in several weeks due to plastic memory effects. Hemispheres made more recently with different thermal treatment appear is be stahle. These initial results are promising: however. much additional development is required to establish a viable molding procedure.

Molecular Beam Levitation and conting. A third possibility for producing the outer shell of the double-shell targets is to assemble a pair of thin hemispheres around the inner capsule. suppor: this preassembly in a levitator (described in "Molecular Beam Levitation During Coating," above), and coat it with $\mathrm{CH}$ ablator material. The advantage of this procedure is that the ablator layer contuins no seam that would disrupt the symmetry of the implosion. Also, piasmi-discharge coating techniques developed for single-shell targets ${ }^{32}$ ran be used to apply the ablator.

The substrate hemishells are produced by coating a hemispherical mandrel with parylene. In this process, dimer gas is pyrolyzed and gaseous monomer is allowed to condense on the cold mandrel. Polymerization takes place during condensation. This process produces a uniform coating about $5 \mu \mathrm{m}$ thick. The coated mandrel is placed in an ajrbearing spindle lathe and the polymeric coating is cut at its equator using a diamond tool. The mandrel is removed by dissolving in an acid, leaving a 
Fig. 4-46. Mold for forming hemispheres: (a) the overall mold - mfiguration (The large shank with "S"' cuzves provides centering of the two mold pleces without binding, ); (b) close up of the mold cavity itself; (c) the extremely small inside radius obtained on the male mandrel with "zero radius" diamond machining tools.

(a)

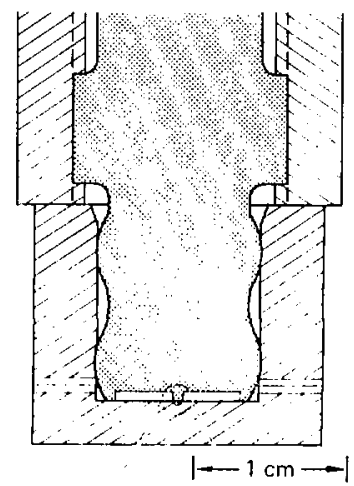

(b)
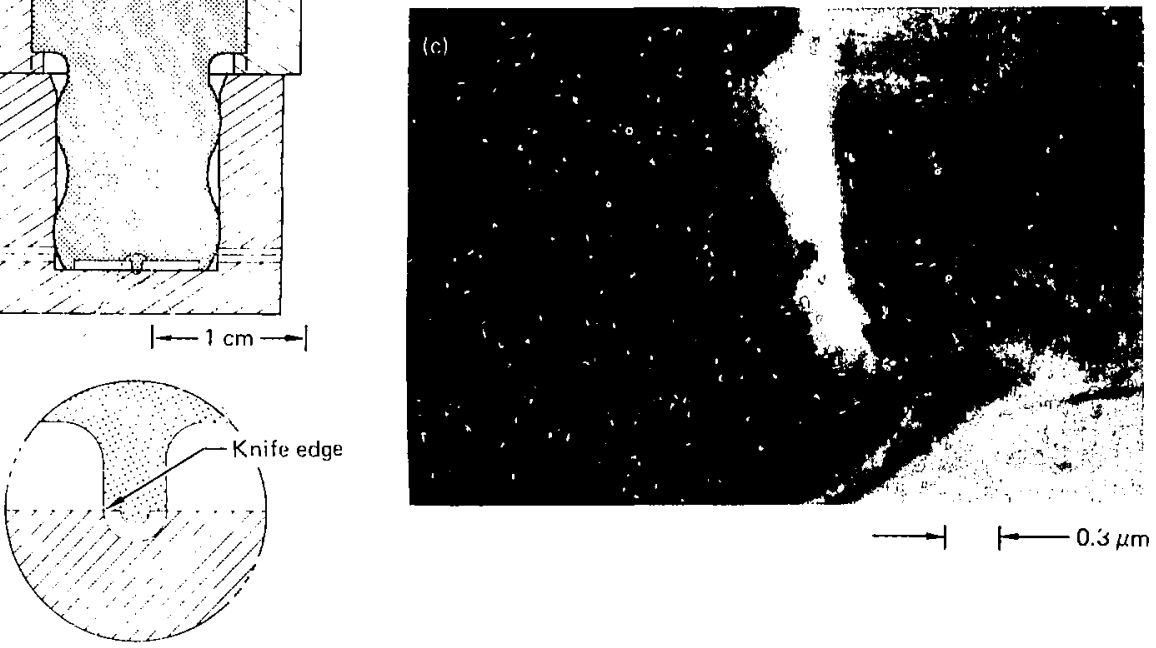

$-1 \quad 1-500 \mu \mathrm{m}$

Fig. 4-47. Double-shell target produced by coating $\mathrm{CH}$ onto a levitated subassembly of two thin parylene hemispheres. Currently, defects in the subassembly seam produce large defects in the coating. However if these defects can be controlled, this process will provide seamless second shells.

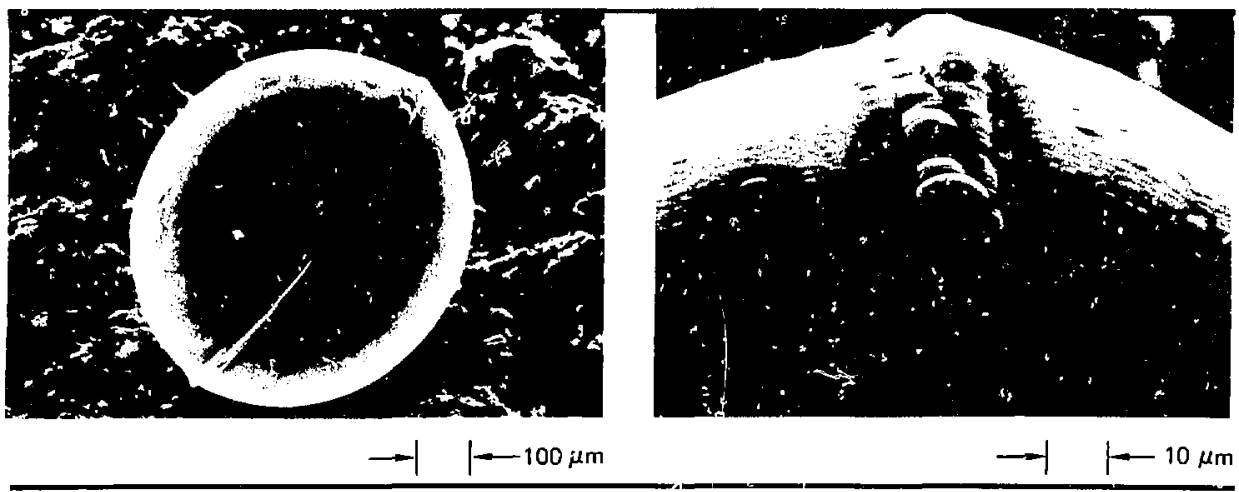


free-standing, thin-wall hemishell. Two of these hemishells are assembled around the inner fuel capsule by the procedure described in the preceding article.

We have successfully levitated and coated several subissemblies using the molecular beam levitator and plasma discharge coating process. Coatings as thick as $50 \mu \mathrm{m}$ have been achieved. Unfortunately, the seano in the subassembly serves as a nucleation point for defect growth, resulting in a continuous band of thick, rough coating around the target. Figure 4-47 shows an example of a target produced by this lechnique.

Future work in this area will conceatrate on eliminuting the defeet caused hy the seam. With improved assembly procedures, we can reduce the magnitude of the initial defect. By controlling the coaling parameters, we can increase the ability of the coating process to "bury" small defects, ather than having these small defects nucleate the growth ol larger ones.

\section{Author: B. W. Weinst tin}

Major Contributors: J. B. Bryan, J. K. Crane, C. W. Hatcher, T. D. Jones, L. L. Lorensen, and W. L. Ramer

\section{References}

31. Later Program Ammal Rejurt-1976, L.ausence 1.nermare lahoriltors. Jinermore. Callil., UCRI..50)21.76, (1977). pp. $4-|6|$ in $4-174$.

32. W. L. Johnom, S. A. I.ells, C. W. Hatcher. 1. H. Lorenten. and C. D. Hendricks, "Plasma Polymerisatton Cobltme at D-T-Filled Cilass Shells for L.iner Furion Targeh," in Plavma polvmerization. M. Shen and A. T. Bell. Eds.. 1979 ACS Symponium Series 10k. p. 313-330 (1979). Sho "Coalings and lasers," this section.

\section{Analysis and Characterization}

Physical, chemical, and structural characterifation of targets is necessary both to provide feedhack for the continued development of fahrication techniques and for measurement of each target prepared for laser experiments. Development of new target designs requires constant improvement of our measurement capabilities.

In "Production Characterization," we describe the role of characterization in the target production sequence. Most of the measurement techniques used routinely in target production have been described in previous annual reports. ${ }^{33-35}$ However the
Fig. 4-48. Fusion target fabrication sequence. Characterization plays a key roie: batches of target spineres must be inspected after each fabrication step. The same characterization techninues are also used to select and individually measure eacur target sphere that is sent to the laser.

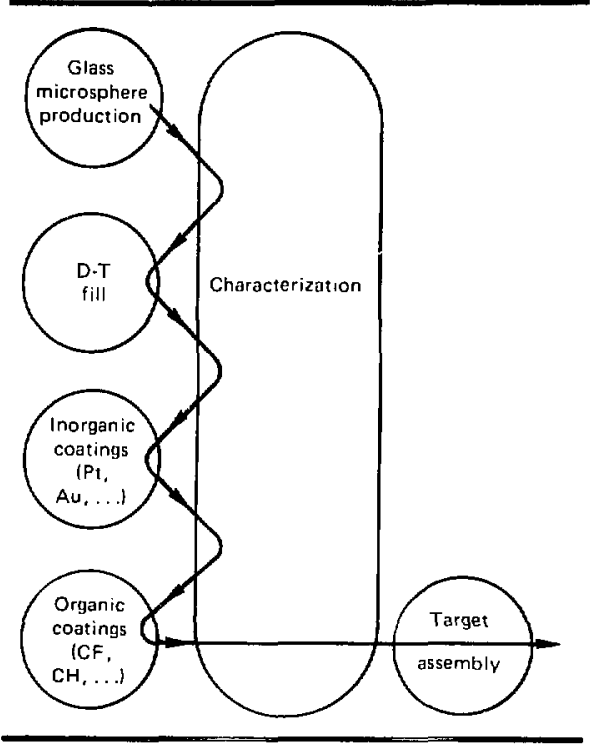

development of nes largets, such as those using nontransparent coatings (metals and thick polsmer layers) and double-shell designs, has resulted in changes in emphasis and in the development of several new procedures.

In "Development of Automated Characterization Techniques" and "Malerials Analysis Developments and Surfice Studies," "se describe new meatsurement sistems that have been developed to meet the need for increasingly rapid and alceurate target characterization. as well as materials analysis.

\section{Production Characterization}

Figure 4.48 illustrattes the Ispical sequence for target production and characterization. Batches of microspheres must be inspected al each step in the fabrication sequence. We measure the size distribution, thickness and uniformity of each layer, surface finish, and the amount of fuel and tracer gas fill to 


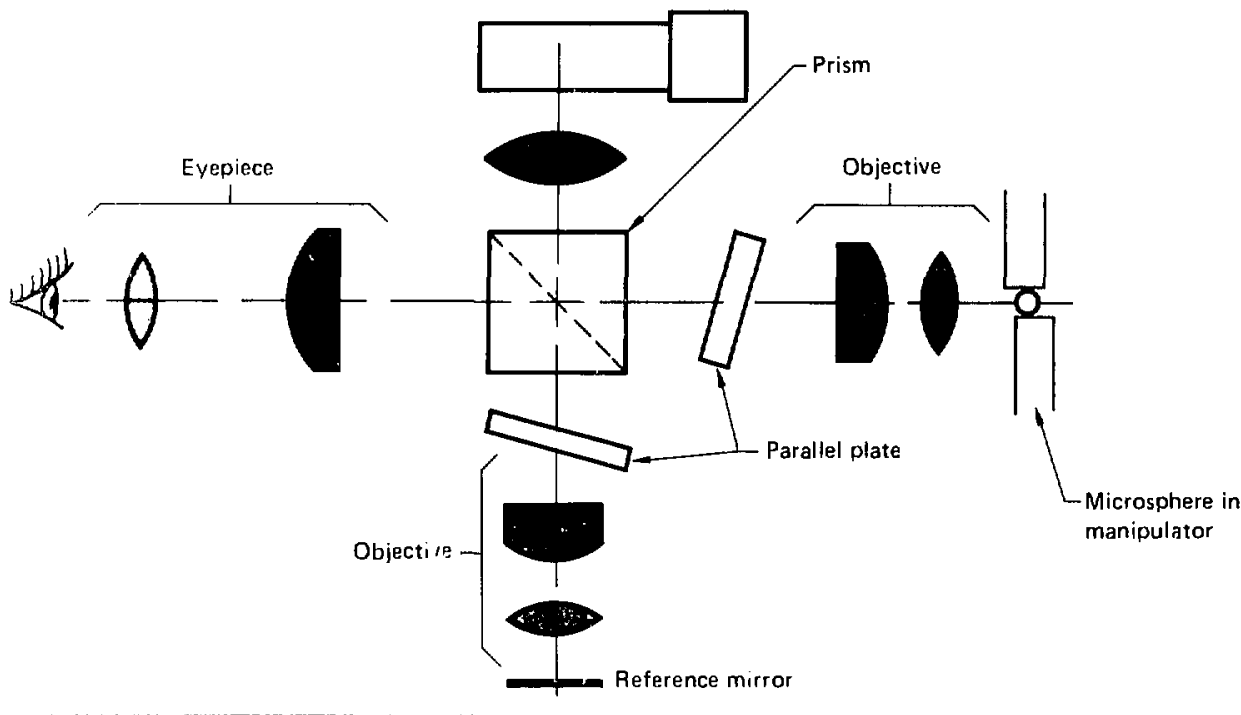

Waluate whether the spheres meet target specifications, We combine these results with measurement of the relevant material parameters such as density, refractive index, chemical composition, crystal structure, and surface contanination to provide feedhack about each fabrication process.

Once the batch of spheres has passed through the final fabrication stage, the target-quality spheres must be selected from the batch. This selection is generally a multistep process tailored to each par. ticular batch. Depending on the most prevalent defects and the speed of the various measurement techniques, the order of the sorting steps is chosen to eliminate unacceptable spheres as rapidly ats possible. For example, if the batch consists of glass spheres with a narrow wall thickness distribution that received a very uniform, transparent coating, the first sorting process will be interferometric inspection for wall thickness nonuniformities. If, on the other hand, the batch of glass spheres has a broad thickness distribution, the first sorting step will be to look for spheres with the proper wall thickness.

Spheres that have no defects discernible in the batch-sorting processes are removed for individual examination. The thickness of each layer, as well as the amount of D-T fuel and tracer gas fill, is measured. The target sphere is examined ir one of our manipulation systems that allow systematic examination of the entire sphere for surface defects and contamination, and measurement of wall thickness variations of transparent targets. As with batch sorting, the order of the measurements is arranged to reject defective spheres as rapidly as possible.

The development of new targets and fabrication processes during the year has resulted in changes in emphasis and the implementation of several new procedures. Microspheres coated with metals or with thick polymer layers $(\sim 50 \mu \mathrm{m})$ require radiographic measurements to determine wall thickness and uniformity. These radiographic measurements are much slower than optical measure- 


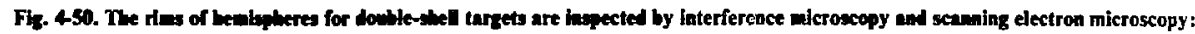

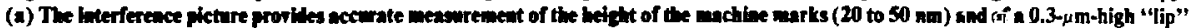
on the inerer rin. (b) the SEM photoniercezryph wows detallel defect strecture below the lifito of optical resulution.

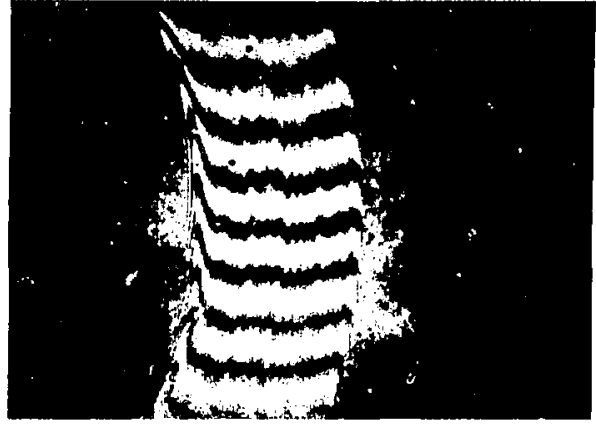

$\rightarrow \quad 50 \mu \mathrm{m}$

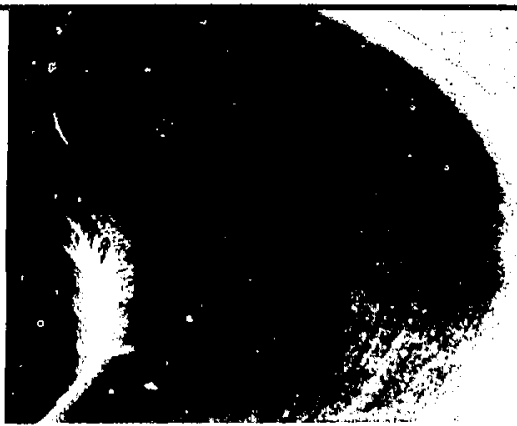

$\rightarrow 10 \mu \mathrm{m} 1-$

ments, so an optical inspection for surface defects is employed as a sereening procedure prior to radiography.

These opaque targets also require an interferometric inspection of the entire surface for small defects that are not measured by radiography. We have modified TOPO I, our semiatumated interferometric inspection system ${ }^{34,36}$ to provide these measurements. Figure $4-49$ shows a schemalic of the modified arrangement. We are able to change from reflection to transmission measurements or back in a few minutes.

Production of hemispheres for multishelled targets has also prompted the development of new measurement procedures. To measure the thickness and refractive index of these hemispheres, we use interferometric techniques similar to those used for measuring glass microspheres. ${ }^{37.38}$ However, because of the large wall thickness of these hemispheres, fringe counting under monochromatic light is no longer practical for measuring optical paths. Instead, we use accurate, position-sensitive probes $t o$ record the position of the sample relative to the optics of an interference microscope, while a white light interference pattern is used to locate each surface or interface. ${ }^{35}$

A particularly important feature for these hemispheres is the condition of the rim, which must be very flat and smooth to permit accurate assembly. We use reflection interferometry to measure the flatness of the rim within $0.05 \mu \mathrm{m}$ [Fig. 4-50(a)]. We also use an SEM to obtain highresolution pictures of the machined surface and to determine the shape of the cormers [Fig. 4-50(b)].

Author: B. W. Weinstein

Major Contributors: J. T. Weir, C. M. Mazuch, D. M. Gouveia, T. D. Jones, and V. C. Chen

\section{References}

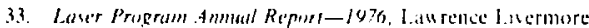

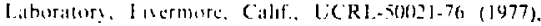
pp. 4-101 is 4.1 .7

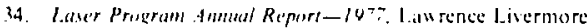

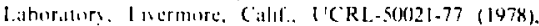
пр. $5-23$ t八 $5-87$.

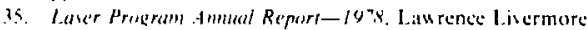
l.ahuritors, I.nermore. Cilat., LCRL 50021.78 (1979). рг. 4.21 10 4.34 .

36. B. W. Wematem. D. I Billenharg, J. T. Weir. and (C. I) Hendricks. "+ Interfermetric Measurements of Laher Fuunn Targets." presented al the Topical Meeting on Inertial Conlinement Furent, Fenruary 7-9, 1978, Sa, Diego, Caluf. Also Law reme l.nermore lahoratory. Livermore. Calif.. LCRL. $-8(0)+21197 ?)$

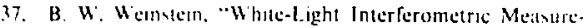
ment of the Wall Thickness of Hollow Glan Mistospheres." f. Appt. Phis., 46, 5303 (1975).

is B. W, Weunstein and C. D. Hendricks. "Interferometric Measurement of Lalaer Fusion Targets," Appl. Opl., 17. $36+1(1978)$ 
Fiz. 451. Inprerement in senolution from 1977 (a) to 1979 (b). The curve ha the aproximate upper lisat of acceptable defects for a 600 -

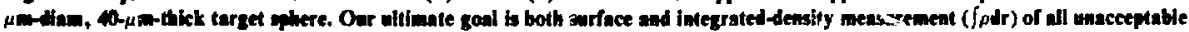
defects for either tranparem or opepue targets.
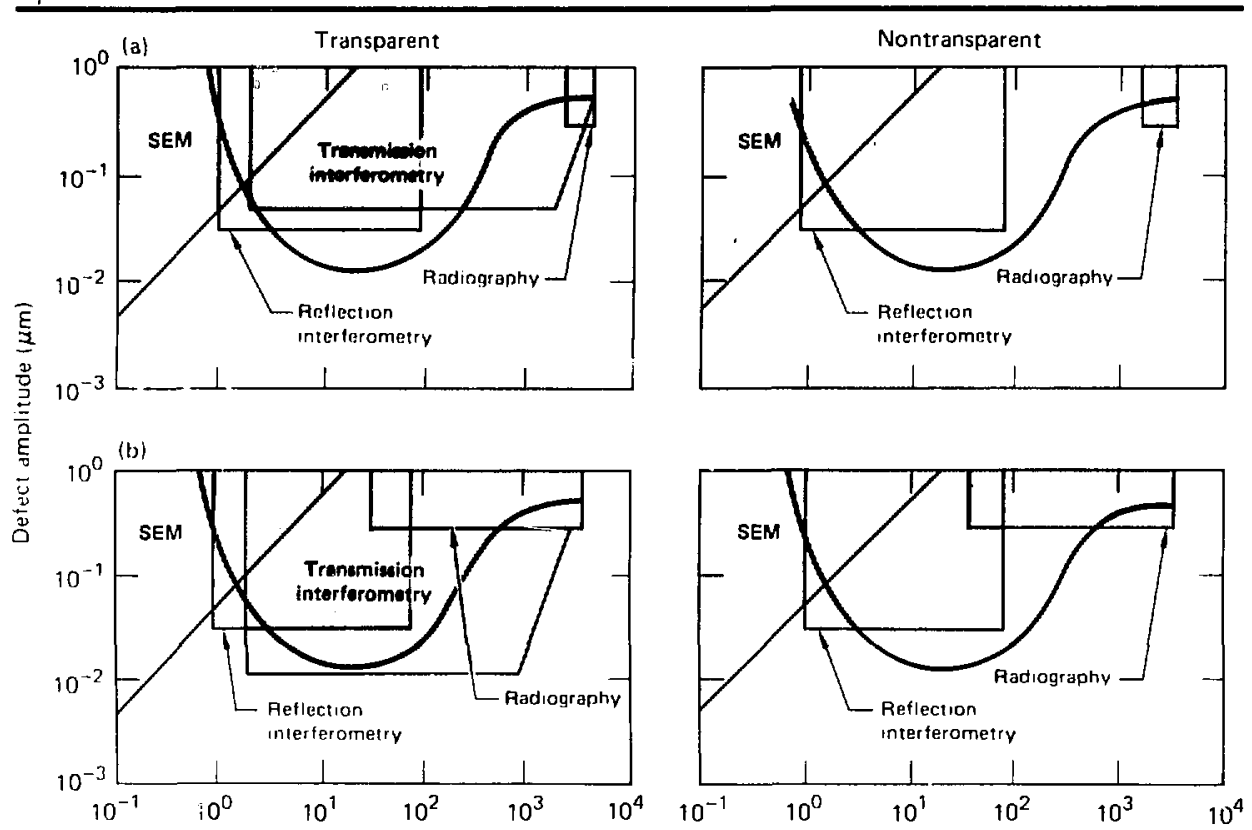

Defect lateral dimensions $(\mu \mathrm{m})$

\section{Development of Automated Characterization Techniques}

To meet the larget charateriation needs of laser fusion restarch, constant improvement is necessaly in both the resolution and speed of measurement. Such improvements are, for the most part, gained by automation and on-line data analysis. During the year, we added automated digitiation and processing of microradiographic images to our capabilities. This has improved the speed and accuracy of our measurements of nontransparent targets. New $x$-ray sources improve the quatity of the images themselves and reduce the processing time. An automated interferometric measurement and sorting system has been devel- oped that reduces by a lactor of five the time required (1) characterize a batch of transparent microspheres. A fully alutomated system for mapping the surface of a target with $0.01-\mu \mathrm{m}$ accuracy is under development. We discuss each of these improvements in detail in this article.

The effect they have made on our target measuring and sorting abilities is shown in Fig. 4-51. which compares the resolution presently available with that of two years ago: and Table 4-1. which shows the improvements in measurement speed that we have achieved in the same time.

Automatic X-ray Image Analysis and Sorting System. Wie have started the development of a microconputer-based system to automatically extract measurements of opaque targets from their respective $x$-ray film images. The microradiographic techniques for recording these $x$-ray images have 
Fig. 4-52. Antomatic x-ray image aralysis and sortiog system ( AXIAS). This systen provides rapid digitization of microradiograph inages and on-line computer analysis. Iange procesing time is less than one minute (vs turnaromed times of a day or mone for ordianry digitization and sulsepwent andysis on a larte time-sharing compoter).

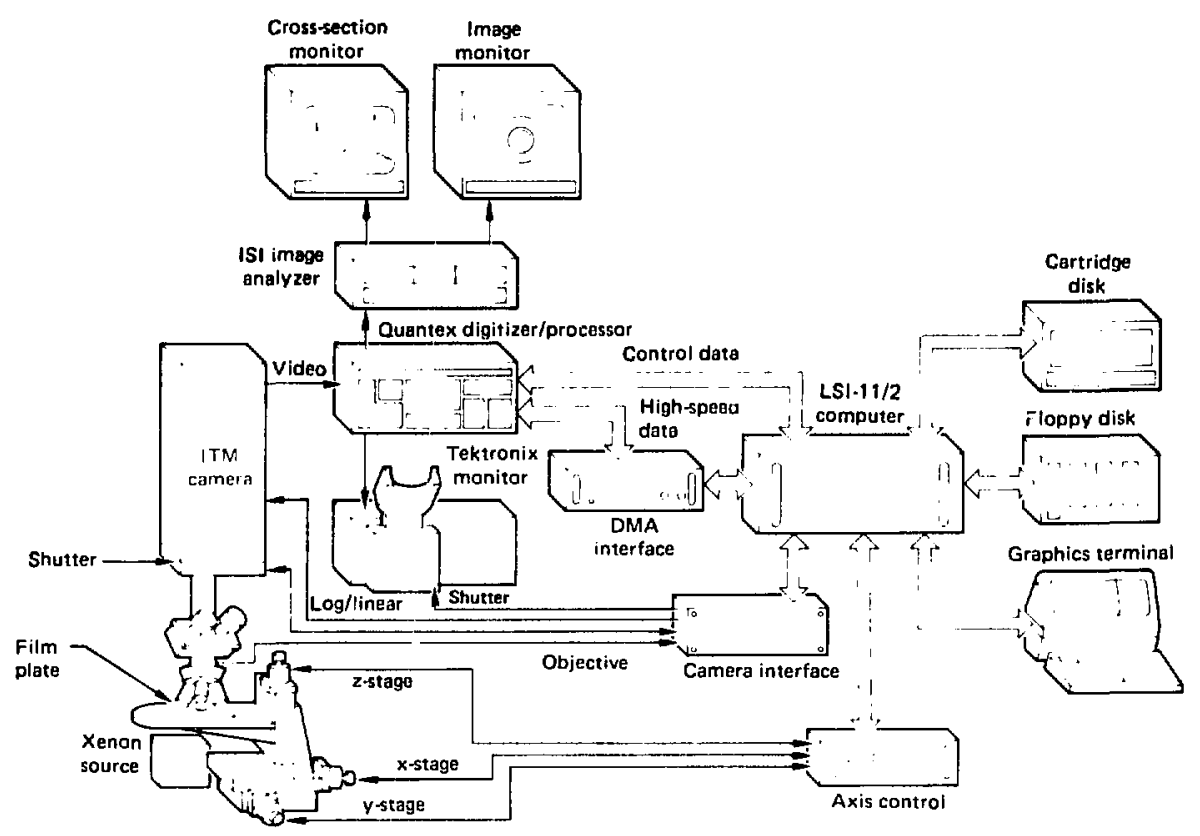

Table 4-1. Improvements in measurement speed from 1977 to 1979 . Both the tianc to measure and sort large numbers of spheres and the time required to completely map a preselected sphere have been reduced by factors of between 5 and 10 .

\begin{tabular}{lrc}
\hline & 1977 & 1979 \\
\hline Transparent targets & $5 \mathrm{~min}$ & $30 \mathrm{~s}$ \\
$\begin{array}{l}\text { Opaque targets } \\
\text { Thickness map } \\
\text { (transparent target) }\end{array}$ & $15 \mathrm{~min}$ & $3 \mathrm{~min}$ \\
$\begin{array}{c}\text { Surface map } \\
\text { (transparent } c \text { opaque) } \\
\text { Thickness map } \\
\text { (opaque target) }\end{array}$ & - & $15 \mathrm{~min}$ \\
\hline
\end{tabular}

been deseribed in the 1977 and 1978 Laser Annual Reports. ${ }^{34.35}$ Previously, the examination of the $x$ ray images has required manual inspection with an image-splitting microscope for coarse measurements. For precision measurements, microdensitometry and computer analysis on the CDC-7600, a large, tomeharing computer yuired. These technigues are lime-comsuming and. cupecialls in the later cance, nos well suited for large numbers of litrgets. Thus, we were motivated to desolop a rapid and accurate x-raly image measurement $y$ vem. Specitically. the Automatic X-ray Image Analysis atnd Sorting (AXIAS) system "als derened to atcomplish the following:

- Reduce meisurement time for 100 spheres (1) a few hours

- Provide alcuracy comparable ta the CDC. 7600 analbuis

- Allow veveral options for speed vs accuracy in order 10 provide automatic sorting of a large arrity of imilges.

This ststem is shoun whematically in Fig. 4-52. A film plate with an x-ray image of an array of targets (ree Fig. 453) is examined by a television camera through a high-power optical microscope. One individual taget image is in view at a time (four ex- 


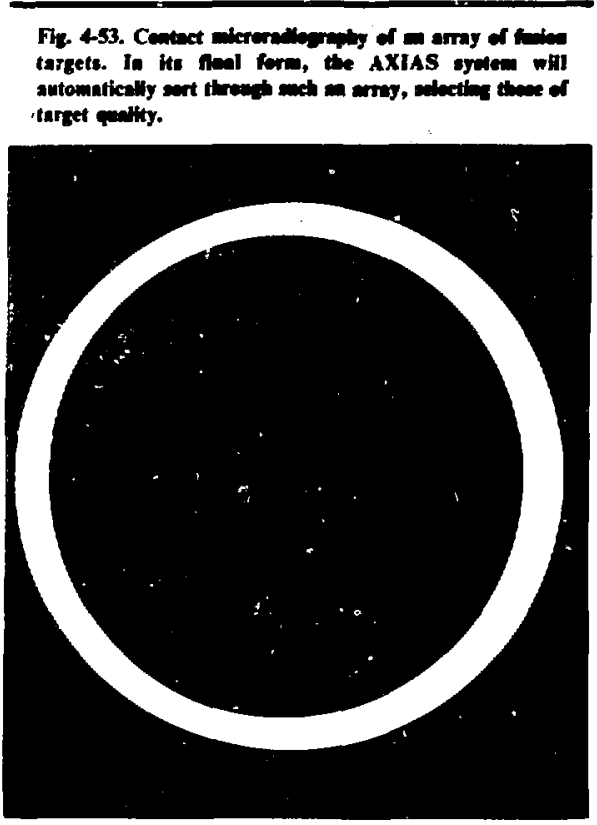

:mples are bown in Fig. 4.54). The video image is digitised at high speed into a special-purpose random access memory from which the computer processe, the imate.

Once the image has been transferred to compuler memory. image processing can be used to make preese, rapid measurements. The system currently provides noise-reduced scans and operatur-controlled cursors for making meatsurcmonts. Provisions for high-accuracy, fullyautomated measurement and sorting are under dev心lupment.

The film plate, camera, and microscope system are show $n$ in Fig. 4-55. The camera (manufactured by Image Technolugy Methods. Inc.) incorporates a logarihmic amplifier 10 deteet optical densities in at linear lashion. B! contrast, most commercially available cameras are linear devices, and they detect optical densities in logarithmic fashion. Because of very high signal-to-noise characteristics, the ITM camera can detect an optical density range of 3 (dynamic range of 1000). A very bright xenon source illuminates the image through the microscope, which enables the camera to be set so that shading error and latent image of the vidicon are extremely small. The microscope uses several objeclives of different powers to enable examination of different sise targets with maximum resolution. The system is capable of viewing target images up to $700 \mu \mathrm{m}$ in diameter with the lowest magnification. At high magnification, the spatial resolution is less than $0.5 \mu \mathrm{m}$.

The video image is digitied by an 8-hit $A, D$ converter in $130 \mathrm{~s}$ and stored direcily into a 512 by-512-by-12-bil random access memory. This digitier processor (mantiactured by Quantex. Inc.) is capable of summing and averaging sequential frames of video images so that the electronic noise is reduced signilicantly. Shading error from the light sourec can be subiracted directly from the image, since this error is additive in logarimmic mode.

We have developed a very high speed data channel from the digitiser memory to the DEC LSI112 microcomputer, so that the computer can read or write image datal either point by point, line by line (both horizontal and vertical), or block by block. The transfer rate is $5.34 \mu \mathrm{s}$ pixel (a pixel is a pitoture element), and an entire image lan be iransfered in 1.4\%. We have also developed the sofinare (1) control the video image digitiser processor lo sontrol such functions as digitie. frame average, and subiract. This enables the computer $(0)$ directly and rapidly digitise noise-reduced images in the video processor ard then to transfer the imalges for analysis.

In a measurement. we extracl a horizontal cross-sectional sean through the center of the sphere. We determine diameter and wall thickness by locating the edges in the cross-section. Our accuracy in locating these edges is limited by filmgrain noise. as shown in Fig. 4-56(a). We can reduce the grain noise by averaging several successive horizontal lines [Fig. 4-56(b)]. However this procedure reduces our resolution because the adjacent lines do not all pass through the center of the sphere and are therefore not identical. We can reduce the grain noise without a loss of resolution by averaging along an arc. This method of averaging, which we refer to as a "wedge scan," is illustrated in Fig. 4-56(c). The resulting cross section, 


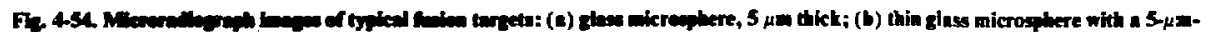

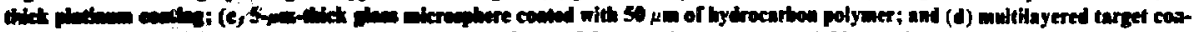

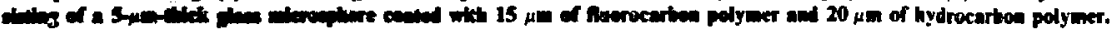
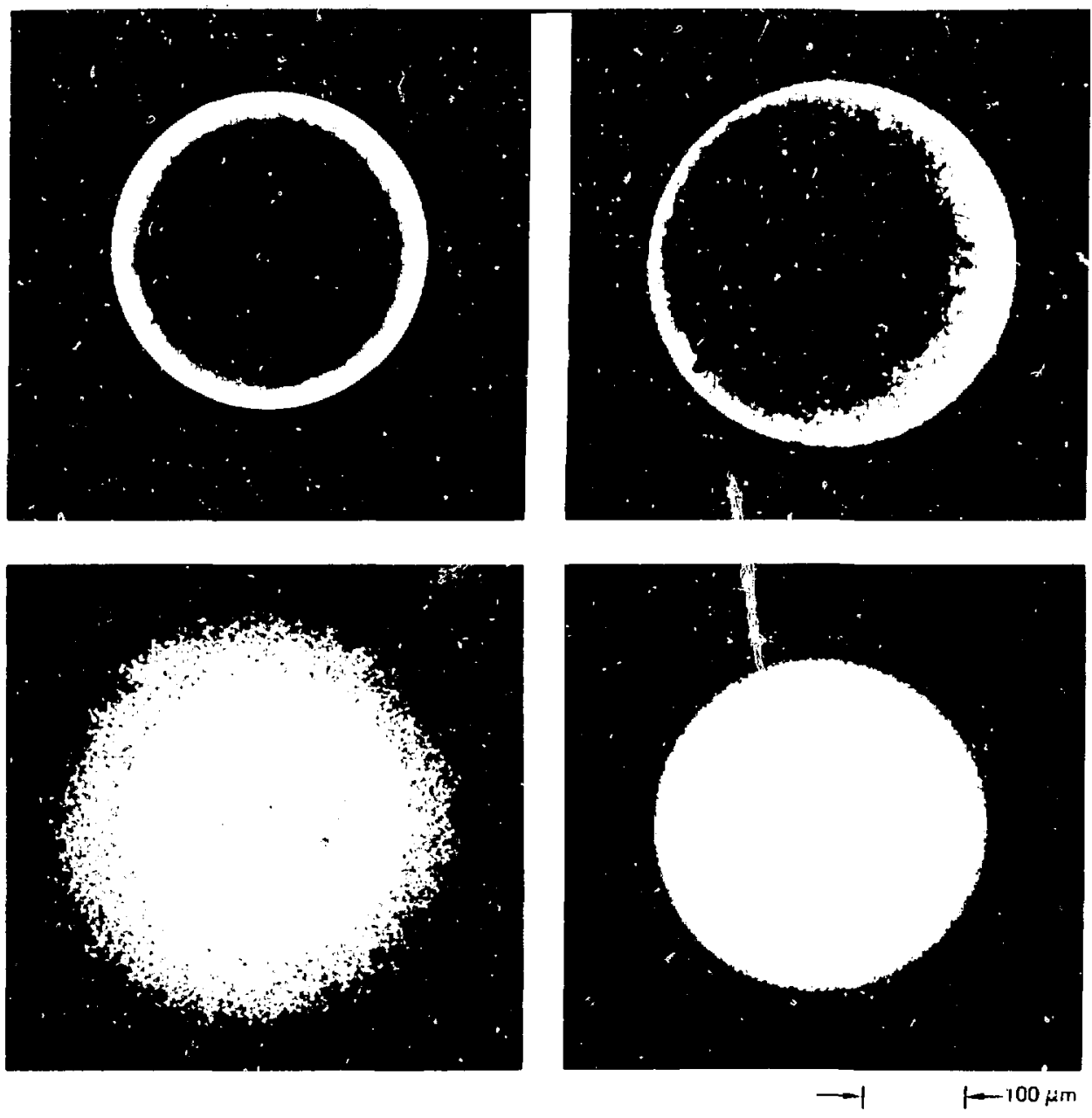

shown in Fig. 4-56(d), is very significantly noisereduced compared with the unaveraged cross section shown in Fig. 4-56(a). Edge measurements are much more accurate after this averaging.

In the present system, an operator measures thickness by positioning cursors, using fast interactive software. These measurements are usually accurate to 0.5 to $1.0 \mu \mathrm{m}$ and can be made in a few minutes lis edch phere. In the next development stage, the computer will be programmed to recognis these edges and make the measurement automalically. reducing measurement time pesphere to a lew seconds. $\mathrm{W}_{\mathrm{e}}$ also plan to implement a least-squares fitting analysis similar to that used 


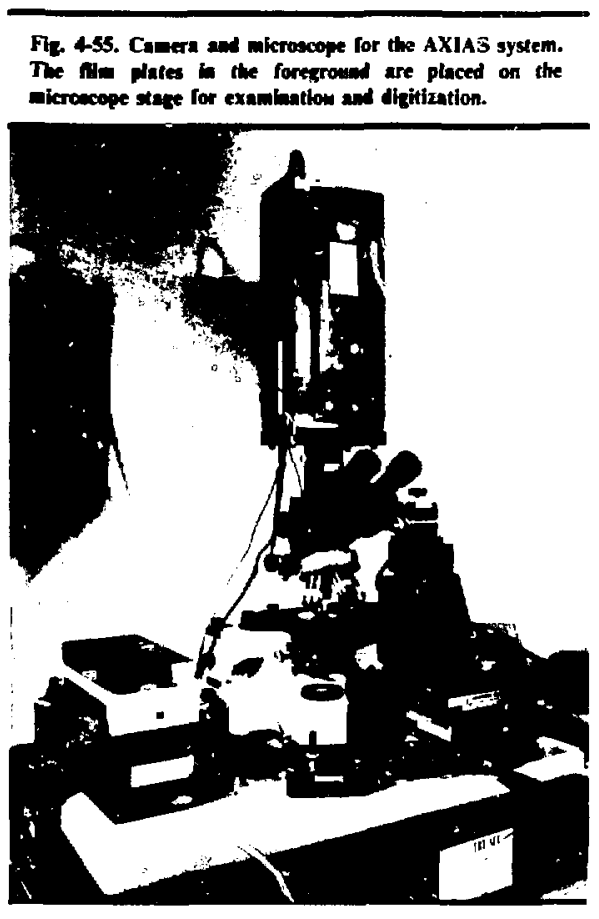

on the ( 1$)(-76010.39)$ This method hats been found accuritte $111.3 \mu \mathrm{m}$ in diameter meisurements, 1). $3 \mu \mathrm{ml}$ if wall-thickness measurements, and $0.1 \mu \mathrm{m}$ III makencentricty meisurements. In a preliminary us! uc hate found that this more delailed analysis lither abour 70, on the LSI-11 = microcomputer. the final development that we have planned at present in 60 provide totally automated sorting of an arril! of images. The computer wiil control the positioning of the film plate and will recognise and meatsure each larget image. Based on at set of criteria prosided by the operatur. Ine system will progress prem ralpid. cor,rse neilsurement and selection Io more accurate. lober meatsurentent of the betler images.

The AXIAS stutem (Fig. 4-57) is convenient to use, and it pro. Ides datia that have important intplications for a icrosphere production. One of the main advantage of the AXIAS system is that it is very easily uperaded. Computerized smatl-scale defecl meacurement or fusion targets has heen demonstrated hy Whitman et al. ${ }^{\text {to }}$ This analysis could be easily implemented on the AXIAS system, using at fast flouting-point processor. Another future use of this system would be to directly analy/e images as they are recorded by a realime $x$ raly system. This type of system nould provide even more rapid lurnatound in the charatcterifation of oprique largets.

Automated Sphere-Mapping System. During the seatr, we reached the final design stage of an automilted uphere-mapping system (ASM). The ASM sysem will provide capabilities nimilat lo the TOPO il incerleromelric mapping 4 stem ${ }^{15} .41 .42$ alreads in use, but with the signtlicant added abilit! to map opitque an well as tramyparent larget spheres. In addition, the system optics have been tolally redesigned for much simpler operation, and solidstatce phateshift generation hats been incorporated 10 improve measurement speed and accuraer.

Tise ASM A microcomputer will control the sphere-rotitor ststem and will coordinate the rotaltion with interferemetric phase measurement of the surface topography. The lopographic dalat can be stored on a computer disk and displased in real lime on a graphice display. A permanem eops of the graphics display can be made lor comparison with other larget spheres. The system will be last enough to malp a 150 - $\mu$ m-diam sphere in less than 1 minute with 5-nm amplitude resolution and $1-\mu m$ spatial resislution.

The ASM optics consist of an optical phaseshift generator. a phase-shift interferometer. and an optical viewing system. The optical phase-shilt generator is diagrammed in Fig. 4-59. A linearly polarized. single-longitudinal-mode HeNe laser is pass. $\mathrm{d}$ through a spatial filter to reduce phase-front distortions. The linearly polarized output beam is passed through a quarter-wave plate and then split by a $50-50$ beam splitter. The resulting beams are passed through flint glass acrustooptic modulators that are driven by if signals with a small, very well defined frequency differencr Typical frequencies are 40 and $39 \mathrm{MH}$. Fach beam exits the acoustoopic nodulator with the frequencies of the first-order diffraction component shifted by the of driving frequencies. The first-order diffraction components are passed through orthogonal polarizers and then recombined by a beam combiner. In this output 
Fig. 456. Significant improvements in resolution are achieved by averaging to remove grain noise. Trace (a) is a single line from the digitized image. Identifying the boundaries between different layers is almost impossible. Averaging successive lines [as shown in (b)] reduces noise, but resolution is lost because the scans do not all lie along a diameter of the sphera A "wedge scan" (c)--in which the data are averaged along ares-provides the higli-resofntion, noise-reduced image shown in (d).
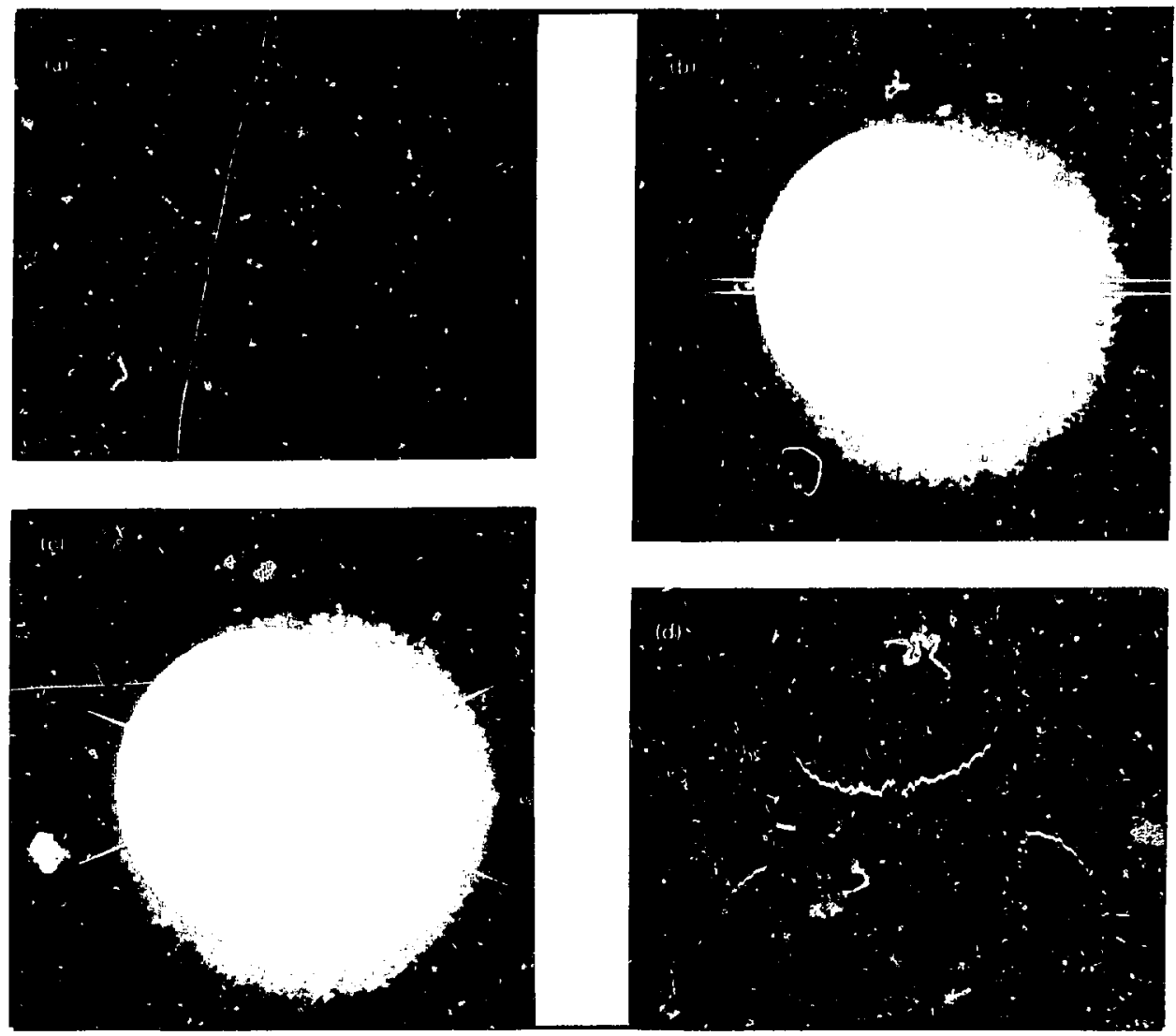

beam. the frequencies of the tramsverse clece tromagnetic (te) and transverse magnetic (tm) coms. ponents differ by 1 Mlla. The resulting heam passes through constantly changing slates of elliptical pola 'zation at a frequency al' I Mll/. This $1-\mathrm{MH} /$ "rotation" can be delected simply hy placing a polarizer at $45^{\circ}$ to the te or $t \mathrm{~m}$ modes, then viewing

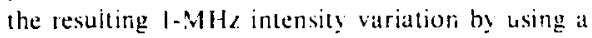
photodiode detector. Any phase shift between the te and $\mathrm{tm}$ polarizations will cause a corresponding shift in the phase of the $1-\mathrm{MH}$ / intensity variation. The ASM interferometer optics / shown in Fig. $4-60(a)]$ cause the relative phase of the te arid $\mathrm{m}$ components to he molulated hy path length challiges introduced hy urface defects on the largets. Ome polariallon component is used as a probe beam. whike the other serves as a reterence. The probe beam is litet low used on a point on the face of the lareet uphere. The retlested prothe beam is then direded around the target onto the reserse side. (On the reverse bide. the beam is focused lowards the center of the larget sphere so it strikes the sphere aser a fairly broad atrea aind is refiected directly back on ilself. Viler refleciion from the 
Fig. 457. Compater and display hardware for the AXIAS system.

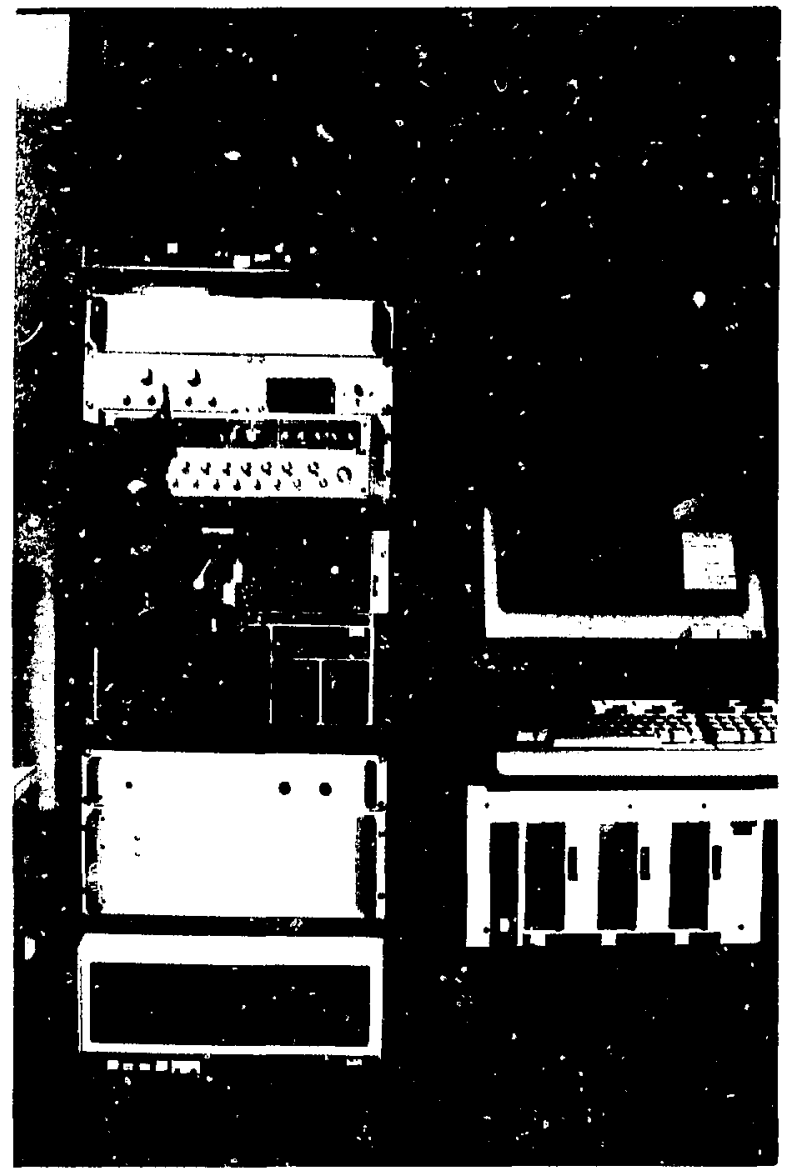

reverse side of the target, the probe beam is recombined with the reference beam.

This output beam is directed through a polarizer at $45^{\circ}$ and orto a photodiode detector. As the target sphere is rotated, any changes in diameter, either from nonsphericity or from small surface perturbations on the front surface, will cause a change in optical path of the probe beam and. therefore. a shift in the relative phase of the te and $\mathrm{tm}$ components of the output beam. This will, in turn, cause a shift in the phase of the $1-\mathrm{MHz}$ intensity variations seen by the photodiode detector.
An electronic phase detector compares the phase of this output signal with the phase of a reference signal taken directly from the phase-shift generator. This measurement can be very rapid and accurate (easily to $1 / 100$ of a cycle in $1 \mu \mathrm{s}$, corresponding to a resolution of better than $5 \mathrm{~nm}$ for detecting defects on the target).

The primary advantage of the interferometer arrangement shown in Fig. 4-60(a) is that it is insensitive to changes in position of the target sphere. Small translations of the target along the beam axis cause no change in path for the probe beam. In an ordinary interferometer, where the probe beam is simply focused on one point on the sphere, any motion of the sphere along the beam axis has the same effect as a defect on the surface. Because our interest lies in defect amplitudes of $10 \mathrm{~nm}$, we would need a manipulator able to maintain the sptiere position to $10-\mathrm{nm}$ accuracy. (Such a manipulator would be extremely expensive.) With the ASM interferometer, the manipulator is required to maintain the sphere position only within $\sim 1 \mu \mathrm{m}$. Another advantage is that we can make minor modifications to the optics of the same system [see Fig. 4-60(b)] to measure wallthickness variations of transparent spheres.

To measure the surface irregularicies of a target in the ASM system, we must place the target between the target rotator tips in accurate coincidence with the optical axis of the measuring branch of the interferometer. To accomplish this, a target-viewing and alignment instrument (shown schematically in Fig. 4-61) has been designed and integrated into the ASM system.

To precisely locate the target in the interferometer, we view the target simultaneously along two orthogonal axes. One viewing arm is coincident 
Fig. 4-58. Schematic diagram of the automated aurface mapping system. The controlling micrecomputer correlates high-speed phasemensarewent dat from the optics with position data from the target sphere to generate a map of the sphere surface. The apparatus can resolve a defect with as amplitude of $10 \mathrm{~nm}$.

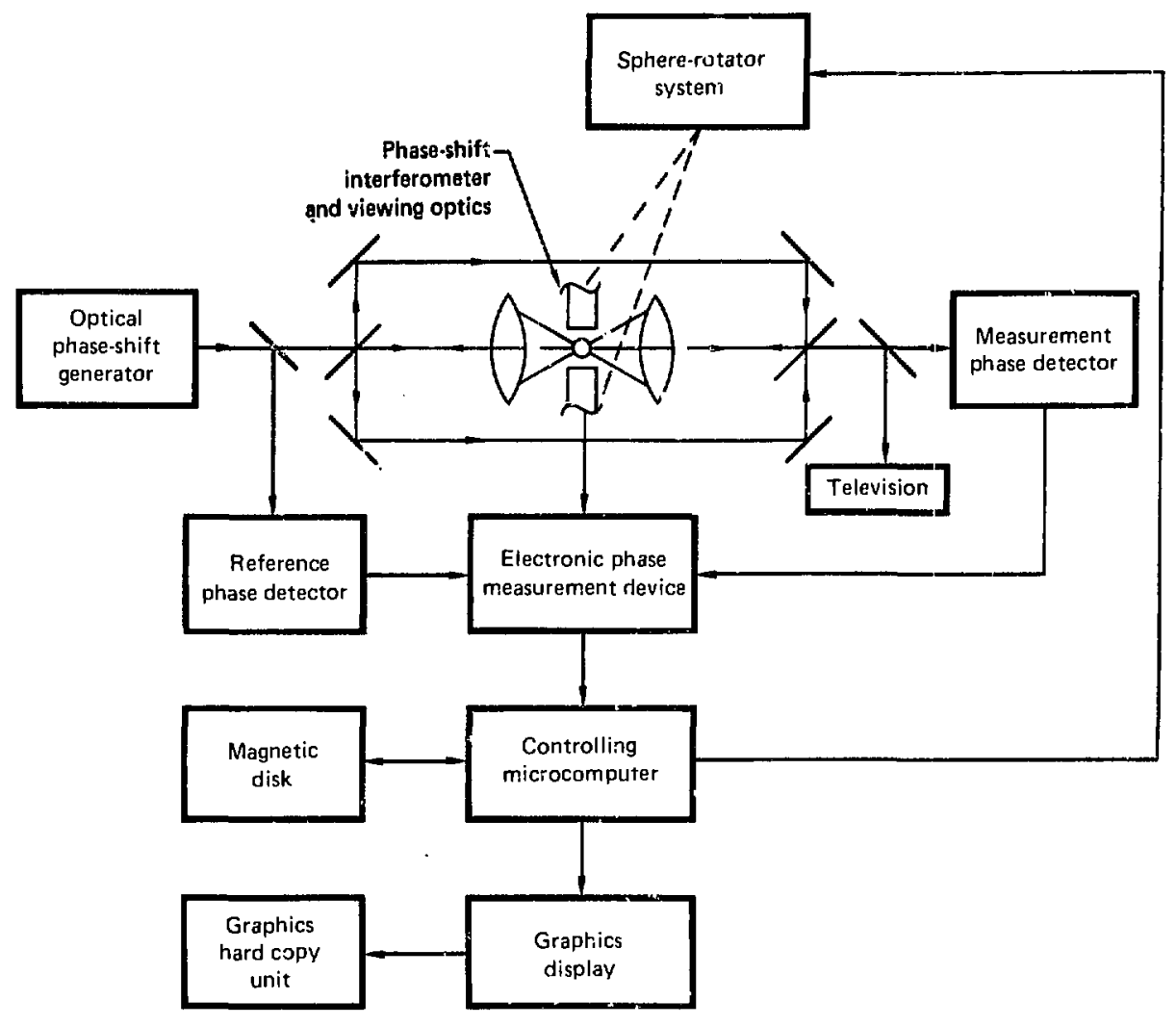

with the interferometer axis and uses the interferometer focusing objective as an imaging objective. The other arm uses an identical objective placed at $90^{\circ}$ to the interferometer axis. A.s Fig. 4-61 shows, the image formed by each of these two objectives is projected onto an alignment reticle. The two targets and reticle images are combined in a central beam splitter so that both images can be seen in ihe same field of view. The beam-splitter output is directed to both an eyepiece and a TV camera. Once the reticles have been aligned on the interferometer axis, they serve as a reference for positioning the target on the rotator tips. The target is centered on each reference reticle as it is viewed by either the eye piece or TV screen. This system has been tested in an opticul bench setup, and the measured resolution limit is $1.5 \mu \mathrm{m}$.

Autornated Sorting Interferometer. We have automated a white-light Mach-Zehnder interferometer that was originally developed for cryogenic targets ${ }^{43}$ to rrovide quick diameter and thickness measurcinents of microspheres (1-1g. 4-62). $T$ is computer-interfaced interference microscope aids the production of glass and transparent coated glass spheres, and it accelerates the presorting of targets.

The Mach-Zehnder interferometer is particularly well suited to this application. The longworking-distance objectives (13 $\mathrm{mm}, 10$-power) 
Fig. 459. Optical phase-shift generator for the surface-mapping system. This device generates a light beam in which the te and tm components change in relative phase at a frequency of $1 \mathrm{MHz}$. The resulting beam passes continuously through different states of elliptical polarization.

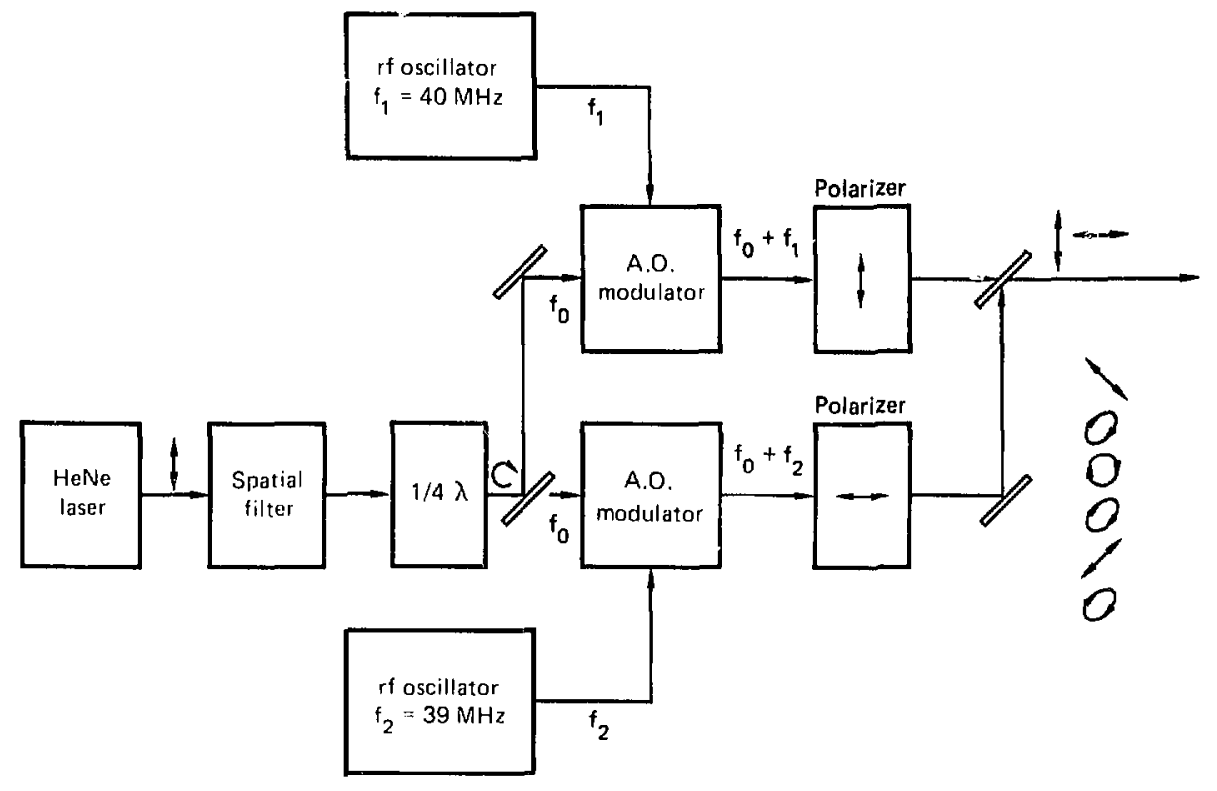

Fig. 4-60. Phase-measurement optics for automated surface mapping. One polarization component is directed onto the sphere, while the other serves as a reference (defects in the sphere cause changes in the optical path of the probe beam, resulting in a relative phase shift between the te and $\mathrm{tm}$ components): (a) optics in a reflection hode; (b) changes necessary for a transmission measurement of transparent targets.

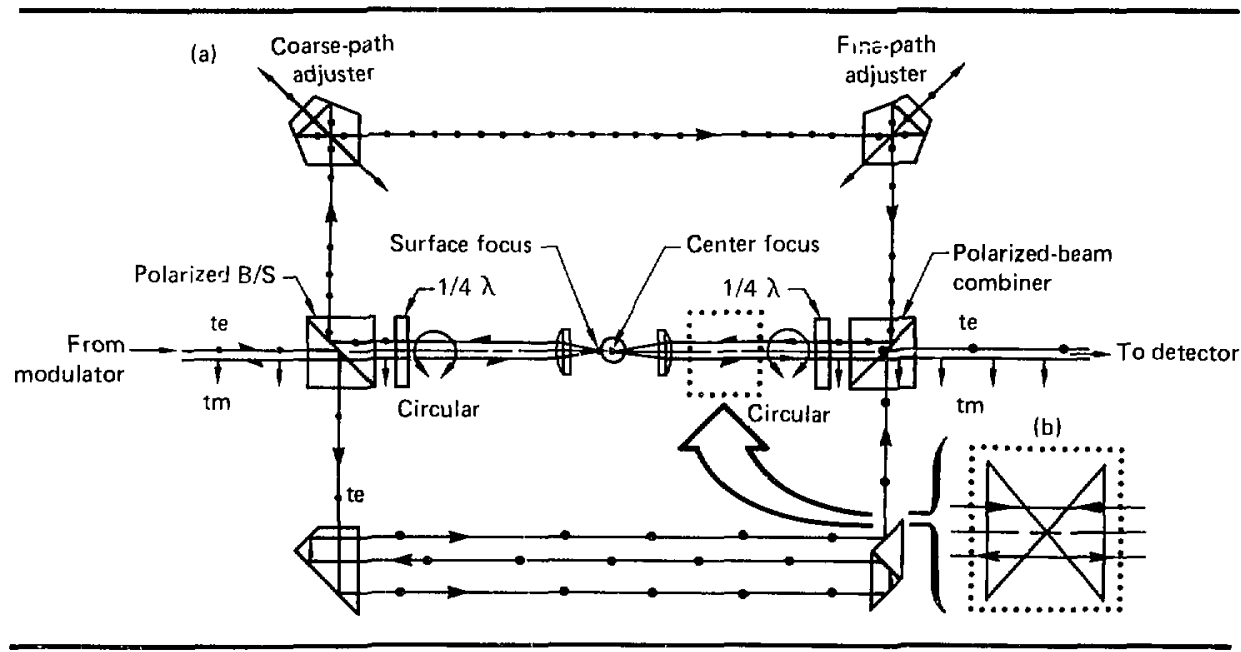


Fig. 4-61. Viewing optics for allgaing a target in the autociated surface-mapping system. The target is viewed along two orthugonal exes. Each arm includes a reference reticle for target alignment (all dimensions in $\mathrm{mm}$; $\mathbf{M}=$ magnification).

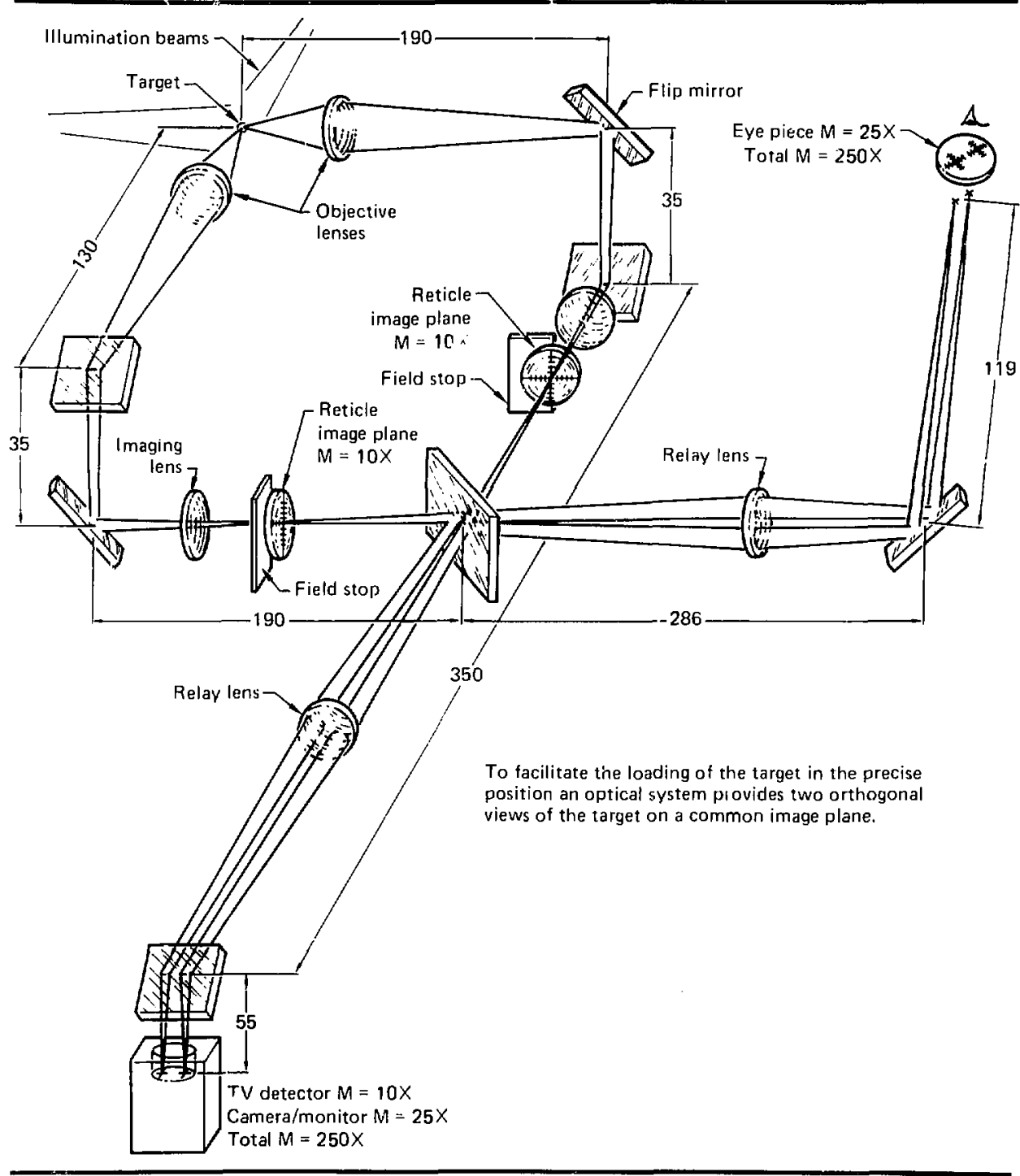

allow ample room for handling the targets and the essentially arbitrary long arm length provides space for phase-measurement hardware.

The spheres to be inspected are placed on a transparent window attached to a molor driven $x-y$ stage. The objective in the stage arm magnifies and focuses the microsphere image, while the compensating objective in the reference arm provides independent control of the interference pattern. 
Fig. 4-62. Autonuted interference microscope diagram. The system sutomaticeily measures the thicknew of each micromphere selected by the operator. She date sre siored in computer memory and can bs plotted as histograms of wall thlekness asd dinater.

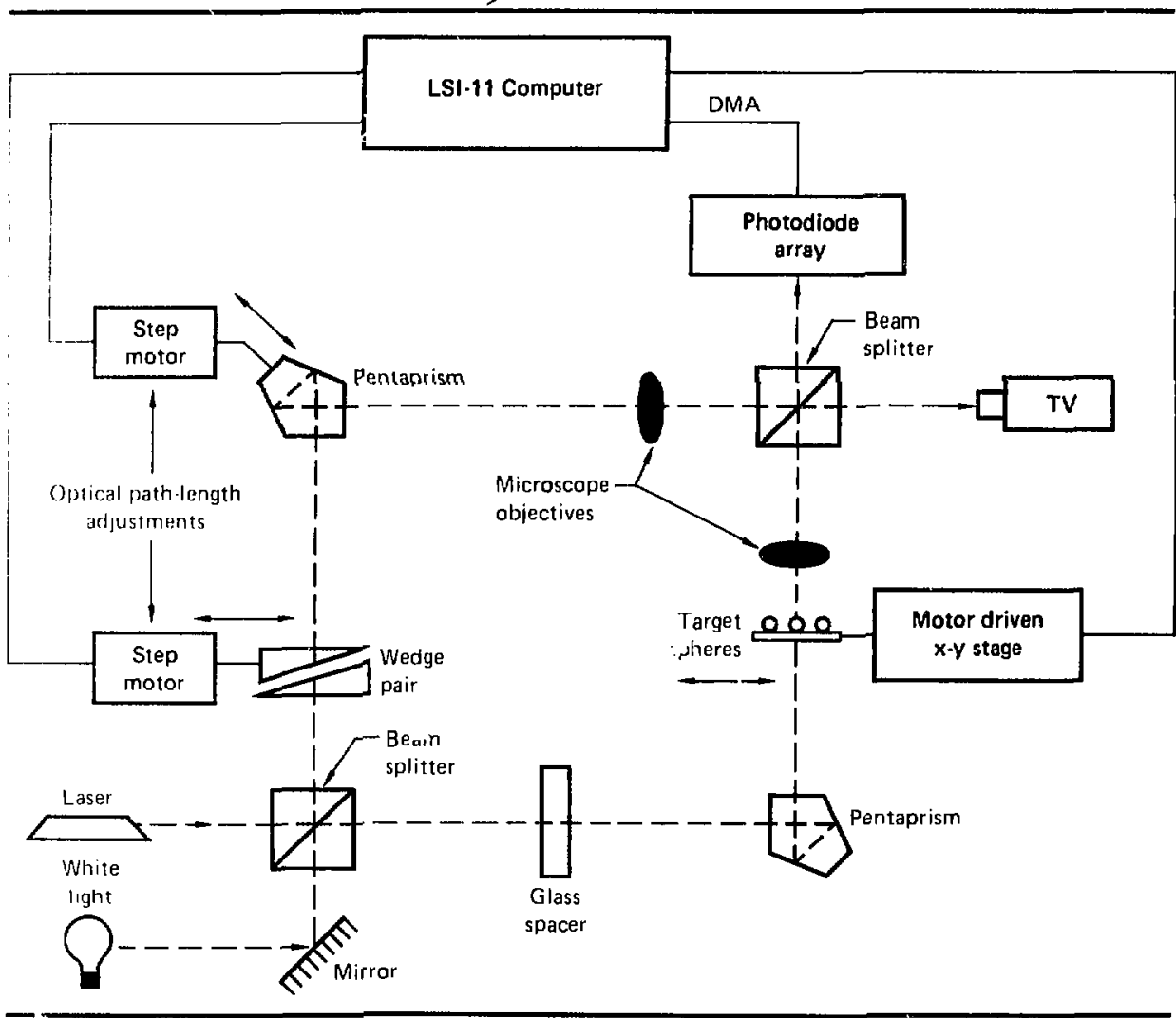

We detect the interference pattern with a phosodiode array (at 1024-linear-element Reticon arrity) interfaced to an LSI-11 computer that also controls path-length adjustments. To measure a shere, we roughl: center the image of the ball over the photo-diode array by viewing a television monitor. The sptiere diameter is deter lined by the number of reticon alements subtended by the sphere image.

Thickness measurements are made by finding the wedge positions for the white-light interference maximum in the background and in the center of the ball. ${ }^{43}$ The change in wedge position corresponds to a change in wedge thickness, and from it we can calculate the thickness of the sphere wall. The system is calibrated by counting fringes of laser light $v s$ wedge rosition. System accuracy is $5 \mu \mathrm{m}$ for the diameter measurement and $0.1 \mu \mathrm{m}$ for thickness. Focusing effects in the single-pass Mach-Zehnder interferometer are only half as severe as in our double pass Twyman-Green interferometers. This allows us to measure very thick, high-aspect-ratio targets. We have successfully measured targets with $\mathrm{CH}$ coatings of more than $100 \mu \mathrm{m}$ on a microsphere with an inside diameter of $100 \mu \mathrm{m}$.

In addition to measuring target diameter and thickness, the computer also controls the $x-y$ stage 
and remembers sphere positions and sizes for later retrieval of the spheres. This, coupled $u$, the short measurement time, enables us to rapidly characterize and sort large batches of spheres.

Authors: J. A. Monjes, R. M. Singleton, B.W. Weinstein, D. L. Willenborg, and R. L. Woerner Major Contributors: D. E. Perkins, G. T. Reel, and A. ... Richmond

\section{References}

39. R, M. Singletom. B W. Heinstem, and ( , 1). Hendrichs.

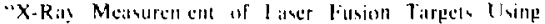

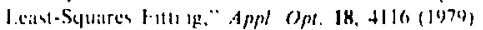

40. R. I. Whilman. R. H. Dil\}, R. P. Krumer. and 1). M.

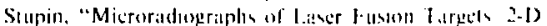
Modeling and Amalysia." Appl. Opt. 18, 1266 (1979).

41. D. I. Willonbrog, D. B., Perkins, J. A. Monjes, B. W. Weinstein. (; T. Recl. and A. 1. Richmond, “Aulumats Transmusion Interferonketac Mapping of $I_{\text {ither Fusion }}$ Targets." presented al the Topical Meeting on Inertal Conlinement I usion, Fehruary 26-2X. I980. San Diego. Callif.

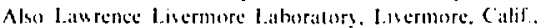
(ICR 1. -433777 (1979).

42. J. A. Munges, B. W. Wouniein, D. L. Willenhorp, and A. 1. Richmond, Micresphere Retater for Aummated inter.

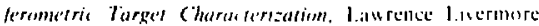

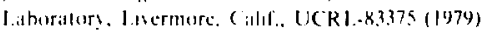

43. Laner Program Ammos Repuri-1978, 1.aurence lincrmese lahoralors, livermore, Calif., UCRI.-5002)1.78 (1979), pp. $4-4 k$ (1) $4+49$

\section{Materials Analysis Developments and Surface Studies}

The Surface and Materials Analysis Group concentrated in three new areas this year: optical analysis, surface analysis, and microlopography mapping by SEM.

Optical Microsenny. We have added a Zeiss Axiomat optical microscope to our complement of tools (Fig. 4-63). It is a polarized light instrument capable of magnifications from 10 to $3200 \times$ using hoth transmitted and reflected light, and it is coupled with a temperature-controlled stage for examining objects immersed in index-of-refractionmatching liquids. Figure 4-64 demonstrates the ability of the microscope to see through an immersed, thick plastic-coated ball. The various levels that we have previously observed only by examining a fractured coating on the SEM are now visible, along with defects at the glass-to-plastic interface. Crossed polarizers are used to further enhance detail and locate birefringent particles.
Fig. 4-63. Zeiss Axiomat microscope

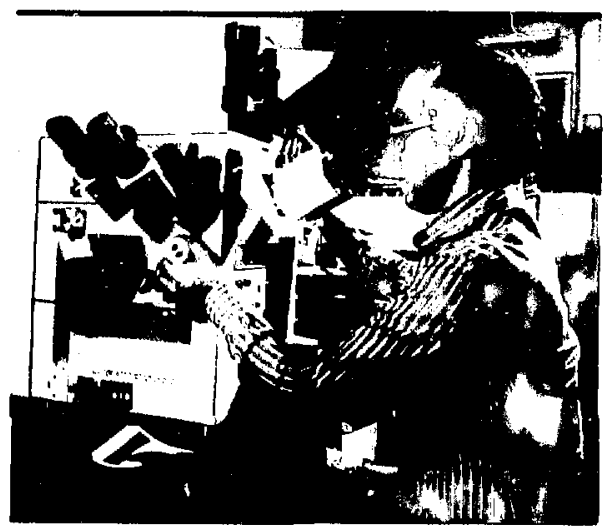

Microtopography by SFM. We routinely use an SEM to examine the surlite of target inaterial. The simplest uay of measuring defect heights is in prolile. but this method suffers from two major problens: we can sec only a snull portion of the surface all one time, and depressions call be ohscured by their edges. We are developing at new SEM system that is based on the bilckscatteredelectron technique ol Lechicdsik th which directly measures height and has a spatial resolution of less than $0.5 \mu \mathrm{m}$.

The system is composed of a conventional SEM with solici-siate diode delectors for hackscattered electrons placed radially about the heam at $0^{\circ}$. $90^{\circ}, 180^{\circ}$, and $270^{\circ}$ to the electron collector. In : plane containing the heam and the sample, these detectors form a $90^{\circ}$ cone angle with the sample, as shown in Figs. 4-65 and 4-66. Backstiltlered electron (BSE) fluxes are strongly dependent on the take-off angle, hence ace can use them to detcrmine a local slope by talking differences belween the signals seen by tho opposing detectors. Because we are using two detector sets. we are able to measure slope in any direction. Since the backscaltered signal is also a function of altomic number of the scatlering surface, we normilise our data by

$$
\mathrm{I}_{\mathrm{B}}=\frac{\mathrm{I}_{0}-\mathrm{I}_{180}}{\mathrm{I}_{0}+\mathrm{I}_{180}},
$$


Fig. 4-64. $\mathrm{CH}_{1.3}$ coatings examined in $\mathbf{1 . 5 0}$ index-0f-refraction liquid showing the ability of the optical microscope to see through $a$ thkck sample: (a) transmitted light and (b) crossed Polaroids.

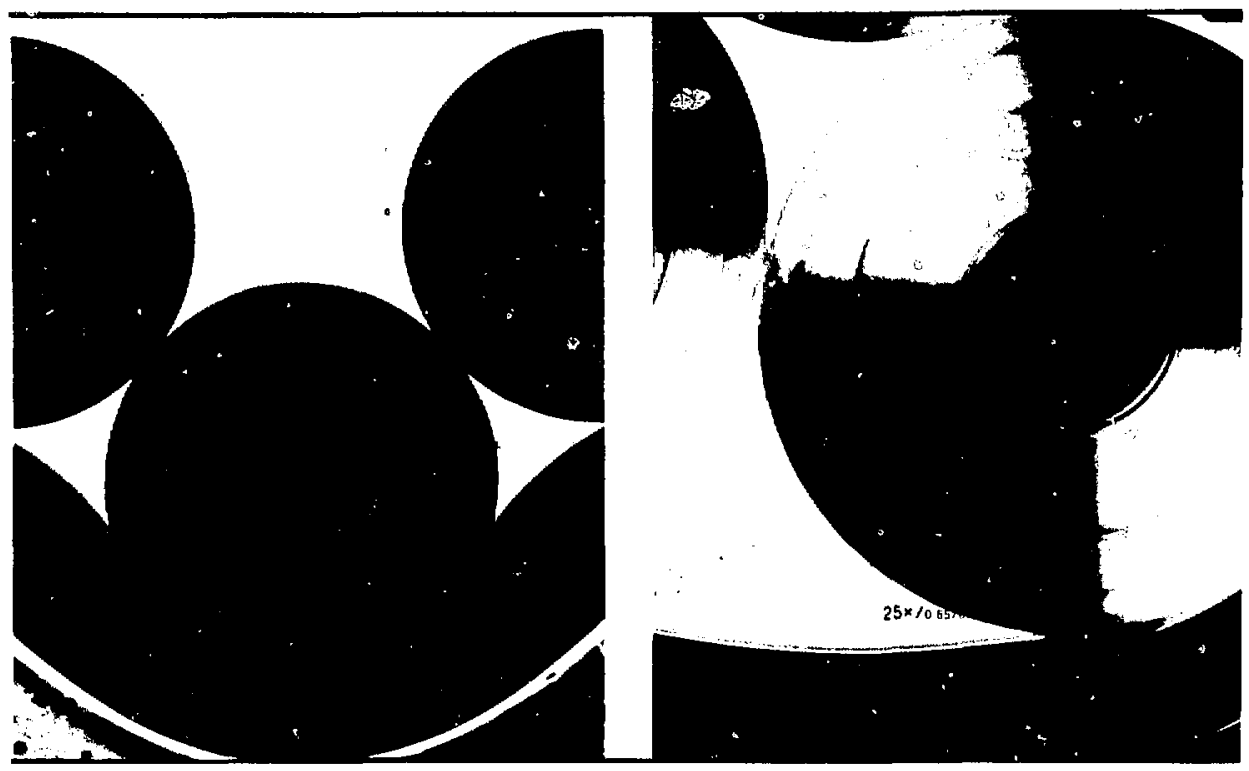

where $I_{B}=$ the normalized difference of BSE signals,

$I_{0}=B S E$ signal from the $0^{\circ}$ detector, and

$1_{180}=$ BSE signal from the $180^{\circ}$ detector.

The slope, $\alpha$, of the surface in our geometry is then given by

$$
\leadsto=\arcsin \mathrm{l}_{\mathrm{B}} \text {. }
$$

This is valid only for slopes up to $\pm 45^{\circ}$.

The computer controlling the SEM receives the slope data and processes them by calculating first a baseline between the detectors and then the height differences, displaying them on a graphics terminal. By scanning a raster pattern over the sample, the computer builds up a topographical image. Figure 4-67 is a topographic plot of aluminum metallization on a silicon wafer; the letters measured $2.5 \mu \mathrm{m}$ high and are clearly resolved.

Initial tests show good correspondence with
Fig. 4-65. Orientation of backscattered decectors to the sample for height measurement.

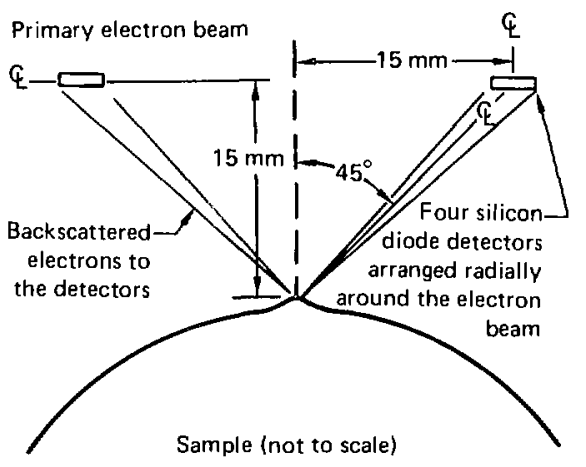

both a roughness standard and surface height resolutions on a sphere better than $0.5 \mu \mathrm{m}$. Our next steps will be to create microstandards for calibrations at $5000 \times$ mignification, establish optimum operating conditions, and evaluate the accuracy of 
the system on glass- and metal-coated spheres. When complete, the instrument will be used routinely for surface measurements.

Surface Analysis. Analysis of the composition of surfaces is important for solving problems encountered in developing new coating techniques or new high- $Z$ glasses. We have, therefore, installed at Physical Electronics Industries 590 , high-resoluti, n

Fig. 466. Fixture to hold the backscattered electron detector uround the electron beam. (The flxture mounts on the final lens of the SEM.)

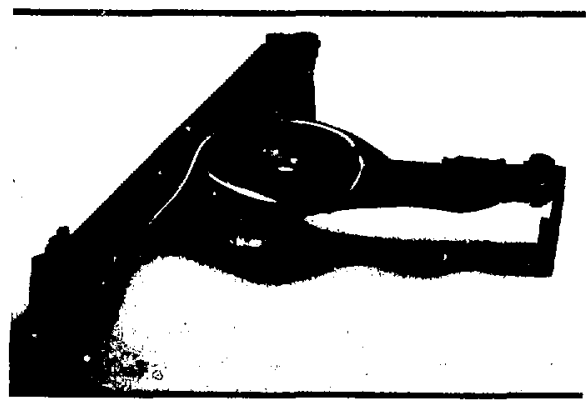

scanning Auger spectrometer for surfacecomposition analysis. (The system is shown in Fig. 4-68.) It has a $0.2-\mu \mathrm{m}$ spatial resolution and allows studies of the top 5 to $20 A$ of a surface, detecting all elements except hydrogen and helium. Figure 4-69 indicates the mean escape depth of Auger electrons for various elements as a function of the energy of the exciting electron beam. The sensitivity of our instrument is typically 0.5 at.\% and a 5-keV argon ion gun is available to make sputteretch depth profiles. Sample exchange can be made in 15 min through an air-lock system.

The simpiest application of Auger spectrometry is for planar surfaces such as metal films and multiliyer composites. We have profiled a gold surface cleaned in an oxvgen plasma. The result, shown in Fig. 4-70, reveals that the surface contamination layer on regular-production gold discs is less than $30 \mathrm{~A}$ thick. Most target applications stre not this simple and can be severely limited by damage from the exciting electron beam that decomposes $\mathrm{SiO}_{2}$, evaporates polymers, and causes mobile ions such as $\mathrm{Na}^{+}$to move. To minimize the exciting-bean current, we use pulse-counting elec-

Fig* 467. SEM topographical plot of an aluminum metallized silicon wafer. The metslization is $2.5 \mu \mathrm{m}$ thick. The inset is an SEM picture of the same aren.

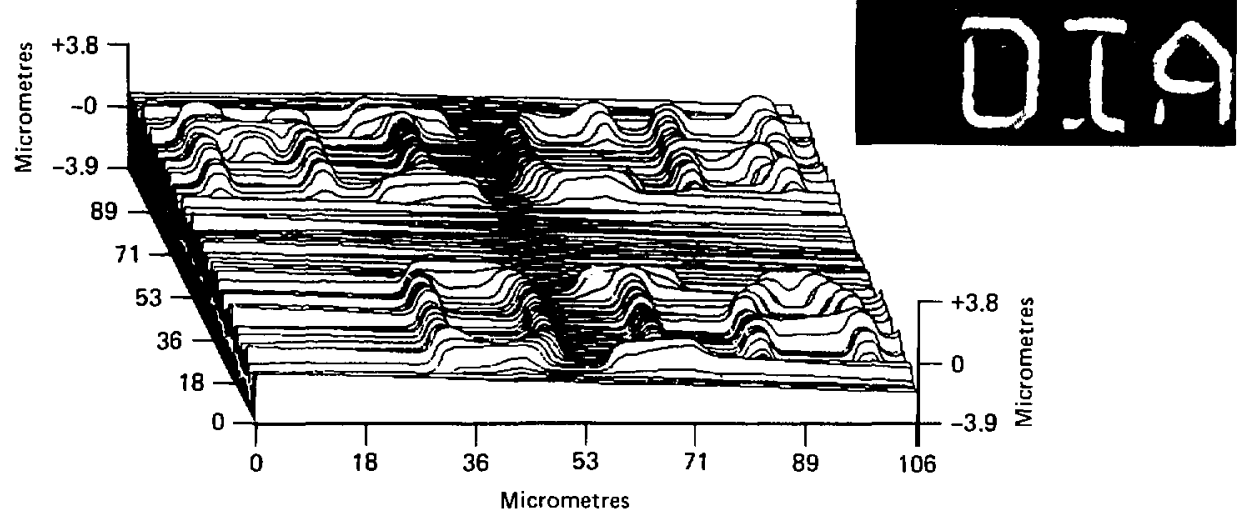


Fig. 4-68, PHI 590 scanning Auger.

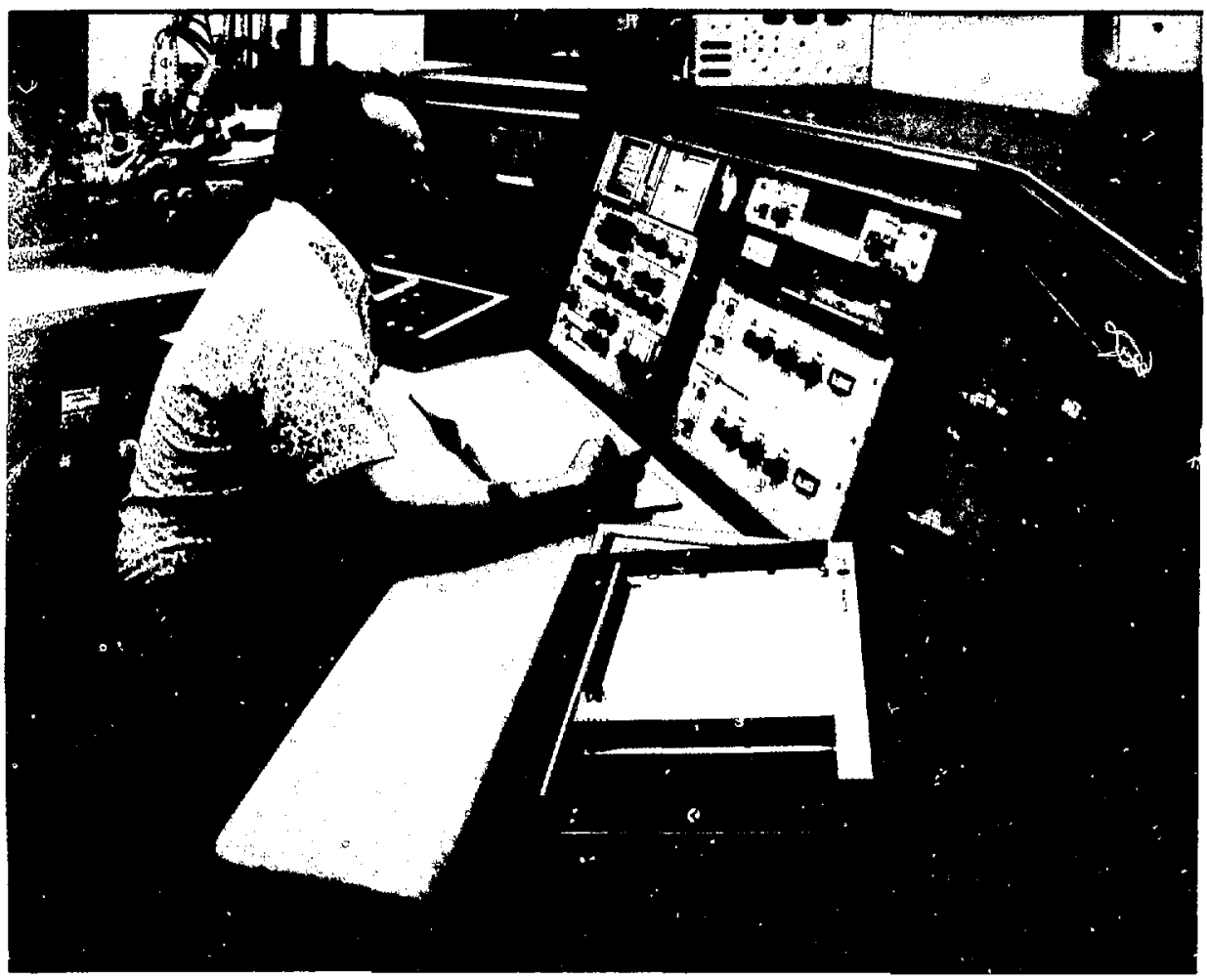

tronics to reduce noise and allow analysis at $1 \mathrm{nA}$ of heam current.

Analysis of metal-coated spheres is more difficult than analysis of planar surfaces. Because the argon-ion etch rate is a function of incicence a.ggle, it varies with location on the sphere. In addition, the etch beam is larger than a single target sphere. hence it sputters substrate material from areas outside the sphere. Some of this material is deposited on the sphere, contaminating the surface and complicating the analysis. To overcome this problem, we use thin silver epoxy as a nonsputtering mounting material. We are also working to calibrate the etch rate as a function of incidence angle so that we can correctly interpret the results of sputtering through the coating on a microsphere. Figure 4-71 shows a 500$A$ platinum coating on a glass sphere that is ad- ditionally covered by a large number of white particles and holes. Analysis with a $0.2-\mu \mathrm{m}$ electron beam reveals these particles to be pure platinum and not a contaminant. (Electron-beam-induced $x$-ray fluorescence cannot make this measurement because the area where $x$ rays are generated is larger than the particles we wish to sample.)

Auger electron energies are affected by the chemical environment of a materi\%l. Oxides and carbides appear as peak shifts: they can be used to determine not only the elemental composition of a sample but also to gain information about chemical compounding. Figure 4.72 shows a comparison of beryllium in an oxidized film with pure $\mathrm{Be}$; the shift from $\mathrm{BeO}$ to $\mathrm{Be}$ is quite evident. Similar effects existing with $\mathrm{SiO}_{2}$ and $\mathrm{PbO}$ are used to analyze glass spheres.

Glass and plastic are the most difficult samples for surface analysis. Not only do they decompose 
Fig. 4-69. Mean escape depth of Auger electrons.

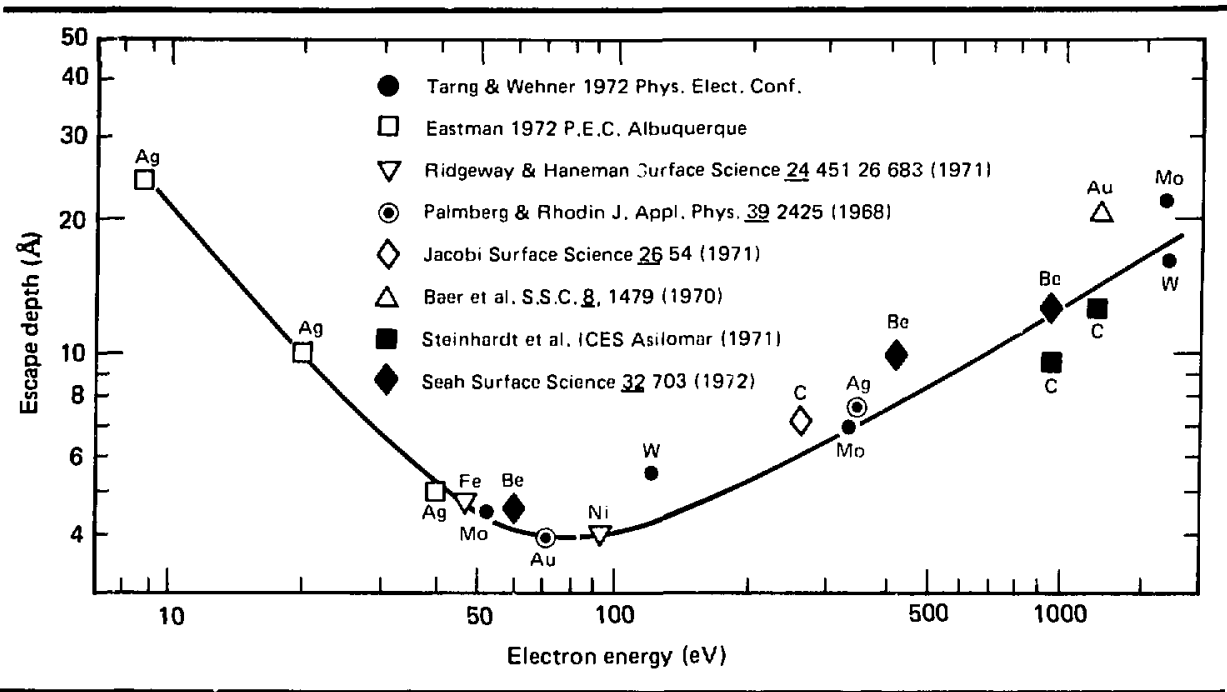

Fig. 4-70. Auger depth profile of an oxygen-plasms-ashed gold surface,

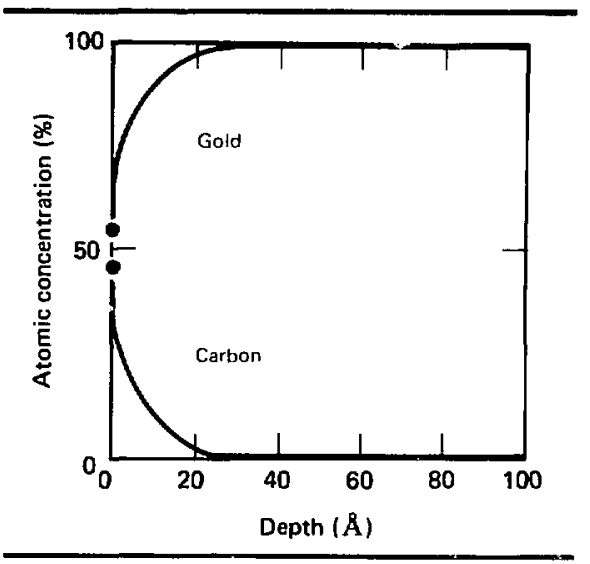

under the electron be:m, but they also become charged, causing instabilities in the measured energies. We can, however, reduce charging by lightly gold-coating before analysis, adjusting the electron-beam voltage, and taking data on an edge (see Fig. 4-73). These precautions make analysis of a crystal growing on a glass surface possible. As Fig. 4-74 shows, potassium and sodium form complexes
Fig. 4-71. Platinum coating $500 A$ thick on a glass sphere. Auger spectroscopy showed the small particles to be composed of pure platinum.

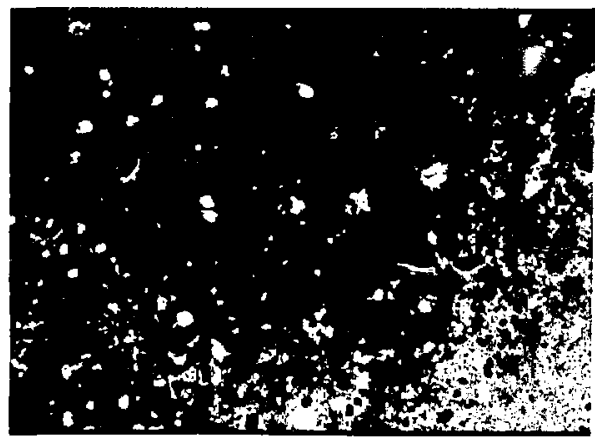

with carbon and nitrogen, which leads to large crystalline defects. Quantitative analysis is not possihle on this sample because the intensity of the sodium peak is rapidly reduced by ion migration caused by electron-beam heating and charge implantation. Experiments are underway with lower electron-bean currents and cooler samples that should improve our ability to handle such analyses. 
Fig. 472. Auger spectre of oxidized and moxidized heryllium. A prowownced Be shif fram beo to Be is vibible.

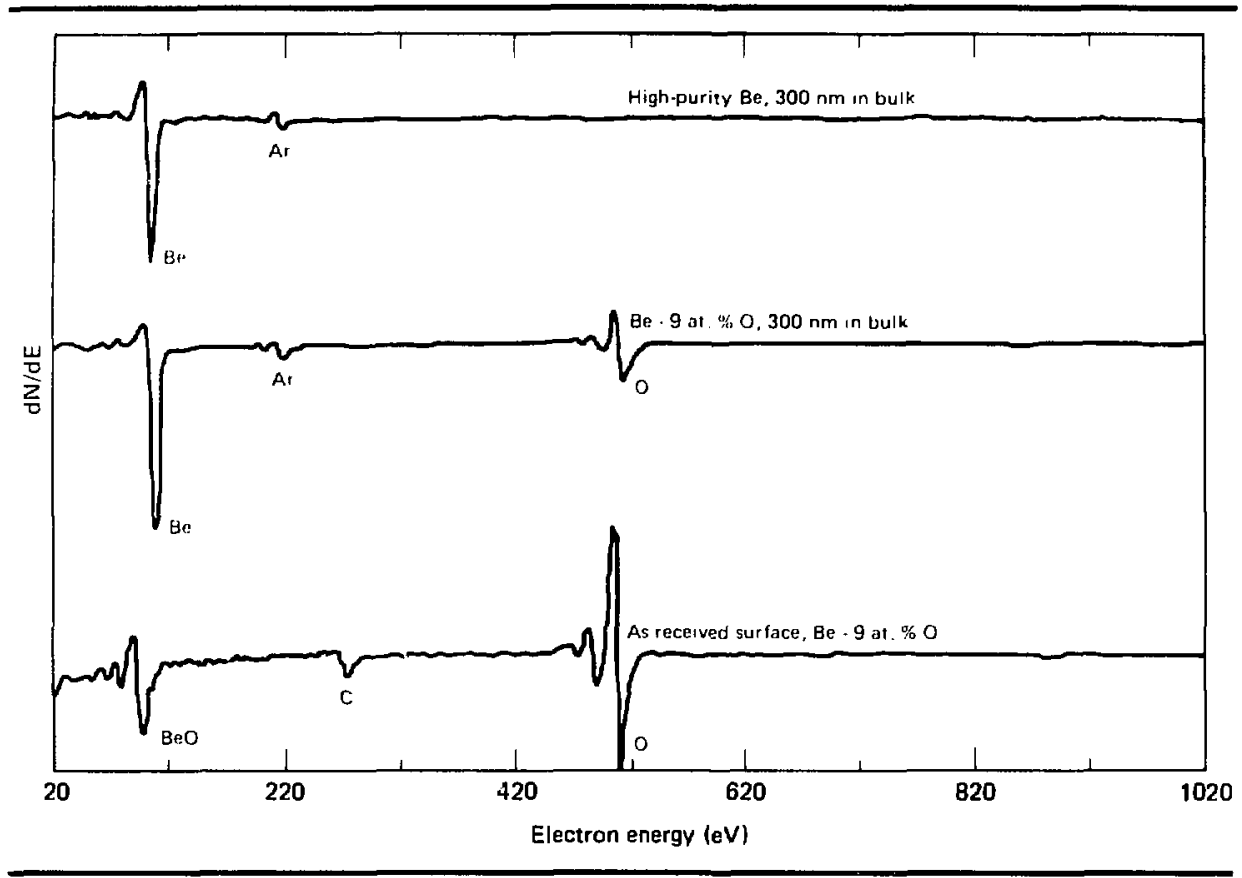

Fig. 4-73. Auger spectrum of a crystal on a glass sphere after sputtering off the surface contamination.

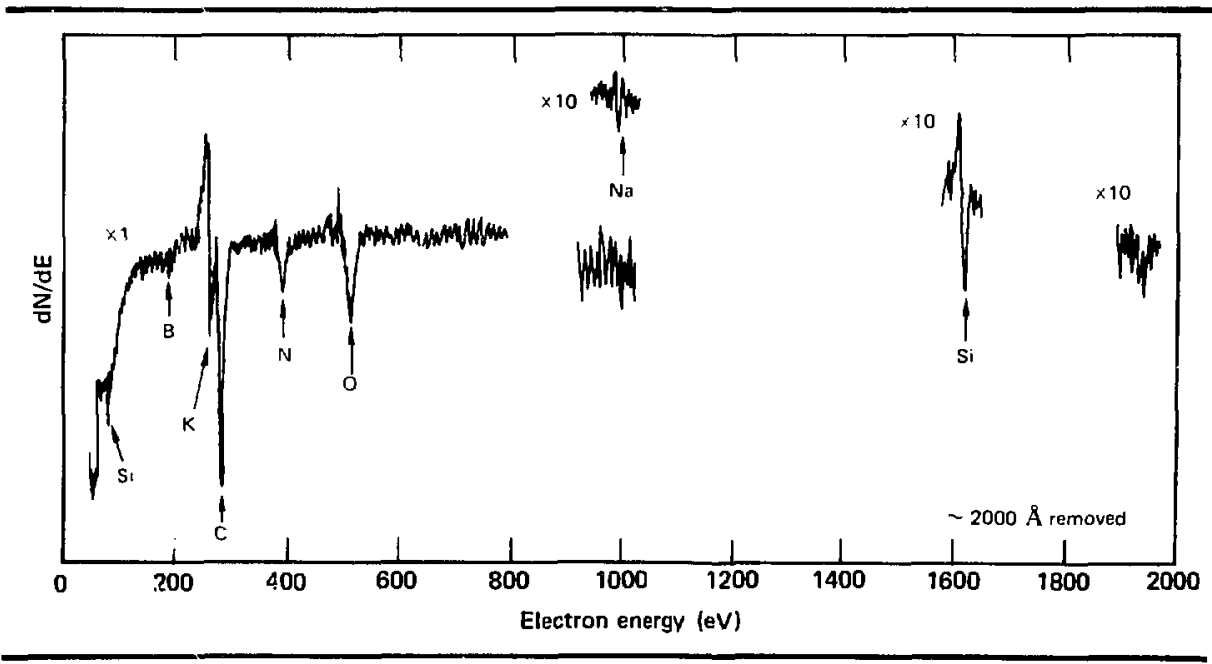


Fiz. 474. SIMS seetre of a washed $4 \mu \mathrm{m}$-wall glass spivere showing alkull depietion ecar the sorface.

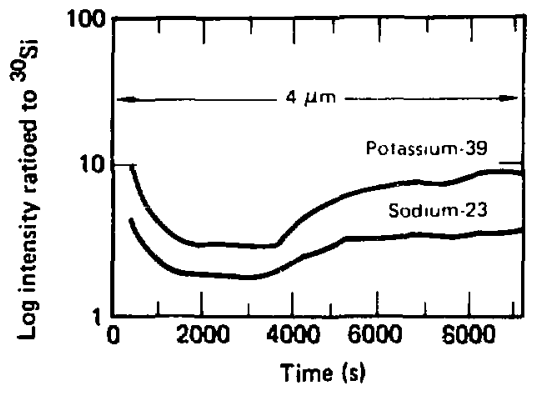

An alternate techneyuc we have used is ecoondar?

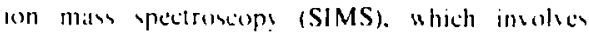
simular sputtering ol at simple and using a mass vectrometer to deted the ions produced. We are currently adding SIMS wo the Auger. Before nom ue hale used an un-prohe mass analy/er at Appliced Research Laboriturat (Los Angeles) lo oblam the data. Figure $4-74$ in a SIMS profile that show surlace alkali depletion from washing. The mosumont s heghly sensitice (1) alkalls but has onls a $2(0)$ $\mu$ m ratial resolution and barts widely in sertsiturles 60 wher elements. Combined with Aluger upectroscopl. howerer. SIMS an he used fo give accurate malerials analsuis ofi a hample.

As with all larget labrication work, surficte analy of of epherical samples is more compliatled than analuse of planar samples. We are descloping new techniques as handle these prohlems and are further exploring the applications of Auger snectroscopt to glassey and metals.

\section{Author: C. M. Ward}

Major Contributors: E. A. Austin, V. K. Chen, D, F. DelGuidice, C. L. McCaffrey, E. A. Pyle, K. L. Montgomery, and D. L. Willenborg

\section{Reference}

44. J. Lehiedrich. "An Autumatic Topongraphisal Sutlase Reconstruction in the SF M." Sianmm 2 (4). 231-23? (1979)

\section{Cryogenic Targets}

The Cryogenics Group has responsibility for labricating targets that contain ligutud or solid D-T fuct and for develoning the technology for placing there tangets for irradiation in the laser target chamher. To these ends. with the participation of NBS, Buslder. "se hitre comtinued work un a cryogenic pylon for holding and positioning eryogenic targeos) and hase bult and lested a protolype apparatu firt rapudls remosing crongenic shichds from around the largels. Adthlomislly, we hate iniliated

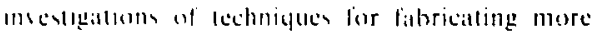

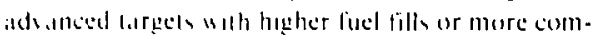
plicaled geomeleses thall simple glans fuel meroppheres. These tehnugues imolsc making. (has ractersing. and wanl whd D-T pellets, and usung lill tuber an formeng thech D)-T liners. Finalls. we harts undertaken , nexperi.n:esial and theoretical

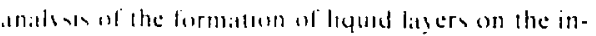
terone of glat pheres. I'nderstanding the profiles

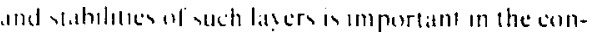

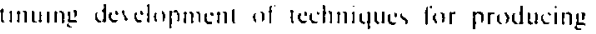
then unform fuel lasers m multilagered largets.

\section{Solid Hydrogen Spherical Pellets}

Sounc adsanced hegh-gann larged desigm are

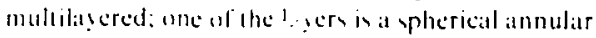
upilec litled wh whed D-T luel, and a central epherkall core is also tilled with whlid D. Tt: (schemallcitl! thown in r-ig. 4-75). Such targets might be regured lor power reaclors. (See also) rig. 4-83.) Targew w 1 h thick annular laners of solid D-T fuel, hut with no fuel core, will probably be investigated with the visal laser lakcility. Solutions to the problem af lorming thick annular fuel laters or boidless cores are difficult. We are investigating two possible approaches: the use of solid spherical fuel peliets, which we disets here, and the use of fill tubes, "hich an dencribed in "Condensed D-T Layer Formation." out next article.

Thick annular spaces in multilatered targets might possibly he filled "ith a "pouder" of very small close-packed D-T spheres whose diameters are much less than the thickness of the annulus. Such close packing necessitutes uniform sphere size in the powder. We have developed a hydrogen isotope spherical pellet generator to investigate and develop these techniques. 


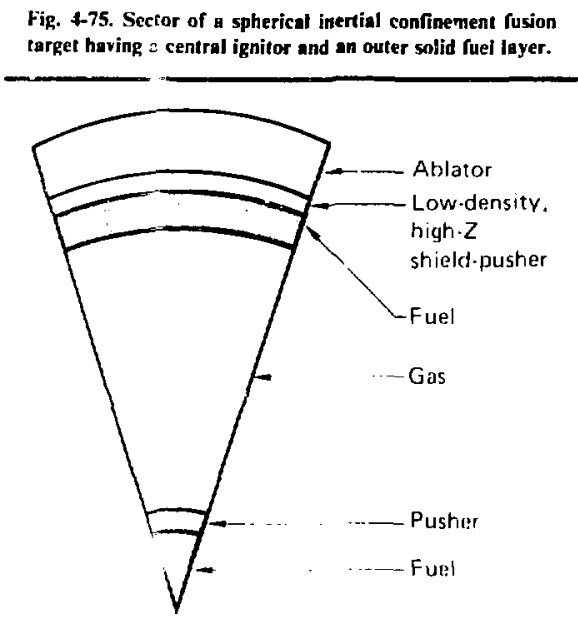

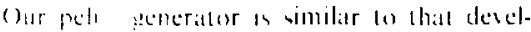
pred he lank Kim. Turnbull, and Hendrick the is

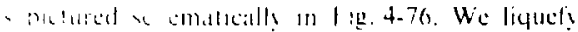

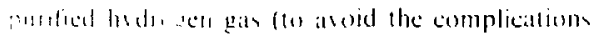

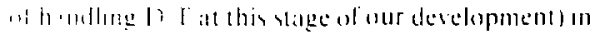

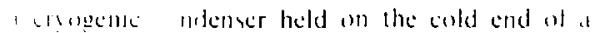

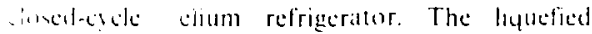
bedrugen is hen forced through an 8 - $\mu$ m-1.d.

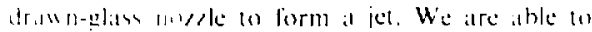
hrest the ice inte a sircian of tunform lequed upheres, each thout $10 \mathrm{~mm}$ in diameler, hy drong

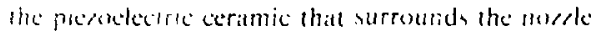
at a frequen: of exeral hundred kll, By manl.1mung the 1. . 1 pressure in the jet chalmter plast helou the errs e point pressure (5t Torr lor Hy). We ate able to situse the liquid drops to rapully saporate, the chy couling and frecing. The frosen pellets aill u mately fall into a cryogenie cattcher milnatined n i $4 \mathrm{k}$. For this reason, ue use helium gats 10 maint it the jet-chamber pressure, since $H_{2}$ would rapidl freere out on the enld catcher and neld the pellets together.

In order lo produce a stable liquid hydrogen jet, we found that the condenser temperallure must be maintained between 14 and $18 \mathrm{~K}$. Temperature is controlled by leeding back an electrical signal from a sensor to a heater on the refrigerator. It is also necessary 10 maintain the jel pressure above 150 Torr absolute and the $j \cdot t$ chamber pressure be-
Fig. 4-76. Ten- $\mu \mathrm{m}$ hydrogen powder generator.

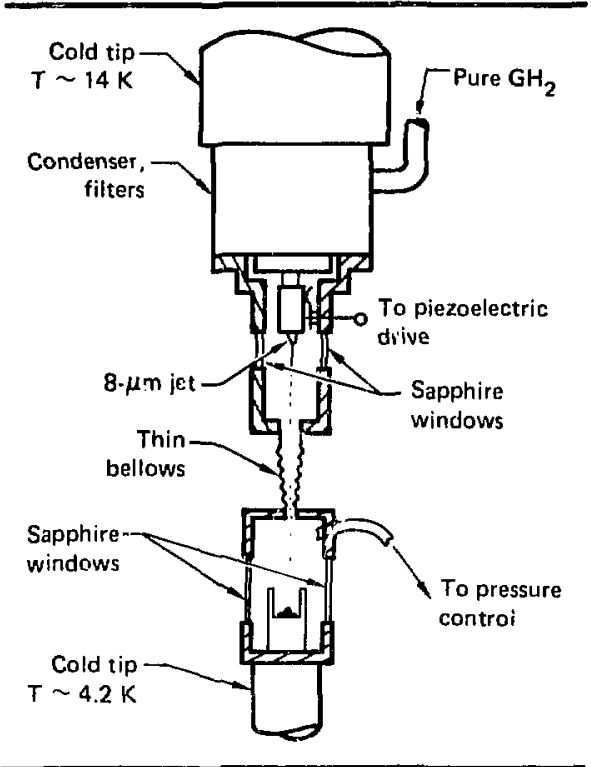

Iween 51 all a 54 Torr. We have designed and consrucled a gits control sylem for purifying control gare athd abaintaining control pressures.

Mantinning the jet issue from the nos/c reyutrus a gund deal ol allention to keeping smatl parinles or frectathle gals contaminants out of the yntem. We therefore inciaded liquid nitrogen traps an the gia and sacuum lines of the gas control butem. Additionally, the norde system was cleaned and anembled in a class 100 clean room. With these precitutions, wa were able to repeatedly produce a liquid-hy dirogen jet, and break up the jet by means of the picsoclectuc transducer. Our opics, however, uere incapable of resolving individual spheres.

In the future. we plan to upgrade our optics lor vingle-particle chatraterization. In addition. we will incorporate the eryogenic catcher into the system to begin characterising the aggregate character of the solid hydrogen powder, and sludy its close-packing properties. We will also increase the pellet diameters for possible use as centrill cores.

Author: T. P. Bernat

Major Contributors: B. H. Ives, B. C. Borman, and K. Kim (University of Illinois) 
Fis. 4-77. Schematic of apparatus to study cryogenic fill tubes and fuel layers.

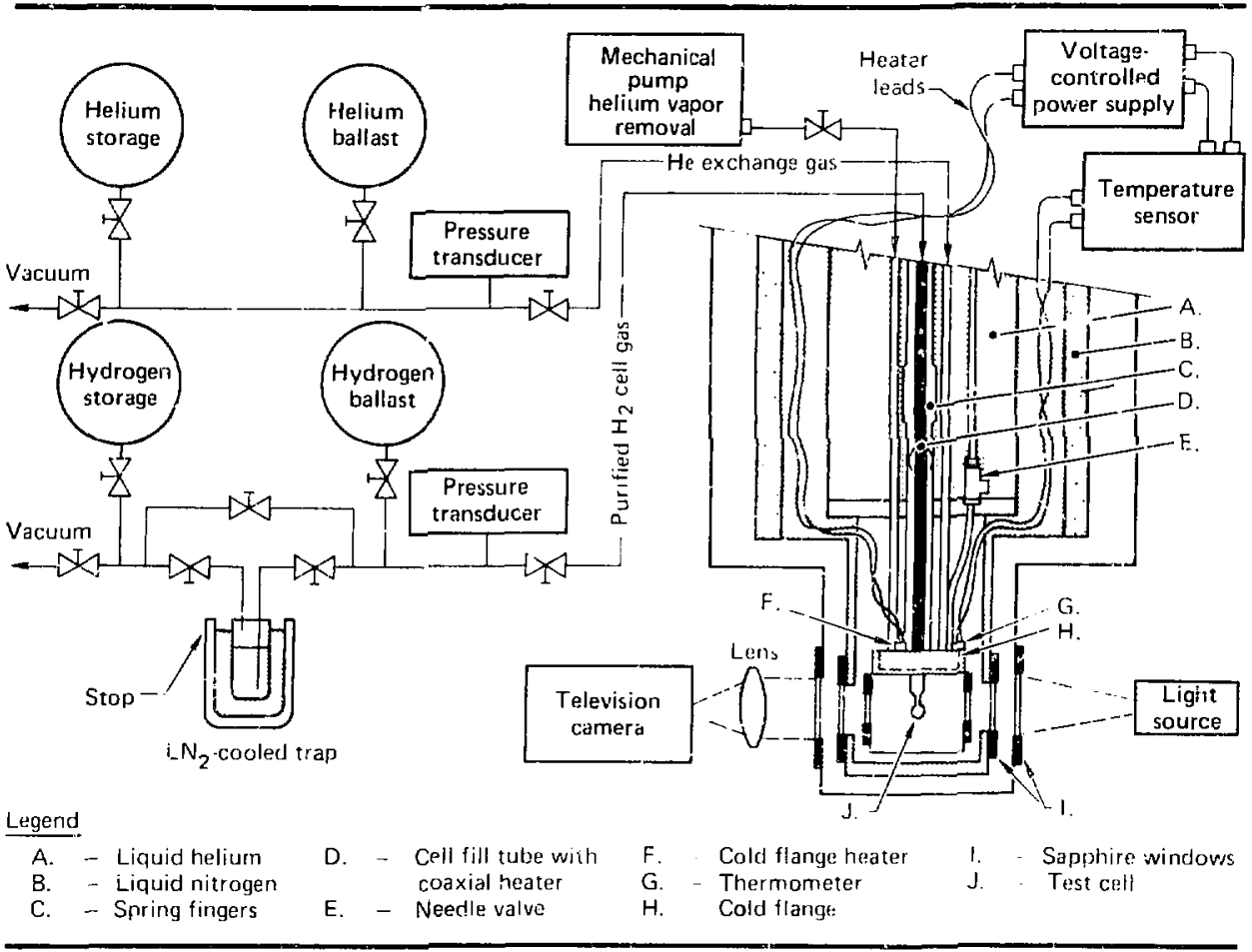

\section{References}

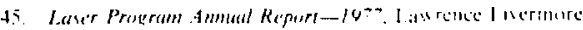

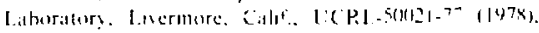
pr. $4.15+10+19$

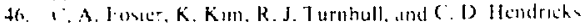
Ro Sis ins $48,625(2,7) 7)$.

\section{Condensed D-T Layer Formation}

Layers Condensed Through Fill Jubes. $A$ second method for forming thick. uniform annular idyers in the multilayer larget shown in Fig. +-75 is to condense the fuel through a small fill tube. Such an approach overcomes the probiem of the very high pressure fills necessary if the fuel were diffused into the glass hall prior to assembly and freezing. It also allows the fuel to be intreduced at a convenient time i: target assembly. It is dificult to imagine how sucin a target would be assembled without this or a similar menod of introducing the fuel. Fill tubes might also be useful in roid-free filling of central cures.
Our ohiectues in ihes studs are lourfold

- in explore the ve limilations imponed an fill lubes hy mechann:all. materials. and fabrication probisms.

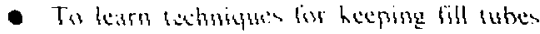
clear during luel lill.

- To suds the limmallem and etructure of thick bisers soliditied from the gits phase

- Tolorm weid-fres central cores.

To achiere there abictises. we have designed and bult a dimble-cemperallare cryostall capable of temperature conterol in the range 2 to $40 \mathrm{~K}$, shown sthematically in Fig. 4-77. Temperature is controlled by halancing the liquid-helium flow-from the main reserwir to the cold hinge through which it circulates--ilgainst electrical heating produced in a resistance wire wound on the cold flange. We 
determine liquid helium flow by setting a needle valve that is located in the helium restrvoir. Temperalture is sensed by a germanium resistance thermometer and the sensing signal is fed back to the heater. The optical windows on the cryogenic shield and chamber are made of sapphire, a material with good thermal conductivity that absorbs well in the inilrared. As a result, room-temperature radiation is frevented from reaching the experimental cell in the interiur of the chamber. Heliun exchange-gas provides thermal contact between the cell and chamher. We can introduec purified hydrogen gas II11 the cell through a vacu m-jacketed capiliary thit parses through the liquid- eliuns reservoir. The gia in cooled by radiation and by "spring lingers" that entiblish thermal transfer between the lower portion of the capillary and the liquid helium. To prevent frozen hydrogen rom clogging the capillary, which would result from the temperature oi liquid helium $(4.2 \mathrm{~K}$; hydrogen freezes below $14 \mathrm{~K}$ ). we have installed a coaxial, small-radius heating wire through the capillary. An additional heater below the cold flange prevents clogging in the ricinity of the llange. By controling the two heaters, we can control the temperature of the hydrogen gas entering the cell.

$A s$ in any cryogenic experiment, all gases introduced to the cryostat must be free of dirt or conden:iible impurities. We have constructed the gashandling system shown in Fig. $4-77$ to provide purified helium and hydrogen. Wi can accurately measure and control gas pressures and can control the hydrogen-gas flow rate.

At the time of this writing, we are just beginning experiments on layering and widless fillung of a l-mm-diam blown glass sphere equipped with a 0.05-mm-i.d. lill tube. After gaining experience, we will attempt smaller spheres with approximately 0.01-mm-diam fill tubes. Techniques must be developed for fabricating these spheres for cryogenic survival.

Liquid Layers on the Interior of Glass Spheres. Current laser fusion targets are fabricated by coating diffusion-filled glass microspheres with one or more layers of organic or inorganic materials. The glass microspheres range in size from 70 to $140 \mu \mathrm{m}$ and contain only a few tens of micrograms of D-T fuel. When such targets are cryogenic, the
D-T fuel must form a thin, uniform, and smooth layer on the interior of the glass microsphere. These layers will typically be 1 to $5 \mu \mathrm{m}$ thick. Two methods for forming the frozen D-T layers on the interior of uncoated glass microspheres developed at LLL and $\mathrm{LASL}^{47}$ a... reviewed in the $1977 \mathrm{An}$ nual Report. ${ }^{48}$ Briefly, the irregular frozen fuel in a cooled target is vaporized by a heat pulse and allowed to "rpidly refreeze on the interior wall directly fom the :apor. This process consistently produces uniform frozen layers in these thin-walled ( 1 to $3 \mu \mathrm{m}$ ) bare-glass microspheres. The process relies on rapid heat removal from the vaporized D-T fuel, however, and the extrapolation of the technique to layered, multishelled targets may not be simple. It is possible that in thickly coated targets the D-T fuel may condense out as a liquid that then freezes. While liquid, the fuel can flow to the bottom of the sphere. In designing specifically cryogenic ICF targets, it is important to know the time- and temperature-dependence of the liquid D-T layer structure, so that the maximum permissible time in the liquid state for al given layer sphericity can be seternined. The thermal transfer properties of the target must then be designed to ensure that the liquid freeses before this time. With this consideration in mind, we have begun a theoretical and experimental study of the structure and stability of the liquid fuel layer. Our objective is to develop a quantitative, physical model of such layers that will be useful in the design of eryogenic ICF targets.

An earlier, unpublished liquid layer model by Camphell ${ }^{49}$ is available, but it is not consistent with the hehavior of $D_{2}$ liquid layers observed in recent experiments performed at the $U$ iversity of Illinois. ${ }^{50}$ In these experiments (see Fig. 4-78), initial liquefaction produced-far above the triple point-a uniform layer of $D_{2}$ that became thicker as the temperature of the sphere was decreased [Fig. 4-78(b)]. Below a certain temperature, the thick layer became unstable and collapsed into a localized droplet that collected at the coldest part of the sphere when a temperature gradient was present [Fig. 4-78(c)], and in the bottom of the sphere when the temperature was uniform. Increasing the temperature reversed the behavior of the liquid laver. Similar behavior has been observed by other groups. ${ }^{51}$

The thick uniform layer of Fig. 4-78(b) is not indicated by Campbell's model, which instead predicts that the layers will always have zero 
Fig. 4-73. Mach-Zehnder interferograms of liquid $D_{2}$ hehavior formed from 186 standard atmospheres in a 200-by-7- $\mu \mathrm{m}$ glass sphere: (a) ao $\mathrm{D}_{2}$ liquefied; (b) a 5 - $\mu \mathrm{m}$ uniform liquid layer far above the triple point; and (c) collapse of die layer to a droplet.

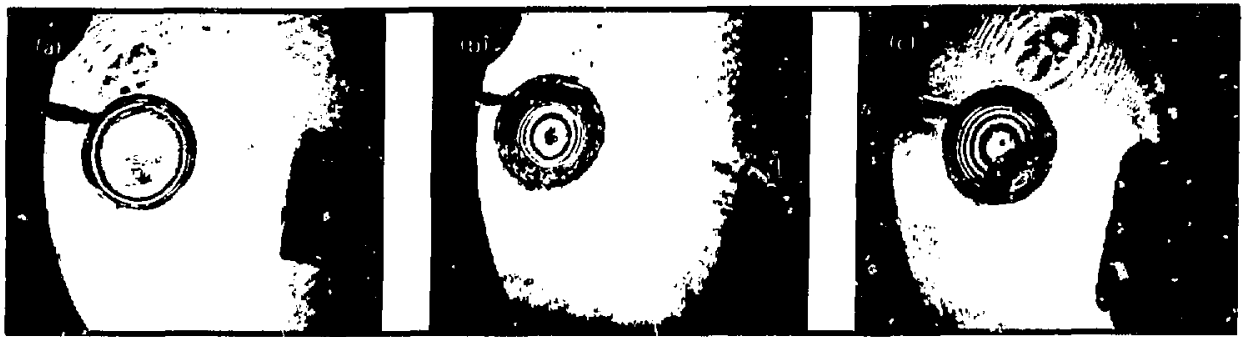

thickness at the top of the sphere. Campbell's model includes the cohesive attraction between the lioutid and gla. : substrate only through the contact angle. The model therefore precludes continuous liquid layers, since, in such layers, contast angle plays no role.

We include the cohesive substrate attraction explicitly in our model, because it is the only possible explanation for the continuous layer of Fig. 4-78(b). We express the attraction through a surface potential of the form $V(r)=-\alpha r^{-3}$. where $\alpha$ is a constant that depends on the substrate material, and $r$ is the distance between the substrate and the liquid surface. This form for the potential is taken from the literature that deals with superfluid helium film profiles on vertical substrates. ${ }^{52} \mathrm{~A}$ simple balance between this surface potential and gravitational energy, with no bulk surface tension effects included (appropriate near the critical point). yields the liquid profile on the inteitor of a sphere shown in Fig. 4-79. To continue this work, we will include the effects of surface ension. which must lead to the collapse of the lay to a spheroid [Fig. 4-78(c)]. We will also study the dynamics of the instability leading to the collapse in order to predict the collapse time scale.

\section{Author: T. P. Bernat}

Major Contributors: K. Kim (Lniversity of Illinois) and $B$. $H$. Ives

\section{References}

47. J. R. Miller, "A New Method for Producing Cryogenic laser Fusion Targets." $A d v$, in Cryog. Eng. 23. K. D. Timmerhaus. ed. (Plenum Press. New York. 1978), p. 669.

48. Laser Program Annual Report-1977, Lawrence Livermore Laboratory. Livermore. Calif.. UCRL-50021-77 (1978). pp. $5-38$ to $5-42$.
Fig. 4-79. Thickness profile of liquid $D_{2}$ layer on the Interlor of a splecre for a substrare potential $V(r)=-u r^{-3}$. The layer is thickest at the bottom ( 180 degrecs down from vertical).

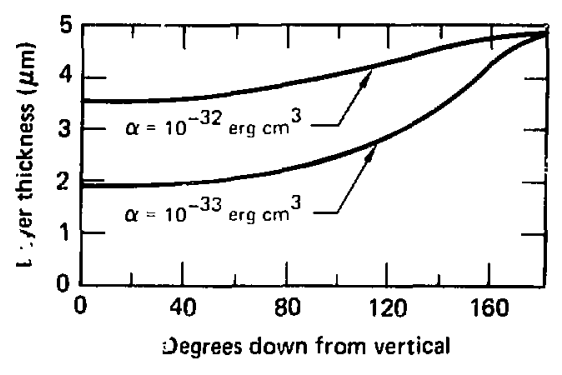

49. I. J. Campbell. Los Alamos Scientific laboratiory. unpublished (1974)

50. K. Kim and II. Reger (both Lniversily of Illinois), unpublished: H. Rieger. "Fabrication and (haracterization of Cryogenic Targets for Inertial confinement I usion." Master's Thesis. Universily of Illintsis at l'rhatha Champaign (1979. unpublisbed).

51. T. M. Ilenderson. R. B. Jacobs, D. L. Musinsk। R. J Simms and (i. H. Wattke, in Ads in Crog. Fing . 4. Vex York. Plenum Press, 1976, p. 690: I: R. (irilly, ihid, 5. 676.

5. K. R. Alkins. "Helium Films." Progress in Low Temperalure Physics. Vol. $V$. . North Holland. 1957.

\section{Cryogenic Target Systems for Shiva Target Chamber}

Cryogenic Pylon. In our last Annual Report. ${ }^{5,3}$ we described a cryogenic pylon that has two capabilities: It can transport a cryogenic target containing a preformed solid D.T layer to the Shiva target chamber. It can also form the layer after the target has been placed in the target chamber at 
room temperature. Workers at the NBS, Boulder, have tested several components of this original design.

They lested the electromagnetic repulsion system for the cryogenic target cell at room temperitlure. The electromagnet inductance was $150 \mu \mathrm{H}$ : discharging a $160-\mu \mathrm{F}$ capacitor at $435 \mathrm{~V}$ through it gave an average velocity of $259 \mathrm{~cm} / \mathrm{s}$ to a $5.1-\mathrm{gm}$ aluminum test eell with a $3.6-\mathrm{cm}$-diam repulsion flange. The flange would thus clear the target in less than $10 \mathrm{~ms}$. This performanee should be improved with both the repulsion coil and the cell at ir negenic temperalures.

VBS. Boulder, also tested the thermal performance of the intermediate cryogenic shield surrounding the target ell (the shield is held to the cryogentc tip of the pylon by gold-plated spring lingers). When the point of attachment of the shield (1) the pylon was at $20 \mathrm{~K}$. the temperature of the vheld itself was $40 \mathrm{~K}$ at the attachment and $43 \mathrm{~K}$ at the free end. No other cryogenic shields were located between the test shield and the roomtemperature vacuum chamber, hence the heat load was due only to radiation. We conclude that this intermediate shield arrangement would be more than adequate in actual use.

As we discussed in the last $A$ nnual Report, ${ }^{53}$ firing any eryogenic target in the target chamber will require the presence of a cold, light, rapidly removable cryogenic cell to maintain the target during final positioning. The cell must be coolable to $10 \mathrm{~K}$ or below while exposed to ambient radiation and must contuin several hundred millitorr of belium exchange gas to provide thermal contact with the target. The exchange-gas escape rate must be less than $0.5 \mathrm{scc}$ to prevent the target chamber pressure from rising above $10^{-.5}$ Torr. The cell seal must, however, be completely dry and easily broken in ensure that the target will not be jarred by the cell removal.

$\checkmark$ ie are developing two types of seal, which are shown schematically in rig. 4-80. The lapped flange seal can be cooled by a cryogenic target mount flange. Thermal transport occurs by means of the exchange gas in the seal gap. whose pressure must be high enough to fall in the gas-conduction regime. For a 10- $\mu \mathrm{m}$ gap, this pressure is above $300 \mathrm{~m}$ Torr. In preliminary work, we measured a leakage rate of
Fig. 4-80. Schematic of two types of dry, demountable cryogenic seals: (a) lapped fange and (b) laynred mylar.

(a)

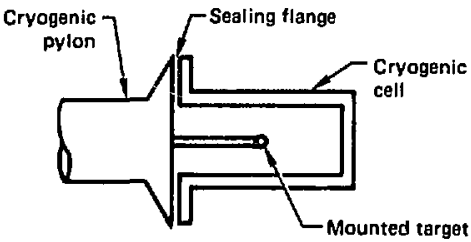

(b)

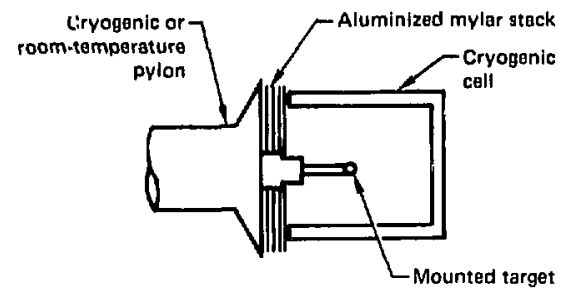

$0.3 \mathrm{sccm}$ with a cell-gas pressure of about $500 \mathrm{mT}$ Trr. The mounting flange was cooled to about $10 \mathrm{~K}$, and the $12.7-\mathrm{mm}-0 . \mathrm{d}$., 6.4-mm-i.d. flange was lapped to a $10-\mu \mathrm{m}$ finish. These results are encouraging. However, the lowest cell temperature obtained was only ahout $20 \mathrm{~K}$, and in continuing work we will attempt $\mathbb{t}^{-}$'ower this by surface treatment of the mating flanges and by varying the geometry of these surfaces.

The layered mylar seal provides thermal insulation, as well as a slow leak rate, and it is very useful when the target is held by the sort of noncryogenic pylons that are currently in use. In this case, all cooling must be provided by the cell retraction mechanism. In our initial tests with the cell around $20 \mathrm{~K}$ the seal leak rate was $0.018 \mathrm{sccm}$ with 1 Torr cell pressure. The leak rate for this type of seal is a strong function of sealing force.

We have embarked on the development of a cell retractor capable of providing cryogenic cooling to the target and target cell. Figure $4-81$ is a schematic of the cryogenic cell retractor. The cryogenic shield is thermally coupled to the cold finger by helium exchange gas. This shield can act as the freezing cell for a target held on a room temperature mount using the layered mylar seal (as shown in Fig. 4-81). Alternatively, it can completely 
Fig. 481. Schematic of cryogenic retractor. Cooling is provided by the retractor mechanism, but can also be supplied by the torget mount.

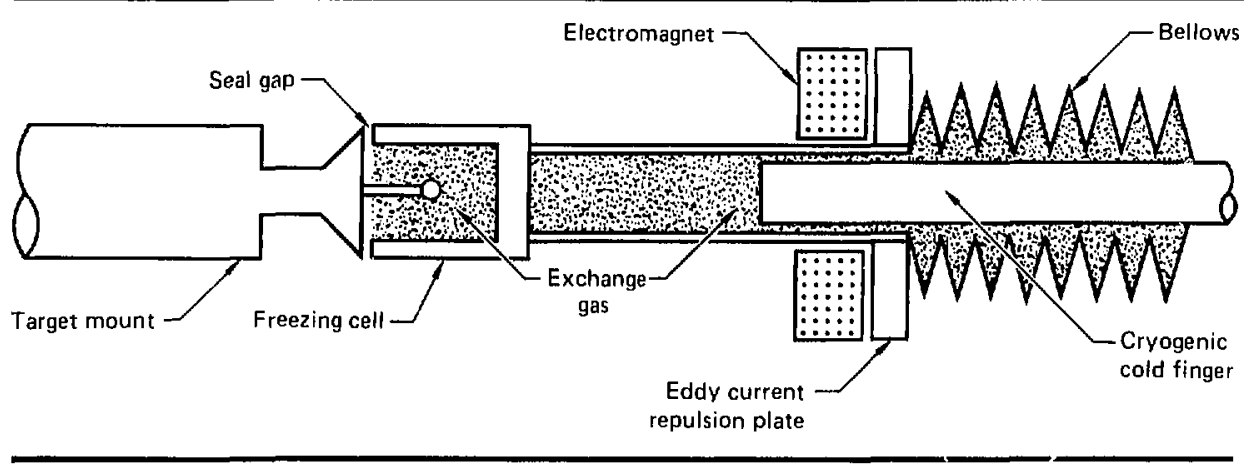

cover a freezing cell held on a cryogenic mount, thereby providing a radiation shield. Again, NBS, Boulder, is participating in this work.

In operation, the cryogenic shield is retracted from the target by an electromagnet repulsion scheme similar to that used in our earlier retractor designs. The retraction compresses the soft bellows, which thus absorbs the impulse from the electromagnet. NBS. Boulder measured the shield position vs time of a prototype retractor at room temperature to obtain the results shown in Fig. 4-82. The time for the tip of the shield to clear a target is about $3 \mathrm{~ms}$. This is at least an order of magnitude faster than the fuel vaporization time in the presence of radiation.

They have also tested the thermal performance of the prototype and found that the cryogenic shield varied between 5 and $14 \mathrm{~K}$, depending on radiant heat load, even though the temperature of the thin sleeve around the cold finger remained at $5 \mathrm{~K}$. This indicates a high thermal resistance between the shield and the sleeve, probably due to an epoxy layer between the two. We will replace this epoxy with a more highly thermal conductive solder.

\section{Author: T. P. Bernat}

Major Contributors: R. O. Voth (NBS, Boulder) and D. H. Roberts

\section{Reference}

53. Laser Program Annual Report-1978. Lawrence Livermore Laboratory, Livermore. Calif., UCRL-50021-78 (1979). pp. 4-45 to 4-49.
Fig. 482. Results of tests on cryogenic retrector: shield position vs time measured in air at ambient temperatures.

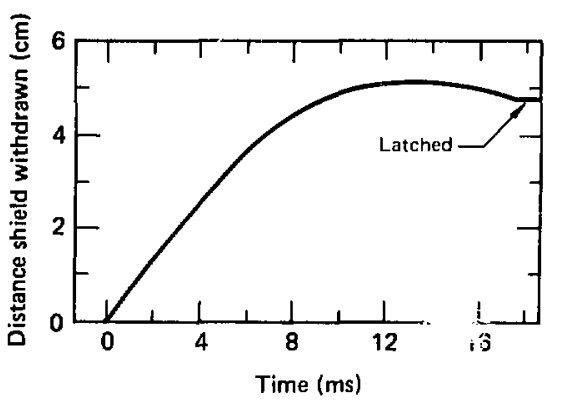

High-Rate Manipulation, Coating, and Transport in Target Production

To continue our efforts of 1977 and 1978, we are studying the problem of producing ICF targets at a high rate and with high yield in a "Target Factory." In this article, we evaluate possible incorporation into a factory scenario of some techniques developed or perfected during 1979: we also attempt to identify areas where further work is needed. In what follows, we consider the double-shelled target shown in Fig. 4-83, which has many of the features 
Fig. 4-83. The target designed for use in reactors in a $0.5-\mathrm{cm}-d i a m$ nested multishell (each shell consists of several layers.)

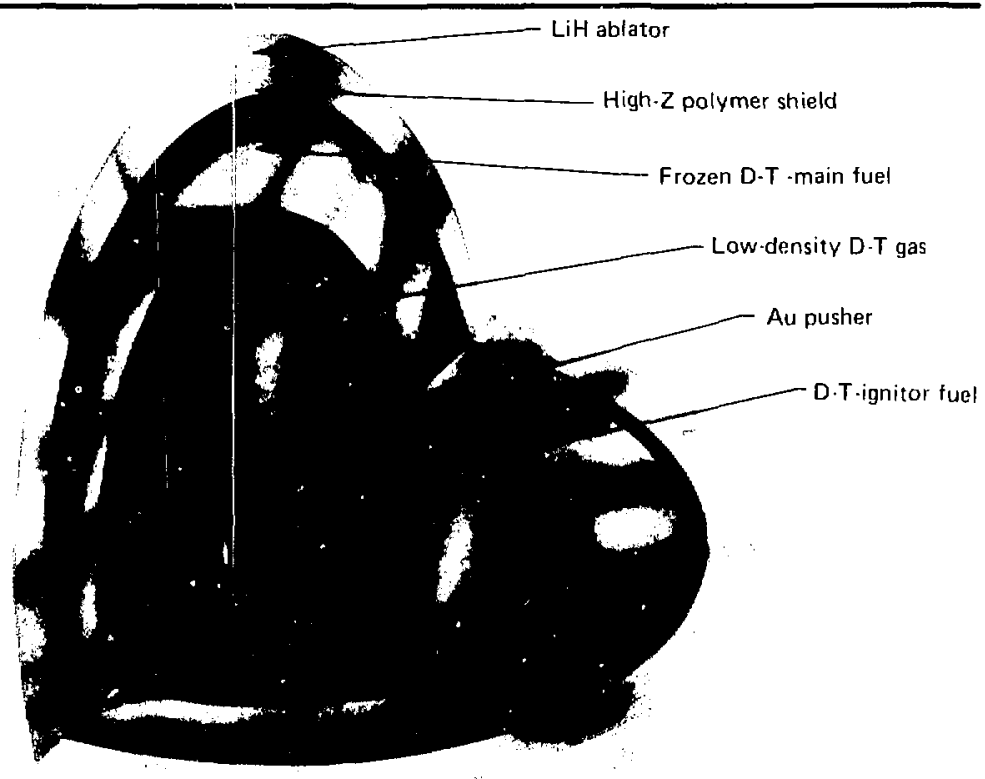

of the complex. reactor-type targets that must be built in an automated processing system. We discuss the production processes for each of the elements of the target in the paragraphs that follow.

Inner Shell Production. The inner high-Z, highdensity coated shell can be fabricated with a system that we outlined in the 1977 Annual Report. ${ }^{54}$ In this system, the shells are formed. filled, characterized, and coated with several layers.

Second Shell Prodiction. The technique of nesting a multilayer shell inside a larger second shell with centering tolerances of $\pm 1 \mu \mathrm{m}$ has been demonstrated (described in "Double-Shell Targets," this section). Considerable development is needed to bring this lechnique to production rates. The inner shell could be delivered to the thin fitms at high rates and sandwiched between two films in much the same way that small parts are packaged. The lower film would constitute a conveyer belt upon which the shells are deposited, and the upper film is pressed over it in a compression system that is shown in Fig. 4-84. The success of this approach would depend on the development of noncontact suspension techniques of very thin films $(0.1 \mu \mathrm{m})$. With this technique of inner-shell suspension, the second shell must be assembled about the inner sheil from hemishells. (Several techniques of forming tite hemishells are described in "Double-Shell Targets.") Machining complete hemishells is a process that could extend to a production system 
but may be very expensive and may preclude our trying to provide these largets for a few cents each. There are other approaches, such as molding, that offer the possihility of reducing the cost of compiete hemishells.

Assembling hemishells accurately about a sandwiched shell has a comparable analog in current production technology. Mask alignment in the semiconductor industry is routinely accurate to well within $5 \mu \mathrm{m}$. Probably an automated optical leedhack manipulator systen could align henishells. though the expense of such a process could again be prohibitive.

Assemhling these hemishells in a high-pressure I)-T chamber can provide the fuel inside the outer shell that is later frozen to a uniform layer. as diszussed in "Condensed D.T I.ayer formation." this section. Since the bulk of the fuel in these targets is contained in this layer, filling towards the end of a target process minimizes the tritium inventory in the target factory. The tritium inventory will then he essentially the fuel in those targets stockpiled against an interruption in target factory output.

The last technique for forming the outer shell is an extension of the tecbnique discussed in "Molecular Beam Levitation During Coating." above. An assembly consisting of the inner coated shell and webbing is nested in a nandrel shell formed from thin hemishells. These hemishells are very thin and are only a small fraction of the thickness of the ablator layer. Tbis complete assembly is then coitled as a shell lo provide a seamless second shell.

Because the mandrel is very thin. it cannot conlain the fill at noneryogenic temperatures. The coating process is then constrained to coat with optimum surface finish at cryogenic temperatures. Physical vapor-deposition coating techniques would be required at low coating rates to minimize heating, as well as in the presence of an exchange gas. These constraints make the coating process very difficult. The resultant low coating rates would require that the targets spend a much longer time in processing. The extended processing time can boost the tritium inventory to an unacceptable level. A better option may be to coat targets unfilled except for the fuel in the inner high-Z coated shell and develop drill and plug techniques to fuel the target late in the processing sequence.

Transport Levitator. Projected laser fusion targets for power reactors will require surface
Fig. 4-84. The shells are placed on a thin plastic film and then sandwiched by the addition of a second film.

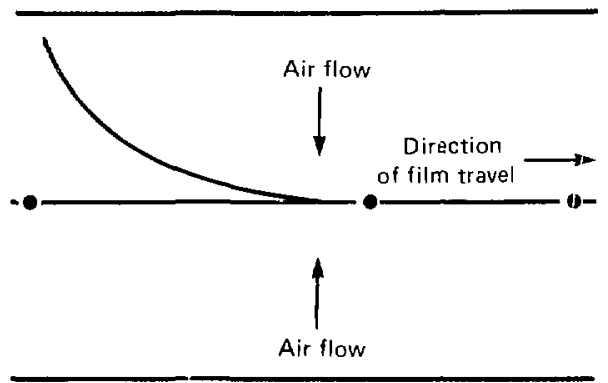

finishes on the order of $100101000 \mathrm{~A}$ : further, the largets nust be delivered with reliabilities approaching $100 \%$ One concept for providing these targets is to sequentially lead a single target through several well-characterized processes. ${ }^{54}$ Contact-free support. manipulation, and transport of these largets ease contamination problems and should improve the controllatility of any process. Several levitation schemes seem capable of this task, but none is universal. The suitability of any scheme is specific to the material of the target and the coating process.

Optical levitation ${ }^{55}$ uses photon momentum to support and move shells. The photon flux capable of supporting targets will heat the target to a few hundred degrees (entigrade in the vacuum environment required in many processing steps. This heating may be unacceptable. Acoustic levitators fail to operate in a vacuum, but they may find use for transport hetween processes because of their simplicity and reliability. This is true even though the target must be brought to near atmospheric pressure before transport and must be returned to a vilcuum environment before a subsequent vacuum process could proceed.

Our efforts in developing the molecular beam levitator ${ }^{56.57}$ show it is capable of providing support in the range $10^{-4}$ to $10^{-5}$ Tors, which is sufficient for many processes. One possible drawback to the molecular beam levitator is that advanced manipulation systems could require elaborate, very low-pressure pneumatic systems.

Magnetic levitators ${ }^{58}$ require some process to provide a high-quality ferric coating before the shell 


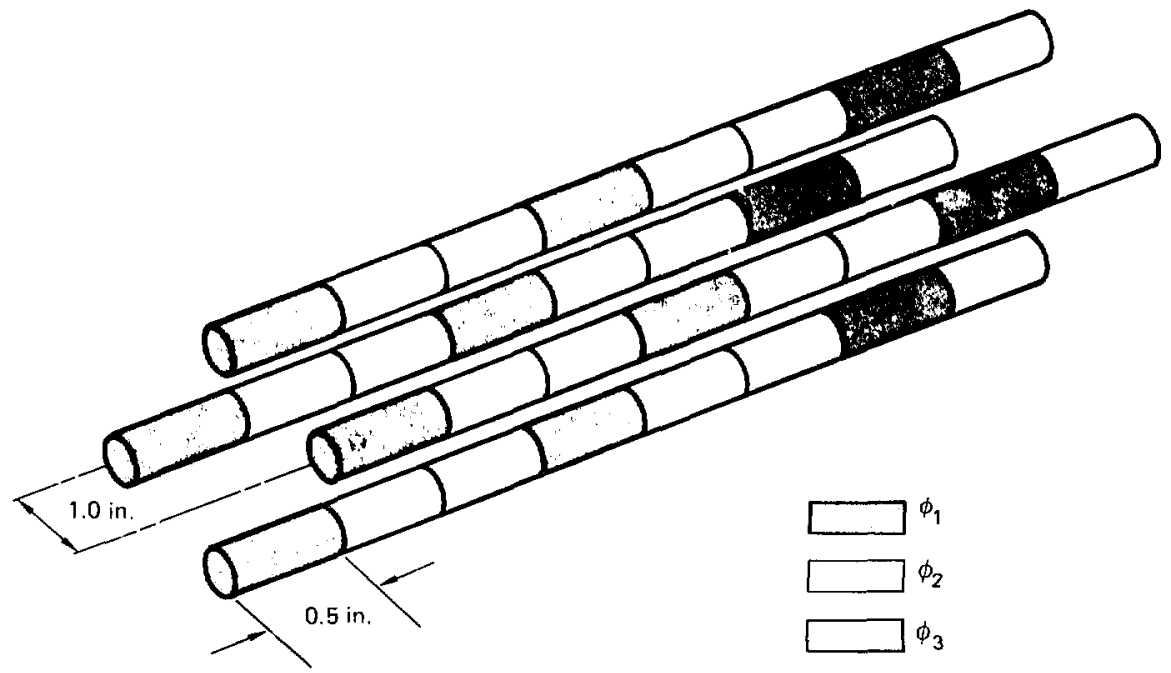

could be injected into the general handling system. The use of ferric material could also be detrimental to the target design.

Hectrostatic and electrodynamic levitators (shown in Fig. 4-85) apply a foree to the charged shell by controlling the electric fields at its strface. ${ }^{59}$ This type of levitator is independent of pressure. matcrial. and temperature. but the shell must remain reliably charged during processing. We earlier demonstrated this type of levitator in a quadrupole ring configuration ${ }^{\text {fll }}$ that was unable to transport charged largets: it could only support them. We have now extended this effort to incorporate transport.

We have again constructed a prototype linear electrostatic levit:1tor around a basic quadrupole rail that is segmented, as shown in Fig. 4-85. As in any quadrupole. the opposite electrodes $A+C$ are driven $180^{\circ}$ out of phase from electrodes $B+D$. In addition, a de potential is applied between the vertical electrodes $A+C$ to offset gravity. With the currently lised charge-to-mass ratio of $10^{-4} \mathrm{C} / \mathrm{Kg}$. this offset potential is 300 to $500 \mathrm{~V}$. Impressed on these electric fields are the fields to transport. The schematic in I ig. 4-86 shows the; voltages delivered to the transport rail. Vvery thi d segment of each rail is drive 1 in phase from a 'ource of three-phase ac at $\pm 1 \mathrm{kV}$ peak to peak. This voltage (whose frequency is variable from 0.008 to $1000 \mathrm{~Hz}$ ) provides possible target velocities of $0.03 \mathrm{~cm} / \mathrm{s}$ to $3.8 \times$ $10^{3} \mathrm{~cm} / \mathrm{s}$, allowing a wide range of transport velocities to be investigated.

With the levitator operated horizontally in air. we have demonstrated :hat velocities of $4.87 \mathrm{~cm} / \mathrm{s}$ can be reached befort the air drig exceeds the transport fields. In the vertical or litt position, the system has been capable of velocities of $3.6 \mathrm{~cm} / \mathrm{s}$,

Production Rate. We anticipate that an: target factory will incorporate a number of sequential processing steps, some of which we can identify already. and some that may lie in the future. Both target production rate and target inventory (including the very important consideration of tritium inventory) will be determined by types of processes and their sequence. We have begun a parametric study of how production rate and inventories are affected by proposed processing systems. A study of this type will lead to an understanding of the factors that have a major influence on the operation and cost of a target factory. By recognizing the production processes that may have difficulty in meeting 
Fig. 4-86. The potentials are applied to the transport levitutor with a very simple system operating at $60 \mathrm{~Hz}$.

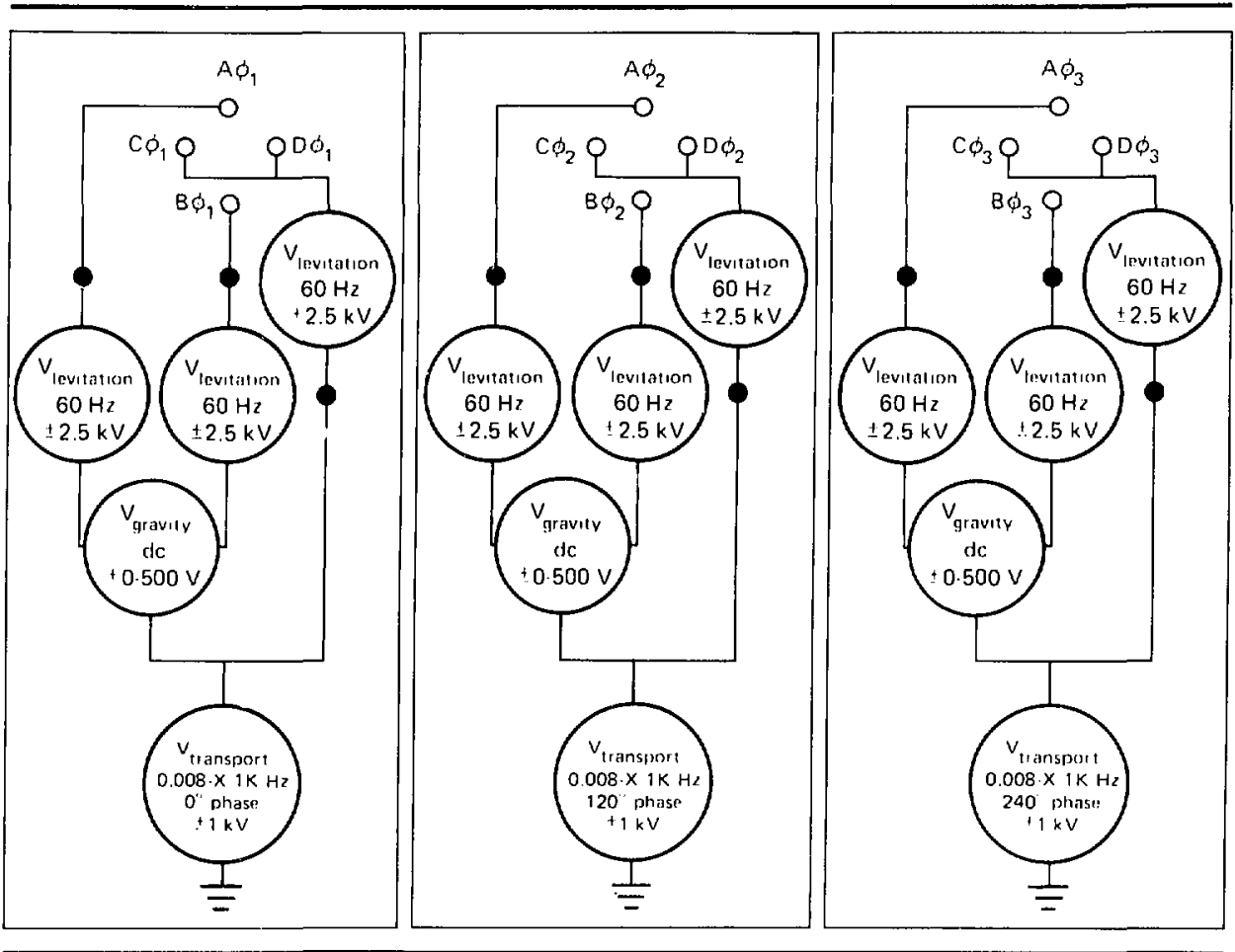

larget production criteria, we can focus our restarch efforts or emphasize more favorable processing systems. This study is now in its infancy. but we anticipate its completion during 1980.

\section{Author: W. 1.. Johnson}

Major Contributor: J. W. Sherohman

\section{References}

54. Laser Program Amisal Report-1977, L an rened lasermore laheratory. Lisermore. (alif. UCRL.50021-77 (1978). pp. 5-42 (a) $5-50$.

55. C. D. Hendrick, and W. I. Johmson, Power Plam Predisofiom of Inertial comfinemant Fervion Targats. Law rence l.ivermore Lithoratury, J.inermore, Cillif., LCKL. 52539 (1979).

56. A. Ashkin and J. M. Drieckic, "Optical Levilatum in Hagh Vacuum," Afpl. Pha. Le'tl. 28, 33,3 (1976)

57. J. K. Crane. Molecular Beam Levilation, Law rence L 1 vermore Lahoralory, Lisermore. Calil., UCRL-\$.3379 \{1979\}.

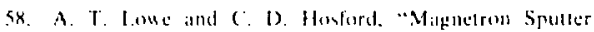

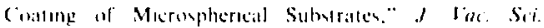
Tildmel 16. $197(1979)$.

51) 1. (jatris and K. (). H. Anch, "A Dumamlälls Regulated

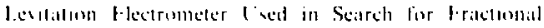

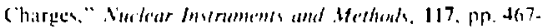
$475(1974)$

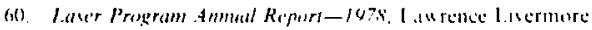

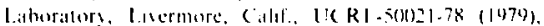
nf. 4.29 $11+52$. 

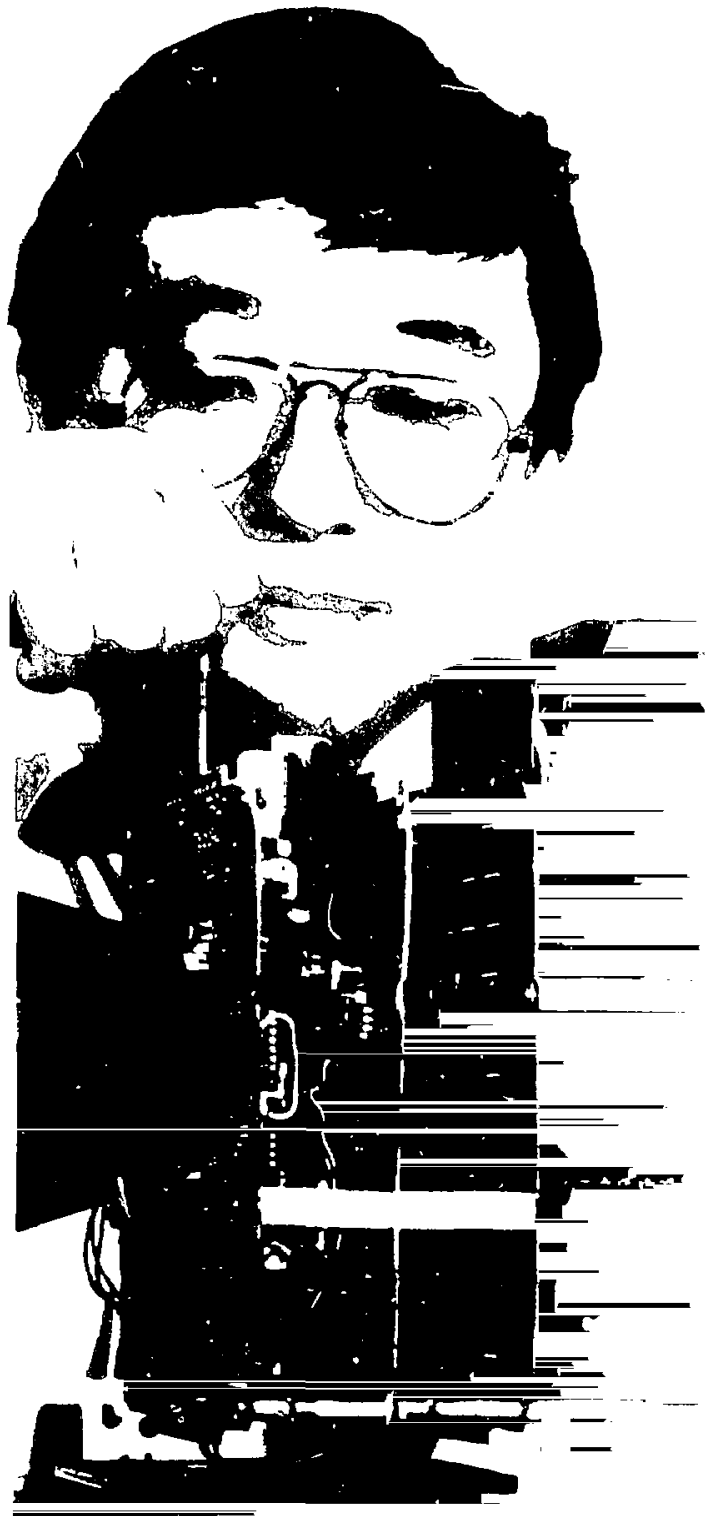


\section{Diagnostics Technology}

\section{Contents}

Introduction

Temporal Response of the XRD-31 X-Ray Diode

Filtered-Mirror Sub-keV X-Ray Measurement System

Develcipment of a Time-Resolved, Broad-Band Sub-Kilovolt X-Ray

Spect.rsmeter for Absolute Flux Measurements

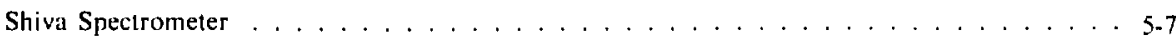

Calibration of the $\mathbf{S X R S C} \ldots \ldots \ldots \ldots \ldots \ldots$

Gold and Cesium Iodide X-Ray Photocathodes $\ldots \ldots \ldots \ldots \ldots \ldots \ldots \ldots \ldots$

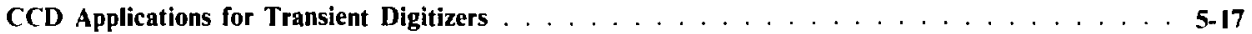

Streak Camera Readout System . . . . . . . . . . . . . . . . 5-17

CCD Ultrafast Transient Recorder . . . . . . . . . . . . . . . . . . 5-19

Reflector-Diffractor Spectrograph Measurements on Shiva $\ldots \ldots \ldots \ldots \ldots \ldots \ldots$

Synthetic Multilayer Structure Characterization $\ldots \ldots \ldots \ldots \ldots \ldots \ldots \ldots$

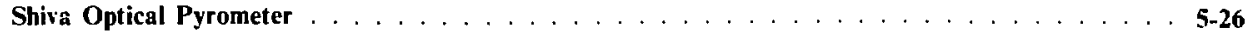

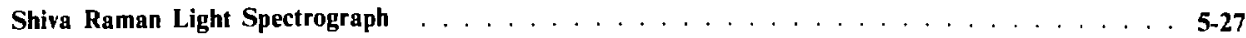

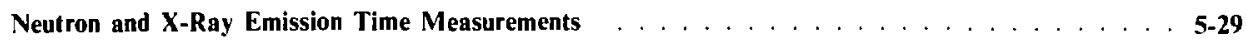

X-Ray Measurements . . . . . . . . . . . . . . . . . 5-31

D-D Neutron Measurements $\ldots \ldots \ldots \ldots \ldots \ldots \ldots \ldots \ldots \ldots \ldots$

Compression Diagnostics for High-Density, Low-Temperature Targets . . . . . . . . . . . . 5-34

X-Ray Imaging of Laser Fusion Targets $\ldots \ldots \ldots \ldots \ldots \ldots \ldots \ldots \ldots \ldots$

Implosion Measurements with Neutron Activation Techniques . . . . . . . . . . . . 5-39

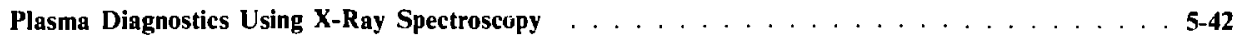

Density Diagnostics . . . . . . . . . . . . . . . . . . . . . 5-43

$10 \times$ Experiment Series at Shiva . . . . . . . . . . . . . . . . . . . . . . . . . . .

Problems with Present Spectroscopic Density Measurements . . . . . . . . . . . . 5-46

Measurements of X-Ray Spectra from Laser-Disk Interactions . . . . . . . . . . . . . 5-49 


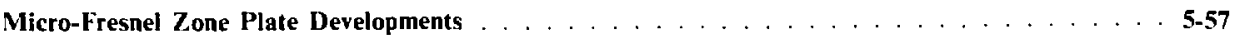

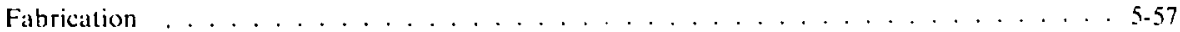

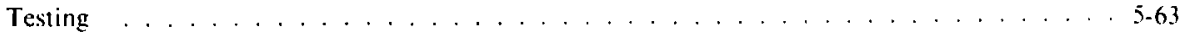

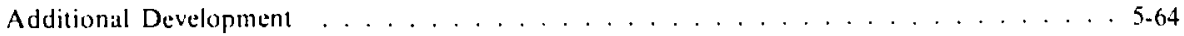

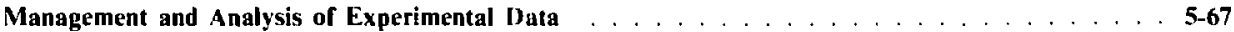

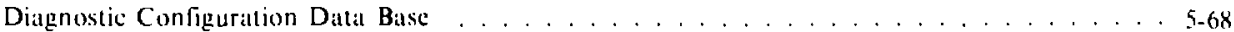

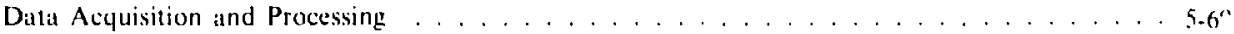

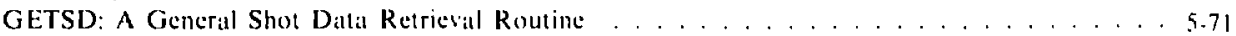

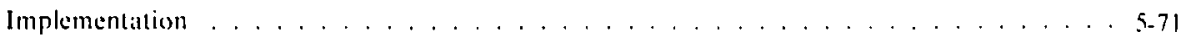

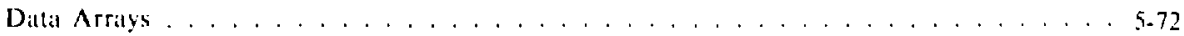

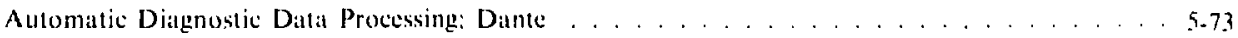

Automatic Diagnostic Data Processing: Filter Fluorescer . . . . . . . . . . . . . . 5.76

Automatic Diagnostic Data Processing: Calorimeters . . . . . . . . . . . . . . . 5.74

Automatic Diagnostic Datat Processing: Photodiodes . . . . . . . . . . . . . . . . 5.81

Automatic Dialgnostic Datal Processing: Energy Balance . . . . . . . . . . . . . . . $5-83$

Film Image Processing . . . . . . . . . . . . . . . . . . . . . . . . . . . . . . $5-84$

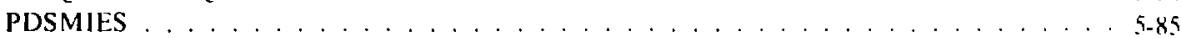

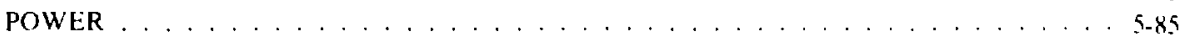

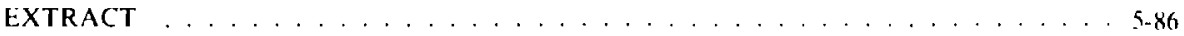

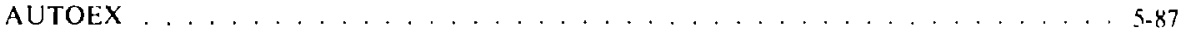

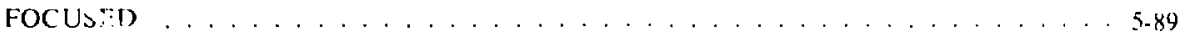

CCD Array Processing . . . . . . . . . . . . . . . . . . . . . . . . . . . . . . . . . . . . .

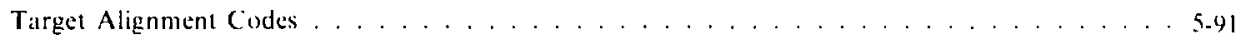

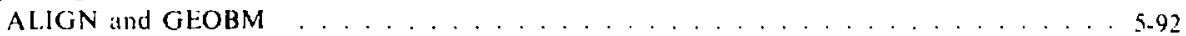

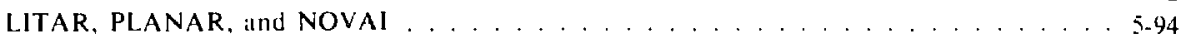

Fusjon Experiments Dita Analysis Facility . . . . . . . . . . . . . . 5-97 


\section{Diagnostics Technology}

\section{Introduction}

The mandate of the Laser Fusion Program's diagnostics effort is to provide appropriate measurement technology at a pace consistent with our rapidly cvolving inleriktion and implosion experiments. At the same time, we must perform diagn'stics on harge! physics and dynamics whose parameters vary on the scale of micrometers and picoscconis To meet these twin goals of extreme accuracy and rapid evolution, our diagnostics progratt. is necessarily both sophisticated and developmentil.

In general we approach diagnostic problems in two ways:

- Using state-of-ths-art (perhaps commercially available) detection systems, whoti provide reliability and modest resolution as on-line diagnostics.

- Pursuing in-house development of high-resolution, high-technology instrumcmittion and techniques, in some cases in collaboration with university or industrial grouph.

Because of the high temperatures involved in fusion experiments, target emissions al primary interest are

- X rays of $100 \mathrm{eV}$ to $100 \mathrm{keV}$.

- Thermonuclear zaction products such as neutrons, alpha particles, and Mel protons.

- Several neutron-activated species.

During the past year we made significant progress in our ability to diagnose drive. preheat, and final-fuel conditions in high-convergence targets. We ciln also field diagnostic: and retrieve data on the large and complex Shica laser system in an alutomated, more reliable fashion.

In high-convergence target designs, we have traded the high temperature of callicr thin-shell targets for higher density and,$R$ in the compressed fuel and surrounding glatss pusher. As a consequence, valuable compression diagnestic techniques such as alphatparticle images and keV-range $\mathrm{x}$-ray images are no lo:nger suitable. because of reduced emissions and increased opacities for both. Several diagnestics which address these problemes are discussed in separate sections below, and are then brought 1 ogether in a discussion of their application to intermediate-density targets.

Several representative articles describe recent advanees in spatially, temporally, and spectrally characterizing $x$-ray emission from laser-irradiated targets. These include

- Improved spectral and temporal characterization of our broad-band sub-kilovolt spectrometer "Dante."

- Similar work with, and absolute calibration of, our sub-kilovolt x-ray streak cameras.

- Contiuued developments of $x$-ray mirrors and lenses for futurc experiments.

Other articles describe

- The mating of an x-ray microscope and streak camera, which will allow us for the first time to study space-time dynamics of selected Shiva targets (Fig. 5-1).

- Work on measuring stimulated scattering and associated target preheat.

- Recent advances in our ability to diagnose neutron yield and relative emission time; this is of particular significance for anticipated experiments next year with velocitymultiplying double-shell targets.

Fast data retrieval and analysis, an aspect of our work which received significant attention during the past year, $: 5$ important to us in two ways. Expeditious daia management 


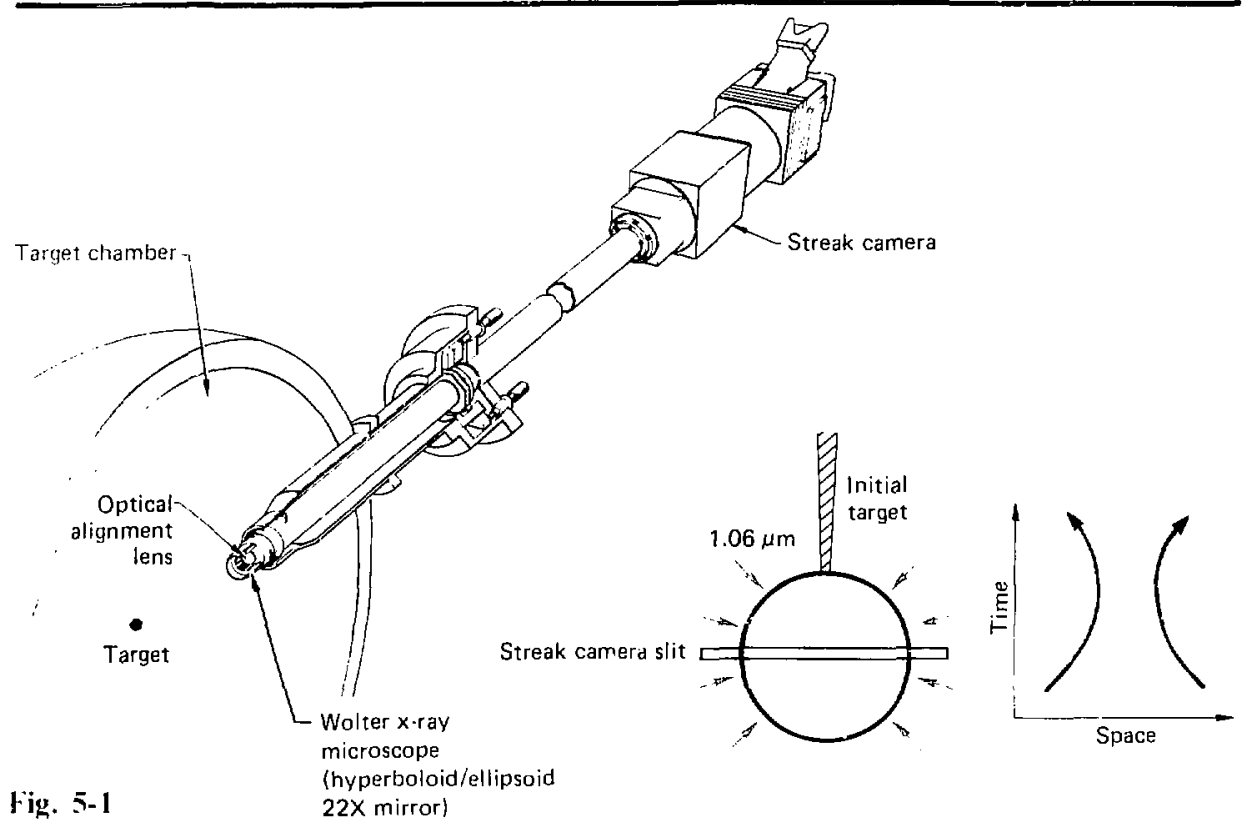

allows us to effectively digest and utilize in a timely fashion the considerable amount of information produced in our rapidly evolving experimental program: this in turn permits more efficient use of valuable manpower. A long-term approach to data handling and storige, a new diagnostic-data computer facility, and a sampling of new data-handling routines are described in this section. A substantial effort has been made, for example, in computer-compatible readout systems employing charge-coupled devices (CCDs); applications of such systems to streak cameras, imaging devices, and transient digitizers are also presented.

We expect a significant payoff from our accrued capability in data retrieval and analysis to manifest itsel $f$ in a more timely, effective and complete digestion of experimental resuits, as well as in a substantial reduction of manpower now invested in relatively routine procedures.

Authors: D. T. Attwood and V. W. Slivinsky

\section{Temporal Response of the XRD-31 X-Ray Diode}

The LLL latser program uses fast $x$-ray diodes (vacuum photoelectric diodes) to provide spectral and temporal information about low-energy $x$ rays emitted from A rgus and Shiva fusion targets. 1.2 For $x$-ray energies below $1.5 \mathrm{keV}$, the typical detector channel consists of a filter, an XRD-31 x-ray diode ${ }^{2}$ (made by EG\&G to LLL specifications), high- quality signal cable, and a large-bandwidth transient recorder. We recently measured the temporal response of a high-quality XRD-31 detector system at the Monojoule laser facility. Those measurements and results are described in this article.

The XRD-3I system being characterized consists of

- A vanadium filter $4000 A$ thick.

- An XRD-3I with 2.I-mm anode-cathode spacing, biased at $5 \mathrm{kV}$.

- $30 \mathrm{~ns}$ of $1 / 2$-in. air-dielect ric coaxial cable. 
- A 4-GHL TSN 660 (Thomson-CSF) oscilloscope.

The impulse response of a fast $i$-ray delector ssstem is best determined using a pulsed x-ray source with in emission time mach less than the detector-system FWHM. The Monojoule laser facility, equipped with $x$-ray divdes and an $x$-ray strcak cilmerat, is a we!l-characterised $x$-ray soures: with an incident 50-ps optical pulse, $x$-raly bursts ol 70 to 110 ps are gencrated $w$ ith suflicient intensity to lest our detectors. We measure the temporal chatralet eristics of the x-ray source with a 1 5-ps x-rit? streak camera, then unlold them from the recorded diode signill to determine the diode system impulse response.

In eur measurements, a $1-J, 56-p s, 1.06-\mu \mathrm{m}$ liser pulse focused on a planar Ti larget generates a nominal 100-ps FWHM x-raly pulse (depending oll energy). An XRD-31 delector system and a soli :ray streak camera (SXRSC) simultaneously detect emitted x raly. For $x$-rity burst durations sigiaficantly longer than the 15-ps resolution, the SXRSC provides a direct temporal ineasurement of the $x$ raly source. Tha $\$ X R S C$ is also fitted with a $4000-A$ thick $V$ filter: with the $V$ filters, foth detectors respond 10 x rays between 300 and $500 \mathrm{cV}$.

$\mathrm{X}$-ray signals meatsured on a typical $1-\mathrm{J}$ hitser shot are shown in Fig. 5-2. Ralu data for the XRD31 system show a 197-ps FWHM, and some ringing [Fig. 5-2(a)]. The SXRSC data [Fig. 5-2(h)] show the s-ray puise on this shot to have a l(12-ps FWHM. To determine the impulse response of the XRD-31 detector system, we unfold the $x-r: 1 y$ temporal distribution from the recorded XRD-31 iignal: the XRD-31 system impulse response thus determined is shown in Fig. 5-2(c). It is about $140 \mathrm{ps}$ FWHM, and shows some ringing.

We also recorded datat using a series of $x$-rall pulses separated by $217 \mathrm{ps}$, to demorstrate the resolving power of the XRD-31 system. To generate the $x$-ray pulse train, the incident laser beam was passed through a 217-ps ctalon. Figure 5-3(a) shous XRD-31 data recorded on al lypical shot. The pitse has : 565 ps FWHM, with $10 \%$ modulation near the peak. From the raw XRD-3l data, it is unclear wh.ll the inpul x-ray temsporal distribution looks like. The modulation near the peak suggests multiple pu'ses. and the broadening suggests the $x$ ray." are emilted over several hundred ps.

Using the previously determined impulsc response function for the XRD-31 system [Fig.
Fig. 5-2. XRD-31 system impulse responsz determined from data recorded on a I-J laser sbot: (a) XRD-3I dara; (b) soft $X$-ray streak camera (SXRSC) data; (c) inferred XRD-31 system impulse response, found by unfolding the $x$-ray temporal distribution (b) from the measured XRD-31 data (a).
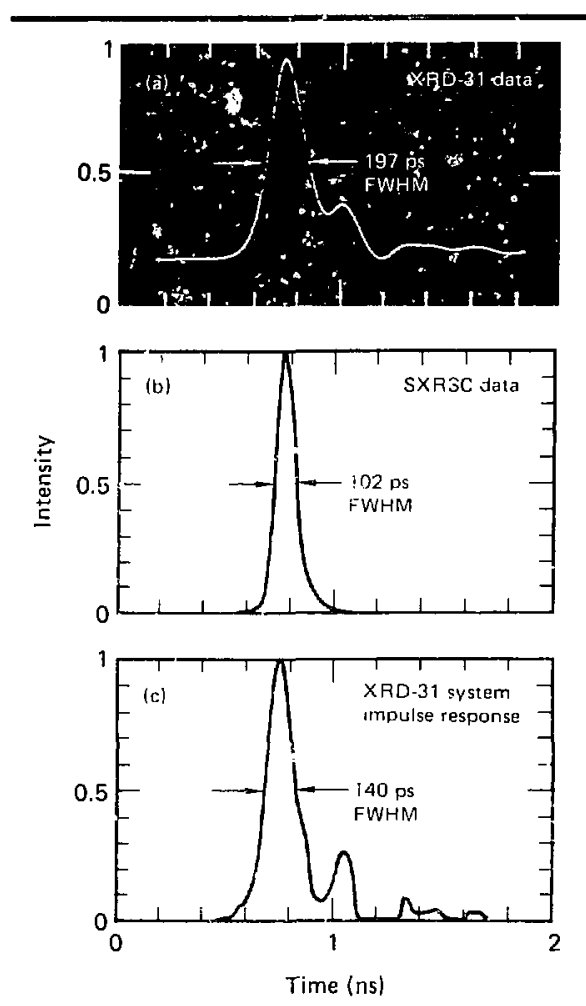

5-3(b)]. the XRD)-31 diata recorded on this shot can he unfolded on determine the a-ral-source lemparal tharateristics [1]g. 5-3(c)l. Temporal ouput of the t-raly source an recorded by the SXRSC is shown for comparisom in Fig. 5-3(d). The unfolded XRD-3i data hous the same batsic detail ats the SXRSC measurement, thus uemonstrang the 200-ps capability of the diacte detection system when properl! charaterised.

In Fig. 5-4, the rumning integrat of the unfolded XRD-31 dalta is compared with that of the SXRSC datta, stowing that retative area under the individual peaks is preserved.

The ability to unfold a set of data, ats demonstrated above. is dependent on the data quality. Typicalls. deiailed strueture on time scales 
Fig. 5-3. XRD-31 gystew repoese of a lever shot producing - series ef X-ray palds separated by 217 pa: (a) XRD-31 dota; (b) XRD-31 system inculse respome, as deternined from single-pribe experibear; (c) x-ray sonce temporal distribution, sonad by unfolling the systen lapule reaponse from the recorded XRD-31 data; (d) $x \rightarrow$ ray sonce temporal distribution mesenred with the SXRSC, for comparison.
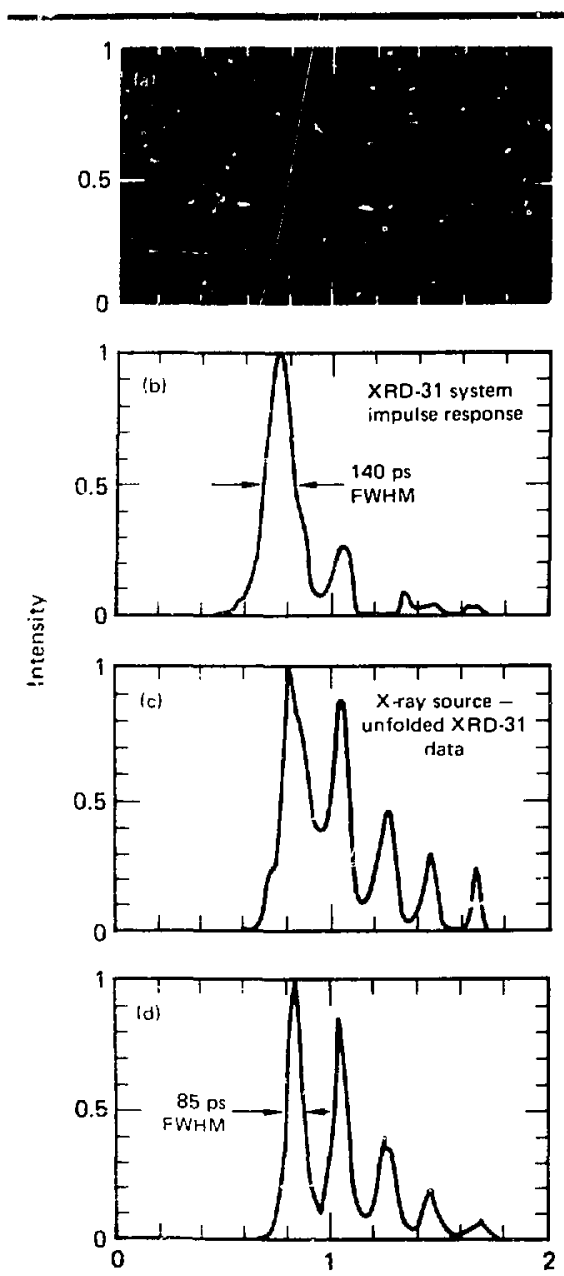

Time (ns)
Fis. 5-4. Fruction of $x$ rays emitted vs tim. A comparieon of the SXRSC data [Fis. S-3(d)] with the unfolded XRD-31 date [Fig. 5-3(c)] shows that the relative intenaities of the Tive puives remnin the sane for both metbods of measurecent.

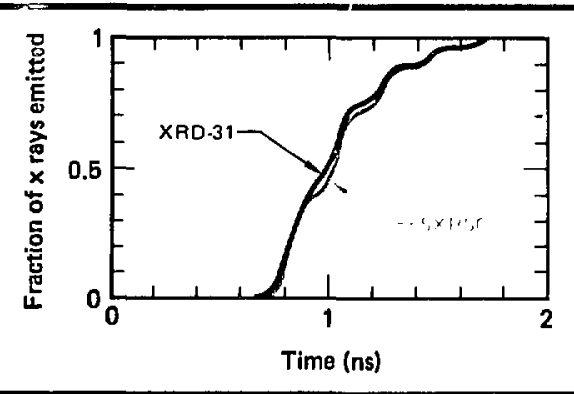

less than $75 \%$ of the width of the system impulse response should be seriously questioned. The unfold in Fig. 5-3(c) is correctly interpreted as a series of short $x$-ray pulses spaced about 215 ps apart. The relative energy in each pulse can be determined from its total integral.

The data presented show what can be done with a well.ch aracterized XRD-31 system in a low'noise environenent. The single-pulse data show that an XRD-31 system with a 4-GHz TSN 660 oscilloscope has an impulse response of $140 \mathrm{ps}$ FWHM. The etalon data demonstrate that such a system can just barely resolve $x$-ray pulses spaced $217 \mathrm{ps}$ apart. We have also demonstrated that under favorable conditions the XRD-31 data can be unfolded to a resolution somewhat better than the system impulse response. With a slower recorder, such as the $1-G H z$ Tektronics 7912 transient digitizer, we would expect an XRD-3I system to show an overall impulse response of $400 \mathrm{ps}$.

Authors: R. A. Lerche and D. E. Campbell

\section{References}

1. Laser Program Annual Reporl-1978. Liswrence Livermure Laboratory. Livermore. Calif., UCRL-50021-78 (1979). pp. 6-5 10 6-7

2. Laser Program Anmual Report-1977, Laurence Livermort: Lahorutory. Livermore, Calif., UCRL-50021-77 (1978), pp. 3-57 to 3-60.

3. Laser Program Annual Report-1974, Lawrence Livernore Laboratory, Livermore, Calif., L'CRL-50021-74 (1975). р. 290

4. Laser Program Annual Report-1978. Lawrenc: Livermore Laboratory, Livermore. Calif., UCRL-50021-78 (1979), pp. 6.2 to $6-5$. 


\section{Filtered-Mirror Sub-keV X-Ray Measurement System}

A ten-channel filtered windowless $x$-ray diode system has been used at the Shiva laser since January 1979.5 This system has a problem, inherent in all filter/detector $x$-ray measurement schemes: because the filter trarsmission above the absorption edge of the filter material makes contributions to the recorded signal, the resolution and accuracy of channels al or below the peak of the spectrum to be measured are significantly degraded. We therefore designed and installed a five-channel fillered-mirror system, using critical-angle reflection by vitreous aarbon and beryllium mirrors to remove the unwanted high-energy response. The mirrors were aligned to an aceuracy of $0.01^{\circ}$ and pinned in position with a rigid cassette mounting to ensure proper alignment / the mirrors and their mounting hardware were designed and built by Lockheed Palo
Alto Research Laboratory (LPARL) to LLL specifications].

Figure 5-5 shows the mirror reflectivity ard the boron filter transmission for the lowest energy

Fig. 5-5. Carbon mirror reflectivity and $1-\mu \mathrm{m}$ boron filter transmission, for lowest energy channel.

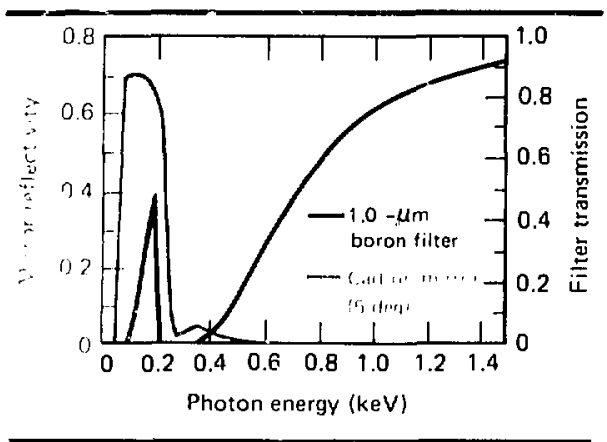

Fig. 5-6. Experimental geometry for Shiva filtered-mirror x-ray diode system.

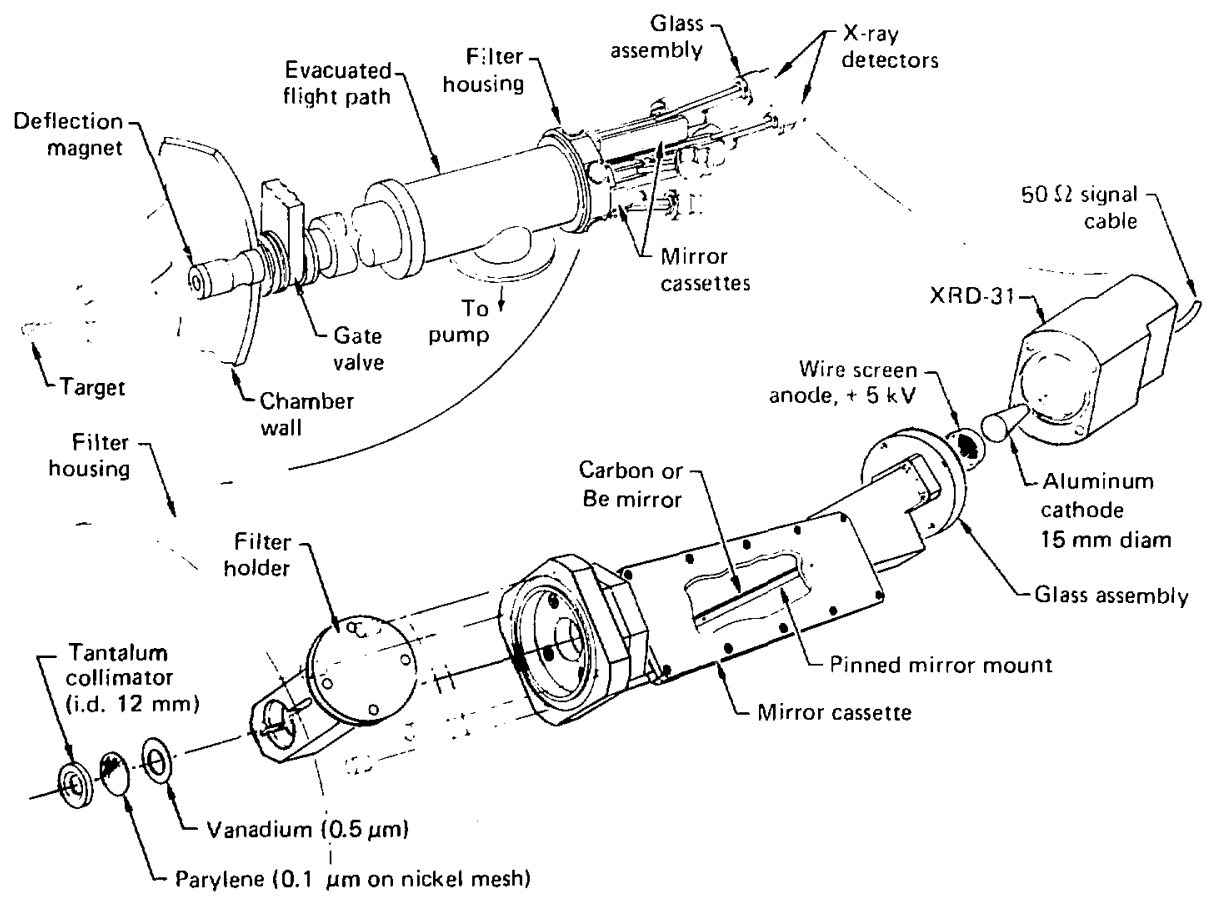


channel. Note how with increasing energy the mirror reflectivity drops to rero as the litter transmission increases. The mirrors suppress the response to energetic photons hy at least an order of magnitude.

The physical arrangement of the filtered-mirror yystem is shown in Fig. 5-6, Tahle 5-1 outlines the

Table 5-1. Characteristics of the Shiva filtered-mirror sub-keV x-ray measurement system.

\begin{tabular}{lcclll}
\hline \multicolumn{3}{c}{ Plitcrs } & & \multicolumn{2}{c}{ Mirrori } \\
\hline $\begin{array}{c}\text { Absorption } \\
\text { edge }\end{array}$ & $\begin{array}{c}\text { Thickness } \\
(\mu \mathrm{m})\end{array}$ & $\begin{array}{c}\text { Edge cnergy } \\
(\mathrm{keV})\end{array}$ & & Material & Angle \\
\hline Boron-K & 1.0 & 0.19 & & Carbon & $5.04^{\circ}$ \\
Carbon-K & 1.9 & 0.28 & & Beryllium $3.41^{\circ}$ \\
Vanadium-L & 0.5 & 0.52 & & Carbon & $2.43^{\circ}$ \\
Chromium-L & 0.6 & 0.58 & & Carbon $1.91^{\circ}$ \\
Cobalt-L & 1.0 & 0.79 & & Carbon $1.98^{\circ}$ \\
Aluminum-K & 9.2 & 1.56 & & \multicolumn{2}{c}{ None } \\
\hline
\end{tabular}

essential parameters of each channel, and Fig. 5-7 gives the response of each channel to a flat spectrum.

Except for the presence of the mirrors, the filtered-mirror system is similar to the ten-channel system mounted direetly below it on the Shiva target chamber. The system consists of six lowencrgy $x$-raty channels; five are filtered-mirror channels and one is a higher-energy (aluminum K-edge) channel that dees not use a mirror. The six-channel system uses 0.1- $\mu \mathrm{m}$ parylene blast shields for UV absorption, and fatst, small, x-ray diodes. Datal recording is donc on Tektronix $\mathrm{R} 7912$ digitising oscilksecopes, for preliminary analysis on the Shivat $11 / 70$ computer and eventual transfer to the Occopus system for final processing.

We have been using the filtered-mirror system routinely since August 1979. The system nol only provides us with a valuable new sub-kilovolt $x$-raty

Fig. 5-7. Filtered-mirror system channel responses to a fat spectrum.

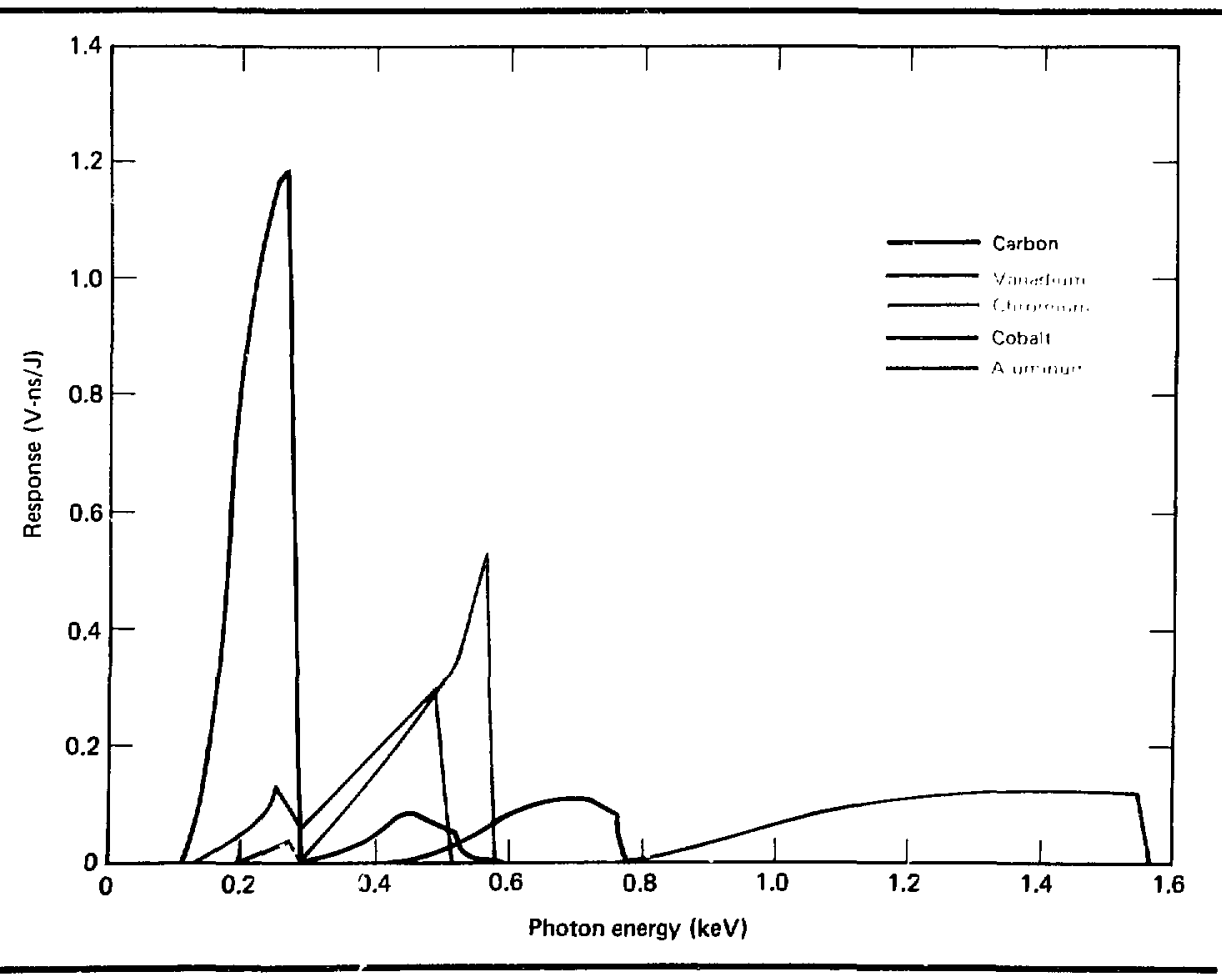


diagnostic instrument, it has provided us with more accurate informition about the low-energy photon spectrum. Further, by comparing spectral results from the six-channel filtered-mirror system with those of the ten-channel system, we have obtained additional information ahout the angular distribution of soft $x$ rays.

\section{Authors: R. A. Heinle and K. G. Tirsell}

\section{Reference}

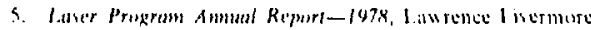
lathoritors. L iscrmore, (alif,, IICRI.-5(1021.78 (1979)), p. 6.5 .

\section{Development of a}

Time-Resolved, Broadi-Band Sub-Kilovolt X-Ray Spectror . .er for Absolute Flux Measurements

We have previously described the development of a soft $x$-ray streak camerat (SXRSC) which is sensitive to $x$ rays $>100 \mathrm{eV}$ and has a time resolution of $15 \mathrm{ps}^{\circ}$. A version of the cameral hats heen used at Argus to measure the time history of the $x$-rity emission in three broad-bano energy channels below $1 \mathrm{keV}$. The SXRSC hals established its vilue ats an important diagnostic tool, recording data on a routine hasis for a variety of targets.

We have now designed and implemented at technigue to perform intensity calibrations of the SXRSC which will make possible absolute fux measurements of $x$-ray emission belou $1 \mathrm{keV}$.
Preliminary results from the calihrations, and the techniques used, are discussed helow: we also describe the SXRSC as it will be fielded on Shivat.

Shiva Spectrometer. The SXRSC is similar to the instrument whose development is deseribed in previous annual reports ${ }^{6-x}$ : as it will ie fielded on Shiva (Fig. 5-8), the SXRSC is designed to make spectrally resolved ahsolute flux measurements of the suh-kilovolt $x$-rily spectrum from laster fusion targets. Using methods similar to those used at Argus. We obtain broad-band spectral resolution using $x$-rily mirrors and transmission lilters. Seven mirror-filter combinations, listed in Table 5-2, have been designed to cover the range from 100 to 930 eV. Citleulated relative spectril responses for the seven channels are compared in ligy. 5-9.

The mirror miterials and angles were chosen lo provide efficient reflection in the r-ray region of interest and discriminate against a rays having energies greater than the lilter cutoff. The seven material/angle combinations require only lus separale mirrors. one at lmo degrees and one al four degrees. The mirrors are fabricated by polishing the carbon and germanium surfaces 10 optically flat linishes and vaper-depositing nickel and aluminum wer part of their respectise wrlites. lising such composite mirrors greally simplifies the alignment procedure, by reducing the number of mirrors which muse be ponitioned.

The SXRSC dial recording medium will be a CCD actise-readeut detection ustemo" directly linked to the Shisi computer strem. Data in the lorm of a digetised mo-dimenuienal image will be avalabie lor allallse immediately aller the shot, bypassing the time-comsumme processing and

Fig. 5-8. A schematic of the SXRSC as it will be fielded on the Shiva laser system.

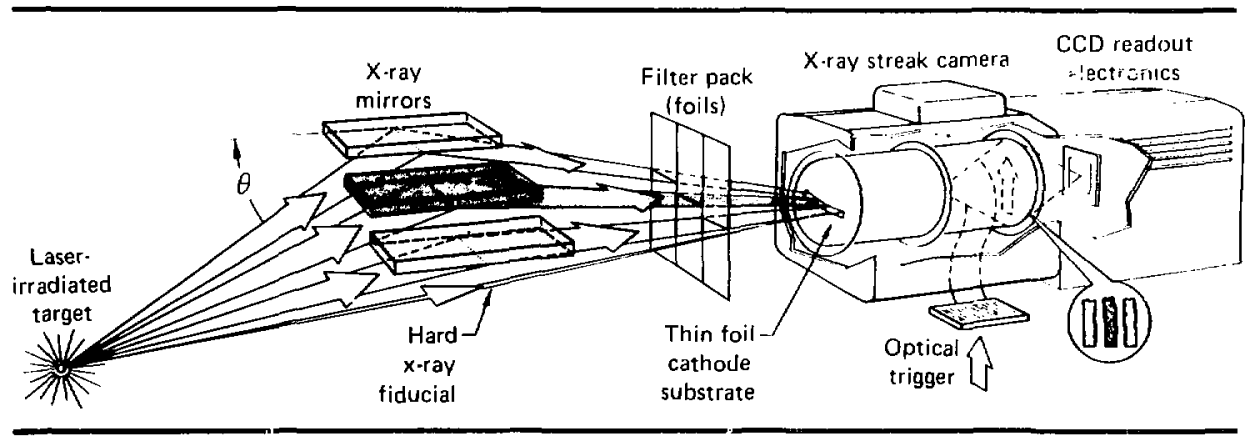


Table 5-2. Mirroz/filter combinations for the SXRSC x-ray channels at Shiva.

\begin{tabular}{|c|c|c|c|c|c|c|}
\hline Mirro & Angle & Fìlter & $\begin{array}{l}\text { Thiclones: } \\
\left(\mu \mathrm{g} / \mathrm{cm}^{2}\right)\end{array}$ & $\begin{array}{l}\mathbf{E}_{\text {abs }} \\
\text { (eV) }\end{array}$ & $\underset{(e V)}{E}$ & $\underset{(\mathrm{oV})}{\Delta \mathrm{E}_{\mathrm{FWH}}}$ \\
\hline $\begin{array}{l}\text { Carbon } \\
\text { Nickel } \\
\text { Nickel } \\
\text { Aluminum } \\
\text { Aluminum } \\
\text { Germanium } \\
\text { Germanium }\end{array}$ & $\begin{array}{l}4^{\circ} \\
4^{\circ} \\
4^{\circ} \\
2^{\circ} \\
2^{\circ} \\
2^{\circ} \\
2^{\circ}\end{array}$ & $\begin{array}{l}\text { Carbon } \\
\text { Vanadium } \\
\text { Chromium } \\
\text { Iron } \\
\text { Cobalt } \\
\text { Nickel } \\
\text { Copper }\end{array}$ & $\begin{array}{l}300 \\
250 \\
300 \\
500 \\
600 \\
600 \\
600\end{array}$ & $\begin{array}{l}280 \\
513 \\
575 \\
708 \\
778 \\
855 \\
930\end{array}$ & $\begin{array}{l}230 \\
476 \\
525 \\
633 \\
700 \\
766 \\
819\end{array}$ & \begin{tabular}{r|}
72 \\
86 \\
114 \\
117 \\
143 \\
168 \\
224
\end{tabular} \\
\hline
\end{tabular}

Fig. 5-9. ('alculated relative spectral responseg of the seven x-ray channels to be used with the SXRSC at Shiva; included in the response rorves is the gold photocathode sensitivity. (Sec Table 5-2 for further characterization of each chamel.)

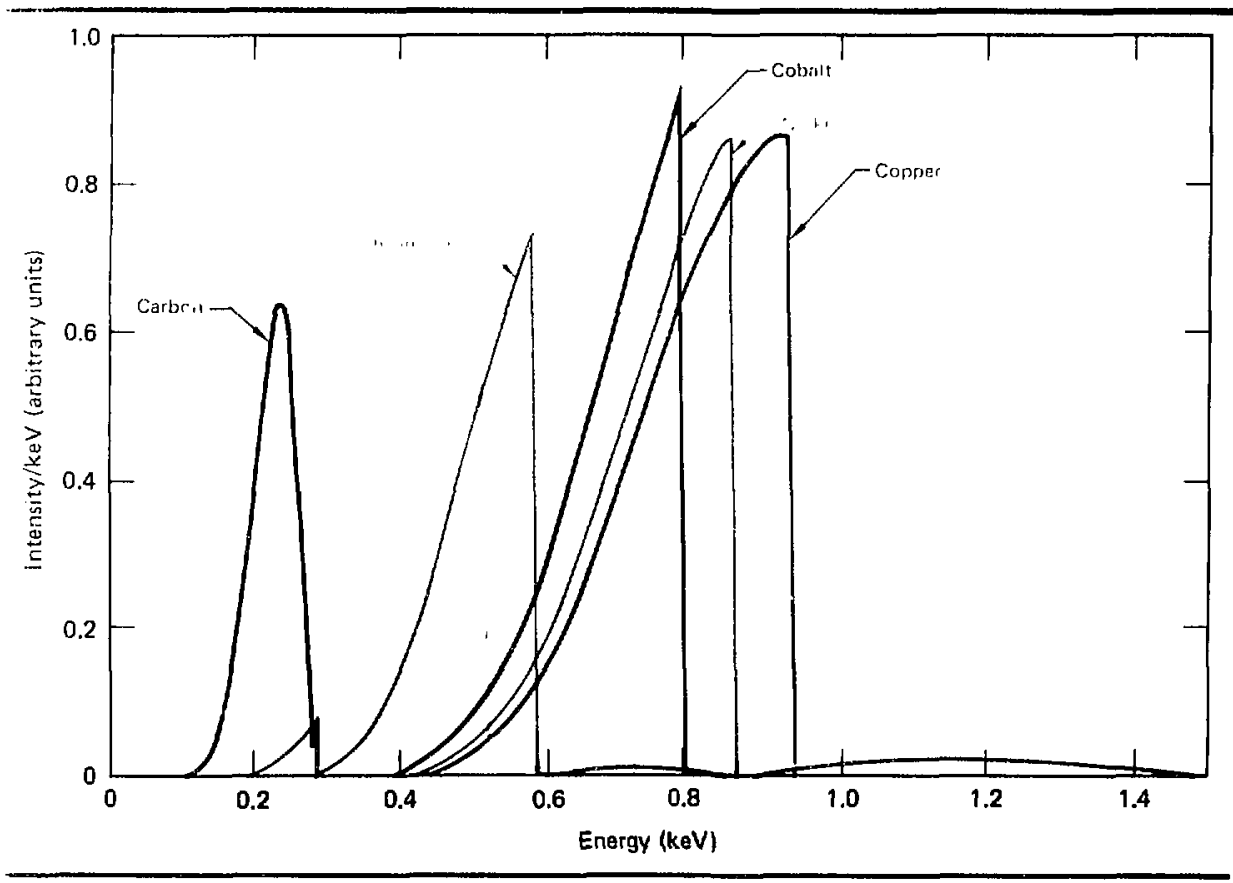

d: rilirmg of film data presently required for quanit, live analysis.

Capacity for a hard $x$-ray fiducial and for $\therefore$ ir ninhole imaging has been incorporated into th: S.XRSC design on Shiva. The hard $x$-ray fiducial Hlius us to determine the time of emission of the wh-kel $x$ ralys relative to $x$-ray energies greater than $1 \mathrm{keV}$. The fiducial is a direct line of sight from the target to the entrance aperture of the SXRSC: the $x$-ray mirror is not used, but a $K$-edge filter can be placed so as 10 define the higher-energy $x$-ray channel. Complemented by other streak camera data, the fiducial will allow us to investigate the timing relationships among the total $x$-ray spectrum. ${ }^{10}$ The pinhole imaging system will permit us to spatially localize observed emission areas.

Calibration of the SXRSC. Calibration of the SXRSC is done in a pulsed mode, approximating as nearly as possible the experimental configuration in 
Fig. 5-10. A schematic of the calibration set-up at the Monojoule laser, for culibrating the SXRSC using a pulsed source.

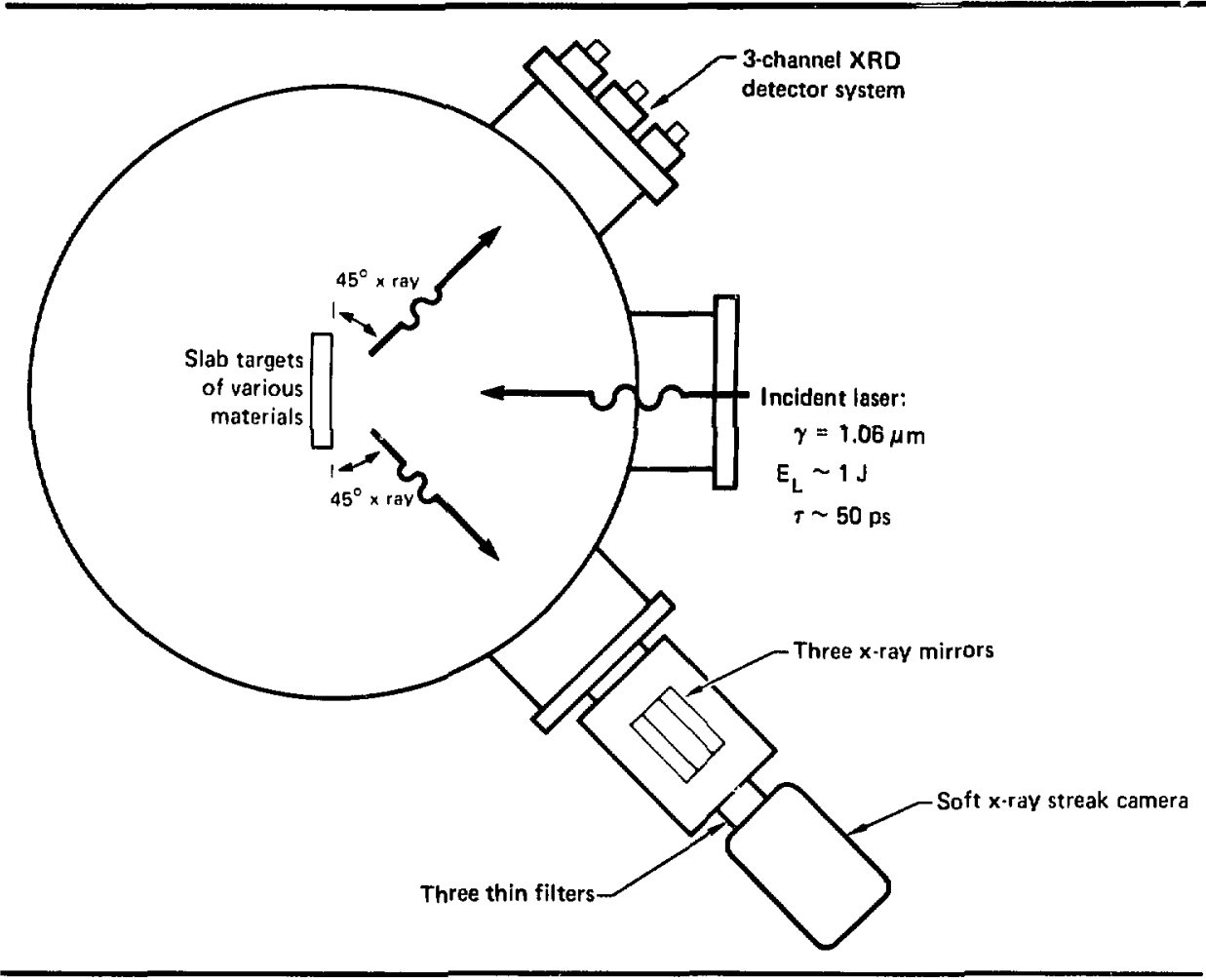

which it is used. Pulsed-mode calibration is inheremty as reliable and aceurate as the steady state method employed with other detectors used for low-energ! x-ray measurements, although source characterization may be more difficult in the pulsed mode. The SXRSC's fast timing characteristics diseriminate agatinst unwanted late signals, which presently preclude calibration in a stealdystate mode.

We have assembled a calibration facility using the Monojoule laser to create a pulsed $x$-ray source. A schematic of the arrangement is shown in Fig. 5-10. A 50-ps pulse of $1.06-\mu \mathrm{m}$ light having energy up to I J irradiates slab targets of various materials at normal incidence. Three absolutely calibrated $x$ ray diode (XRD) detectors on an angle of $45^{\circ}$ to the larget normal measure the sub-kilovolt $x-r a y$ fluence from the target. The SXRSC is also placed at $45^{\circ}$ to the target normal, at $9\left(1^{\circ}\right.$ from the XRDS. To chtalin the calihratoms, lime-resolved sy!nats from the SXRSC are inlegrated and compared with integralled fluences measured by the KRDs.

The SXRSC is calibrated in three x-rat energs channels defined b! thin $x-r a l$ transmission hilters and graing-incidence a-ray mirrors. Similar channels are defined for the XRDs. also using sets of 8 raly transmission filers. $A$ eomparison of the spectral shannels for the SXRSC and the X.DS is shown in Fig. 5-11: responses of the SXRSC and XRD channels have been matched as closely as possible in order to reduce errors due to variation in the spectral emission shape. 
Fig. 5-11. A comparison of normalized channel responses for the XRD detectors and SXRSC used for the calibrations. The response functions hetween the different detectors have been matched as nearly as possible to minimize effects due to structure in the $\mathrm{x}$-ray spectrum.
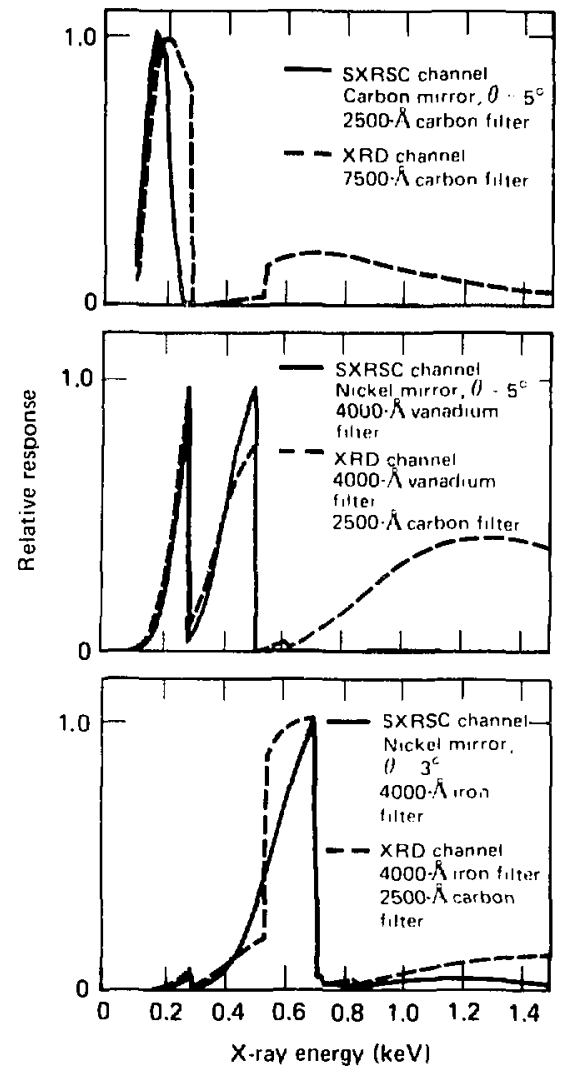

We hate meivured eiteh of the components of the channel to alecuraltely determine the spectral dependence of the cameral response ffigs. $5-1210$ 5-14). X-rot trimmision by the filter foils is meisured using proton-induced line soureses in the sub-kilovole region. Figure 5-14 slous data for al lypical sel of liters: the datia are lit using tabulated absorption coefficients to determine foil thickness.
Fig. 5-12. Representative measured $x$-ray fuences from the various laser targets used to calibrate the SXRSC. Large fuence variations among the targets at higher energies are evidence for emission in this region.

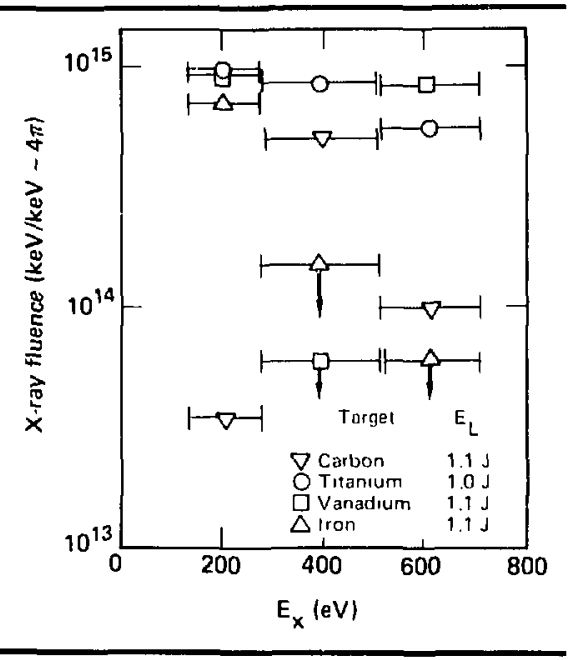

We hate atho meisured the reflection elfifeience of the v-ris merore (the. 5-13). Belou I kev we we protem-induced line emission: at higher energies ue une at continuum s-rity source prodused hy electron bremsstrahlung. The curves drian n through the dalat are predietion of the reflection efficiency, using a semiclassieal calculation adjusted to fit the datat." The three $X R D$ ) detectors with aluminum cathodes have been ealibralled in the normal fashion and alerec with withle and reproducible calibrations from similar imalruments.

Vertious target materials ate used for the calibrations, for our laser operalting parameters, the larget upectrum above $250 \mathrm{eV}$ is dominated bs line emissions. By using various largets we can change the energy of the line emission and thus test the dependence of the callihrations on the source spectrum. Source spectra from various targets measured by the threechannel XRD system are shown in Fig. 5-12. Near $200 \mathrm{eV}$ the fluences from titanium, vanadium, and iron are similar, while the earhon targer fuence is greatly reduced. At higher energies the fluences vary greatly depending on the target beciase the spectrum js dominated by lines. For example, by changing the target atomic number $Z$ by one, from titanium to vanadium, the fluence in 
Fog. 5-13, Measured reflectivity of the three SXRSC x-ray mirrors; curves were generated from a semiclassical reflectivity theory adjusted to fit the data.

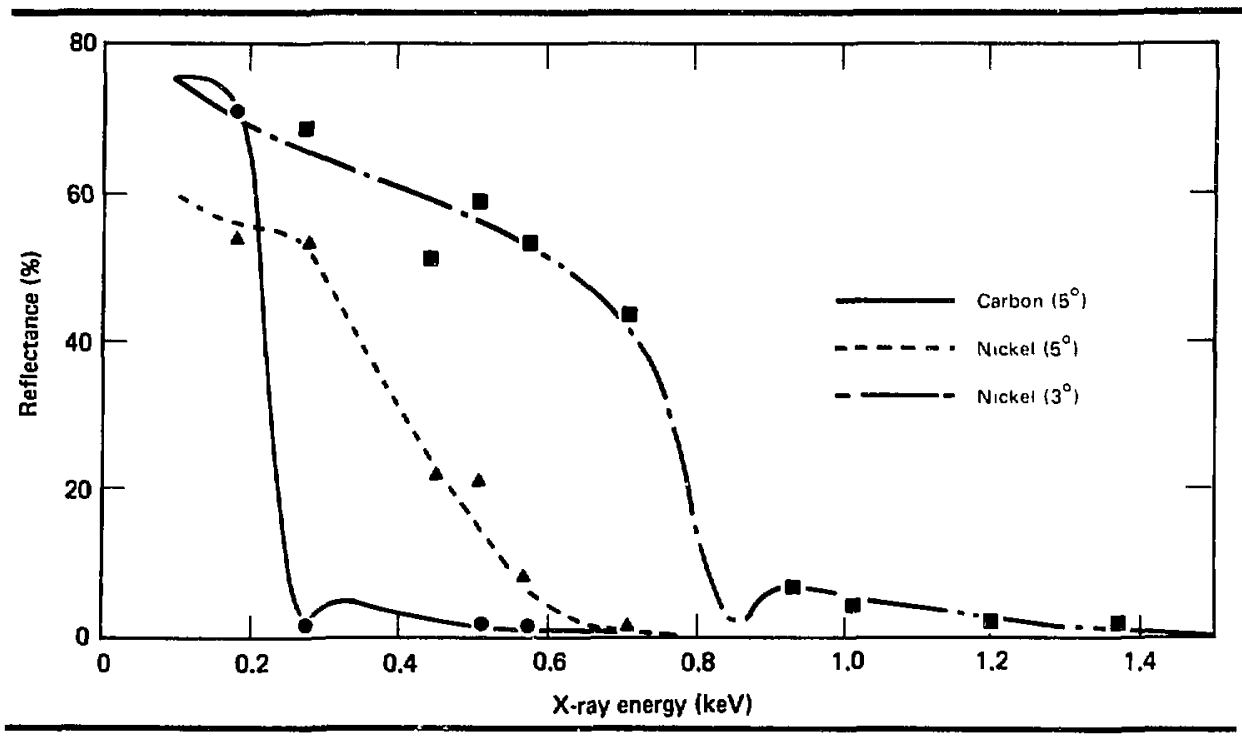

Fig. 5-14. Measured transmission of the filter foils used in the SXRSC calbrations.

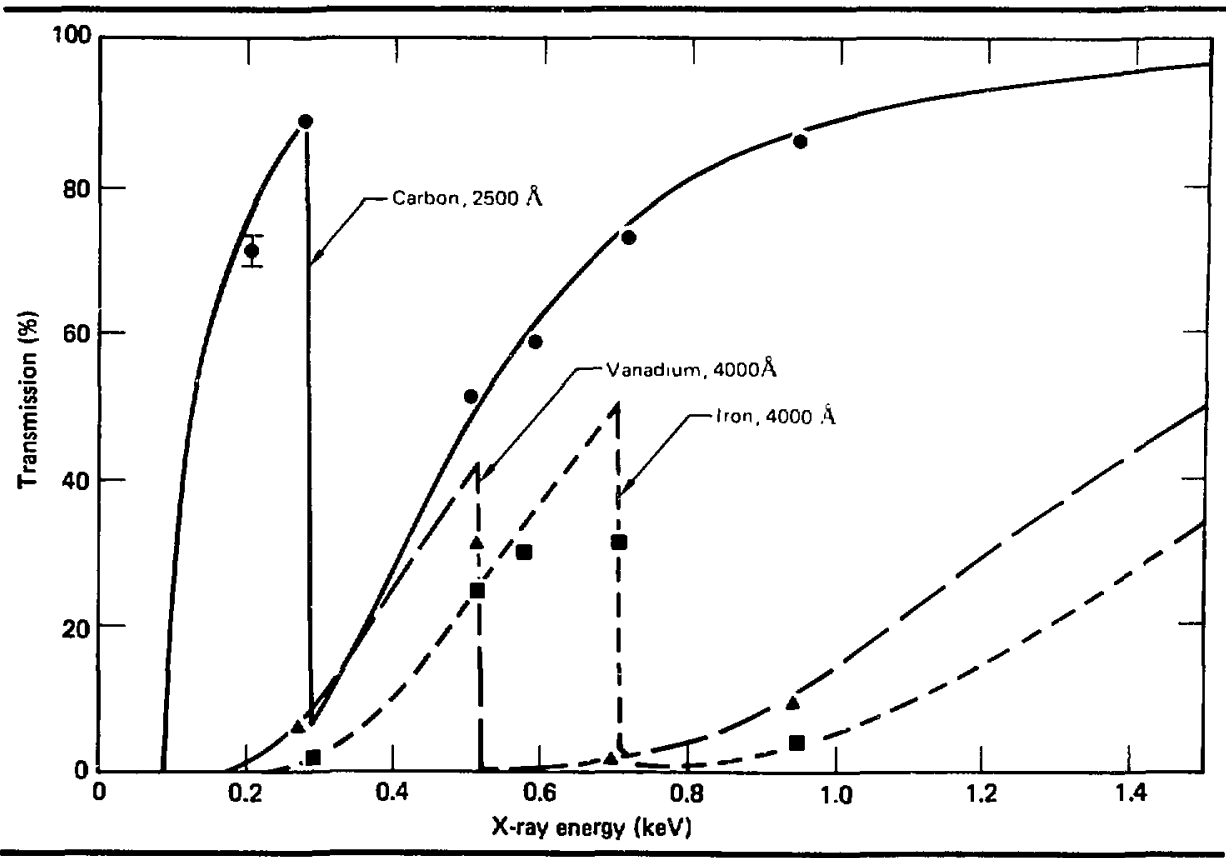


Fig. 5-15. The two-dimensional streak image of typical SXRSC calibrclion, showing (a) the film image and (b) a contour plot of the twodimensional digitel image. Calibrated streak records of the three $x$-ray channels are shown in (c).

Film image of soft $x$-ray streak data

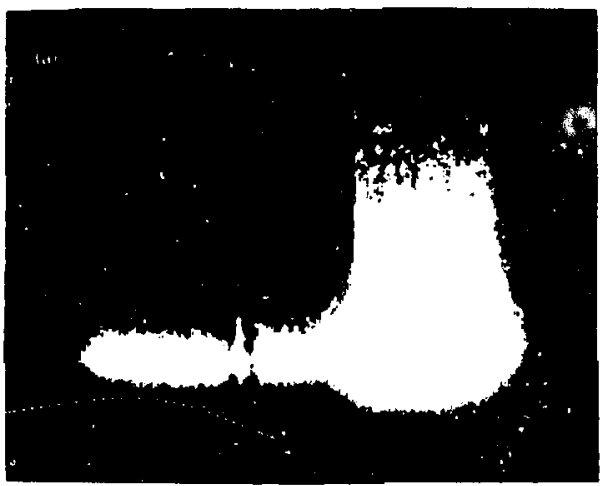

Digitized contours of film record

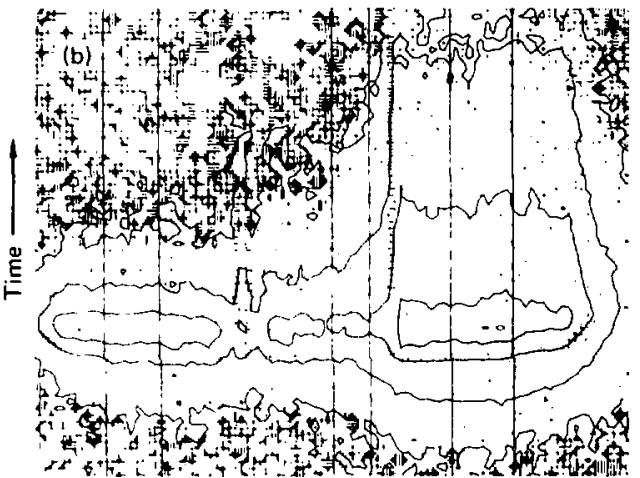

Iron

Vanadium

Calbon

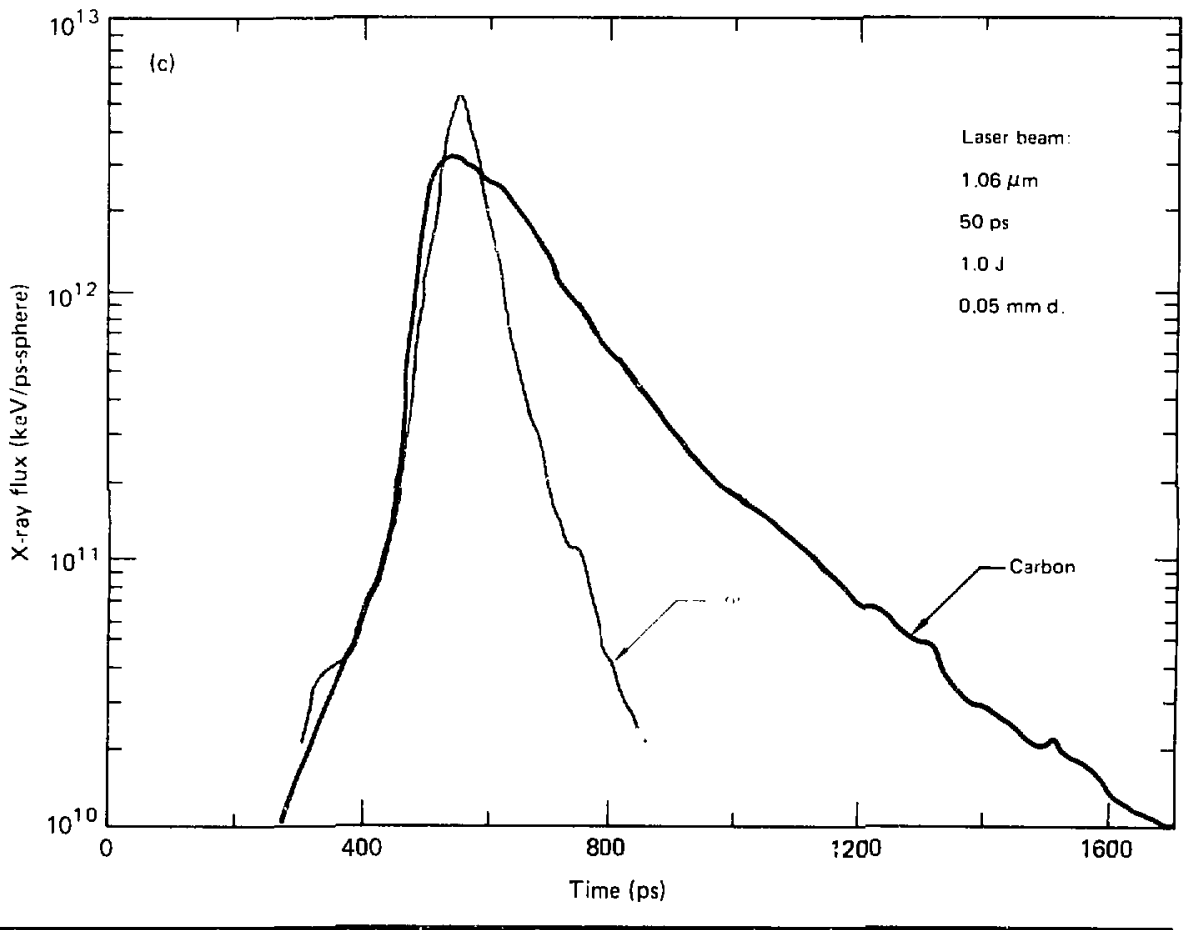


Fig. 5-16. Typical SXRSC calibratioi date from various tangets, for three soft $X$-ray regions; lines represent fits to the data.
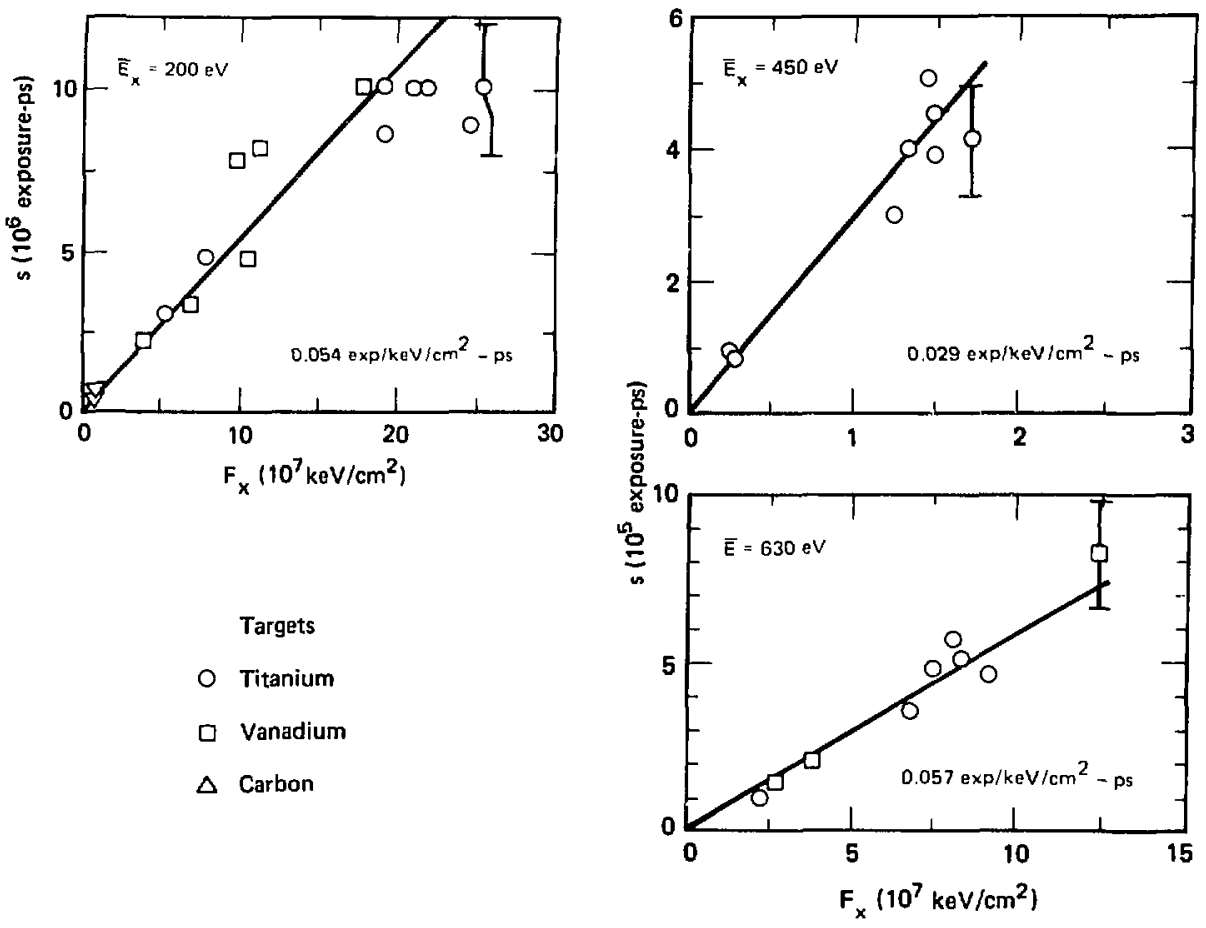

the $500-\mathrm{eV}$ channel decreases by more than an order of magnilude. Line emission from highly ionised vanadium is at a higher $x$-ray energy than that from cold vanadium: the strongest vanadium lines shilt above the $L$ absorption edge of a vanadium filter. while the hot titanium lines lie below the edge. Similarly, an iron target signal observed through an iron filter is also greatib reduced. For the carbon target, peak output is observed in the 280-t0-510-ev channel which includes the highly excited carbon lines.

An example of SXRSC calibration is show $n$ in Figs. 5-15 and 5-!6. Each data set consists or a number of laser shots taken with various targets. Figure 5-15(a) gives film data fronr a titanium target (calibrations have also been performed using a CCD active readout system). Film density is converted to light exposure, using a calibration curve produced for each shot on the film. The film data is processed and converted te a digital contout plot [Fig. 5-15(h)] using a microdensitometer: the pairs of certical lines parallel to the streak direction denote the x-raly challinels. The Jata are further reduced [ Fig. $5-15(\mathrm{C})]$ to time histories of the three $x$-ras bands, by averaging across each x-ray channel: the curves have been plotted using calibration filctors determined for each channel.

The calibration factors are determined by comparing the integralted time history of each SXRSC channel with XRD measurements of total fluence in the corresponding energy bands. The data are then corrected to account for the difference in channel spectral response between the SXRSC and the XRDs. Th.se corrections are usually less than $10 \%$, but can be as large als $50 \%$ depending on the target 
material. Figure 5.16 shows plots of the integrated response of the SXRSC vs fluence into the instrument for three $x$-ray energy channels.

Errors in the plots, including errors in film data ieduction and integration of the XRD output, are - vimated at 20\%; this is consistent with the scatter $i_{1}$, this set, which is ahout $25 \%$. Systematic errors in . mithrating chisnnel cor ponents increase the eslin...tcel irror of the measurement to $35 \%$. Potential vertatce errors in the method of data reduction,

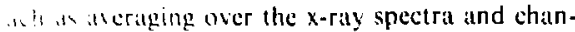
11 1:- ponse, are being investigated. These uncer"116 111 data analysis are due to using broald-hand . rimels in the calibration, and can he reduced by

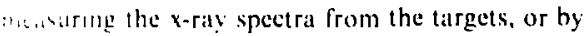
wall mach narrower channels which reduce the uprest of encrgies over which the channels must be arerated

In vimmary, we have developed a calibration procedure to neasure the absolute spectral senvianity of the SXRSC. A further improvement, inwhily a more accurate characterization of the lines cmilled lrom the source, is presently underwiay; when this spectrum is measured, a more thorough datia analysis will be possible. We will continue to refine the calibrations and investigate the $x$-ray response of the camera. Other photocathode materials are also being investigated, as discussed in the following article.

Author: R. L. Kauffman

Major Contributors: H. Medecki, E. L. Pierce, and (i. L. Stradling

\section{References}

6. Laser Program Anmal Report-1978. Lawrence Livermore Lahoratory, Livermorc, Calif., UCRL-5002/-78 (1979), рг. 6-2 to $6-5$.

7. Laser Program Annual Report-1974, Law rence Livermore Lahoratory. Livermore. Calif., UCRL-50021-74 (1975).

8. Laser Program Annual Report-1975, Lawrence Livermors Laboratory, Livermore, Calif., UCRL-50021-75 (1976).

9. Laser Prugram Annual Report-1978, Lawrence Livermore Luboratory, Livermore, Calif, UCRL-50021-78 (1979), pp. 6-54 10 6-59.

10 D. T. Attwood, L. W. Coleman, J. T. Larsen, and E. K. Storm, Phys. Rev. Leli. 37, $499-502$ (1976).

11. A. H. Compton and S. K. Allison, $X$ Rays in Theory and Experiment, 2nd ed. (Van Nostrand Co.. Princeton, N,J., 1935). pp. 263-315.

\section{Gold and Cesium Iodide X-Ray Photocathodes}

Time-resolved $x$-ray measurements using $x$-ray streak cameras are an important means of diagnosing inertial confinement fusion experiments. To meisure incident $x$-ray flux intensity over time, our streak cameras utilize the emission current from a transmission x-ray photocathode. Gold films $\sim 100 A$ thick are commonly used for this purpose. In some applicitions, however, such as $x$-ray backlighting or time-resolved irystal spectroscopy, insufficient s-ray flux can limit the effectiveness of gold photocathodes. Improving the sensitivity of the streak camera photocathode can thus extend the range of application of $x$-ray streak cameras.

Initial efforts to establish the optimum thickness of gold photocathodes under standard streak camera operating conditions have heen previously discussed. ${ }^{12}$ Cesium iodide, a photocathode material whose sensitivity and secondary-electron temporal-emission characteristics are reported to compare favorably with those of gold, has also been tested. 13.14 We have utilized the soft $x$-ray streak camera's convenient interchangeable-photocathode design to make side-hy-side sensitivity and temporal-response comparisons of $\mathrm{Csl}$ and $\mathrm{Au}$ in typical picosecond-scale pulsed operations. For these comparisons we used composite photocathodes, consisting of up 10 six CsI and/or Au samples of different thicknesses, vapor-deposited on a single cartoon-foil substrate.

We used the Monojoulc laser as our x-ray calibration facility. $\mathbf{X}$ rays were produced by irradiating $8-\mu \mathrm{m}-$ thick titanium foils in an evacuated chamber with short, intense $\left(10^{13}\right.$ to $10^{14}$ $W / \mathrm{cm}^{2}$ ) laser pulses. The target emission was filtered using $\mathrm{x}$-ray absorbers and reflectors, to define a moderately narrow energy channel. A 4000 $A$ vanadium [oil and a $3^{\circ}$ nickel reflector were used to form a spectral channel between 400 and $500 \mathrm{eV}$. The $x$-ray pulse width, which varies significantly with channel energy, was measured at $\sim 70$ ps FWHM.

A composite-photocathode profile of relative $\mathrm{x}$-ray intensity over time, obtained with 1080- $A$ thick CsI and 150- $A$-thick $\mathrm{Au}$, is shown in Fig. 5-17. The curves confirm the greater sensitivity of CsI and show its temporal response to be comparable to that of Au for this pulse width. The response curves of 
Fig. 5-17. Plot of relative intensity over time for the 1080- $A$ Csl and 150 $A$ Au composite photocathode. $\mathrm{X}$ rays are from a target heated with a leser putse train produced by sending a 50-ps, 1.06- $\mu \mathrm{m}$ laser pulse through a 50R-ps, 50\%-transmitting etalon. The temporal respoase of cesium iodide matches that of gold, with nearly identical pulse shapes down to $\sim 1 / 30$ of pulse peak intensity; all pulse widths are FWHM.

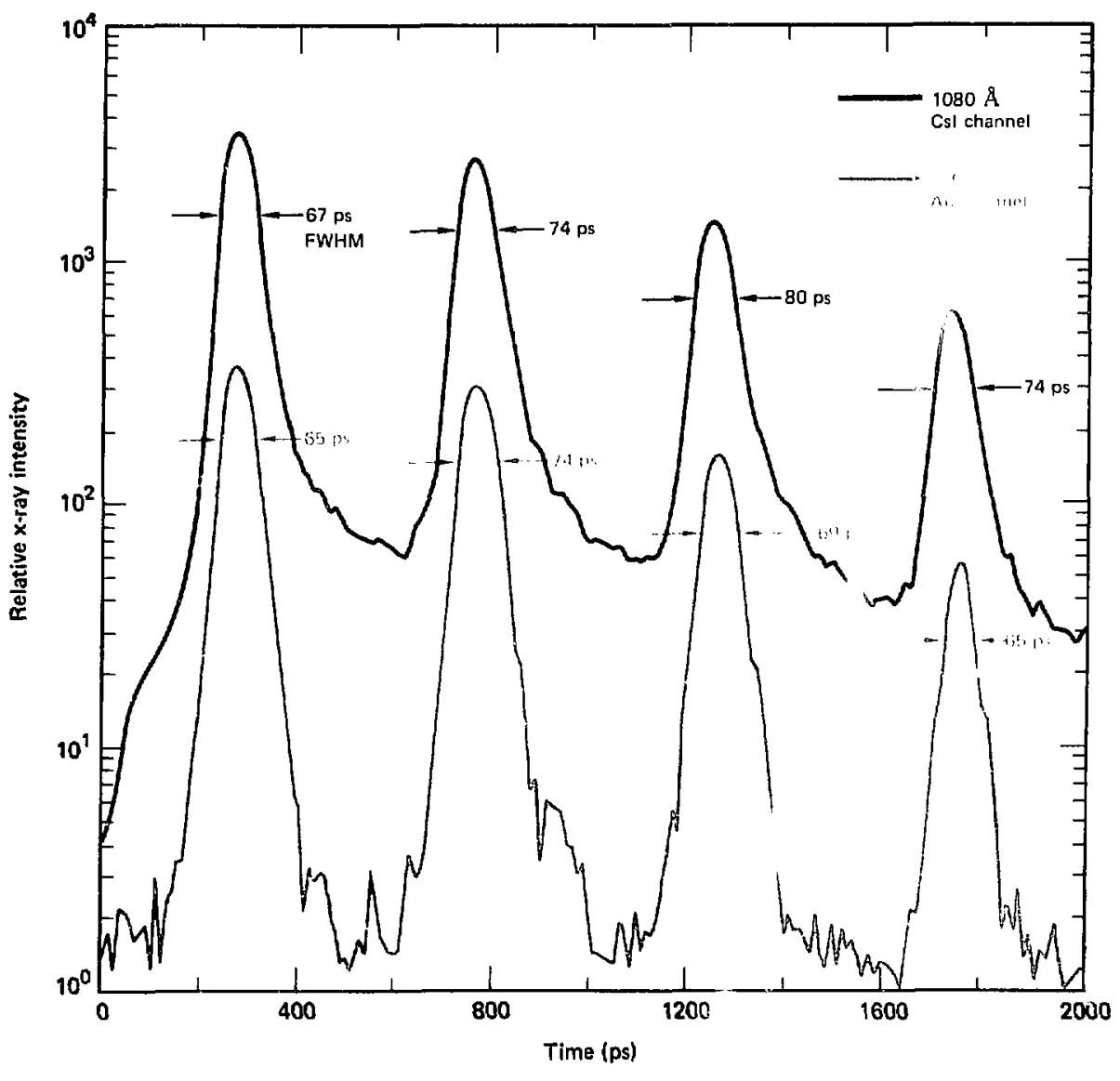

both materials to each incident $x$-ray pulse are nearly identical down to $1 / 30$ of pulse peak intensity, at which point the CsI curve begins to exhibit a low-intensity tail. This late tail miay indicate some electron straggling from cesium iodide which is not present in gold, although other possibilities have not been discounted.

The cesium iodide and gold photocathodes we tested range in thickness from 50 to $4000 A$. The relative sensitivitics of all these photocathodes were correlated with component samples common to dif- ferent composite photocathodes, to scale the Au and $\mathrm{C} \cdot \mathrm{f}$ photocathode response levels. The correlate relative intensities of both materials, for a nominal $\mathrm{x}$-ray energy of $500 \mathrm{eV}$ as described above. are plotted as a function of thickness in Fig. 5-18.

The data points in Fig. 5-18 represent the relative-response measurements of indıvidual samples for given material-thickness combinations. The iertical bars indicate the shot-to-shot $s_{F}$ read in each sample's response. These variations stemned from 
Fig. 5-18. Relative response-vs-lhickness of Csl and gold in response to $500-\mathrm{eV} x$-ray excitation. The data points represent the relativeresponse measurement of individual samples of a given material-thickness combination. The vertical bars indicate the shot-to-shot spread of each saniple respoise; the bonizonial bars show the measurement uncertenty ( $\pm 30 A$ ) in sample thickness. Also plotted are intensityversus-thickness data, taken with de, monochromatic, $573-\mathrm{eV}$ excitation and scaled for comparison.

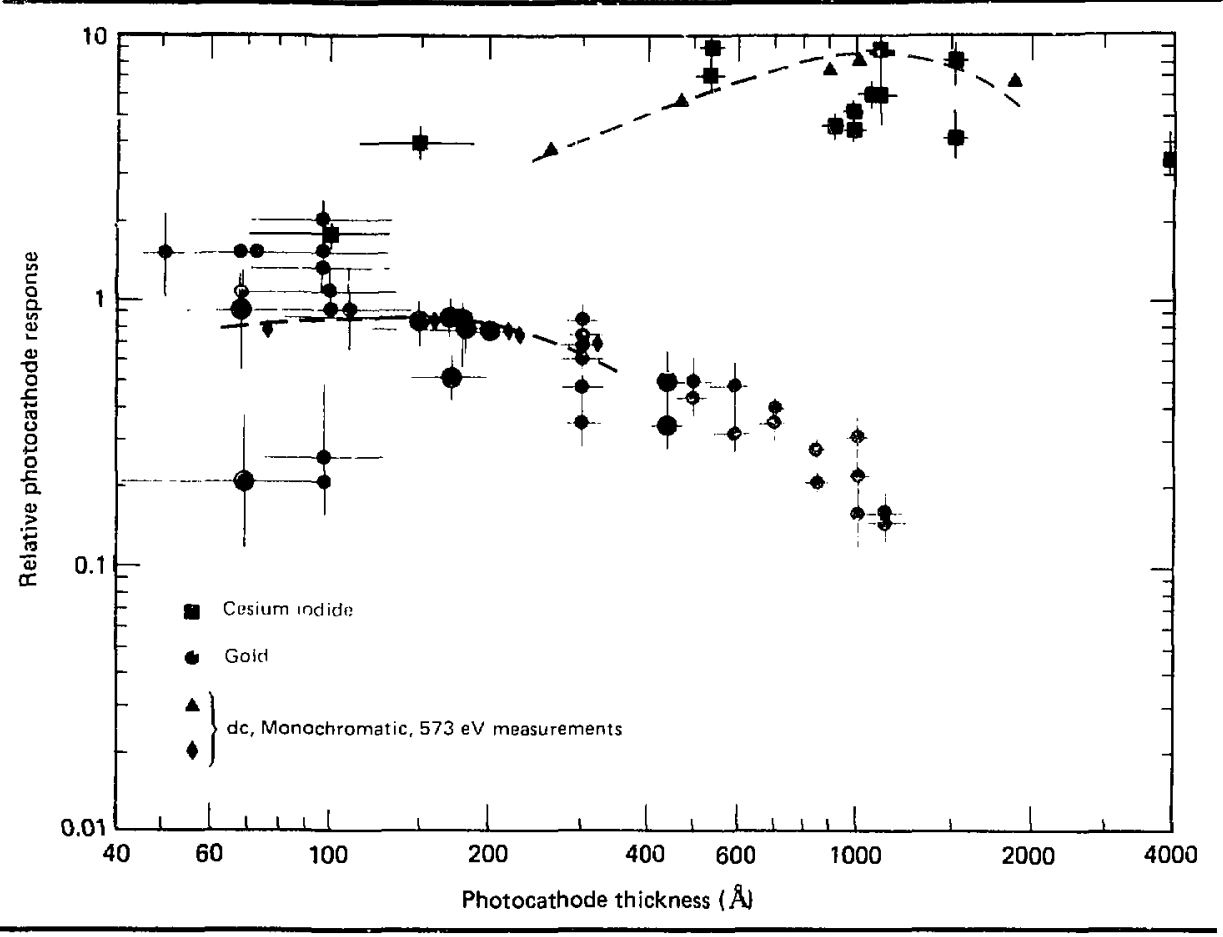

17uctuations in the target emission spectrum and from camera noise. Other variations arose when the range ol flux levels required to measure the span of component sensitivities on several composite photocathodes exceeded the camera's dynamicrange linitations.

Horizontal bars in the figure show the measurement uncertainty $( \pm 30 A)$ in photocathode thick nesses. Very thin $(\sim 100 A)$ gold samples show a wide sensitivity range: this variation is seen among thin samples from any of the several evaporation fucilities used, and even among samples produced in the same evaporation run. The lack of consistency in thin photocathode production may be due to variable surface properties, uncertainties in thickness measurements, or other unknown effects characteristic ol very-thin film depositicn. Because of the variable reproducibility of very thin samples. we recommend $200 A$ as a minimum thickness jor gold photocathodes.

Also plotted in Fig. $5-18$ are previously measured intensity-versus 4 hick ness data taken with dc, monochromatic, $573-\mathrm{eV}$ excitation, ${ }^{1 \downarrow}$ which have been scaled for comparison with our measurements. Our observations of sensitivity-versusthickness maximi correspond well with these findings. At $500 \mathrm{eV}$, the optimum CsI thickness of $1100 A$ is more sensitive than $200-A$ Au by a factur of five; larger factors $(\sim 30)$ are expected at photon energies of $1.5 \mathrm{keV}$. The difference between the two sets of data, regarding the relative sensitivity of cesium iodide and gold. may be attributable to the mix of $x$-ray energies in our source. Efforts are in 
progress to measure the source spectra, and to confirm the larger sensitivity ratios above $1.5 \mathrm{keV}$.

Cesium iodide photocathodes are seen to significantly increase $x$-ray streak camera sensitivity without appreciably affecting the camera's temporal response to $\sim 70$-ps structure. We are now implementing these photocathodes in selected applications requiring increased sensitivity.

\section{Authors: G. L. Stradling, R. L. Kauffman, and B. L.} Henke (University of Hawaii)

\section{Referen :s}

12. Leiker Program Anmut Report-1978. Lawrence Livermore Lithuritur, Livermere, Calif., UCRL-50021-78 (1979), P. 6.2.

13. B. I., Henke, J. Lengang, ind S. D. Smith, Phrs. Rer, $B 19$. $350+(1979)$

14. B. L Herike, J. P. Knatuer. and K. Permerane, Ball. Alm Phis. Set 24, 1098 (1979).

\section{CCD Applications for}

\section{Transient Digitizers}

Streak Camera Readout System. The ultrafast streak camera is one of the most valuable hightemporal-resolution diagnostic tools available in laser fusion research. The need for a totally computerized data acquisition system with which to take full advantuge of the ultrafast camera hat been previously addressed. 15 This year we report the development of a high-resolution, large-dynamicrange, all-solid-state data acquisition syst'm designed specifically for the LLL laser lusion program"s $x$-ray and $1.06-\mu \mathrm{m}$ ultrafast streak cameras.

Our system design philosuphy seeks to acbicte three primary goals:

- Minimization of computer processing ol raw data.

- Immediate viewing of raw and processed data on a standard TV monitor, to determine the validity of the information captured.

- Minimization of the overall external hardware and software support requirements.

Based upon these criteria and our previously reported research, ${ }^{16}$ we have chosen a newly developed RCA all-buried-channel charge-coupleddevice (CCD) as our sensor. This two-dimensional solid-state silicon CCD has a resolution of 512 lines, each of which contains 320 individual pixels.
Becaluse of the all-buried-channel construction of the CCD. its dynamic range is greuter than 256:1. We have also previously determined that this type of sensor provides linear response to both photon and electron excitations.

Using the RCA SID53601-XO CCD sensor satisfies our first goal, which is the minimization of computer processing before the acquired information is valid. Since the CCD is a lineat device and shows no reciprocity fialure even in the picosecond time domain, ${ }^{17}$ no computer processing, analogous to D-log(E) corrections of lilm, is required. These ideal properties of the CCD thus transiate directy to at higher level of conlidence in the atcquired streak (iatia.

Our second design goal for a streak cumera readout system is the ability to display streak data on a standard $\mathrm{TV}$ monitor immediately after the event. This instant playback is especially importan! during the initial streak-camera setup and or calibration period. during which prompt leedback is required for sioh critical parameters as tining. jitter. and inpul-beam alignment. In many earlier systems. analog video recording devices (such as video disks) were employed to accomplish this task. ${ }^{\text {is }}$ ('nfortunately. Lhese analog techniques require a compromise between instuntaneous playback and the dynamic range of the instrument.

To circumvent this trade-off while preserving the all-important instant playback feature. we have developed a unique all-solid-state. random-acces.s. high-speed image memory capable of recording and subsequent playback of all $330 \times 512$ pixels of $C C D$ data with an intensity resiolution of 256 gray levels ( 8 bits). Furthernore. the image-memory readout unit is designed to the specific requirements of the stieak cantera application: timely computer processing of the large volume of CCD duta is ably promoted by such leatures as high-speed directmemory-access data transfer in the column, row, or diagonal read write modes.

A number of design features combine to satisly our third goal. that of minimizing the data acquisition system's external support requirements:

- With a set of special control lines we can program the instrument's operating characteristics totally by means of software control.

- To integrate the readout system into a large, instrument-laden laser facility like Shiva, we 
Fig. 5-19. General róew of the CCD date ecanisition eyste- for an exiating LLL ultrafust x-ray sareak cenera.

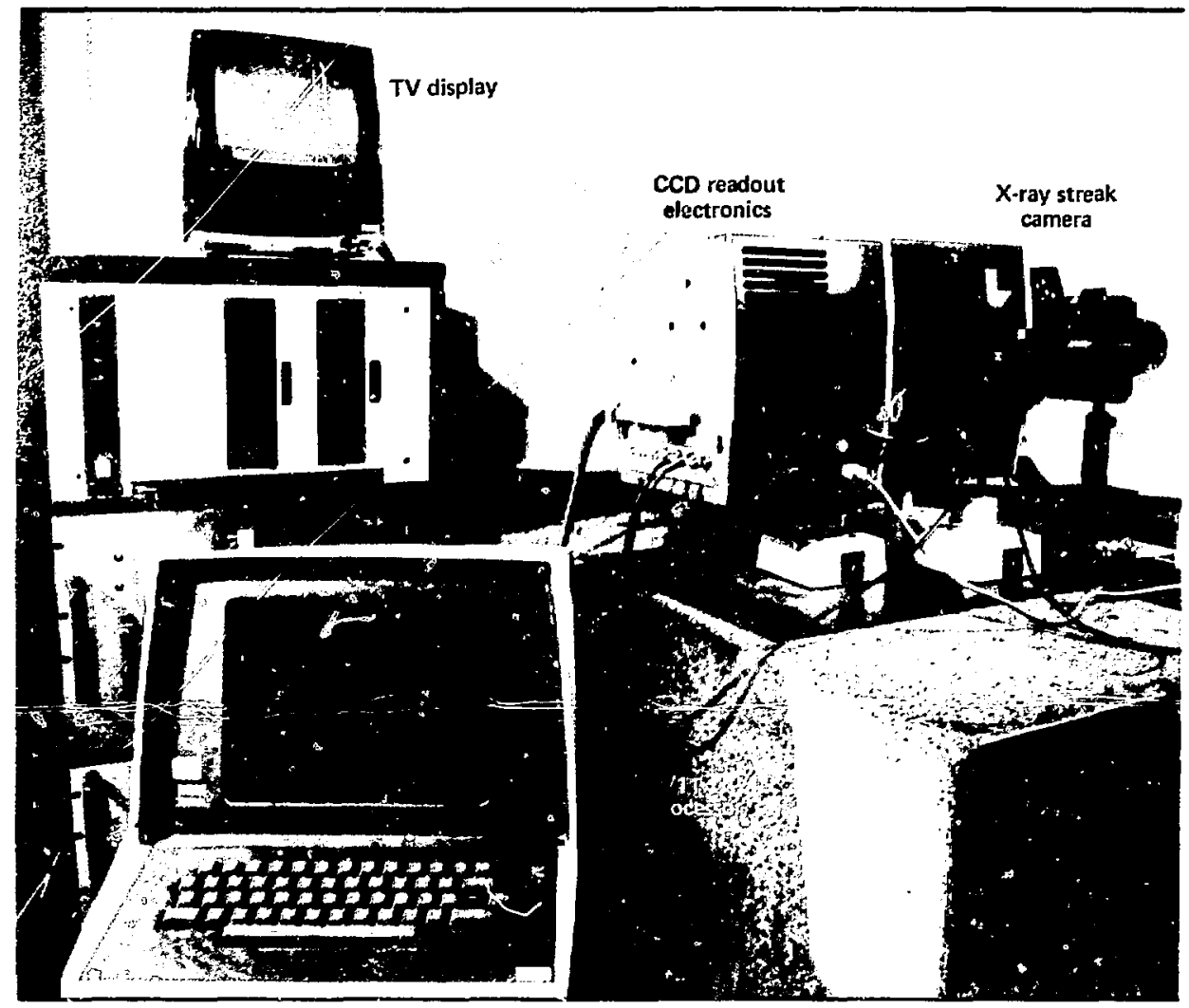

have included options which initialize. enable computer. triggei the system. and provide calibrated tackground. gain, and sweep speeds.

- To simplify the operation of the readout ıni:. we have developed a new CCD scanning mude "nich removes the need for a pre-event trigger. This simplification is of great importance in a large laser diagnostic systern where various other instruments may require complex and precise pre-event triggers: an additional load to the master timing network mat be inconvenient or difficult to achieve. With the advent of this new $C C D$ continuous scanning mode. the readout instrument need only be triggered at event time (zero time trigger). The source of this zero time trigger can be conveniently. derived directly from the streak camera's own sweep circuit.

Higure 5-19 shows a general view of one version of the LLL streak camera diagnostic system. In this version, the CCD camera portion of the readout system is optically coupled to the phosphor end of a standard LLL 10-ps streak camera with a 2.5-10-1 coherent fiber-optics minifier. (This external coupling technique of course offers the advantage of minimal hardware redesign in retrofitting existing sireak cameras with a $C C D$ readout system.) $A$ cable connects the camera portion of the instrument to the image memory unit: the two sections can be separated by at least 100 feet. and data transmissions between the two sections are totally digital. Figure 5-19 also shows the instant-playback TV 
Fit. 5-20. Schenatic of an x-ray atreak camern data acquisition syoten, implezented in a stand-alone configuration.

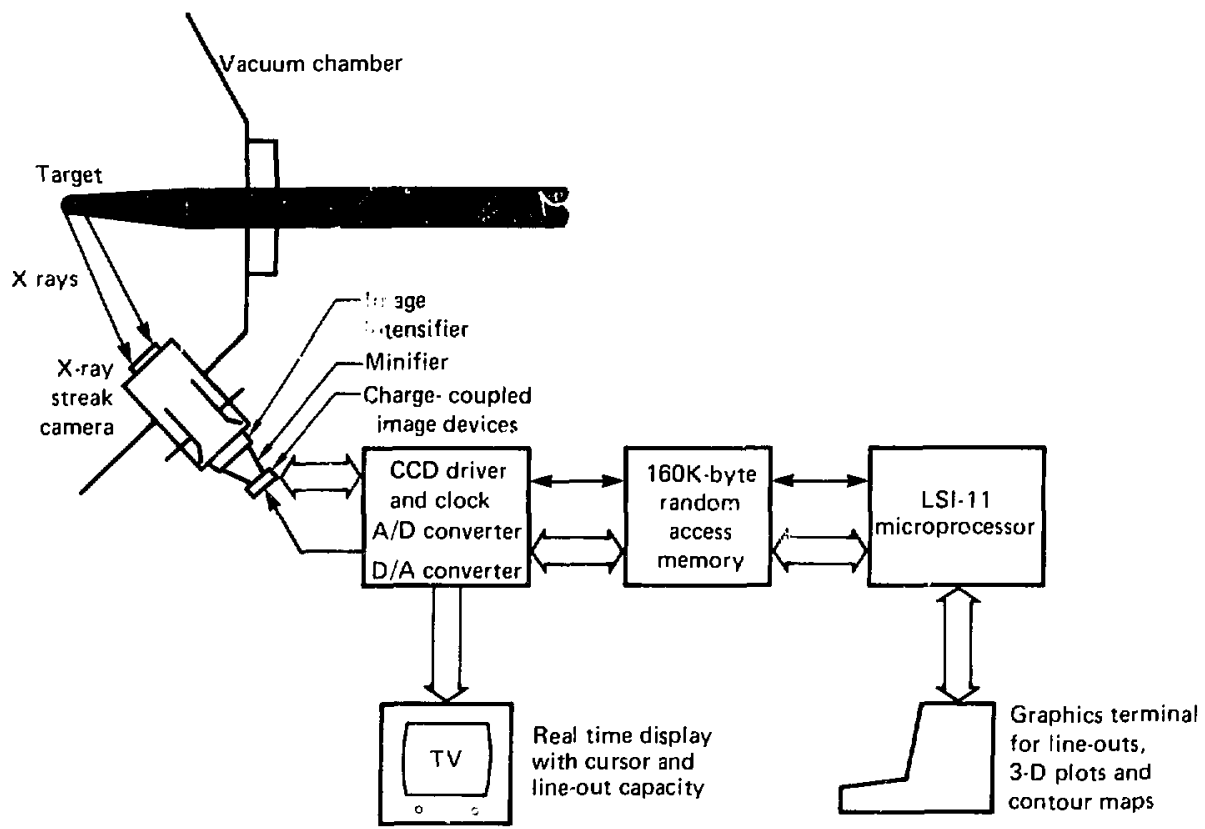

monitor, a local LSI-1I processor, and a Tektronix 4006 graphics terminal. In this case, the system has recorded a series of 1.06- $\mu \mathrm{m}$ etalon pulses, and has displayed the recorded data on the video monitor both as a two-dimensional intensity-modulated picture and as a computer-generated line-out profile (also visible on the graphics terminal).

Figure 5-20 shows a schematic of the main segments of a complete $x$-ray streak camera data acquisition system and computer interface network:

- $\mathrm{X}$ rays generated by the target in an experiment are recorded by an $x$-ray streak camera.

- The phosphor image is transmitled by a fibcr-optics minifier to an all-buried-channel CCD sensor.

- The analog CCD data is digitized to an intensity resolution of 8 bits at standard video rate $(6 \mathrm{MHz})$ and stored in the solid-state image memory unit.

- The digitally recorded data in memory are accessible either by a computer (in digital format) or by the TV monitor (in analog format) as a standard interlaced video NTSC picture.

- The memory unit can be interfaced to a local LSI-11 computer hus, for example, or to a custom bus (such as the CMOS power conditioning bus ai Shiva; see Section 2, "Engineering Summary and Update: Power Conditioning") which totally bypasses the need for a local microprocessor.

- Finally. the system is capable of external composite synchronization. Video information displayed either from memory or at real time can be joined witk all other vidco data from diverse sources (streali cameras, TV cameras. etc.), and viewed through a standard studio TV switching network.

(Cl) IItrafast Transient Recorder. Multigigahertz oscilloscopes, like streak cameras, are a primary diagnostic tool for many laser fusion experıments. From studies using intensified (i.e., electron-in mode) (CD readout for an EG\&G 
Fig. 5-21. The I.LL/Tek 4GHZ R7912/CCD transient digitizer system, along with the associated ISt-1I microprocessor, image memory, TV, and araplites terminal.

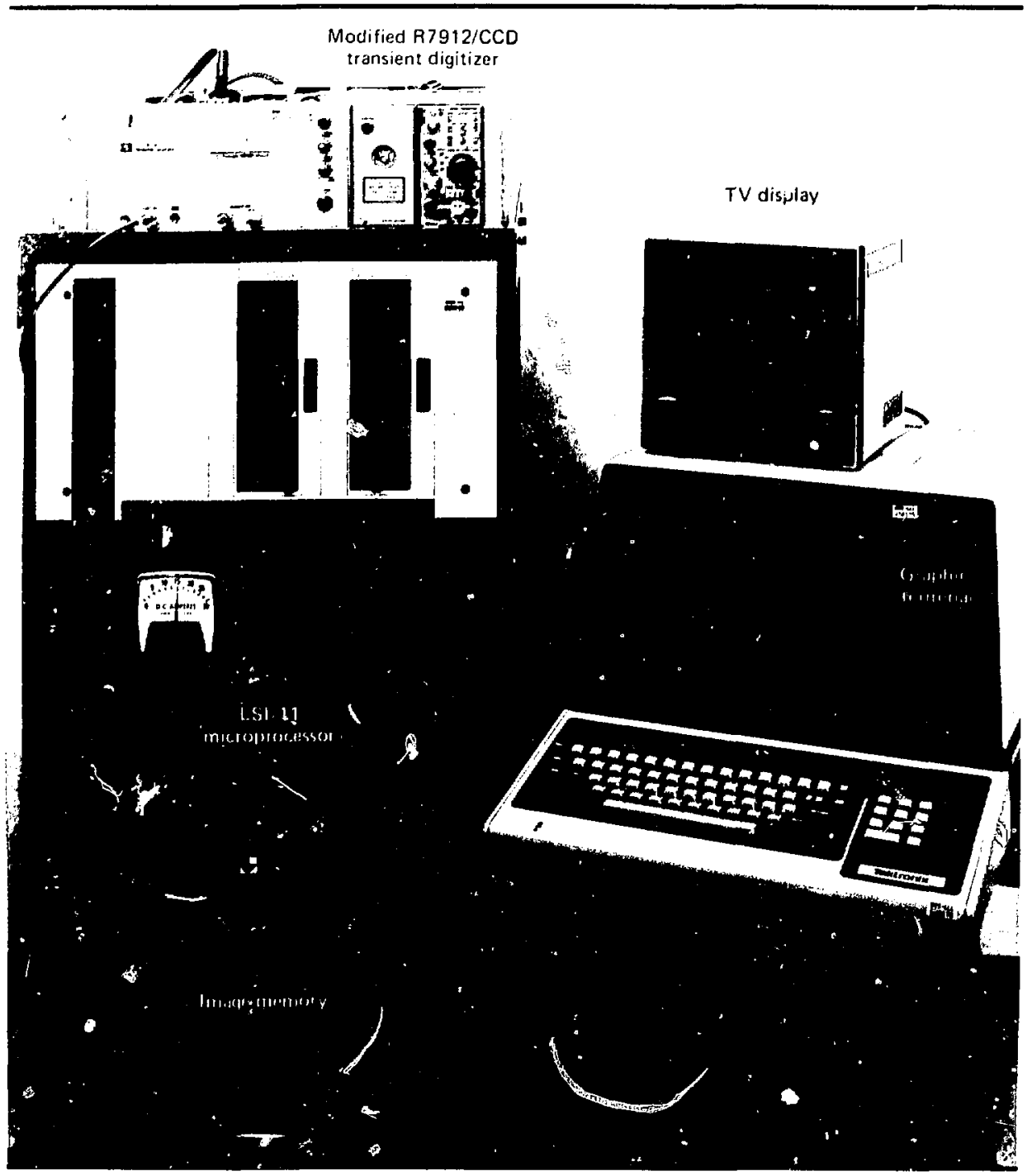

KR23 oscilloscope. it became clear that a CCD sensor has many advantages over ronventional phosphor film readout techniques. ${ }^{14}$ During the past year we have made significant progress toward producing a practical CCD transient digitizer for use in a CCD multigigahertz oscilloscope system: advances were made possible by joint efforts of LLL, RCA, and Tektronix research groups.

The 4-GHL CCD ultrafast oscilloscope we developed uses many components already developed for the $\mathrm{CCD} /$ streak camera data acquisition 
ynem. as a general wew of the I I I lek ( ( 1)

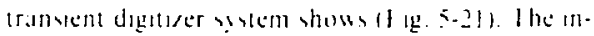

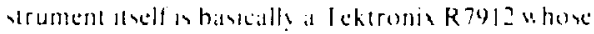
argenal deade target arra! and reid gun hase heen replated wh a satum-compallable bateh-thmed

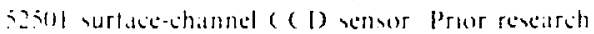
determaned that the ( (1) emore ehobited mant ad

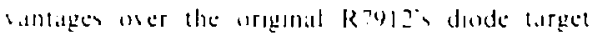

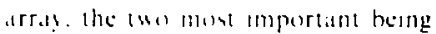

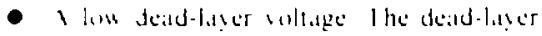

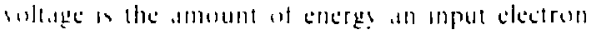

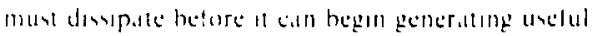

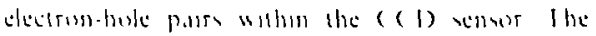

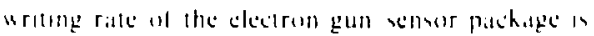
derectls relaled the the lonal number of semal electrom eneneraled per incideme electron since the

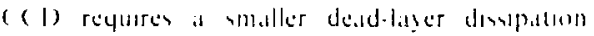

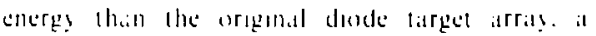
correspondingly lower electron heam enerty can mantan the same wronge ralle: thos lower clectrongun energe tramblaten directly to a higher delketom handudah ior the modelied R79l? ( R 1 . He are athe (1) operate the electron gun at $7 \mathrm{ket}$. rather than the orgemal $11 \mathrm{kel}$. whech produced a beller siatch belween the etectron propagittion velocits and the dellectorn whages phase belocily within the helix tratcheng wate deflectom structurt.

- Superior andubleoming properly. The anuble oming property of at ( ( 1 ) is the semsor a ability (1) present hoth sertical and horisontal charge spillage moto adjacent (CD) cells when the sensor is oserloaded. In the original R7912. a disde target array "as uned to record the electron beam. In that design. there was a trade-olf between the latget's antiblooming proper's and its sensitivity to electron excitatione. Vou, with the incorporation of a ( ( C into the R 7912. the excellent antiblooming property and the high electron sensitivity can be achieved simultaneously. which will signilicantly improve the overall performance of the transient recorder.

Figure 5-21 also shows a 160K-byte solid-state image memory and an LSI-II local microprocessor, desigricd to he incorporated into the existing R7912 commercial housing unit. The system has here recorded a 100 -ps electrical pulse and is redisplaying the captured data from menory onto a TV monitor. A corresponding computer-generated scope trace of the pulse also appears on the Tektronix 4006 graphies terminil.
In summats. the major advances in the ( (I) ( R I areal the sear are

- Ihe encecrsial incorporation of a (c)

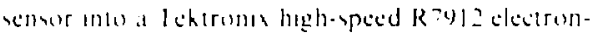
gen enchere. than combintalon has resulted an it

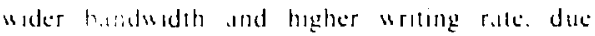

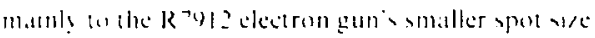
$1+11 \mu m+$ and hegher heam current $15 \mu$ il.

- Ihe desclopment and unige of the feok

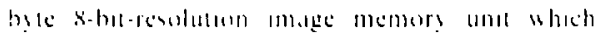
collbles the combined molrument to capture.

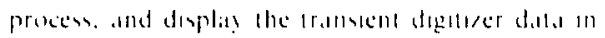

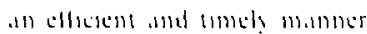

Author: J. C. ( hengh

Vlajor Contributors: (i, R. Tripp, J. T. Voonan. R.J. Schenete, F. Saroug (RCA), and I., Rile? (T'Tektronis)

\section{References}

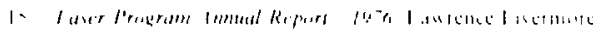

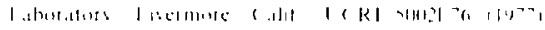

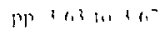

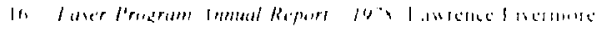

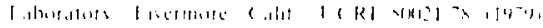

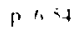

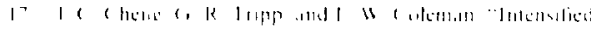

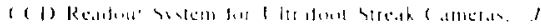

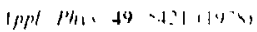

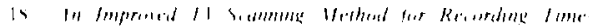

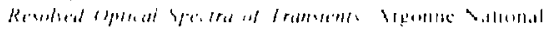

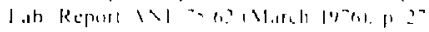

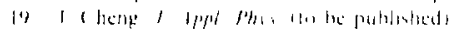

\section{Reflector-Diffractor Spectrograph Measurements on Shiva}

Wle are interested m makng hight-resolution $x$ raly upectroncons? moanurements in the photon energy region from $13 . .1$ lo $1.2 \mathrm{kcV}$. hecause this region contains chatrateteristic lines (in the $v$ and $O$ serics) of gold and other heavy elements. There are lwo ypes of metruments that can be used in this region, the diffraction crystal spectrograph and the grating-incidence grating spectrograph, although it is difficult to obtain accurate quantitative data from either instrument in the region of interest.

Despite the practical advantages of the erystal spectrograph. including lower initial cost, easier 
Fig. 5-22. Schemafic of the Shiva reflector-diffrector spectrograph, with grezing-incidence mirror reflectivity limited to 1.2 keV: actual size of mirror is $4 \mathrm{in.} \times 4 \mathrm{in}$.

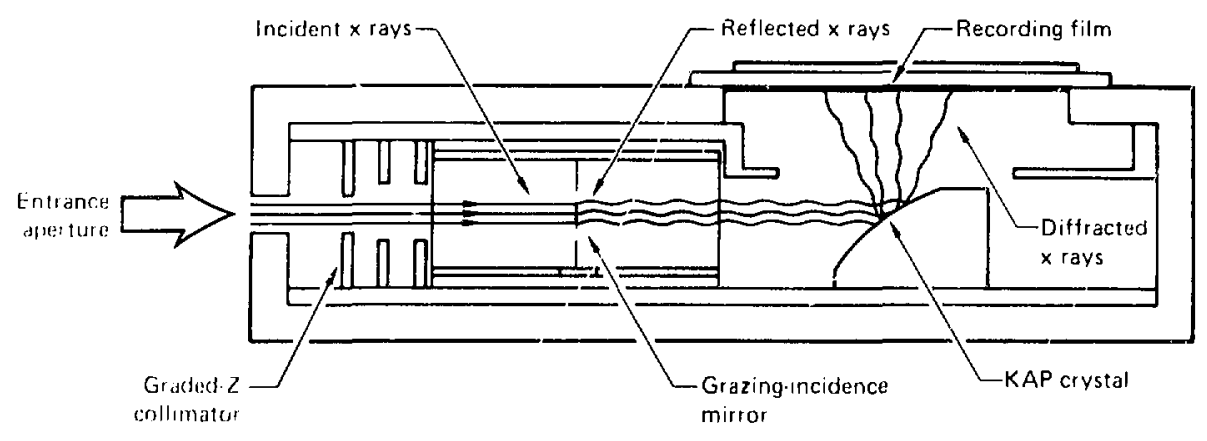

Fig. 5-2.3. X-ray response of key components of the crystal spectrograph.
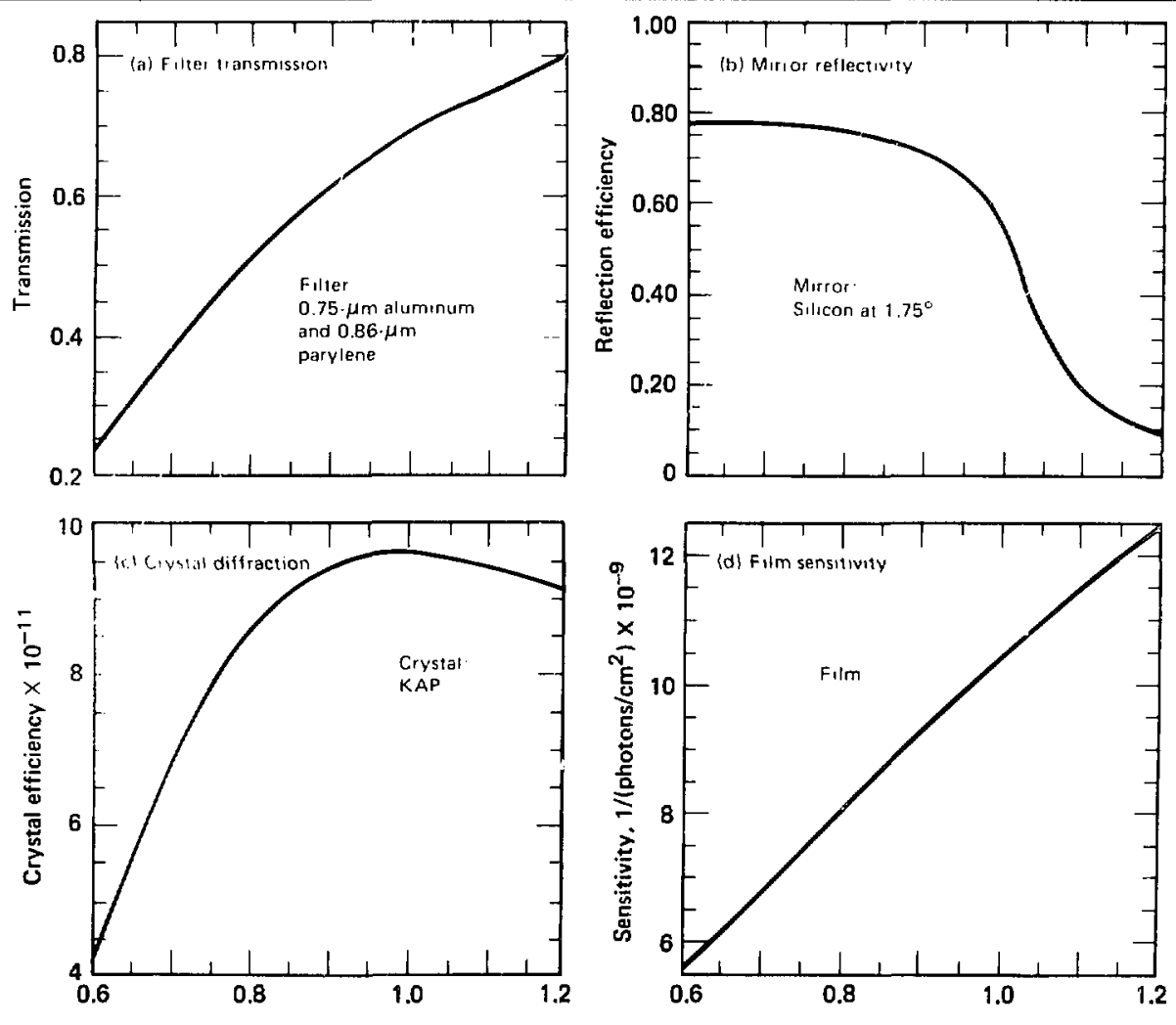

Photon energy (keV)

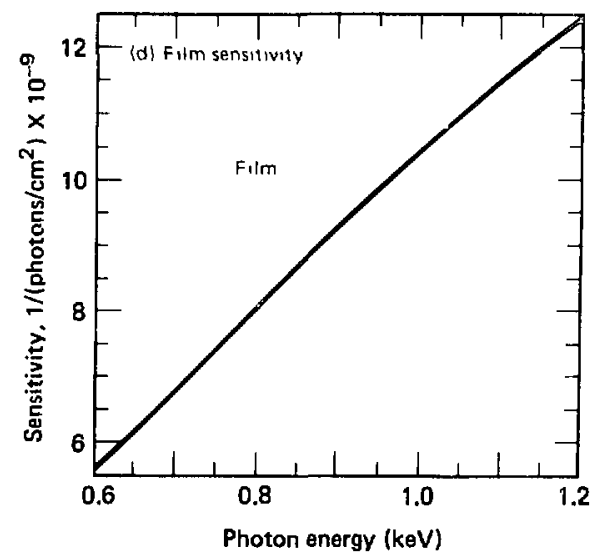


alignment and maintenance. and higher $x$-ray sensitivity. previous attempts to use crystals to record sub-kilovolt spectra have demonstrated a basic problem with the method: the senstivity to $x$ rays of greater photon energy than desired (such as the $M$ lines of gold near $2.5 \mathrm{keV}$ ). This unwanted response results from the nonnegligible second- and thirdorder diffraction efficiency of crystals. and from the greater $x$-ray transmission and sensitivily of filters and photographic film. respectively. at higher photon energies. To suppress unnecessary sensitivity, a reflector-diffractor spectrograph in operation at Shiva (Fig. 5-22) uses a grazing-incidence mirror whose reflectivity falls abruply above $1.2 \mathrm{kel}$. effectively filtering the $\mathrm{x}$-ray beam reaching the KAP crystal. Measurements obtained with this instrument for litinium. vanadium. and gold disk shots at Shiva have provided useful qualitative information, indicating whether significunt energy in the region from 0.6 to $1.2 \mathrm{keV}$ is contained in characteristic lines.

l.on response characteristics also make it difficult to obtain aceurate quantitative data with the spectrograph. because small errors in the calibrattion of the components can produce significant errors in the reduced spectral data. Figure 5-23 shous the $x$-ray response of the spectrograph 's key components. Ai low photon energies (near $0.6 \mathrm{keV}$ ) the light-light filter does not transmit well, the crystal reflectivity is lon, and the photographic film is not sensitise: Fig. 5-24 shous the same general result for the total instrument response. Under these conditions, small calibration error may translate into significant error in reduced ditlit. To date agreement between the spectra (Fig. 5-25) obtained

Fig. 5-24, Complete reflector-diffractor spectrograph sensitivlty.

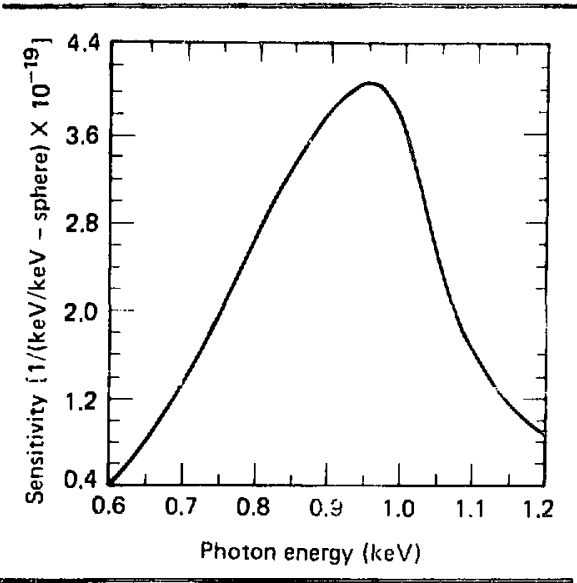

Fig. S-25. X-ray spectra in the region of 0.6 to $1.2 \mathrm{kev}$, obtained for (a) titanium and (b) vanodium disk shots at Shiva.
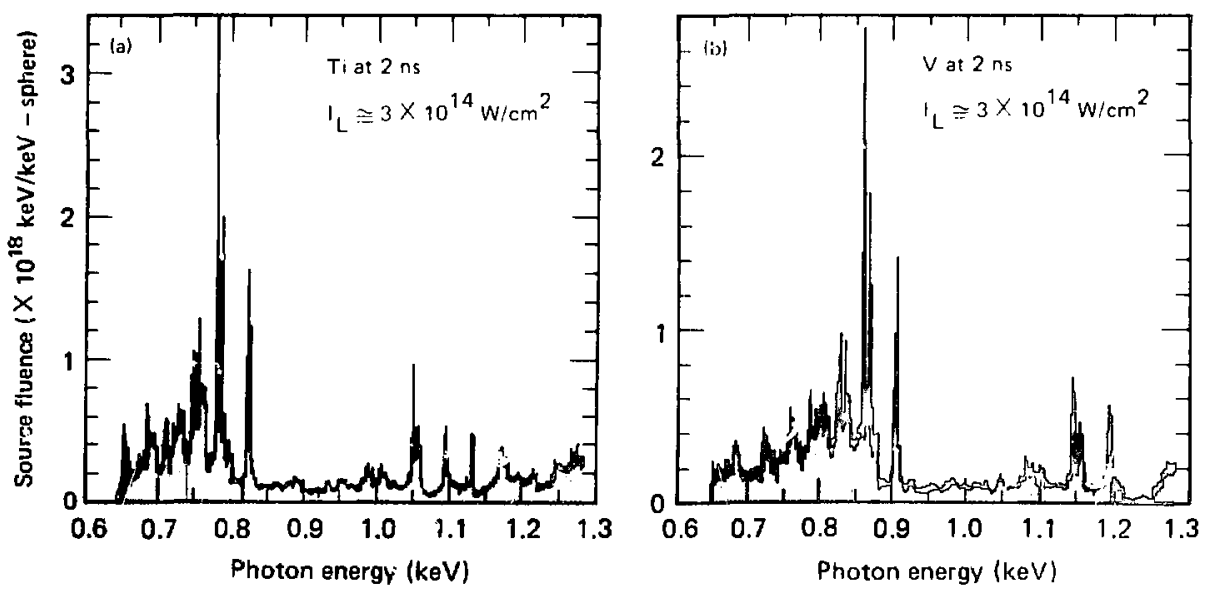
with the crystal spectrograph and with the Dante rectrometer systenis has not been good. A careful recolibration of the spectrograph components is maceding, and the situation shouid improve.

spectra obtained with the instrument for seral lypes of disks are shown in lig 5-25. The dish uere irradiated with $3 \times 10^{14} \mathrm{~W}^{2} \mathrm{~cm}^{2}$, in pulses of 2 ns. The data for the titanium and vanadium disks show lany sharp lines in the $\mathbf{L}$ series. due to the berylliun.-like and lithium-like species ( 4 or 3 electrons remaining bound to the ion). Despite a wide range of irradiation conditions. however. distinct lines for gold in the $\mathrm{N}$ and $\mathrm{O}$ series have never been observed.

\section{Author: I. \. Koppel}

Fig, 5-26, X-ray reflectivity of a tungsten-carbon synthetic multilayer structure (SMS) mirror, in the region from 4 to $12 \mathrm{keV}$; each profile results from diffraction at a particular $\theta$ from $1.4^{\circ}$ to $3.5^{\circ}$.

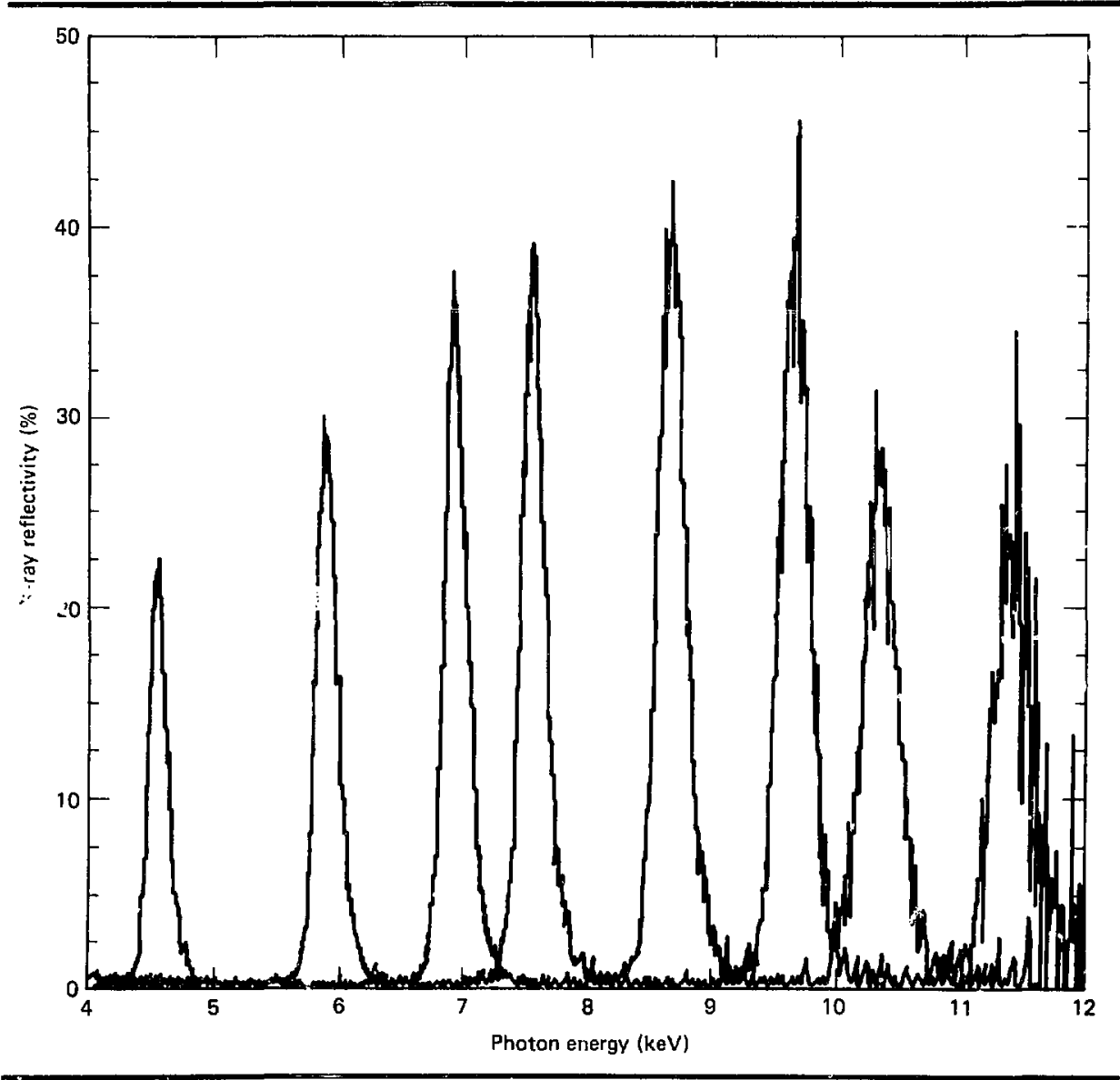


Fig. 5-27. Comparison of tungsten-carbon and lead myristate multilayer $x$-ray mirrors in the region fmom 500 to $1000 \mathrm{eV}$, for various layer $p$ airs. Reflection probability is plotted vs $2 \theta$.
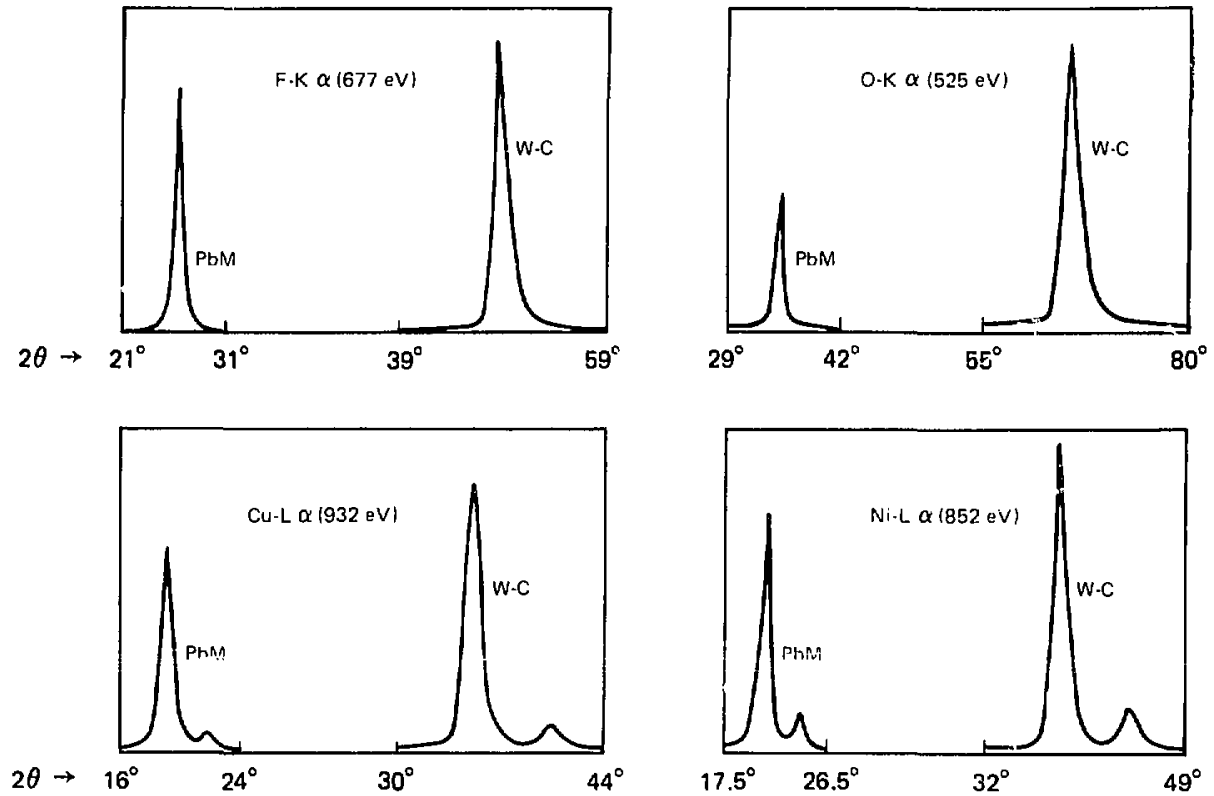

Collimation FWHM (Gaussian) $-0.77^{\circ}$

\section{Synthetic Multilayer Structure Characterization}

We are participating with the Center for Materials F search (CMR) at Stanford University in a prejeet to develop and refine a new type of $x$ ray mirror based on $x$-ray interference within a synthetic multilayer structure (SMS). On a smooth substrate we use vacuum sputtering deposition to build up a stack of films. where each film is 7 to 15 or more $A$ thick. Each layer of the stack is actually a pair of films (called a layer pair), one each of a highand low- $Z$ material: a typical layer pair consists of tungsten and carbon. The spatially-periodic variation in coherent scattering power produced by this construction results in efficient medium-bandwidth $x$-ray diffraction. We have observed, for example, peak $x$-ray reflectivities of 20 to $40 \%$ and resolutions of $E / \triangle E=30$ to 40 for tungsten/carbon structures.

Dispersion by the SMS is controlled by the Bragg re $\therefore \cdots$ : $\lambda=2 \mathrm{~d} \cdot \sin \theta$, where $\theta$ is the grazing incidence angle of an $x$-ray beam and $d$ is the thick ness of a liayer pair. Simples with values of $d$ as small as $14.5 A$ have been built and tested, making feasible the use of the SMS in the wavelength region from 1 to $100 \mathrm{~A}$. Figure 5-26 (opposite) illustrates the x-ray response of a tungsten-carbon Sir? ${ }^{2}$ with a d value of $22.4 A$ : eilch profile results from diffraction at a particular $"$ in the iange from $1.4^{\circ}$ to $3.5^{\circ}$

$X$-ray lesting was performed in a vacuum dilfractometer equipped with a Henke tube $x$-ray source (ihis facility is maintained by the L-division $x$-ray Measurenents (iroup at LLL). The $x$-ray beam was continuous in energy in the region from 4 to $12 \mathrm{keV}$ and was collimated to $1 / 2$ arcmin in the plane of dispersion. Spectra of the beams incident on and reflected by the sample were obtained with a high-resolution $\mathrm{Si}(\mathrm{Li})$ detector. Al each of several values of incident angle, a raw band-pass curve was oblained by dividing the reflected spectrum by the 
incident spectrum: the curves in Fig. 5-26 were oblaned in this manner.

Multilayer mirrors have the following advan$\therefore g(s)$ when used as spectrometers in the region from 110104 :

- The resolution of the SMS bridges the gap : ween those of natural crystals and spectrometer bianels defined by Ross filter techniques.

- The dispersion and resolution of the SMS an whored by adjusting the value of $d$ and num. i o liver pairs

- In SMS with equal layer thicknesses and ". aded interfaces suppresses high-order diffraction isponse.

When used in $x$-raty imaging instruments, the . Mractive properties of the SMS are

- Ifficient reflection of $x$-rays whose photon energies exceed the practical limit of total external reflection.

- Adaptability to curved substrates, making kissible x-ray optic elements of complex cirvature.

Multilayer mirrors can also be used to good adantage in the region from 10 io $100 \mathrm{~A}$, whet the choice of $x$-ray dispersion elements has previously been limited to grazing-incidence ruled gratings and stearate-type (soap film) artificial crystals. For measurements requiring high s,yectral resolution $(\lambda / \lambda \mathrm{s}>100)$ there is no alternative to gratings. The SMS, however, appears to be better suited than the stearate-type crystal for medium-resolution applications in this region, because

- The d value for the SMS is not controlled by the carbon chain length of high-melting-point fatty acids, but can be freely selected.

- The particularly pernicious higher-order diffraction response of stearate crystals can be avoided by proper SiMS fabrication.

- Proper selection of SMS component elements preserves sensitivity in the region from 25 to $50 A$ where the reflectivity of stearate crystals is very low.

Measurements at ihe University of Hawaii have provided the most thorough characterization of the SMS in the soft $x$-ray region. As shown in Fig. 5-27, the reflectivity of a tungsten-carbon SMS is about twice as high as that of a lead myristate crystal.
Author: L. N. Koppel

Major Contributors: T. W. Barbee (Center for Materials Research, Stanford University) and B. L. Henke (University of Hawaii)

\section{Shiva Optical Pyrometer}

The Shiva optical pyrometer is designed to measure visible light emitted by a target. An achromat telescepe focuses light magnified by about ten onto the slit of a streak camera: the beam is split to provide two spectral channels, usually chosen to be in the orange and blue regions of the spectrum (Fig. 5-28). Also, a portion of one of the incident laser beams is doubled to provide a $5320-A$ timing fiducial for the streak camera. This $5320-A$ fiducial is necessary because the streak tube has an S-20 photocathode. Each streak camera photograph will thus have three concurrent, but spatially separate, streaks.

The fiducial is limed by irradiating foils with rod shot of short pulse lengti. for linese shuls buth spectral channels are set up to respond to $2 \omega$ light from the target. We assume that the peak intensity of the $2 \omega$ light is coincident with the peak intensity of the $1.06-\mu \mathrm{m}$ light.

The KIDP doubling crystal is aligned using the collimated beam from the Argus oscillator: the phase-matching direction is indexed by two crosshuirs. The entire optical assembly is on a kinematic mount.

The streak camera detects light coming from a part of the target that is typically only several hundred micrometers across: such a source requires that we check the alignment of the optical assembly before every shot if our tesults are to be believable. For alignment, the target is illuminated at the specular angle. as shown in Fig. 5-28 (plan view). We view the target in reflection, rather than simply shadowing it, because the laser-irradiated side of the target is usually masked off by a large shield many millimeters in size: this shield is necessary for the complementary low-energy Dante diagnostics, which presently have no imaging capability.

We have measured the transmission of the two optical paths as a function of wavelength, using unpolarized light, and have measured the spectral response of the streak camera photocathode. With this information, we can calculate preheat temperature from the ratio of the orange and blue chan- 
Fig. 5-28. Plan and side views of the Shiva optical pyrometer; the two achromat lenses in the telescope are not shor'n.

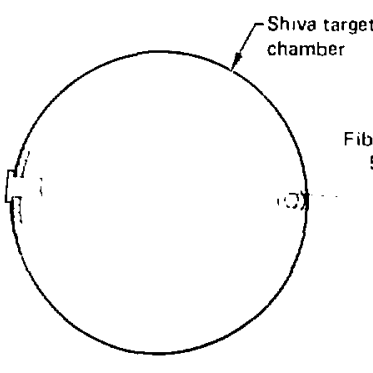

Fiber

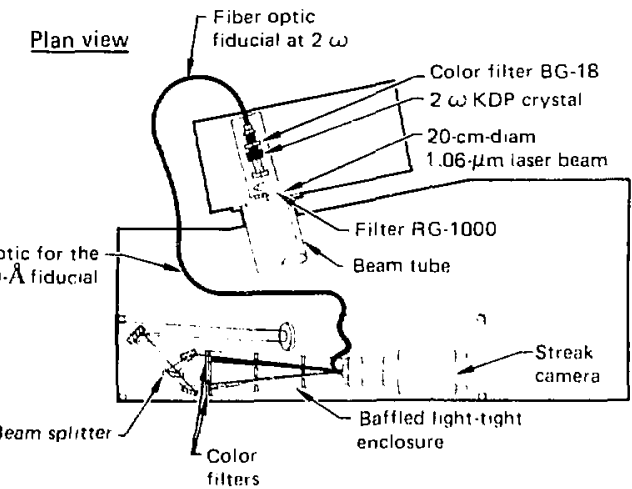

Side view
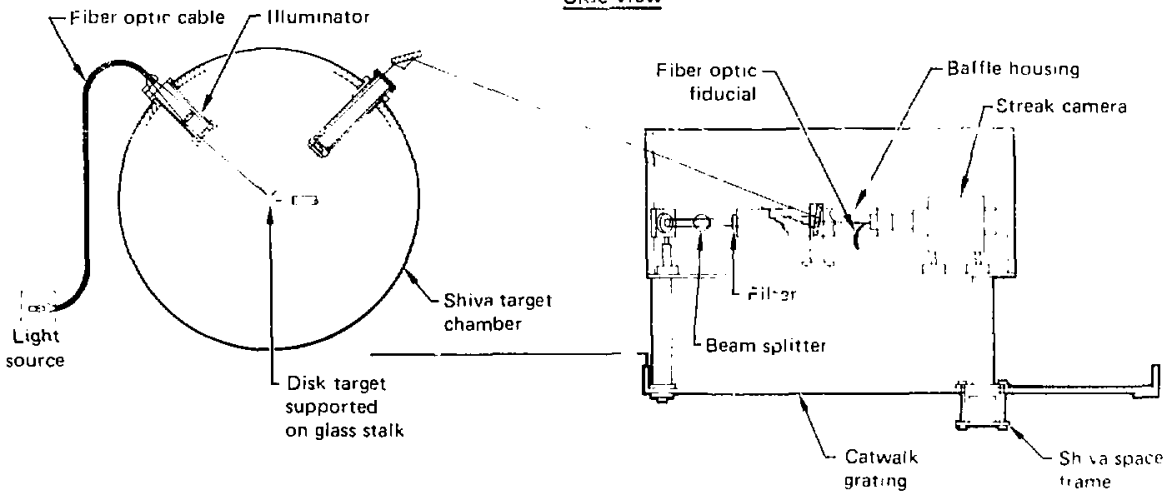

nel fluences, provided $T ₹ 1 \mathrm{eV}$.

If the temperature is too high. both channels see emission in the Rayleigh-Jeans portion of the blackbody spectrum, where the flux is simply proportional to the temperature: the ratio ol the two Ruences will then tell us only that the perature is 'igh. The streak cantera has been absolutely calibrated, however, so that we can also deduce a temperature from the film exposure, using the calibrated step-wedge exposed on the film: the spectrum and duration of the light exposing the step-wedge match those of the light radiated by the P-11 phosphor at the output of the streak camera microcbunnel plate.

\section{Author: D. W. Phillion and M. W'. Kobierecki}

Major Contributors: D. 1. Banner and R. D. Rudd

\section{Shiva Raman Light Spectrograph}

We are setting up an optical experiment at Shiva that will give far grealer spectral resolution of the Raman-scattered light than was achieved at Argus, where the spectrum had to be obtained from many laser shots by changing interference filters. The heart of this experimental diagnostic is a 25 element indium arsenide array fabricaled by Judson Infra-red. The array is mounted in a stainless steel Dewar flask and cooled to the liquid nitrogen temperature of $77 \mathrm{~K}$. The array will be read out by 
Fig. 5-29. Experimental setup to spectrally resolve Raman-scattered light at Shiva.

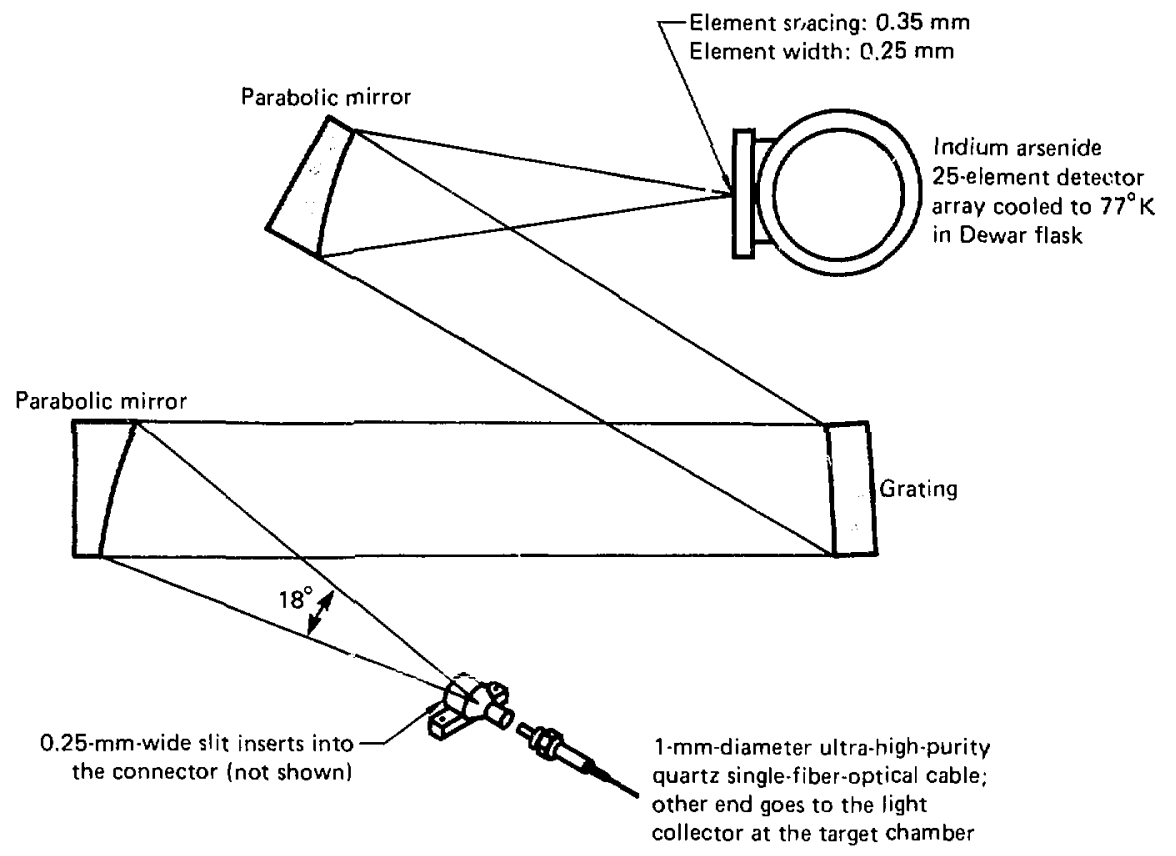

programmable clarge-integrator modules interfaced with the Shiva data acquisition system. Each single-width module contains four channels, whose sensitivities are set by computer control.

The spectrograph (Fig. 5-29) is compact: the Dewar flask is $2-1 / 2$ in. in diameter and $5-1 / 2$ in. high, the optics if? $? \mathrm{n}$. in diameter, and the effective focal length "the two parabolic mirrors is 6 in. (f/3.2). A $2 \lambda_{0}$ wavelength fiducial is provided by the $1.06-\mu \mathrm{m}$ light in second order; the light is brought by fiber optic from one of the $\mathrm{cw}$ alignment lasers.

The light coliector (Fig. 5-30) is designed to fit on a dome port of the Shiva target chamber at either $\theta=20$ or $\theta=160$; we use the folded design because all dome ports at Shiva are located under lens positioners. allowing only about 2 in. of vertical clearance. The Raman-scattered light is expected to be highly collimated by refraction along the densitygradient direction. For most of the experiments we plan, this demands a location as close to the axis as possible. Mirrors are used instead of lenses so that we can align the light collector using visible light and still be confident it is also aligned for the Ruman-scatterer infrared light. A diamond-turned ellipsoidal mirror focuses the light from the target onto the fiber optic with a magnification of 0.0825 . The ellipsoidal mirror that determines the lightcollection solid angle is $40 \mathrm{~mm}$ by $40 \mathrm{~mm}$. and is placed $1265 \mathrm{~mm}$ from the center of the target chamher: the light-collection solid angle is about $1.0 \times$ $10^{-3}$ steradians.

Since the quartz optical fiber is one millimeter in diameter, the field of view is $12 \mathrm{~mm}$ across laterally: longitudinally, the field of view is hundreds of millimeters deep. A dielectric coating on the Infrasil window is highly reflective of $1.06-\mu \mathrm{m}$ light, but transmits in the spectral region of interest from 1.2 to $2.6 \mu \mathrm{m}$. Color filters (such as the Corning 7-56 visible-light-absorbing filter and the Corning 4-64 near-infrared-abs , bing filter) will also be 
Fig. 5-30. Light collector for a Shiva dome port, to be fit at $\theta=20^{\circ}$ or $\theta=160^{\circ}$.

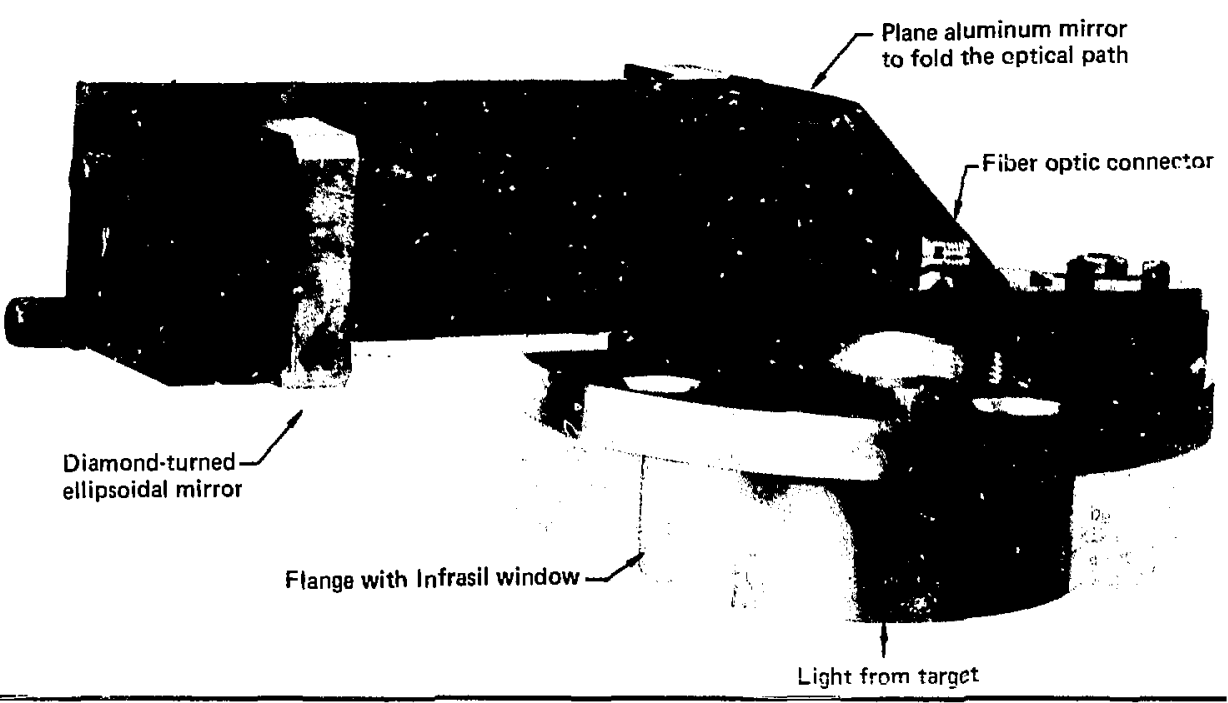

used to ensure that only the Raman-scattered light is collected.

The entire system is calibrated with a black. body source placed in the field of view of the light collector. Since the blackbody sourc has an emissivity known to $\pm 1 \%$, an aperture area known $100.1 \%$ and a temperature (variable up to $1273 \mathrm{~K}$ ) which is known to $\pm 2 \mathrm{~K}$, the absoluie spectral intensity in watts $/(\mu \mathrm{m}$-steradian $)$ can be accurately calculated. We will m.ke a dc measurement of the current generated by each indium arsenide detector using a nanovoltmeter shunled by a small resistance. The background current can be cancelled with a high-impedance adjustable-current source, so that with the blackbody source blocked, no voltage appears across the small shunt resistance. This measurement technique has worked well in calibrating other indium arsenide deteciors and has agieed with the pulsed calibration within error. We can thus calibrate the entire system with the same filters as are used in the experiment.

We make the assumption that the cw calibration will agree with a pulsed calibration in the lowpower, low-energy limit. The somewhat nonlinear response to a short high-energy light pulse can be corrected for by measuring the curve giving the output charge as a function of the relative energy of the incident light pulse. The relative energy scale can te made absolute by assuming that the low-energy linear asymplote agrees with the low-po. " $\mathrm{cw}$ calibration. Should questions arise, however, a pulsed calihration will be carried out off-line.

Author: D. II. Phitlion

Najor Contributors: W, B. Laird, R. K. Reed, D. K. Walton, and T. D. Schwinn

\section{Neutron and X-Ray \\ Emission Time Measurements}

Neutron diagnostics have been used since the beginning of the laser fusion program at LLL to measure thernonuclear yield and reaction temperature in "exploding-pusher" ICF targets. With optimum exploding-pusher targets, the laser pulses were 30 to 100 ps FWHM: implosion times were typically less than 200 po. For these conditions we determined implosion times using our $x$-ray streak camera. ${ }^{20}$ Now, as we change our emphasis to colder targets with thicker shells and higher $\rho \mathrm{R}$ 's, $x$ ray studies become inore difficult and our diagnostics become much more dependent on 
Fig. 5-31. (a) Schematic of the experimental setup for neutron emission time measurement. (b) Example of raw data; $x$-ray, neutron, and $1.06-\mu \mathrm{m}$ light signals are delayed and combined to give a convenient order and spacing on the oscilioscope trace.

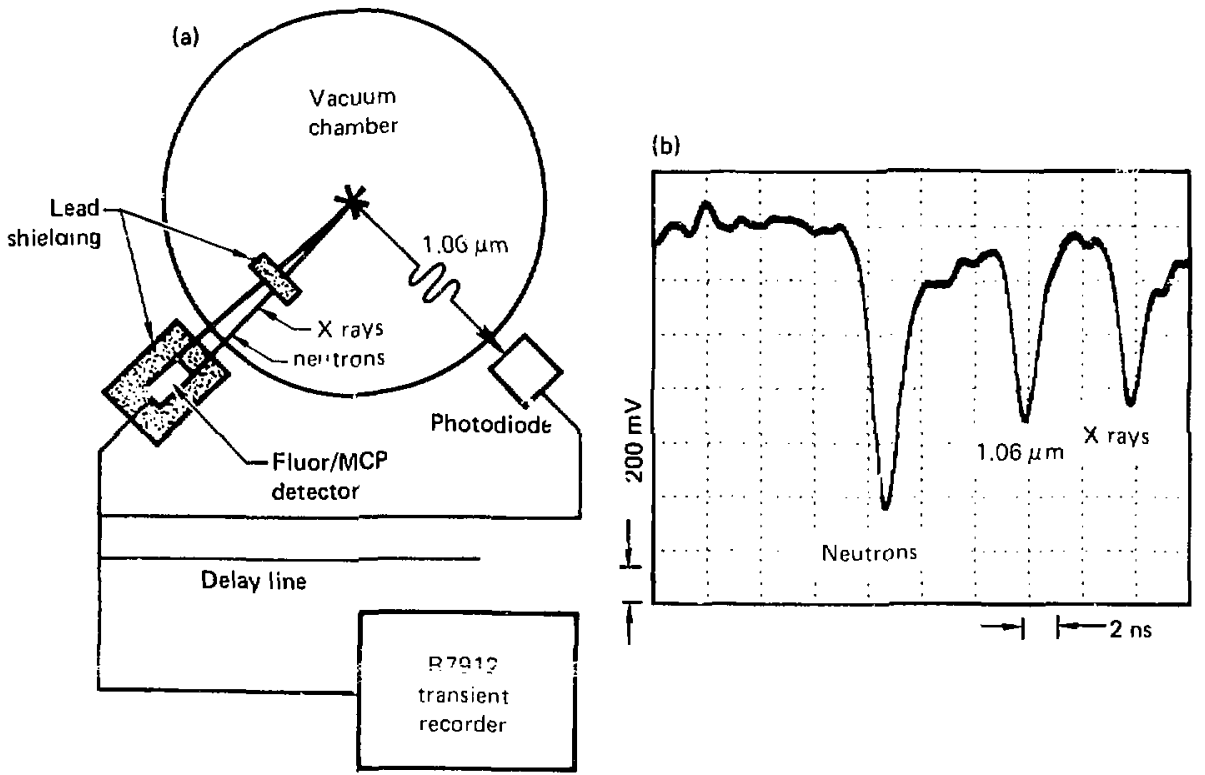

Fig. 5-32. (a) Schematic of the fast neutron/x-ray detector. (b) Detector impulse response.

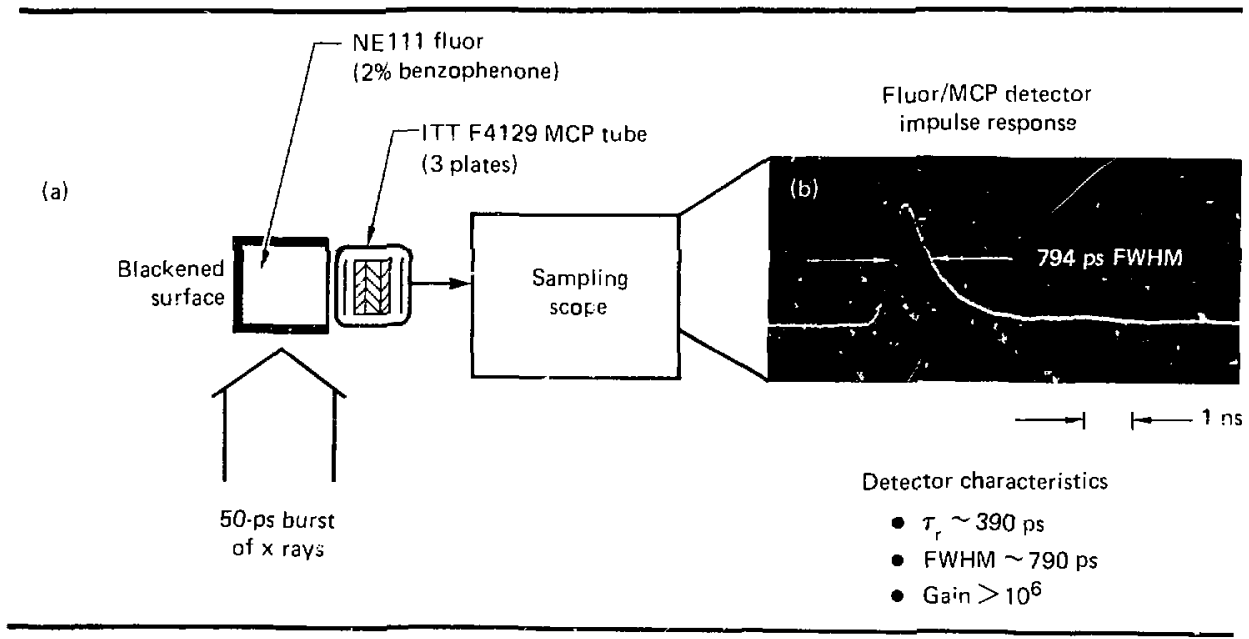


Fig. 5-33. Neutren emission time relative to the laser pulse peak, for 10 shots that had similar laser and target characteristics. For these shots, the average emission time is 295 ps (solid line) \pm 85 ps (shaded area).

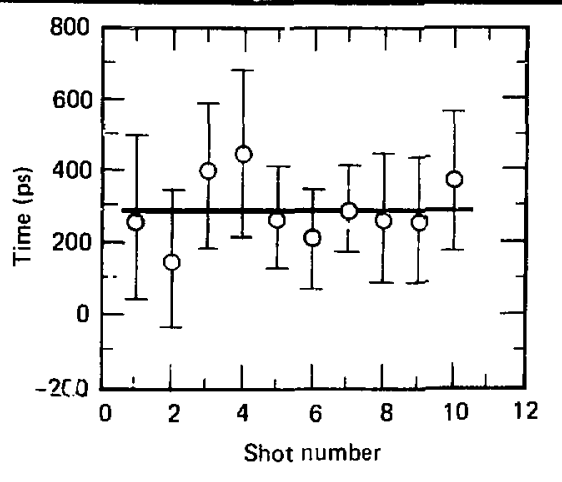

penetrating radiaticns su.h as neutrons (sce "Conpression Diagnostics for High-Density. LowTemperature Tarete" later in this stction!

A diagnostic which can be particularly valuable for providing information about the implosion dynamies of double-shelled largets is "neutron interval timing." whereby an implosion time is deduced from the time differenc." between laser and neutron signals recorded with a single trainsient recorder. We have built and used such a device. coupling $\downarrow$ fast neutron detector and a separale fast photodiode (for laser light) 10 a single recording instrument. This neutron system ${ }^{21}$ is designed to

- Measure neutror and high-energy $1>+6$

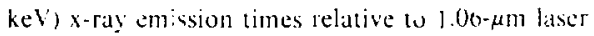
pulses, wit: , urecision of $\pm 100 \mathrm{ps}$.

- Be seful with neutron yields in the range from $10^{\text {h }} 1010$

- Function in the presence of large x-ray bursts.

Figure $5-31$ shows (a) a schenatic of the system and (b) an example of raiw data. The fast neutron detector used in our studies is an NEll! plastic scintillator to mon in diameter and $25 \mathrm{~mm}$ thick. qunc.ed with 29 benzophenone. 21 the scintillator is coupled to a three-stage ITT F4129 mierochannel plate $(A R(P)$ phoiomultiplier tube. as shown in Fig. 5-32(a). The external scintillator surfacts are painted biack to reduce dispersio , oy internal light reflections: lead shielding attenuates the intense 1 . ray bursts and concomitant lluorescence
The detector response, shown in Fig. 5-32(b). has a 390-ps rise time. a 790-ps FWHM. an electron gain of $10^{6}$. and recovers to baseline in several ns. $A$ yield of $10^{7}$ neutrons produces approximately 300 interactions in the scintillator. located $0.9 \mathrm{~m}$ from the target.

The detector setup to monitor scattered light from the target consists of an ITT 14018 biplanar vacuum photodiode with an $S-l$ photocathode. Signal levels in the 300-ps rise-tine detuctor are controlled with a 100-1-wide band-pass ilter centered on $1.00 \mu \mathrm{m}$, and appropriate neutral-density filters. $x$-roy and neutron signals irom the neutron detector and the scattered light signal from the photodiode are mised with s(l)-! pomer-spliters and recorded by 500-11ll, Icklronic 7912 transiemt recorders. We seled atppropriale cable lengths and use an open-cireuil delaty stuh lo display the three signals with optimal swee, speed. in convenienty displaced positions | Iig. 5. 3lib)|.

I'mission time meanurements are subjeet to

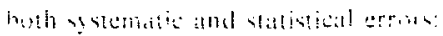

- Sistenatic errors are ssociated with instrument time-delay uncel limlies. which affect the: absolute position of all timing datie in the same manner. Sinkemalic errors are estumated to be fess than \pm 175 pr for neutron datia and \pm 120 ps for $x$ ral dalia.

- Sialisical crrors are assciciated with uncertainties in neutron interation with the detector. ceconbolution of the sustem response. and viatat reduction of the $x$ pe traces. These uncertanties arc l!picall: $\pm 175 \mathrm{ps}$ and are primarily associated with making ino reildings from a single transient recorder !race.

Initial experiments have demonstrated the capabilitien of our noutron interval timing technique. ligure 5.3.3 snows the implosion time deduacd for 10 shots that had similar laser and target characteristics: in these tests the ir erval time was determined to be $295 \mathrm{ps}$. with a reproducibility ol $\pm 100 \mathrm{ps}$. This clearly indicates that the technique will be one of great: value for the longer time intervals expected with larger, double-shelled largets.

X-Ray Measurements. Along with measuring reialice r-ral specira and pulse shapes. We are currents interested in monioring the $x$-ray emisvion times relause to the incident $1.06-\mu \mathrm{m}$ laser 
pulse. We have implemented two similar systems for this purpose, one for $x$ rays from 300 to $800 \mathrm{eV}$, another for $x$ rays from 40 to $70 \mathrm{keV}$ :

- To detect low-energy $x$ rays from 300 to $800 \mathrm{eV}$, we use a filtered XRD-31 x-ray diode, ${ }^{23}$ and an ITT F4014 vacuum photodiode to monitor a small fraction of the incident laser beam. Signals from the two detectors are fed tr signal and marker inputs of a 4-GHz TSN 660 (Thompson-CSF) oscilloscope. Used with a TSN 660 oscilloscope, these detectors have impulse responses of about $200 \mathrm{ps}$ IWHM. Representative outputs, for gold disk largets irradiated at two different intensities, are shown in Fig. 5-34.

- To monitor $x$ rays in the 40 to $70 \mathrm{keV}$ range. we use an ITT F4128 MCP photemultiplier tube with about a 400-ps FWHM time response. X rays pass through a filter pack, then interact in the MCP tute: the resultant signal is recorded on a TSN 660 oscilloscope, along with a photodiode signal applied to the marker input.

The object of data analysis for the $x$-ray systems is to detect small shot-to-shot shifts in $x$-ray emission time relativi to the incident laser pulse, using the photodiode signal as a precise time fiducial. (No attempts have been made to determine the absolute emission times from these systems.)

Two digitized oscilloscope traces produced by our data analysis technique are compared in Fig. 5-35. The delayed photodiode signals (negative pulses) are normalized to facilitate alignment of the trace time axis. Then we translate the trace of interest so that the leading edge of its fiducial signal coincides with that of the reference shot. Next, the $x$-ray signals are normalized to permit better comparison of the signal shapes and relative shifts in emission times.

With this method we can detect relative time shifts of about $100 \mathrm{ps}$. We have arbitrarily chosen the peak of one of the $x$-ray pulses as the zero reference time; we have not yet attempted to determine the absolute $x$-ray emission time using the present systems because of the relatively large uncertainties in transit times through the various system components.

Authors: R. A. Lerche, H. N. Kornblum, and K. G. Tirsell

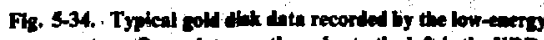

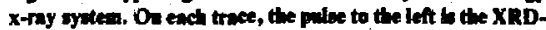

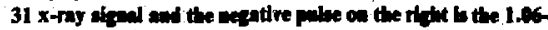

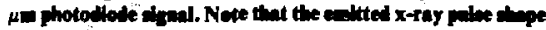

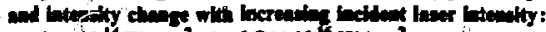
(a) $3 \times 10^{14} \mathrm{~W} / \mathrm{c}^{2} ;$ (b) $1.5 \times 10^{\mathrm{k}} \mathrm{W} / \mathrm{c}^{2}$.
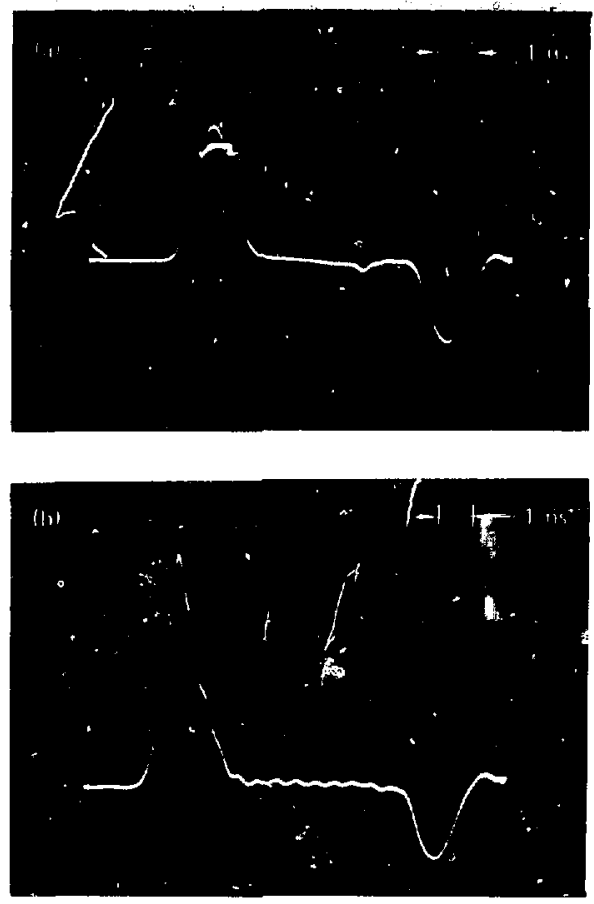

\section{References}

20. D. T. Attwood, L. W. Coleman, J. T. Larsen, and E. K Storm. Phys. Rev. Letr. 37, 499 (1976).

21. R. A. Lcrchc and J. T. Ozawa. "Neutron and X-ray Emission Time Measurements," Lawrence Livermore Laboratory, Livermore, Calif, UCRL-83092 (1979).

22. J. C. Cheng. K. G. Tirsell, G. R. Tripp, E. M. Lent, and R. A. Lerche, Rev. Sci. Instrum. 49(5), 650 (1978); P. B. Lyons, S. E. Caldwell, L. P. Hocker, D. G. Crandall, P. A. Zagarino, J. Cheng, G. Tirsell, and C. R. Hurlbut, IEEE Trans. Nucl. Sci. 24, 1 (1977), p. 177.

23. See Section 5. "Temporal Response of the XRD-31 X-Ray Diode."

\section{D-D Neutron Measurements}

In 1979 we shot the first LLL laser-irradiated target with a significant D-D neutron yield. Of the four techniques we use to monitor D-T neutron yields - fast time-resolved scintillator/photo- 
Fig. 5-35. Cosparivon of two TSN GGD oscllowecpe traces for goild disk shots. The digitized laser. (nezatiye pulsea) and x-ray (positive pulses) atyals are cormalized to faclitiate compartion.

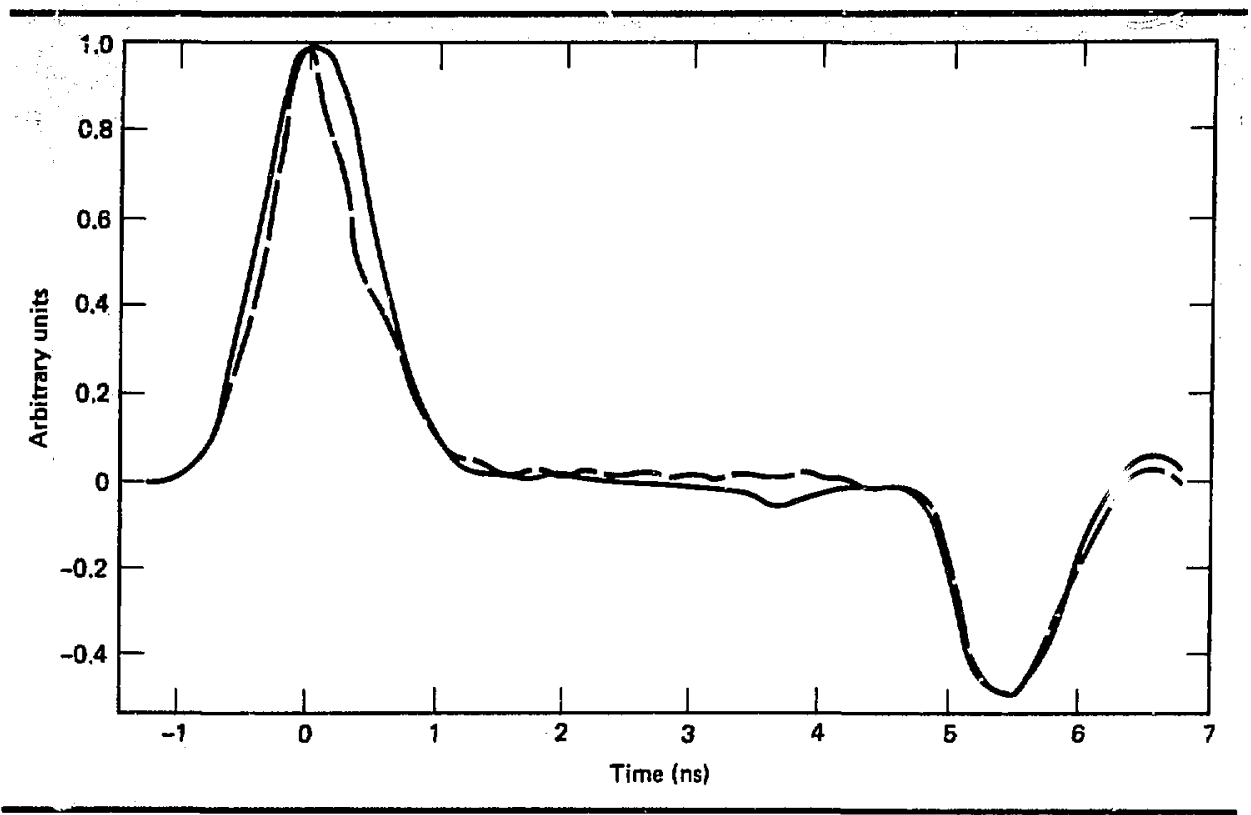

multiplier detectors, ${ }^{24,25}$ copper activation, ${ }^{26,27}$ lead activation, ${ }^{28}$ and silver activation-all but copper activation can also be used to determine D-D neutron yields. The 10.9-MeV neutron activation threshold of copper renders it useless for measuring the $2.45-\mathrm{MeV}$ D-D neutron yields.

The fast scintillator/PM tube system is perhaps the most important diagnostic technique. For a neutron source of subnanosecond duration, distinguishing which neutrons are truly D-D and which neutrons are D-T is easily accomplished by time-of-flight (TOF) separation. Signal strengths are used to determine the relative yield of the two species. We calibrate our scintillator detectors for D-T neutrons by comparing their output with lasershot results obtained with a copper activation system carefully calibrated previously at the LLL RTNS facility; we then estimate the scintillator's D-D neutron sensitivity using Monte Carlo simulations and light response curves for D-D and D-T neutrons. For our scintillator/PM tube detectors, D-D neutron sensitivity is 12 times less than D-T neutron sensitivity. Figure 5-36 shows a represen- tative scintillator detector output for a D-D neutron signal.

We deseloped our lead activation system as a sensitive monitor of low D.T neutron yields. The lead detector is calibrated for D-T neutrons by comparison with copper activation results on laser shots. To estimate the D-D neutron sensitivity of lead activation, we multiply the D-T sensitivity by the ratio of the cross section for forming $\mathrm{Pb}-207 \mathrm{~m}$ with $2.45-\mathrm{MeV}$ neutrons to the cross section for forming $\mathrm{Pb}-207 \mathrm{~m}$ with $14-\mathrm{MeV}$ neutrons. Using this technique. we determine the lead detector to be 62 times sensitive to D-D neutrons than to D-T neutrons.

A silver activation system mounted at Shiva has been monitoring neutrons from D-T targets since late 1978. D-T neutron calibration is determined by comparing the silver activation output with copper and lead activation results on laser shots. Monte Carlo calculations for the silver detector show the D-D sensitivity to be 1.25 times greater 
Fig. 5-36. Oscilloscope trace for time-resolved scintillator/photomsltiplier detector locared $7.47 \mathrm{~m}$ from the target, showing $7.1 \times 10^{8} \mathrm{D}-\mathrm{D}$ neutrons; this trace shows no evidence of D-T neutrons. $X$ rays arrive 119 ns before $D-T$ neutrons and 319 ns before $D-D$ neutrons. Sensitivities are $50 \mathrm{mV} / \mathrm{div}$ and $50 \mathrm{~ns} / \mathrm{div}$.

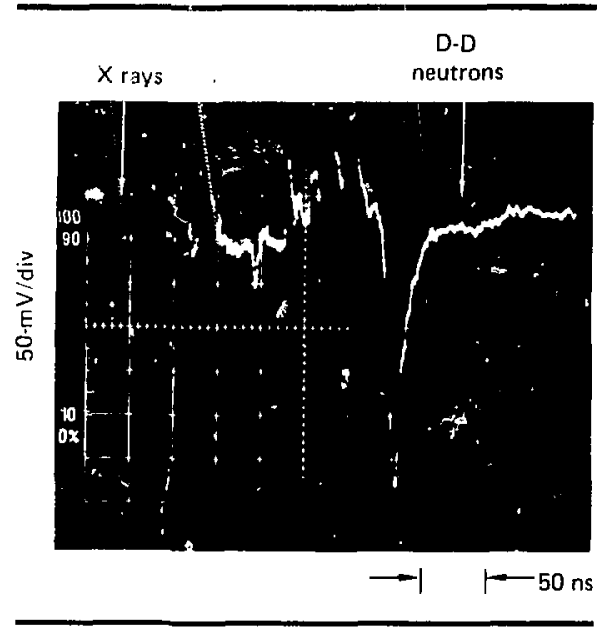

that its D-T sensitivity. D-D calibration of the detector hats heen done with D-D neutrons from a neutron generator, which provides a D-D calibration independent of the D-T calibration of the other detectors.

The D-D neutron yield values determined by the three systems are in good agreement; any one of the three can he used for diagnosing the yicld fron at D-D neutron source. If there are D-T neutrons present, however, the analysis is less simple. A D-T yield of only $1.6^{\circ}$ \% of the total neutron yield produces a $50 \%$ error in the lead activation system: the silver system shows a sintilar inability to distinguish beween D-D and D-T neutrons.

The mosi accurate D-D neutron measurement when D-T neutrons are also present is obtained with a combination of the scintillator, silver, and copper detectors. Estimates of D-D and D-T neutron yields can be made from scintillator data, and an accurate D-T yield determined from copper aciivation data; the silver counter output corrected for D-T yield gives the D-D yield. The lead system can duplicate the silver system, but has significantly poorer D-D sensitivity.
Authors: R. A. Lerche, M. S. Singh, and G. E. Phillips

\section{References}

24. Laker Pregram Anmal Reporl-1974, Lawrence Livermore L.aboratory. livernore, Calil.. UCRL-50021-74 (1975). ре. 337 to 339

25. Laser Program Anmal Repuri-1975, law rence Livermure latbotatory, Litermore, Callif., UCRL.-50021-75 (1976). pp. 407 (1) 409

26. Leaser Program Ammal lieport-1976, Lianrence Livermore L.iburators, Livermure, Calit., UC'R1-50121-76 (1977). pp. 3. 10.5 to 3.108 .

27. Laser Pringram Ammal Repmert-1977. Law rence L iscrmore laboraturs, I.ivermore, Calif, UCRL-50)(121-77 (I978), nn. 3-82 10 3-84t.

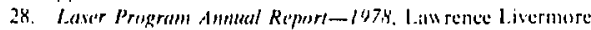
Liburitury, Livermore. Citlil., UCRL-50)21-7K (1979). пp. $61-5,3$ to $6-54$.

\section{Compression Diagnostics for High-Density, Low- Temperature Targets}

A significant lask of our diagnostic program is developing techniques to detormine the final fuel conditions in laser-compresied targets. The measurements of final fuel density and $p R$ have posed a particular challenge during the past year, as our program evolved from an earlier interest in thin-walled exploding pushers to concentration on thicker-walled largets designed to achieve higher fuel densities. As a consequence, present imploded cores are colder, but more dense, and are surrounded by a significantly less transmissive pusher.

These new conditions adversely affect the usefulness of previous diagnostics in two ways:

- Temperatures are lower, and thus largets emit fewer keV-range $x$ rays and fewer thermonuclear reaction products such as neutron and alpha particles, each of which provided valuable diagnostic information for thin-walled targets.

- Because the surrounding glass pusher is of a higher $(\rho \Delta R)_{n}$ (our shorthand for $\int_{0} \int^{R} p d r$ ), fewer $x$ rays and reaction products can escape and be used for diagnostic purposes.

These problems are summarized in Figs. 5-37 and 5-38. Figure 5-37 shows the transmission of $x$ rays of 2,3,4,6 and $8 \mathrm{keV}$, as a function of glass $(p \Delta \mathrm{R})_{\mathrm{p}}$, for glass temperatures from 100 to $500 \mathrm{eV}$. 
Fig. 5-37. Calculated $x$-ray transmission coefficient as a function of areal density of a compressed and heated glass pusher. The vertical yellow band labeled "EP"' represents typical behavior for exploding pushers; targets which might achieve higher fuel densities are represented by the yellow bands labeled "10X" and " $100 \times$," indicating 10 and 100 times the liaguid D-T density of $0.5 \mathrm{~g} / \mathrm{cm}^{3}$.

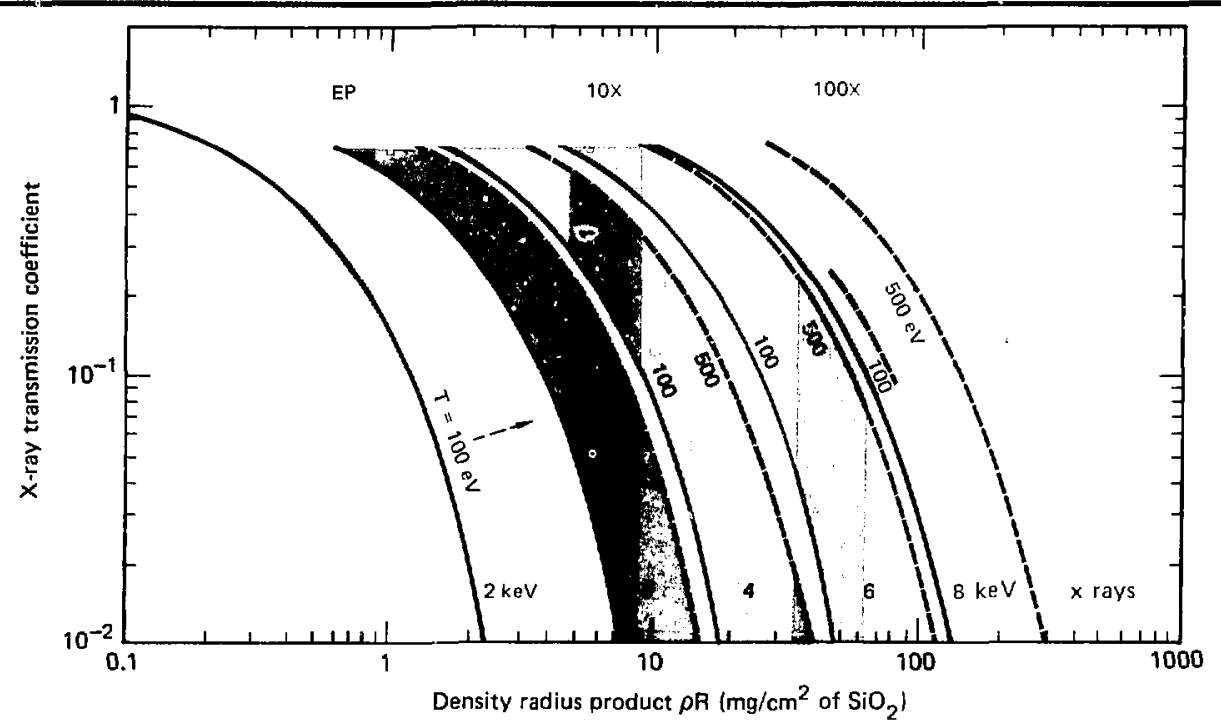

Fig. 5-38. Particle energy loss as a function of glass pusher areal density, for some common thcrmonuclear renction products.

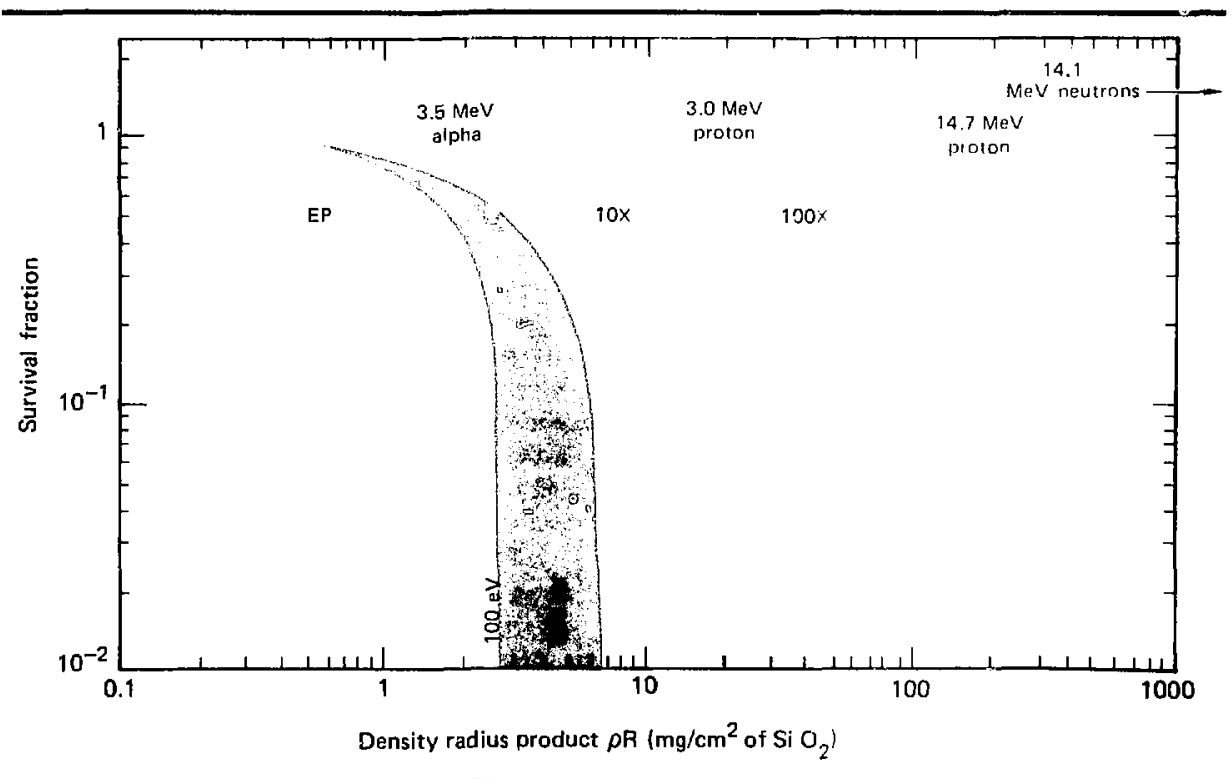


Fig. 5-39. Calculated operating rezimes for various density diagnostics for ranges of experimental interest.

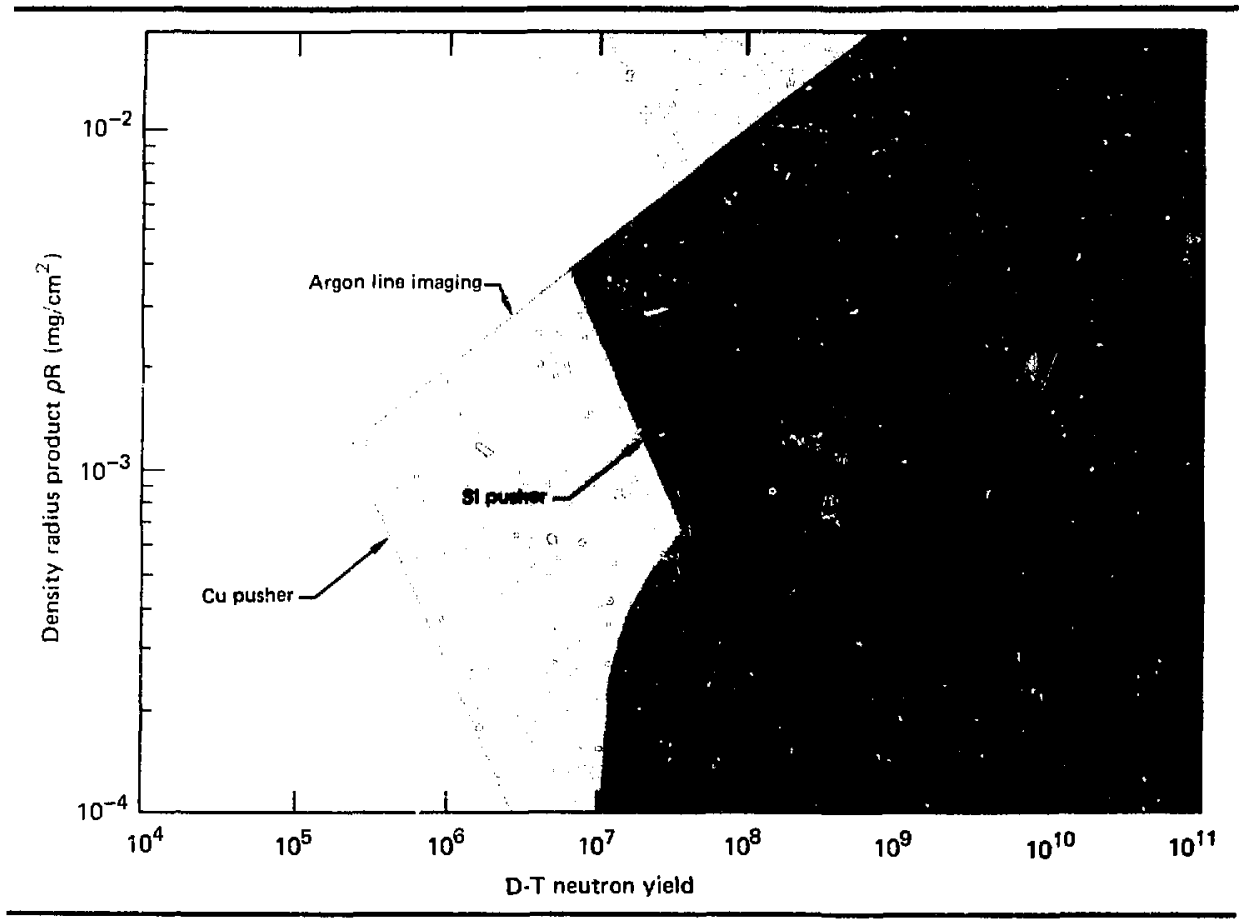

Vote for instance that whereas $2-k e V \times$ rays are approprate for diagnosing thin-shelled targets achieving final $(\mu \Delta R)_{p}$ s of $<1 \mathrm{mg} / \mathrm{cm}^{2}$. largets that would ahieve values as high as $30 \mathrm{mg} / \mathrm{cm}^{2}$ require a photon energy of 6 to $8 \mathrm{keV}$ to provide information siarding the fuel region or fuel-pusher interface.

The situation is similar for reaction-product $\therefore$ tal particles and protons, which also must pass !hrough the compressed pusher if they are to provitc diagnostic information on final fuel condiuons. In Fig. 5.38 we see, for example, that $2.5-\mathrm{M} \cdot \mathrm{V}$ alpha particles readily pass through the sompressed glass of an exploding pusher $\left[(\rho \perp R)_{p} \simeq\right.$ $0.5 \mathrm{mg} \mathrm{cm}^{2} 7$. permitting alpha imaging of the thermonucleat burn: with targets achieving $(\mu \Delta R)_{p}$ s $>$ $10 \mathrm{mg} / \mathrm{cm}^{2}$, however, the alphas no longer escape. Thus our compression diagnostics must take advantage of higher-energy photons and more penetrating particles, such as multi-MeV protons and neutrons. Several such diagnostics are discussed in other articles in this section.
While Figs. 5-37 and 5-38 address the issue of photon and particle transport through the surrounding glass pusher, they do not address the issue of temperature-sensitive enission rates. Figure 5-39 includes such information in providing a simple guide to diagnostic operating regimes, given the parameters of (temperalure-sensitive) neutron yield and target $p R$ fof either fuel or pusher, as appropriate). Although only a sampling of possible diagnostics is shown, the parameler space is well overlapped. Note that not only is the value of $(\rho \Delta R)_{p}$ important for alpha imaging. but the final fuel temperature, and thus neutron yield, must be high enough to produce a detectable image.

The role of neutron-activated bromine and argon tracer gases for measurement of fuel region $(\rho \mathrm{R})_{\Gamma}$ is also shown in Fig. 5-39, with an explicit inverse relation between neutron yield and achieved $(\rho \mathrm{R})_{\text {. }}$. We can sec that for the assumed seed conditions, a $(\rho R)_{f}$ of $10 \mathrm{mg} / \mathrm{cm}^{2}$ would require a neutron yield in excess of $10^{7}$. Threshold for silicon pusher 
$(\rho \Delta R)_{p}$ measurements is ten times lower, but these diagnostics require modeling to relate pusher and fuel conditions. Not shown in the figure are variable-energy $x$-ray images which can provide two-dimensional compression symmetry as well as stability information, but whose emission levels are quite sensitive to fuel-pusher interface :inperatures-a situation difficult to summa:lze in a single illustration.

Our program is presently pursuing a broadbased approach to compression diagnostics, emphasizing the need for multiple, complementary diagnostics, such as

- Two-dimensional broad-band images.

- Spectral broadening and one-dimensional imaging.

- Neutron activation.

- The use of numerical simulations to consistently explain the various observable phenomena peaking at different times during implosion.

A preliminary example of our work in this area is described in Section 6, "10X Liquid Density Target Experiments."
Authors: D. T. Attwood, N. M. Ceglio, E. M. Campbell, and J. T. Larsen

\section{X-Ray Imaging of Laser Fusion Targets}

Zone-plate-coded imaging cameras now routinely provide multispectral $x$-ray images of intermediate-density laser fusion targets. ${ }^{29}$ Figure 5-40 shows representative multispectral data: a series of two-dimensional $x$-ray ımages in distinct spectral bands within the range from 2 to $20 \mathrm{keV}$. The isointensity contour maps are of $x$-ray emission in four spectral bands centered at 2.7, 4.6.6.1, and $16 \mathrm{keV}$. Adjacent to the contour maps are linear plots of $x$-ray intensity versus position along a slice through the target center. The images are from a D-T-filled 145- $\mu \mathrm{m}$-i.d. glass microsphere target whose $5-\mu \mathrm{m}$ glass wall was coated with $16.5 \mu \mathrm{m}$ of teflon: the carget was irradiated at the Shiva facility. with $3.4 \mathrm{~kJ}$ in a 200-ps, 17-TW pulse.

Such an array of image data is useful in the diagnosis of intermediate-density targets:

Fig. 5-40. A series of $x$-ray images in discreet spectral bands, for a laser-irradiated intermediate-density microsphere target. (In these inages, the two I0-bean clusters of the Shiva laser facility were incident on the tefon-conted (areet from the tor and bottom.)

Isointensity contour maps

$(2.7 \mathrm{keV})$
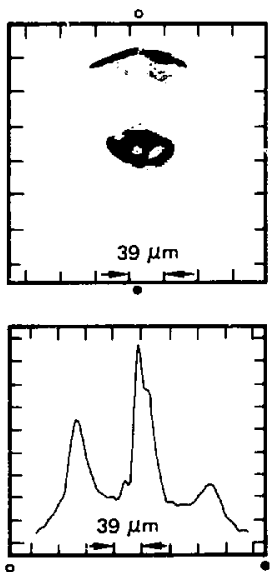

$(4.6 \mathrm{keV})$
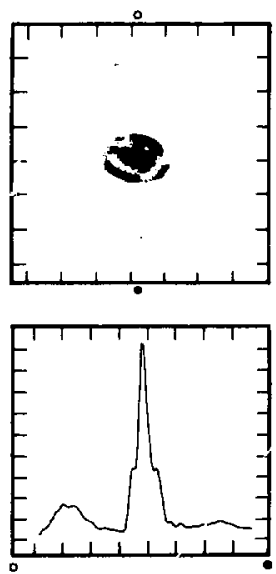

$(6.1 \mathrm{keV})$
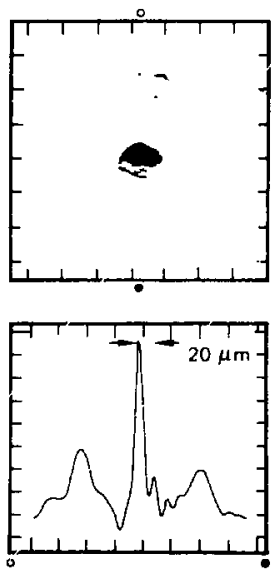

$(16 \mathrm{keV})$
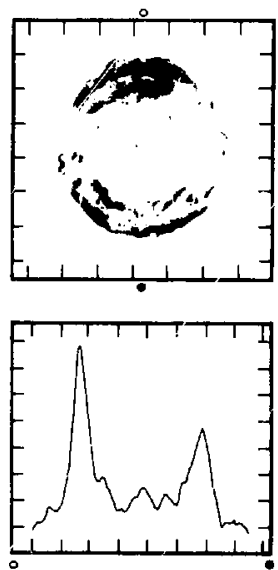

Intensity profiles 
Fig. 5-41. A representative pair of x-ray images from two nominally identical target experiments. The variations in the geometry of the depression in the central core suggest that in some of these experinents, iwo-dimensional effects perhaps compromise the integrity of the fuel-pusher interface at compression.

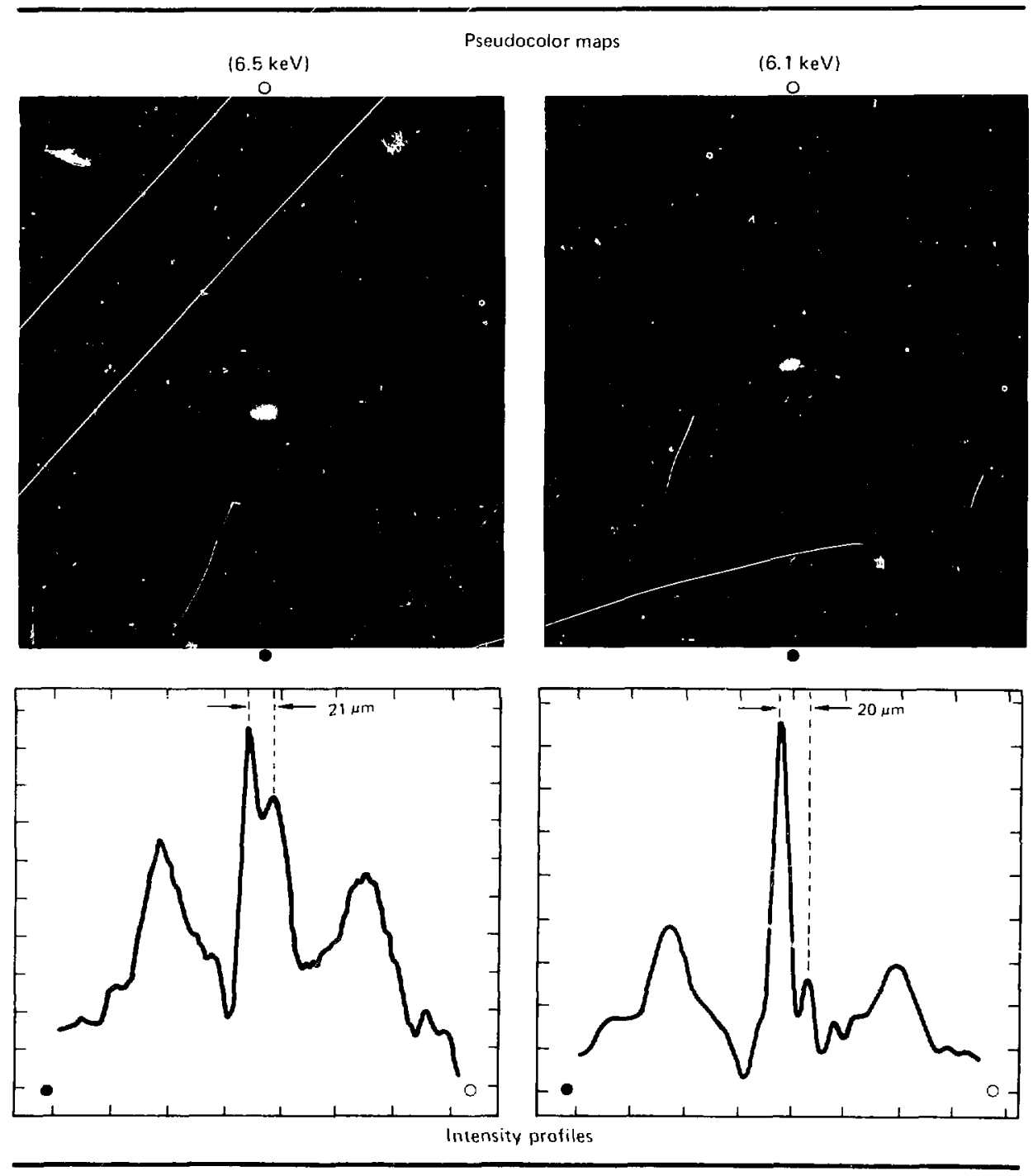

- The low-energ! ( $\approx$ I keV) image shous thermal emission from the laser-illuminated teflon coating. thereb! providing an indicition of the illumination symmetry on target: it also shous the compressed core. To unambiguously "see" into the compressed core of intermediate-density largets, which are designed to achieve a fuel density of ' to $10 \mathrm{gm}$; ce and a glass,$\Delta \mathrm{R} \approx 51010 \mathrm{mg}$; $\mathrm{cm}^{2}$, requires higher-energy $x$-ray images.

- The intermediate-energy $(\approx 4106 \mathrm{keV}) \mathrm{im}$ alges allou us to see further into the compressed 
target core. and perhaps evaluare the quality of compression: the synimetry and volume of the cure, and the integrity of the fuel-pusher interface (interpretation of time-integrated images of the compressed cores of intermediate-density targets is discussed later in this article).

- The high-energy image ( $\approx 16 \mathrm{keV})$ shous the hollon shell of suprathermal $x$-ray bremsstrahlung arising from interaction of long-range (approximate larget a all thickness) suprathermal electrons with the high-/ pusher material. The suprathermal image provides a snapshol of the pusher stell at around the linse of intense laser irradiation. that is, in the early stages of target implosion. Such an image allous us to monitor the initial stages of pusher motion. to diagnose larget itlumination as mmetries through local inhomogeneitics in STX. and therefore in STE. productions. and to detect an eariy hreak-up of the pusher shell if it should occur. ${ }^{31)}$ There is no apparent hreak-up of the pusher shell during this experiment's 200-ps laser irradiation.

As mentioned athese. this class of experiments is particularl! concerned with the quality of compresion of the laser-impleded target. Becaluse local parameters such as electron and ion density and lemperature sars on a acale of mierometers and lens ol peomeconds, it is important to combine sereral independent direct diagnoutic methed in order lo provide a comprehensise description of the larget in it compressed vitle.

Although a time-integrated a-ra! image cimnol be expected an tell the whole vory. intermediate-

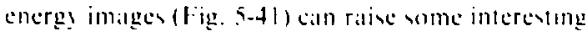
quexurom. For esimples a one-dimensionit cialculation of the larget implesion leads us to expect a dip in the center af the compressed core, the locus of the D-T fuel: in such a cine we cian "see" the fuelpurher interface, and extimate the volume of the encloned fuel. In this regard. Fig. S-4l shoms $\approx 6-k e V$ image from two nominalls identical target shots. In image (a) the compresied core shous a distinct central dip. as copected, whereas in (h) the evidence for such a depression in the central core emossion is less consincing: this indicates that tuo-dimensional effeets naty compromise the integrity of the fuelpusher interface at compression. Note that the target shot in image (a) exhihits a higher degree of symmetry than that in image (b). The illumination pattern on the target-as monitored hy the outer ring of suprathermal enission-is more symmetric; this results in a more syn metric $(21 \mathrm{~b} ! 25 \mu \mathrm{m})$ compressed core, with a dip in the x-ray emission all its center. It should he further noled that a closer inspection of fig. 5-4l(a) shows that the dip or depression in the centrat core emission is not full! enclosed hy regions of higher emission: thall is, if 1 e are indeed looking al a luel-pusher interfited it is one that does not appear wo fulls enclone the compressed fuel.

Although a clear interpretatton of the phe-

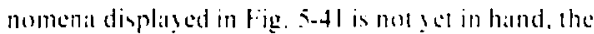
datta weem to sugges that ino-dimemsunal eflect play an important role in this cliss ol target experi. ments: one-dimemsional simulatioms of target performance mily he insuflicient. Such conclusiom should not he surprising. cornsidering the illumination an mmetry al the Shis facilit!. The vers inlerewing maller of repcalling these incermediatedensit! targel experiments wing sy momere target il. lumination is being pursued.

Author: X. M. Ceglio

Major Contributors: (i. I.. Howe. (;. Wurden, (i. Stone, $C$. H. Dittmore, W. ( , Hermann, and D. R. ( iarlo

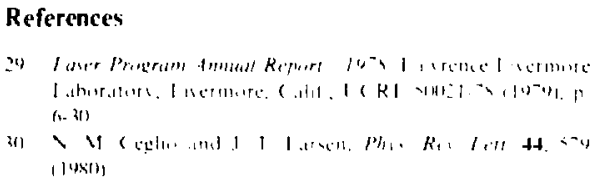

Implosion Measurements with Neutron Activation Techniques

In an imploded laner fumbon target. comsisting of comprened D.T gir encapulated in a glass microwhell. the thermonuclear newrom acmate the

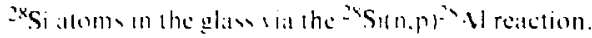
Bi measuring the neutron yeld and the lotit numher of ${ }^{2 x}$ Al allom crealed. "se cinn delermine the areal demsits " $\triangle R$ of the comprened glatso shell at the lime of pealk neutron production. ${ }^{31-35}$ The compressed-glits p. $\mathrm{R}$ e call then be used in simple models or computer vmulatorn as estimate the compressed densty of the luel. Thi article describes experiments at the Shra laser facilit! in which the activils found in the collected target debris was 


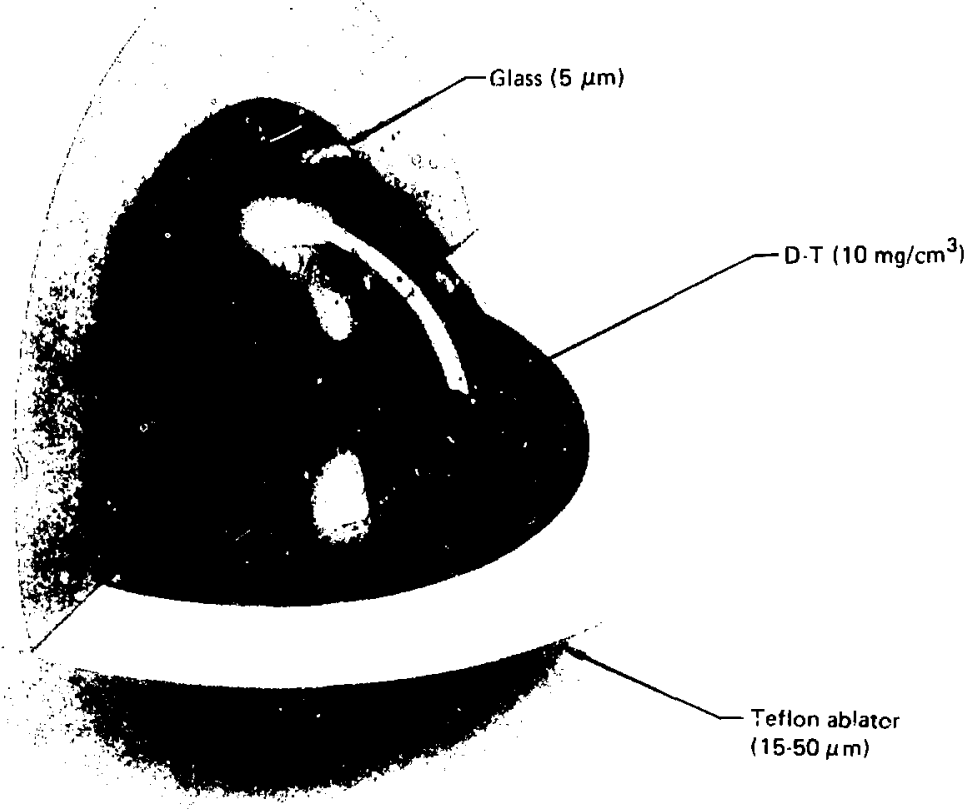

identified by its decaly rate as ${ }^{28} \mathrm{Al}$. The amount of activity was then used to derive the compressedglass $\mu \Delta R$.

The turgets we used in these experiments (described in greater detail in Section 6. "10x Liquid Density Target Experiments") were glass microshells with inner diameters of $140 \mu \mathrm{m}$ and wall ihncknesses of $5 \mu \mathrm{m}$, filled with a $10-\mathrm{mg}, \mathrm{cm}^{3}$ - uimolar mixture of D-T gas and coated with

in of teflon (Fig. 5-42). The glass constituents $\therefore$ by weight, $76 \% \mathrm{SiO}_{2}, 7.5 \% \mathrm{~B}_{2} \mathrm{O}_{3}, 14 \% \mathrm{Na}_{2} \mathrm{O}$. $\therefore ? 25 \% \mathrm{~K}_{2} \mathrm{O}$

$\mathrm{i} \cdot \mathrm{i} \cdot \mathrm{kJ}$ of laser energy in a 200-ps Gaussian . re directed by 20 individually focused ' the teflon ablation layer of the target.
The healing and subsequent blow off of the ablator compressed the glass pusier and the fuel. It was estimated from both optical and plasma calorimetry that $20 \%$ of the incident laser energy was absorbed. Typical neutron yields of 3 to $6 \times 10^{8}$ were obtained.

To collect a portion of the debris from the exploding target, we used an aluminum collector cylinder placed $10 \mathrm{~mm}$ from the target, opposite a dish-shaped tantalum reflector placed $25 \mathrm{~mm}$ from the target (Fig. 5-43). The cylinder was lined with 20- $\mu \mathrm{m}$-1hick, $99.9995 \%$.pure titanium roil, and measured $50 \mathrm{~mm}$ in diameter by $165 \mathrm{~mm}$ long: the reflector was also $50 \mathrm{~mm}$ in diameler. The front $30 \mathrm{~mm}$ of the cylinder were covered with an additional $50-\mu$ m-thick layer of tantalum foil to 


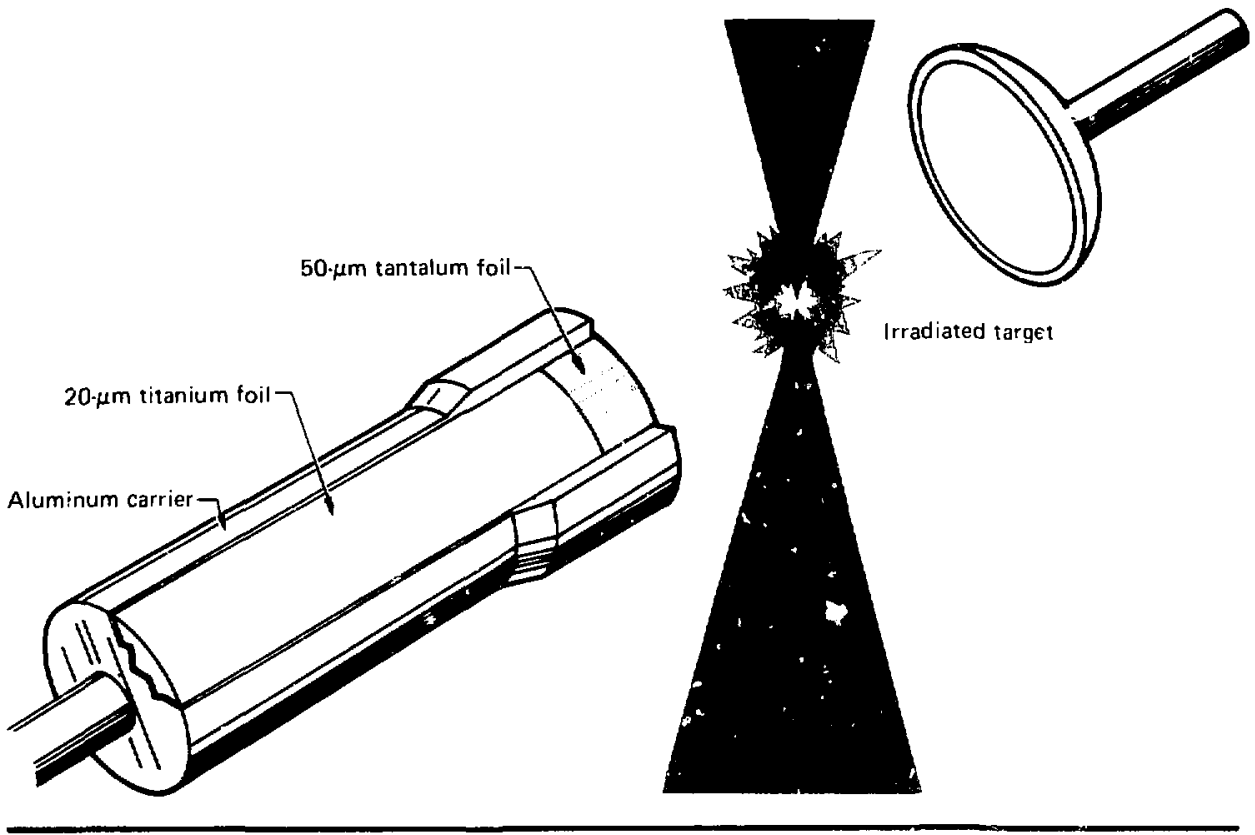

protect against blast damage. It took 17 seconds for an automated system to withdraw the collector from the evacuated target chamber and transfer it to the counting facility.

The amount of collected debris was determined in two auxiliary experiments using targets and laser conditions identical to those described above. In these experiments, ${ }^{36}$ however, the microshells were made slightly radioaetive by placing them in a light water reactor and allowing thermal neutron capture 10 create radioactive ${ }^{24} \mathrm{Na}\left(t_{1}{ }_{2}=15\right.$ hours $)$ from the ${ }^{23} \mathrm{Na}$ present in the glass. We were then able to measure the fraction of target debris collected by taking the ratio of the ${ }^{24} \mathrm{Na}$ activity found on the collector foils following a laser shot to that known to be present in the target. The average amount of target debris collected in these two experiments was $55.3 \% \pm 0.5 \%$. We also found in other experiments that $<1 \%$ of the debris was collected in the absence of the reflector, and that $<0.5 \%$ of the target debris adhered to the reflector.

We measured both the ${ }^{24} \mathrm{Na}$ and ${ }^{28} \mathrm{Al}$ activities with a $\mathrm{NaI}(\mathrm{Tl})$ detector $250 \mathrm{~mm}$ in diameter and
$250 \mathrm{~mm}$ long. having a well $150 \mathrm{~mm}$ decp and $50 \mathrm{~mm}$ in diameter. ${ }^{34}$ We surrounded the detector with it 100-mm-thick lead shield to reduce the backeround in the 30 )-keV-wide I.78-MeV window to 88 counts minutc. Aluminum decals with a 2.24 min half-life hy emutting at it particle and a 1.78 MeV gamma rat: this gamma ral wal detceled hy the Nal(Tl) erystal with a measured efficiency of $33 \% \pm 4 \%$.

In one of the the a $\triangle R$ ciperiments. we loaded the detector with the collector foil and were able to start coulting the $1.78-\mathrm{Mel}$ gammia ras $1.36 \mathrm{mir}$ after the laser shot. Figure $5-44$ shows that the measured activity decilyed with a half-life of $2.16 \pm$ $0.16 \mathrm{~min}$. This, together whth the fact that the observed level of activity is consistent with the neutron yield and reaction cross section, gives us conlidence that we are indeed measuring the 2.24min decay activity of ${ }^{2 x} \mathrm{Al}$ : presious experiments reported in the literature have not had sufficient activity to identify the radioactive nuclide.

Equation (1) relates the detected number of decays $\left(N_{c}\right)$ to the total number of activated atoms 
Fig. 5-44. Decay curve obtained from gamma activity (1.63 to $1.93 \mathrm{MeV}$ ) detected in the collected target debris. The approximately 2.2-min half-life indicates that we gre observing 28 Al.

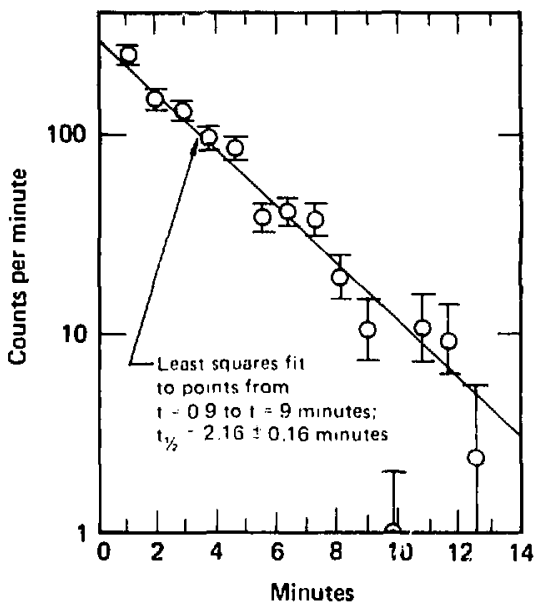

created $\left(\mathrm{N}^{*}\right)$, by accounting for the collection eflicienty $\eta_{\infty}$, the detection efficiency $\eta_{d}$ the delay time (betueen laser shot and commencement of counling) t, the counling interval do (taken to be $5 \mathrm{~min})$, and the ${ }^{24} \mathrm{Al}$ decall constant $\lambda$.

$$
N^{*}=\frac{\kappa_{c}}{\left[\eta_{c} n_{d} e^{-\lambda t}\left(1-e^{-\lambda \Delta 1}\right)\right]}
$$

In Fig. 5-44. the 770 net counts delected in the lirst 5 min of counting indicatc that $7960^{28} \mathrm{~A}$ atoms. "were created. Thus, we are able lo detect nearly $10 \%$ al the total activation sield of the larget, which clearly demonstrates the extreme sensitivity of neutron actiattion lechnique. Using our more sophisticated $\beta$-) coincidence counting technique ${ }^{3-4}$ which reduces the backeround rates by a factor of 200. we are able to detect total activation yields of only 100 atoms.

We can now relate the number of activated atoms created to an atverage,$\downarrow R$, using the equation

$$
\rho \Delta R=\frac{N^{*}}{Y_{n} \sigma \hat{f} \frac{A_{0}}{A_{w}}},
$$

where $Y_{11}$ is the neutron yield $\left(6.7 \times 10^{8}\right), \sigma$ is the ${ }^{28} \mathrm{Si}(\mathrm{n}, \mathrm{p})^{28} \mathrm{Al}$ cross-section $(0.250 \mathrm{~b})$, $r$ is the fraction of ${ }^{28} \mathrm{Si}$ atoms in the pusher $(0.25), \mathrm{A}_{u}$ is the average atomic weight of al pusher atom ( $20 \mathrm{~g})$, and $A_{0}$ is Avogadro's number.

In our experiments, we found the pusher areal density to be $5.9( \pm 1.5) \times 10^{-3} \mathrm{~g} / \mathrm{cm}^{2}$. This represents al 4.8. fold increase in $p \triangle R$ from its initial value of $1.2 \times 10^{-3} \mathrm{~g}, \mathrm{~cm}^{2}$, and indicates a fucl density at burn time of 1 6) $2 \mathrm{~g} \mathrm{~cm}^{\text {? }}$.

Author: S. M. Lane

Major Contributors: k. M. Camphell, W. M. Ploeger, C. K. Bennett, and (. E. Thompson

\section{References:}

31. F, J. Mayer ant W. B. Romel, "Mlanlac Bubbles and Tam.

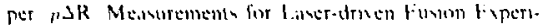
ments." J App/ Phat. 47. 149) (i976).

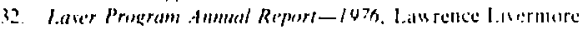

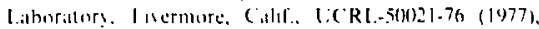

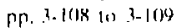

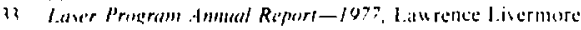
l.ahur.llsrs. I isermore, Calif., (UCRI.5(1021-77 (1978),

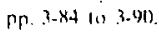

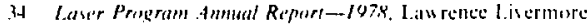
lithoralurs. Inermore. (illif. UCRL.-501021-78 (1979). एр. 6-45 $116-52$ ?

35. I. Y Camphell, W. M. Ploceter, P. II. Lec, and S. M. I.anc.

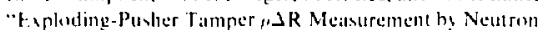

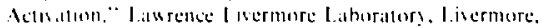

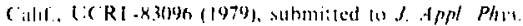

36. H: M. Camphell, H. (i. Hick, W, C Me:ad, 1. W. Coleman. (. W. Hatcher, J. H. Dellis, M. J. Bogle, J. T.

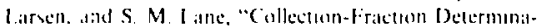

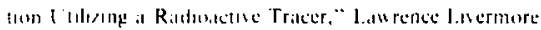
l.aburalury. I cermore, Cillf., L:C RI.-8.3072 (1970), whmitled lat $A$ Apl $\mathrm{Pm}$

\section{Plasma Diagnostics Using X-Ray Spectroscopy}

During 1979 we utilized $x$-ray line spectroscopy to meit ure compressed-fuel density achieved in exploding-pusher and ablativel: driven targets. Owing to the small amouit of argon mixed with the D-T in these microballoons, we were unable to make density determinations from Stark-broadened Ar $x$-ray lines. We have instead concentrated on one-dinensional line imaging to measure fuel $x$-ray emission volume, which can then be related to the fuel density: two separate series of experiments were performed, one at Argus and the other at Shiva. We 
Fig. 5-45. Schematic of argon lime iraging crystal spectrograph (ALICS), with typical data.

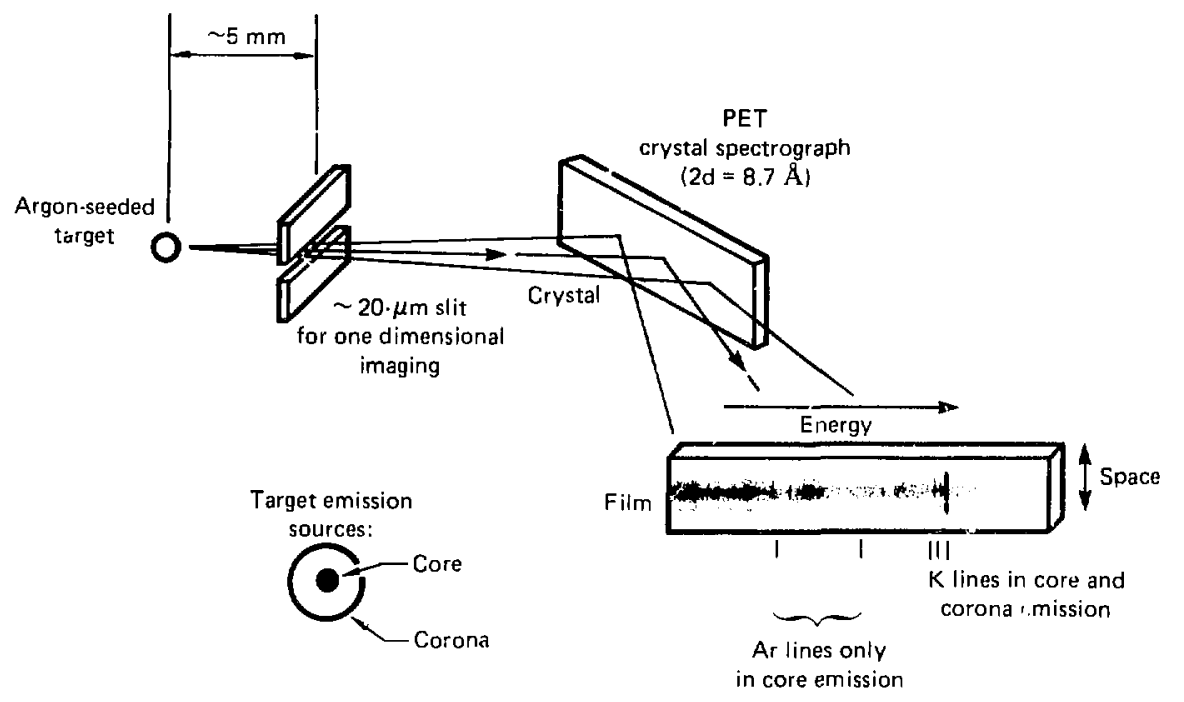

discuss some problems with the present imaging diagnostics as well as some plans for future improvements.

We also studied line emission intensity from various disk materials as a function of laser paramelers such as pulse length and intensity. This effort was begun in order to characterize efficient, intense, $x$-ray line sources for future $x$-raly backlighting or time-resolved $x$-ray radiography experiments. We oblained encouraging results concerning the production of suitable r-ray lines by lasergenerated plasmas. We discuss these experiments in the second part of this section.

Density Diagnostics. We have developed argon $x$-ray line imaging as an ICF target diagnostic technique. This technique measures the diameter of the compressed fuel core to provide an independent and direct measurement of fuel comprission achieved by the imploded target. A small amount of argon gas is loaded into the target along with the D-T fuel. Near the time of maximum compression the argon radiates its characteristic and penetrating $x$-ray lines. A spatially-resolving $x$-ray crystal spectrograph then measures the extent of the line emission region in one diaisension, producing an estimate of the size of the compressed core. This technique has been tested
- In Argus exploding-pusher target shots for which zone-plate-camera alpha-particle images were also obtained. ${ }^{37}$

- In experinents at Shiva in which the compression achieved by ablatively driven targets was measured with the pusher neutron atctivation lechniqua described in the previous article.

It is expected that the argon $x$-ray technique will be useful only for largets whose final fuel density is no greater than 50 times that of liquid D-T. Targets expected to achieve higher compressions require fuster shells that are 100 optically thick at stagnation to permit transmission of argon $x$-ray lines. The valuc of argon imaging. then, is to corroborate and increase confidence in the neutron activation technique for medium-density targets; results from neutron activation measurements on higher-density targets can then be interpreted more confidently.

The miniature erystal spectrograph shown schematically in Fig. 5-45 was used in the argon line measurement. Because high resolution is required in order to isolate the narrow $x$-ray lines of argon from the continuous spectrum radiated by the glass of the 
targei, the spectrograph wals designed for deployment very close to the target. The recording film is shiclded from direet exposure to target radiation by at hick lantalum block localced above and formard of a flall diffraction erystial.

A fine slit placed between the target and the analyeing erybtal produces information abould the extent of the $x$-ray surece in space, hy contritining $x$ rits from any given region of the largel to fill an a unlyue area of the spectrograph lim. The axis of the thl. located $5 \mathrm{~mm}$ from the tarpet and $6.5 \mathrm{~mm}$ lrem

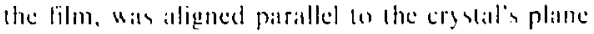

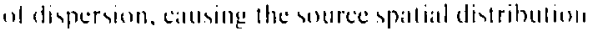
lo be recorded on the lilm frem side to side with an image magnilicalton al 12 (slits, with widthe of 20 )

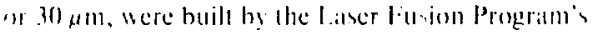
larget lisbrication group).

As $x$ raly emerpe from the targel, they strike the ergetal oner a limited range of angles of incidence. for each angle value, the crystal selects a unigue a-rils photon eneres for reflection to the film. This biand of plooton encrgics frem 2.6 to $3.7 \mathrm{keV}$ is analy aed along the length of the film, for the instrument geometry shown. The bind includes the moss intene belium-like and hydrogen-like argon lines (demoted as Ar-Hee and $\therefore:$ : I 1 ), whose encrgies are $3.14 \mathrm{keV}$ and $3.32 \mathrm{keV}$, respectively.

The argon liate inaging lechnique was lested "ith exploding-pusher largets al the Al culs laser likeilits. The purposes of the lest series were

- To determine il the x-ray lines radiated by helium-like and hjdrogen-like argon were strong enough to he visible abole the comtinuum radiation.

- To compare the argon line images with those produced by the proven alpha-particle zoneplate images.

It was important to verily that the electron temperature in the compressed lucl "ats high enough to poduce nearly complete stripping of the argon, because only the lines radiated by the helium-like and hydrogen-like ion species (with two or one electrons remaining hound) could penetrate the glass shell. We hoped to show in the Argus sho! series that the targets did perform as typical exploding pushers, while stiil radiating argon $x$-ray lines of sufficient intensity to allow the compression measurement. Thus the amount of argon gas seeded into the fuel in all of our targets was very small, so that the implosion dynamics of the fucl and other observables (such als the neutron yield) would be only slightly altered by the presence of the high- $Z$ gas.

One important question about the argon inaging technique could not be answered directly in the Argus series, that question being whether or not the limes ol argon $x$-raly line emission and of D-T fued burn conincided. The houndary of the fuel volume converges very quickly during an implosion, and unlew the timing in good, the xarly images sill be larger than the linly compressed fucl core. Comnpuler smolutatums of the implesion (which showed that the high electron temperatures required to st rip the argun would be achieved only late on the implosinul) sugpested that there would indeed be no semfifiam errors intreduced hy this liming ficctor-il conclusion we hoped a verify by correlation of the $x$-raly and alpha-particle images.

The argon imedeing spectrograph and the ane plate camera were posilioned diametrically . sposite each other in the Argus target chamber. A second inaging spectrograph, modified to image the silicon lines emilled by the target's glans shell, was locited in a third guadrant: the mission of this spectrograph wats lo determine the extent to which the glass mixed with the fuel during the implusion. The plane containing these instruments was normal to the direction ol propagation of the two opponed laser beams.

The glass pellets used in the lest series nere formed hy the liguid droplet method, in a "drop tower" sulfused with argon. The density of argon in the largets was about $0.15 \mathrm{mg} / \mathrm{cm}^{3}$, while the density of D-T fuel subsequently loaded into the pellets was $5.0 \mathrm{mg} / \mathrm{em}^{3}$. The initial diameter of the pellets wals $140 \mu \mathrm{m}$; the wall thickness was $2 \mu \mathrm{m}$. When exposed to the Argus laser heams, each delivering an encrgy of 300 joules in $140 \mathrm{ps}$. it was expected that the pellets would be compressed to a final diameter on the order of $30 \mu \mathrm{m}$ and healled to a temperature of 16 nillion Jegrees Kelvin. Potassium is a significant constituent of the target glass (11\% by weight) and the $x$-ray lines of this element, with photon energies near $3.5 \mathrm{keV}$, were observed in the argon spectrograph data.

For a typical test series snot. $x$-raly images of argon and potassium were recorded by the two spectrographs. While the lines of the glass constituent, potassiun, were radiated at both the initial target boundary (the region heated by the laser 
Fig. 5-46. Direct size measurements of the compr sced target core, obtained by (a) ALICS and (b) an alpha-particle zone plate camera (LPC).
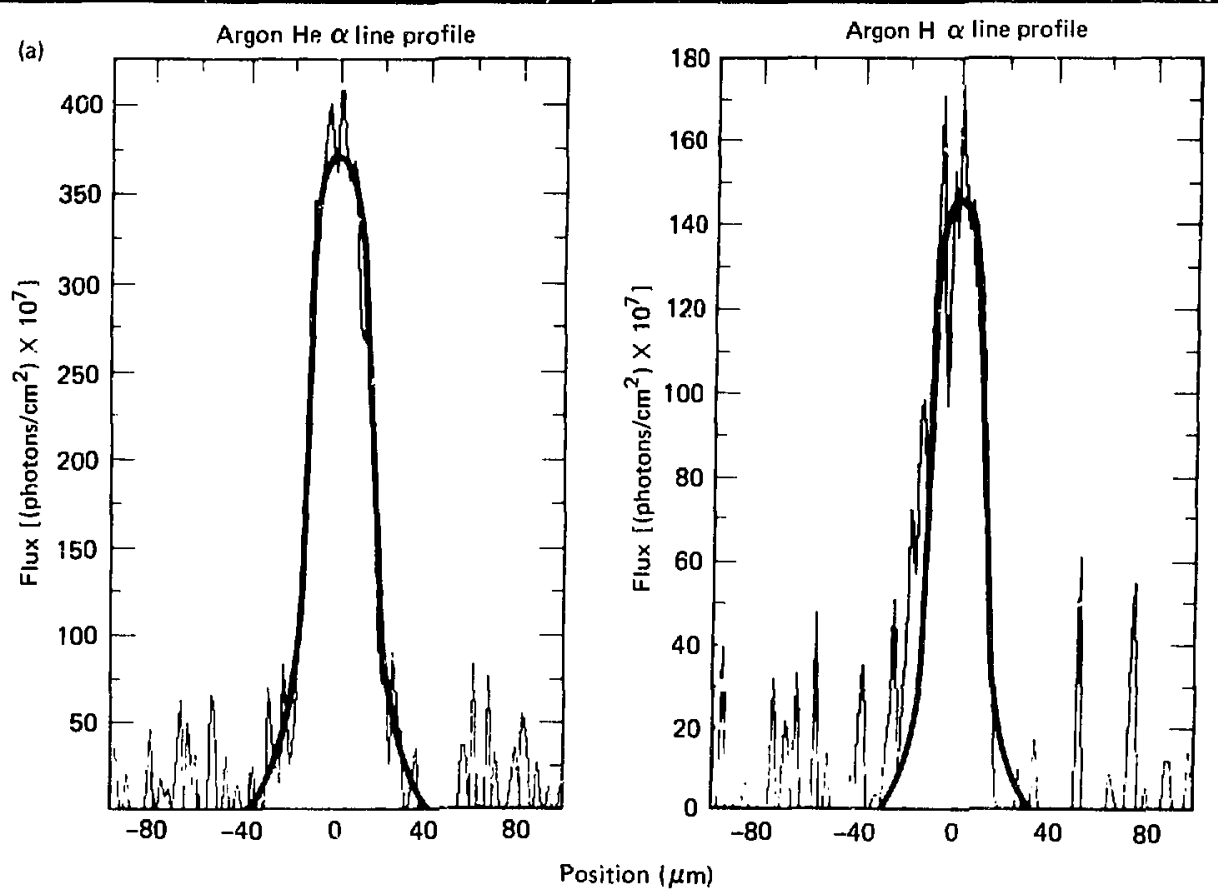

(b)
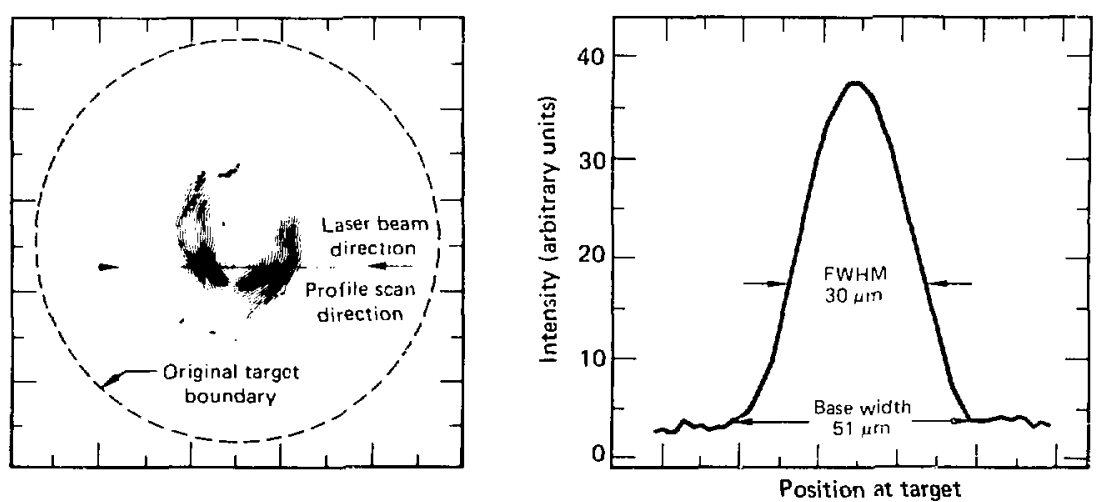

heams) and at the core, the source region for the argon lines is obviously confined to the compressed core. The ratio of intensities of the Ar-Heo and ArHo lines indicates a core electron temperature on the order of 16 million degress. Stark broadening of the lines was not observed.
The argon line inages were analyzed by computer. lo correcl for the width of the slit al sther effects of the imaging technique. The spatial profiles produced by the analysis are shown in Fig. 5-46(a). 
The width of the Ar-Ho image is about $35 \mu \mathrm{m}$, while that of the Ar-Her image is somewhat larger. Because the electron temperature in the core increases during implosion, the radiation from the lower-ionisation-state He-like ions necessarily precedes, and thus occurs within a larger volume than the H-like species. An alphit-particle emission profile, scitnned in the same direction as the argon imiges, is shown in Fig. 5-46(h). The widlh of the profile, which is an accuralle measure of ihe hurnregion diameter for expleding-punher targets, is about $30 \mathrm{~km}$. Thus there is icisomalhy good agrecment hetueen meisurements of the sise of the compressed-lucl region oblained hy Ar-Ho imaging and by alphatpartacte imiging. The targets performed as typical exploding pushers in terms of compression and neutron bicld achieved, which suggests that the argon seed had no effect on the impiosions.

I0X Experiment Series at Shiva. The argon line imaging cryblal spectroglaph (AI.ICS) wals tised at Shiva in experiments whose main purpose was to correlate diflerent lypes of density diagnostics. The no-cual!ed "I()X" series (Ref. 38: ste also Section 6. "I0X I.iquid Densily Target Studies") embodied the study of densily athieved for 140- $\mu$ m-diam microballooms with at glass-pusher wall thickness of $5 \mu \mathrm{m}$ and an ablator thickness typically of $15 \mu \mathrm{m}$ $\mathrm{Cl}_{1.4}$. These targets were filled with $\sim 10 \mathrm{mg} / \mathrm{cm}^{3}$ of D-T and usually about 0.05 to 0.06 almospheres of argon. Becaluse of Shiva's asymmetric irradiation geomely we assumed the compression would be nonsphericil. Therefore, we oriented Al.ICS such that its spatial resolution dimension was parallel to the cirrection of irradiation.

rigure 5-47 shows a typical argon $x$-ray spectrum obtained from one of ihe shots. Note that we observe only one line. the He-like $A_{r}$ resonance transition. Because of the poor signal quality we could make only spatial measurements to determine the diameter of the $\mathrm{Ar}$ emission region: because of the width of the slit $(\sim 20 \mu \mathrm{m})$, the spatial resolution is also certainly nol optimum for our imploded target sizes. Figure 5-48 further illustrates the difficulty in data reduction. We assume different sizes for a uniformly emitting spherical source which is conveluted with a slit function, then plotted and compared to the measured spatial distribution: this method was essentially the same one used to analyze
Fig. \$-47. X-ray spectrum in region of He-like argon lines from $10 \times$ shot series.

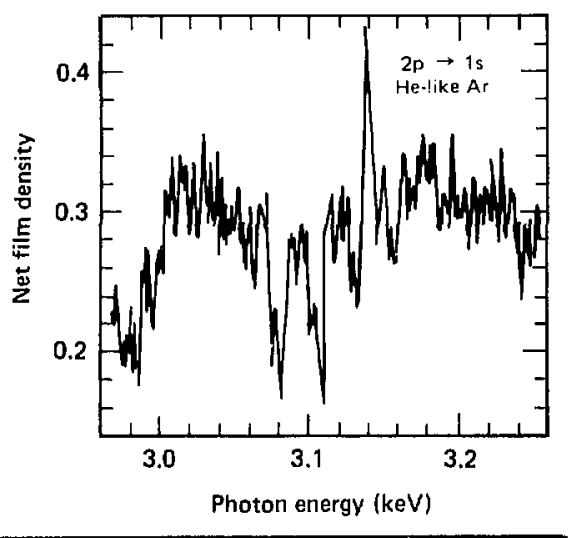

our exploding-pusher target data. Since the data are of poor quality as well as statistically uncertain, it is difficult to assign a source size to represent the data. Best guesses give a value of $\sim 30 \mu \mathrm{m}$, since that value corresponds to the orange curve (in the figure) that more or less traverses the mean of all the data points. Tahle 5-3. however, does outline our best estimales of the source sizes for all the $10 \times$-series shots. Since Al.IC'S gives only a one-dimensional image we hald to assume spherical symmetry for the source. If we assume 2-10-1 asymmetry with the narrow axis being the dimension measured, then we obtain final density values which are $\sim 4$ limes less than in the spherical case: these values are also tabulated in the lable. Future imaging measurements will employ two ALICS instruments imaging along orthogonal dimensions so as to circumvent uncertainties introduced by asymmetric sources.

Problems with Present Spectroscopic Density Measurements. Present targel-capsule designs have jeopardized the utility of using Ar $\mathrm{x}$-ray spectral and spatial line widths to determine density. The combination of too much glass (thick pushers) and too little argon (an average of $0.05 \mathrm{~atm}$ in the $10 \mathrm{X}$ series) in the target capsules results in a small line/continuum ratio (continuum is the Si freebound emission). When this small ratio is coupled with normal spectral noise, it becomes very difficult to separate important line wings (line intensity far from line center) from continuum and film noise. As a result, the ( $10 \times$ series) data obtained thus far are of poor quality. 
Fig. 5-48. Spatial emission profile for argon He-like resonance transition. The three colored curves are calculated for indicated source sizes, convoluted with a $20-\mu \mathrm{m}$ slit and imaged onto the spectrograph film plane.

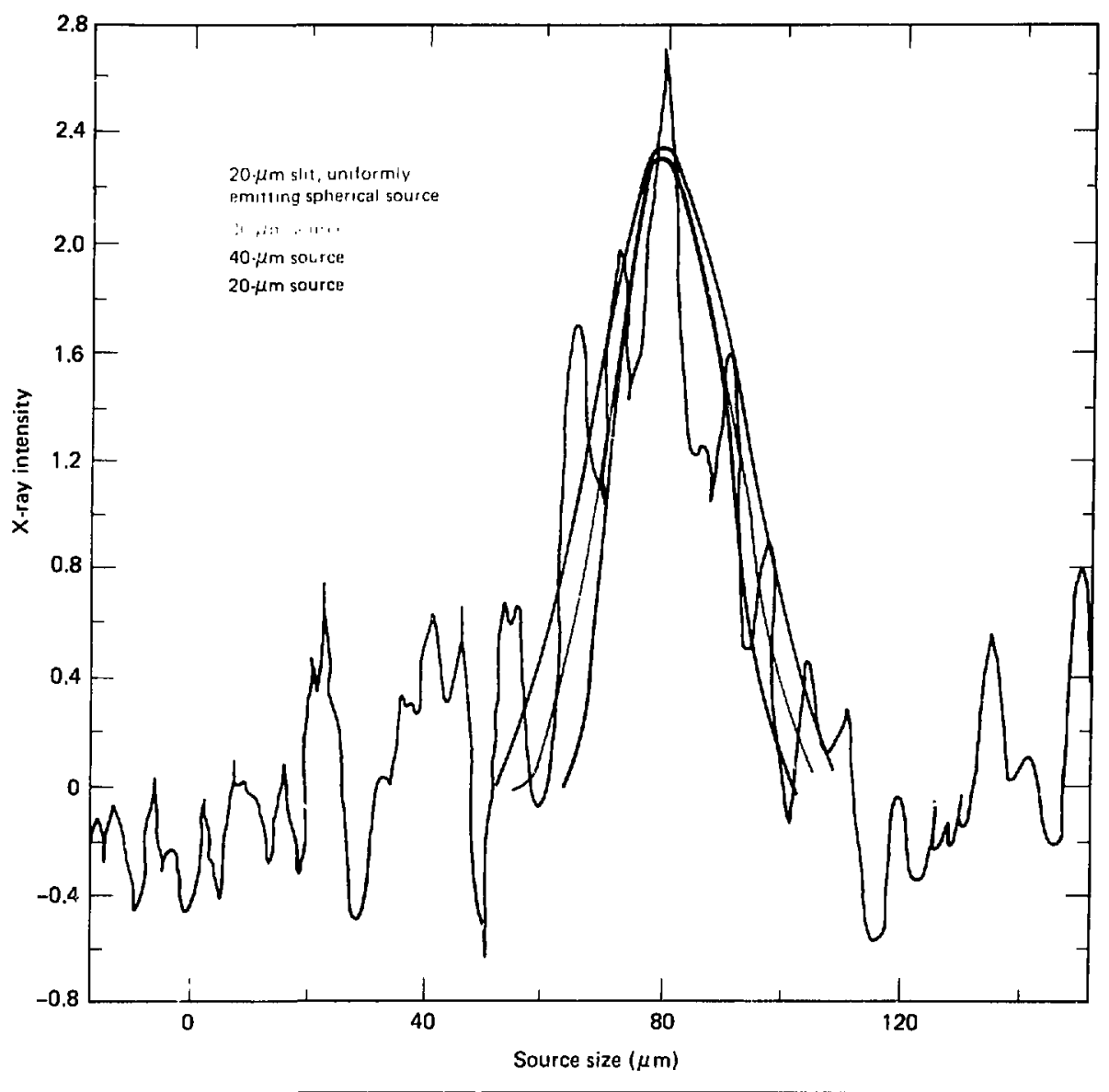

Table 5-3. ALICS results on $10 \times$ targets.

\begin{tabular}{ccccc}
$\begin{array}{c}\text { D-T fill } \\
\left(\mathrm{mg} / \mathrm{cm}^{3} \text { ) }\right.\end{array}$ & Ball size & $\begin{array}{c}\text { Dia from } \\
\text { ALICS }(\mu \mathrm{m})\end{array}$ & $\begin{array}{c}\rho_{\text {uniform sphere }} \\
\text { (units of } \rho \mathrm{D}-\mathrm{T} \text { liquid) }\end{array}$ & $\begin{array}{c}\rho_{2: 1} \text { ellipsoidb } \\
\text { (units of } \rho \text { D-T liquid) }\end{array}$ \\
\hline 10 & $152 \times 3.7 \times 15$ & $30-40 \mu \mathrm{m}$ & $7-3$ & $1.8-0.8$ \\
8.7 & $136 \times 4.6 \times 15$ & $15-20 \mu \mathrm{m}$ & $32-14$ & $8.0-3.5$ \\
10 & $141 \times 4.6 \times 15$ & $30 \mu \mathrm{m}$ & 5 & 1.3 \\
10 & $135 \times 4.3 \times 15.4$ & $30 \mu \mathrm{m}$ & $4.5(=5)$ & 1.3 \\
\hline
\end{tabular}

aDimensions represent outer diameter, glass thickness, and CF ablator thickness, all in $\mu \mathrm{m}$.

bALICS measures diameter of semiminor axis of ellipsoid of revolution. 
The most obvious solution to the weak signal problem is to put more argon into present target capsules. LASL has had good success observing $A r$ lines when using a 0.2 -atm loading. ${ }^{39}$ Their largets are twice the diameter of ours and they report achieving the same final $\mathrm{D}_{2}$ (in our case. D)-T) densities. Since $x$-ray yield is proportional (1) compressed-argon density. they can expect a minimum of 32 times our signal strength taking into account the difference in initial argon loading). Scaling their results would suggest an initial $\mathrm{Ar}$ loading of our largets with $1.5 \mathrm{~atm}$. although we believe that $\sim 1$ alm is a reasoniable compromise that should yield numerous lines (resonance and higher-series memhers) from both lle-like and HIlike $A r$ charge states. Fach line offers an independent check of density by using hoth its spatial and Stark profile: this provides redundancy in the measurement.

Fig. 5-49. He-like $\operatorname{argon} 2^{1} P_{1} \rightarrow 1^{l} S_{0}$ transition intensity ys electron temperature.

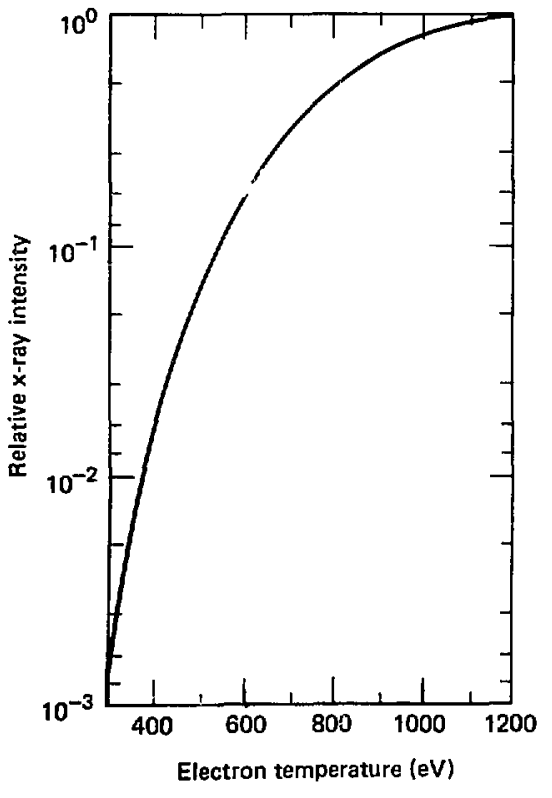

Argon may not always be the most prudent choice as a seed gas. Since some of our present larget-cupsule designs do not achieve significant final-fuel electron temperature ( $T e \simeq 500 \mathrm{eV}$ ), maximum He-like $A r$ line emission is down by a factor of ahout 15 (see lig. 5-49). liven more importint is the lact that Ile-like argon lines are readily ahsorbed by the silicon in the glass pusher. 1'igure 5-50 illustrates the elfect of the giass pusher opacity on $\mathrm{Ar}$ line Iritnsmission.

Our first choice among solutions to the abovementioned problems would he to replace argon with phosphorus as a seed gals. lgnoring the obvious largel fabrication problems with handling it. phosphoress would make an ideal choice since it has one lle-like and one H-like $x$-ray line each below the ahourption edge in heated glass (1:ig. 5-50). The phosphorus $\mathrm{KV}$ line (2'P1 - I'S $\left.\mathrm{S}_{0}\right)$ at $2.152 \mathrm{keV}$ and the phosphorus XVI line $\left({ }^{2} P_{\mid} 2_{2}, 2^{2} \cdot 1^{2} S_{\mid}\right)$at 2.303 hev could provide hoth Stark and spatialprofile analyses for final-density determination.

Bromine is a more pratcical seed gas candidate. and would improve both the ahsorption and production problems encountcred with argon: bromine can also he utilied by radiochenistry methods to measure fuel „R. I Infortunately for our purposes. bromine call only provide a measurement of fuel $x$-raly spatial extent. sinee at present fuel temperatures we ealn only excite the hromine Ne-like

Fis. 550. Opentey of l. L: microballoon glass at a temperature of $500 \mathrm{eV}$ and normal density.

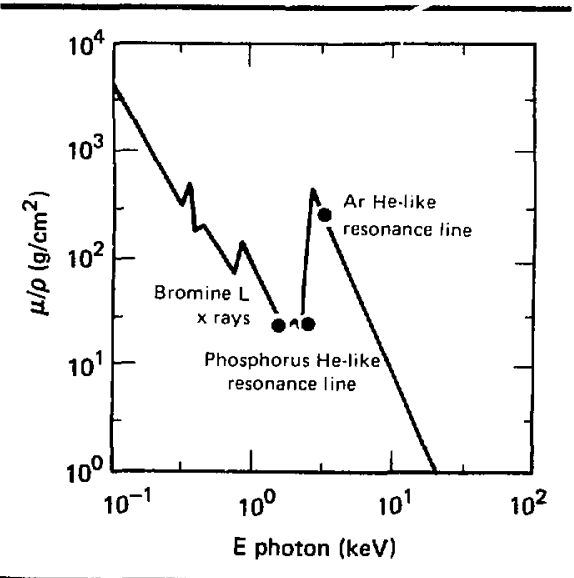


lines to Be-like L. $x$-ray lines at $\sim 1.5 \rightarrow 1.9 \mathrm{keV}$. whose Stark profile is presently not modeled. Bromine will. however, produce much better signal strengths than argon.

Measurements of X-Ray Spectra from LaserDisk Interactions. In last year's report ${ }^{40}$ he discussed $x$-ray probing diagnostics for studying ablative implosions. In order to image the pusherfuel interface of near-term target designs. it will be necessary to use well-characterired $x$-ray sources with energies from o to $8 \mathrm{keV}$. X-ray line sources will be utilized. since

- They provide for casier discrimination against target self-emission. and hence betler signallu-noise ratios and sensitivity.

- Narrou r-rily lines eliminate the dependence of target opacity on probing $x$-raly energy: this leads to simpler and more accurate determination of target component intei laces.

In this section we describe the results of a serie" of measurements of $x$-ray line intensity $I$, versus $k$. 1. and $M \times$-ray emitlers. as well as versus 7 of the

Table 5-4. $X$-ray line intensity and signal-to continuum ratio for $K, L$, and $M$ emission types.

\begin{tabular}{|c|c|c|c|}
\hline & $\begin{array}{c}\text { K X rays } \\
\text { (titanium) }\end{array}$ & $\begin{array}{l}\mathbf{L} x \text { rays } \\
\text { (tin and } \\
\text { barium) }\end{array}$ & $\begin{array}{l}\text { M x rays } \\
\text { (gold } \\
\text { uranium- } \\
\text { niobium) }\end{array}$ \\
\hline $\begin{array}{l}\text { Ix }_{\text {(photons }} \\
\text { ner sphero) }\end{array}$ & $T i=10^{15}$ & $\mathrm{Sn}=4 \times 10^{14}$ & $\Delta u=10^{l S}$ \\
\hline Signal & $\sim 100$ & $\sim 5$ & $\sim 6$ \\
\hline $\begin{array}{l}\text { continuum } \\
\mathbf{E}_{\mathbf{X}}\end{array}$ & $4.8 \mathrm{kcV}$ & $3.8 \mathrm{keV}$ & $2.5 \mathrm{keV}$ \\
\hline
\end{tabular}

target, versus laser pulse lengths $\lambda$, and versus intensity $I_{1}$.

The first series of experiments a as performed to ohtain $\mathrm{I}_{\mathbf{\gamma}}$ versus 7 and to study emission-lype $\{\mathrm{K}$. I.. or M) x rays. The experiments were done on Irgus with 1 -ns 1 WHMl laser pulses and $l_{1} \simeq 3 \times$ 10 ${ }^{\text {it }} 11 \mathrm{~cm} \mathrm{~cm}^{2}$. We made thesc and subsequent measurements with our time-integrated $x$-ray line spectrograph: Tathe s-t gives the results. The obvious conclusion is that $x$-raly intensity is constant for $\mathrm{k}$. I or $M$ - ra! emission. The big difference is in the strength of the line continumm ratio: titanium produced aes strong and narrou lone emission. This is a significant discovers, since It implies that if a narrow-energy-band imaging device is ased. the sensitivity and contrast ratlo can be greatly increitsed.

Io achiese this semitisily we therefore chose wo lud $k x$-ray emitter versus target $/$. . 1 , and $l_{1}$. A series of measurements to study some of the above wats performed at Shw al. using from one to ten of its beams 10 achieve $I_{1}=3 \times 10^{16} 11 \mathrm{~cm}^{2}$ in source sises frorn 140 to $500 \mu \mathrm{m}$ in diameter: a summary of the dalit obtained 10 date is shoun in Table 5-5. Nlthough incomplete with regard (o measurements at all pulse lengths and laser intensilies, present ditta does indicate that prolific line emission cian be produced at $\mathrm{L}$, as high as $9.0 \mathrm{kel}$ (/.n linest. (real. lion of the high-energy $/ n$ and $v i$ lines does. however. require hgls laser inconsity. In measurements so far. the laser snot diameter was $1+10,0 \mathrm{~m}$ and $\mathrm{I}_{\mathrm{I}} \simeq 3 \mathrm{~kJ}$ : these shots required 10 Shiva beams. In the coming year we plian to complete this measurement series to determine ly versus litser

Table 5-5. K x-ray line emitters.

\begin{tabular}{|c|c|c|c|c|c|c|}
\hline Element & $\begin{array}{c}\text { Laser intensity } \\
\left(w / \mathrm{cm}^{2}\right)\end{array}$ & $\begin{array}{l}\text { Pulse } \\
\text { length } \\
\text { (ps) }\end{array}$ & $\begin{array}{c}\text { Strongest line } \\
\text { energy } \\
\text { (keV) }\end{array}$ & $\begin{array}{l}\text { Strongest line } \\
\text { intensity } \\
(\mathrm{keV} / \mathrm{keV}-\mathrm{sp})\end{array}$ & $\begin{array}{c}\mathrm{E}_{\mathrm{L}} \text { total energy } \\
\text { all lines } \\
(\mathrm{J} / \mathrm{sp})\end{array}$ & $\begin{array}{c}E_{v} / \varepsilon_{\text {laser }} \\
(\mathscr{E})\end{array}$ \\
\hline Titanium & $\begin{array}{l}3 \times 10^{14-A r g u s} \\
3 \times 10^{14-A n g u s} \\
3 \times 10^{15} \text {-Shiva }\end{array}$ & $\begin{array}{r}1000 \\
2000 \\
600\end{array}$ & $\begin{array}{l}4.75 \\
4.75 \\
4.75\end{array}$ & $\begin{array}{l}\text { film saturated } \\
5.8 \times 10^{16} \\
2.6 \times 10^{17}\end{array}$ & $\begin{array}{l}-2 \\
0.27 \\
1.6\end{array}$ & $\begin{array}{l}- \\
0.05 \\
0.06\end{array}$ \\
\hline Vanadium & $3 \times 10^{14}$ & 2000 & 5.20 & $1.5 \times 10^{16}$ & 0.09 & 0.02 \\
\hline Nickel & $\begin{array}{l}4 \times 10^{14} \\
3.3 \times 10^{15} \\
2 \times 10^{16} \\
3 \times 10^{16}\end{array}$ & $\begin{array}{l}600 \\
600 \\
600 \\
600\end{array}$ & $\begin{array}{l}7.80 \\
7.80 \\
7.80 \\
7.80\end{array}$ & $\begin{array}{l}\text { no lines observed } \\
\text { no lines observed } \\
\text { no lines observed } \\
1.2 \times 10^{17}\end{array}$ & 245 & 0.08 \\
\hline Zine & $\begin{array}{l}1.5 \times 10^{16} \\
3 \times 10^{16}\end{array}$ & $\begin{array}{l}600 \\
600\end{array}$ & $\begin{array}{l}8.9 \\
8.9\end{array}$ & $\begin{array}{l}\text { not analyzed } \\
1.4 \times 10^{16}\end{array}$ & 0.50 & 0.02 \\
\hline
\end{tabular}

${ }^{2}$ Spot size at $3 \times 10^{15} \mathrm{~W} / \mathrm{cm}^{2}=500 \mu \mathrm{m}$ at $3 \mathrm{~kJ}$; spot size at $3 \times 10^{16} \mathrm{~W} / \mathrm{cm}^{2}=140 \mu \mathrm{m}$ at $3 \mathrm{~kJ}$. 
Hig. 5-51. Titunlum $\mathrm{K}$ x-ray spectrom frotu Shiva disk shot.

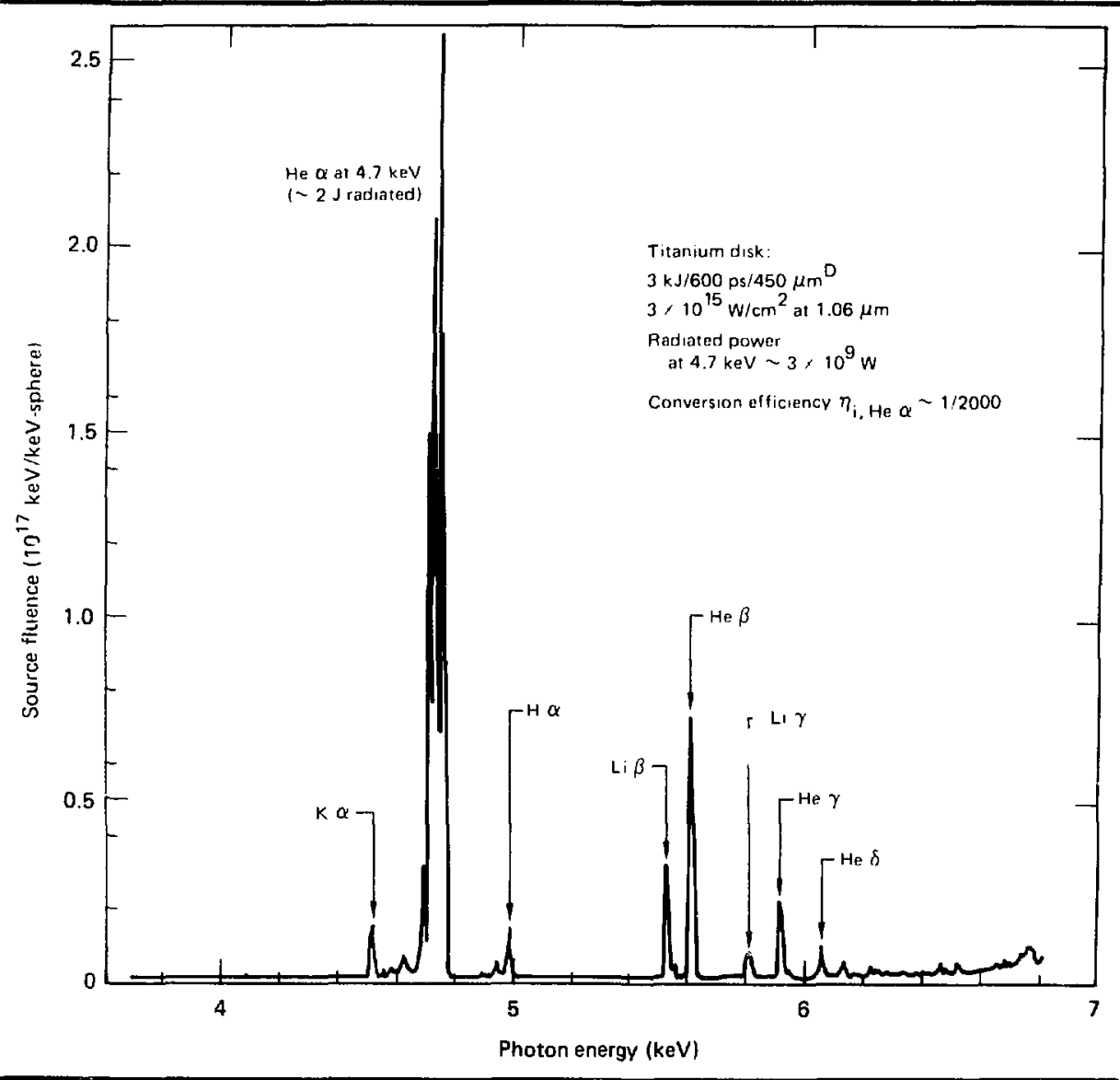

pulse duration $\tau_{\mathrm{I}}=100 \mathrm{ps} .600 \mathrm{ps}$. and $2000 \mathrm{ps}$. and $\mathrm{I}_{1} \simeq 3 \times 10^{14} .3 \times 10^{15} .3 \times 10^{16}$, and $1 \times 10^{17}$ $W / \mathrm{cm}^{2}$. Knowledge of the scaling of $I_{x}$ with these parameters is essential to the design of future backlighting efforts at Shiva and Nova.

We have initiated a modest effort to understand the mechanism(s) by which the higher-erergy $x$-ray lines are produced. Figures 5-51 and 5-52 show the $x$-ray spectra observed for some laserproduced $\mathrm{Ti}$ and $\mathrm{Ni}$ plasmas. The experimental results indicate that

- Strong line emission is observed for Ti at $\mathrm{I}_{L}$ $\simeq 3 \times 10^{15} \mathrm{~W} / \mathrm{cm}^{2}$ and for Ni with $\mathbf{I}_{\mathbf{L}} \simeq 3 \times 10^{16}$ $\mathrm{W} / \mathrm{cm}^{2}$.
- The conversion efficiency (energy in line to encrgy in the laser) is high.

- For $\mathrm{Ti}$, the $\mathrm{He}$-like to $\mathrm{H}$-like line intensity ratios indicate a time- and volume-averaged electron temperature $T_{\mathrm{et} \text {.volume }} \simeq 1.5 \mathrm{keV}$.

- A strong characteristic $K \boldsymbol{\alpha}$ (cold matter $K$ $\mathrm{x}$-ray line) was observed for both $\mathrm{Ti}$ and $\mathrm{Ni}$ : this cold line was produced by high-energy electron and photon-induced ionization of target atoms residing deep in the slab.

Careful examination of the Ti $x$-ray spectrum near the $\mathrm{n}=2$ to $\mathrm{n}=1$ resonance line indicates that the plasma from which it is emitted can only be described by a non-LTE model. For long-pulse 
Fig. 5-52. Nickel $\mathrm{K}$ x-ray gectron from Shiv disk shot.

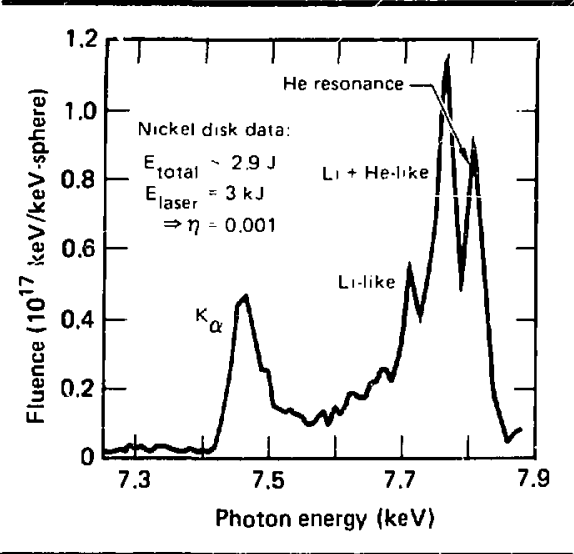

Fig. 5-53. Colculated titunium seetrum, assuming local thennodynamic equilibrium (LTE) in Jasan.

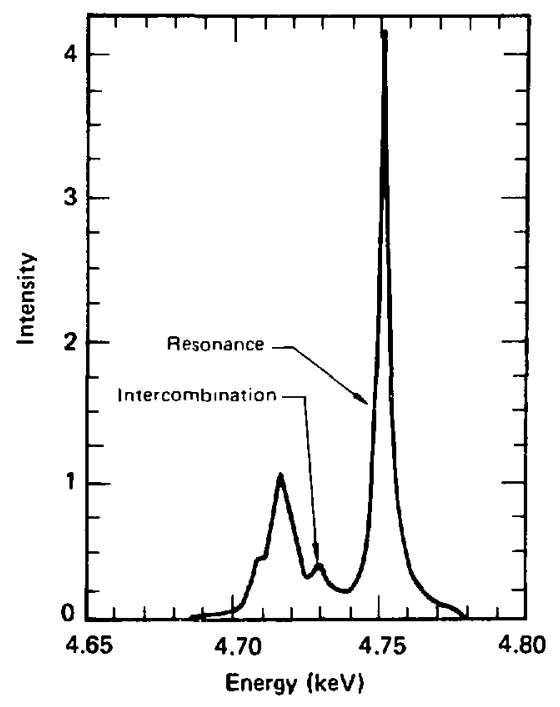

irradiation. however, the line spectra indicate that the plasma approaches LTE. Figure $5-53$ illustrates a theoretical $T_{1} \times$-ray spectrum in the region of the $\mathrm{He}$-like resonance line, derived assuming local thermodynamic equilibrium. The two lines of interest in this spectrum are labeled as the resonance transition
Fig. 5-54. Meavured titanlum spectrum at two different laser palse lengths: (a) 600 ps and (b) 2000 ps.
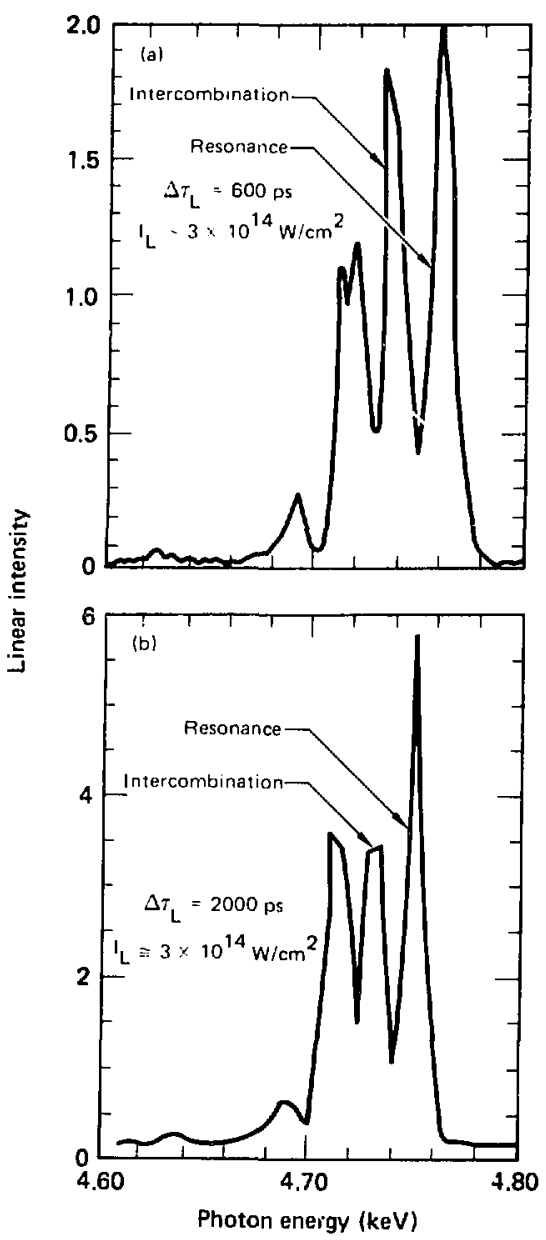

$1 \mathrm{~s}^{2}{ }^{1} S_{0}+1 \mathrm{~s} 2 \mathrm{p}{ }^{i} \mathrm{P}_{1}$ (Resonance) and the intercomhination line $1 s^{2}{ }^{1} S_{0}+1 s 2 p{ }^{3} P_{1}$ (Intercomb). In LTE. line intensitics are proportional to their oscillator strengths: the calculated rattio ${ }^{41}$ of the oscillator strength of the intereombination line to the resonance line is 0.04 . Therefore, the intercombination line is very weak in this theoretical spectrum. In the measured spectrum of Fig. 5-54(it), however, we 


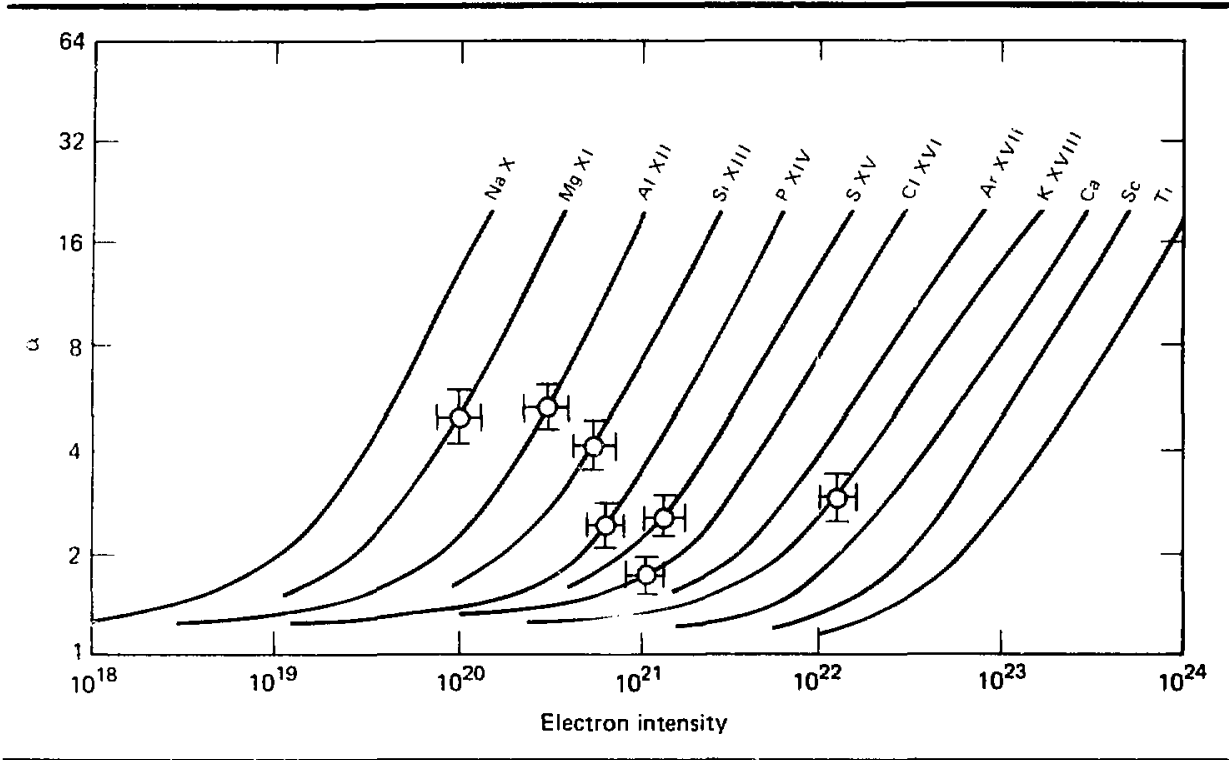

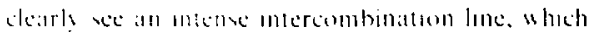

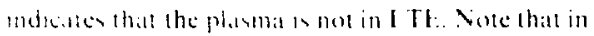

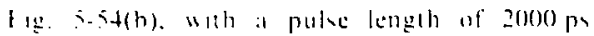

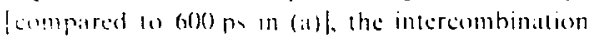

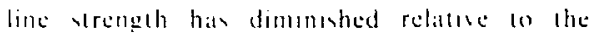
reomance transitum. This mat indiate that the plasma is an liate equalibrating during the longer laser pulse. It could alus mean that the emission in commln from a high average electron densits plasmat.

Boiko et at "2 have modeled the ralio of of intercombination-to-resonance line intensity for non-L.TI: plasmas where the resonance line is optically thin: the ratio of the lines is strongly dependent on electron density over certain density intervals. A sample of their calculations is given in rig. 5-55. Analysis of our data using their calculations would indicate electron densities $\mathrm{N}_{\mathrm{c}}>10^{22}$ $\mathrm{cm}^{-3}$ for Ti at $\Delta \tau_{1} \simeq 600 \mathrm{ps}$ and $\mathrm{N}_{\mathrm{e}}>6 \times 10^{22} \mathrm{~cm}^{-3}$ for $\mathrm{Ti}$ at $\Delta \tau_{1} \simeq 2000 \mathrm{ps}$. We have simulated these plasmas using our conventional (L.S.SNFX) hydrodynamic computer code: of course this crude analysis does not comsider line transfer effects or the fact that the plasma mat be approaching 1.TF: at 2000 ps.

Figure $5-56$ is a "snapshot" of the plasma conditions at the peak of the laser pulse $\left(\Delta \tau_{1}=\right.$ $1000 \mathrm{ps} . \mathrm{I}_{1}=3 \times 10^{1+} \mathrm{W} \mathrm{cm}^{2}-1$. giving the electron density. teetron temperature. and Je-like resonance-line emission strength as a function of distance normal to the surlace of the Ti disk. These calculations indicate that the majority of the $x$-ray emission comes from the coronal plasma instead of the denser plasma near the disk's surface. This could explain why the line emission indicates a plasma more in coronal rather than local thermodynamic equilibrium.

Note that the plasma emission region or "scale length $"$ in Fig. $5-56$ is very large $(\sim 100 \mu \mathrm{m}$ for the FWHM of line intensity). This large emission scale length is beneficial for producing copious line radiation. This benefit is not present for lower- $Z$ elements at the same laser parameters. because lower$Z$ elements will be ionized past their He-like states 
Fig. 5-56. LASNEX calculation of electron density and temperature at peak laser pulse intensity vs distance normal to surface of a disk, and nom-LTE calculation of He-like argon resonance line intensity as function of electron density and temperature.

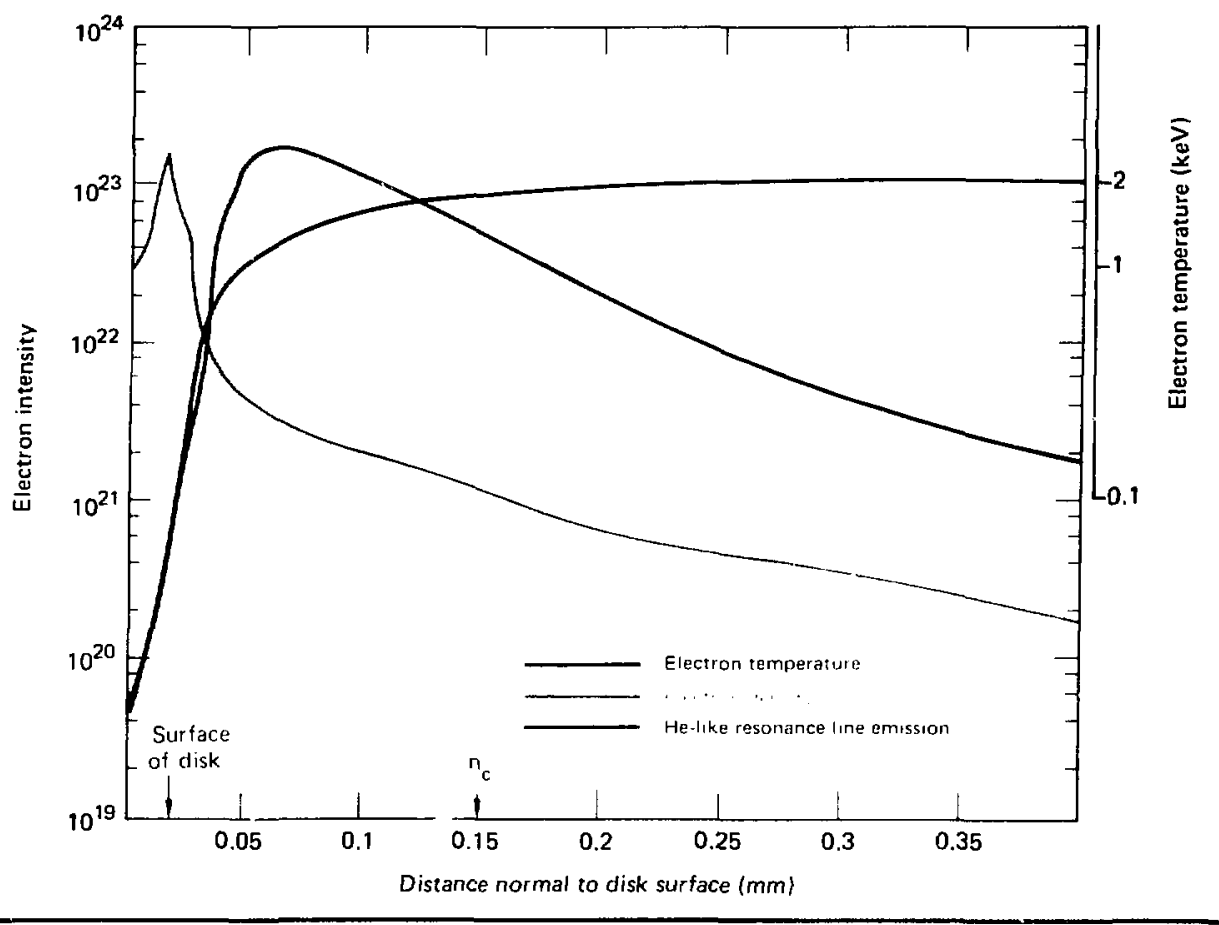

al at lower electron temperalure and hence al at malle distance from the disk s surlace. Thus thes will emir radiation frem a smaller scale length $\mathrm{We}$ should add that lower-/ elements also suffer from an intensits suppression owing to the $/^{+}$scalling of

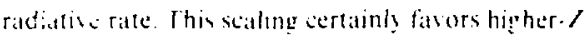
clements prosided one hils emough latser power to sufficientl ionise the high./ atoms.

The linal point concerning these calculations is that the aterage electron temperature at the peitk of the laser pulse is $-1.5 \mathrm{kel}$. Thes is identical to the value derived hy the line ratios taken from our experiment. and indicates that the effects of time ateraging on experimental line intensities are mininual.

Authors: D. I.. Matthess and I.. V. Koppel

\section{References}

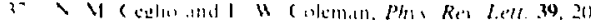
(l)

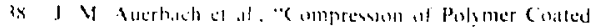

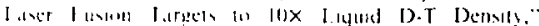

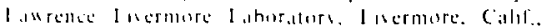
( ( RI.X1984 (19)(1)

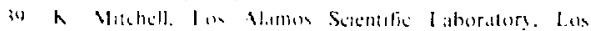

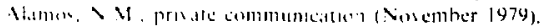

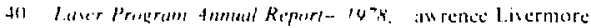

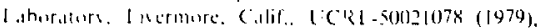
एр 6. 38 is $(1-411$

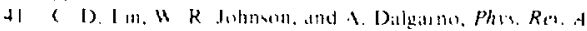
15. $154119 \% 9$

t. I Buhnel.t. I Ph, B 12. 1889 (1979). 


\section{$\mathrm{X}$-Ray Backlighting with a Wolter Microscope and X-Ray Streak Camera}

The process by which laser light in used 10 compress D-T fuel and cause thermonuclear hurn caln he broken doun into four parts: absorption. enetgy transpert, larget hydrodynamics, and thermonuclear hurn. Oser the last several sears, we hase de.oted signilicant effort to improsing our theoretical and experimental understanding of

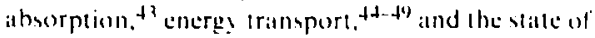

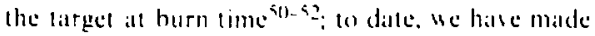
feu measurements of target hadrodynamic be-

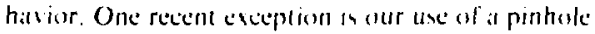
rerat streak camera dialgnostic 10 measure radius1. - inte hehasior uf expleding-pusher largets."

NRI. has presiousl! used isible-light shadowgraphs (o) measure ablative acceleration of slath targels. 5 optical probing. howeser, is limited b! relraction effech and the light can penetrilte onls to the sritical densil! surface, which is itself moving with respect wo the target viab. The use of raly. which can penetrate dene matterial, permils determination of the densits profile of the mosing object through the relation between areal density and the attenuation of a rats. Measurements of this type hase been initiated at the Rutherford Laboratery in England ${ }^{55}$ and at Limeil in France. ${ }^{57}$ These experiments hase provided proof of principle. and hate also previeued some of the problems (such as dy namic range, resolution, and target allignment) which must he addressed hefore x-rat backlighting caln provide high-qualily, interprelable datal from a variets of hargets.

W'e have decloped an s-ra! backlighting sistem (Fig. 5-57) designed to minimize the problems of dynamic range, target alignment. and resolution. The system uses a $22 \times$ magnilication Wolter (hyperboloid-ellipsoid) axisymmetric grazing-incidence $x$-ral microscope as its objective element and an $x$-ray streak camera of LLL design as the detecting device: the development of the Wolter microscope $e^{58,59}$ and sireak camera ${ }^{60}$ have been described in previous annual reports. The Wolter microscope currently being used has a resolution of 2 to $3 \mu \mathrm{m}$, depending on the mode of operation. In the apertured mode, the depth of field can be greater than $\pm 100 \mu \mathrm{m}$. The solid angle of the full-aperture y-ray microscope is approximately 1.1 $\times 10^{-6}$ sphere, with a high-energ! cutoff of $2.1 \mathrm{keV}$. Other Wolter microscopes heing fabricated are designed to hale high-energy cutoffs in the range from 3 lo $+\mathrm{kev}$.

Because the streak cameral hats a thin photocathode it in semsitive 6o xat? with energien from lol) eV to more than $10 \mathrm{keV}$. The oserall spalial resolution of the s!stem is limited to ahout $4.5 \mu \mathrm{m}$ at the object plane b! the $22 \times$ malgnification of the v-rity microscope and the $1001-\mu \mathrm{m}$ resolution al the r-rily treatk camera. The lime resolution of the strealk callmerat a 15 ps: te dy latmic range in approxmalcly lour orders of mignitude. In priatice. the werill dynamic ringe of the sylem is limited b? basklighter sluree intemvits and imigeng uplice to athout three urders of magnitude.

The - -rat backlighting s!stem in, aligned using a wible-light target iewer mounted coavially along the eral! merescope line of sight (Fig. s.58). A visible-hght kens is motnled in the center al the ayisometrie aras merosione mirror such that its principal plane and optciel axis soincide with the principal plane and opecical asis of the s-ras! mic: mespe. The focell lengtho of the lens and the $x$ rats mirror are the same, so that the object and image focall points also coincide. During an initial alienment phasc. the v-rat microscope is adjusted in tilt $( \pm 20$ arcseconds) to minimise coma. Then the image of at single-mode optical fiber, mounted at the center of the chamber, is projected onle: the streath eamera slit: the streak camera is then moned wh that the imige is centered on the slit.

A pellicle splits the beim and projects it atho onto a second image plane, mounted rigidly on the st reatk eamera at right angles 10 its face: this risibleimage plane then moves when the streak camerat moves. A set of cross hairs in the visible-image plane is rroved to coincide with the imatge of the oplical fiber and then locked into position. From this point, aligning targets to the streak camera slit is equivalent to aligning them to the cross hairs in the visible-image plane: the alignment is accurate 10 $10 \mu \mathrm{m}$ and reproducihle to $5 \mu \mathrm{m}$ from shot to shot. The visible-image plane is cauipned with a Polaroid film pack for documentary photos of target alignment, though the secondary inlage is bright cnough that real-time alignment can be performed with the unaided eye.

The target is illuminated by a hand-passfiltered mercury arc lamp (5461 $A$ ) with an annular 
Fig. 5-57. Schematic of the $x$-ray backlighting system at Shive. The main components are a $22 \times$ magnification Wolfer $x$-ray microscope, an x-ray streak camera, and a mercury are lamp illominator.

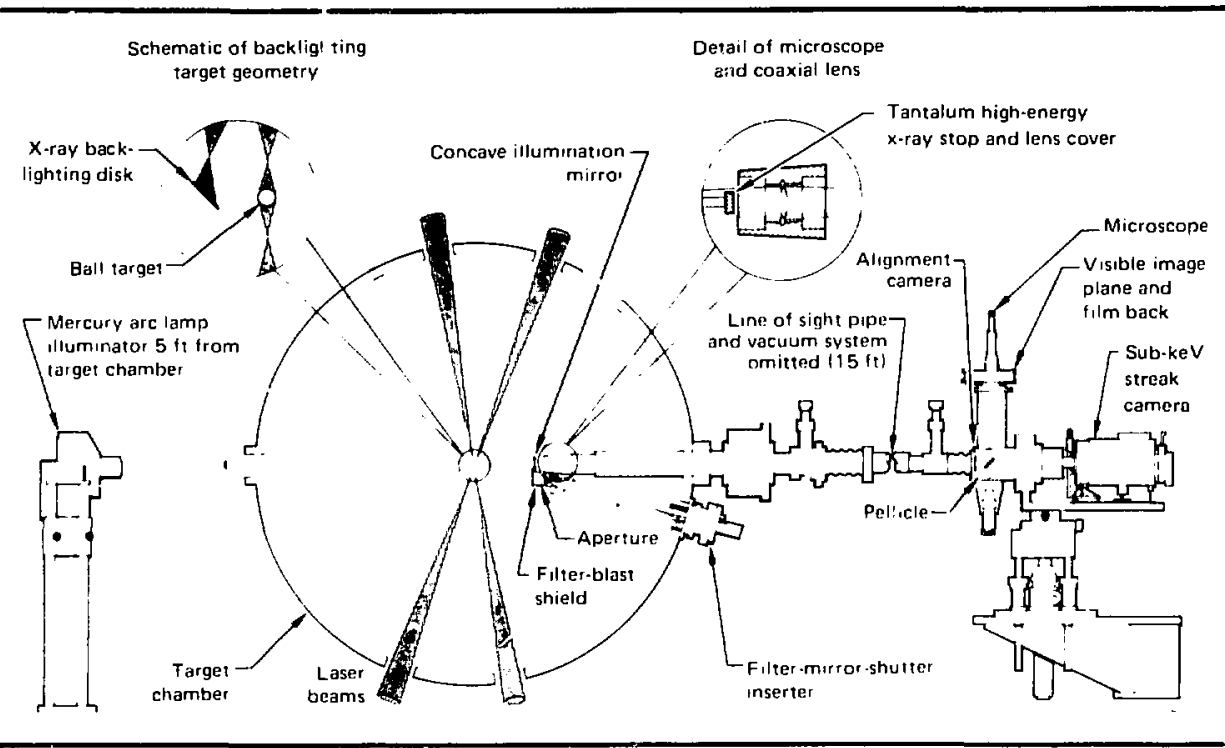

Fig. 5.58. Sctrematic of the x-rny backlighting optical alignment configuration.

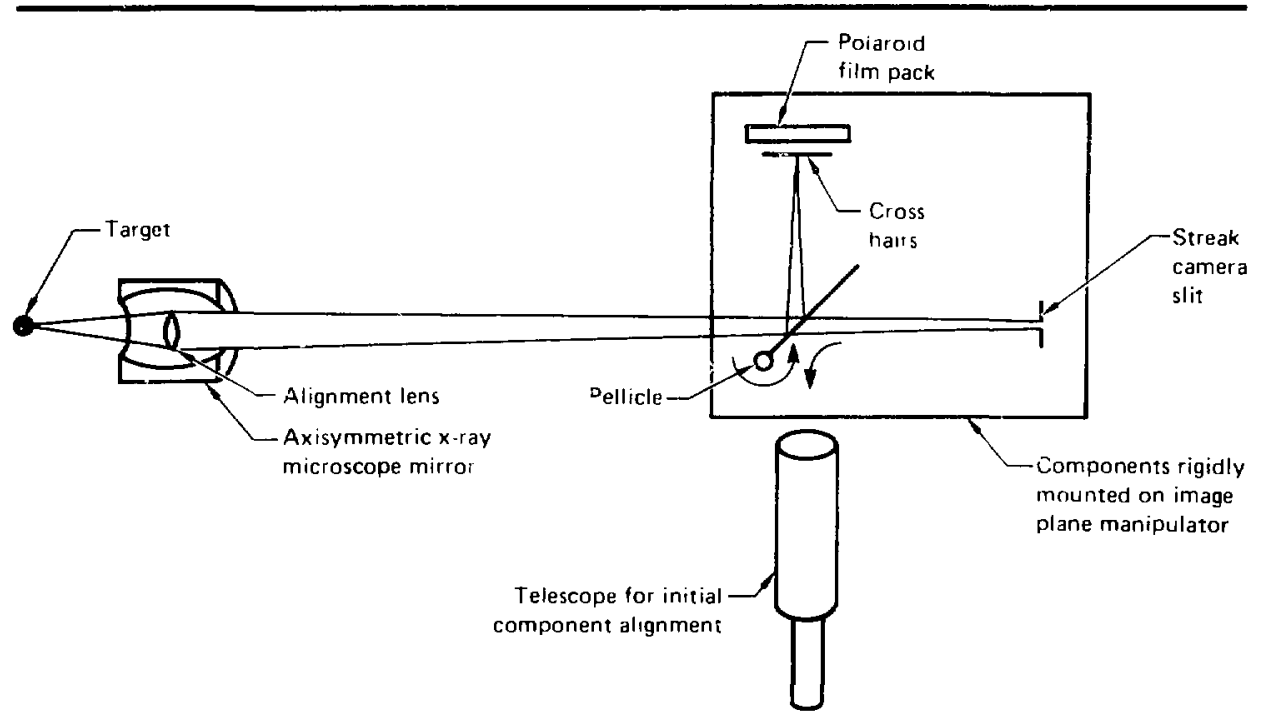


Table 5-6. X-ray backlighter intensities and $x$-ray 6 -ptic solid angles required for streaked $x \cdot$ ray backlighting.

\begin{tabular}{|c|c|c|c|c|c|c|}
\hline Materinl & $\begin{array}{c}\text { Laser } \\
\text { intensity } \\
\left(W / \mathrm{cm}^{2}\right)\end{array}$ & $\begin{array}{c}\text { Spot } \\
\text { diameter } \\
(\mu \mathrm{m})\end{array}$ & $\begin{array}{c}x+\text { ray } \\
\text { line }\end{array}$ & $\begin{array}{c}\text { hv } \\
\text { (keV) }\end{array}$ & $\left(\frac{\mathrm{keV}}{\mathrm{cm}^{2} \text { sphere }}\right)$ & $\begin{array}{c}\text { Figure of } \\
\text { merit } \\
\left(\Omega R^{2} / M^{2}\right) \\
\text { (sphere) }\end{array}$ \\
\hline $\begin{array}{l}\text { Ta } \\
\text { Au } \\
\text { U.Nh } \\
\mathrm{Sn} \\
\mathrm{Ti} \\
\mathrm{Ni}\end{array}$ & $\begin{array}{l}2 \times 10^{15} \\
6 \times 10^{14} \\
6 \times 10^{14} \\
6 \times 10^{14} \\
3 \times 10^{15} \\
3 \times 10^{16}\end{array}$ & $\begin{array}{l}250 \\
400 \\
400 \\
400 \\
440 \\
130\end{array}$ & $\begin{array}{l}\mathbf{M} \\
\mathbf{M} \\
\mathbf{M} \\
\mathbf{L} \\
\mathbf{K} \\
\mathbf{K}\end{array}$ & $\begin{array}{l}2.0 \\
2.5 \\
3.5 \\
3.9 \\
4.7 \\
7.9\end{array}$ & $\begin{array}{l}4 \times 10^{28} \\
4 \times 10^{27} \\
1 \times 10^{27} \\
1 \times 10^{27} \\
1 \times 10^{28} \\
2 \times 10^{29}\end{array}$ & $\begin{array}{l}5 \times 10^{-11} \\
2 \times 10^{-10} \\
8 \times 10^{-10} \\
8 \times 10^{-10} \\
7 \times 10^{-11} \\
1 \times 10^{-11}\end{array}$ \\
\hline
\end{tabular}

.1 की mon a.a. and $35 \mathrm{~mm}$ i.d. The lens

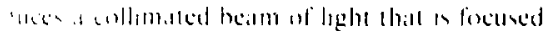

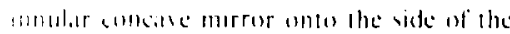

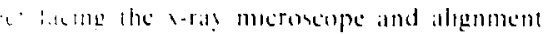

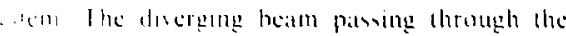

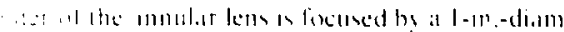

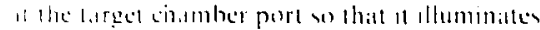

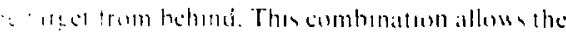

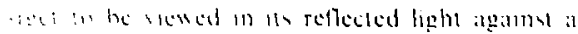
anith hatherumed

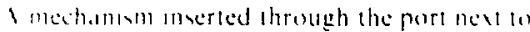
$\because$-1.1! mersetupe allows the concase anmular

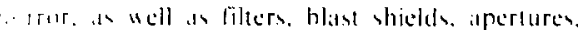
and hullers, lo he mosed in and out al position in front of the r-rat meroscope. At shot lime. at lan-

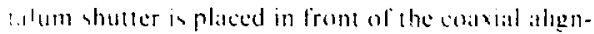
memt lens, we protect it from blast damage and to thetd the sleith camerit from high-eneres x rass 1. I MeV) thall would pass deswn the center of the |ane-nf-slght pipe wh wout being focused by the x-ras morer. Additional x-rat filless can he unserted al wost time with : pnesmatic filter inserter, wilhout merfering with the optical alignment system.

The strealk camera photocithode stopent loil is 'r.ggle and can withstitnd only slight pressure dif'entiak. An automated vacuum system controller an heing conseructed will cyele the system lo and '.im atmosphere at a controlled rate, and with also iverlock the valves. filter inserter, shutter mechiasm. and pellicle to prevent damage to the !enostics, target chamber, and vilcuum system.

lise of the streaked x-ras microscope for inlit slab acceleration experiments is presently - fer intensive in estigation. Measurements can he mike which will follow the target sample from a :. Indsti.l, through its initial acceleration phase, to

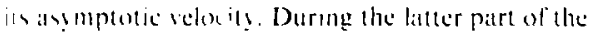
sreak record, it mal he possible to meisure the demots protile of the simple is it disassemblen.

The a-ra! meromeape cain allow be used with

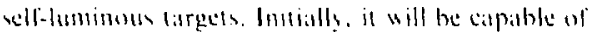
sicwing largess an radiagion from $270 \mathrm{eV}$ (o) $2 \mathrm{koV}$.

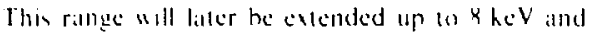
perhap dow $n$ to $180 \mathrm{eV}$. The "ysem will be used lo mecisure the self-lummous intertace near the aborplion region (ill hall largets as thes implonde. With x.het hatklighter sources, we expect in measure the dymamics of the sore during the ithlative implombun al high-densit! bill largets.

To :alcud hacklighting capahility whe $x$ keV ratgec reyuires holl iniense batcklighter sources and high-encres s-r.us microscones with large solid angles, We bace measured a number al hacklight:r sesurces al Iregus and Shiva: resules are gasen in

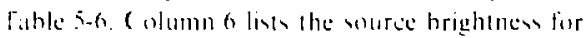
the most miense line (or hand of lines) at the wats encrys lesed an columns for the gisen laser patramelers. A combination of arget sutput and the semsitivity of the streak catmera used with the microstope seth the parameters of the figure of merit $\Omega R^{2} M^{2}$ required of an imaging device, where k. 82. and $M$ are reflectivity, solid angle, and magnification, raspestively. Column 7 lists the ligure of meril required to abtain a dyamic range of three orders of malenitude for the various lines listed in bolumn 5 .

We are currentl construcling a $22 \times$ magnifiealion Kirkpatrick-Bats-lype s-ray mieroscone with multilaver interference $x$-ray mirrorsh for use in the range from 3.5 to $8 \mathrm{keV}$. Multilater interference $x$-ray mirrors allow reflection of high-energy x.ays at much larger angles than single-layer grasing-jncidence $x$-raly mirrors: prelinuinary eslinates are that the instrument under construction 
can oblatin a figure of me. should he satisfactory for bals al $8 \mathrm{keV}$.

In summary, we are des' , ong x-ray backlighting at 1 !: to address questions abe : : the dynamics of iaser fusion targets. A system presently being assembed ";l! perform preliminary asperiments using ?-keV a-ral backlighting sources; 14 plan lo deselop instruments which will allew us to

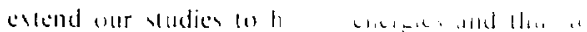
probe the dense core reguns of laser fusion tarects.

\section{Author: R. H. Price}

Major Contributors: A. Cappahianea, B. A. Jones, M. W. Kohicrecki, H. B, Laird, W, M. Ploeger. J. R. Zickuhr, R. D. Rudd, IJ, 1., Holeman

\section{References}

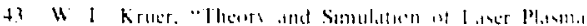

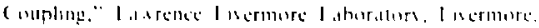

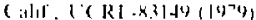

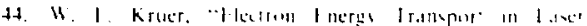

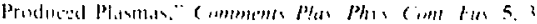
(1974).

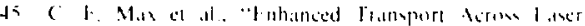

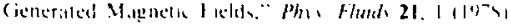

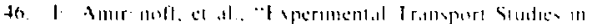

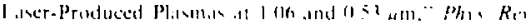
l.8? 4., 7 (1979)

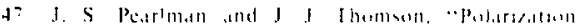

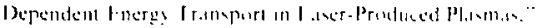

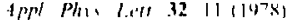

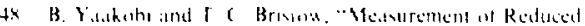

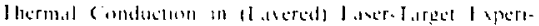

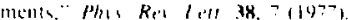

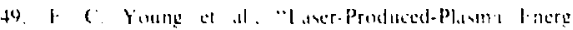

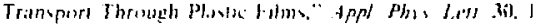
(1977).

5i) F. 11. ( . .

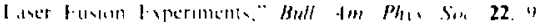
110?7) (ahstr.te1 and )

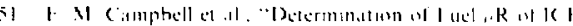

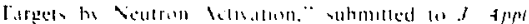
PMW

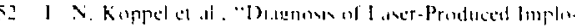

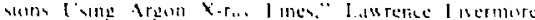

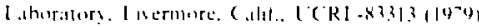

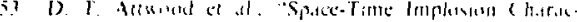

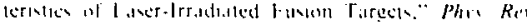
lats 38. 6 (15?7)

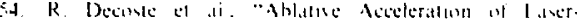

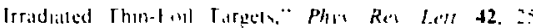
119791

is M H. Key et al, "Pulsed-X-rils Shoddougraph al Dene.

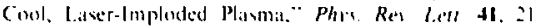
$(197 k)$.

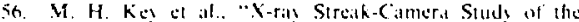
Dy namice of Laser-Imploded Microballonms." Appl Phle Ledt. 34. 9 (1979)

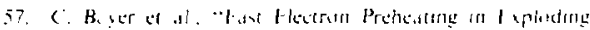

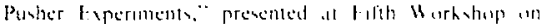

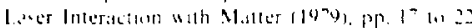

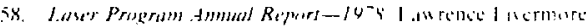

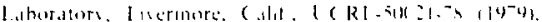

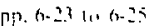

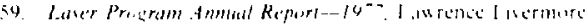

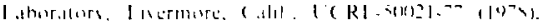

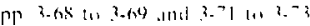

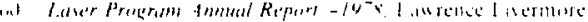

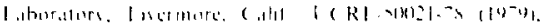

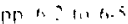

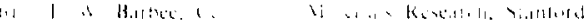

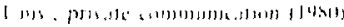

\section{Micro-Fresnel Zone} Plate Developments

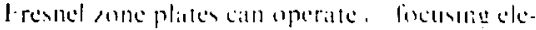
ments that use diffraclunt rather thatul refraction ar rellection. Our goill his been to fibricile and

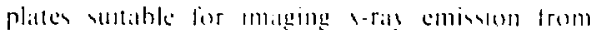
liser-produced planmas. I lypicial applicitlom

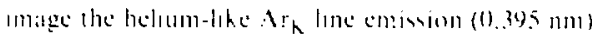
from al laser-compresued D-T i.trel containing all argan seed gats: such a meanurement prosides informallon athull the vise and sommetrs of the compressed flee] requit

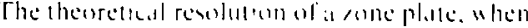
uned with monochromatio ruditiom, is af the arder

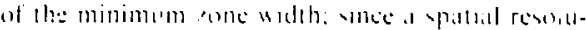

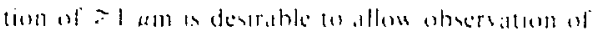
the smallest strututes of interest. "we chese at

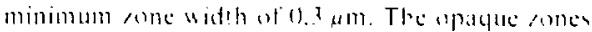

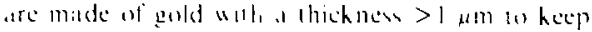

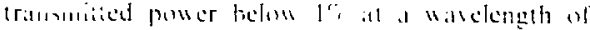

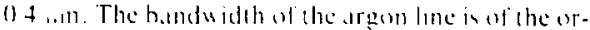
de. al a few electron whe centered at 3.1 kev, w the solle alate sould be hamled to a feu hundied coles la montmuse chromathe atherratum.

Fabrication. Minimum ane widthe near

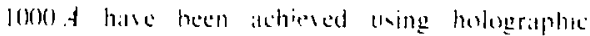
lithograph. electron-projection-lithographs, and diamond-iurming lechmques to genterate some plate paltern. thent The ante plate produced bs these methods, howerer, were limited to gold thicknessen on the order of long f, which has restricted their

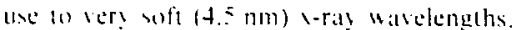

We have deseloped a process which allow sone plates with submicrometer line widthe whe 
fitbricated in thick gold. The rone plate patlern is first generated on an $x-r a y$ mask, using scarningelectron-heam lithography (SEBL); the pattern on the $x$-ral mask is then replicaled using carhon $K$ line soft-x-raty tithegraphy. Though the originat mask absorber patlern is fabricaled in gold less than Ioof $A$ thick. the x-rity-lithography replication produces very high aspect-ratio resist profiles which serve as a "mold" during a subsequent electroplating step that produces :he linil thick gold structure.

Although in principle any ol the methods menlioned above for fitbricating sone plates in thin gold soruld he used to form the $x$-rity mask absorber pattern, scanning-clectron-heim lithegraphy has several advantages:

- Il is possible to extent the SI:BI. technicue to line widths of 1000 A or less; at the present time, wher methods have not demonstrated comparahle resolution.

- Changes in rone plile parameters, such as focal length or minimum fone width, are simply entered from a keyhoard on the SEBl compuler control; holographic coll plate generation requires timc-consuming modificition of the opticel selup.

- Frec-standing conc plates require support structures: using SEBI. the support strueture is writfen at the same time as the rome plate pattern. Com. . x support structures are eitsily generated and modified through software changes.

- SEBL. exposure can casily be varied across the cone plate to compensilte for cooperative exposure effects due 10 changes in periodicity; this atlows good line width control and a uniform resist profile across the sone plate.

- Spherical aheration can he casily avoided. With holographic lithography, the rone plate is used alt a different wavelength than the exposure wavelength, resulting in spherical aberration unless special correcting optics are used during exposure of the zone plates. ${ }^{62}$

- As described helow, it is possible to ensure that the electron beam sean raster is free of distortion which would impair the optical performance of the zone plates.

In order to produce accurate zone plate patterns, we require the distortion of the electron beam scan raster to be less than the minimum zone width
$(3160 A)$ over the tone plate dianeter $(0.632 \mathrm{~mm})$ : about 5 pars in 10000 . Ling a Moire lechnique. ${ }^{6,4}$ we have reduced sean distortion to -2.5 parls in $1000 \%$. The FTEC Corp model !EBES-D Scanning Filectron Beam Lithography system has a distortion correction module which allows small correction terms to be added to the deflection signats. to compensate for predominant irregularities such as pineushion and traperoidal distor(iion. By abserving on the display (RT a Moiré patlern produced by ritstering the electron beim over a distartion-free gold reference grating, any distortion in the sciln ritster is easily visualised and can be corrected using the distortion correction controls. Our reference grating were holographically generilted, and consisted of $50100(-A$-period etched gold lines on a silicon substrate. The grating lines could be orienled parallel to either the $X$ or $Y$ deflection axis. lo check distortion in hoth axes. Wo also used grids of geld dots, formed by holographic douhle-exposure of orlhogonally oriented grating lines.

Figure 5-59(a) shows a Moiré paltern indicating that there is some pincushion distorlion in the scian raster. In fig. 5-59(h), the pincushion distortion has been corrected and only straight, tequally spaced fringes are visible: the lringes appear hecause the spacing between the raster lines does not match the grating period exiclly. In Figs. 5-59(e) and (d), the scan-line spacing has heen adjusted to match the grating period exactly: hy transiating the scan raster one-half period $(2500 A)$ with respect to the reference grating, the entire pattern can he ma de dark (c) or bright (d) as the sean raster faitis exactly between or on the gold lines, respectively (a similar result is ohlained by scanning with an orthogonally oriented ratster/grating combination). These scans, of a field $1 \mathrm{~mm}$ square, indicate that the SEBL machine has a distortion of less than $2500 A$ over $1 \mathrm{~mm}$, or $<2.5$ parts in 10000 . Raster distortion could also be reduced to approximately $2500 A$ over a $1-1 \mathrm{~nm}$-square field.

The Moiré distortion correction technique brings the $X$ and $Y$ deflection axes into precise registration with the lines of the $X$ - and $Y$-oriented reference gratings. For our zone plates, we require that the scan axes be orthogonal to within $7 \mathrm{~min}$ of arc and that the $X$ and $Y$ axis gains rnatch to 1 part in 1000 . The orthogonality of our $X$ and $Y$ reference gratings is within $1^{\circ}$, but is nc. : : recisely controlled. Slight adjustments were performed to reference the 


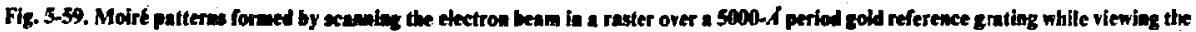

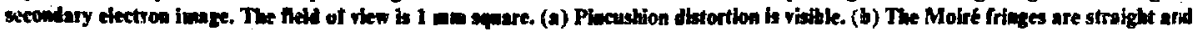

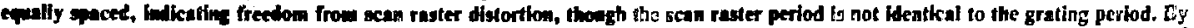

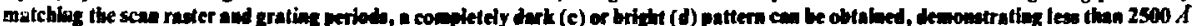
of distortion over the entire $1-\mathrm{en}^{2}$ nith.
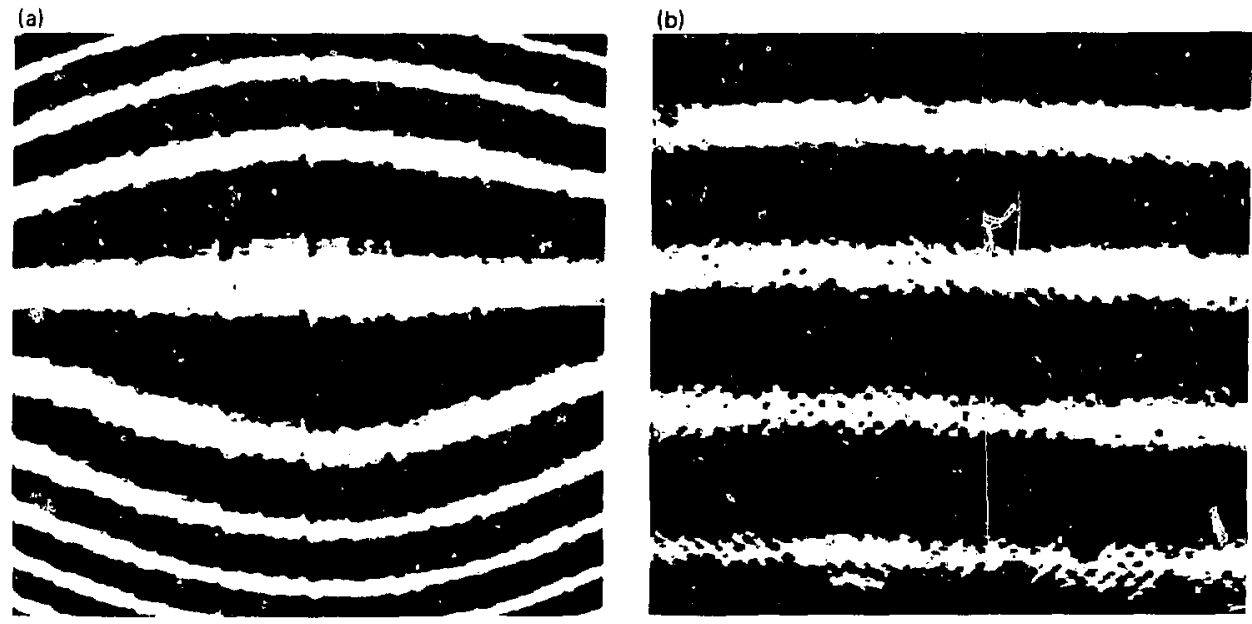

(c)

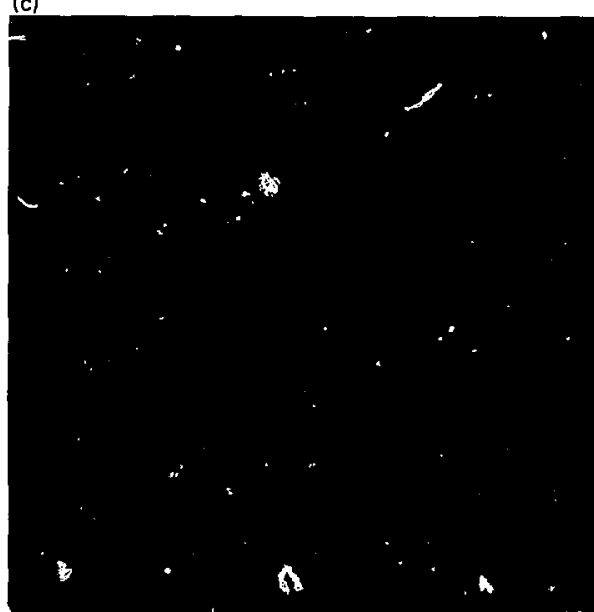

(d)

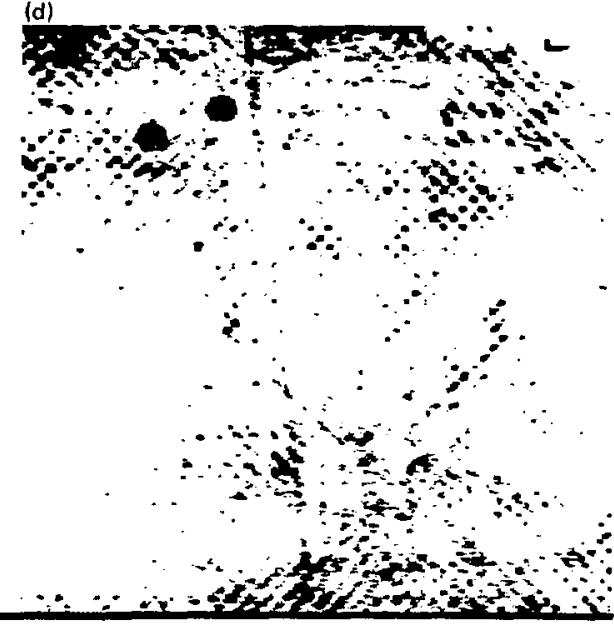

scan axes to the $\mathrm{X}$ and $\mathrm{Y}$ axes of our laser interferometer which medsures $X$ and $Y$ sample stage motion: the interferometer axes are orthogonal to $<1$ min of arc.

Beam position drift during writing is another potential source of pattern distortion. Large zone plates with minimum zone widths of $3000 A$ take about 1 hour to write, and may be affected slightly by drift. Beam drift in our system is typically less than $5000 A$ per hour, as measured by observing shifts in Moire fringe patterns.

Our zone plate patterns were written in a layer of Polymethylmethacralate (PMMA) 1500 to 
Fig, 5-60. A 4000- $A$-period grating exponed in PMMA wsing scanaig-electron-benn lithography (SEBL).

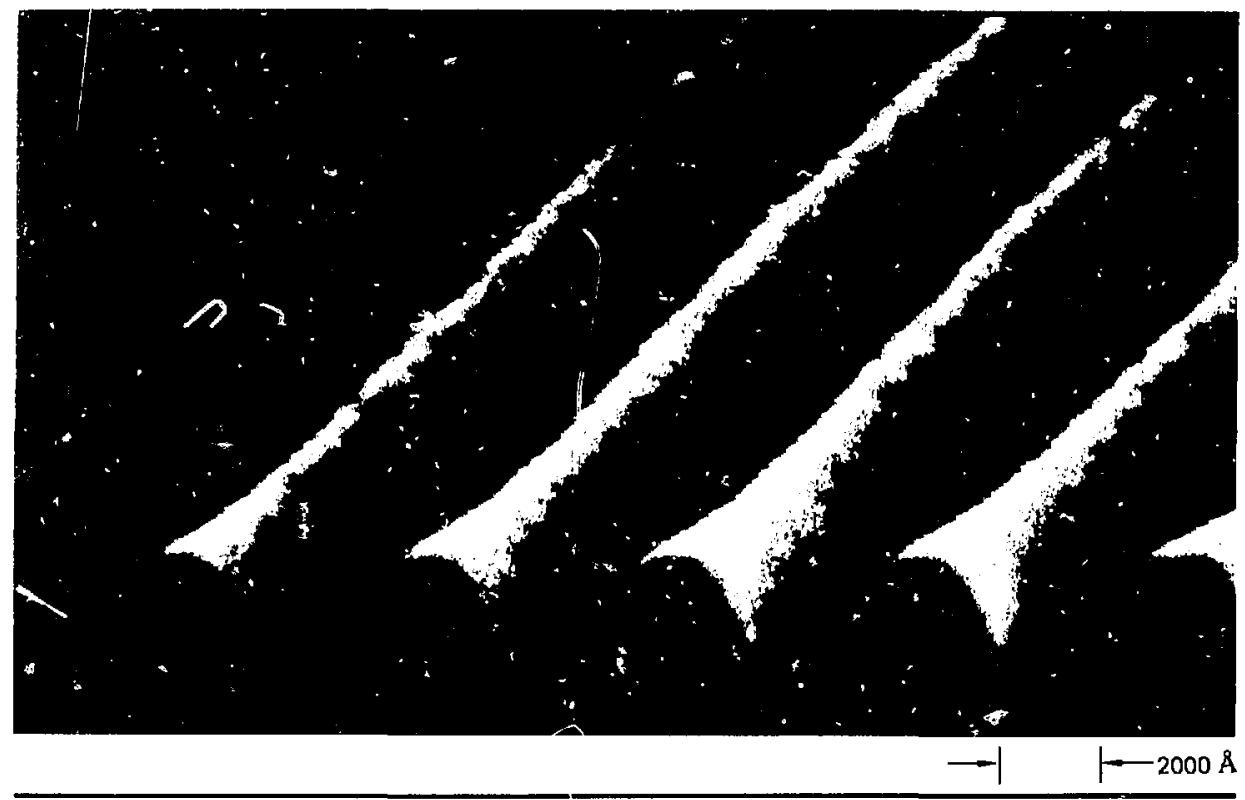

$3000, A$ thick, over a 9000-A-thick layer of polsimide on a $76-\mathrm{mm}$-diam silicon suhstrate. The molyimide linger serves as the support memhrane of the X-ray matsk: we use the 76-mm-dian silicon suh'ratle hecatuse of its convenience in our SEBL $\therefore$ stem. We operated the SEBL system with a 1. hesten filament, and used a beam current of 0.2 11) For all writing to ensure a beam diameter of - $1000 \mathrm{~A}$. Patterns were written in 950000 molecular-weight PMMA at a dose of $1.8 \times 10^{-4}$ $C, \mathrm{~cm}^{2}$ : development took one minute in a solution of 2 parts methyl isobutyle ketone (MIBK) to 3 parts isopropanol. The resist profile in Fig. 5-60 shows $2000-A$-wide lines with vertical sidewalls, produced by SEBL: lines as narrow as $1000 A$ have also been exposed in 3000- $A$-thick PMMA with vertieal sidewalls suiv tble for liftoff.

The zone plate pattern is written by drawing concentric circles with the system's minicomputer, functioning as a point-plotting circle generator. Since the circles are generated on-line, the entire zone plate (including the struts) can be represented algorithmically, providing data compaction and allowing exposure to he varied radially to improve line width control. A sone plate $0.63 \mathrm{~mm}$ in diameter is composed of ahout $3 \times 10^{7}$ discrete points, and takes ahout 1 hour to write.

After the PMMA has heen developed, a gold ahsorber paltern $500 A$ thick is formed on the polyimide layer, using standard liftoff techniques. Figure 5-61 shows scanning-electron micrographs of some gold ahsorber patterns: note [Fig. 5-61(d)] that we have fahricated line widths as small as $1000 \mathrm{~A}$.

The polyimide layer with the absorber pattern is then transformed into a polyimide-membrane $x$ ray mask. We used a procedure similar to that used with glass substrates ${ }^{65}$ :

- The entire top side and most of the underside of the silicon wafer, except for about a $30-\mathrm{mm}$ diam region under the zone plate patterns, is protected while the wafer is immersed in a solution of $4 \%$ concentrated nitric acid, $96 \%$ concentrated HF. This etches a hole through the silicon to form a polyimide membrane supported by the surrounding unetched silicon. 
Fig- 5-61. Scaming-electron micrographs (SEMs) of zone piate gold absorber patterns on an $x$-ray mask. The pattems were written by SEBL, followed by a liftoff of $500 A$ of gold. Micrographs $(k)$ and (b) show the inter and outer zones of a zone plate with a minimum zone width of $2000 A$; (c) and (d) show sections of a zone plate with a minimum zone width of $1000 \mathrm{~A}$.

(a)

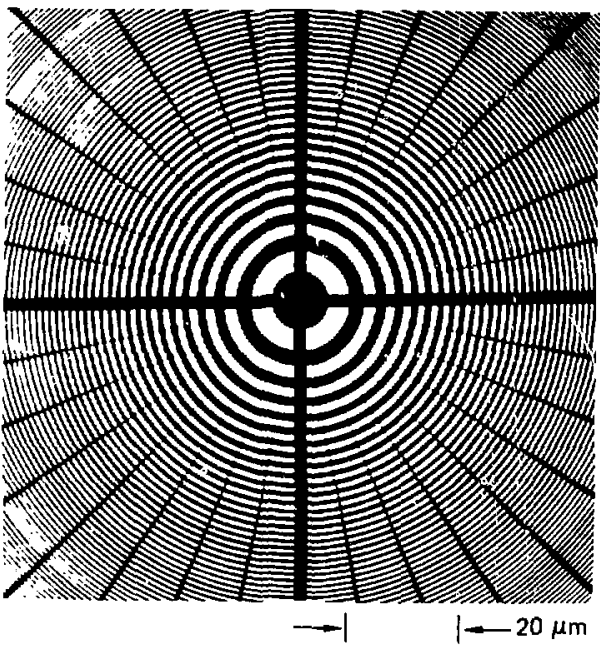

(c)

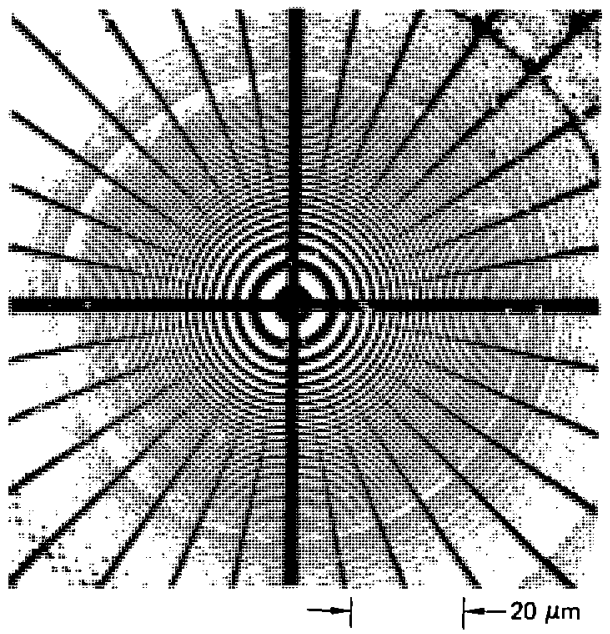

- A Mat plastic ring is then epoxy-bonded to the polyimide on the absorber pattern side.

- The polyimide membrane surrounding the plastic ring is cut away, leaving a polyimidemembrane mask on a plastic ring. (b)

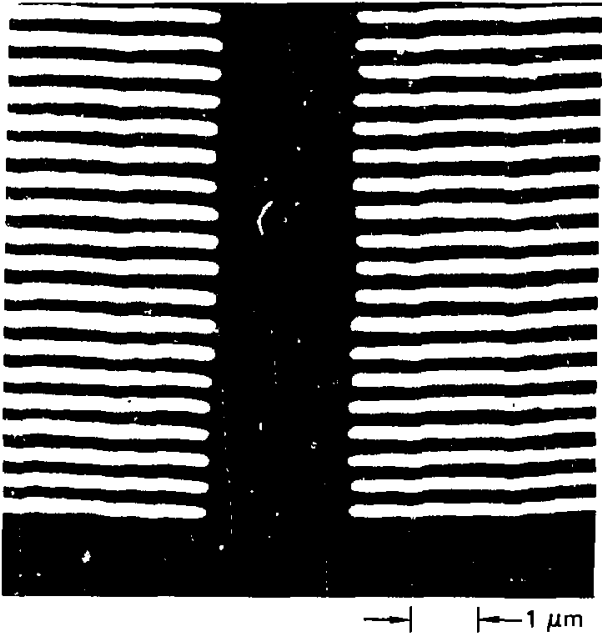

(d)

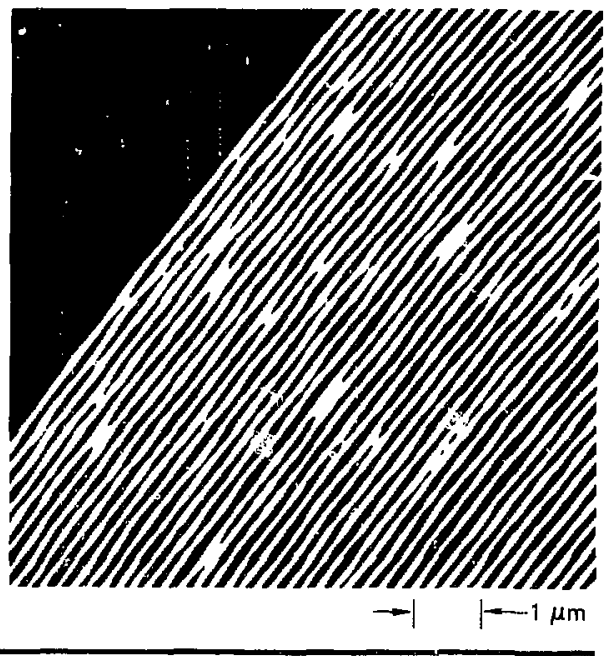

- A $400-\not$-thick layer of aluminum is then cvaporated onto the polyimide on the ahsorber side. This aluminum layer serves as an electrode, to hold 
Fig. 5-62. Gold lines electropłated between very ligh aspect-ratio PMMA Iimes exposed by x-ray litwography. The wight beading of the tops of the PMMA lines occurred during SEM observation.

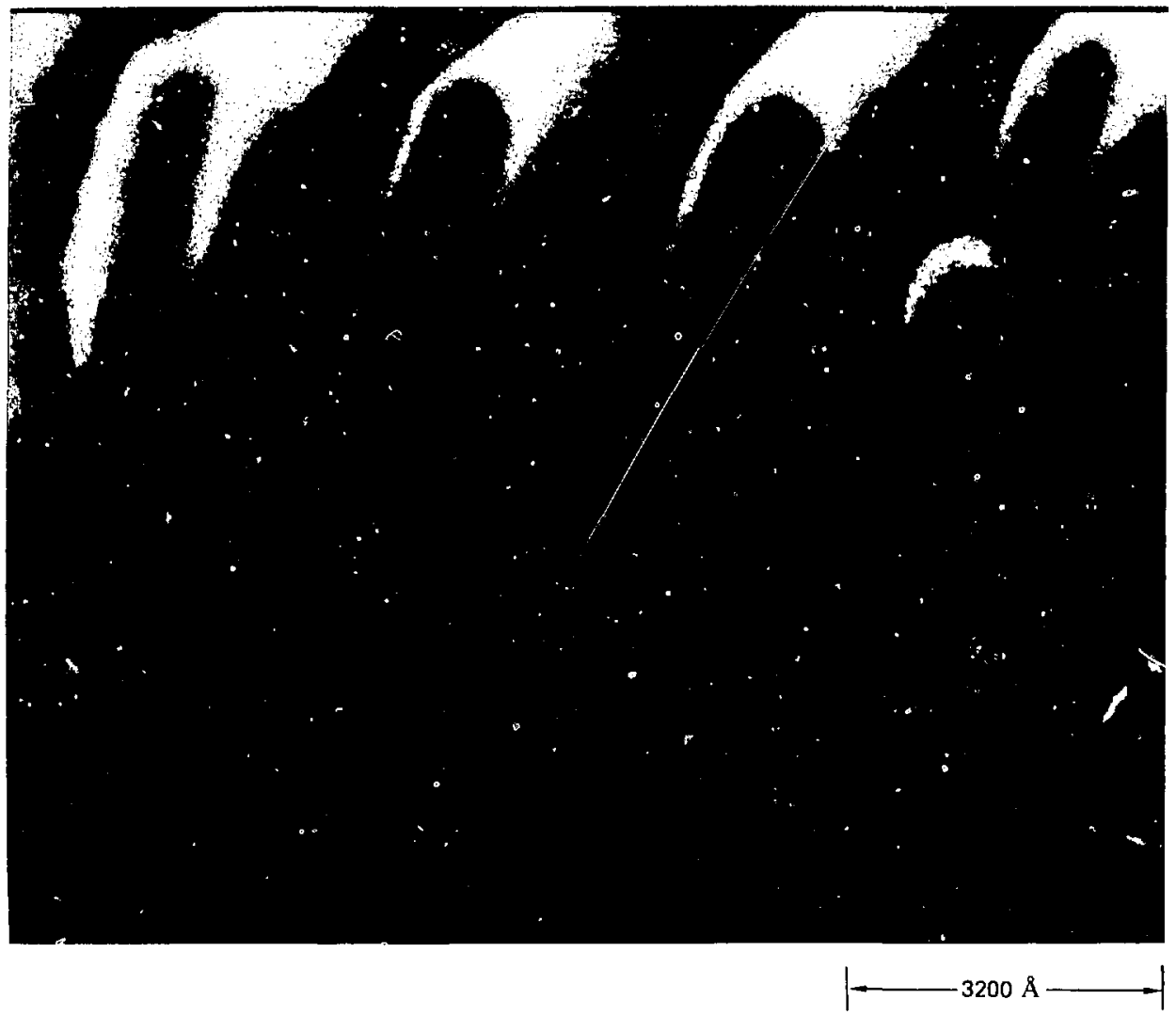

the matsk electrostatically in intimate contact with the substrate during $x$-ray exposure: the polyimide membrane serves as a dielectric.

Using this $x$-rity mask, the zone plate pattern is exposed onto $225-\mu \mathrm{m}$-thick Corning 0211 glass substrates coaled with. in order, $50 A$ of chromium, $50 A$ of gold, $2 \mu \mathrm{m}$ of PMMA, and $100 \AA$ of chromium. The chromium on top of the PMMA serves as the second electrode for electrostatic holddown of the $x$-ray mask; the chromium is etched away before development of the PMMA. The thin gold under the PMMA serves as a plating base. We used the carbon $K$ wavelength $(4.5 \mathrm{~nm})$ to expose the resist: is 7 -h exposure was required at it $60-\mathrm{mm}$ source-to-substrate distance. The $\mathrm{C}_{\mathrm{K}} \times$ raly was used in order to obtain high contrast with our thin $(500 A)$ gold absorber patterns, and to minimize problems with photoelectrons generated by $x$-ray absorption at the plating base. With harder $x$ rays and a higher bremsstrahlung background, the partial exposure produced by these photoelectrons results (during development) in rapid undercutling of resist profiles at the plating base surface, causing adhesion loss for small structures. All $x$-ray exposures were developed in 2:3 MIBK:isopropanol, with typical development times of 5 to $10 \mathrm{~min}$.

After development, patterned slots extending down to the plating base are present in the resist. 


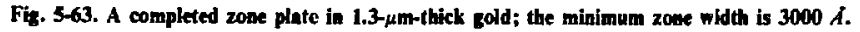

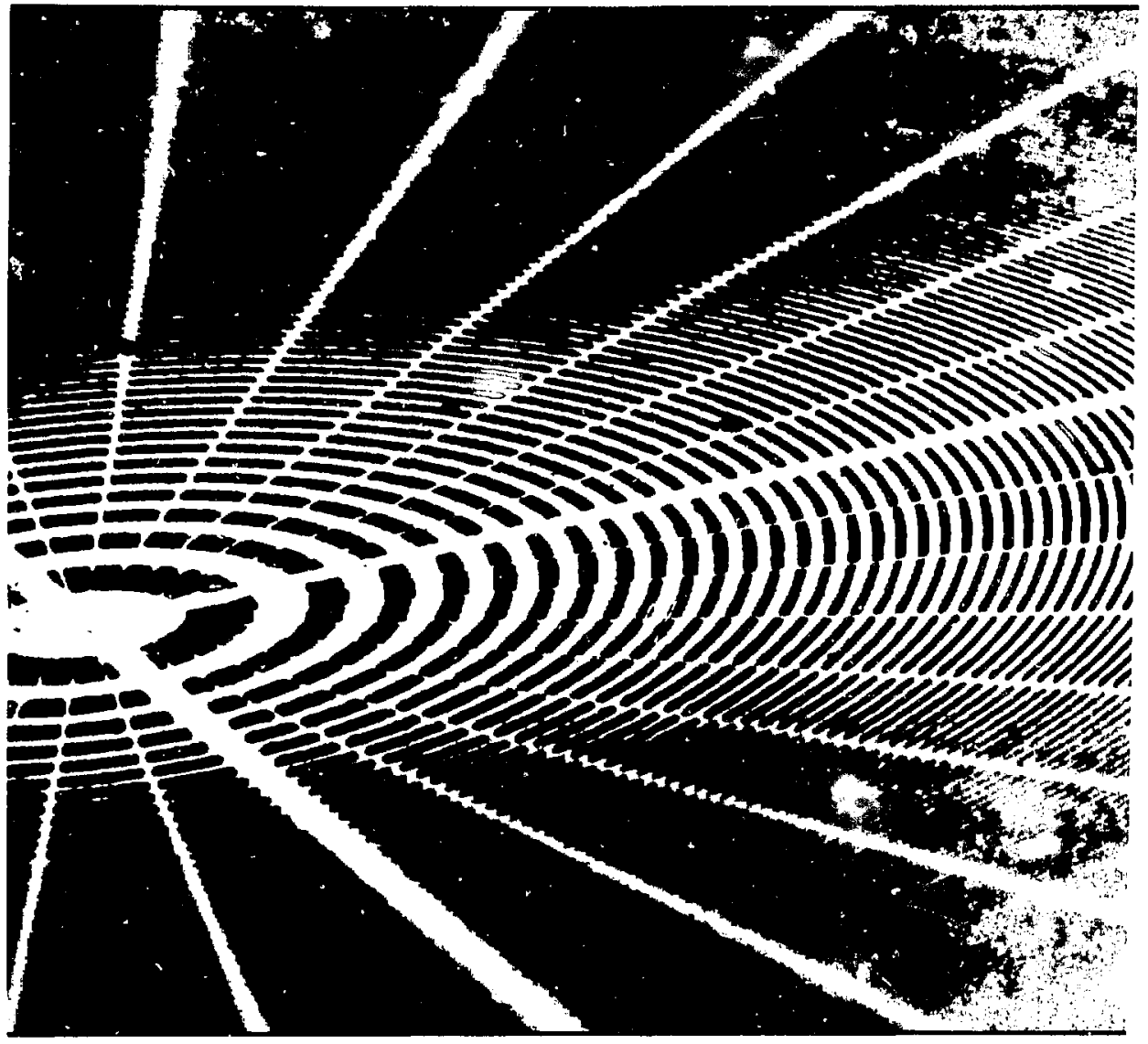

After a 10-s exposure 10 a $3 \%$ oxygen plisma to remove a thin resist sium which covers the plating hase, gold is electroplated to a height of $1.3 \mu \mathrm{m}$, using BDT-510 plating solution maintained at $40 \mathrm{~K}$ with a plating current of $1 \mathrm{~mA} / \mathrm{cm}^{2}$ or less. Using this plating solution. we have been able to produce bright gold films with no graininess obserwable under the seanning electron microscope. Our plated films are under considerable conapressive stress, which can lead to deformation of the completed free-standing zone plates. Figure 5-62 shows 1600$A$-wide gold lines plated to a height of $7200 A$ using a $3200-A$-period grating exposed in PMMA as the "mold" for the plating.

After electroplating, the PMMA is dissolied away, and a copper support tube $1.5 \mathrm{~mm}$ bore in

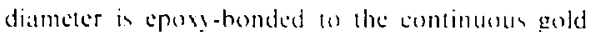
film surrounding the ante plate. The glas substrale is then etched ats.1! in concentrated HF lo produce a lree-standing gold some plate supported an a copper tube fin some cases, the thin chromium and gold of the plating hase, which have litte elfect on the rone plate pertormanee, were also remoned by chemical etchingl. Figure $5-6,3$ shom a free-

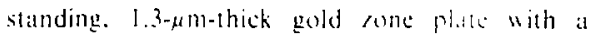
minimum $/$ onc width of 3000 .

Testing. Preliminary $x-r a$ ! cesting of these rone plate lensing elements hals alreads begran. Figure 
Fig. 5-64. Resolution tests of a Fresnel zone plate $x$-ray lersing element are conducted on a rigid optical bench. The lensing element being tested has a minimum line width of $3200 \mathrm{~A}$, a gold thickness of $1.5-\mu \mathrm{m}$, and a $0.632-\mathrm{mm}$ diameter. It is used to image a free-standing crude fresnel zone plate with a 2.5-jm minimum zone width backlit by an aluminum Ka $\mathrm{x}$-ray source. The radial strut patierus on both the zone plate lens and the zone plate test pattern are used to support the free-standing gold microstructures.

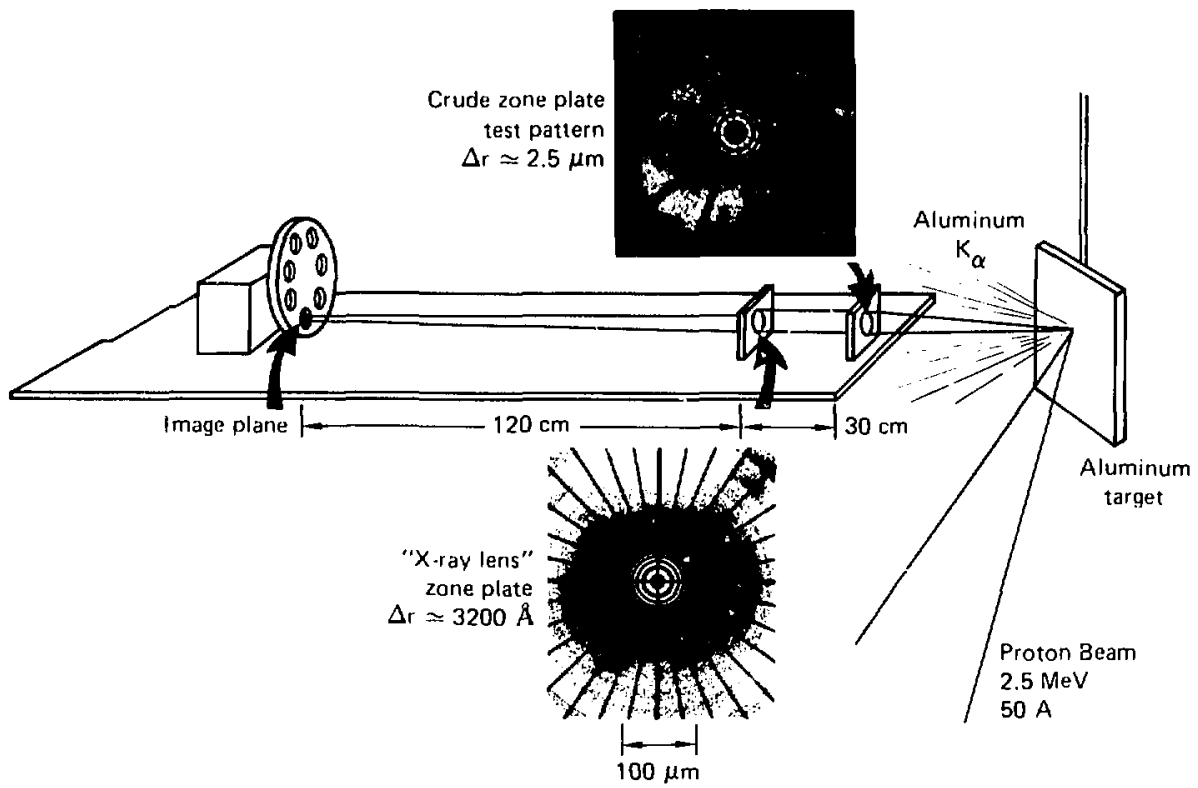

$5-64$ illustrates the test selup in which the rone plate "lens" is used as the optical component of a 4 power mieroseope. In this experiment the lens is used lo image a $1-\mathrm{mm}$-diam crude $(2.5-\mu \mathrm{m}$ minimum line width) Fresnel rone plate used as a test mask. The rone plate test mask acts as an ideal resolution pattern since the line widths valy predietably over a wide range. The test pattern is backlit with atluminum $\mathrm{K}_{n}$ radialion at $i=8.34 \mathrm{~A}$. produced by irradiating a water-cooled aluminum target with a $2.5-\mathrm{MeV} 50$ - $\mu$ anp proton beam from a Van de Grafl generator. The x-ray images are recorded on single-sided type $M$ x-ray film. A representative $x$-rity image of the $2.5-\mu$ m-line-width test pattern is shown in Fig. 5-65: note the resolution of the outermost zones of the test pattern. This is more clearly illustrated in the data of Fig. 5-66, in which the $x$-ray image has been scanned by a PDS microdensitometer and a plot made of the radial density distribution over the outermost 20 dark comes (from " $A$ " to " $B$ " in the inzige). The individual fones are clearly resolved, while also apparent are modulations in the image densily level produced by nonuniformity of the backlighting source and spattial shot noise in the image. Additional simple tests of the rone-plate-lens field of view and depth of focus (in the image plane) were made by translating the test mask off the optical axis $( \pm 1 \mathrm{~mm}$ ) and by varying the image plane position $( \pm 20 \mathrm{~mm})$. As expected, these variations had no affect on the ability of the lens to resolve the 2.5 . $\mu \mathrm{m}$ lines of the test pattern.

Additional Development. Also included in this $x$-ray lens development program are the labrication and testing of off axis micro-Fresnel zone plates intended for use as imaging spectrometers for 
polychromatic $x$-ray sources. The imaging characteristics of an off-axis Fresnel zone plate are illustrated in Fig. 5-67. The off-axis zone plate is simply a circular, off-axis segment of a circularly symmetric (on-axis) Fresnel sone plate. The off-ixis tone plate has the same focal length as its "parent" on-ixis one plate $(f=r i / \lambda)$ and similar chromatic aberrations. Due to its off-axis geometry, however. the olf-ilsis cone plate holds the possibility of spattially separating chromatically distinet emissions from the sabe source, therehy providing twodimensional, ehromatically distinet, multiple imagen of a polychromatic source (fig. 5-67).

In order for an imiging spectrometer employing an ofl-ixis one plate as its opticit slement to stceessfully separate spectrally distinet images from a given source. certain design criterial must be met for both the source and the instrument. Using simple raty-tracing cialeulations, we arrive at the following qualitative criteria:

- Spectral lines $\left(\lambda_{1}, \lambda_{2}, \ldots\right)$ must be well sepiralled.

- Source sire (in the offset direction) must not be too larget.

- Zone-plate offsel distance must he sufficiently liarge $\left(r_{0}<\lambda_{0}\right)$.

- Minimum line widh must be as small an possible.

Representaltive parameters, for an imaging spectrometer currently being fabricated for use with lasser fusion targets, are listed in Table 5-7.

A practical consideration for the imaging spectrometer is the apparent need for microscopic recording medial w capture the separated images without blocking shorter-wavelength images to be recorded dounstream (sce Fig. 5-67). This problem can be obvialed and macroscopic recording media used if the imaging planes for the long-wavelength images are appropriattely tilted hack toward the borizontat. The maximum angle of tilt loward the horiontal is linited by the depth of focus (in the

Table 5-7. Qualitative criteria for an off-axis zone plate imaging spectrometer.

\begin{tabular}{ll}
\hline Maximum zone width & $\Delta_{\mathrm{r}}=1600 \AA$ \\
Off-axis zone plate radius & $\mathrm{r}_{0}=0.375 \mathrm{~mm}$ \\
Offset distance & $\Delta_{0}=0.5 \mathrm{~mm}$ \\
Speciral lines & $\lambda_{1} \simeq 4 \AA$ \\
Maximum source size for & $\lambda_{2} \simeq 6.9 \AA$ \\
separable images & $\mathrm{s} \simeq 500 \mu \mathrm{m}$ \\
\hline
\end{tabular}

Fig. 5-65. Initial resolution tests of the rone plate lens were conducted using a test pattern (a) with a mininaum line width of $25 \mu \mathrm{m}$, even though the anticipated resolution of the instrument is roughy $0.5 \mu \mathrm{m}$. The enlargement (c) of the "lens" image in (b) shows the 2.5- $\mu$ m outermost lines of the test poitern are clearly resolved.

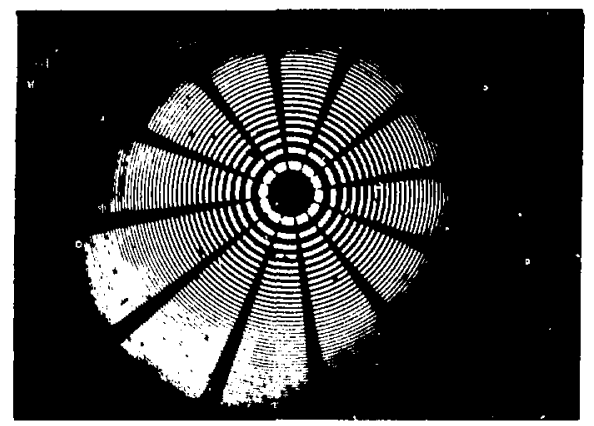

Test pattern (2.5- $\mu \mathrm{m}$ lines)

(b)
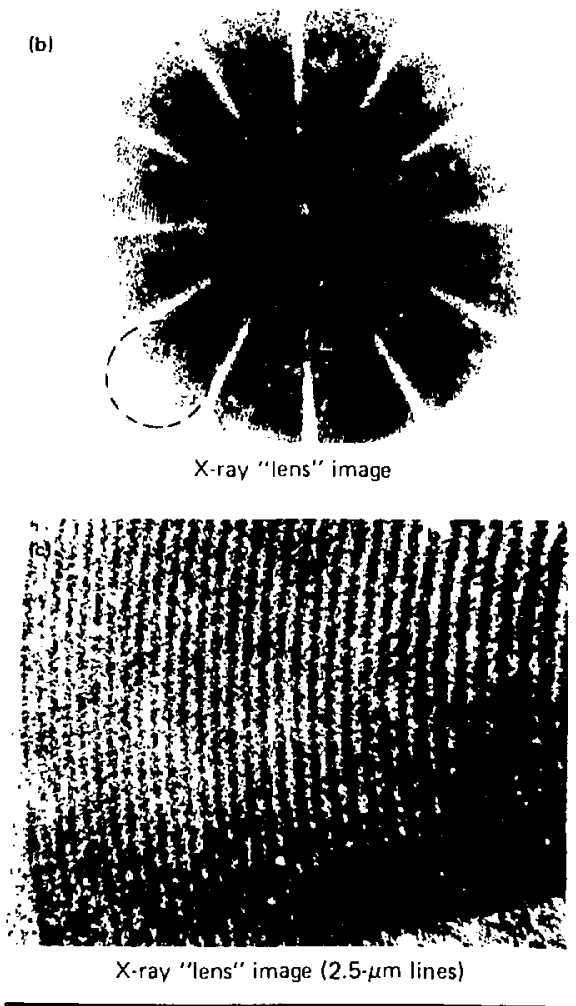


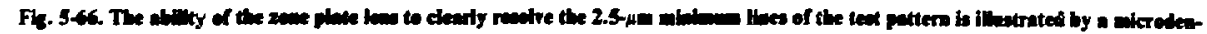

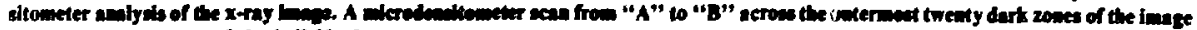

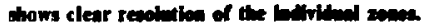
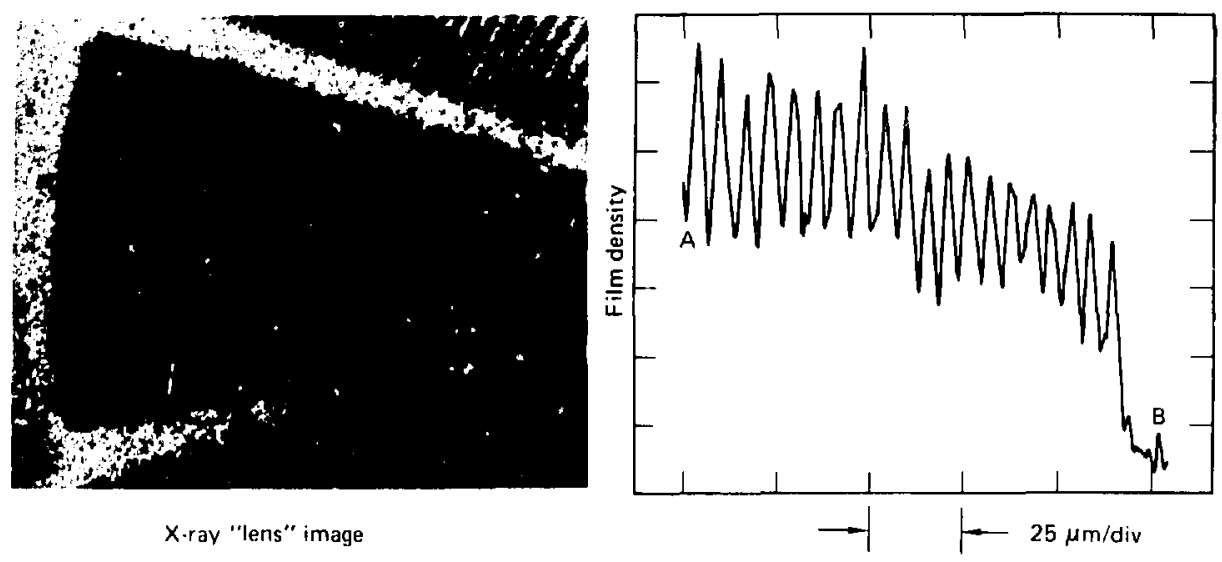

Fig. 5-67. An of-axis segment of a Frenel zone plate can be used as an imaging spectrometer to spatially separate distiact spectral emissiuns from palychronatic source.

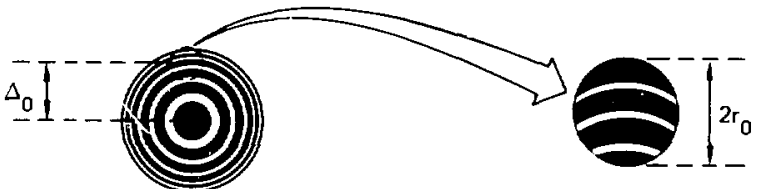

Parent zone

Off-axis

plate

zone plate

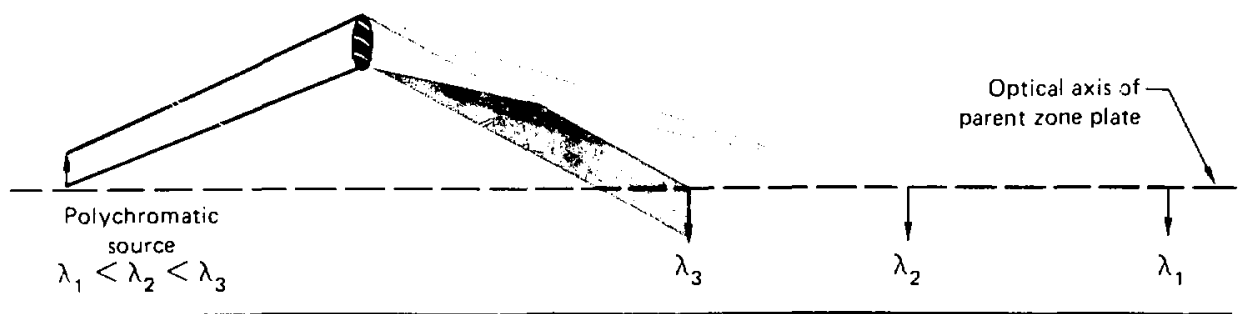

:mage plane) and can be determined from simple arlulations. For the example in Table 5-7, the maxinum tilt angle is roughly $81.5^{\circ}$, yielding a recording-medium width $\simeq 2.5 \mathrm{~mm}$ for the image at $\lambda_{2}=6.9 \mathrm{~A}$.

Off-axis zone plates used as imaging spectrometers will very likely find a wide range of application in the laser fusion program (and otber 
Fields as well). In the particular examples cited above, the spectral lines chosen are the argon and silicon $\mathbf{K}_{n}$ emissions from the multiply-1onized laser fusion target. Argon is added as a tracer element in the D-T fuel, and silicun is at natural constituent of the glass microsphere. The imaging spectrometer. therefore. Will provide separalte and distinct images of the glass pusher and the enclosed fuel during the laser-driven target implosion.

\section{Authors: X. M. Ceglio, D. C. Shaver (MIT), D. C. Flanders (MIT), and H. I. Smith (MIT)}

\section{References}

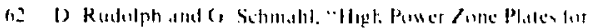

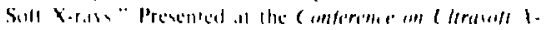

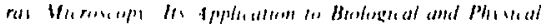

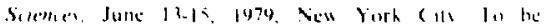

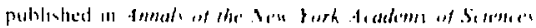

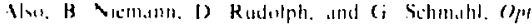
(imminar 12. 1601 11474)

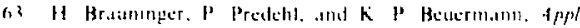
Opt 18. 3the (147)

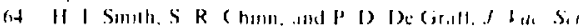
lacherl 12. 12t: 21975$)$

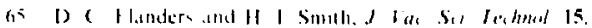
บyร $(197 x)$

\section{Management and Analysis of Experimental Data}

Laser experiments at LLL are designed to study particular aspects of liser-target interatctions and fusion larget physics. texperiments by pically have one or a leu printary diagnestics on which the experimenter expects to rely for his main measurements. Often several diagnostics are included in a "desirable but not essential" cettegor!: occassionally diagnostics are used on a target experiment purcly for diagnostic development purposes not necessarily directly related to the primary measurements being attempted. The food of data so produced must be intelligently managed so tha: important data are at:curately, completely, and promptly reduced. Less critical data must not consume the experimenter's time to the detriment of programmatic progress.

At the point of taking data. the operations group continuously moniturs the diagnostic instrumentation. If they judge that a diagnostic system deemed primary for a given study is inoperative, they delay the shot until the fault has been corrected. Such attention can only be expended on the most critical diagnostics; those judged merely desirable are simply dropped for the shot if they are questionable. These precautions reduce the chances that laser-target irradiations will be wasted.

Given a shot considered successful in any sense. the data produced take several standard forms:

- Rall dala from detectors exist as records in computer memory or on film that must be processed and densitometered or digitized.

- The configuration of the diagnostics on the target chamber. including their angular orientations, sensitivity settings, and the like, is preserved as a configuration dala base.

- Finally, the experimenter's notes on alignment and orientation of the target al recorded in a shot book provided far each target irradiation.

Within about two weeks of any target shot. results from the shot are distributed in several locations. The best summary is contained in the experimenter's shot book. By this time the responsible investigator will have accumulded and stored in the shot book most of the relevant information gleaned from the shot. These dallat are reduced using the characteristice of the parlicular diagnontice (stored in the configuration data basc), and whalever additional cillibration datal are required. Fach of several diagnosicians retain copies of the datal provided by the measurement systems for which thes are responshle. Digitied photographic data exist in memory in the L.LL. central computer facility: ratu electronce datat are stored, along with the configuration datil batse. in the memory of the Shiva compuler ystem. In some cases, all steps heyond the initial collection ol the rals data are acecomplished using L.I.I. V contral computer system.

At thi point. dilla collected in the shoi hooks are compared with preshut expectations, the dominant issues being adequate experimental controls, significant differencen belween datal sets, diagnostic cillihration and reliability. and the rest of the concerns which are the expermonter's stock in trade. Meanuhile, the datat lechniciam in the Data Management and Analyss Group archive the shot hooks in at central sault.

In former years. entire experimental sequences were grossly modified or aborted after the first target shots when results devided too wildly from 
predictions. As knowledge has grown and dhanostic systems have become more sophisticated. however. instances in which laser-plasma interaci, ..: espersments produce unintelligible results have 'amme rare. (ienerally, sequences of several tens of ". . . ire designed and executed more or less as ?manct. sets of shot books are produced. lateled ath the II Ince assigned to the experimental series.

Hher it is complete. an experimental sequence 1. 1 jorrted. when on the archival literature. Conlact "mike with pant work fatnd work at other l.thusulesen here and abruad These experimental repure legether wh the shot books. Lonstitute the ifesdil: abllable) promary record of the experimatil. and are hored together in the same areat. (neler is. lew addutions or corrections are made lo He sher benk atfer the experimental report is writeetl (ampleted reports and shot books are used by target deseners to correct and or atugnent simulit-

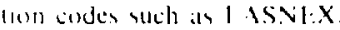

Summarke of each hot are written and stored In the shos books. During the coming year we will whithlish and maintain a summary data base on the Dalta Vanalgement and Analysis (iroup VAX computer wstem such a data base will contain results from . Ill eur caperiments and will permit statistical trend amalsus Partial datat bases have been malnlained in past icars but these. while helpful, were tume-consumme. alten incomplete. and never entired ratisfactors "Ith the advent of better data reduction endes. mure of the shor dalla ean be atulomallealy entered into the data base. This develupment will greatly facilitate the discovery of parametric fits. scaling relations. and weak trends. as well as the reevaluation of old datat in the light of new results.

Author: k. R. Vanes

\section{Diagnostic Configuration Data Base}

There :Ire 220 openings, or ports, in the Shiva target chamber. Twenty of these ports are used as windows for the twenty laser beams, one is used for the target posiluner. and the remainder are available for target diagnostic instruments. Because of this large number of ports. each of which usually contains a diagnostic device, we have developed a configuration data base. TDB. to keep track of them.

The configuration data base has three different purposes:

- from the operations point of view. TDB permits easy identification. modification. or retrieval of different experiments or detectors in the target chamber.

- It allows the data analysis programs to deltermine data paths and the computer location ahere ras larget diagnostic data is stored

- IDls provides a record of the position and characteristles of the diagnostic instruments during at shot: this then becomes a part of the arehived ditlit.

IDR contains all the information neessary 10 describe the following items:

- Port position. dimensions. and function.

- I:xperiment use. position. dimensions. detector and electronic specifications and data paths

- I egical and physical interaction between several experiments placed at several locations.

One of the man characterstics of this data base is its llexibility in terms of adding. changing. or deleting instrunents. Because of its special sharateristics, we will give a briet description of TDB.

Ti) $B$ is a commented lile structure (like a texu) in which every entry (line) starts with an t.nglish abbreviation that allows immediate identification of the numbers. keywords or comments that follow. I very line in the TDB file is a logical record. Words. comnents or numbers are delinited by blank spaces or tabs. and maly be located anywhere in a line A ny blank or tab is assumed to be a word terminator: any string of characters started and terminated by blanks or tabs is assumed to be a word. Any line can have up to a maximum of 120 characiers and or 15 nords: words can be up 108 characters long.

The first uords on a line are usually reserved for keywords or flags, with the rest reserved for identifiers or numeric values. There can be any number of entries between $k$ sywords. Lines started with the exclamation symbol (!) are assumed to contain comments, and ate ignored by the retrieval subroutines.

There are two basic types of keywords: descriptives and globals. Descriptives indicate the characteristic. its ID number. and amplifier gain or position in the target chamber. Descriptives usually 
refer to information co tained in one line. such as DI:THC VLMBER I.C 27 148, which translates as "detector type I.C 27. unit number 148."

Global indrate that all entres that follow helong wo the sime global group. For csamples the global keywork "PORT" indicilte tht the rest ol the line, and all suheyuent entries untul an "F:OP" lend af portlentrs is encountered. contann inform: lan dircelly related lo that part.

liter the "PORI" entry there wan be a comment entry to descrihe the specific ust of the port. which mughe include an "I X.P. levperument entry In I XPentry indecites that all subsequent entres. until an "F(s) xp" and of experisments entry. dentribe the experinent named in the following word I nder the prexent lormatt. any number of eniris can be made to describe the experment in detall Ihe P(OR I entry mal contatin several expertments each expermem mat comtan sereral deles. tars

fable 5- - show at small lacesmile segmen: of II)B for one POR I entry. whteh is interpreted as follows 1 he port at angles thetal $=148^{\circ}$ and $\mathrm{ph}_{1}=$

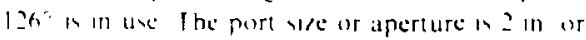
$5.18 \mathrm{~cm}$. and the outsde dimension to the neatrest obstructoon is $x$ in. or $20.32 \mathrm{~cm}$. The eaperiment or instrument $X R \backslash D O)$ currently in use is mounted on the port and belong to the $X 1$ (a-rat) logecal nodule: Dr Wang in the indirdual respomste for the experiment. Deltat $X=5.04 \mathrm{~cm}$. delta $Y=$ $50 \times \mathrm{cm}$. and radial distance $=(1.0)$ are the dimensions of the instrument inside the larget chamber The instrument XR $10(0)$ uses detector type I. PD whth serial nuniter 10.0 .

Since TDB has a text-like steucture, dit? Lext editor can be uned to make thanges. Queries or searches can be done by a specially developed subroutine named Pl'L.I. which will search TDB for any given port. experinent. or instrunient and return all the relesant information. For example. TDB and Pl 1.1. are used to produce larget chamher djagrams containing port and experiment usage. TDB and PLLL. can also be used to obtain target chamber statisties. such as the latest configuration at the time of $\mathrm{x}$ riting of this report:

- Number of used ports $=114$.

- Vumber of free purts $=73$.

- Number of blocked ports = 25 .

- Number of reserved ports $=8$.
Table 5-8. Facsimile of readout from Shiva configuration data buse TDB, for sumple PORT entry.

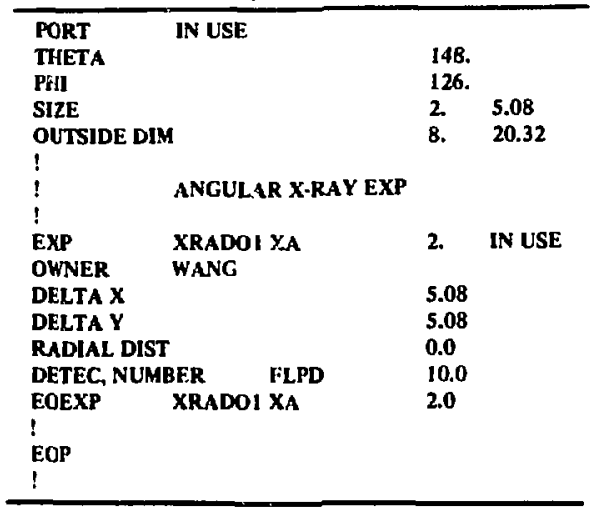

Suthor: J. F. I ter pays

Vajur ( ontributo: J. VI. Iuerbach

\section{Data tcquisiti in and Processing}

During the I st sear. ue have made substantlat

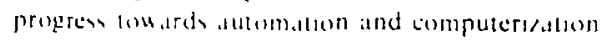
ol data from laser fuvon experments Our goal an 61 relese the cepormenter of ume-consumeng routine datat reductoon wh that more ume can be devoted to moterpelatton. It the hegennung of 1979 . almol all datal processng was done on detopus through manual data input since thall tame. He hase im. plemenled aummatied procesang of vereral

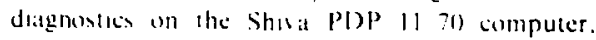
which reyures much less user interilctun than $\mathrm{Oc}_{-}$ lopus llor a comparison of data processing steps on

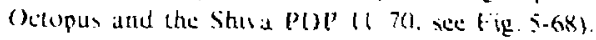
He are also alcquiring a new 1 ix computer to proside more elficient lusun esperiment data processing and analysis.

Data ar: currenly recorded electronically (by digitiration of analog dalat and on film. Fevenually. (CD array cimeras mas replace nearly all of the film recording. Hectronic datia are transmitted via a ( A.MAC serial highway and stored on a floppy disk by the TAC Al computer system: T ICAI. standing for Target Acyuisition. Control. and Instrumentation. has been describca in previous annual repors. fin The TIC I! zomputers at Shiva and 
Fig. 5-68. Comparieon of functocai diagranes of data procesdas steps for the Octopus aod Shiva PDP 11/70 computer systems.

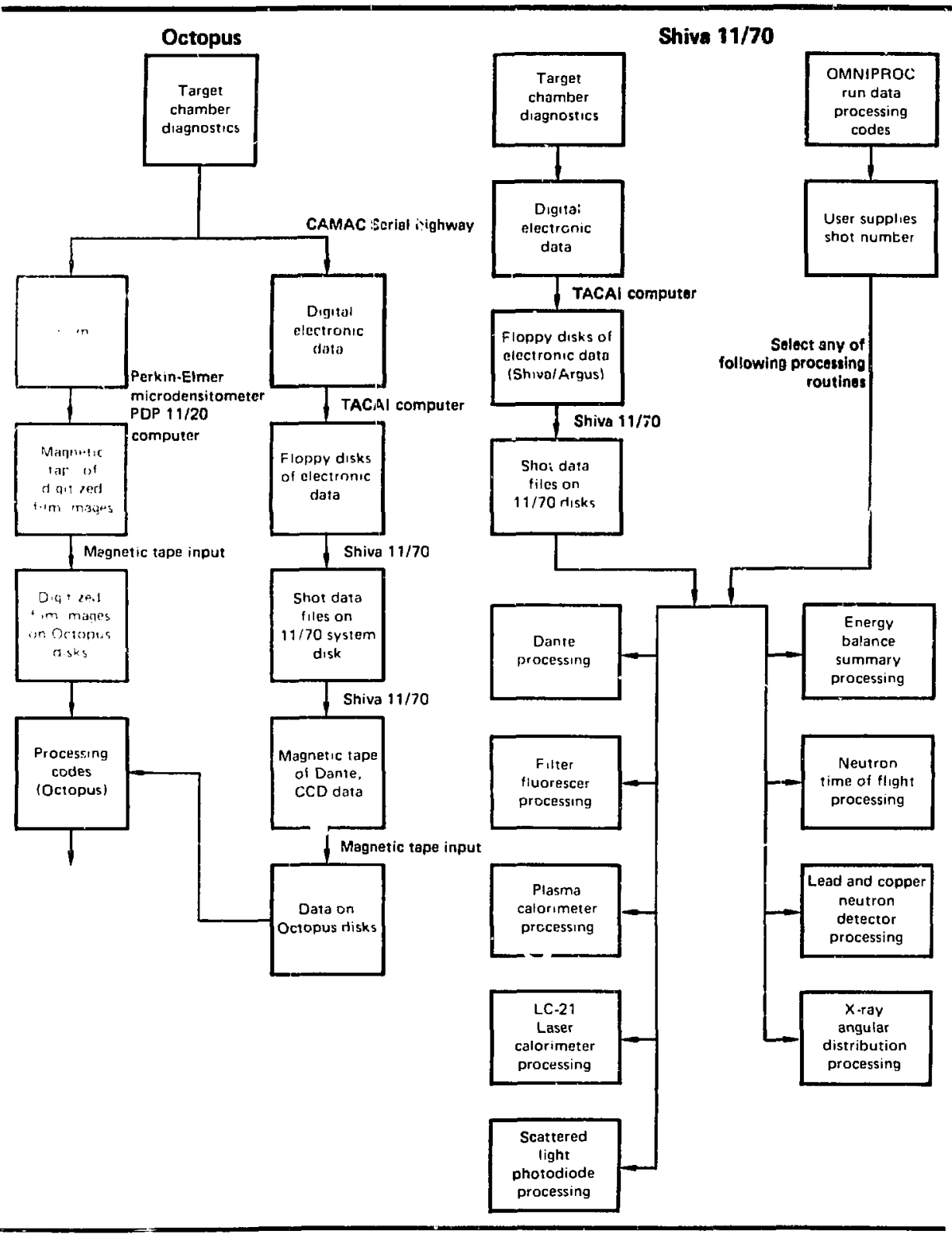


Argus write experimental data on floppy disks. which are ther read onto the Shiva PDP 11/70 system disks for iurther processing. At Shiva we aiso have the capability for transmitting datid directly from the TAC Al computer to the $11,70 \mathrm{com}$ puter. using Digital Equipment Corp.'s intercomputer communications software. DECNET.

Fxamples of electronic data for which final processing is done on the Octopus System include data in the shot file for the Dante spectrometer. and data from our soft $x$-ray streak camera equipped with a (CD arraly readout. These data are transfered daily from the 1170 to Octopus on magnetic lape reels hand-carried belween computer centers.

f-ilm intages are converted into digitied data arrays using a Perkin Filmer Microdensitometer controlled by a Digital Fouipment Corp. PDP II 21 computer. Diaia are written on magnetic tape. transported to the Octopus tape readers. and stored on the system disks: processing is then accontplished through user terminals. (Details of film data processing are given in "Film Image Processing" later in this section.)

Processing of fusion experiments data on the Shiva PDP |I 70 is a highily automated sequence. due (o) the nature of the operating system and the lack of need for the manual data transfers required for film processing. Various levels of automated rocessing have been implemented for the following diagnostics:

- Dante low-energy $x$-ray spectrometer.

- Filter fluorescer high-enersy x-ray spectrometer.

- Plasma calorimeters

- 1. (-21 laser calorimeters for scattered light.

- Scattered-light photodiodes.

- Veutron time-of-flight detector

- Lead and copper activation neutron yield detectors.

- X-ray angular distribution detectors.

in following subsections we will describe the processing software for the first five of these diagnostics. Processing for the last three of these five diagnostics are incorporated with the processing of laser system calorimetry to give an energy balance, also described in a following section.

To allow automated processing, all processing codes utilize files on the PDP $11 / 70$ system disk for setup and calibration parameters. These files are up- dated daily or as required to reflect the current diagnostic configuration. In the sections that follow. these files are referred to with their standard Digital Fquipment Corp. nomenclature. This is the form NAMFXXX. where NAME can be up to al nine-tetter iame and $X X X$ is a three-letter type: thus SETLP.PAR is a parameter (P.IR) file named SrTLP.

Auconitted processing is aceomplished by use of command files which contain groups of processing commands. In the sections which follow, these command liles have the lypical label NAMF.CMD. where (MII) is the abbreviation for command. The processing lor all ditgnostics is controlled through one command lile called OMNIPROC CMD. I pon execution. this code lists the various diagnostics that can be processed, asks the user to choose the diagrostic(s) of interest. and alsks for an (eight-digil) shoi number so the appropriate rak data can be alcessed

Authoi: J. M. Iuerbach

Ilajor (ontributor: .J. T. Ozasa

\section{Reference}

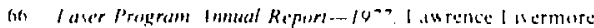

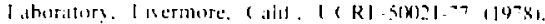
एр

\section{GETSD: A General Shot Data Retrieval Routine}

Digital data from the Argus and Shiva diagnostic data acquisition systems are organized by CAMAC crate. CAMAC station, and a disk file containing all the shot data. For subsequent higherlavel analysis, processin ${ }_{z}^{\prime}$ programs must extract certain parts of the data .om the disk. To avoid duplication. with each experimenter/programmer writing 'is own routine for datal extraction. we have written a single versatile data extraction routine called (ifTSD (GFT the Shot Data).

implementation. (iF:TSD is implemented using ISHOT. a 9-4ord integer array containing the 8digit shot number (used to access the appropriate shot data file) and one additiona! word reserved for future expansions. Users then einploy ILOC. a 3 - 
word integer array, to specify the location parameters of the device producing the data of interest

The werall assignments for ILOC(I) are as follows

- $11 . O C(1)=0-99$ indicates target diagnimuss.

- $11.0(1)=100-199$ indicates laser diagnostics.

- II $9(1)=200-299$ indicates alignment.

- $I 1()(1)=300-399$ indicates power con(1:uning.

- $I I . O(1)=400-499$ indicates central conmoln.

Itse meaning of the elements in the ILOC array 1) dependunt on the diagnostic system containing I.c particular device. For larget diagnostic data produced by the Shiva or Argus TACAI systems. $110(1), 12)$, and (3) are the CAMAC crate, station. and unit numbers, respectively. For data produced by the laser diagnostics system at Shiva. Il.o(11) provides the following parameter assignments:

- $100=$ Beam energies.

- $101=$ Detector sensitivities.

- $102=$ Amplifier gain.

- $103=$ Amplifier offset.

- $104=$ Autozero setting.

For the laser diagnostics system at Shiva II.OC(2) is the coded number of the laser component coscillator. preamps, Pockels cells, rotators, calorumeter groups, etc.) from which the ILOC(1) data were recorded: each componeat :so carries a 4-character name for call by other GET SD routines. [ILOC(3) is ignored for laser diagnostic devices.]

The meaning of elements in MODNAM, an 8word integer array containing the ASCII name of the device producing the data of interest, is also system-dependent. For data produced by target diagnostic systems at Shiva and Argus, device names are CAMAC interface module names. Device names for, laser diagnostic data are the 4character component names used in ILOC(2). Users must also supply the abbreviations for the types of unalog-to-digital converter's on each diagnostic: examples are "TK 7912" for a Tektonix Model 7912 transient digitizer, and "PRCHIN" for a programmable charge integrator.
Data Arrays. The four data arrays in GETSD are called IPARM, SCALE, IDATA, and BUF:

- IPARM is an integer array, receiving parameter data from the specified shot data file for the specified diagnostics device.

- SCALE is a real array, receiving scale data. 1 LOC $(1)=100$ returns beam energy data: $\operatorname{LOC}(1)$ $=101$ returns detector sensitivity data. In the future. SCAI.F, will also receive data on amplifier gains, amplifier offsets, and autozero settings.

- IDNTA is an integer array, receiving data sets returned by GETSD.

- BUF is an integer array, receiving the common buffer data returned from a common buffer CAMAC device.

The first three arrays need be dimensioned no greater than 128, though this limit is arbitrary : $1: \mathrm{d}$ may be changed in the future: BUF must be dimensioned at least 3000 to accommodate most common buffer sets.

Table 5-9. Status calls retumed by subroutine ISTAT of the shot data retrieval routine GETSD.

\begin{tabular}{|c|c|}
\hline ISTAT $=1$ & Succ \\
\hline ISTAT $=-1$ & $\begin{array}{l}\text { Error detected while attempting to } \\
\text { open the shot data file; most likeiy } \\
\text { cause is locked flle or no file. }\end{array}$ \\
\hline ISTAT $=-\mathbf{2}$ & $\begin{array}{l}\text { Data in shor filc out of sequence or } \\
\text { not found; caused by a bad shot file } \\
\text { or bad calling arguments. }\end{array}$ \\
\hline ISTAT $=-3$ & $\begin{array}{l}\text { Error encountered whitie reading the } \\
\text { shot data file; this is likely an I/O } \\
\text { error, but may also arise becausc } \\
\text { end-of-file was found before requested } \\
\text { data was found or recognized. }\end{array}$ \\
\hline ISFAT $=-4$ & $\begin{array}{l}\text { Premature end-of-file found while } \\
\text { accessing the shot file; occurs when } \\
\text { end-of-file is found before all data } \\
\text { are complete. }\end{array}$ \\
\hline ISTAT $=-\mathbf{5}$ & $\begin{array}{l}\text { Bad shot number; nonnumerics not } \\
\text { currentiy allowed in shot number. }\end{array}$ \\
\hline ISTAT $=-6$ & $\begin{array}{l}\text { Bad ILOC value(s); negative values } \\
\text { not allowed for locatien parameters. }\end{array}$ \\
\hline IST & $\begin{array}{l}\text { More than one of the four data arrays } \\
\text { was too small to hold all the data } \\
\text { found for the specified device; check } \\
\text { values passed for array sizes. }\end{array}$ \\
\hline ISTAT $=-11$ & $\begin{array}{l}\text { IPARM array is too small for number } \\
\text { of dit ta points found. }\end{array}$ \\
\hline ISTAT $=-12$ & $\begin{array}{l}\text { SCALE array is too small for number } \\
\text { of data points found. }\end{array}$ \\
\hline ISTAT $=-13$ & IDATA array is too small. \\
\hline ISTAT $=-14$ & BUF array is too small. \\
\hline ISTAT $=-15$ & $\begin{array}{l}\text { Common buffer data were found to } \\
\text { be flagged as incomplete or in error. }\end{array}$ \\
\hline
\end{tabular}


Each data array has an associated integer variable routine which is both an input and output parameter for the GETSD routine: these subroutines are called. respectively, NPARM. NSCALE. NDATA, and NBUF. The value of the integer variable is set to the size of the actual data array on entry: then, using IPARM and NPARM as an example.

- Ir the number of data points read Irom the shot data file is less than the number of elements in the IPARM array. GETSD sets the value of NPARM to the number of points read.

- If the number of data points in the shot data file exceeds the number of elements in the IPARM array, the IPARM array will be filled to its limit and an error code will he returned by the status subroutine ISTAT.

- If NPARM is set to zero before the GETSD call, GETSD will not read parameter data and the IPARM argument may be omilted from the call. Similarly, if any integer variable is set at zero before the GETSD call. the argument of its data array may be smitted and no data will be returned to that array.

GETSD's status subroutine. ISTAT, is an integer variable whose values are listed in Table 5-9.

Authors: J. T. Ozawa and J. M. Auerbach Major Contributors: J. E. Krammen and J. Wilkerson (Bendix)

\section{Automatic Diagnostic Data Processing: Dante}

The Dante system ${ }^{67}$ uses fast $x$-ray diodes as detectors. Output currents from these detectors are related to the incident $x$-ray energy llux through the detector and filter response functions. The $x$-ray spectrum emitted by the target in the direction of the instrument can be obtained from approximately $200 \mathrm{eV}$ to $2 \mathrm{keV}$ with ten appropriately filtered channels.

At Shiva, the detectors are impedence-matched to $50 \Omega$ and their outputs are recorded by Tektronix R7912 transient digitizers. Each digitizer is associated with a given crate, station, and unit in the CAMAC system through which the digitized data are transferred to the shot file. Preprocessing of the Dante data is then performed on the Shiva 11/70 according to the process described in this article.
A data file named DANCHN.SET contains the crates, stations, and channels connected to aclive Dante channels: the program TKDANT (adapted from programs written at LLL. to handle R7912 transient digitizers datal uses these pointers to extract from the main shot file the data from each of the R7912's. A subroutine of TKDANT. TD7912. then unpacks the data and, using subroutine N7912Z (Ref. 68). determines the centroids of the trace: representative raw and fitted data are given in Fig. 5-69.

The output of TKDANT is a single file, DANRAW.DAT, contianing $x$ blocks of data where $x$ equals the number of Dante channels: the output structure of one such block is shown in Tuble 5-10. An identical file named TK7NORM. BSL contains similar information, obtained during a rod shot immediately preceding the shot to be analyzed. This file. created by TK7BASE. a general R7912 baseline program which follows the same steps as TK 7912, is used to establish baseline levels.

The two files, DANRAW.DAT and TK7NORM.BSI., are used by program ANDANT2 (ANalyze DANTe) to obtain voltage-vs-time information from the recorded counts: to do so, the baseline is subtracted from the data. Tests are then porformed to determine whether the remaining haseline is indeed zero. Should this not be the case.

Table 5-10. TKDANT output structure.

\begin{tabular}{cl}
\hline Word number & \multicolumn{1}{c}{ Content } \\
\hline 1 & Crate number \\
2 & Station number \\
3 & Channcl number \\
4 & Not used \\
5 & Total number of data points $=\mathbf{5 1 2}$ \\
6 & R7912 vestical sensitivity $(\mathrm{mv} / \mathrm{cm})^{\mathrm{a}}$ \\
7 & R7912 horizontal sensitivity $(\mathrm{ns} / \mathrm{cm})^{\mathrm{a}}$ \\
8 & Number of dats, sints \\
9 & Index of first valid vertical datum \\
$16-23$ & Shot number \\
257 & First vertical value (centroid) \\
& \\
768 & 51 2th vertical value (centroid) \\
\hline
\end{tabular}

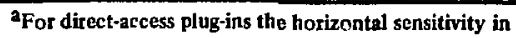
$\mathrm{ps} / \mathrm{cm}$ is in word 6 ; word 7 is not used. 


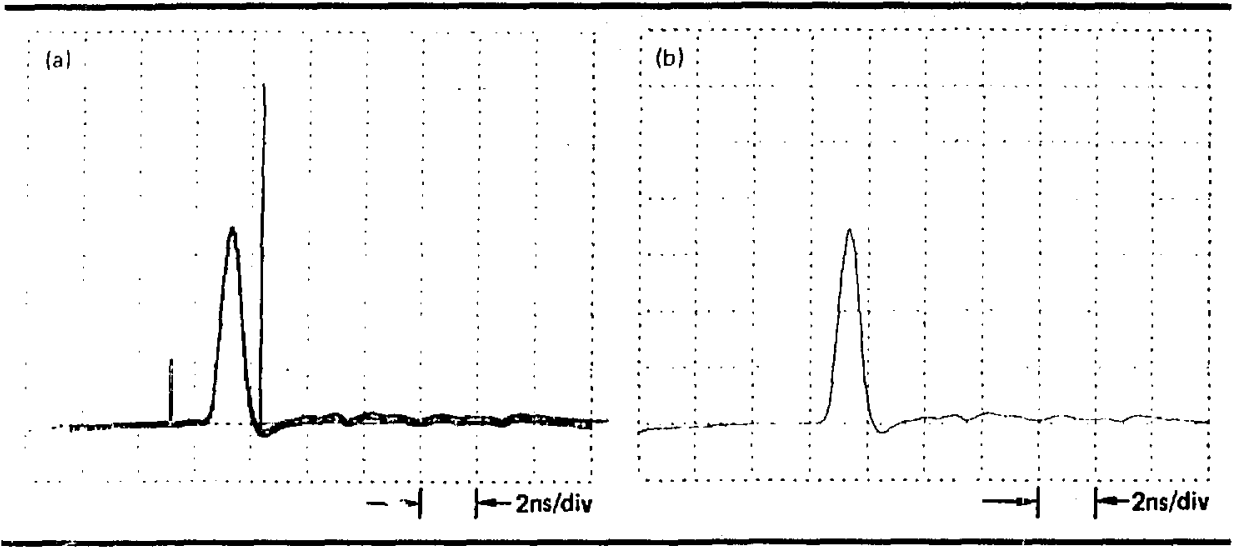

a dita-derived baseline is obtained and the process repciled. The following quantities are then calculated:

- The location of the peak of the pulse with respect to a fiducial.

- The voltage at the peak.

- The FWHM about the peak.

- The integral of the signal over the full range excluding the liducial.

- The integral of the signal for one FWHM on each side of the peak.

- The index location of the fiducial peak.

- A flag indicating the data quality.

Two files are generated to utilize these quan(ities: DANDAT.INP is used for further processing on the 11/70: (shot \#).DAN is transferred via tape to the I.L.L Octopus system. A third file. DANIRR.TMP, provides the experimenter with information on the program's handling of baseline data. as well as characteristics of the fiducial pulse.

The final preprocessing step is performed by UNDANT2 (UNfold DANTe). The basic iole of this program is to combine the data accumulated in DANDATINP with the characteristics of the associated detectors and provide a summary file (Table 5-11) for inclusion in the shot book. The column labeled "Integral v. ns" provides the necessary input for the UNSPEC (UNfold SPECtrum) routine which is used through Octopus on a
Fig. 5-70. Representative unfold Dante spectrum.

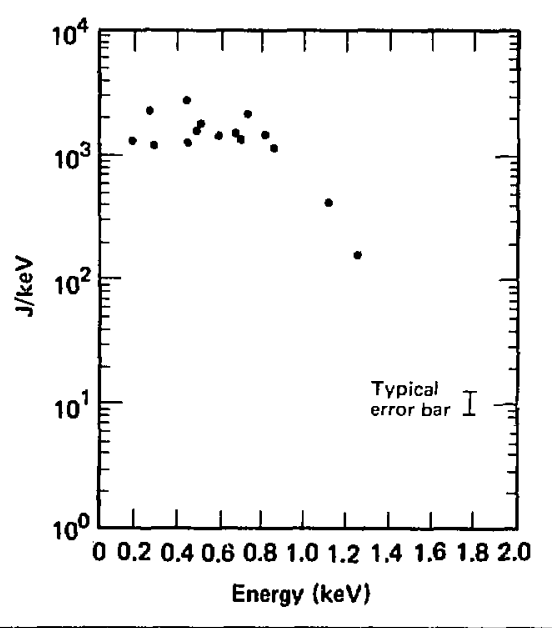

CDC 7600 to obtain the final unfolded spectrum: and example of this final output is shown in Fig. 5-70. Given a reference spectrum, this program is also capable of providing, for quick-look purposes. a ratio spectrum based on the current shot data (Fig. 5-71): this option is normally not exercised, however, since appropriate reference spectra are not generally available.

The (shot:f).DAN file is read into the Octopus system where is operated on by the frequency 
Table 5-11. Representative Dante summary for Shiva target shot.

POSITION: THETA $=90, \mathrm{PHI}=234$

BIAS: $5 \mathrm{KV}$

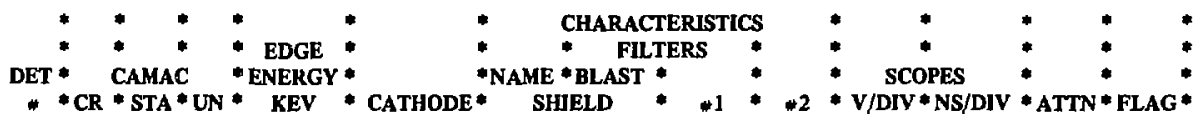

\begin{tabular}{|c|c|c|c|c|c|c|c|c|c|c|c|c|}
\hline $\begin{array}{l}11 \\
* 11\end{array}$ & $\begin{array}{r}\text { * } 4 \\
* \quad 4 \\
* 4\end{array}$ & $\begin{array}{r}7 \\
-6\end{array}$ & $\begin{array}{r}* 0.28 \\
* \quad 0.52 \\
* \quad 0.58 \\
* \quad 0.71 \\
* \quad 0.79 \\
\quad 0.86 \\
\quad 0.94 \\
\quad 0.94 \\
\quad 1.56 \\
\end{array}$ & $\begin{array}{l}\text { * AL43 } \\
* \quad \text { ALS2 } \\
\text { * ALS3 } \\
\text { * AL54 } \\
\text { AL49 } \\
\text { * ALS0 } \\
\text { CR19 } \\
\text { * CR23 } \\
\text { * NI15 } \\
\text { NI16 }\end{array}$ & $\begin{array}{l}\text { FV2 } \\
\text { * VV1 } \\
\text { CR1 } \\
\text { FE2 } \\
\text { FO1 } \\
\text { CO11 } \\
\text { NCU1 } \\
\text { * CU2 } \\
\text { * AL9 } \\
\text { AL2 }\end{array}$ & $\begin{array}{l}\text { * PY.1 } \\
\text { * PY.1 } \\
\text { * PY.1 } \\
\text { * PY.1 } \\
\text { * PY.1 } \\
\text { * PY.1 } \\
\text { * PY.1 } \\
\text { * PY.1 } \\
\text { * PY.1 } \\
\text { * PY.1 }\end{array}$ & $\begin{array}{l}\text { PV.03 } \\
* \\
* \\
* \\
\text { ALS1. }\end{array}$ & 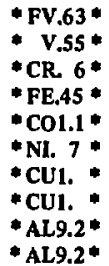 & $\begin{array}{l}4.000 \\
4.000 \\
1.000 \\
1.000 \\
1.000 \\
1.000 \\
1.000 \\
4.000 \\
0.500\end{array}$ & $\begin{array}{l}2.000 \\
2.000 \\
2.000 \\
2.000 \\
2.000 \\
2.000 \\
2.000 \\
2.000 \\
2.000\end{array}$ & 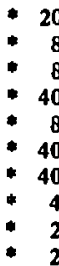 & $\begin{array}{l}20000^{*} \\
20000^{\circ} \\
+20000^{*} \\
* 20000^{*} \\
* 20000^{\circ} \\
20000^{*} \\
* 20010^{*} \\
* 20000^{*} \\
+20000^{*}\end{array}$ \\
\hline
\end{tabular}

FLAG = XXXXI IF BASELINE WAS SHCT DERIVED

$=$ XXX1X IF FWHM*IHAX IS LESS THAN 90\% INTEGRAL

$=$ XX1XX IF MAX DEFLECTION $<10$ COUNTS

$=$ XIXXX IF FWHM OR INTEGRAL $<0$

$=1 \times X X X$ IF NO BASELINE AND CANNOT DERIVF IT FROM SHOT

\begin{tabular}{|c|c|c|c|c|c|c|c|c|}
\hline DET & 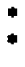 & $\begin{array}{l}\text { FIDO PEAK } \\
\text { CDUNTS }\end{array}$ & $\begin{array}{l}\text { * PEAK LOC -NS } \\
\text { - REL TO LASER }\end{array}$ & $\begin{array}{c}\text { FWHM } \\
\text { NS }\end{array}$ & $\begin{array}{c}\text { PEAK VALUE } \\
\text { VOLTS }\end{array}$ & $\begin{array}{c}\text { INTEGRAL } \\
\text { V.NS }\end{array}$ & $\begin{array}{c}\text { * SUM/ } \\
\text { FWHM*PV }\end{array}$ & $\begin{array}{ll}-\quad \text { SUMP } \\
-\quad \text { V.NS }\end{array}$ \\
\hline 1 & $\bullet$ & 418 & $-0.108 E+02$ & $\bullet \quad 0.254 E+01$ & $0.401 E+02$ & - $0.118 \mathrm{E}+03$ & 1.16 & $-0.104 E+03$ \\
\hline 2 & * & 440 & $-0.114 E+02$ & * $0.266 \mathrm{E}+01$ & $0.113 E+02$ & * $0.312 \mathrm{E}+02$ & 1.04 & $0.308 E+02$ \\
\hline 3 & * & 436 & $-0.104 E+02$ & - $0.261 E+01$ & $0.992 \mathrm{E}+01$ & $* 0.266 \mathrm{E}+02$ & 1.03 & - $0.259 \mathrm{E}+02$ \\
\hline 4 & * & 444 & $-0.110 \mathrm{E}+02$ & * $0.267 E+01$ & $0.147 E+02$ & * $0.361 \mathrm{E}+02$ & 0.92 & - 0.331E+02 \\
\hline 5 & $*$ & 448 & $-0.935 E+01$ & - $0.262 \mathrm{E}+01$ & $0.468 E+01$ & - $0.119 \mathrm{E}+02$ & 0.97 & * 0.109E+02 \\
\hline 6 & $*$ & 445 & $-0.105 E+02$ & $-0.274 E+01$ & $0.134 E+02$ & - $0.351 E+02$ & 0.96 & $0.341 \mathrm{E}+02$ \\
\hline 7 & 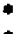 & 503 & $-0.122 E+02$ & * $\quad 0.282 E+01$ & $0.126 \mathrm{E}+02$ & * $0.369 E+02$ & 1.04 & - $0.333 \mathrm{E}+02$ \\
\hline 8 & $\bullet$ & 450 & $-0.102 E+02$ & - $\quad 0.206 E+01$ & $0.174 E+01$ & * $\quad 0.541 \mathrm{E}+01$ & 1.51 & - $0.339 \mathrm{E}+01$ \\
\hline 9 & 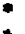 & 459 & $-0.119 \mathrm{E}+02$ & * $0.250 \mathrm{E}+01$ & $0,499 \mathrm{E}+01$ & $* 0.120 E+02$ & 0.96 & $0.113 E+02$ \\
\hline 10 & $\star$ & 442 & $-0.116 \mathrm{E}+02$ & $* 0.251 \mathrm{E}+01$ & * $\quad 0.607 E+00$ & * $0.146 \mathrm{E} ; 01$ & 0.96 & $0.143 E+01$ \\
\hline
\end{tabular}

Fig. 5-71. Representative ratio spectrum for Dante data.

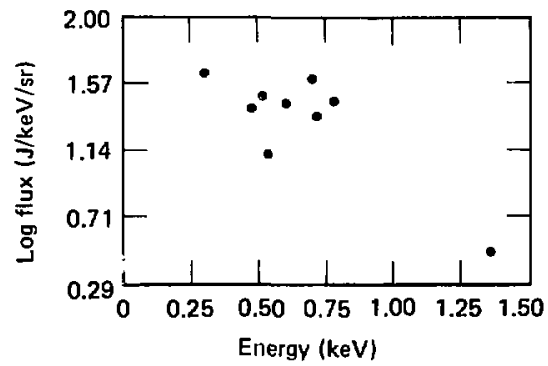

domain deconvolution code DCON, which deconvolves the temporal response of the detectors. cables. and oscilloscopes in the Dante diagnostics system. After removal of the temporal response. spectral unfolds of "snapshots" in time are done with UNSPEC. Using this method, we have produced time-dependent spectral unfolds with a resolution of $120 \mathrm{ps} \mathrm{FWHM}$ on direct-access R7912 data and $300 \mathrm{ps}$ on amplified R7912 channels.

Authors: V. C. Rupert and R. A. Haisle Major Contributor: G. S. Chinen

References

67. Laser Program atmual Report-1978, lawrence Livermore I.aboratory. I.jucrmore, (alif. LCRL-50021-78 (1979), pp. 6.5 to 6.7.

4.8. J. Sireenwood and J. Ozawa. V79/2Z-A Normalized Routine for the R7Q/2 Transient Digitizer on the PDP 11 . I.awrence l.ivermore laboratory. Livermore, Calif.. l'CID-17810 (1978). 


\section{Automatic Diagnostic Data Processing: Filter Fluorescer}

The filter fluorescer high-energy $x$-ray (FFLFX) spectrometer, described in detail in last vear's annual report, ${ }^{69}$ is used to produce a spectrum of the high-energy $(>\mid \mathrm{keV}) \mathrm{x}$ rays from laserplisma interaction experiments. The detector signals (given amounts of charge from photomultiplier tubes) can be processed automatically to yeld the desired spectra. Before the spectra are enenerated, routines are executed that detect very low or saturated detector channels and establish the signal-noise ratios for all channels. This preprocessing requires that we maintain a set of parameter and setup data files, which will be described in conjunction with the processing steps enumerated below.

The PM tube output of each detector is connected via three different attenuators to three channels of a LeCroy Research Systems Charge Integrator/ADC module (LS2249W). Each module is assigned a crate and station in either the Shiva or Argus CAMAC systems. In addition, the high voltage applied to each PM tube is set and monitored through a computer interface, with a LeCroy Research System HV 4032 programmable high-voltage power supply; the voltage-sensing circuits of the supply are interfaced to the TACAI computer through a CAMAC crate and station.

Thus the recording of shot data and detector voltages has been automated. At shot time the outputs of all the active charge integrator modules are transferred via the CAMAC serial highway to the TACAI LSI-I1, where the data are written on Moppy disks and then transferred to the Argus or Shiva data acquisition computers along with other diagnostic data. The FFLEX data are incorporated into the shot dati: file on the Shiva PDP 11/70 using the routine PROCESS, which transfers the TACAI raw data from the floppy disk to the main computer disk.

The LS2249W module outputs generally have non-zero baseline values; that is, they show nonzero dc outputs with no signal input. This necessitates "clry-run" data processing before a shot, so that baseline values can be subtracted from the total detector charge signals obtained during a shot.

With the 11/70 shot data file as input, data processing proceeds in the sequence described below (and summarized in Fig. 5-72).

The processing program FFBDP checks a disk file which gives the active detector channels for the shot. The program then opens a data file containing CAMAC crate, station, and $A D C$ channel numbers for each detector channel. Only the CAMAC parameters corresponding to the active channels are extracted.

Using the GETSD routine (described in a previous article) and the CAMAC data, a baseline processing routine is run which extracts from the dry-run file the baseline values for the active detector channels. Still using GETSD and the CAMAC data, the main processing program extracts from the actual shot data file the high-voltage readings and LS2249W ADC outputs for the active channels: there are three $A D C$ readings for each detector, corltsponding to the three attenuators. Next the program opens a file which contains the maximum linear current of each PM tube as a function of bias voltage.

A summary of system performance is now calculated, a sample tabulation of which is given in Table 5-12. For each attenuator value, the summary provides the total channel ADC output, baseline, and charge output in picocoulombs. Each ADC count corresponds to 0.25 picocoulombs. If an ADC count is within 10 counts of baseline, it is flagged as NOISE LEVEL; if an ADC count exceeds 1900 it is nagged as SATURATION, which occurs in the i.S2249W between 1800 and 2000 counts. The program then selects the value farthest removed from NOISE LEVEL or SATURATION as the BEST SIGNAL. Readings are also printed for the HIGH VOLTAGE, the MAXIMUM LINEAR CHARGE (maximum linear current at the bias voltage times scintillator pulse width), and the ratio of BEST SIGNAL to MAXIMUM LINEAR CHARGE. A ratio greater than or equal to $I$ indicates that the PM tube may have been in the nonlinear regime of operation, in which case the calibration factors used in processing would be inaccurate.

Channels 1 and 10 of the filter fluorescer are "background" channels. Channel 1 is used to obtain a background figure for detectors with NE 111 
Fig. 5-72. Summary of automatic filter fluorescer diagnostic data processing.

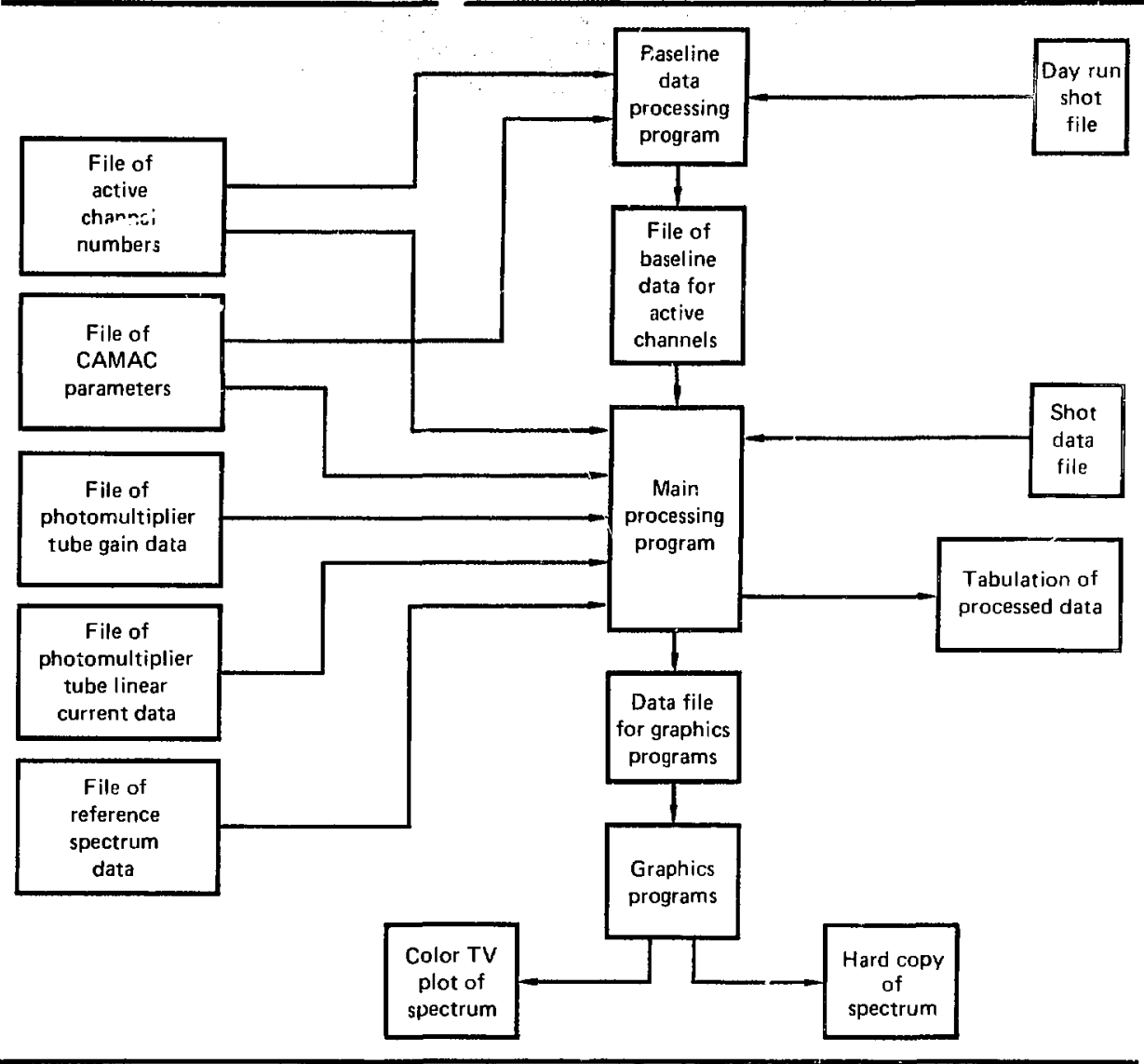

scintillators: channel 10 is used to obtain a background figure for detectors with $\mathrm{NaI}$ scintillators. These background channels contain no fluorescer and hence produce a charge signal arising from electromagnetic pickup and high-energy $x$ rays impinging directly on the PM tubes. The ratio of these signals to the signal on each active channel is an indication of noise to signal ratio. Using a file which contains the gain of each PM tube as a function of voltage, the main program normalizes the background signals to the gain of each active channel tube at 2500 volts, and calculates a noise to signal ratio. This summary for all active channels is displayed in the second section of the program output.

The last part of the data processing involses computation of the $x$-ray spectrum from the "good" channels, that is, from those detectors having outputs that are neither in SATURATION nor near the NOISE LEVEL. If $S(E)$ denotes the $x$-ray spectrum, $Q_{n}$ the output from the nth channel, and $R_{n}(E)$ the response function of the $n$th channel as a function of energy $E$, then we must soive for $S(E)$ from the set of integral equations 
Table 5-12. Sample FFLEX channel summaries.

\begin{tabular}{|c|c|c|c|c|}
\hline \multicolumn{5}{|l|}{ CHANNEL NO. 7} \\
\hline $\begin{array}{c}\text { ATTENUATOR } \\
1 . \\
10 . \\
100 .\end{array}$ & $\begin{array}{c}\text { ADC OUTPUT } \\
1995 . \\
535 . \\
30 .\end{array}$ & $\begin{array}{c}\text { BASE LINE } \\
35 . \\
37 . \\
23 .\end{array}$ & $\begin{array}{c}\text { PICOCOULOMBS } \\
0.147 E+04 \\
0.374 E+04 \\
0.525 E+03\end{array}$ & $\begin{array}{l}\text { REMARKS } \\
\text { SATURATION } \\
\text { NOISE LEVEL }\end{array}$ \\
\hline $\begin{array}{l}\text { HIIGH VOLTAGE } \\
3000 .\end{array}$ & \multicolumn{3}{|c|}{$\begin{array}{l}\text { BEST SIGNAL (PC) } \\
0.374 E+04\end{array}$} & \\
\hline & $\begin{array}{l}\text { MAX. LIN. CHGE, (PC) } \\
0.115 E+06\end{array}$ & & $\begin{array}{c}\text { BEST SIGNAL/MAX. LIN. CHGE.) } \\
0.032\end{array}$ & \\
\hline \multicolumn{5}{|l|}{ CHANNEL NO. 8} \\
\hline $\begin{array}{c}\text { ATTENUATOR } \\
1 . \\
10 . \\
100 .\end{array}$ & $\begin{array}{c}\text { ADC OUTPUT } \\
1995 . \\
447 . \\
46 .\end{array}$ & $\begin{array}{c}\text { BASE LINE } \\
32 . \\
27 . \\
29 .\end{array}$ & $\begin{array}{c}\text { PICOCOULOMBS } \\
0.147 \mathrm{E}+04 \\
0.315 \mathrm{E}+04 \\
0.128 \mathrm{E}+04\end{array}$ & $\begin{array}{l}\text { REMARKS } \\
\text { SATURATION }\end{array}$ \\
\hline $\begin{array}{l}\text { HIGH VOLTAGE } \\
\text { 3000. }\end{array}$ & \multicolumn{2}{|c|}{$\begin{array}{l}\text { BEST SIGNAL (PC) } \\
0.315 E+04\end{array}$} & & \\
\hline & $\begin{array}{l}\text { MAX. LIN. CHGE. (PC) } \\
0.110 E+05\end{array}$ & & $\begin{array}{c}\text { BEST SIGNAL/(MAX. LIN. CHGE.) } \\
0.029\end{array}$ & \\
\hline
\end{tabular}

$$
Q_{n}=\int_{0}^{\infty} S(L) R_{n}(E) d E: \quad n=1,2, \ldots N
$$

Finding at rigorous solution for $\mathrm{S}(\mathrm{t})$ requires much interaction between user and computer, accomplished using the unfolding code UNSPEC2. for experiments having similar targels and laser irradiation conditions, however, the rigorous unfold procedure can be supplemented by a ratio calculation using one rigorous unfold as the reference spectrum: this procedure, known as a ratio spectrum calculation. is often used in our automated data processing.

Let $S_{0}\left(E_{11}\right)$ be the value of the reference spectrum at energy $E_{n}$ (usually the mid-energy-point of at detectur channel). Let $Q_{\text {an }}$ be the calculated or measured charge for channel $n$ due to the reference spectrum (they should be equal) and $Q_{n}$ be the measured signal in channel $n$ due to the $x$-ray spectrum to be determined. Then the new spectrum $S(E)$ can ie determined for a similar class of experiments by

$$
S\left(E_{n}\right)=S_{0}\left(E_{n}\right) \times \frac{Q_{n}}{Q_{0 n}}
$$

In calculating a spectrum, all signals $Q_{n}$ are referenced to a PM-tube voltage setting of 2500 volts. The processing program opens a file contain- ing the PM tube gains as a function of voltage, and extracts gain data for the active channels. Then if $Q$ v is the measured signal at voltage $V$ and $G(v)$ the gain at $V$, the processing program calculates $Q_{n}$ in Fi. (4) by

$$
Q_{n}=\frac{G(2500) Q_{v}}{G(v) Q_{0}}
$$

The ratio calculations, tabulated in the third part of the program output, give the values of $Q_{n}$. $E_{n}, S_{n}\left(E_{n}\right)$ and $S\left(E_{n}\right)$ for each detector channel. The

Fig. 5-73. Sample printout of an x-ray spectrum obtained with the FFLEX spectrometer.

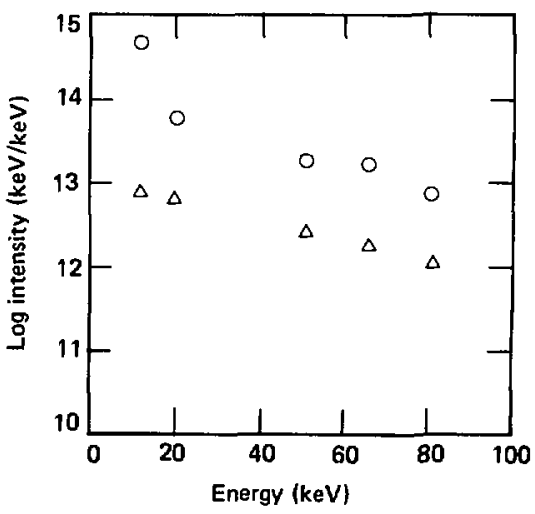


program also plots the experimental and reference spectra on both a color TV monitor and a Versatec printer: a sample plot is shown in Fig. 5-73.

Automation of this simple set of calculations for processing filter fluorescer data has reduced the experimenter's workload by 2 to 3 hours per experiment.

Author: J. M. Auerbach

Major Contributors: (i. S. Chinen, H. X. Kornblum, B. I.. Pruett

\section{Reference}

69. Laser Program flmal Repurt-147,8. I.ilw rence I ivermors 1 aboratury. 1 isermore. (allf. I ( R I -50021-78 11979). PP $6-5100.12$.

\section{Automatic Diagnostic Data Processing: Calorimeters}

Various types of ealorimeters have been described in Ref. 70 as well ats in previous annual reports. 71.72 For a given heat input, the sensing clements of calorimeters provide a lime-varying roltage which is amplified in two stages. The first stage is a fixed-gain (1000) preamplifier? the second stage is a 16-channel programmable-gain amplifier of LLL design. ${ }^{73}$ The gain of this latter amplifier is selected through a computer interfiase so that its value is atrailable in the shot datat file and can be aceessed directly for automatic datat processing. The amplified daltat are digitised by "cialorimeter modules," designed at LLL. ${ }^{74}$ "hich provide multiplexed data from up to sixteen data channels.

Processing of all calorimeters (plasma, light, $x-$ ray, and special-purpose) is conducted simultaneously. The progranmable amplifier module and eath calorimeter module are assigned a crate and a station in the CAMAC system through which datal are recorded in the shot file; each of the amplifier channels is also assigned a unit number in the CAMAC system.

Program CALRED uses CALCHN,SET. which contains the crate, station, and unit numbers, to extract appropriate data from the shot file. For each crate and station, data from the sixteen channels are demultiplexed and the corresponding amplification factors determined. The main output of
CALRED is a single file, CALRAW.DAT, containing blocks of data. Each block includes the crate and station number, followed by the amplification fictors for the 16 channels, and the 16 sets of denultiplexed data headed by the channel number. CALRED also contains an option (normally not exercised) which ploss the ciata from each thannel.

Program CALIN2, a simplified version of at thermopile data analysis program, ${ }^{\text {is }}$ uses the shot datia from CALRAW.DAT and associalles each channel with a silibration falctor, detectur ID, and leciltion provided by dilla lile CALNAM.SET. Tho basic role of CALIN2 is to calculate an encrgy thes from the time-dependent raw data. Two output liles are generated:

- CALFIT.DAT provides the experimenter with various indicators of the data yualits, and information concerning the fits used.

- CAloUT.INP comains, for cach channel (in the order recorded), the calorimeter ID, polar and armuthal angles, the energy flux measured. and an "error" value indicalive of the smoothness of the datat and its deviation from the filted curve: CALOUT.INP also provides a halg indicating barious possible datal conditions, such as noise level and silturation.

Final dala for plasma and special calorimeters are provided b! program CALOUT: dala for light cillorimeters are further procensed by program ENERGY (described later in this section). CAIOUT perlorm an separate tasks. The first consists in sorting the various types of cilorimelert atcording wo three cillegeries: "plasmal," "light." and "others." Data from pliama and light calorimeters are further sorted according 10 arimuthal angles and for each asmuth according to polar angle. The datal are then printed out in the shot-book format displayed in Table $5-13$.

The second task consists in ubtaining an absorbed energy value hased on plissma calorimeter datia. This procedure is vatid lor axisymmetric configurations only, such as balls or untilted disks. Since the plasma calorimeter data are strongly affected by any diagnostic extending close enough to the target to seriousls distort the plasma distribution, the calculation of ahsorbed energy is usually suspect if any such diagnostic has been used. For the same reatson. energy halances obtained from scattered-light diagnostics are also suspect under these conditions. 
Table 5-13. Kepresentative Shiva phasma calorimeter data, as printed out in shot-book format.

\begin{tabular}{|c|c|c|c|c|c|c|c|c|c|c|}
\hline * & $\begin{array}{l}\text { THETA } \\
\text { DEGREES }\end{array}$ & $\div$ & $\begin{array}{l}\text { PHI } \\
\text { DEGREES }\end{array}$ & $\bullet$ & $\begin{array}{l}\text { ENERGY FLUX } \\
\text { J/SR }\end{array}$ & 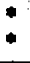 & $\begin{array}{c}\text { DE/E } \\
\not\end{array}$ & 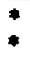 & COMMENTS & 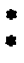 \\
\hline$=$ & 160.00 & $\div$ & 18.00 & $\bullet$ & 3.21 & $\bullet$ & 6.36 & * & & $\bullet$ \\
\hline$\neq$ & 45.00 & 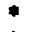 & 36.00 & $\bullet$ & 0.00 & $\bullet$ & 100.00 & $\bullet$ & LOW SIG & $\bullet$ \\
\hline * & 60.00 & $\bullet$ & 36.00 & $\bullet$ & 8.38 & $\bullet$ & 1.95 & $*$ & & - \\
\hline$*$ & 75.00 & 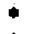 & 36.00 & $\bullet$ & 0.00 & $\bullet$ & 100.00 & $\bullet$ & LOW SIG & - \\
\hline 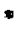 & 90.00 & $\bullet$ & 36.00 & $*$ & 4.32 & $\bullet$ & 4.10 & $\bullet$ & & - \\
\hline 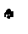 & 105.00 & $\bullet$ & 36.00 & - & 3.49 & * & 3.36 & $\bullet$ & & * \\
\hline 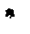 & 120.00 & 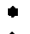 & 36.00 & $\bullet$ & 2.20 & $\bullet$ & 8.11 & $\bullet$ & & - \\
\hline$\bullet$ & 45.0 .1 & $\bullet$ & 90.00 & $\bullet$ & 0.00 & $\bullet$ & 100.00 & $\bullet$ & LOW SIG & $\bullet$ \\
\hline * & 45.00 & 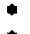 & 126.00 & * & 36,84 & $*$ & 0.43 & $\bullet$ & & * \\
\hline * & 60.00 & $\bullet$ & 126.00 & $\bullet$ & 29.88 & $\bullet$ & 3.02 & $\bullet$ & & - \\
\hline 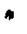 & 75.00 & $\bullet$ & 126.00 & $\bullet$ & 0.00 & $\bullet$ & 100.00 & $\bullet$ & LOW S1G & - \\
\hline * & 90.00 & $\bullet$ & 126.00 & $\bullet$ & 0.37 & $\bullet$ & 100.00 & $\bullet$ & & $\bullet$ \\
\hline * & 105.00 & $\bullet$ & 126.00 & $\bullet$ & 0.97 & $\bullet$ & 100.00 & $\bullet$ & & $\bullet$ \\
\hline$\bullet$ & 120.00 & $\bullet$ & 126.00 & $\bullet$ & 0.16 & $\bullet$ & 89.73 & $\bullet$ & & $\bullet$ \\
\hline \# & 135.00 & $*$ & 126.00 & $\bullet$ & 3.10 & $\bullet$ & 3.97 & $\bullet$ & & $\bullet$ \\
\hline$\bullet$ & 45.00 & $\bullet$ & 162.00 & $\bullet$ & 46.73 & - & 0.36 & $\bullet$ & & $\bullet$ \\
\hline$*$ & 160.00 & $\bullet$ & 16200 & 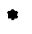 & 255 & $*$ & 7.80 & $\bullet$ & & * \\
\hline * & 45.00 & * & 216.00 & 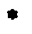 & 107.18 & $\bullet$ & 0.81 & $\bullet$ & & 中 \\
\hline * & 60.00 & $\bullet$ & 216.00 & $\bullet$ & 60.04 & $\bullet$ & 1.58 & $\bullet$ & & $\bullet$ \\
\hline * & 75.00 & $\bullet$ & 216.00 & $*$ & 51.84 & $\bullet$ & 0.42 & $\bullet$ & & $\bullet$ \\
\hline * & 90.00 & * & 216.00 & $*$ & 42.16 & $\bullet$ & 0.33 & $\bullet$ & & - \\
\hline$*$ & 105.00 & $\bullet$ & 216.00 & $\bullet$ & 29.49 & $\bullet$ & 0.48 & $\bullet$ & & $\bullet$ \\
\hline * & 120.00 & $\bullet$ & 216.00 & $\bullet$ & 20.69 & * & 0.92 & $\bullet$ & & $\bullet$ \\
\hline * & 45.00 & $*$ & 270.00 & $*$ & 54.91 & $\bullet$ & 0.92 & $\bullet$ & & $\bullet$ \\
\hline * & 45.00 & $\bullet$ & 305.00 & 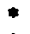 & 39.43 & $\bullet$ & 1.54 & $\bullet$ & & • \\
\hline • & 60.00 & $\bullet$ & 306.00 & $*$ & 37.15 & $\bullet$ & 1.25 & $\bullet$ & & $\bullet$ \\
\hline * & 75.00 & $\bullet$ & 306.00 & - & 35.97 & $\bullet$ & 0.68 & $\bullet$ & & $\bullet$ \\
\hline • & 90.00 & " & 306.00 & + & 20.72 & $\bullet$ & 1.03 & $\bullet$ & & $\bullet$ \\
\hline * & 105.00 & $*$ & 306.00 & $*$ & 8.20 & $\bullet$ & 1.50 & $\bullet$ & & * \\
\hline * & 120.00 & 中 & 306.00 & $*$ & 5.14 & $*$ & 3.68 & * & & * \\
\hline * & 135.00 & * & 306.00 & $\bullet$ & 2.59 & $\bullet$ & 10.18 & $\bullet$ & & $\bullet$ \\
\hline * & 45.00 & $*$ & 342.00 & $\bullet$ & 32.84 & $\bullet$ & 1.33 & $\bullet$ & & • \\
\hline
\end{tabular}

For appropriate configurations, CALOUT fits the arimuthally averaged data for energy flux versus polar angle to a curve of the form $\Sigma a_{n} \cos n i t$. Each coefficient of this fit is then tested for statistical significance based on the collected data, and deleted if necessary. The final fit, containing only significitnt coefficients, is then integrated to yield tota' absorbed energy: an example of such a fit for a disk shot is given in Fig. 5-74, along with the collected datit.

Because the requirements for a meaningful calculation of total absorbed energy are often not met in an experiment, this value is not automatically printed in the output intended for the shot book, but is manually entered (when appropriate) on the plasma calorimeter summary page. This value is also extracted from CALOUT's output data by program ENERGY, which prints it on its owl summary page. It must be emphasized that this value is not to be considered useful unless verified by the experimenter.

Author: V. C. Rupert

\section{References}

70. S. R. Gunn and V. C. Rupert, "Calorimeters for Measurements of lons, $X$ rays, and Scatlered Radiation in Laser Fusion Experiments," Rev. Soi. Ihst. 48(1I), (1977).

71. Laser Program Anmal Reporl-1975. Lawrence Livermore Laboratory, Livermore, Calif., UCRL-50021-75 (1976), pp. 404 to 407.

72. Laser Program Anmal Repori-1976, Lawrence Livermore Laboratory, Livermore, Calif., UCRL-50021-76 (1977). pp. 3-55, 3-93 to 3-95.

73. D. Campbell and J. Severyn, The Electronics of the Target Diagnosics System for the Shiva Laser Fusion Facility. Lawrence Livermore Laboratory, Livermore, Calif., UCRL-5252! (1978).

74. Laser Program Anmual Report-1976. Lawrence Livermore Laboratory, Livermore, Calif., UCRL-50021-76 (1977), Pp. 3-131 10 3-133.

75. S. R. Gunn, On The Calculation of the Corrected Temperature Rise for Isoperibol Laser Calosimeters, Lawrence Livermore Laboratory, Livermore, Calif., UCRL-52019 (1976). 
Fig. 5-74. An example of collected and fit data for the Shiva plasma calorimeters, used to estimate total absorbed energy. Triangles are the collected data (ali aximuth and polar angles); the solid line is of fit of the form a, $\cos n \theta$ to the aximuthally averaged data. The disk was located normal to the Shiva benm claster and irradiated by the lower 10-beam cluster only.

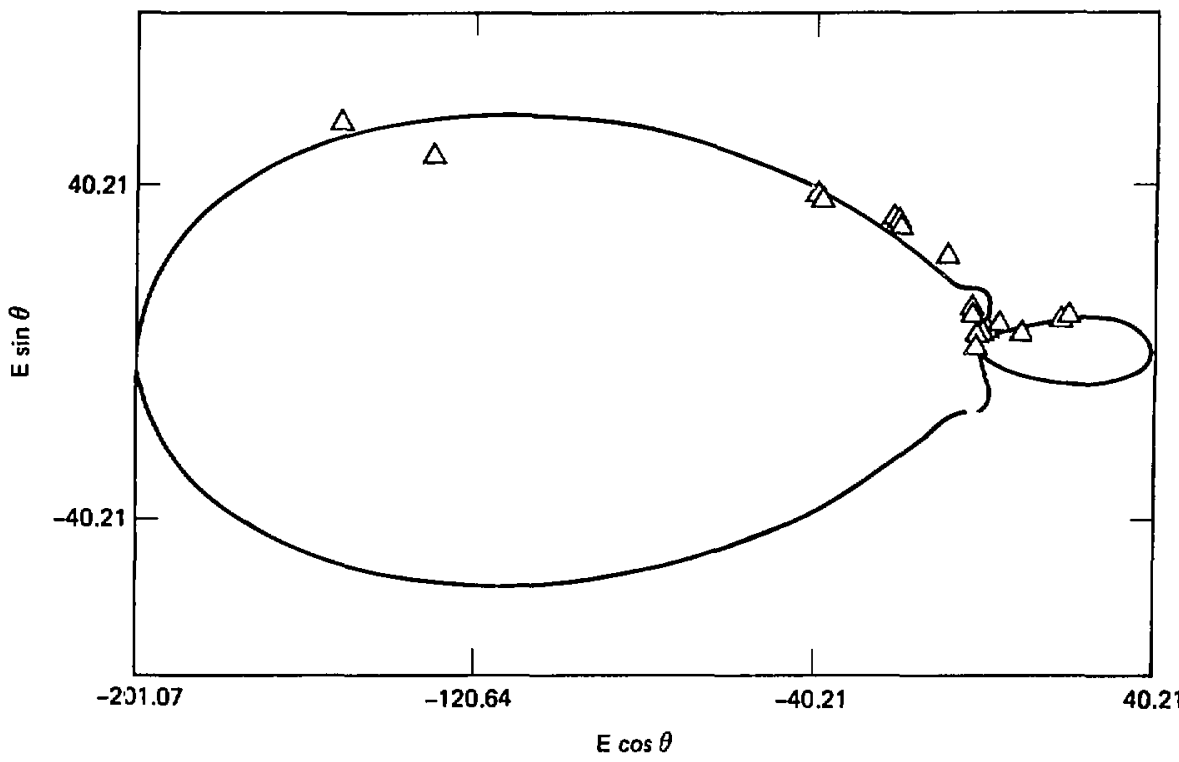

\section{Automatic Diagnostic Data Processing: Photodiodes}

Automatic reduction of the photodiode data required for the St.iva energy balance measurement (EBM) is processed by program LIGHT (Fig. 5-75) on the Shiva PDP 11/70. LIGHT is controlled by the processing command tile EBF.CMD, which also controls the calorimeter data processing and energy balance routines. LIGHT gets raw shot data from the TACAI system, and employs two input files:

- SETUP.PAR (read into the program at run tine), containing parameters necessary for the reduction of the photodiode data.

- CAL.FAC (incorporated into the program at compiling), containing the programmable charge integrators' sensitivity calibrations.

SETUP.PAR contains, for each photodiode detector, its

- Number, CAMAC crate and station, serial number (denoting the programmable charge integrator module), and channel.

- Spherical angular coordinates, distance $\mathbf{R}$ from the target, $x$ indow transmission index, and sensitivity factor $\mathrm{R}^{\prime}$ (in picocoulomb/Joule $/ \mathrm{cm}^{2}$ ).

SETUP.PAR also contains

- The total number of active diode detectors.

- A conversion faltor of 5.0 volts per 4095 full counts: any reading grcatcr than 4092 is considered (and Magged as) saturated.

- A flag to denote a blocked detector and to specify the diode used to measure the $x$-raly hackground.

CAL.FAC contains the data stattements for two arralys in LIGHT:

- SENS(8), which contains the detectors' sensitivity per volt full scale, in picocoulomb/volt. This value is obtained from the TACAI file and passed to LIGHT via the data acquisition routine GETSD.

- $P C(8,4.20)$. The first dimension of this array corresponds to the sensitivity setting of the amplifier: the values are the elements of the array SENS described above. The second dimension corresponds to four detectors grouped into one module, and is thus the channel assignment read in from SETUP.PAR. The third dimension corresponds to 20 modules of amplifiers, thus corresponding to the serial number read in from 
Fig. 5-75. Flowchart of automatic photodiode data processing at Shiva.

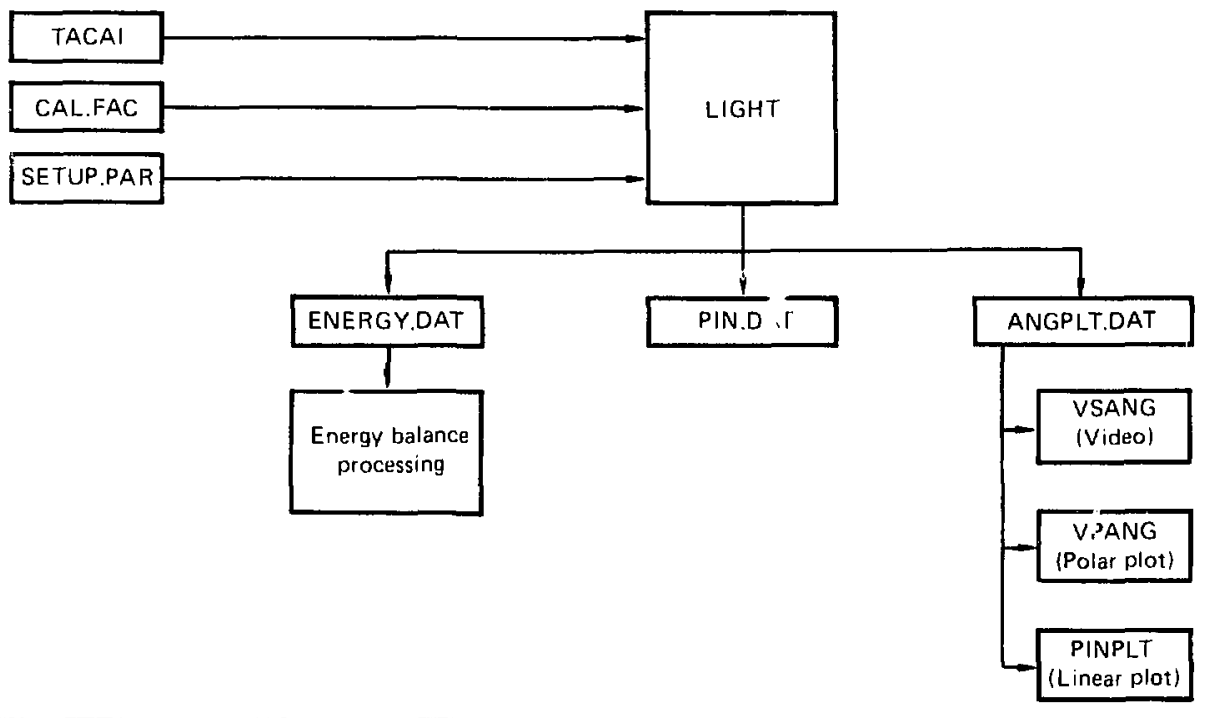

Table 5-14. Sample tabular output of subwutine PINDAT of Shiva seattered light photodiode data automatic processing program LIGHT.

\begin{tabular}{|c|c|c|c|c|c|c|c|c|c|c|c|c|c|c|}
\hline DETR & RPRIME & TRANS & $\mathbf{R}(\mathbf{C M})$ & PHI & THETA & SR & $\mathbf{C H}$ & POS & $\mathbf{C R}$ & ST & PC/VOLT & PC/CNT & ADC & ENERGY (J/SR) \\
\hline $\begin{array}{l}020 \\
010 \\
034 \\
035 \\
033 \\
032 \\
031 \\
037 \\
017 \\
024 \\
030 \\
027 \\
028 \\
013 \\
070 \\
011 \\
080 \\
050 \\
038 \\
010 \\
016 \\
019 \\
021 \\
015 \\
060 \\
023 \\
029 \\
014 \\
039 \\
022 \\
040\end{array}$ & $\begin{array}{l}0.10 E+07 \\
0.69 E+06 \\
0.12 E+05 \\
0.12 E+06 \\
0.12 E+06 \\
0.11 E+06 \\
0.12 E+06 \\
0.77 E+06 \\
0.12 E+07 \\
0.88 E+06 \\
0.12 E+07 \\
0.11 E+07 \\
0.11 E+07 \\
0.75 E+06 \\
0.11 E+07 \\
0.13 E+07 \\
0.11 E+07 \\
0.74 E+06 \\
0.12 E+06 \\
0.75 E+06 \\
0.78 E+06 \\
0.66 E+06 \\
0.11 E+07 \\
0.84 E+06 \\
0.11 E+07 \\
0.10 E+07 \\
0.11 E+07 \\
0.82 E+07 \\
012 E+06 \\
0.93 E+06 \\
0.11 E+06\end{array}$ & $\begin{array}{l}0.95 \\
0.95 \\
0.95 \\
0.95 \\
0.95 \\
0.95 \\
0.95 \\
0.95 \\
0.95 \\
0.95 \\
0.95 \\
0.95 \\
0.95 \\
0.95 \\
0.95 \\
0.95 \\
0.95 \\
0.95 \\
0.95 \\
0.95 \\
0.95 \\
0.95 \\
0.95 \\
0.95 \\
0.95 \\
0.95 \\
0.95 \\
0.95 \\
0.95 \\
0.95 \\
0.95\end{array}$ & $\begin{array}{l}92.1 \\
92.1 \\
110.8 \\
110.8 \\
110.8 \\
110.8 \\
110.8 \\
92.1 \\
92.1 \\
92.1 \\
92.1 \\
92.1 \\
92.1 \\
92.1 \\
92.1 \\
92.1 \\
92.1 \\
92.1 \\
92.1 \\
92.1 \\
92.1 \\
92.1 \\
92.1 \\
92.1 \\
92.1 \\
92.1 \\
92.1 \\
92.1 \\
92.1 \\
92.1 \\
92.1\end{array}$ & $\begin{array}{r}126.0 \\
126.0 \\
54.0 \\
198.0 \\
90.0 \\
306.0 \\
216.0 \\
126.0 \\
126.0 \\
126.0 \\
126.0 \\
126.0 \\
216.0 \\
216.0 \\
216.0 \\
216.0 \\
216.0 \\
216.0 \\
54.0 \\
54.0 \\
54.0 \\
54.0 \\
54.0 \\
54.0 \\
306.0 \\
306.0 \\
306.0 \\
306.0 \\
306.0 \\
306.0 \\
306.0\end{array}$ & $\begin{array}{r}120.0 \\
105.0 \\
20.0 \\
20.0 \\
160.0 \\
160.0 \\
160.0 \\
45.0 \\
60.0 \\
75.0 \\
90.0 \\
135.0 \\
45.0 \\
60.0 \\
75.0 \\
90.0 \\
105.0 \\
120.0 \\
45.0 \\
60.0 \\
75.0 \\
90.0 \\
105.0 \\
120.0 \\
45.0 \\
60.0 \\
75.0 \\
90.0 \\
105.0 \\
120.0 \\
135.0\end{array}$ & $\begin{array}{r}1 \\
1 \\
1 \\
1 \\
2 \\
2 \\
2 \\
2 \\
5 \\
5 \\
5 \\
5 \\
15 \\
15 \\
15 \\
15 \\
12 \\
12 \\
12 \\
12 \\
17 \\
17 \\
17 \\
17 \\
20 \\
20 \\
20 \\
20 \\
11 \\
11 \\
11\end{array}$ & $\begin{array}{l}0 \\
1 \\
2 \\
3 \\
0 \\
1 \\
2 \\
3 \\
0 \\
1 \\
2 \\
3 \\
0 \\
1 \\
2 \\
3 \\
0 \\
1 \\
2 \\
3 \\
0 \\
1 \\
2 \\
3 \\
0 \\
1 \\
2 \\
3 \\
0 \\
1 \\
2\end{array}$ & $\begin{array}{l}6 \\
6 \\
5 \\
5 \\
5 \\
5 \\
5 \\
6 \\
6 \\
6 \\
6 \\
6 \\
6 \\
6 \\
6 \\
6 \\
6 \\
6 \\
4 \\
6 \\
6 \\
6 \\
6 \\
6 \\
6 \\
6 \\
6 \\
6 \\
4 \\
6 \\
4\end{array}$ & $\begin{array}{l}5 \\
5 \\
5 \\
5 \\
5 \\
5 \\
5 \\
5 \\
5 \\
5 \\
5 \\
5 \\
5 \\
5 \\
5 \\
5 \\
5 \\
5 \\
5 \\
5 \\
5 \\
5 \\
5 \\
5 \\
5 \\
5 \\
5 \\
5\end{array}$ & $\begin{array}{r}3 \\
3 \\
3 \\
3 \\
5 \\
5 \\
5 \\
5 \\
7 \\
7 \\
7 \\
7 \\
9 \\
9 \\
9 \\
9 \\
11 \\
11 \\
11 \\
11 \\
13 \\
13 \\
13 \\
13 \\
15 \\
15 \\
15 \\
15 \\
17 \\
17 \\
17\end{array}$ & $\begin{array}{l}0.30 E+05 \\
0.30 E+05 \\
0.10 E+05 \\
0.10 E+05 \\
0.10 E+05 \\
0.10 E+05 \\
0.94 E+04 \\
0.30 E+05 \\
0.30 E+05 \\
0.30 E+05 \\
0.30 E+05 \\
0.30 E+05 \\
0.30 E+05 \\
0.30 E+05 \\
0.30 E+05 \\
0.30 E+05 \\
0.30 E+05 \\
0.30 E+05 \\
0.30 E+04 \\
0.30 E+05 \\
0.30 E+05 \\
0.30 E+05 \\
0.30 E+05 \\
0.30 E+05 \\
0.30 E+05 \\
0.30 E+05 \\
0.30 E+05 \\
0.30 E+05 \\
0.30 E+04 \\
0.30 E+05 \\
0.30 E+04\end{array}$ & $\begin{array}{l}0.37 E+02 \\
0.37 E+02 \\
0.12 E+02 \\
0.12 E+02 \\
0.12 E+02 \\
0.12 E+02 \\
0.11 E+02 \\
0.37 E+02 \\
0.37 E+02 \\
0.37 E+02 \\
0.37 E+02 \\
0.37 E+02 \\
0.37 E+02 \\
0.37 E+02 \\
0.37 E+02 \\
0.37 E+02 \\
0.37 E+02 \\
0.37 E+02 \\
0.37 E+01 \\
0.37 E+02 \\
0.37 E+02 \\
0.37 E+02 \\
0.37 E+02 \\
0.37 E+02 \\
0.37 E+02 \\
0.37 E+02 \\
0.37 E+02 \\
0.37 E+02 \\
0.37 E+01 \\
0.36 E+02 \\
0.37 E+01\end{array}$ & $\begin{array}{r}240 \\
162 \\
51 \\
68 \\
63 \\
58 \\
58 \\
17 \\
248 \\
241 \\
388 \\
200 \\
-4 \\
137 \\
265 \\
354 \\
257 \\
124 \\
428 \\
171 \\
261 \\
285 \\
328 \\
229 \\
210 \\
241 \\
243 \\
178 \\
327 \\
203 \\
227\end{array}$ & $\begin{array}{l}0.79 E+02 \\
0.77 E+02 \\
0.69 E+02 \\
0.88 E+02 \\
0.85 E+02 \\
0.84 E+02 \\
0.73 E+02 \\
0.73 E+01 \\
0.69 E+02 \\
0.95 E+02 \\
0.10 E+03 \\
0.58 E+02 \\
-.12 E+01 \\
0.60 E+02 \\
0.81 E+02 \\
0.91 E+02 \\
0.74 E+02 \\
0.55 E+02 \\
0.12 E+03 \\
0.75 E+02 \\
0.11 E+03 \\
0.14 E+03 \\
0.10 E+03 \\
0.89 E+02 \\
0.61 E+02 \\
0.78 E+02 \\
0.75 E+02 \\
0.71 E+01 \\
0.90 E+02 \\
0.71 E+02 \\
0.68 E+02\end{array}$ \\
\hline
\end{tabular}


Fig. 5-76. Fiow chart of Shiva energy balance measurement (EBM) automatic data processing.

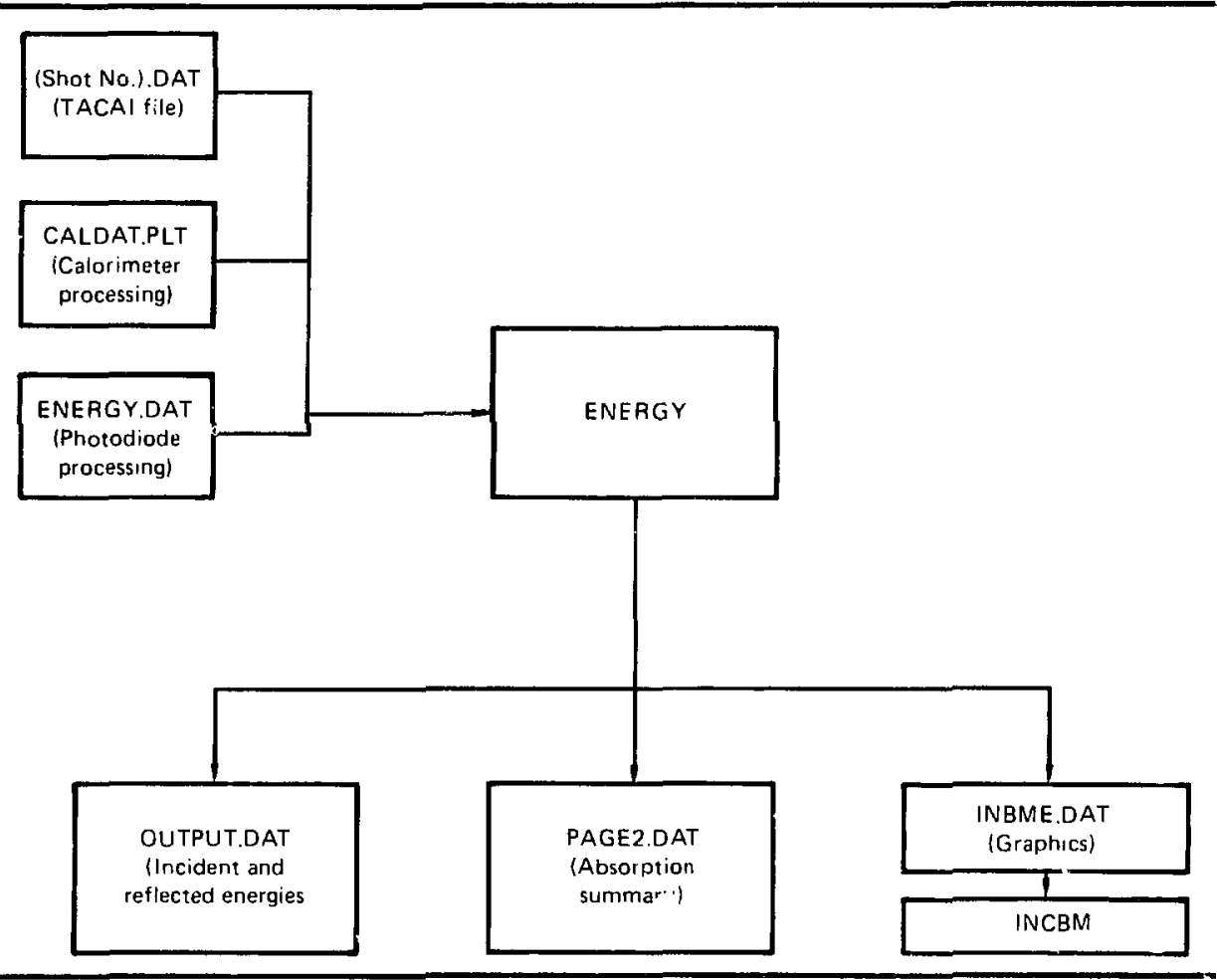

SFTLP.PAR: the elemeits of this dimension are essemtially the seating factors necessary to scale the delector to full voltage.

Hasing identified the nroper detector. conversion cocfifients, and scaling factors. LIGHT proceeds to calcubate the energy llux per counl and to subtract the r-ray hackground. This is done by the algorithm $E\left(J_{\text {ouls }}\right.$ (count $)=\left(R^{2} R^{\prime} \times\right.$ Transmission) times (picocoulombs sount), where $R$ is the distance (in $(n)$ between the target and the diode and $R^{\prime}$ is the diode sensitivity factor in terms of charge per energy flux (picocoulomh, Joule $\mathrm{cm}^{2}$ ). Th. result of this cialeutation is in turn multiplied by the counts corresponding to the integrator output for each detector.

LIGHT then creates three output files:

- ENERGY.DAT, an input ise to the energy balance routine.
- PIN.DAT. a hard-cops tabular lile (sect Table s-it for a ritmple PIVIDAT outpuis.

- ANGPL.T.DAT, an inpul file to the graphi s routines VSA V(i. VPANG. and PINPLT. These routines generalte, respeonels, a color vides polar distrihution displas. " hard-cops polar plot. and a hard-cops linciar plos.

Author: G. S. Chinen

\section{Automatic Diagnostic Data Processing: Energy Balance}

The primary purpose of the energy summary routine ENERGY (Fig. 5-76) is to integrate the angular distributions of scattered light and plasma energ: in order to obtain a relative measurement of 
the laser energt abourbed by the target from the top and hottom heid clusters. ENERGY is controlled by the indirece ammand lile EBF.CMD (on the PIPP $1 / 7(0)$, wh requires three input files:

- iShot $\Rightarrow$ DAT, which contains the incident "Im encrgies a d the hack-reflected light energies anded hy Rulected Buam Diagnostics (RBDs). line latier d.h ariginate from al laser diagnostics Pup I1 34 al are appended to the end of the f y d ditital hol time

- IVRR Y.DAT, which contairs the phowhek enters lukes and spherical coordinates +3 iar and . 1/11, thal angles).

- CA.DA I Pl.T, which contains the plasma 1) deghe calorimeter energy lluxes and spherical . "sirduntes. als, Il at the integritted totill plasmat incergy.

FNERCiY ppends the bick-reflected heim encrgies to the fortodiode intensity averalges and calculates energ: 'ntensity at $"=0^{\circ}$. where $"$ is the polar angle of th. largel chamber. The anlensity at" $=9.7^{\circ}$ is assume to be the same as at $11=0^{\circ}$. and the intensity itt $"=17.7^{\circ}$ is extrapolated 10 be at Mraight-line fit $1 \mathrm{rom} A=45^{\circ}$ io $\mathrm{A}=20^{\circ}$. This calculation is th ' done for the lower beams.

The output :olats are appended to the light II.C.21) calorimeter intensity atverages and then both the photodinde intensities (with back-reflected energies) and light calorimeter intensities ( $w$ ith output total energie 1 atre sorted and integrated over the entire sphere by a straight line lit.

ENERGY then culeulates

- Total out put energy (in kJ).

- Total b. ck-reflected energy (in kJ and as a pereentage of the cotal output).

- Beam balance, i.e., the relative output energies from th upper and lower beam clusters, using the formu :

Be:am $\left.{ }_{h i l 1}=r_{I}-E_{B}\right) /\left(E_{T}-E_{B}\right) \times 100 \%$.

where $E_{T}$ is total upper output energy and $E_{B}$ is sotal lower output energy

- The absorption percentages of the photodiodes and light and plasma calorimeters, as well as their average absorptions.

- The absorption deviations of the photodiodes and calorimeters.
Table 5-15. Represenmative summary of Shiva automatic energy balance dinglostics.

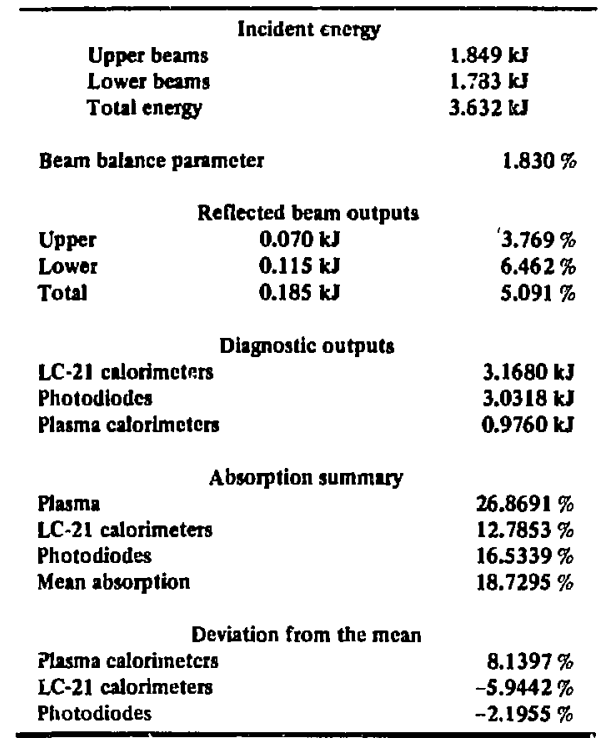

A sample output summary is given in Table $5-15$. The at sorption parameters indirectly summarise the amount ol energy absorbed by the target, and provide a measurement of energy balance ats indicaled by the relative contribution of the various energy components.

\section{Author: G. S. Chinen}

\section{Film Image Processing}

Photographic lilm has long been recognised as one of the most versatile detectors for recording electromagnetic encrgy signals. Film produces an archivable record of the signal and is relatively immune to undesirable electromagnetic interference. Film also has a dynamic range of over 5 orders of magnitude of input energy-a range which can be further extended using different kinds of film and/or thanging the development parameters-and can therefore be trusted to record signals under conditions of large uncertainty about the intensity of the inpul energy.

We process film images by first developing the film under highly controlled conditions, and then digitizing the density values very accurately with a 
photomicrodensitometer. The resulting record or file is then read into a computer for processing. We have written several MATHSY programs ${ }^{\text {th }}$ to manipulate our film data files and to plot the results (Figs. 5-77 10 5-82). Some of these programs are discussed helon.

film has a nonlinear respone to energh, and must be calibrated so that its nonlinear density value output translates into the input energy huence used for evaluations. We use film for three regioms in the electromatenetic spectrum:

- X rals from $200 \mathrm{eV}$ lo $>50 \mathrm{keV}$ : images in this region are produced by $x$-ray micrescopes, $x-$ rity spectrographs, pinhole cameras, and Frennel cone plate ciameras.

- Green light (from 2u litser light and the wsible light output of $x$-rily struak camerals).

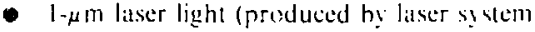
diagnostics).

The ealibrations for each of these regions are quite dilferent.

The calibration procedure lor x-raty film uses an x-ray mathine and requires many hours of exposure time and much interpretalion of the dittit. We presently use Kodak Iypes AA. M. R, and nosereen for $x$-ray detection. Of these we hate a fair calihration on type $M$ : quite a lot of wark has been done on types $A A$ and no-screen: very feu relatible calibration data have been ohlained for lype $R$,

There are several variables relating the aposure of $x$-raty film to the resulting film densits, including the photon energy of the $x$ ray, the fluence of the exposure, and the film development parameters (lime, chemical strength, and chemieal temperature). By keeping the development paramelers constant we can produce experimental curves for converting film datal to energy exposure.

To calibrate film used in the $x$-ray streik camera for imaging green light, we pre- or postexpose the film with a known light source and a step wedge. A plot of the digitized step "aedge provides the necessary ealibration eurve. Since green $2 w^{\prime}$ laser light is often produced by a pulsed source, however, we derive its calibration using the same technique as that used for $1-\mu \mathrm{m}$ light.

To derive a calibration curve for $1-\mu \mathrm{m}$ light we use a multiple-image camera at the jaser output that produces two images with known differences in intensity $(\sim 50 \%)$. These exposures are on the same film, thus eliminating the errors introduced by variations in development parameters. We then produce for ealch image a hislegram. or plot of number of points is litm densily. These plots are compared, using the known sor; intensily dilference in the exposures. to produce plots of densily is energ! (exposure): this is done with a MATHSY command program called CALiB2.

PIDSMES. PISSMIES is a MATHSY command file writlen 6 do the necessar! unlolding of the densilometer-produced datit file from an s-rals

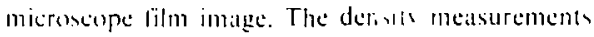
are ilfeceled b:

- Source, intensits, and speciral distrihutuon.

- Viller tramsmismion.

- Mirror reflectirits.

- Villia sensitisits is photon energy.

- Film development paratmeters.

Typical larget spectral distributions were not "ell known at the lime this code " ats writen and are therelore nut considered in the unfold. Spectras distribution is nonetheless certitinly one of the prime parameters for consideration in improsing the code.

The source intemsits seen by the film is deternined hy largel emission, reflecter-filter combinitlions, and microscope geometry: PDSMIES is alliare of micrencope geometry al a geometrical face10r. Both the filter transmission and the mirror reflectiv ity are uned by PDSMIES when it unfolds the datta: values for these parameters come from the ditat in the L-Division Library and are stored as a file in the tape library. Parameder files are read into MATHSY program HFF, where ae calculate

- The arerige channel energes.

- The effective encreg width of the channel.

- The intrage channel efficiene?.

These satues are stored in the datid fite, MSPY.

Film ensilivils data are contained in PDSMIFS in mo forms. The original program sonlained it lit for M-lype film: AA-lype film was atded later. M-type litting is done from a set of lables generated from three parameter curves that were compuler-lit to experimental datid. Data for A A-lype fitting is stored in a functional form. derived from a best fit to experimental curves. Film development parameters are controlled to match as nearly as is practical those used for the experimental curves.

PDSMIES produces output as shown in Fig. 5-77, in which ine - w and column scans are taken through the centroid of the data.

POWER. A MATHSY command file called POWER takes the ciensity tile from a streak camera 

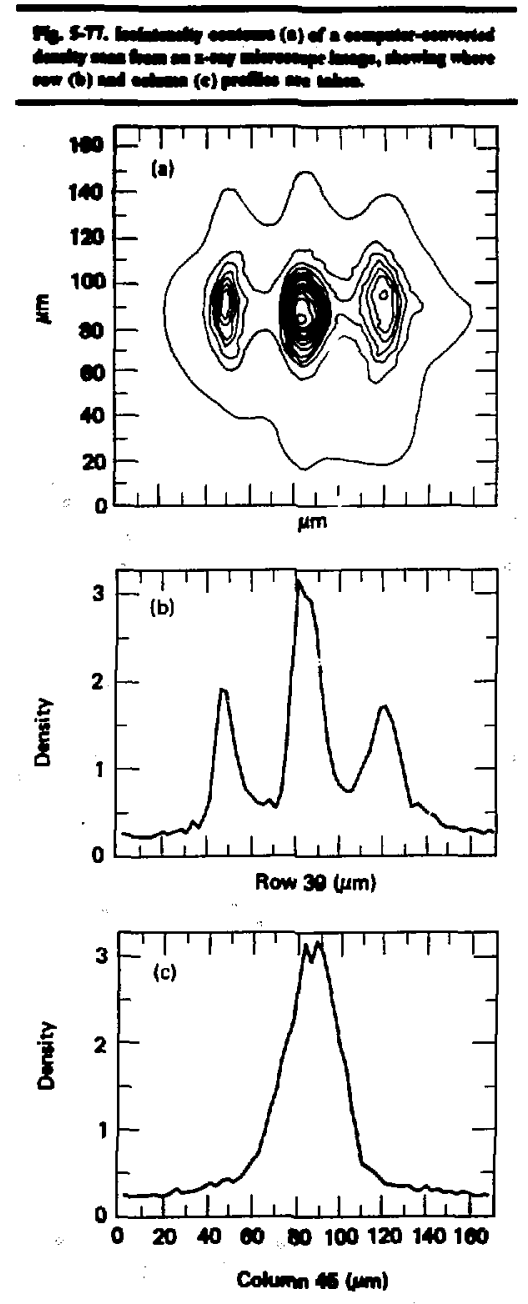

image of a laser beam and produces a plot of intensity vs time. A step wedge is also exposed onto the film; these images are developed together to control for all development parameters. From the step

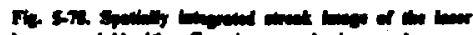

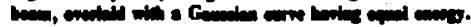

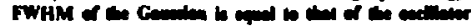
mine wim.

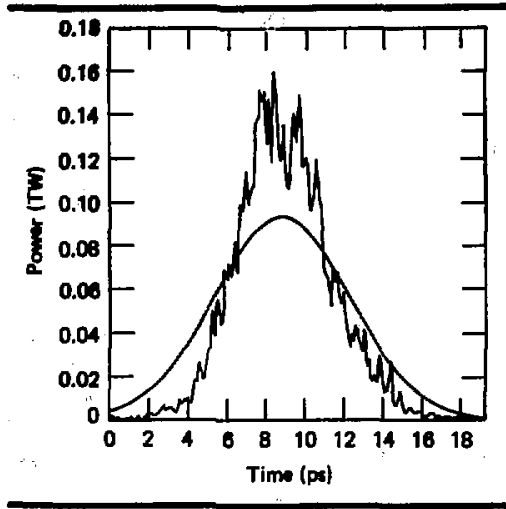

wedge we produce a $\mathrm{d}-\log \mathrm{E}$ curve which is used to unfold the relative exposure value from the density file produced by the densitometer reading of the streak image. This relative exposure is then inlegrated in the spatial direction and normalized to input energy from a laser system autput calorimeter; thus we produce a curve showing power vs time (Fig. 5-78).

Having recorded the FWHM of the Gaussian liser oscillator pulse, we then overlay a Gaussian curve for comparison with the measured temporal pulse. In this way we can easily evaiuate the temporal change in a pulse that passes down a chain of amplifiers.

EXTRACT. There is always a compromise in computer reduction of film data; if the densitometer sample size is 100 small the number of samples in the file becomes large, and data processing efficiency deteriorates. On the other hand, if the sample size is 100 large, thus keeping the file small, the data statistics suffer. To minimize errors and maximize speed, we have written the program EXTRACT, which allows us to display any part or all of the processed data. With this capability we can choose a section of the file, write it into an output file created by EXTRACT, and then trim and average the output file to the optimum size for both maximum processing efficiency and minimum statistical distortion. The cutput binary file created 
Fig. 5-79. Isointensity contour of the laser bean characterization, converted by FOCUSED from the measured density scan.

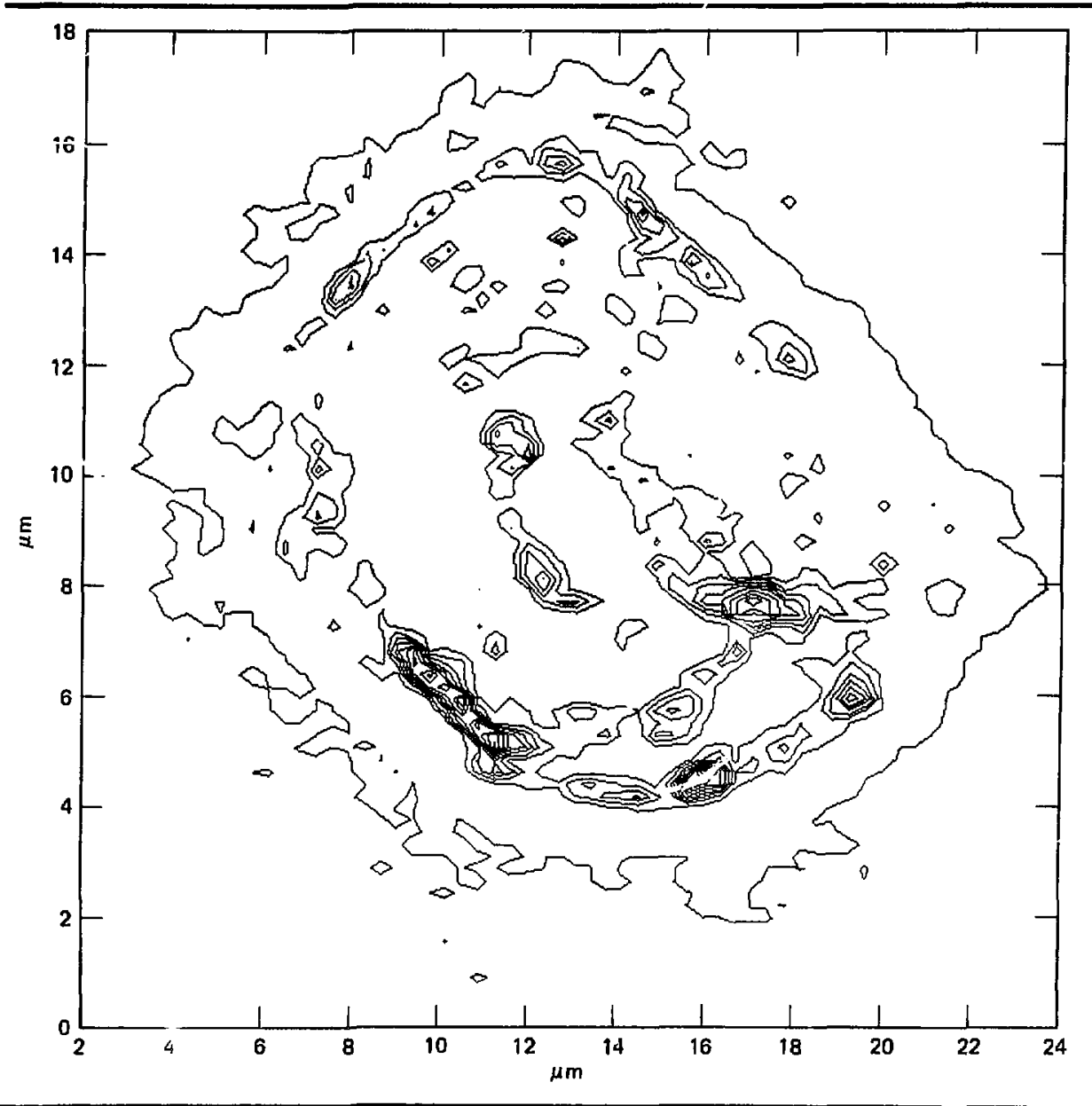

by EXTRACT can i: so be read by any of our other film image processin routines.

ACTOEX. The data file that characterizes our $x$-ray microscope consists of an array of point sources projected through the microscope; we use the file to determine the resolution of the microscope as well as the distortion in its field of view. ${ }^{78}$ The file is so large, however, that the computer memory cannol contain it and do processing on it at the same time: we must therefore read in and process small sections of the file one at a time. AUTOEX is a MATHSY code that was written to process a very large file in an automatic mode, but which also has a manual mode enabling us to see the data as it is processed.

The first problem to be solved by AUTOEX is locating the point source near the center of the array, to be defined as the starting point for the atutomatic processing. This is done with a patternsearch algorithm that looks for three points located within a total maximum spacing differential; the points are located using a routine that finds the certroid of the small file. based on the density values 
Fig. \$-80. Laser beam radial power profle, showing power radius normalized with energy as read from the calorimeter.

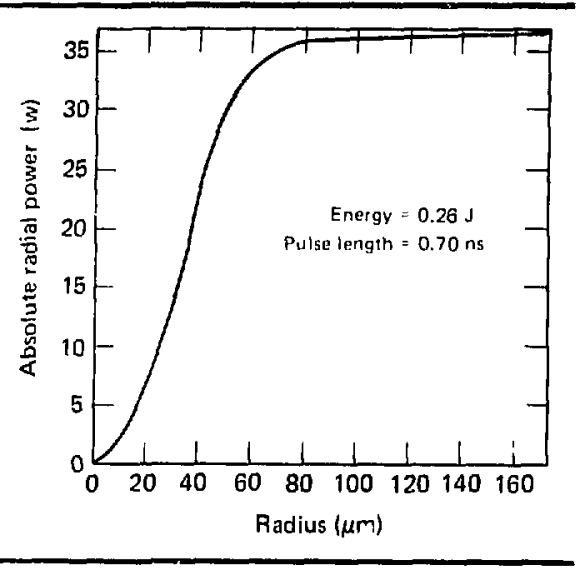

ahout the 50\% of maximum value of that small file.

The output file created by AUTOEX consists of the following listing derived for each point:

- Background, found by averaging the intensity value found in the corners of the small file.

- Peak value of the signal with noise removed

- X-position in row number and micromelers. as found hy the eentroid routine.

- Y-position in column number and in micrometers.

- The FWHM value of a row taken through the centroid.

- The FWHM of the column through the centroid.

- The diameter of the radial average of the intensity ahout the centroid taken at the half maximum value.

Hig. 5-81. Three-dimensional plot of the laser beam profile.

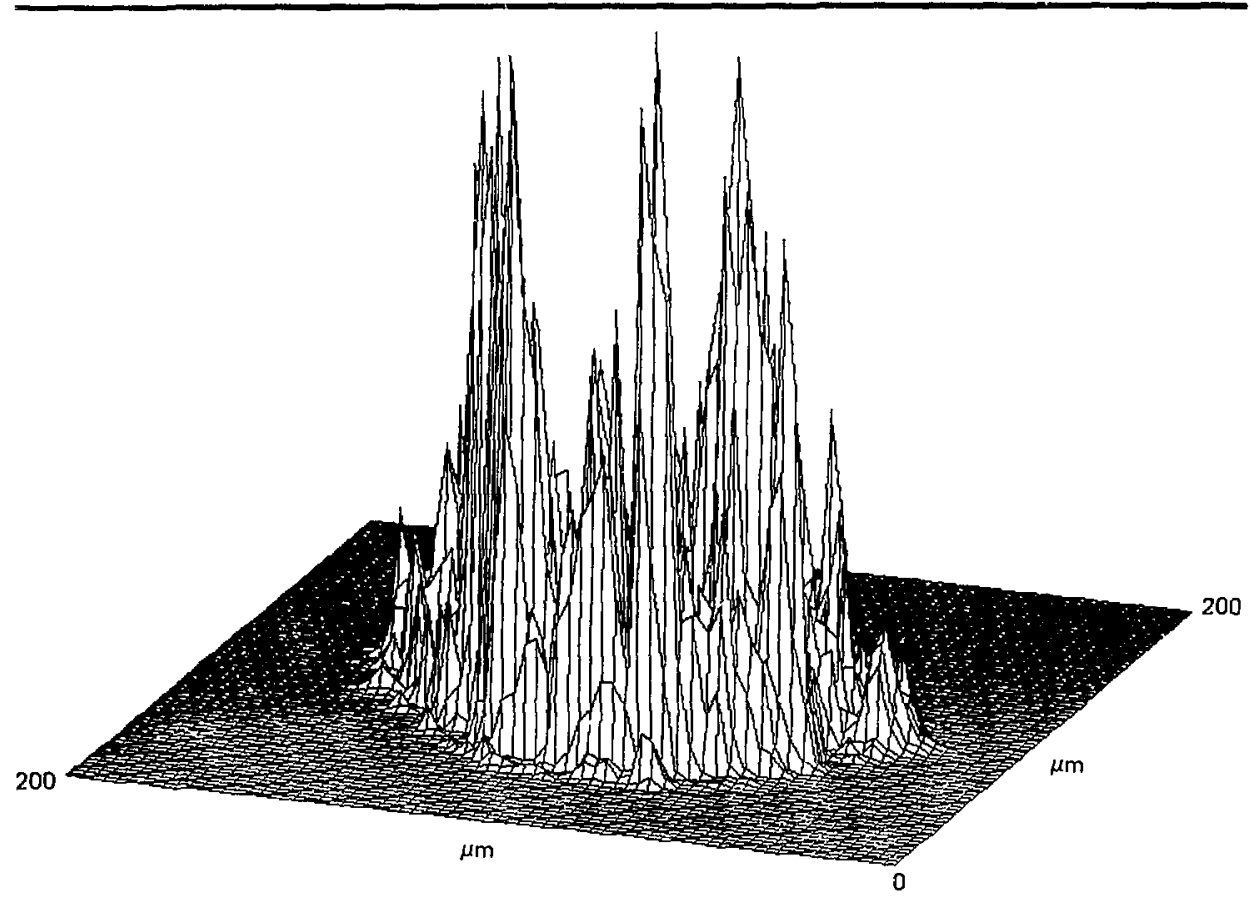


Fig. 5-82. Intensity histogram, showing the number of samples at a given Intensity vs the intensity of the samples.

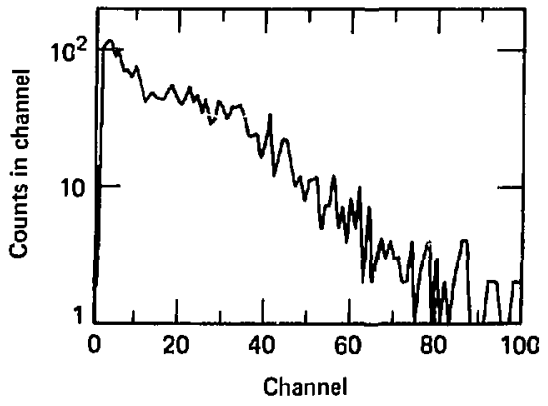

We compare the position of these points at ditferent places in the arraly to look for field distortions; by comparing widths and diameters we can see the spatial resolution of the $x$-ray microscope.

FOCUSED. FOCUSED was written to do heam analysis. CALIB2 is used to create al callibraltion file: FOCUSED then employs the file to convert the densuly data into intensity values and present a display of the calihrated data with isointensity lines (Fig. 5-79). Superimposed on this display is a series of concentric cireles; if the image appears to be more elliptical, the eircles can be changed to ellipses. These circles or ellipses ar: then used to calculate a radial average of the hedm intensity. By integrating and normalising witt, the known input energy we can calculate and plot the radial power profile of the beam as in Fig. 5-80.

We can delete bad spots in the data with FOCUSED by using the command CEN 10 center about the point to he operated on. The command AVOUT will then remove the values from the area inside the inner diameter and fill it in with the average value of the datla contained hetween the inner and ouler diameters.

FOCUSED also allows us to

- Look at either a single data row or column, or at the average of several rows or columns from any place in the array.

- Do a three-dimensional display (Fig. 5-81).

- Do an intensity hisicgram (Fig. 5-82) which displays the numbei of data points contained in each channel of energy vs the energy of that channel.

\section{References}

76. Laser Program .4mmal Report-1976. Laturencis I inore Laboratory, Livermors, Calif., UCRI.-5002!, , (ig77), pp. 2-359 to 2-364, 3-136.

77. T. L. Harper, Kudal. $M X$-Ray Film Calibraim Calculavions wed for Mireserope Data Analwix, UOPB-76-7.3, May 1976. J. P. Stuering and A. Toor, K-Ral Calibration of Nodak No-Sirean. Type A.A, and Tipe M in the l-4.5 hel Region, l(J)-16775, Mily 8. 1975. A. Toor, A Plision Motel for f:-Rat Film Sinwitivitis, XRM-79-14.3, Sept. 20, 1979.

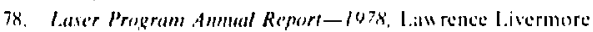

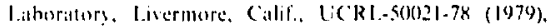
p). 0.27 1110.29

\section{CCD Array Processing}

In laser fusion experiments, target and laser parameters are measured using various camerals, inchuding oscilloscope cameras, ultafast streak cameras, and $x$-raty microsene camerals. Film has been the usual recording medium, but as more instruments are added to diagnose target experiments, the need for automatic digital readout hecomes essential. Transient digitizers such as the Tektronix R7912 have replaced some oscilloscopes, aiding our efforts to implement digital readout of hoth streak camera images and oscilloscope traces. We have successfully oblained a direct readout of streak camera images using a CCD (charge coupled device) array camera, which bypasses film retrieval. development, scanning with a microdensitometer, and datti correction for film response. The hardware description for the CCD streak eamera is given earlier in this section, and in last year's annual report ${ }^{79}$ : this article eovers CCD image processing and our plan to handle all image processing in a unified manner.

Becaluse we anticipated that a number of these CCD arraly cameras would be institled at the Shiva facility, we have undertaken to acquire the images from all the CCD canteras through one system. using Shiva's existing computer architecture. ${ }^{x()}$ Uur solution is to attach the CCD memories to the bus implemented under Shiva's power condilioning system ${ }^{81}$ :

- Data are accessed through an LSI and at four-port memory from either the PDP 11 .3t or PDP $11 / 70$. 
Fig. 5-83. SXRSC data from a Shiva shot. Color contours of intensities sit overlayed with temporal distribution from a channel.

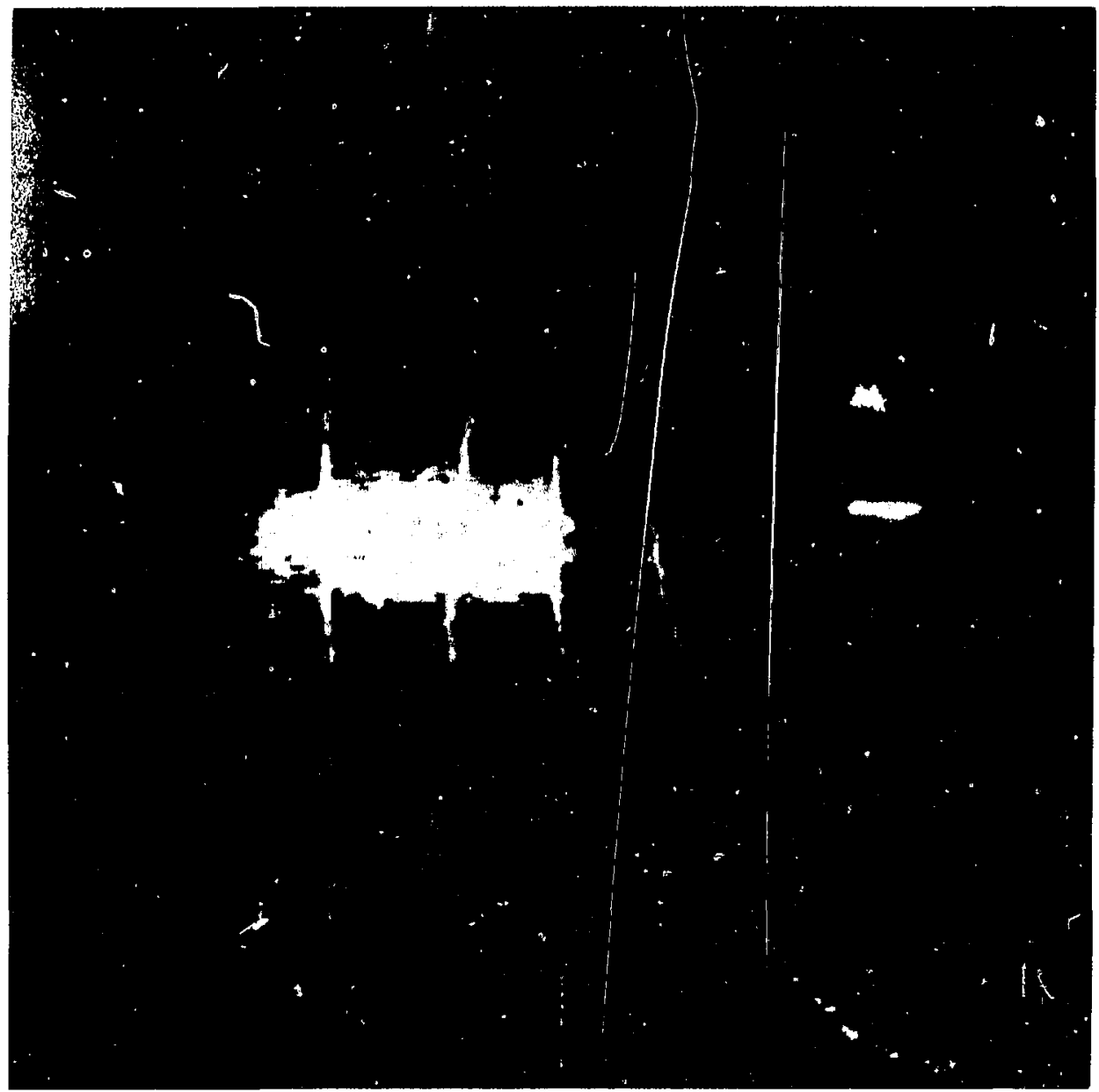

- Data are transferred from the CCD memory in either column or row mode: normally, $160 \mathrm{~K}$-bytes of data are transferred in approximately $30 \mathrm{~s}$.

- Data can be manipulated in the PDP $11 / 70$; the data format is given in Table 5-16.

We presently operate CCD camera and memory systems on output-beam-diagnostic optical streak cameras and on a target-diagnostic soft " - ray streak camera (SXRSC) ${ }^{82}$ : we discuss processing for the SXRSC in this article.
Initial quick-look processing of SXRSC data is performed on the streak image immediately after the shot, using the Shiva PDP I1/70. The image is displayed on a color monitor, with intensity values converted to color contours. Lineouts in one spatial direction can be obtained to display the temporal profile of the $x$-ray distribution. These car: be superimposed on the color-enhanced distribution or drawn by the Versatec printer/plotter (Fig. 5-83).

The final unfolding of the data is performed through Octopus on the LLL central computer system. The image data are converted into an 8-bit 
Table 5-16. CCD memory data format (160 K-bytes of image).

\begin{tabular}{|c|c|c|}
\hline \multicolumn{2}{|c|}{ Byte offsets } & \multirow[b]{2}{*}{ Contents } \\
\hline Decimal & Oetal & \\
\hline 1 & 1 & Last shotnumber (ASC II) \\
\hline 9 & 11 & ASC II time of dey (HH:MM:SS) \\
\hline 17 & 21 & $\begin{array}{l}\text { Taskname of task generating the } \\
\text { file }\end{array}$ \\
\hline 19 & 23 & (RADSO) \\
\hline 21 & & Mode: $1=$ continous, $0=T V$ \\
\hline 23 & & Number of records written (binary) \\
\hline 25 & & Error status of return (blnary) \\
\hline 27 & & Memory number $(0.99)$ \\
\hline 29 & & \\
\hline & & \\
\hline 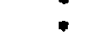 & & \\
\hline 1024 & & \\
\hline
\end{tabular}

ASCII file and written on magnetic tape as a PDP $11 / 70 \mathrm{RSX}-11 / \mathrm{M}$ file. An interface program is then run on the Octopus system 10 decode the tape into a standard Octopus 6-bit ASCII file. The analysis program, SXRSCX, is written in MATHs: ${ }^{83}$ SXRSCX obtains lincouts for each energy channel. and then folds the temporal profiles with responses for each channel to oblain x-raly flux as a function of time. Integration of this distribution gives the total flux.

Streak camera data still recorded on film and digitized are analyed with a program called ATTWOOD. ATTWOOD is identical to SXRSCX except for the input routines and the fact that the CCD inage no longer needs to he corrected for the many nonlinear effects which complicate the analysis of film images.

The ultimate goal is to obtain all target diagnostic data, including images, in computercompitible digital form. In the meintime, since some film images and oscilloscope traces will need to be digitired after intermediate development steps, the interim goal is to integrate all digitized data in a comprehensive analysis package. Progress was made loward both goals by implementing the CCD camera and its software on the Shiva SXRSC, and by using existing fi!m datal processing programs for dita analysis.

\section{Author: J. T. Ozawa}

80. Laser Program Anmual Repert-1978. Lilu rence Livermore Laboratory, Livermore, Catlif., UCRL.-50021.78 (1979), pp. 2-45 to 2-54.

81. Laser Program Anmal Report-1977. Law rence I sermore Laboralory, Lisermore. Calif., U(CRI.-50021.77 (1978). рp. $2-41$ to $2-47$.

82. Laker Pragram Anmul Report-1978, L.an rence L.Acermurc Laboraturs. Livermore, Calif. (UCRl-50021-78 (1979), me. 6.2106 .5$.

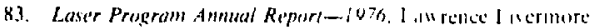

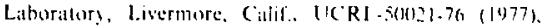
pp. $2-359$ in $2-364$.

\section{Target Alignment Codes}

With the advent of the Shiva laser system, we have heen challenged with the problem of aligning many laser beams to achieve specified illumination patterns on microscopic largets. Solved mantally, the probiem requires hours of drawing complicated graphs of targets and beam cones, als well as tedious computing of the results using diffraction theory: checking several alignment schemes manually would become prohibitively costly in terms of the manpower required. The need for an erlicient alternative provided the motivation for the larget alignment codes described in this article. The codes cin be divided into two cattegories:

- Those which produce beam position information.

- Those which produce the intensity distribution on a given larget als a result of a preselected beam alignment and power scheme.

All of the codes are based on relationships between the target and focusing-lens coordinate systems diagrammed in Fig. 5-84. Normally the origin of the target coordinate system is at the center of the chamber. Pasitive $X$ is directed along the target positioner, with $Z$ pointing up. Eitch focusing lens coordinate system is defined by the axis along which the focus moves lowards or allay from chamber eenter: the origin of each letrs coordinate sustem is the chamber center. As shown in the figure, the $Z_{i}$ axis is defined by lwo spherical angles. $H_{1}$ and $w_{1}$.

For the $j^{\text {th }}$ lens system, the, courdinate $Z$, lies outwards along the beam axis. The len can be offset in two perpendicular directions from the $Z_{1}$ axis; these are the $X_{1}$ and $Y_{1}$ coordinate axes. Positive $Y_{i}$ points towards the nearest pole of the

\section{References}

79. Laver Pregram Anmual Report-1978. Lawrenet Livermore Laboratory, Livermote, Calif, UCRL-50021-78 (1979), np. 6. $5410 \quad 6-59$. 
Fig. 5-84. Target and lens coordinate systems useci by the Shive target alignment codes.

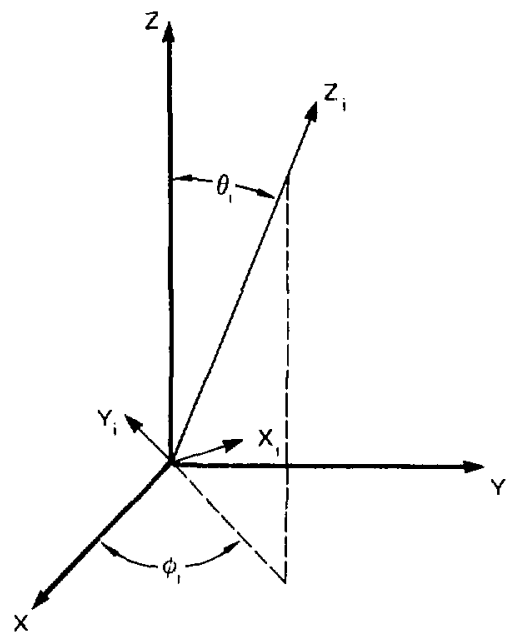

chamber, while posilive $X_{1}$ is such as lo lorm a righthanded system with $Y_{1}$ and $Z_{\gamma}$.

The targel and lens coordina' $s$ are related by a transformation matrix $T_{1}$, the elements of which are given in Table 5-17, along with the 20 values of $\|_{1}$ and the 20 values of $\Rightarrow$ for the Shiva illumination system. All the heam-position and intensity calculattions involve the use of this transfiormation matrix.

ALIGN and GFOBM. Most Shivit alignments are done under conditions of no tangential offset $\left(X_{1}=0\right)$ and the same $Y_{1}$ and $Z_{1}$ values for all heams in a cluster. In this case one has to work with only (wo) (an inner beam and an outer hean) of the ten beams in at cluster. For this situation, we bave developed a simpie computer code named ALICN. Given a set of lens offsets $\left(Y_{i}, Z_{j}\right)$. and either a spherical target or al disk larget of specified diameter and tilt angle, ALIGN

- Draws a graph showing the cones of an inner and outer beam positioned on the target.

- Allows one to rapidly change the lens coordinates (presets).

- Can position beams on an arbitrarily tilted disk so that all energy is incident on the target.
Table 5-17. Transformation matrix $\mathbf{T}_{\mathbf{i}}$, and Shiva illumination coordinates.

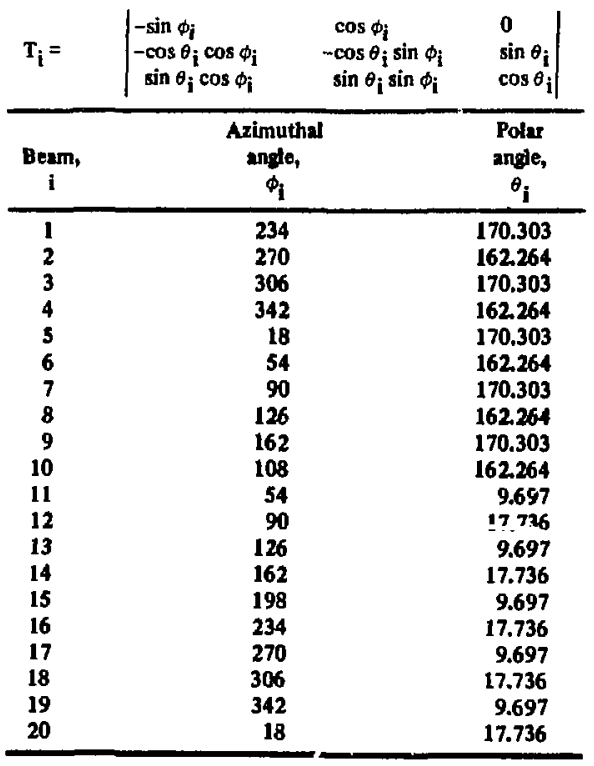

Fig. 5-85. Sample Versatec ryinter output from ALIGN, showing th parameter for tangential focusing on a sphere.

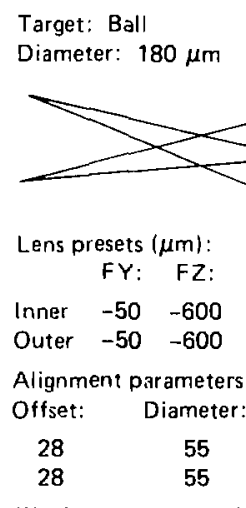

(\% of target diameter)

- Draws various diagrams showing, for example, when the beam cones are positioned so that the outer ray of each cone is tangential to the surface of the sphere: Fig. 5-85 is a sample diagram for the case of such "tangential focusing." 
- Computes the displacement of the center of each heam axis from larget center and the beam diameter at the target central plane, expressed as fractions of the target diameter. These data are useful for allignment verification and are displayed on each alignment diagram, as illustrated in Fig. 5-85.

The second computer code which produces beam positioning information is named GEOBM. GEOBM has 1 wo independent routines: GEOSPH, which gives data for alignments on at spherical tatrget, and GEOPLN, which gives data for alignments on a planar target. The computational method for GEOPLN is explained here in detail: GEOSPH uses very similar methods.

The GEOPLN user specifies both the lens presets $\left(x_{1}, y_{j}, t_{i}\right)$ for the 10 beans in one of the Shiva clusters and the diameter, tilt angles, and vertical displacement of a planar larget in the target coordinate system whose origin lics at the chamber center. Tilts can be specified as an angle of rotation about the stalk and a tilt angle about an axis perpendicular to the stalk. The disk larget is then subdivided into a polar grid with a maximum azimutha! resolution of $2^{\circ}$ and a maximum radial resolution of $1 / 45$ of the radius. For GEOSPH, spherical coordinate nesh is set up. The maximum arimuthal resolution and the maximum polar angle resolution are both $2^{\circ}$.

On polar grids having radial lines every $20^{\circ}$ and circles of equal radii at intervals of $1 / 10$ of the target radius. GEOPLN draws diagrams showing the intersection of the geometric optic cone of each heam with the planar surface. These intersections are calculated according to the following procedure.

Each point on the dise $\left(x_{1}, y_{1}, x_{1}\right)$ is transformed into the coordinate system of the $i^{\text {th }}$ lens; any point $X_{t}$ may lie out of the $X-Y$ plane of the target coordinate system (see Fig. 5-84) as a result of specified tilts or displacements.

The corresponding lens coordinates $X_{1}$ are given by

$$
\vec{x}_{i}=T_{i} \vec{x}_{t} .
$$

If we let $\vec{F}_{j}$ be the focus of $i^{\text {th }}$ lens, then relative to this focus the point $\overrightarrow{\mathrm{X}}_{\mathrm{i}}$ has the coordinates

$$
\overrightarrow{\mathrm{x}}_{\mathrm{i}}=\overrightarrow{\mathrm{x}}_{\mathrm{i}}-\overrightarrow{\mathrm{F}}_{\mathrm{i}}
$$

Fig. 5-86. Dlagram showing the basis of the GEOPLN calcosution for fiading the istersection of a beam cone with a planar surface.

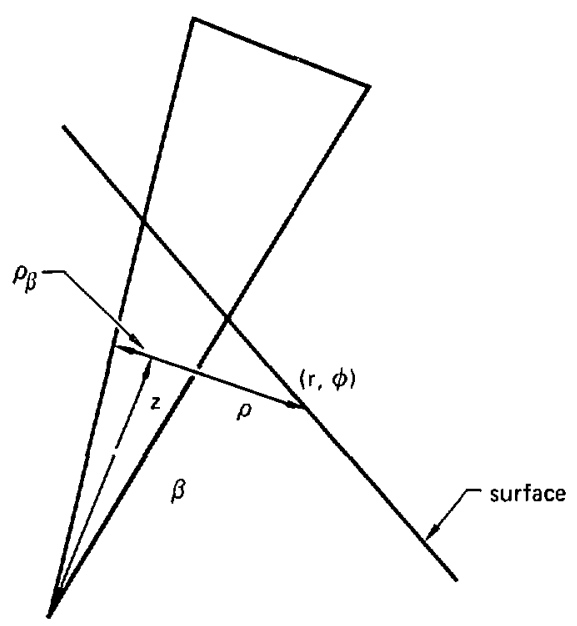

Focus of ith beam

$\vec{X}_{i}$ is next expressed in terms of polar coordinates ( $\%$. 1), where

$$
\rho=\sqrt{X_{i}^{2}+y_{i}^{2}}
$$

and $/=l_{1}$.

At this point. the code determines whether or not the point $\vec{x}_{1}$ is intercepted by the $\mathrm{i}^{\text {th }}$ heam cone. The radius of the cone correspanding to the distince $l$ is given by

$$
\rho_{\beta}=|z| \tan _{\beta},
$$

where of is the fens half-angle for the ith bean. Then the point $\vec{X}_{1}$ is on the cone if

$$
\rho=\rho_{\beta} .
$$

A diagram showing the conditions expressed by the above equations is given in Fig. 5-86. 
Fig. 5-87. Sample GEOPLN outpat showing the intersection of 10 Shiva beams with a disk tited $30^{\circ}$.

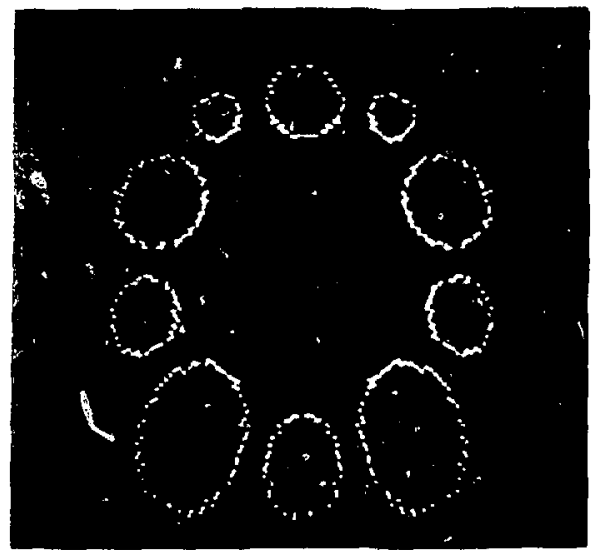

Fig. 5-88. Sample GEOSPH output showing the intersection of 10 tangentially focused Shiva beams with $180 \mu \mathrm{m}$ diameter hemisphere.

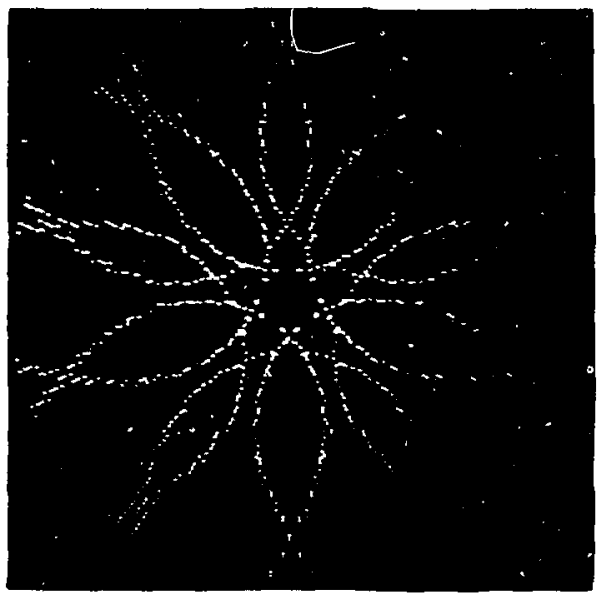

Alter determining all points of the polar mesh which intercept any of the 10 beam-cones in a cluster, GEOPLN draus beam-position polar diagrams (Figs, 5-87 and 5-88). Figure 5-87 shows a GEOPLN output for beams positioned on at disk tilted $30^{\circ}$. Figure 5-88, a sample GEOSPH output, shows a plot for 10 beams tangentially aligned to a sphere. Recall that this is a polar plot of a hemisphere:

- The radial coordinate corresponds to the spherical angle $\#$.

- The azimuthal cesordinate corresponds to the sphericit angle $/$. $10^{\circ}$

- Circles of constant theta are separated by $20^{\circ}$.

- Radial lines on the grid are separated by

Using GEOBM, the user can rapidly select several sets of lens presets and view the resulting beam positions on target until the desired alignment is ohtained.

LITAR, PLANAR, and NOVAl. The next set of codes to be described produces more than beam position information; these codes calculate and display the incident and ahsorbed intensity distribution for preselected target type, lens presets, and power in each beam. The three codes and their functions are

- LITAR, which computes the incident and absorbed inlensity distributions on a hemisphere irradiatted by a Shiva heam cluster.

- Planar, which computes the incident and absorbed intensity distributions on a plane irradiated by a Shiva bean cluster.

- NOVAI, which computes the incident and absorhed intensity distribution on a hemisphere irradiated by both proposed beam clusters on the Nova laser system. This code allows the user to specify the lens axis angles $\#_{\mathrm{i}}, \Phi_{\mathrm{i}}$ and thus evaluate various proposed beam geometries.

Each code divides a hemisphere or disk of userspecified diameter into a spherical or polar grid, respectively. The grid can have a maximum of 2700 points. For each point the codes calculate its distance from the focus of the $\mathrm{i}^{\text {th }}$ lens, using Eqs. (6) through (8) above. To calculate the intensity at that grid point, the code provides a model of the beam profile using a series of Gauss-Laguerre polynomials $\Psi_{i}(\rho, Z)$, where $n$ is defined as in Eq. (8) and $z=Z_{i}$. 
Fig. 5-89. (a) Sample PLANAR output showing the incident intensity distribution on a disk tilted $30^{\circ}$; (b) absorbed intensity distribution of (a) based on a resomance absorption model.

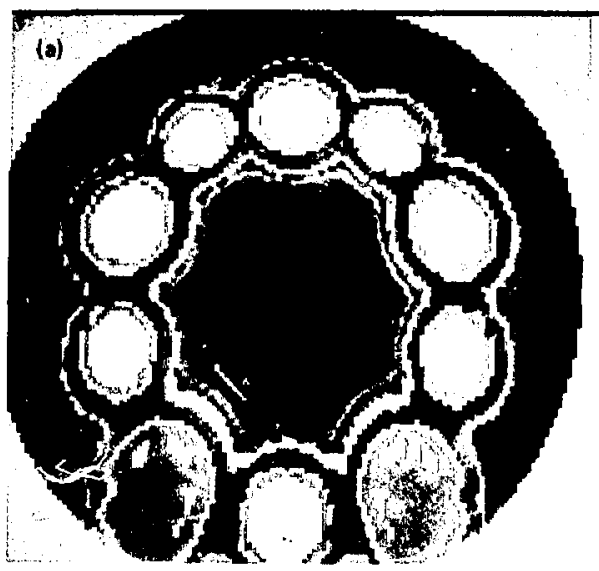

$\begin{array}{ll}\text { White: } & 1.5 \times 10^{16} \mathrm{~W} / \mathrm{cm}^{2} \\ \text { Pink: } & 7.5 \times 10^{15} \mathrm{~W} / \mathrm{cm}^{2} \\ \text { Yellow: } & 3.8 \times 10^{15} \mathrm{~W} / \mathrm{cm}^{2} \\ \text { Orange: } & 1.9 \times 10^{15} \mathrm{~W} / \mathrm{cm}^{2} \\ \text { Red: } & 9.5 \times 10^{14} \mathrm{~W} / \mathrm{cm}^{2} \\ \text { Brown: } & 4.8 \times 10^{14} \mathrm{~W} / \mathrm{cm}^{2}\end{array}$

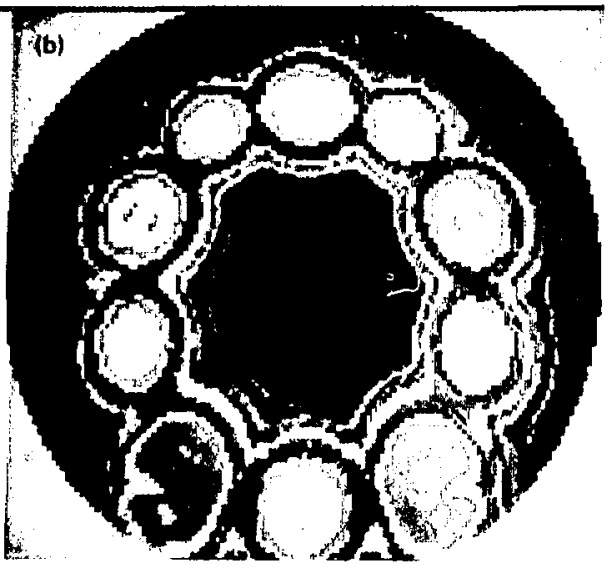

$\begin{array}{ll}\text { White: } & 4.6 \times 10^{15} \mathrm{~W} / \mathrm{cm}^{2} \\ \text { Pink: } & 1.5 \times 10^{14} \mathrm{~W} / \mathrm{cm}^{2} \\ \text { Yellow: } & 2.5 \times 10^{15} \mathrm{~W} \mathrm{~cm}^{2} \\ \text { Orange: } & 5.75 \times 10^{14} \mathrm{~W} / \mathrm{cm}^{2} \\ \text { Red: } & 2.89 \times 10^{14} \mathrm{~W} / \mathrm{cm}^{2} \\ \text { Brown: } & 1.45 \times 10^{14} \mathrm{~W} / \mathrm{cm}^{2}\end{array}$

The user specifies the power $P_{1}$ in each beam. The function $\Psi$ is normalised so that at any value of $\mathrm{Z}$.

$$
\mathrm{P}_{\mathrm{j}}=2 \pi \int_{0}^{\infty}\left|\psi_{\mathrm{i}}(\rho, Z)\right|^{2} \rho \mathrm{d} \rho .
$$

The function $\Psi_{\mathrm{i}}(\rho, Z)$ is also parameterized by the beam waist size al focus, $W_{0}$ : for example the Shiva $\mathrm{f} / 6$ lenses focus spatially filtered $1.06-\mu \mathrm{m}$ beams to an effective waist size of $12 \mu \mathrm{m}$.

A1 each grid point the angle between the surface normal and the local bean direction is conputed. The iricident intensity at the $\mathrm{N}^{\text {ih }}$ grid point is computed as follows:

$$
\mathrm{I}_{\mathrm{n}}=\sum_{\mathrm{i}=1}^{\mathrm{N}}\left|\psi_{\mathrm{i}}(\rho, \mathrm{Z})\right|^{2} \cos \phi_{\mathrm{i}},
$$

where $\phi_{i}$ is the angle of incidence.

The codes also calculate total incident and absorbed power using the formulas

$$
P_{\text {inc }}=\sum_{j=!}^{M} I_{n} s A_{n}
$$

and

$$
P_{a b s}=\sum_{j=1}^{M} n_{n} n_{n} A_{n}
$$

where ${ } A_{11}$ is the area nucrement lor the $N^{\text {th }}$ grid increment and ${ } n$ is the ahourption fraction based on either a resonance absorption or inverse bremsstrahlung model.

The codes calculate and produce incident and absurbed intensity distributions, in either tabular or graphic form: graphic ouputs can be either sym. bolic contour plots from al Versatec printer or at color-coded intensity plot produced on at color TV monitor. Examples of the latter are shown for PLANAR (Fig. 5-89), LITAR (Fig. 5-90), and NOVAI (Fig. 5-91). 


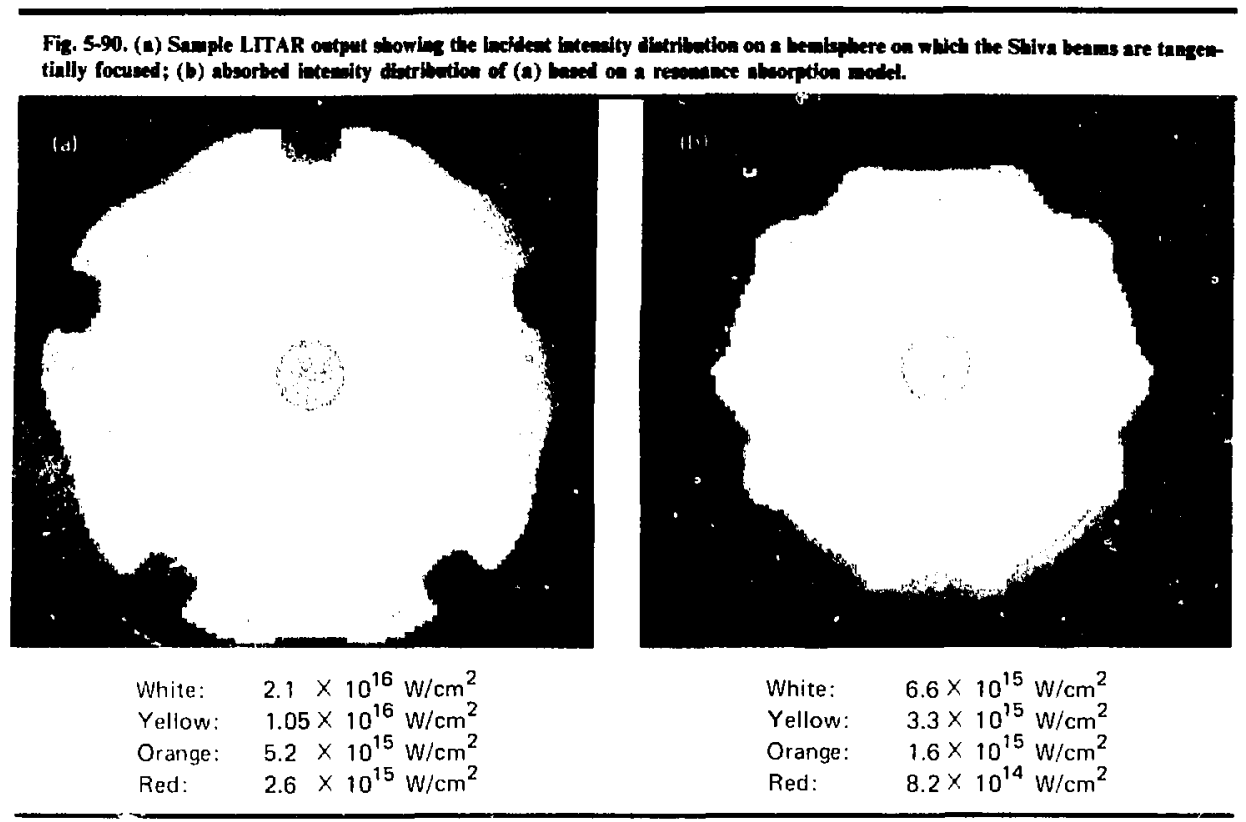

The intensity plot generated by PLANAR is in pular courdinate form. For PLANAR outputs, the onter ratdius of a plot corresponds to the outer ridius of the target. For LITAR and NOVAl, the ples is a representation of a hemisphere in polar corrdinalles. The radial coordinate corresponds to the polar angle $\omega$. The center of the circle is the pole (1) $=0^{\circ}$ or $H=180^{\circ}$ ) and the outer radius is the equatur $\left.(")=90^{\circ}\right)$ : other values of $A$ are found by linear interpolation hetween the two. The azimuthal spherical angle $\phi$ corresponds one to one with the angular coordinate around the circle. The $\theta=0^{\circ}$ radial is the line at the three $0^{\circ}$ clock position on the plots that follow. Correspondence between colors and the intensities they represent appear with the plots.

Figures $5-89(a)$ and (b) present color-coded intensity plots for a PLANAR calculation; (a) shows the incident intensity distribution, and (b) shows the absorbed intensity distribution based on resonance absorption. The lens presets correspond to the beam positions shown in Fig. 5-87. Each of the 10 beams in the Shiva cluster is given an output power of
$0.5 \mathrm{TW}$. The effeet of the disk tilt on the internsit? distribution is very evident. Of the tolal power ( 5 TW) incident on the disk, 26 ' was atsorbed.

Figures 5-90(al) and (h) are the incident and ahsorbed intensity distributions for the "tangential focusing" alignment on at 180- $\mu$ m-diam hemisphere previously described in Figs. 5-85 and 5-88. Note the rapid decrease in intensity from pole to equator. This is a consequence of the high angle of incidenee hetween the beam and the target normal in the equatorial regions. Of the $10 \mathrm{TW}$ incident, $99 \%$ intercepted the target. The calculated absorption fraction using a resonance absorption model wats $33 \%$.

Figures 5-91(a) and (b) present results from the recently developed alignment code NOVAl. Shown are the calculated incident and absorbed intensity distributions for a Nova Phase 1 irradiation geometry un a $2000-\mu \mathrm{m}$-diam sphere. In the Nova Phase I configuration there are five $f / 4$ lenses with beam axis angles of $\theta_{\mathrm{i}}=55^{\circ}$ and $\Phi_{\mathrm{i}}=$ a multiple of $72^{\circ}$. The resulting large beam-cone angles allow a more uniform illumination than with Shiva, as a comparison of Figs. 5-90(a) and 5-91(a) shows. Figure 5-9l(b) shows an absorbed intensity dis- 
Fig. 5-91. (a) Sample NOVAl output showing the incident intensity distribution upon a 2000- $\mu$ m-diameter hemisphere irradiated by a cluster of Nova beams. (b) Absorbed intensity distribution of (a) based on an inverse bremsstrahlung model.

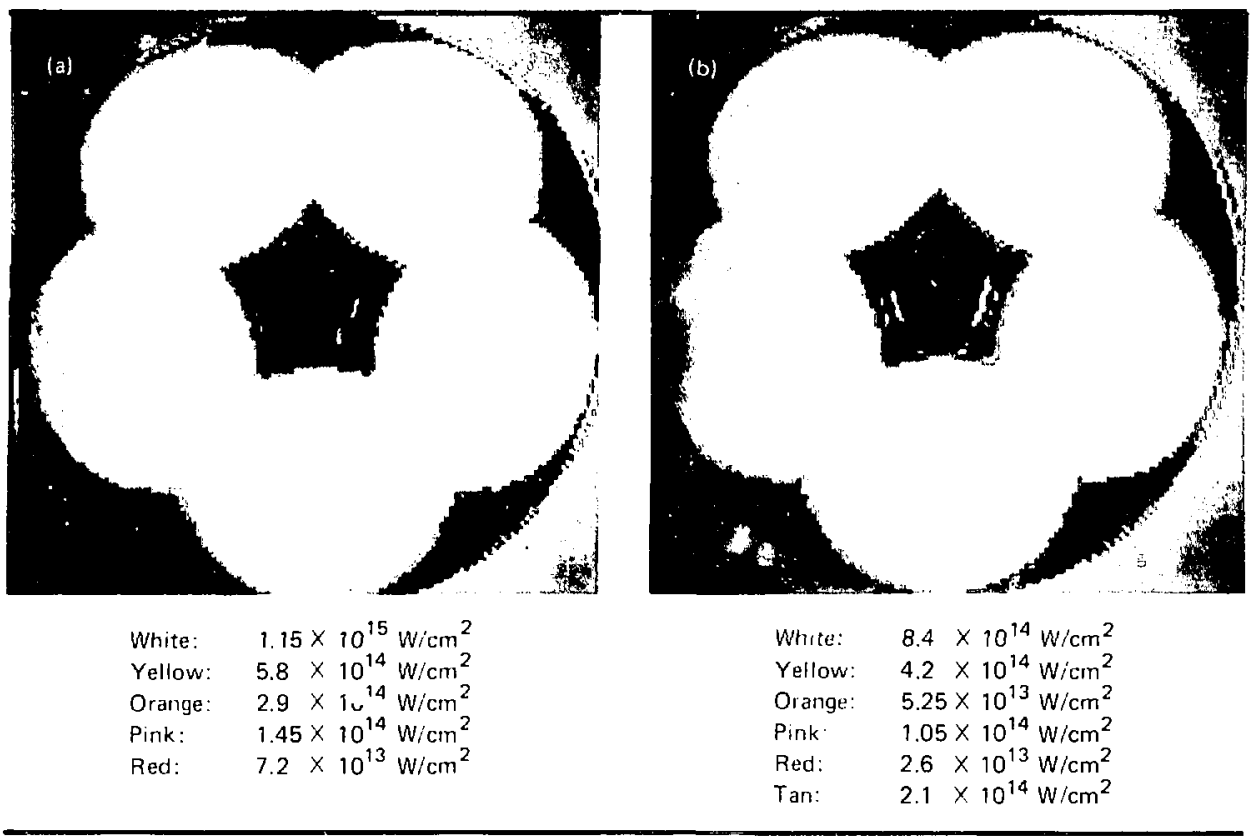

tribution: of the 50 TW which irradiated the hemisphere. $78 \%$ wals absorbed hased on an inverse bremsstrahlung calculation.

In the past year the ALIGN, GEOBM. LITAR, and PLANAR codes have become indispensible cools both for determining proper lens positions and for saving mans hours of experimenters' time.

Author: J. M. Auerbach

Major Contributors: F. O. Feiock and K. R. Manes

\section{Fusion Experiments Data Analysis Facility}

As more diagnostics have been added to the Shiva and Argus laser lacilities, the volume and complexity of the data collected have increased rapidly. During the past year we reviewed the procedures and techniques used to analyze the target and laser diagnostic data, identifying areas where improvement was possible. At the same tine we were concerned with integrating any planned improvements into future needs arising from the Nova facilis! mon under construction.

We ennicuded that the most efficient way to hamalle mal analye our larget and laser diagnostic dallit $x \ln (1)$

- Concentrate in onc location the analysis resoures and facilitien eurrently scattered through. out the laboratory site.

- Ensure that the personnel responsible for dala analysis should have easy alcoess to such a cenIralised facilily.

To achieve our goals, we plan to set up a data analysis facility (Fig. 5-92), the center of which will be a DEC VAX 11; 990 computer system; this system is compatible with the existing Shiva and Nota, DEC computers, thus permitting transfer of existing software without nodifications. Delivery and operation are scheduled for the third quarter of fiscal 1980. One of the major tasks this facility will 
Fig. 5-92. Schematic of the phanned Fusion Experiments analysia facility.

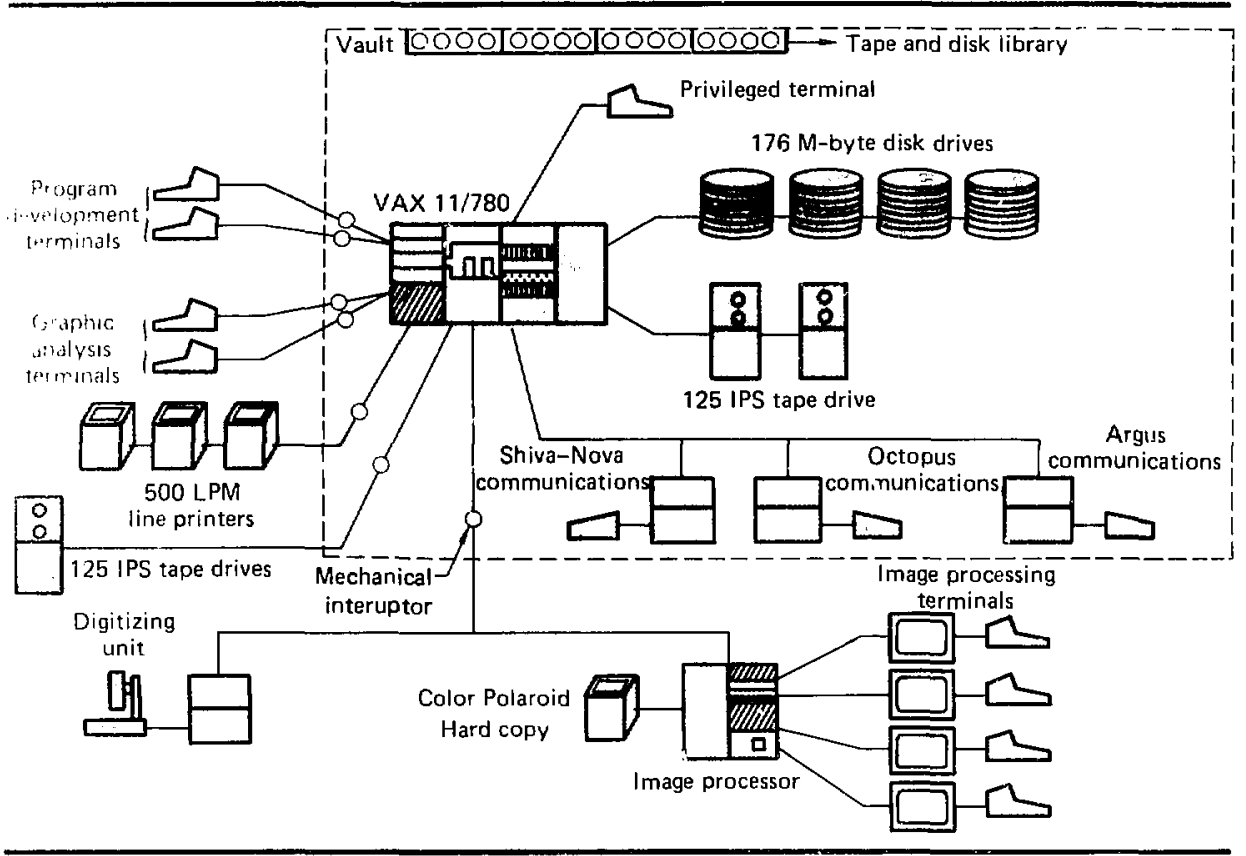

support is digital image processing. The image processing system will be used to analyze all twodimensional datia, in particular $x$-ray and streak camera data, and will be equipped with a directly linked digitising unit for rapid digitizing of film data. In uddition to standard contour and lineout capabilities, the system will have color display and color hard copy capabilities.

Once this analysis facility is operational, it will vatstly improve the efficiency and increase the volume of data processing and analysis, by

- Increasing the capacity and throughput for analyzing most of our data and results from the Shiva and Argus facilities.

- Permitting the operation of Nova as an unclassified facility except during the brief periods when classified targets are shot (this is the current practice at Argus and Shiva): this will reduce operation costs and enable us to maintain an open facility.

- Improving analysis capability and reducing the effort and time required to process $x$-ray imuging and streak camera data.

- Reducing the load on the overtaxed Shiva PDP 11/70 control and analysis computer. Most of the analysis codes now at Shiva will be moved to the new facility, which will provide the needed additional capacity to handle various operational codes for Shiva.

- Linking the Fusion Experiments data analysis effort with Octopus (the laboratory central computer system) whenever greater computational capacity is required. This link (via Octopert) will make the computer facility part of the Octopus Far Support Network.

During the first six months of operations, raw target and laser diagnostic data will be hand-carried to the analysis facility on magnetic tape. Later a 
Fig. 5-93. Schematic of the planned communication network for laser fusion data transfer.

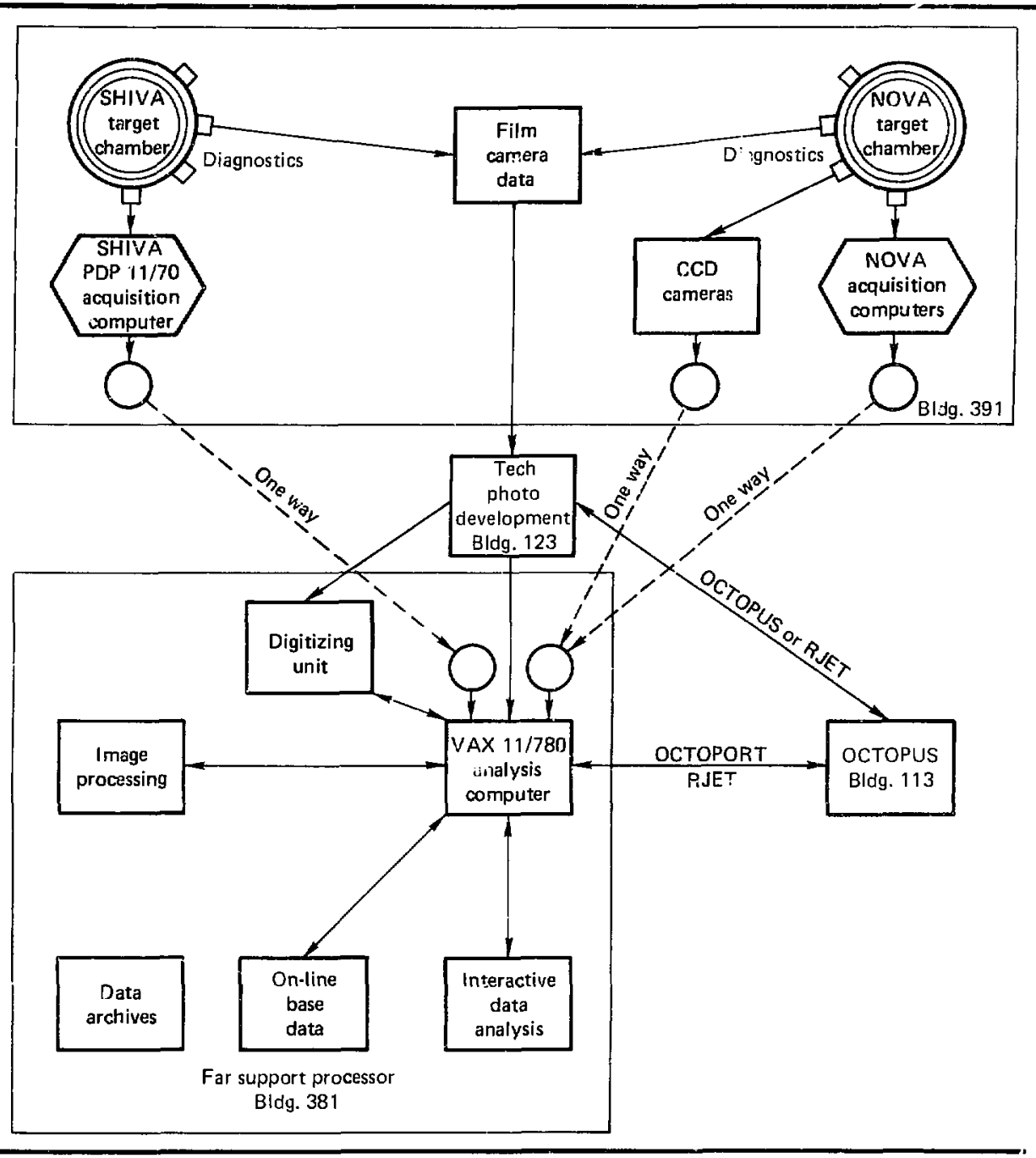

one-way fiber optics communications link will be established to carry data from Shiva directly to the analysis computer. Figure $5-93$ shous a diagram of the planned communication network.

It is envisioned that in its initial stage this system will support approximately 25 interactive users perlorming dala reduction and analysis in the fusion experiments area.

Author: J. E. Vernazza 


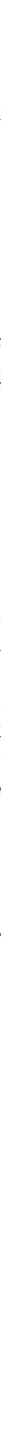




\section{Laser Fusion Experiments and Analysis}

\section{Contents}

Introduction

Shiva 2-ns Disk Experiments

Sidescatter in Laser-Irradiated High-Z Target

Diagnostic Configuration

Experimental Results

Other Experiments

Summary and Interpretation

Electron Transport Analysis Using Layered Slab Targets

Experimental Results

Analysis and Simulations

Summary

Z-Dependence of Sub-keV X-Ray Emission and Laser Intensity

Threshold for Inhibited Electron Thermal Conduction

Experimental Data

Analysis

Conclusion

High-Energy X-Ray Measurements from Disks of Different $Z$

Angular Distribution of Suprathermal X Rays

Stimulated Raman Scaltering Experiments

Experimental Contiguration of the $2 \omega_{0}$ Experiments

Beam Diagnostics Packages

Target Alignment and Spot Size Determination

Diagnostics During Target Shots

Preliminary $2 \omega_{0}$ Results

Summary of Disk Experiments 
D-T Fuel Density Determination from Measurements

of $\mathbf{P}$ usher Areal Density $(\rho \mathbf{S} \mathbf{R}) \ldots \ldots \ldots \ldots \ldots \ldots \ldots$

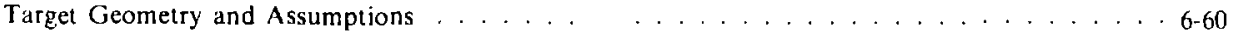

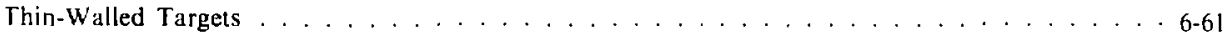

Thick-Walled Targets in the Absence of Mixing . . . . . . . . . . . . . . . . . . . . . . . . .

Nonuniform Pusher Density Distributıons . . . . . . . . . . . . . . . . . . . 6-64

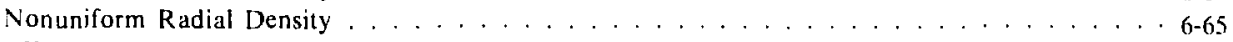

Effects of Mixing . . . . . . . . . . . . . . . . . . . . . . . . . . . . . . . . . . .

Comparison of Model with Experimental Results and

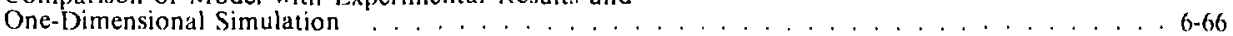

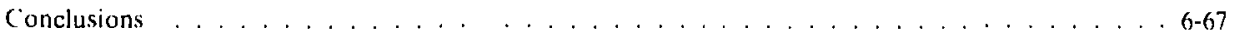




\section{Laser Fusion Experiments and Analysis}

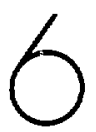

Introduction

The primary goal of our laser fusion program is to demonstrate the feasibility of inertial confinement fusion. In conception, planning, and execution we have found it useful to group our calculations, experiments, and analyses into six catagories:

- Absorption and scattering experiments, designed and conducted primarily to measure and characterize the amount of incident laser energy absorbed by the target, and the fraction, angular distribution, and other characteristics of laser radiation reflected and scattered by the target plasma.

- Plasma conditions experiments, performed to determine the density and temperature distributions of the plasma in laser interaction and ablation regions.

- Shell dynamics experiments on accelerated slabs and cylindrical and spherical shells, to measure the dynamics, symmetry, and stability of their motion.

- High-density implosion experiments, conducted for the express purpose of achieving and diagnosing high-density fuel conditions.

- Drive and preheat analysis, to explore the preheat of shells and fuel and the transport of energy in the capsule that produces the pressure driving the implosion.

- Diagnostics development, to develop atsd implement instruments and dataacquisition systems appropriate to the experimental efforts outlined above.

Our experimental program for the first five areas is discussed in this section; the sixth category is discussed in Section 5 of this report.

Generally, one of the above categories represents the primary goal of planned experimental sequences, athough in almost all cases data are obtained in one or more of the other categories as well. This structuring of objectives helps us balance and prioritize our program.

Disk experiments have extended our data base and understanding of absorption to include the effects of pulse lengths of $2 \mathrm{~ns}$ at $1.06 \mu \mathrm{m}$, and we have begun to obtain data at 600 ps and $0.53 \mu \mathrm{m}$. Long pulse lengths $(0.5$ to $10 \mathrm{~ns})$ appropriate to the achievement of highdensity fuel conditions produce long gain regions for stimulated Brillouin and Raman scattering; we continue to study these phenomena so that our target designs minimize their deleterious effects.

Implosion experiments performed on several target types have achieved fuel densities ranging from $10 \times$ liquid-density D-T to nearly $100 \times$. Figure 6-1 shows high-energy $x$-ray images from a target which achieved $10 \times$ liquid D-T density. Density was measured using pusher activation, by diagnosing $x$ rays emitted by seed argon in the fuel, ard by the 6.5keV-continuum $x$-ray image. The suprathermal (16.5 keV) image shows soniniform heating of the pusher by the suprathermal electrons. Advances in our unde sranding of suprathermal electrons, including particularly the confirmation of the conjecture that they may be temporally delayed with respect to the laser pulse, have made possible significant improvements in our ability to model high-density experiments.

Energy transport has been studied in disk targets with $Z$ varying from $B e$ to $U$, and with CH- and Al-layered targets. LASNEX calculations require both inhibited-transport and non-LTE physics to reproduce the experimental observation on these absorption and transport disk experiments.

The categories of plasma conditions and shell dynamics will receive more emphasis in the future, as diagnostics technology enables us to gather more and better target data. Until 
Thermal $x$-ray image

$(6.5 \mathrm{keV})$

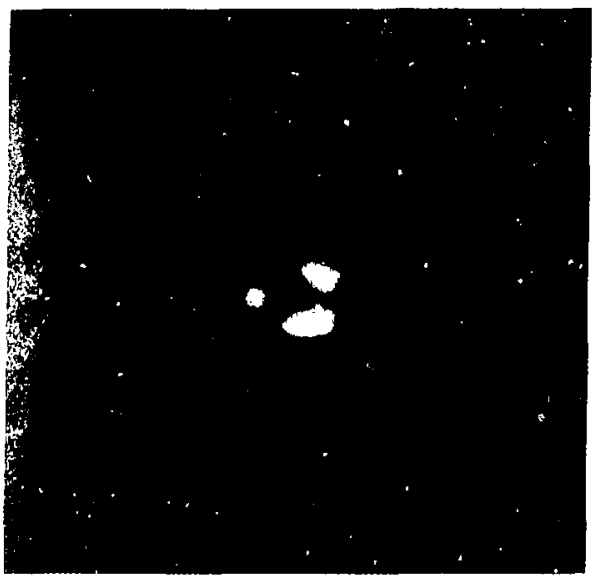

Fig. 6-1

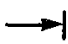

$1 \leftarrow 40 \mu \mathrm{m}$
Suprathermal x-ray image

(16.5 keV)

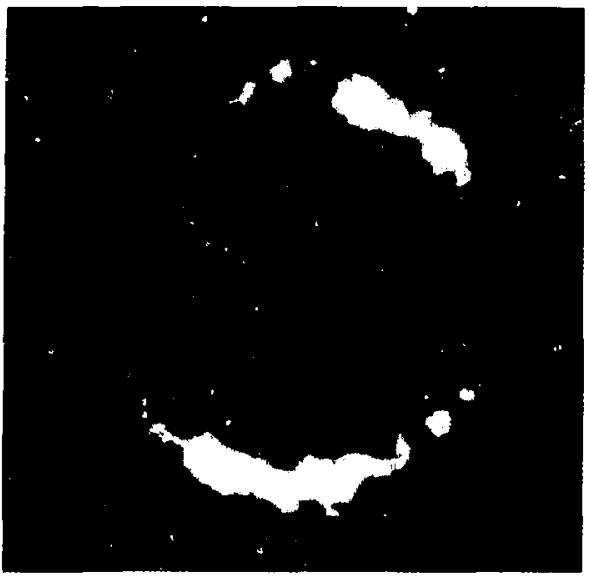

$1 \longleftarrow 41 \mu \mathrm{m}$

plasma condit ons are better known we will not be able to fully quantify our understanding of ahsorption and scattering processes. Diagnostic techniques are now beconing available which will allow sophisticated shell dynamics experiments in the coming y:-...

\author{
luthors: H.G. Ahlstrom and J.H. Nuckolls
}

\section{Shiva 2-ns Disk Experiments}

l.ascr-healed ICF targets capable of achieving hreakeven energs oulput will most probably be driven by carefully shaped pulses many nanosecond in duration. During the last year we conducted disk experiments with 2-ns (FWHM) laser pulses in investigate several of the conditions that arise when very large regions of plasma are irradialed with such long laser pulses; these conditions include such dangerous instabilities as Brillouin scaltering. filamentation, and Raman scattering. Efficient Brillouin scattering would mean low light absorption, while filamentation and Raman scattering would produce high-energy electrons which can contribute to target preheat. At longer pulse lengths, however, inverse bremsstrahlung absorption is also expected to increase. There is thus a competition between inverse bremstrahlung absorption and the instabilities; inverse bremsstrahlung absorption dominates if the light is absorbed before Brillouin scattering or filamentation can oceur.

Earlier 1-ns experiments ' were performed with a single Gatussian optical pulse, while the 2-ns experiments we report here were carried out with a stacked pulse oblained by using a Mach-Zehnder interferometer to coherently add two I-ns Gaussian optical pulses separated in time by $1.2 \mathrm{~ns}$. Since the eoherent addition of two pulses requires the difference in optical path lengths to be stable to better than $\lambda / 10$, the optics for combining the two pulses were mounted on an NRC super-Invar table. The optical phase of one of the two pulses was adjusted by tilting an etalon in one arm of the MachZehnder interferometer; the etalon's surfaces were plane-parallel and antireflection-coated.

The correct optical phase for coherent superposition was located by first finding two successive etalon angular positions for which a null occurred in the center of the pulse (as record:d by a streak camera with an automated readout). The etalon was then adjusted to approximately the midpoint. If the two optical pulses are not nearly colinear, their 
Fig. 6-2. Absorption mensurements for 2-ns gold and parylene disk experiments. The "swaller spot" data are from experiments in which the spot dismeter was reduced from 900 to $450 \mu \mathrm{m}$, the same spot sine used for the $1-\mathrm{ns}, 3 \times 10^{14} \mathrm{~W} / \mathrm{cm}^{2}$ experiments.

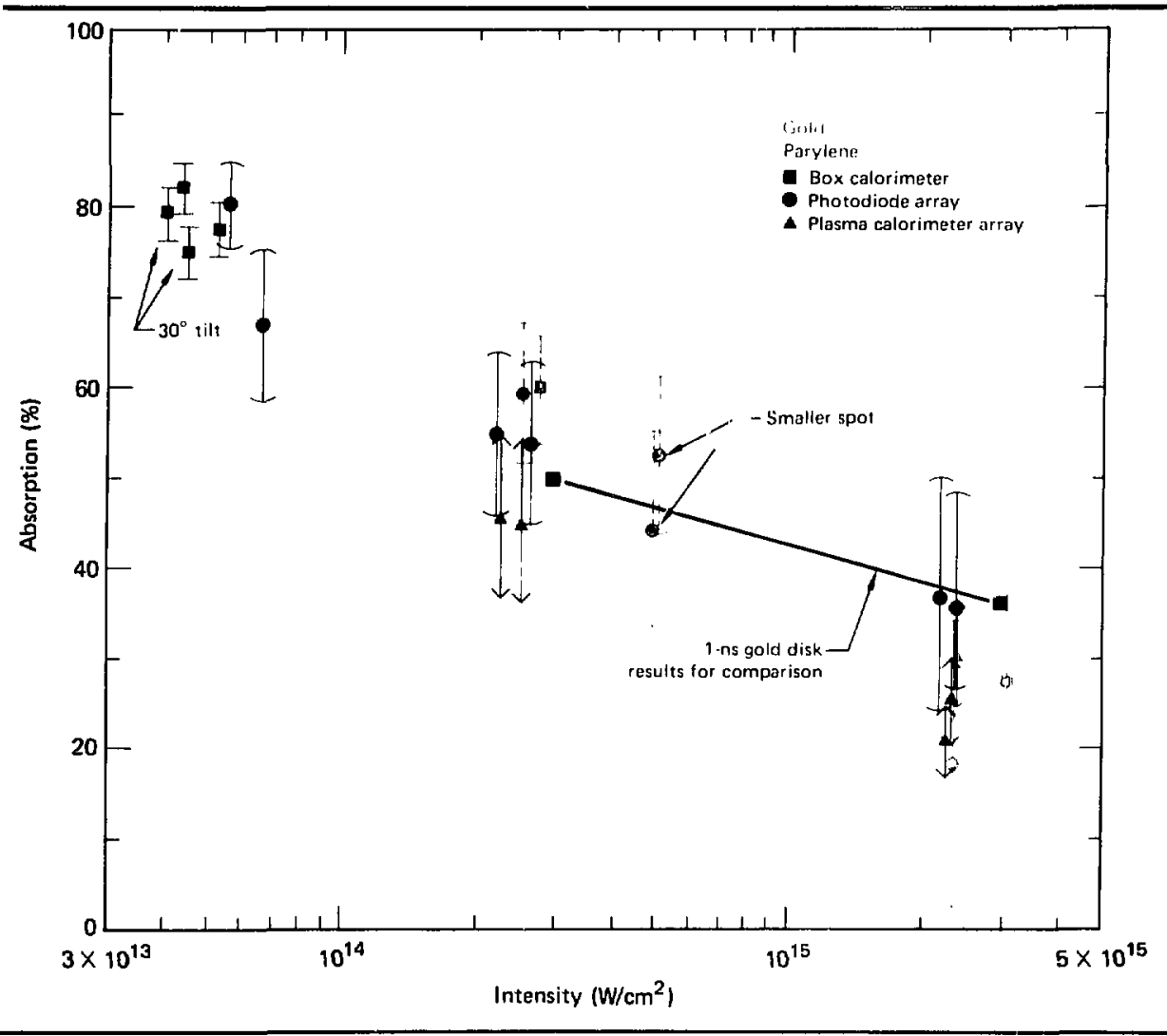

relative phase will vary significantly across the wave front. Given the beam diameter $d$ and a maximum phase error $\lambda \phi$ across the wavefront. we can find the maximum angular alignment error $\epsilon_{\max }$ from $\Delta_{\phi}=k_{0} \epsilon_{m}$ axd. For $\Delta_{\phi}=k_{0} \lambda / 10$ and $d=5000 \lambda_{0}$ (the beam diameter at the pulse stacker), we have $t_{\max }=20 \mu \mathrm{rad}$. A computer-controlled pointing and centering sensor enabled us to attain this alignment accuracy. On most of the shots the output puise shape of one or two beams was measured by the output streak camera and a fast photodiode located in the target room.

Parylene and gold disks were irradiated at three intensities: $5 \times 10^{13} .3 \times 10^{14}$, and $3 \times 10^{15} \mathrm{~W} / \mathrm{cm}^{2}$. The spot diameter was $900 \mu \mathrm{m}$ for the two lower intensities and $250 \mu \mathrm{m}$ for the highest intensity. The principal measurements made were of the fraction of light absorbed. of the radiative losses into soft $x$ rays $(\leqslant \mid k e v)$. and of the high-energy $x$-ray spectrum. We found little difference between the 2-ns results and our earlier $1-n s$ resuits for the two intensities we could conpare. The spot diameters for the 1-ns experiments were $450 \mu \mathrm{m}$ for $3 \times 10^{1} \mathrm{~W} / \mathrm{cm}^{2}$ and $150 \mu \mathrm{m}$ for $3 \times 10^{15} \mathrm{~W} / \mathrm{cm}^{2}$. Absorption (Fig. 6-2) was measured either by a box calorimeter or an array of photodiodes, calibrated to betier than $5 \%$ relative accuracy and 5 to $10^{r}$ absolute accuracy.

An understanding of the measurement limititions is imporlant for Fig. 6-2. The photodiode measuremer $t$ of the scattered light is nominally $15 \%$ 
Table 6-1. Scattened fight measurements showing the existence of a beck-reflected peak for a 2-rs gold diak experiment in which beams 16 and 18 irradinted the diak at $5 \times 10^{13} \mathrm{~W} / \mathrm{cm}^{2}$ and were incident at $30^{\circ}$ to the target normal. The light was p-polarized, the major-axis spot diameter was $900 \mu \mathrm{m}$, and the total enersy on target was $585 \mathrm{~J}$. Meanurements were made with photodiodes (PD) and a reflected-beam diagnostic (RBD) calorimeter.

\begin{tabular}{|c|c|c|c|c|c|}
\hline \multirow[b]{2}{*}{ Detector } & \multirow{2}{*}{$\begin{array}{r}\text { Intensity } \\
\text { in }(J / s r) \\
\end{array}$} & \multicolumn{2}{|c|}{$\begin{array}{c}\text { Target chamber } \\
\text { coordinates }\end{array}$} & \multicolumn{2}{|c|}{$\begin{array}{c}\text { Rotated } \\
\text { coordinates }\end{array}$} \\
\hline & & $\phi$ & $\theta$ & $\phi^{\prime}$ & $\theta^{\prime}$ \\
\hline $\begin{array}{l}\text { PD-38 } \\
\text { Beam I6 RBD } \\
\text { Beam 18 RBD }\end{array}$ & $\begin{array}{l}113 \\
100.3 \\
\text { No data }\end{array}$ & $\begin{array}{l}270^{\circ} \\
234^{\circ} \\
306^{\circ}\end{array}$ & $\begin{array}{l}20^{\circ} \\
17.74^{\circ} \\
17.74^{\circ}\end{array}$ & $\begin{array}{c}0^{\circ} \\
-72.9^{\circ} \\
+72.9^{\circ}\end{array}$ & $\begin{array}{r}2.3^{\circ} \\
10.8^{\circ} \\
10.8^{\circ}\end{array}$ \\
\hline $\begin{array}{l}\text { Beom } 17 \text { RBD } \\
\text { Beam 15 RBD } \\
\text { Beam } 19 \text { RBD }\end{array}$ & $\begin{array}{l}59.6 \\
54.5 \\
30.2\end{array}$ & $\begin{array}{l}270^{\circ} \\
198^{\circ} \\
342^{\circ}\end{array}$ & $\begin{array}{l}9.70^{\circ} \\
9.70^{\circ} \\
9.70^{\circ}\end{array}$ & $\begin{array}{c}0^{\circ} \\
-\mathbf{3 2 . 5 ^ { \circ }} \\
+\mathbf{3 2 . 5 ^ { \circ }}\end{array}$ & $\begin{array}{r}8.04^{\circ} \\
17.4^{\circ} \\
17.4^{\circ}\end{array}$ \\
\hline $\begin{array}{l}\text { P'D-35 } \\
\text { PD-40 } \\
\text { PD-37 } \\
\text { PD-34 }\end{array}$ & $\begin{array}{r}35.2 \\
27.5 \\
4.7 \\
3.6\end{array}$ & $\begin{array}{r}198^{\circ} \\
342^{\circ} \\
126^{\circ} \\
36^{\circ}\end{array}$ & $\begin{array}{l}20^{\circ} \\
20^{\circ} \\
20^{\circ} \\
20^{\circ}\end{array}$ & $\begin{array}{l}-60.3^{\circ} \\
+69.9^{\circ} \\
-20.1^{\circ} \\
+30.1^{\circ}\end{array}$ & $\begin{array}{l}22.0^{\circ} \\
20.3^{\circ} \\
35.8^{\circ} \\
33.5^{\circ}\end{array}$ \\
\hline
\end{tabular}

. Murate for atn avially symetric light distrihution. whle the incident energy is known $105 \%$. Thus the error in the meanured absorplion fraction f can he a high a $20 \%$ of (1 - h). The more accurale bux alorimeter measurement is expected lo be in error he lew that $10 \%$ of (1 - 0). We have also included plasmal ailarmeler resulds when abailahle. When the sheoroten fraction is mall. the plasma

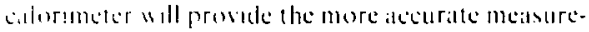
mont.

Shorption an normal incidence is slightls

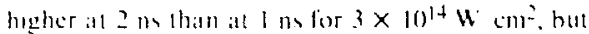

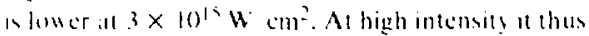
sems likely that stimulated Brillouin sattlering domimates. Perhaps the mast surprising result of these experiments is the fialure of the abserplion lo var! sienificantly with $Z$. The inverse bremssrahlung incensils absorption coeflicient in given b!

$$
\left.K_{C I}=\frac{4 \sqrt{2 \pi}}{3} \frac{\left(\overline{2} \mathrm{r}_{0}^{2}\right)}{\beta_{\mathrm{te}}^{2}} \frac{\mathrm{n}_{\mathrm{c}}^{2} / \mathrm{n}_{\mathrm{c}}}{\sqrt{1} \mathrm{n}_{\mathrm{c}} / \mathrm{n}_{\mathrm{c}}}(0) \mathrm{cm}\right) .
$$

where $r_{0}$ in the classical electron radius 2.8/8 $x$ $10^{-1} \mathrm{~cm}$. $n_{2}$ is the cisceron density, $n_{c}$ is the eritical electron demsils. As is the thermal selocity expressed as a fraclion of light speed $\left(\beta_{\mathrm{t}^{\circ}}=\right.$ $\sqrt{\left.\mathrm{KT}_{\mathrm{L}} / \mathrm{ms}^{2}\right)}$, and $\mathrm{F}(\mathrm{a})$ is a function which depends upon $v_{\text {as. }} v_{10}:$ for $v_{n x} \ll v_{10}, F(a)=1$. The nean ionization $\overline{\bar{Z}}$ for gold exceeds that for parylene hy an order of magnilude.
There are several possible partial explanations of whs we do not observe an order-of-magnitude difference in the inlegrated inverse bremsistrahlung absorption coelficient:

- The intograted absorption coefficent is propurtumat of the density seale length L. and we would expece l. for parylene plasmas to he much lomper than for gold plasmas. The sound speed

$$
c_{s}=\sqrt{\frac{\bar{Z} \mathrm{kT}}{\Lambda \mathrm{N} \mathrm{T}_{\mathrm{N}}}}
$$

will be higher for a parblene plasma than for a gold plasmat of the same comperature. In Ev. (2) $m_{N}$ is the mean nucleon mass, $Z$ is the mean ionization itatte, $A$ is the nucleon number, $k$ is Boltemann's comstitnt, and $T_{c}$ is the electron temperature.

- Heat-flax inhibition is expected lo be more sere lor higher /.. Strong inhibition will stecpen the demsits profile and raise the electron temperatture.

- Slow clectrons, which are principally responsible for inverse bremsstrahlung absorplion, collide with ions $Z$ times more often than they collide with other electrons. But electron-electron collisions "re necessary to repopulate this group of slow electrons. When $\bar{Z} v_{\text {osd }} / v^{2}$ te $>1$, we find the electron distribution is no longer Maxwellian hut more that-lopped. This parameter is much smaller for parylene than for gold.

As these partial explanations show, a full explanation for the small difference in measured ab- 
Fig. 6-3. Angelar diatribution of the x-ray energy for the 2 -ns gold disk experiments at $3 \times 10^{14} \mathrm{~W} / \mathrm{cm}^{2}$. The major-axis spot diameter was kept cowstant at $900 \mu \mathrm{m}$.

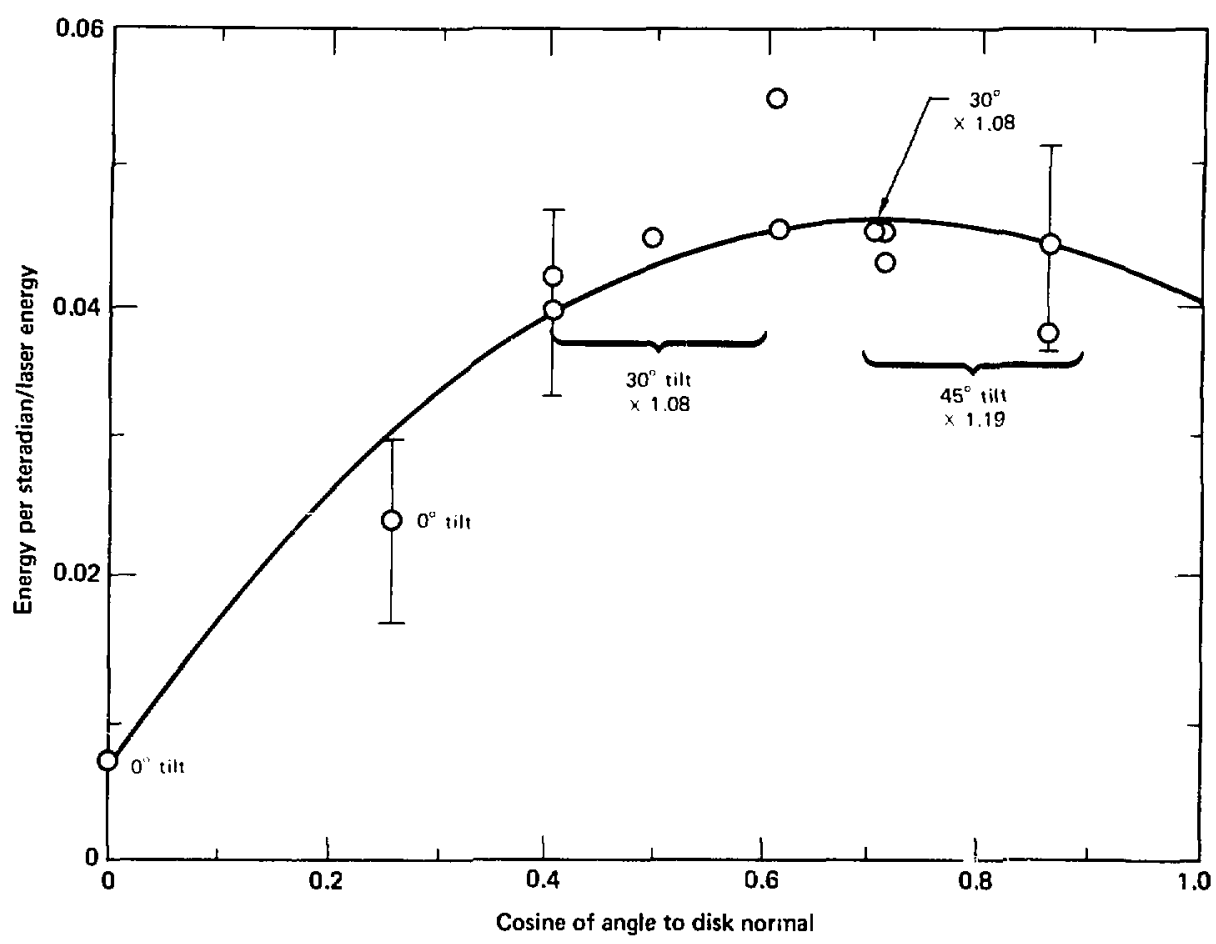

sorptions belween parylene and gold will probably he a complicated one, arrived at only after eonsiderahle computer simulation.

Even al $5 \times 10^{13} \mathrm{~W} \mathrm{~cm}^{2}$ some Brillouin scaltering must occur. We see a highly collimiled peak in the backuard direction (sec Tahle 6-1). in addition to the much broader specular peak when the target is tilted $30^{\circ}$ from the laser heams. To oblain the data in Table 6-1, the target was irradiated only by two adjacent outer lieams of the upper 10-heam cluster. nos. If and 18. which have asmuths $\phi=$ $234^{\circ}$ and $s=306^{\circ}$. respectively: their common polar angle is $:=17.74^{\circ}$. and the lenses are ${ }^{\prime}, 6$ with a marginal ray half-angle of $5.05^{\circ}$ (the heam is somewhat smaller). We have also labulated the rolated coordinates ${ }^{\prime}, \theta^{\prime}$ ' which are based upon an axis at $\omega=270^{\circ}, A=17.74^{\circ}$. The a cinuth $\omega^{\prime}$ is ese on the plane of incidence. We find that about 5 to 6\%. of the incident light is seattered into the
Tabie 6-2. Summary of the fraction of the absorbed energy radiated as soft $x$ rays from a gold-disk target, determined from the date in Figs, 6-3 and 6-4.

\begin{tabular}{cccc}
\hline \multirow{2}{*}{$\begin{array}{c}\text { Time } \\
\text { (ns) }\end{array}$} & \multicolumn{3}{c}{ Intensity Icvel $\left(W / \mathrm{cm}^{2}\right)$} \\
\cline { 2 - 4 } & $5 \times 10^{13}$ & $3 \times 10^{14}$ & $3 \times 10^{15}$ \\
\hline 1 & - & $35 \% \pm 7 \%$ & $35 \% \pm 7 \%$ \\
2 & $62 \%$ & $38 \% \pm 7 \%$ & - \\
& $(64 \%)^{2}$ & $(43 \%)^{9}$ & - \\
\hline
\end{tabular}

\footnotetext{
anased upon multiplying the Dente measurement at $60^{\circ}$ to the turget normal by a $2 \pi$ solid angle.
}

bitck wards peak by fitting the light distribution to the equation $I=I_{1} \cos ^{n} n$. We find $n$ is quite high, about 15 to 18 . For a gold disk tilted $30^{\circ}$ and irradiated by the upper ten heams at $3 \times 10^{14}$ $W$. $\mathrm{cm}^{2}$. about 9 to $10 \%$ of the incident light appeared in the back-reflected peak. 
Fig. 6-4. Angular distribution of the x-ray eaergy for the 2-as gold disk experiments at $5 \times 10^{13} \mathrm{~W} / \mathrm{com}^{2}$. An targets were irradiated by iwo adjacent beams hockeat at $30^{\circ}$ to the target nocmal (p-polerization). The major-axis swot diameter was $900 \mu \mathrm{m}$.

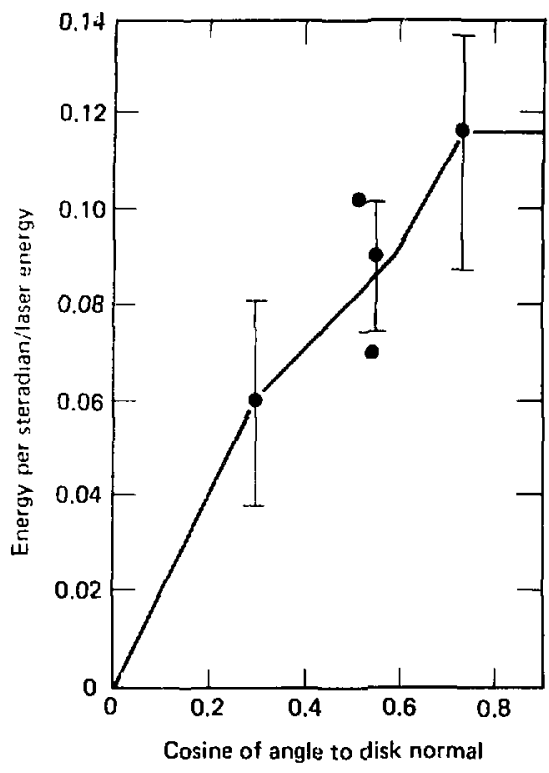

Table 6-3. Summary of the LASNEX predictions for $x$-ray conversion efficiency, expressed as a percentage of the absorbed energy, for both the 1- and 2-ns gold disk experiments. Both the inhibited (IL) and nonimhibited (NIL) LASNEX model results are included.

\begin{tabular}{|c|c|c|c|}
\hline \multirow{2}{*}{$\begin{array}{c}\text { Time } \\
\text { (ns) }\end{array}$} & \multicolumn{3}{|c|}{ Intensity level $\left(w / \mathrm{cm}^{2}\right)$} \\
\hline & $5 \times 10^{13}$ & $3 \times 10^{14}$ & $3 \times 10^{15}$ \\
\hline $2(\mathrm{IL})$ & $50 \%$ & $45 \%$ & $36 \%$ \\
\hline 2 (NIL) & $67 \%$ & $58 \%$ & $44 \%$ \\
\hline 1 (IL) & - & 33\% & $36 \%$ \\
\hline 1 (NIL) & - & $62 \%$ & $58 \%$ \\
\hline
\end{tabular}

For gold a large fraction of the absorbed light encrgy is radialed away in soff x rays. Table 6.2 summari/es these results and compares them to the I-ns results.

Figure 6-.3 shows the angular distribution for the 2 -ns, $3 \times 10^{14} \mathrm{~W} \mathrm{~cm}^{2}$ gold disk experiments: a third-power polynomial in cos $\theta$ has been fitted to these data. To obtain this angular distribution. targets werc tilted to different angles in different shots. Unfortunately, we have no 2-ns absorption dala al angles other than normal. We do have a measurenient for the $45^{\circ}$ angle al $1 \mathrm{~ns}$, however, for which the absorption is $42 \%$, compared to $50 \%$ at normal incidence. We assume the ahsorption at $2 \mathrm{~ns}$ (1) be reduced proportionally al a $45^{\circ}$ incidence angle; the $45^{\circ}$ results shoun in Fig. 6-3 therefore equil the actual measurements multiplied by 1.19. while the $30^{\circ}$ measurements hase heen multiplied by the in-hetween value of 1.08 . Upon doing the angular integration, we lind the v-rat energy is $23^{\circ}$ of the incident laser conergy: if wa do not correct for target till, the figure in $\approx 21 \%$. This percentigge is then divided by the 0.60 asharption fratetion lo find the listion of the absorhed eneres appearing in

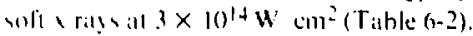

In assigning the error bratckets (in parentheses on Table 6-2) we have atssumed the Dante measurement of the inlegral at at particular angle to be 20 "; acturate. This error is added in quadrature hoth with our evimale for the probable error in integrating wer the angular distribution and with the probatice error in the absorption measurement. For $3 \times 10^{1+} 11$ c.m². the absorption is known to be $60 \%$ $( \pm 5 "$,$) at 0^{\circ}$. The ingular inlegrallion is probably $10^{\prime} ;$ accuralle.

At $5 \times 10^{1: W}$ cm? we hatue the necessary ab. sorplion datal, but lewer measurements (Fig. 6-4). Thus the 62" radialted efficiency measurement is probabls uncertain to $\pm 10 \%$ just lirom inadequate knowledge of the angular distribution. The absorption measurement for the $5 \times 10^{1.3} \mathrm{~W}: \mathrm{cm}^{2} 30^{\circ}$-litt goid disk experiment is $77^{\circ} \%( \pm 4 \%$ ). We feel the angular inlegration is ahout 20\% accurate; thus we lind it unlikely that the radialed percent for gold at 2 us and $5 \times 10^{13} \mathrm{~W} / \mathrm{cm}^{2}$ is outside the range of 48 (1) $80 \%$.

Tiable 6-3 gives LASNEX predictions for both strong heal-flux inhibition (IL) or none at all (NIL). Again, these $x$-ray conversion efficiencies are expressed as a percent of absorbed energy. Strong heat-flux inhibition is conclusively required in the mad- $10^{14} \mathrm{~W} / \mathrm{cm}^{2}$ intensity region and above. Some inhibition may be required for $5 \times 10^{13} \mathrm{~W} / \mathrm{cm}^{2}$, but the strongly inhibited model gives a conversion percent which is at the extreme lower bound of the experiment:" "rror bracket.

The "wrathermal $x$-ray spectra for several representative shots on gold disks at several intensities are plotted in Fig. 6-5. For $3 \times 10^{14} \mathrm{~W} / \mathrm{cm}^{2}$ we estimate the fraction of the laser energy appearing 
Fig. 6-5. Fllter fluorescer meusurements of the high-energy $x$-ray spectrum for several representative gold disk shots.

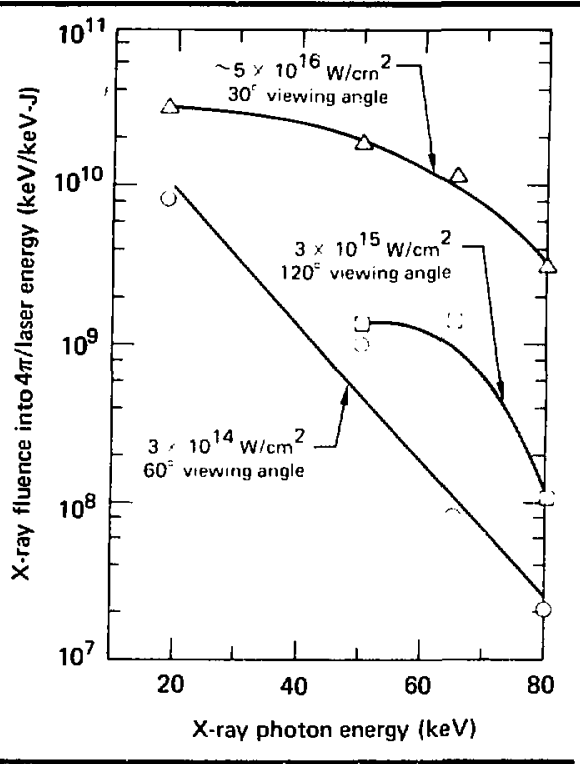

in suprathermal electrons to be approximately 59 $( \pm 3 \%)$ : the "hot" temperalure is ahoul $17 \mathrm{keV}$. 1 ... $3 \times 10^{15} \mathrm{H}$ em? the high-energy $\mathrm{x}$-ray spectrometer looked through the $18.5-\mu \mathrm{m}$ thick gold disk at an angle of $30^{\circ}$ to the disk face. and thus all that could be measured was the level of the high-energy tail al 50 10 $80 \mathrm{keV}$.

We lind that where there are datat to compare. lengthening the lase pulse from 1 to $2 \mathrm{~ns}$ changes the experimental pieture litile. It therelore sems unlikels that absorption or stimulated scatter will thange dramatically al someshat longer pulso lengths. The "leak $Z$-dependence of the absurption. even at long pulse length, has also been obserwed by $\mathrm{AWRE}^{2}$ in their experiments at $\mathrm{I}$ and $3 \mathrm{~ns}$

Authors: 1). W. Phillion, V. (., Rupert, and M. D. Rosen

\section{References}

I. M. D. Ruen. D. W. Philliun. V. C Rupert, W. C. Mteud.

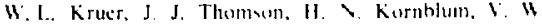
Slavinsk!, G. J. Caperalse, M. J. Busle, and K. G. Tirell, "The Interatcion of $1.06 \mu \mathrm{m} \mathrm{I}$ iner Raduiation wath High $Z$ Disk Targes." Phis. Fiud 22. 2020) (1974).

2. B. R. Thomas, prisate communication.
Sidescatter in LaserIrradiated High-Z Targets

Since stimulated scattering is a potential loss mechanism in the coupling of laser light to an ICF larget, it has been studied extensively. Most experiments have concentrated on the characteristics (spectral. temporill. ete.) of backscaltered light. generally defined as the light collected by the focusing aptics. Datta hate also been obtaned on the angular distribution of scattered light, show ing that more light is scattered out of the plane of polariatlien st the incident light thatr in the plane. An discussed he Phillion. the observed latge an! mumetries call be ascribed to Brillouin sidescallering, hat little datat have heen gathered on hou sideseatler varies with the characteristic farameters of the laserplasma interaction.

Recent experiments with high- $Z$ disks at long pulse iengehs (conditions w nich enhance stimulated sealtering) hatse added some infermation regatding the variation of sidencalter with larget orientation relattes to the laser beam. Indeed se determined

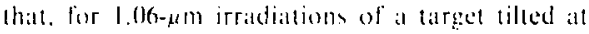

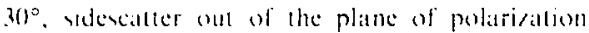
becomes latge enugeh to be comparainie to the sum of the speculat and batkscittered radiation. Since sidescattering in at stong function of inciden inkensils." the datal divemed below are limited 10 result of experiments conducted at $3 \times 10^{14}$ W $\mathrm{cm}^{2}$.

\section{Diagnostic Configuration}

During ahsorption measurements on gold and titanium disks all $1.16 \mu \mathrm{m}$. We obtained an indication of the magnitude of sidescitter out of the plane of polarication. When a linite number ol discrete instruments, such as PIN ciicodes." are used, the spatial extent of the specular peak cannot be accurately determined: thus the required spatial interpolation of the measured flux can mask or distort the effect which we seek to observe. This problem does not arise when experiments are conducted in a box calorimeter. Since each of the six panels of the Argus box calorimeter are read independentiy. a value of the total light scaltered into a given sector can be obtained. 


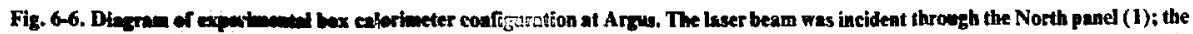

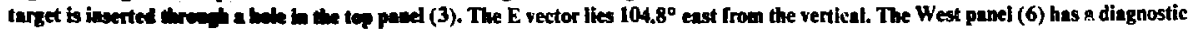
bole, whereas the Eent (5) and lowe (4) panels are integral.

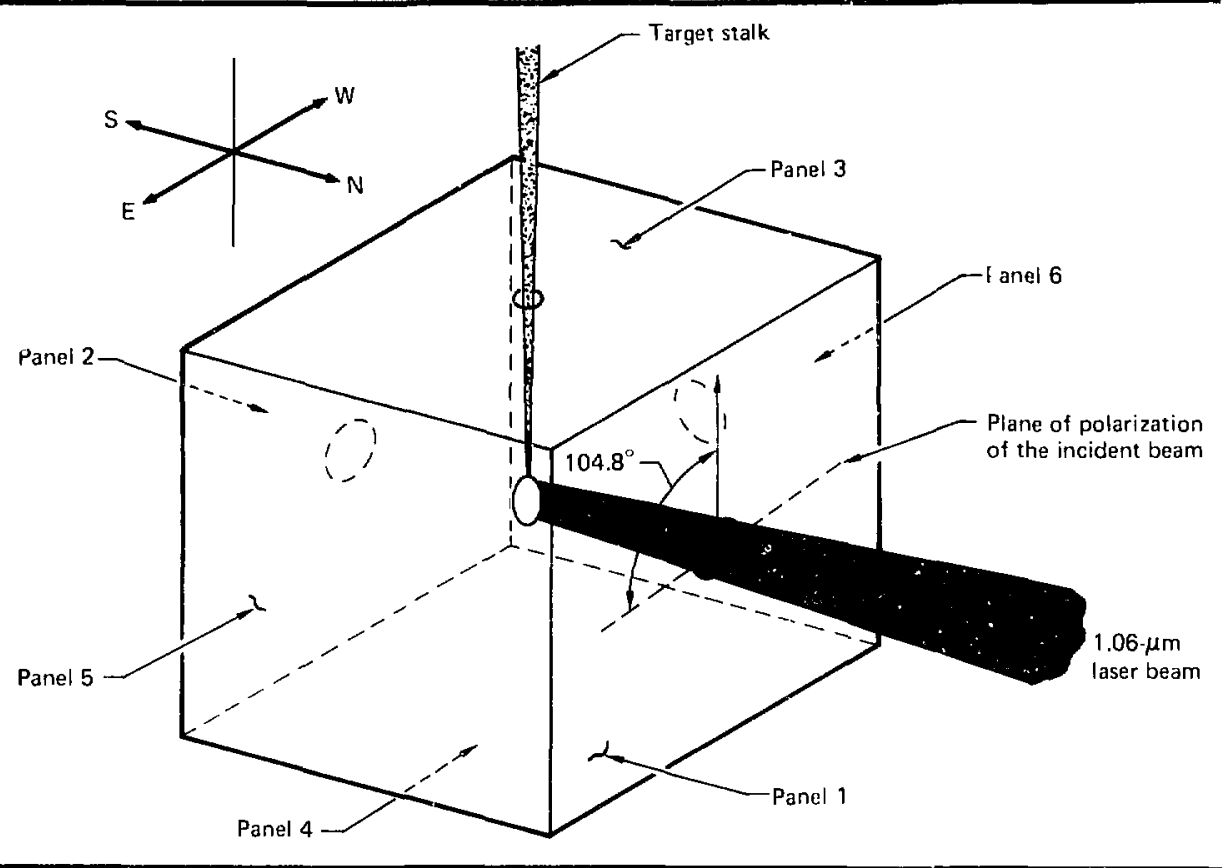

We performed our box calorimeter experiments at Argus, where the plane of polarization of the incident light (measured from the vertical target positioner) is $104.8^{\circ}$ (Fig. 6-6). Panels 3 and 4 of the calorimeter are almost parallcl to the plane of polarization of the incident beam. and therefore collect the light scattered "out of the plane of polarization" of the incident light. Conversely. panels 5 and 6 collect the light scattered "in the plane of polarization." These four panels receive all the light scattered between $55^{\circ}$ and $125^{\circ}$ from the incident beam in their respective directions, and receive part of the light scattered between $45^{\circ}$ and $55^{\circ}$ or $125^{\circ}$ and $135^{\circ}$ : this contribution to the total scattered light will be called "large-angle scattering."

Panel I receives the light scattered between the edge of the $f / 2.2$ focusing lens and $45^{\circ}$, as well as light scattered between $45^{\circ}$ and $55^{\circ}$ that is not absorbed by panels $3,4,5$, and 6 ; this contribution will be referred to as "small-angle backscattering" to distinguish it from the contributions of panels 3 through 6 and the backscitter collected by the focusing optics. By analogy. panel 2 receives "small angle forward-scuttering." while the "transmitted" or "forward-scattered" light is collected by the lens set behind the panel. With the thick $(13 \mu \mathrm{m})$ targets used in these experiments. hovever, the amount of "forward-scattered" ligh: collected by either panel 2 or this lens is small and will not be considered in the following discussion. Note that we will continue to refer to "large-angle scattering" and "small-angle backscattering" in the special sense defined above

\section{Experimental Results}

For the box calorimeter experiments we irradiated high- $Z$ (mainly gold) disks at $3 \times 10^{14}$ $\mathrm{W} / \mathrm{cm}^{2}$ with a $\sim 900$-ps pulse of $1.06-\mu \mathrm{m}$ light. Three target orientations were used:

- The first experiment was conducted with the disk normal to the incident beam. In this case the small-angle backscattering and backscatter ac- 


\begin{tabular}{|c|c|c|}
\hline Scatter characteristics & $\begin{array}{c}\text { Gold } \\
\text { target }\end{array}$ & $\begin{array}{c}\text { Titanium } \\
\text { target }\end{array}$ \\
\hline $\begin{array}{l}\text { Large-angle, out-of-plane } \\
\text { Large-angle, in-plane; and } \\
\text { specular peak } \\
\text { Small-angle and } \\
\text { backscatter }\end{array}$ & $\begin{array}{r}23 \\
9 \\
20\end{array}$ & 30 \\
\hline
\end{tabular}

counted for $36 \%$ of the incident light. Pancls 3 and 4 recovered $3 \%$ (out-of-plane large-ingle seattering) of the incident light: panels 4 and 5 recovered $2 \%$ (in-plane large-angle scaltering).

- The next set of experiments had the disk tilted $45^{\circ}$ towards pance 4 . Here the specular rellection adds to the expected larger out-of-plane seattering, so that little information on the magnitude of the scittered (ats opposed to specular) light could be ohtained. For this configuration smatilangle backscaltering and backscalter accounted for $24 \%$ of incident light, in-plane large-angle scattering acecounted for 5\%, out-of-plane iarge-angle seattering with specular reflection atcounted for $18 \%$, and $4 \%$ was recovered on the panel which looked mostly at the back of the target in the out-of-plane large-angle scattering direction.

- The most interesting experiments however, were conducted with disks (both gold and titanium) tilted $30^{\circ}$ towards panel 5. The specular peak ${ }^{8}$ now adds to the in-plane large-ingle scaltering, so that we would have expected a much larger increase in the light absorbed by panel 5 than by either panels 3 or 4 . In fact, the amount of light seattered tomards panels 3 and 4 was comparable both to the sum of light scattered towards panels 1,5 , and 6 , and to the direct backseatter (Table 6-4). In other words, the large-angle scattering out of the plane of polarization was comparable to the sum of scuttering in all other directions.

\section{Other Experiments}

Data were also collected with large numbers of discrete detectors (PIN diodes) located at various polar angles both in and out of the plane of polarization of the incident light. When comparing these data with the box calorimeter results we must remember that

- Each panel of the box calorimeter represents a spatial integration over a large azimuthal range $(\pi / 2)$, whereas the PIN diodes are centered on the azimuth corresponding to maximum or minimum flux and have an acceptance angle of $1^{\circ}$. Hence, differences in the scattered light with respect 10 the plane of polarization are emphasized by PIN diode measurements.

- For tilted largets, PIN diodes will mealsure specularly reflected light that cruld lie in a relatively narrow region. Although the effect of the specularly rellected light might mask all o'ne for diodes, its total contribution to a callorimes panel mal be smaill.

- A difference between a meisurements will arise due to the respective h; fwidths of the insruments: PIN diodes are fitle with 100)-A bandpass tilters about the main liser trequency, whereas the box cialorimeter panels absorb light from 270 nom to several micromete ?.

Figure 6-7 shous an : Ireme case (for PIN diode measurements) of (iifferences between inplane and out-ol-plane scitlering for a disk irradiated at normal inci nlee by an $5 / 2.2$ lens under the same condition. "the box callorinteter experiments $\left(3 \times 10^{14}, \mathrm{~cm}^{2}\right.$ and a $900-\mathrm{ps}$ pulse). Note the logarithmic ux scas. These data show that for large-angle scattering the maximum ratio between out-of-planc and in-plane flux is of the order of 2.5 compired to 1.5 meisured by the box calorimeterl, and that the integrated llux is of the order of $10 \%$ of " e incident light. As for the hox calorimeter dalt: I large fratition (approximately 45\%) of the in jent light is recovered in "smallangle backscas ring" and backscatter.

Figure 6-s shows another example, but for a shorler $(200 \mathrm{~ns})$ pulse length. Here we used one beam of the whis laser, obtaining an effertive angle al inciden of $9.7^{\circ}$ since the target normal was along the hiva beam cluster axis als shown on the ligure. If this configuration. specular reflection is combinut with small-angle scattering. The ratio of out-of-nlane to in-plane large-ingle scattering is on the 1 . ker of 3 , while the integrated flux is approximat 513 ? of the incident flux. No PIN diode data arc wailable for a larger angle of incidence in p-polarized irradiance at $10^{14} \mathrm{~W} / \mathrm{cm}^{2}$.

\section{Summary and Interpretation}

More experiments, both with the box calorimeter and PIN diodes, are necessary to study 


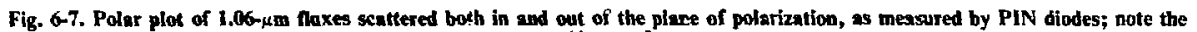
logarithmic scale. The target was a gold disk irradiated at $3 \times 10^{14} \mathrm{~W} / \mathrm{cm}^{2}$ for a 900 -ps pulse.

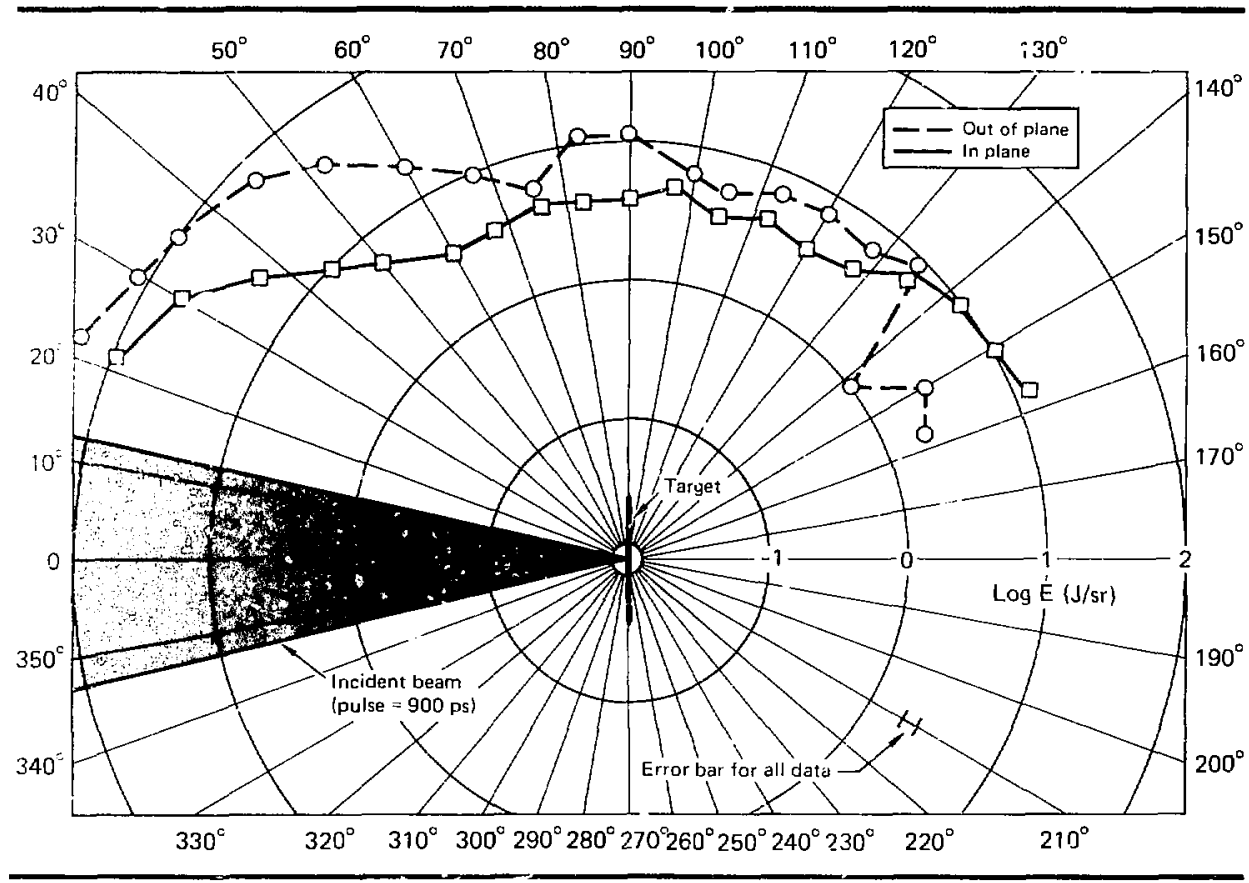

the inlensity dependence of sidescatter, as well as hou it varies with angle of incidence at a fixed intensily.

The datil available at this time (for $3 \times 10^{14}$ $W / \mathrm{cm}^{2}$ and $1-\mathrm{ns}$ pulses of $1.06-\mu \mathrm{m}$ light) indicate that large-angle scattering increases rapidly with angle of incidence relative to the specular and bickscaltered or small-angle backscattered flux, from about $10 \%$ at $0^{\circ}$ to more than $80 \%$ at $30^{\circ}$ for gold targets. Recent experiments at $0.53 \mu \mathrm{m}$, however, show a large-angle scattering increase of less than a factor of 2 for targets tilted at $30^{\circ} \mathrm{com}-$ pired to normal in idence.

Since other evidence shows less Brillouin backscaltering at the shorter wavelength (see "Preliminary $2 \omega_{0}$ Results" later in this section), it is tempting to attribute the observed increased flux at $1.06 \mu \mathrm{m}$ to Brillouin sidescatter. This assumption is consistent with observed larger $3 / 2 \omega$ emission out of the plane of polarization of the incident light than in the plane, which would otherwise be difficult to explain. Raman sidescatter is unlikely to contribute to the observed flux, since experiments ${ }^{9}$ at 1 ns and $3 \times 10^{14} \mathrm{~W} / \mathrm{cm}^{2}$ have shown it to be on the order of only $0.005 \%$ efficierit. We note th it the box calorimeter panels are capable of absorbing several harmonics of the incident light (including $\omega / 2$ ). Finally, an instability which ripples the plasma density contour near the classical reflection point can also play a role. ${ }^{10}$

Theoretical studies of the large-angle enhancement in sidescatter normal to the piane of incidence of p-polarized light are in progress. We may speculate, however, that as the target is tilted Brillouin scattering occurs at lower densities where refraction is less severe. At normal incidence refraction would collimate the scattered radiation towards the small-angle backscattering direction $(\theta$ $<45^{\circ}$ if scattering occurs at or above $0.5 n_{c}$ ). The observed differences in large-angle sidescattering would then be related not only to actual differences 
Fig. 6-8. Polar plot of $1.06-\mu m$ fluxes seattered both in and out of the place of polarization, as measured by PIN dicdes; note the logarithmic scale. The target was a gold disk irradiated at $3 \times 10^{14} \mathrm{~W} / \mathrm{cm}^{2}$ for a $200-p s$ pilse.

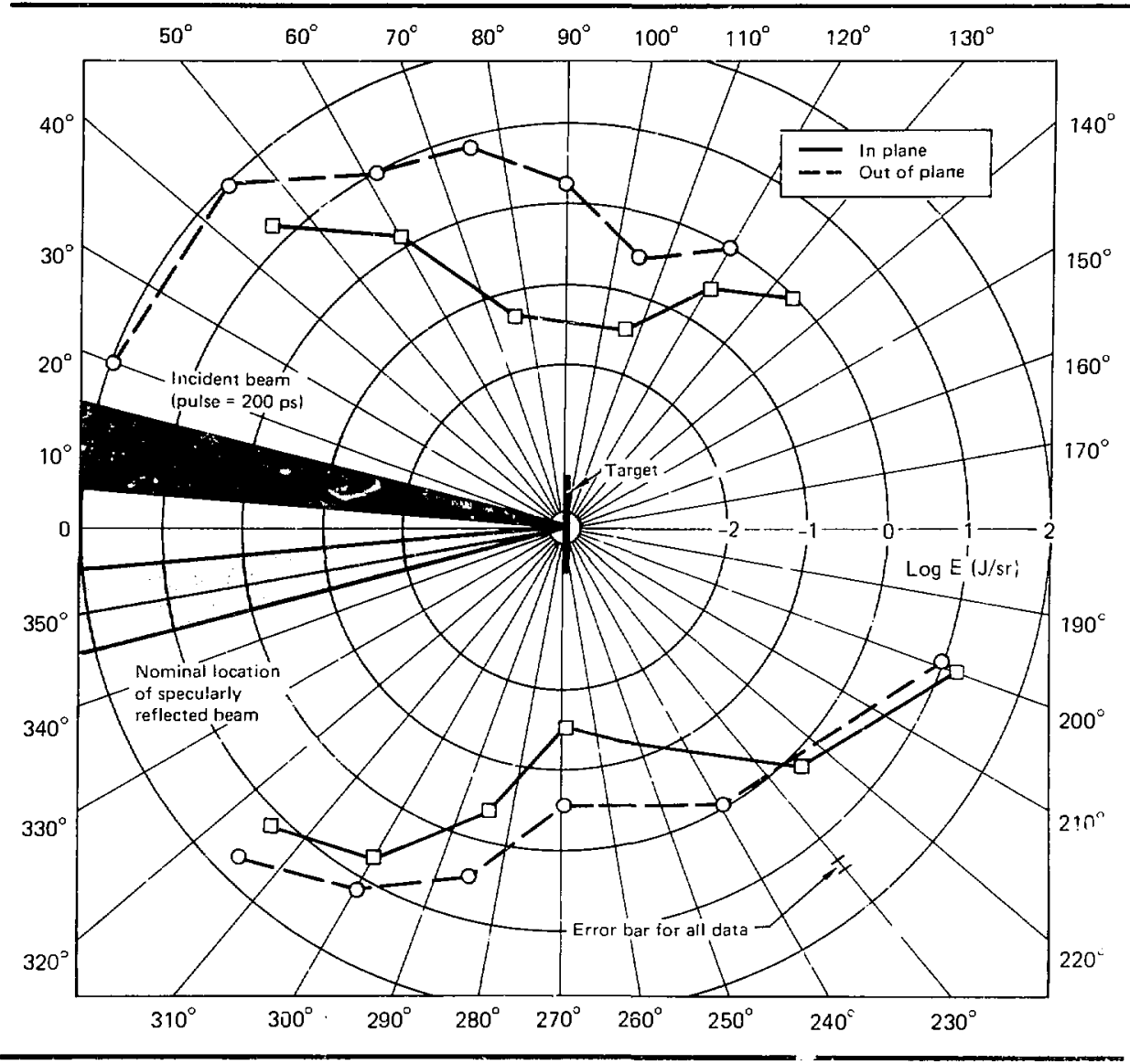

at the location where scattering occurs but also to the direction of propagation of the scattered wave as modified by refraction.

Authors: V. C. Rupert, W. I. Kruer, and D. W. Phillion

Major Contributors: K. M. Campbell, K. (;. Fstabrook, and the Argus Operating Staff

\section{References}

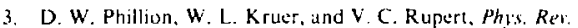
Lett 39. 24 (1977), P. 1529: B. H. Ripin, J. M. MoMahon. E. A. Mclean. W. M. Minheimer. und J. A. Situmper. Phys. Rev. Le't 33, 11 (1974), p. 634: B. Grek, H. Pepin,

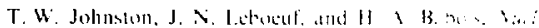

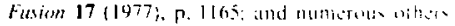

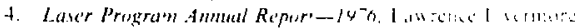

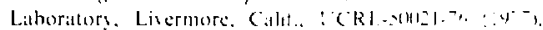
ก. 5-156: D. W. Phillion, R. 1. ! crehe. I ( Ruper: R . 1

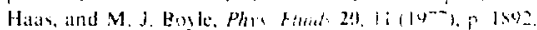

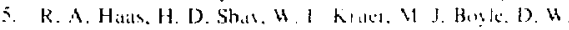

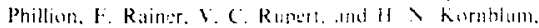

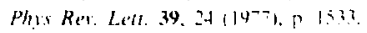

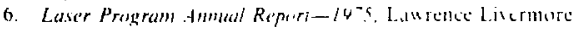

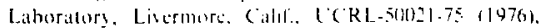
pp. 402 to $4(0)$ ?

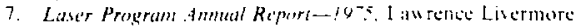
Laboraltors, Losermure. Callt. CCRL-30021-75 11976). pp. 40410405 
8. C. Ginuedard, A. Saleres, and M. Decrobette. Polarizalion and Angle-rof-Incidence Dependence aj Scattering and Absorphun on Short Laser Pulse Interaction Experimems. presented at the Fifth Workshoj) on Laser Interactson with Matter. Rochester, Veu York, 1979; R. Sigel, K. Fidmatnn, A. G. M. Matinunkel. R. Pelsh. G. Takirm and S. Wilkousi. Laser Plaima Interacriom Siudies of Pl.F Branching. presented it the XllI Fouropean Conference on Laver Interatuon wath Malter. L.uprig, 1979.

9. D. W. Phillon. Laturence I.sermore Lahorators. I.Aermose. (alif. porkitle communkation (Mar 1979).

10. K. (i. Latatrook, Phus. F/uds 19 (1976). p. 1733

\section{Electron Transport Analysis Using Layered Slab Targets}

Experiments using disk targets composed of layers of dissmilar materials have provided the most detalled information available on clectron transport in laser-heated plasmas. The materials and layer ihicknesses can be chosen so that their tharacteristic $x$-raly emissions indicate where, when, and by how much the plass a is heated.

Experiments employing varying thicknesses of one material covering a target substrate of another material hate teen reported by several werkers. ${ }^{11.12}$ Generally, the results have been interpreted as supporting the existerce of some inhibition mechanism for eleetron thermal transport. In $1976 \mathrm{G}$. Dahlbacka performed LASNEX calculations ${ }^{13}$ 10 model the experiments of Young et al. "1 that employed $\mathrm{CH}$-coated $\mathrm{Al}$ disk targets; he found support for inhibited electron conduction. He further noted some aifferences between the model required to "fit" the experimental results and the usual LASNEX clectron conduction model eriployed to simulate other experiments.

Our recent analysis using LASNEX has likewise shown that the usual electron conduction rodel simulates the $\mathrm{CH}-\mathrm{on}-\mathrm{Al}$ experiment poorly. and that very strong transport inhibition is indicated. We also find that even with revised transport modeling the calculations and experiment still show significant disagreement. We have thus performed similar expcriments to further expiore the nature of these differences.

We report here the results of our $\mathrm{CH}$-on-Al disk irradiations using the Argas laser. Resuits of the experiments are presented first: these support and extend previous experimental results. Second, we analyze some of the surprising and interesting results from the viewpoint of LASNEX simulations.

\section{Experimental Resuits}

A series of twelve flat-disk experiments was conducted with the Argus laser to study electron transport at a nominal laser intensity of $10^{15}$ $W / \mathrm{cm}^{2}$. The laser pulses were approximately Gaussian, with FWHM of $\sim 100 \mathrm{ps}$ and a verage energy of $122( \pm 23) \mathrm{J}$. Target irradiation was done with a $\mathrm{p}$ polarized converging beam, focused through $f / 2.2$ lenses: the target normals were rotated $30^{\circ}$ relative 10 the incident laser pulse direction. The targets were aluminum disks $600 \mu \mathrm{m}$ in diameter by $25 \mu \mathrm{m}$ thick. coated with $0,0.25$, or $0.67 \mu \mathrm{m}$ of parylent. We also irradiated pure ; arylene targets $600 \mu \mathrm{m}$ in diameter and $25 \mu \mathrm{m}$ thick.

From previous studies of the ahsorption of $1.06-\mu \mathrm{m}$ laser light by low-Z planar plasmas. ${ }^{14}$ we estimate that the absorhed energy wats $30 \%( \pm 10 \%)$ of the incident taser energy. The fraction of hackscattered light was constant. within experimental error, at $\sim 10 \%$ of the incident energy, independent of target material. The FWHM of the hackscattered light was $65 \%( \pm 6 \%)$ of the incident laser pulse FWHM.

Properties of the heated plasmas were inferred from varicius measurements of $x$-ray emissions from $150 \mathrm{eV}$ to $50 \mathrm{keV}$. The array of $x$-ray diagnostics used for these experiments included a 10-channel

Fig. 6-9. Integrated soft $x$-ray emission into $2 \pi$, rormalized by incident laser energy, vs CH thickness. Measurements were made with a fiat calorimeter and a Dorte spectrometer. The "attenuation" depth [see Eq. (3) and text] is 0.11 $( \pm 0.07) \mu \mathrm{m}$.

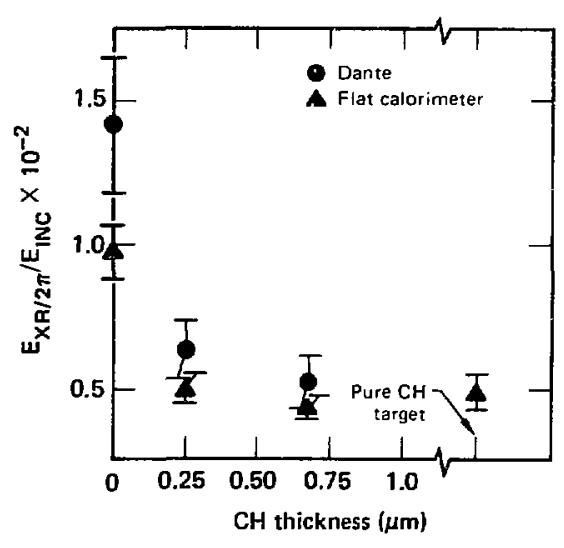


Fig. 6-10. Llae tmission spectra for a bare aluminom target and $0.25-\mu \mathrm{m}$ CH target, detected by a crystol x-ray spectrograph.
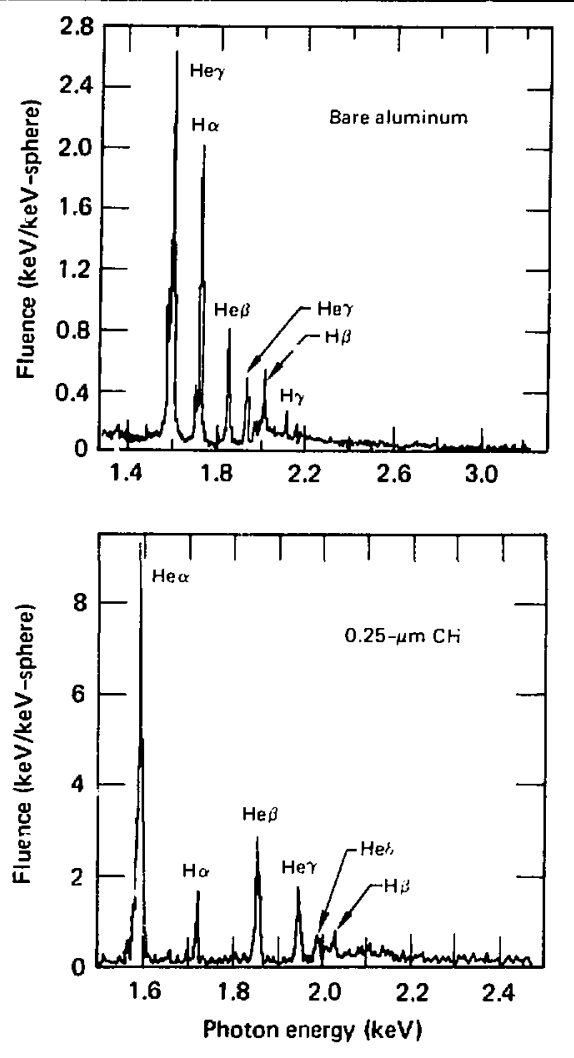

Dante spectrometer ${ }^{15}$ : a fast, flat $x$-ray calorimeter ${ }^{1 f}:$ a soft $x$-ray streak eameral ${ }^{17}$ : a 7-shovter (7 Si PIN K-edge filters); and an FFLEX (filter fluorescer) spect rometer. ${ }^{18}$

Figure 6-9 shows temporally and spectrally integrated emiscion energy $\left(0.15 \leqslant h^{\prime \prime} \leqslant 1.5 \mathrm{keV}\right)$ plotted against $\mathrm{CH}$ thickness, as measured by the fast. flat calorimeter and the Dante spectrometer. and normalized by the incident laser energy: the Dante and calorimeter data are plotted independently in the figure. (Though both sets of data indicate the same trends, the calorimeter data are lower than the Dante data; this relative difference between the two instruments is typical and so far is not explained.) Both detector sustems, which were
Fig. 6-11. Aluminum line radiation vs $\mathrm{CH}$ thiciness; radiltion was detected by the crysial spectrograph, integrated from 1.5 to $2.2 \mathrm{keV}$, and normalized by incident laser energy.

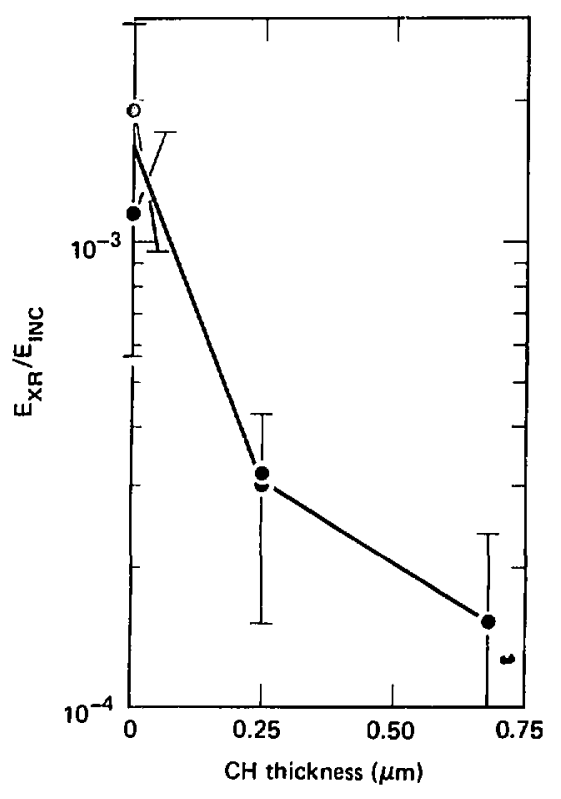

at $60^{\circ}$ to the target normal, show that the soft $x$-ray emission drops off by a factor of $2.4( \pm 0.4)$ in going from hare $A l$ to pure $C H$. It atso appears that $0.25 \mu \mathrm{m}$ of $\mathrm{CH}$ on $\mathrm{Al}$ behaves more nearly as pure CH than as Al. This indicales very steep thermal gradients and very shallou hurn-through depths, consistent with previous measurements at NRL."

If ue describe the soft s-ray emission vs $\mathrm{CH}$ thiskness by the relation

$$
I_{R}=I_{A l} c \frac{-\Delta x}{L}+I_{C H}\left(\begin{array}{ll}
e & \frac{-\Delta x}{L}
\end{array}\right) \text {. }
$$

where $E_{R} \equiv\left(\left.E_{X R}\right|_{2 \pi}\right) / E_{I X(\cdot,} E_{A l}$ and $E_{C I I}$ are the fraction of the total emission from the aluminum and parylene, respectively, $\Delta \mathrm{x}$ is the thick ness of the $C H$, and $L$ is an "attenuation" depth, then the data give the remarkably small value, $L=0.11( \pm 0.07)$ $\mu \mathrm{m}$.

The soft $x$-ray streak eamera recorded $x$-ray pulses whose duration was $2.0( \pm 0.2)$ times the 
Fig. 6-12. Hiph-energy x-5ay fuence as measured with the FFLEX and 7Shooter datector systema, normalized by incident faser energy.

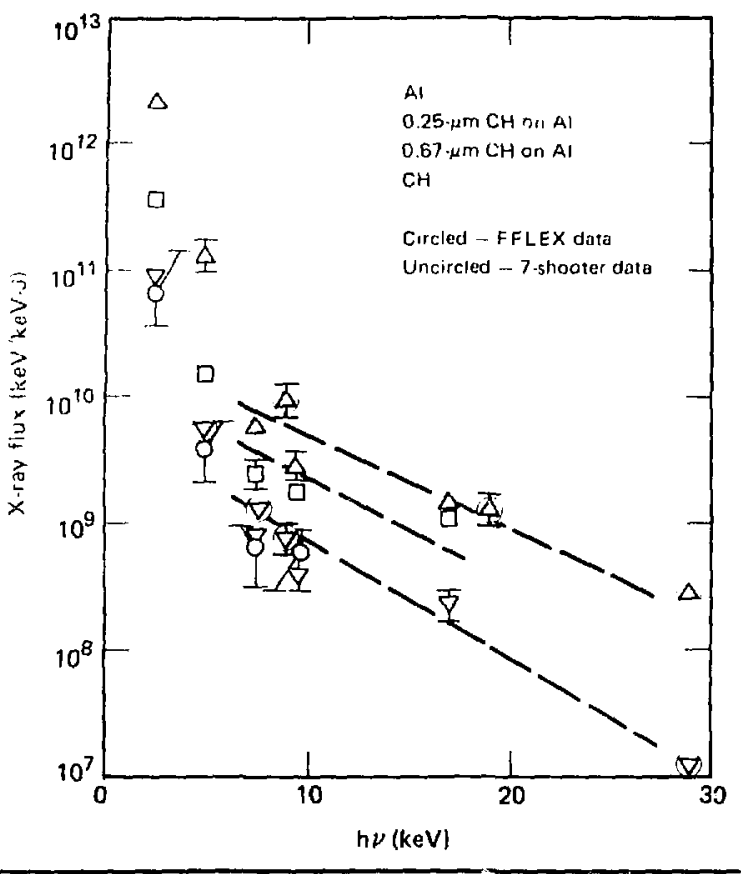

low-energy $x$-ray data. The spectrum for $0.67 \mu \mathrm{m}$ of $\mathrm{CH}$ on $\mathrm{Al}$ is the same as for pure CH, while the spectrum for $0.25-\mu \mathrm{m} \mathrm{CH}$ on $\mathrm{Al}$ is intermediate between pure $\mathrm{Al}$ and pure CH.

The suprathermal $x$-ray spectrum implies a hot electron temperature of $T_{h} \simeq 4$ to $6 \mathrm{keV}$ and a fraction of incident light converted into hot electron energy of l:14)/lime $\approx 10$ to $15 \%$ for all targets. This, logether with an assumed absorption of $30 \%$ $\left( \pm 10^{\prime \prime \prime}\right)$, suggests that the suprathermal electron distribution contains $\sim 1 / 3$ to $1 / 2$ of the energy absorbed by the targets. Corrections for hydrodynamic losses are small and are accounted for in the betailed calculations discussed below. Anisotropic x-ray emission ${ }^{16}$ could alse incroduce an uncertainty of at factor of 2 or so.

\section{Analysis and Simulations}

We were surprised by the low measured flux and slope of the high-energy $x$ rays in these mea-

fWllM of lise lise s pulse, but the $x$-rily pulses were nol at simple, slandard (i.c., Gaussian) shape.

ligeure 6-10 shows rypical line emission spectrat detected by an $x$-ray crystal spectrograph for a pure aluminum arget and for a largel coated with $0.25 \mu \mathrm{m}$ of parylene. Hot aluminum $\mathrm{He}$ - and $\mathrm{H}-\mathrm{like}$ lines are prominently visible. Though cold Al lines swold have been detected if present, none wero seen, which implies that energetic electrons capable of exciting these lines were not getling in a cold $\mathrm{Al}$ to any appreciable degree. The Al $\mathrm{He}$-and $\mathrm{H}$-like lines occur above 1.5 and $1.7 \mathrm{keV}$, respecilively, and below the respective suries limits of 2.086 and $2.304 \mathrm{keV}$. The energy in the integrated spectrum from 1.5 :s $2.2 \mathrm{keV}$, normalized by the incident fitser cnergy and plotted in arbitrary units (Fig. 6.11), drops by al factor of 10 in going from bare $\mathrm{Al}$ to is $0.67-\mu \mathrm{m}$ coating of $\mathrm{CH}$.

The high-energy $x$-ray fluences shown in Fig. 6-12 follow the same trends, qualitatively, as the surements, ligure 6-13 compares the 10-to-50-keV $x$-ray fluence measurencents obtained several years ago in ( 11 disk irradiations near $10^{15} \mathrm{~W} / \mathrm{cm}^{2}$ on Janus ${ }^{20}$ with those of the Argus experiment reporled here. Jach experimental point from Janus represents the average of three or more measurements. while only one $\mathrm{Cll}$ disk shot was available from these Atgus transport experiments. The Janus data have been corrected very slightly, using $T_{110 T} \propto 1^{0.3}$ scaling to shift intensity by a factor of two to $10^{15}$ $W / \mathrm{cm}^{2}$. Compared to the earlier Janus experiments. we find in these Argus experiments that the fluence of 10-10-50-keV $x$ rays per incident joule is down by a factor of 4 to 10 .

The simulation of hot electrons produced by resonance absoption in the LASNEX sode is governed by the relation

$$
\mathrm{T}_{\mathrm{HOT}} \approx \alpha\left\langle\mathrm{T}_{\mathrm{e}}\right\rangle+\left(\mathrm{II} \mathrm{\lambda}^{2}\right)^{\gamma}\left\langle\mathrm{T}_{\mathrm{e}}{ }^{6}\left(1+3 \frac{\mathrm{T}_{\mathrm{i}}}{\mathrm{ZT}{ }_{\mathrm{e}}}\right)^{\varepsilon} \mathrm{Z \phi},\right.
$$


Fig. 6-13. Hot $x$-ray fuence comparioon of curreat measurements on Argus with earlier resalts inferred from Janus disk experiments. A stgailicant decrease in lot $x$-ray fuence and slope is indicated.

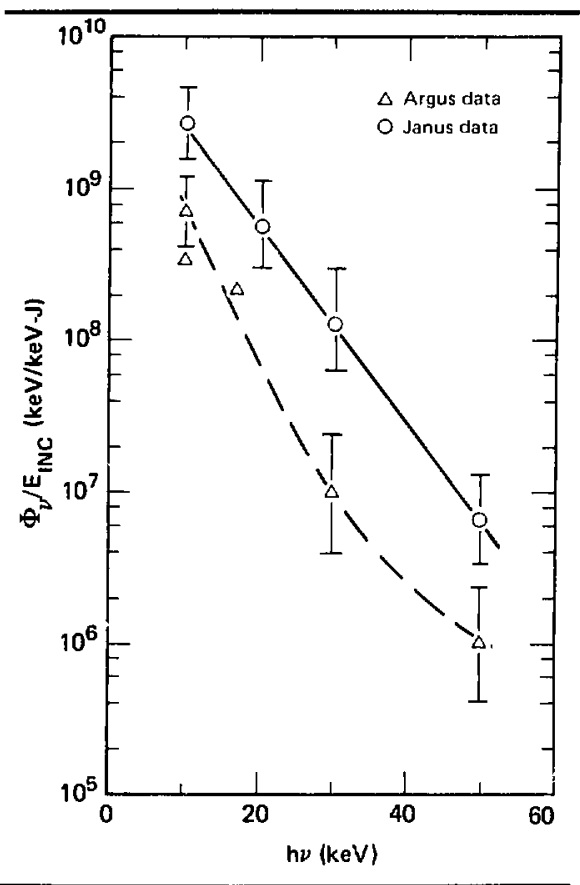

where $T_{e}$ is the mean temperature in (keV) of all electrons. $I$ is the laser intensity in units of $100^{17}$ $W / \mathrm{cm}^{2}, \lambda$ is the laser wavelength in units of $1.06 \mu \mathrm{m} . T_{e}$ and $T_{i}$ are the temperatures (in keV) of thermal electrons and ions. and $Z$ is the nuclear $Z$ of the plasma. From normalization to plasma simulation, and from recent high- $Z$ disk experiments ${ }^{21}$, the coefficients are determined to be $\alpha=1, \beta=50, \gamma=$ $0.42 . \delta=0.04, \epsilon=0.25$ and $r: 0.25$. Checking the model against the low- $Z$ disk experiments on Janus. we find the calculated $T_{O T}=13 \mathrm{keV}$. compared with the measured ThOT $=8.3( \pm \mid .0) \mathrm{keV}$. Good agreement with the Janus data is obtained using $\beta=$ 30. and depositing 20 to $30 \%$ of the incident energy into hot electrons.

The hot $x$-ray fluence calculated with LASNEX is plotted in Fig. 6-14, together with the A rgus data for various absorption models; the four models plotted are summarized in Table 6-5. To fit the Argus data we were forced not only tr reduce
Fig. 6-14. Hot x-ray fluence comparison of verious calculational absorption models (detailed in Table 6-5) with recent Argus measurements. To fit the Argus dath, the spectral hardness parameter must be decreased by $a$ factor of $\sim 2$ and the enercy absorbed into hot electrons must be decreased by $a$ factor of $\sim 2$ to 3 .

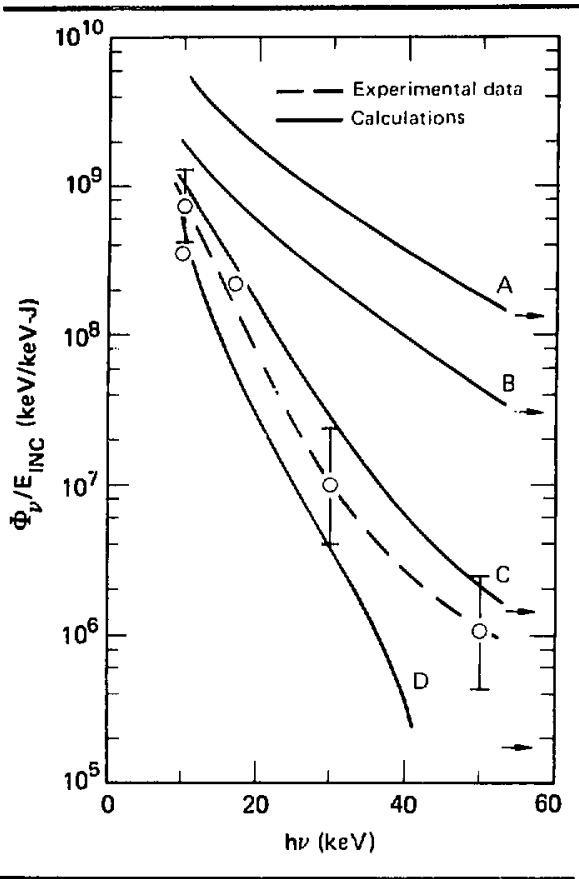

the spectral hardness of hot electrons by reducing $\beta$ 10 20, but also to reduce the hot electron energy to only $\sim 10 \%$ of the incident energy, as indicited by the simple estimates above. This apparent decrease in the number and temperature of hot electrons is intriguing. and further verification of the data is clearly essential.

The infornation on electron transport is mainly contained in the absolute radiation emission levels for $\mathrm{Al}$ and $\mathrm{CH}$, and in the rate of fall-off as increasing thicknesses of $\mathrm{CH}$ are applied to the $\mathrm{Al}$. The data points in Fig. 6-15 show the absolute sub$\mathrm{keV}$ emission determined experimentally; the curves show the same quantity calculated by LASN $r$.X using various models (summarized in Table 6-6). The models are characterized by the values used for the "anomalous" flux-limit reduction of the thermal $\left(f_{t}\right)$ and suprathermal $\left(f_{s}\right)$ electrons, where, as usual, $\phi_{1, s}$ 
Table 6-5. Summary of various absorption models compared with high energy $x$-ray emiscion in Fig. 6-14. The parameters varied are $f_{D}$, the fractional dump of light at the critical surface (mocking up resonant absorption), and $\beta$, the coefficient of the $1 \lambda^{2}$ term in the formula for $T_{\text {HOT [see Eq. (4)]. The thermal and auprathermal flux }}$ limits have been reduced in all models by $f_{e}=f_{s}=0.03$. Also given for the $\mathrm{CH}$ target are the energy absorbed by resonance absorption $\left(E_{R_{A}}\right)$ and the total absorptlon (E $\left.\mathrm{E}_{\text {ABS }}\right)$.

\begin{tabular}{ccccc}
\hline Model & $\mathbf{f}_{\mathrm{D}}$ & $\beta_{\mathrm{D}}$ & $\mathbf{E}_{\text {RA }}(\mathrm{J})$ & $\mathrm{E}_{\text {ABS }^{(J)}}$ \\
\hline $\mathrm{A}$ & 0.3 & 50 & 32 & 36 \\
$\mathrm{~B}$ & 0.1 & 50 & 11 & 16 \\
$\mathrm{C}$ & 0.1 & 20 & 11 & 16 \\
$\mathrm{D}$ & $0.4^{\mathrm{a}}$ & 20 & 11 & 14 \\
\hline
\end{tabular}

${ }^{\text {a }}$ Model D utilized a preliminary treatment of Brillouin scattering, which reflected about $75 \%$ of the incoming light bufore it reaclied the critical surface.

$=f_{1,4} n_{1,3} m v_{t . s}^{3}$ is the salurated heat flux carried by either thermal or suprathermal electrons.

Many previous experiments have been adequately interpreted using $f_{1}=0.03, f_{s}=1$, and with absorptions into suprathermal electrons $\sim 30 \%$. As seen in Fig. 6-15, however, this model (E) does poorly when compared with the Argus experimental data: the absolute emission levels are too high and the scale-depth for emission fali-off is too great. Similar discrepancies had been seen in a comparison of I.ASNIX calculations with the NRL layeredslab experiments.

We have atlempled several ways of varying the model's parameters to improve its correspondence to experimental values. Two obvious techniques are reducing the transport coefficients (Fig. 6-15, Table 6-6) and reducing the absorption (Fig. 6-16, Table 6-7). Fither of these modifications can improve the agreement between calculated and measured attenuation depths. Using flux-limit reduction factors of $f_{t}=f_{s}=0.01$ (Fig. $6-15$ ) gives about the same results as reducing the absorption to $10 \%$ with $f_{1}=$ $0.03, f_{s}=1$ (Fig. 6-16). Neither modification, however, produces an attenuation depth as small as that seen experimentally. Further, as some improvement is made in the calculation of the attenuation depth, the calculated ratio of emission from pure Al to emission of pure $\mathrm{CH}$ becomes discrepant with experimental results. Finaliy, either model alteration
Table 6-6. Summary of transport models compared with sub-keV $x$-ray data in Fig 6-15. Parnmeters varied are the fux.dimit multipliers for thermal and suprathermal electrons. These models hive fxed absorption purameters: the reionance absorption dump fraction $f_{D}=0.3$, and the suprathermal spectral hardnes parameter $\beta=20$. Energy deposited vi resonant absorption was about $32 \mathrm{~J}$ throughout. The total absorbed energy $\mathbf{E}_{\mathrm{ABS}}$ is tabulated for Al and CH didks, and varies wenkly.

\begin{tabular}{|c|c|c|c|c|}
\hline \multirow[b]{2}{*}{ Model } & \multirow[b]{2}{*}{$\mathbf{f}_{\mathbf{t}}$} & \multirow[b]{2}{*}{$\mathbf{f}_{s}$} & \multicolumn{2}{|c|}{$\mathrm{E}_{\mathrm{ABS}}(\mathrm{J})$} \\
\hline & & & Al & CH \\
\hline E & 0.03 & 1.0 & 40.7 & 36.8 \\
\hline $\mathbf{F}$ & 0.03 & 0.03 & 40.3 & 36.0 \\
\hline $\mathbf{G}$ & 0.01 & 0.01 & 38.4 & 35.3 \\
\hline H & 0.003 & 0.003 & 39.7 & 36.2 \\
\hline
\end{tabular}

Table 6.7. Summary of absorption models compared with sub-keV x-ray data in Fig 6-16. Parameters varied are the resonance absorption dump fraction $f_{D}$ and the coefficient of the inverse bremsstrahlung absorption opacity $K_{I B}$. Theso models havo fixed transport parameters: the thermal flux limit $f_{t}=0.03$, and the suprathenmal fux limit $f_{8}=1,0$. The suprathermal spectral hardness parameter $\beta=20$. Energy absorbed for Al and CH disks is tabulated separately for resonant absorption and total absorption.

\begin{tabular}{|c|c|c|c|c|c|c|}
\hline \multirow[b]{2}{*}{ Model } & \multirow[b]{2}{*}{$\mathbf{f}_{\mathrm{D}}$} & \multirow[b]{2}{*}{$\mathbf{K}_{\mathbf{I B}}$} & \multicolumn{2}{|c|}{$\mathbf{E}_{\mathbf{R A}}$} & \multicolumn{2}{|c|}{$\mathrm{E}_{\mathrm{ABS}}$} \\
\hline & & & Al & $\mathbf{C H}$ & Al & CH \\
\hline $\mathbf{E}$ & 0.3 & 1.0 & 31.3 & 32.2 & 40.7 & 36.8 \\
\hline I & 0.1 & 0.5 & 10.7 & 10.9 & 16.1 & 14.0 \\
\hline $\mathbf{J}$ & 0.05S & 0.35 & 5.9 & 6.0 & 9.9 & 8.3 \\
\hline $\mathbf{K}$ & 0.03 & 0.25 & 3.2 & 3.3 & 6.2 & 5.0 \\
\hline
\end{tabular}

alone seems somewhat extreme from a theoretical point of view.

An intermediate model, involving some reduction in absorption and some reduction in transport, produces nearly equivalent results, and is perhaps more justifiable. Using a preliminary Brillouin scattering model, ${ }^{22}$ we calculate $20 \%$ absorption. Combining this with $f_{t}=f_{s}=0.03$ results in agreement as good as has been achieved to date. This LASNEX modeling is compared in Table 6-8 with the three roughly equii !lent models discussed here; a comparison of the results obtained using each of these models with the results of the experiment is presented in Table 6-9. We have performed calculations using reduced electron conductivity (instead of reduced flux limit), and obtained results very similar to the experimental data reported here. Still another alternative is modifying the collisional coupling rate of suprathermal electrons, but this is not noticeably better. 
Fig. 6-15. Comparison of models with varying transport coefficients with sub-keV emission measurements from Al targets with varying Ch coating thekness; model detwils are given in Table 6-6. Best agreement for 35\% absorption is obtained with flux-timit reduction by a factor of 0.01 for both thermal and suprathermal electrons.

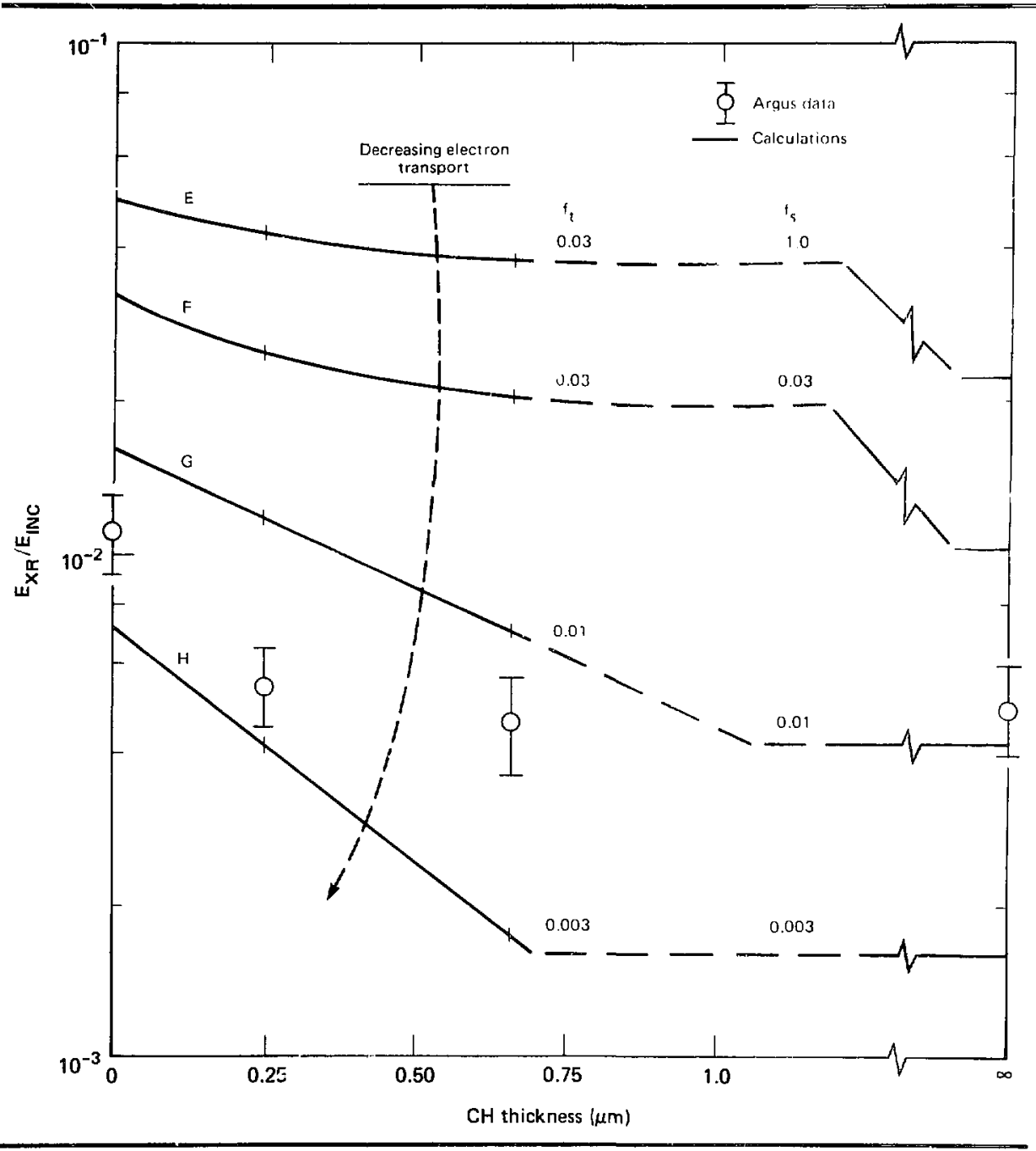

\section{Summary}

The recent layered-slab transport experiments have provided further, more quantitative evidence of strong transport inhibition in $100-\mathrm{ps}, 10^{15} \mathrm{~W} / \mathrm{cm}$. I.06- $\mu \mathrm{m}$ laser-produced plasmas. The experiments at Argus, under larger-spot irradiation conditions and using stor-ke) i-ray diagnosties, have verified the altenuation depth measuremers obtained at NRI. In addition. new high-energy $x$-ray measurements using the Fil.t.X and 7-shooter detector systems have raised intriguing questions about the 
Fig. 6-16. Comparison of models with varying absorption with measured sub-keV emission from Al tarets with rarying CH coating thickness; madel detalls are given in Table 6-7. Best agreement for $10 \%$ absorption is obtained with flax lbait of 0.03 for thermal elecirons and $\mathbf{1 . 0}$ for suprathermal electrons.

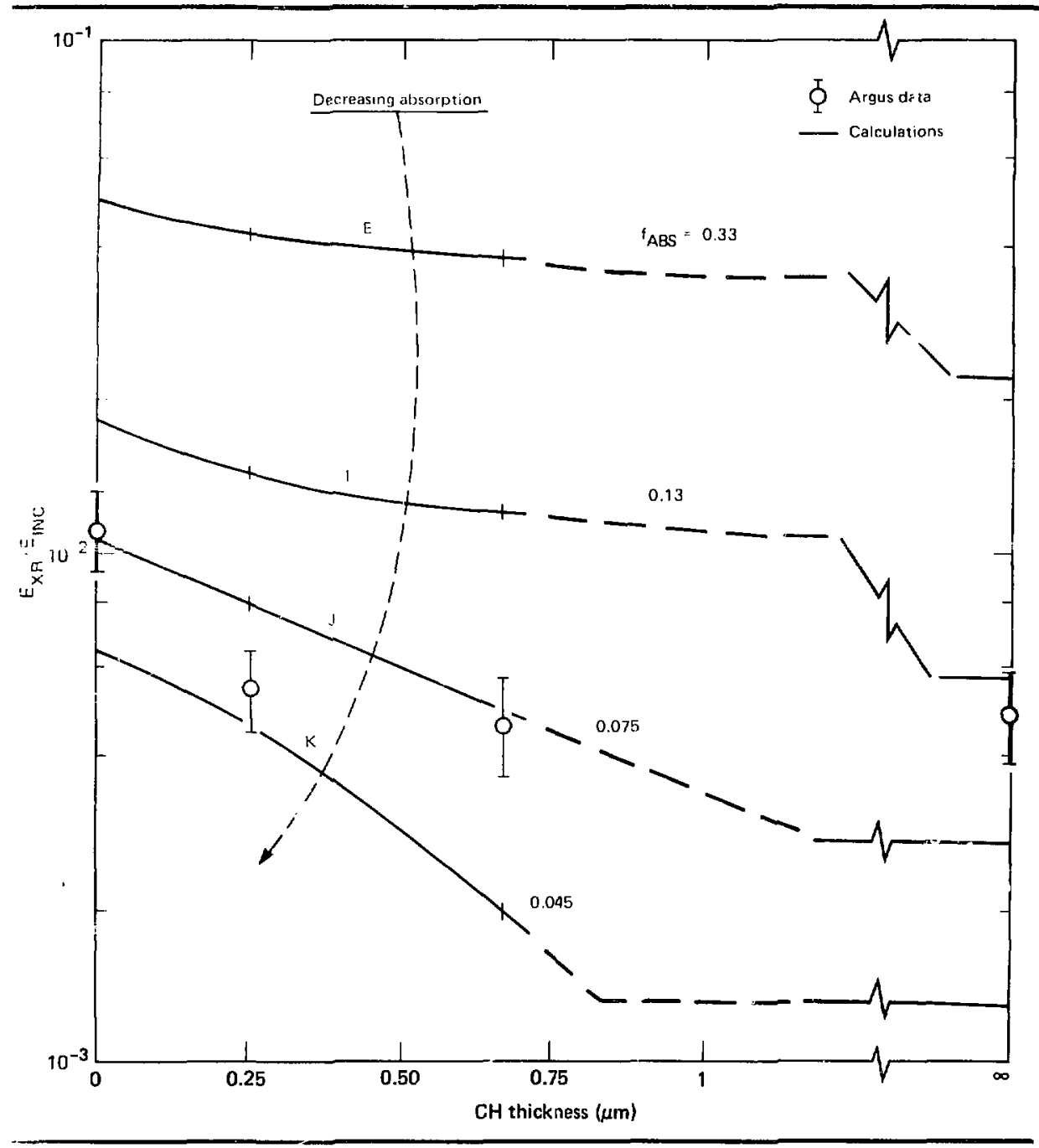

mb-fraction aid temperature of hot electrons. ational work is necessary, however, in order to .... both uncertainties in the experimental data wit discrepancies between the data and the results of I ISNIXX simulations.
LASNEX calculations of soft $x$-ray emission versus $\mathrm{CH}$ thickness that use standard model assumptions overestimate the attenuation depth by $\therefore$ factor of about 6 , well outside the experimental uncertainties. Alternative assumptions can improve the attenuation-depth calculation somewhat, but 
Table 6.8. Comparison of "standard" LASNEX model (A) used for prcvious interaction experiments with three alternative mode's which improve the modeling agreement with the Argus electron-transport experiment.

\begin{tabular}{lllllll}
\hline Model & $\mathbf{f}_{\mathrm{D}}$ & $\boldsymbol{\beta}$ & $\mathrm{K}_{\text {IB }}$ & f $_{\mathrm{t}}$ & $\mathbf{f}_{\mathrm{s}}$ & Comment \\
\hline A & 0.3 & 50 & $0.5-1.0$ & 0.03 & 1.0 & Previous work \\
$\mathbf{G}$ & 0.3 & 20 & 1.0 & 0.01 & 0.01 & Reduced transport \\
J & 0.055 & 20 & 0.35 & 0.03 & 1.0 & Reduced absorption \\
L & $0.4^{\mathrm{a}}$ & 20 & 1.0 & 0.03 & 0.03 & Mixed, moderate chianges \\
\hline
\end{tabular}

This model utilized a preliminary treatment of Brillouin scattering, which reduces the energy incident on the critical surface by a factor of $1 / 3$, thus reducing sesonant absorption by $1 / 3$ and inverse bremsstrahlung by $1 / 2$.

Table 6-9. Sumnary of LASNEX model results and comparison with experiments. The models are described in Table 6-8 and the fext. The attenuntion depth measures tho depth of a CH layer required over an $\mathrm{Al}$ target to make the sub-keV emission drop to CH-target levels [see Eq. (3)]. The emission ratio is tho ratio of sub-keV emission energy from a pure Al target to the cmission from a pure $\mathrm{CH}$ target.

\begin{tabular}{cccc}
\hline Model & $\begin{array}{c}\text { CH disk } \\
\text { absorption } \\
\text { fraction }\end{array}$ & $\begin{array}{c}\text { Attenua- } \\
\text { tion depth } \\
(\mu \mathrm{m})\end{array}$ & $\begin{array}{c}\text { Emission } \\
\text { ratio }\end{array}$ \\
\hline A & $\mathbf{0 . 3 3}$ & 0.7 & 3.0 \\
G & $\mathbf{0 . 3 5}$ & 0.5 & 3.9 \\
J & $\mathbf{0 . 0 7 5}$ & 0.6 & 4.0 \\
L & $\mathbf{0 . 1 5}$ & 0.5 & 5.2 \\
Experiment & $\mathbf{0 . 1 5}$ to 0.35 & $0.11 \pm 0.07$ & $2.4 \pm 0.4$ \\
\hline
\end{tabular}

overall agreement with the sub-koV x-ray measurements is not much improved.

liurther work needs to be done in several areas: experintental checks of absorption and high-energy $x$-ray fluence are required: calculational thecks and other models must be pursued: still other parameter regimes must be examined as well. to determine electron-transport properties in situations more relevant to fu,ure laser fusion targets.

Authors: 1). I.. Banner and W. (. Mead

Major Contributors: F. M. Camphell and W. $\mathrm{W}$. kiruer

\section{References}

II. I C. Young. R. R. Ii hillock. R. Decoste. B. H. Ripın. D. I. Vagel. 1. A. Stamper. 1. M. MeMahon, and 5.1 . Bodner, "I aser-P'odaced Plasma lenergy Trunsporl Through Plastic lilms." Appl. Phys leet 30. 49 (1977).

12. B. Yaakobi and T C. Bristow, "Measurement of Reduces Thermal conduction in (layered) Laser-Target Fxperiments." Phys. Rev lent. 38. 350)(1977).

13. CiH. Dahibacka. "Catculation of e Tramsport Coef ficients in Layered Target Laser Plasmas." Bull. Am. Phys Sor. $1028(1976)$

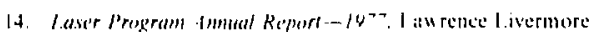

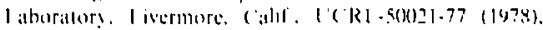
pe $6-7$ 10 $6-11$.

15. Taser Program Immal Repon--1978. I an rence I jermore

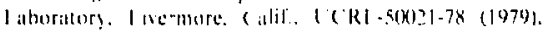
pr. 6.5 to $(1-7$.

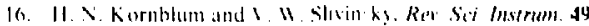
$12(1)+1978)$

17. Laser l'rogram thmal Repert-14:- I au rence lisermore

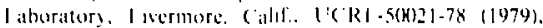
bp. (1-2 $106-5$

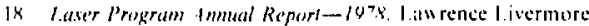
I aboralory. I ivermore. (iatlf.. I ( $R 1$ - $-50(121-78$ (1979). ne 6.5 to $(1-12$

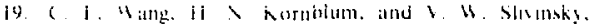
fagular biscributam of Suprathermal I Rave from Laser Produced Plasma. prexented al the Il'S Plasma Physics Meeling. Hoston, Milss. Vinember 12 to 16.1979

20. R. A Hals, M. (C. Mead. M. I. Kruer, D. M. Phillion.

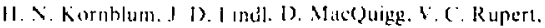
and $k$. (i lirsell. "Irriddiation bi Parylene l)isks with is 1.06 $\mu \mathrm{m}$ I aser," Phas Fhed, 20. 322 (1977).

21. M. 1) Ronem. 1) W. Phillon. I' C. Rupert, W. C. Mead. 11. I. Kruer, I I Thompent. II. V Kurnblum. V. W Slinink!. (i. I Caporasu. If I Boyle. and k. (i. Tirsell "The Interatiosn of lon $\mu$ m I axer Radiatuon with 1 ligh 7

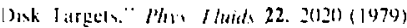

$2 \quad$ 人 (i. I Nithrosk and I b. Harte, "On Redueing Brillouin Scitler." I awrente l iscrmbre I.aburaturs. Iivermore. (:lllf. I ( RI-K262(1. 1979) (ti) be published).

\section{7.-Dependence of Sub-keV X-Ray Emission and Laser Intensity Threshold jor Inhibited Election Thermal Conduction}

Fectron thermal conduction inhibition is a simple mechanism for regulating $x$-ray emission. ${ }^{23}$ The laser light is absorbed at and below the critical density surface, which two-dimensional LASNEX ${ }^{24}$ numerical simulations indicate is 50 to $100 \mu \mathrm{m}$ from the original target surface at the time of peak laser intensity. On the other hand, most of the sub-keV $x$ riys are emitted from the denser ablation layer 
within $20 \mu \mathrm{m}$ of the original disk-target surface. The absorhed laser energy must be transported by eleciron conduction from the underdense plasma to the overdense plasma. A decrease in eiectron thermal conductivity reduces the energy available for lowchergy $x$-ray emission from the dense ablation layer.

Tro-dimensional LASNEX simulations also indicate that when the heal Mux is inhibited, the plasmat corona becomes hotter by 50 to $100 \%$ compared to the uninhibited case; the corona temperallure is 3 to $4 \mathrm{keV}$ instead of $\sim 2 \mathrm{keV}$. When inhibum: aceurs and the low-energy $x$-ray emission Irum the overdense region is therefore reduced, the ane in the corons temperature will result in ad(lllwhil v-raly emission in a higher energy range.

there is substantial experimental evidence (see Rets 23, 25-3.3) (hat electron heal conduction is inhibited in plasmas produced by $1.06-\mu \mathrm{m}$ laser intensities above $10^{14} \mathrm{~W}^{1} \mathrm{~cm}^{2}$. Discussions of mechamusms likely to inhibit electron thermal conduction in laser-irridialed targets have suggested selfgenerated magnetic fields, ${ }^{34}$ ion acoustic

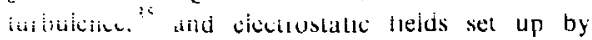
suprathermal electrons. ${ }^{36}$ Datd concerning the dependence al inhibition on paramete ${ }_{1}$ s such as laser intensity, pulse width, wavelength, spot size. largel material. and beam ur.formity should contribute to a better theoretical understanding of conduction inhibition and help determine the dominant mechanism(s). Here we reporl our study of one such parameter. the dependence of inhibition on larget $/$

\section{Experimental Data}

We have conducted a preliminary study of the I-dependence of sub-keV $x$-ray production from laser-produced plasmas at Argus. ${ }^{37}$ using one beam at a nominal laser pulse of $800 \mathrm{~J}$ for I $\mathrm{ns}$ l.WHM: the typical intensity on the target was $5 \times 10^{14}$ it $\mathrm{cin}^{2}$. The targets consisted of disks from 600 to $700 \mu \mathrm{m}$ in diameter and from 12.7 to $25 \mu \mathrm{m}$ thick. Target materials consisted of beryllium, aluminum. litanium, tir, sold. and uranium, with atomic numbers (7) ranging from 41092 . The targets were irradiated witt f:2.2 focusirg optics: the incidence angle was $30^{\circ}$. and the incident beam was linearly polarized $12^{\circ}$ out of the plane of incidence.

A 10-channel filtered $x$-ray detector system called Lante-T (Ref. 38) was used to record the sub-
Fig. 6-17. Typical x-ray emission signals from disk targets ranging from $Z=4$ to $Z=92$, illuminated with $1.06-\mu \mathrm{m}$ laser light at $5 \times 10^{14} \mathrm{~W} / \mathrm{cm}^{2}$ with a pulse of $1 \mathrm{~ns}$ FWHM. A vanadium filter (L-edge at $520 \mathrm{eV}$ ) was used with an aluminum photocathode; time resolution is $190 \mathrm{ps}$.

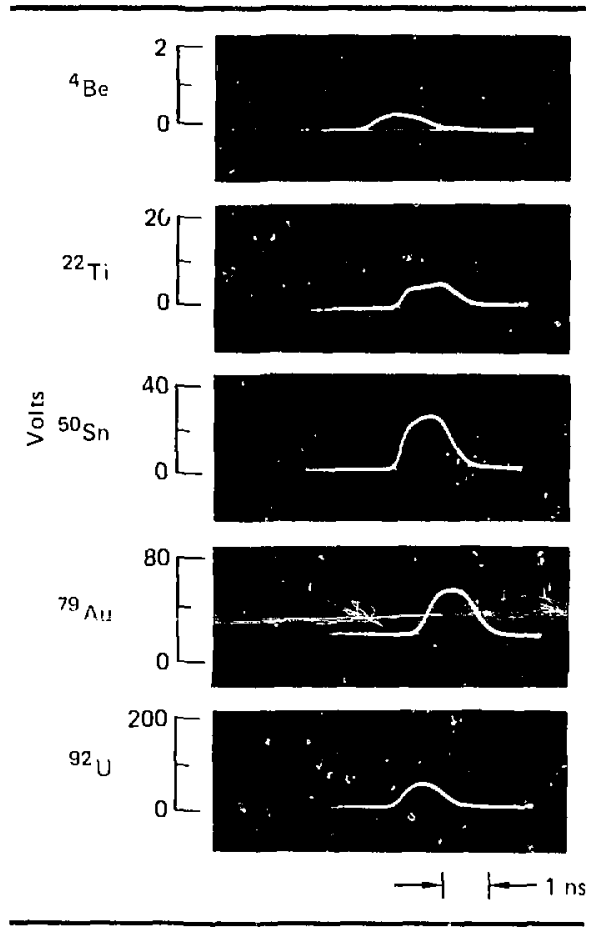

keV emission from the laser-irradiated targets. Dante- $T$ viewed the $x$-ray emission at an angle of $60^{\circ}$ from the target surface normal, in the plane of incidence away from the incident beam. The solid angle subtended by each detector channel was 2.11 $\times 10^{-5}$ steradians for the four lower energy channels and one flat response channel, and $4.36 \times 10^{-5}$ steradians for the live higher energy channels.

One of the interesting results of this experimental series is the systematic Z-dependence of the timeresolved sub-keV x-ray emission, as shown in Fig. 6-17. The data were obtained for the 300-to-520-eV channel, with a time resolution of better than 190 ps. Note that as the larget $Z$ decreases (from uranium to beryllium, for example), for the same Gaussian laser pulse shape the $x$-ray puise shape deviates from a smooth "Gaussian" to an irregular shape; the peak of the pulse becomes progressively 
Fig. 6-18. The results of two Ti disk targets, for the sume laser conditions as in Fig. 6-17. A copper filter (L-edge at $940 \mathrm{eV}$ ) was used with a chromium photocathode; time resolution is $170 \mathrm{ps}$. Note the oscillations in the "nattened" portion of the $x$-ray emission pulse.

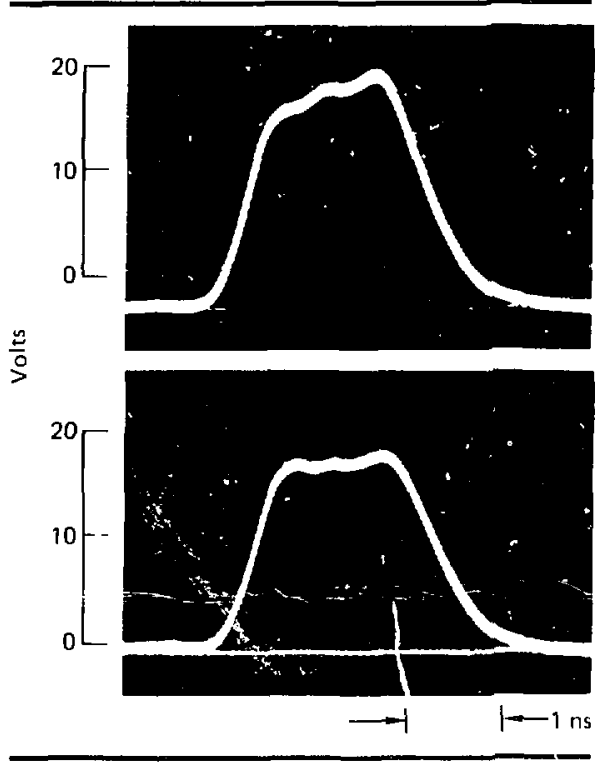

flattened as $Z$ decreases. The transition is shown most clearly by a titanium target, whose $x$-ray emission starts to increase rapidly (presumably following the rise of the incident laser intensity), then at a well-defined point changes abruptly to a show "lincar" rise, and then drops after ] ns (a channel with higher resolution shows that there are oscillations in the "linear" rise region: see fig. $(x-18)$

$X$-ray emission pulses for the same $Z$ material can be quite different at different laser intensities: this is shown in Fig. 6-19 for gold disks. The dita in this figure were obtained from the 650-10-940-eV channel, with a time resulution of better than $170 \mathrm{ps}$. Note that the $x$-ray emission pulse 11 the lower laser intensity $\left(5 \times 10^{14} \mathrm{~W} / \mathrm{cm}^{2}\right)$ is fairly smooth. but as a higher laser intensity $\left(3.3 \times 10^{15}\right.$ $\left.W / \mathrm{cm}^{2}\right)$ the $x$-ray emission pulse rises normally at first, then breaks away abruptly.

A simple of some typical low-energy x-ray spectra is given in Fig. 6-20. The spectral shapes of different materials are quite distinct and can be
Fig. 6-19. X-ray emission sigals from gold disks illuminated with $800 \mathrm{~J}$ for 1 ns with intensity of (a) $5 \times 10^{14} \mathrm{~W} / \mathrm{cm}^{2}$ an.J (b) $3 \times 10^{15} \mathrm{~W} / \mathrm{cm}^{2}$. A copper filter (Ledge at $940 \mathrm{eV}$ ) wa: used with a chromium photocathode; time resolution is $170 \mathrm{ps}$. The negative-going siganl in exch trace is a time reducial.
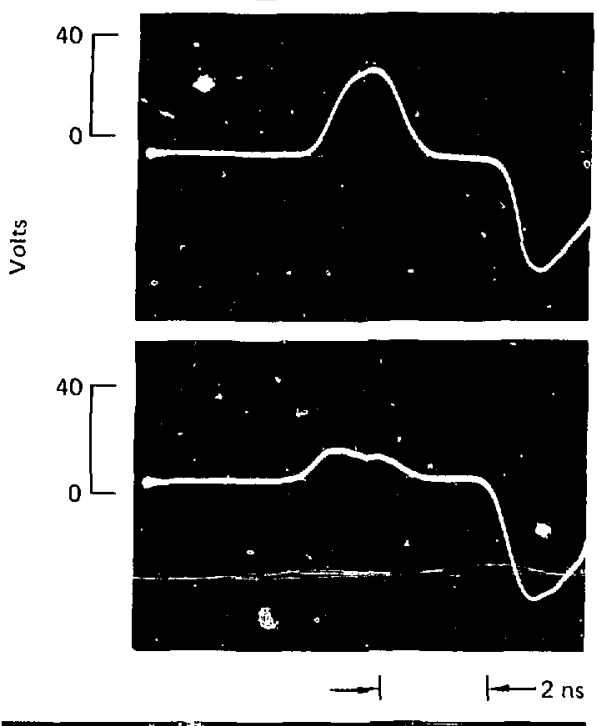

readily reprosed by code calculations. The same is true for low- $\not$ materials such as aluminum and beryllium, which latter material shows a much fister spectral decay with ir "reasing $x$-ray energy. The titanium spectrum is quite different from the other spectra in the figurs. in the sense that in the energy band from ahout 500 to $800 \mathrm{eV}$ there ate strong l. fine cmissions. On the other hand. the small hump near $700 \mathrm{eV}$ in the aluminum spectrem is most probably due to an oxygen line. since an aluminum-oxide layer forms quite readily on an aluminum target.

figure 6-21 shows how well code calculations are able to reproduce the features of the measured spectrum for is titanium target. Because of the presence of the L-lines of a titanium target, it was possible to use the data on relative line intensities of Kelly and Palumbo ${ }^{39}$ to obtain a dominant charge state from the measured spectrum, which in this 
Fig. 6-20. Sample low-energy $x$-ray spectra for $U$, Ti and Al disk targets, illumionted at $5 \times 10^{14} \mathrm{~W} / \mathrm{cm}^{2}$ with a pulse of 1 ns FWHM. Note that the lowest energy channel has the Itregest error bar.

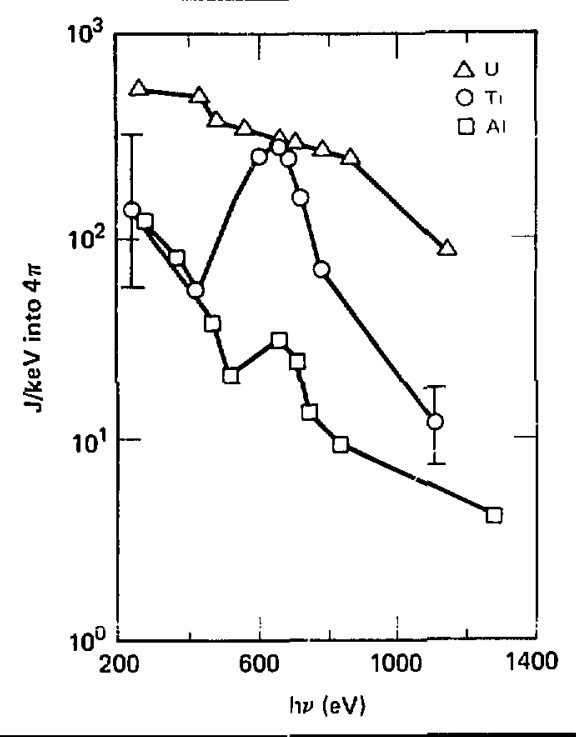

case was $Z_{\text {exp }}=16( \pm 1)$. Also, numerical simulations can obtain the average ionic charge in a computational zone with nxaximun x-ray emission power. The computed charge state was $Z_{\text {code }}=16$ $( \pm 2)$; the \pm 2 does not represent an error bar in the calculation, but indicates the variation in $Z$ over the FWHM of the $x-r a y$ emission region.

The integrated $x$-ray energy from $\sim 100 \mathrm{eV}$ to $1.8 \mathrm{keV}$ can be obtained from the Dante- $T$ signals. The integrated $x$-rav etiergy normalized by the incident laser energy is protted against target $Z$ in Fig. 6-22. Since sub-keV $x$ rays away from the energy regions dominated by line emission are presumably generated by bremsstrahlung of low-energy electrons, these low-energy electrons actually see a screened nucleus: therefore it would perhaps be more meaningful to plot the normalized $x$-ray energy as a function of $Z$, where $Z$ is the codecalculated charge state. $1 t$ is interesting to note that the normalized $x$-ray energy is almost linear with respect to $Z$. This result is quite similar to the bremsstrahlung data obtained with an ordinary
Fig. 6-2i. Comparison of measured and code-calculnted Ti spectrum. $Z$ is the charge state of the $T$ plasana; intensity and polse length are sume as in Fig. 6-20.

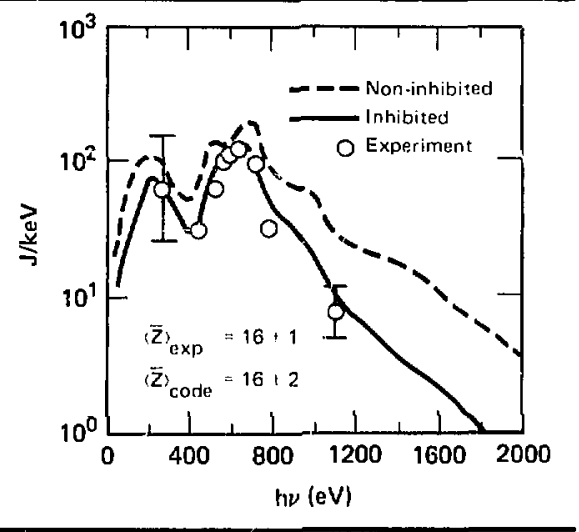

thick-target $x$-ray tube with $x$-ray energy proportional to iarget $Z .^{40}$ the difference being that the $x$ ray encrgy produrod per init $r$ inctest lase: energy is 1 to 2 orders of magnitude larger than the $x$-ray energy produced per unit of cathode-ray energy. ${ }^{41}$

\section{Analysis}

The existence of an intensity threshold for electron transport inhibition has been clearly demonstrated by Pearlman and Anthes, ${ }^{27}$ with measurements of the front and rear plasma thermal expansion velocities from a thin polystyrene film. We extend the idea of an intensity threshold for inhibited conduction to include a Z-dependence of the threshold.

From the time-resolved $x$-ray data in Figs. 6-17, 6-18, and 6-19 we interpret the temporal behavior of the sub-keV $\mathrm{x}$-ray emission as showing the onset of strongly inhibited electron thermal conduction during the rise of laser intensity. As the $Z$ of the larget material is reduced, this strong conciuction inhibition occurs sarlier, that is, at lower laser intensity.

The systematic variation of the $x$-ray pulse shape with $Z$ (Fig. 6-17) indicates that the plasma process involved has a laser intensity threshold which increases with Z. Figure 6-19 can now bc explained: initially the two pulses rise similarly, but the emission from the highe .ensit, shot abruptly 
Fig. 6-22. X-ray energy for various targets as a function of target $Z \mathrm{a}^{-1}$ charge state $\bar{Z}$, at incident intensity of $5 \times 10^{14} \mathrm{~W} / \mathrm{cm}^{2}$. Error bars for values of $E_{n-r a y} / E_{\text {liser }}$ are all $\sim 25 \%$.

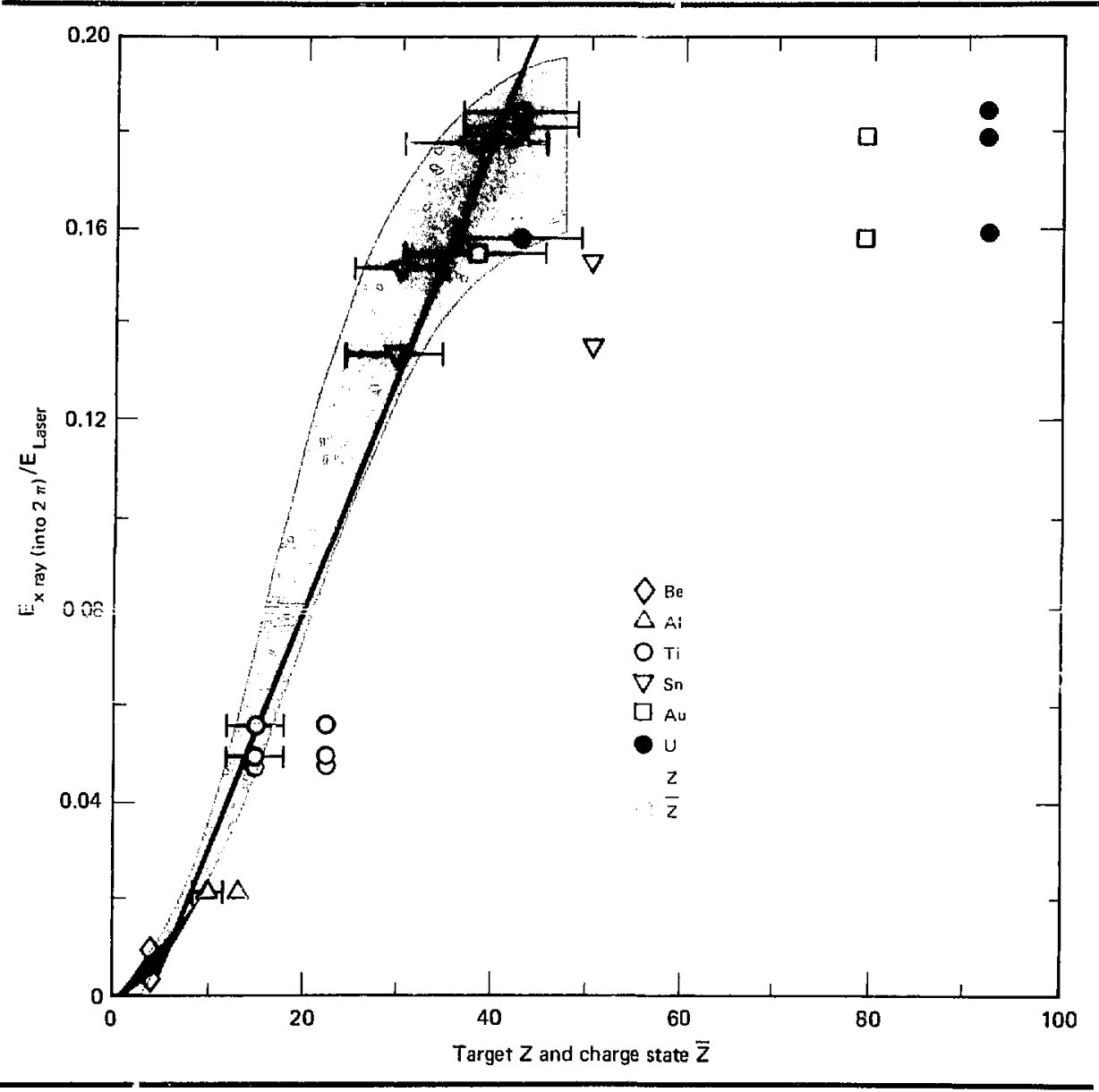

falls away as the threshold is crossed. This kind of behavior appears in all the Dante-T x-ray energy channels. Only a small part of this reduction in $x$ ray emission at the higher intensity is due to reduced absorption of the incident lase: light, since the timeintegrated $\mathrm{x}$-ray emission drops by $60 \%$ while the time-integrated absorption ${ }^{23}$ drops by only $25^{5} \%$ of course a change in the absorption mechanism has not been ruled out. Anomalous absorption in the far underdense plasma would shift the energy flow away from the overdense plasma toward more energetic plasma blow off: a buildup of ion acoustic turbulence with increased laser intensity. for example, might simultaneously increase inhibition and catuse anomalous absorption. ${ }^{\text {? }}$

There are both theoretical and experimental reasons for believing that the variations in pulse shape shown in Fig. 6-17 are not u consequence of atomic structure differences of the targets. Numerical simulations with classical electron thermal conduction and non-LTE ionization physics 
Fig, 6-23. Sub-keV $x$-ray emilssion spectra from gold disks at different intensities.

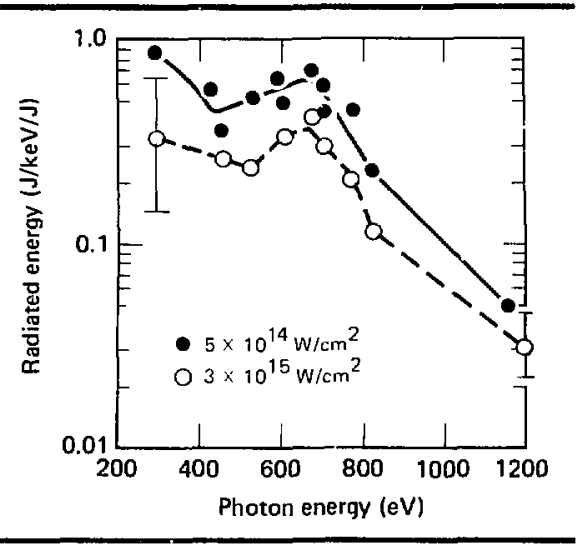

reproduce the qualitative features of the observed $0.2-10-1.5-\mathrm{keV} x$-ray spectra for all of the elements (see. lor example, the Ti spectrum in Fig. 6-21), but

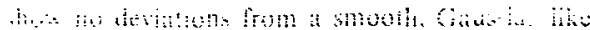
time-dependence for the $x$-ray entission pulses. ${ }^{24.42}$ lixperimentally, the data for gold disks above and below the laser intensity threshold are decisive: in spite of a reduction in radiated energy by a factor of 2.5 for the higher intensity and a dramatic change in the $x$-ray emission pulse shape (Fig. 6-19), we obse-ved only $\pm 15 \%$ changes in the $0.2-$ to- $1.5-\mathrm{keV}$ ppectral shape (Fig. 6-23). Thus, there is no correliaticn between atomic structure (as indicated by $x$-ray spectra) and the temporal behavior of the $x$-ray cmission.

From the data of Fig. 6*19, the laser intensity ihreshold for gold is estimated to be $\left(6_{-3}^{+4}\right) \times 10^{14}$ is $\mathrm{cm}^{2}$. Even though the data of Fig. 6-17 lack time iducia!s. it is clear that the threshold for uranium is Erisuler than $5 \times 10^{14} \mathrm{~W} / \mathrm{cm}^{2}$ and that rough esantites of the thresholds for titanium and tin may he made. These results, and the threshold for rolystyrene from Ref. 27, are plotted in Fig. 6-24.

1 lfects such as $Z$-dependent absorption of laser light and angular distribution of $x$ rays can inHuence th. interpretation of experimental results: preliminary absorption measurements, however, indicate that absorption has a weak Z-dependence (Fig. 6-25) Also. code calculations seem to indicate that the $Z$-dependent angular distribution does not
Fig. 6-24. Strongly inhibited electron thermal conduction threshold us $Z$ of the target material; solid data point in lower left is from Ref. 27.

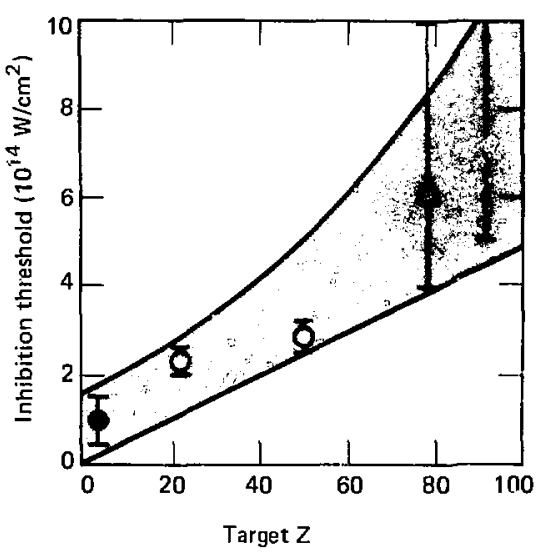

aflect the data interpretation too much. because of

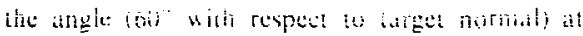
which the measurements were made. The numerical simulation result of 300-to-500- $\Delta \mathrm{V} x$-ray angular distrihutions is shown in Fig. 6-26.

Calculated $x$-ray emission pulses from $\mathrm{Ti}$ are shown in Fig. 6-27 for three nodels of the plasma electron thermal conduclivity. Curves $A$ and $B$ are typical of standard models in that they produce Giaussian-like pulses only 10 or $20 \%$ wider than the incident laser pulse. Curve $\mathrm{C}$ was produced by applying a time-'yarying multiplier to the Spitzer conductivity chosen to reproduce the 1.8-ns FWHM and flat-topped shape of the $x$-ray data. Above threshold, the conductivity nultiplier required by the simulation varied inversely with the $3 / 2$ power of absorbed intensity for both the rising and falling parts of the laser pulse. with a value of about 0.2 at $10^{14} \mathrm{~W} / \mathrm{cm}^{2}$ absorbed intensity. This result is not unique, since the laser light absorption vs time has not been measured. It does show, however, that intensity-dependent conduction inhibition can produce the observed $x$-ray emission.

Sirce more than one inhibition mechanism may be operating. we do not rule out inhibition below the threshold of Fig. 6-19. In fact, the data presented here are consistent with previous results ${ }^{23}$ which showed the need for some inhibition in gold even at $3 \times 10^{14} \mathrm{~W} / \mathrm{cm}^{2}$. 
Fig. 6-25. Plot of absorption fraction (at $5 \times 10^{14} \mathrm{~W} / \mathrm{cm}^{2}$ ) ys $Z$; note that suprathel'mal electrons constitute only a few percent of the absorbed energy for 1-ns pulizes.

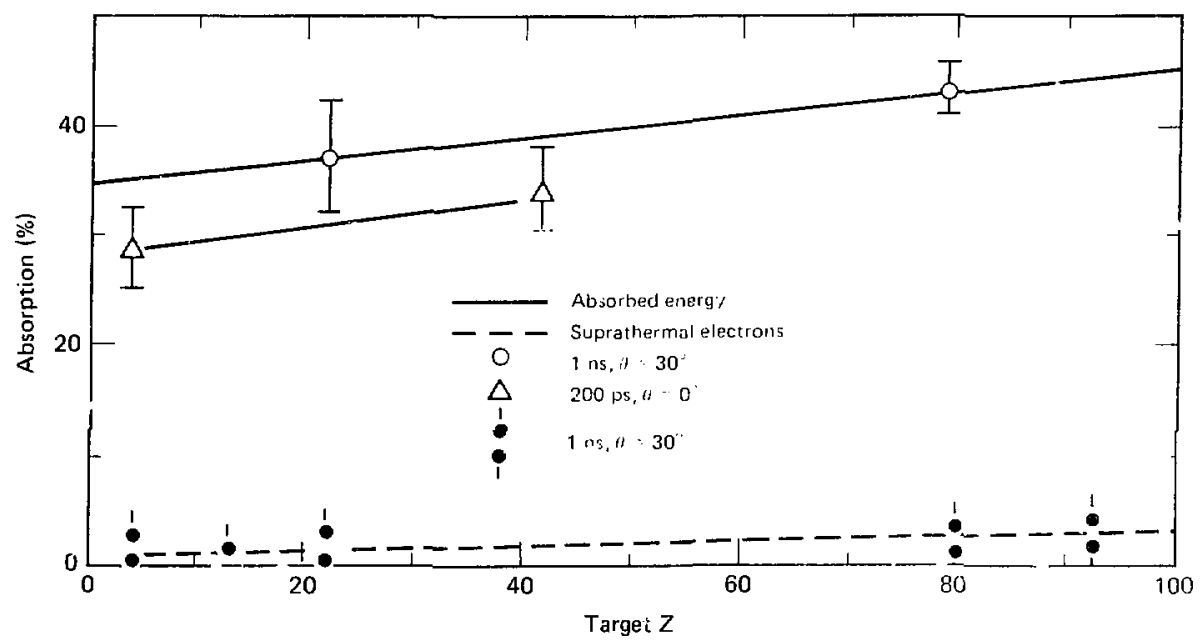

Fig. 6-26. Numerical simulations of the 300-to-500-eV x-ray angular distributions. The Dante-T value at $\theta=60$ extrapolated to $2 \pi$ sr gives total radiated energy within a few percent.

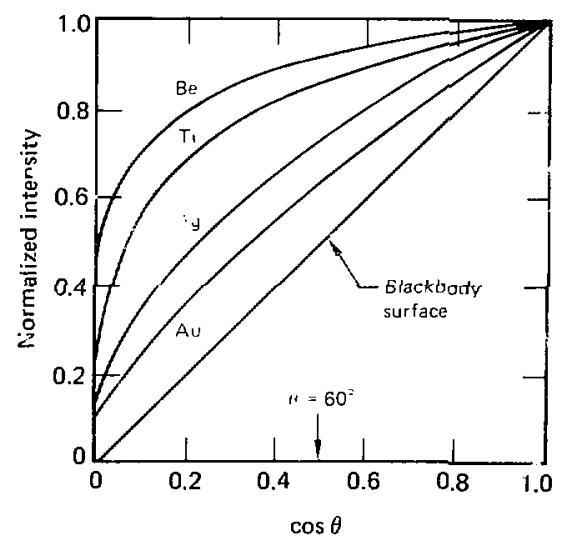

\section{Conclusion}

We have described the temporal behatior of the sub-kev $x$-ray enission from $1.06-\mu m$ laserilluminated disk targets of lixed spot, $1 / 2$, at at in-
Fig. 6-27. X-ray emission is time, taken fronı numerical simulations of a Ti disk illuminared with a I-ns $\mathrm{FWH}$ Caussian pulse with peak intensity of $5 \times 10^{t-1} \mathrm{~W} / \mathrm{cin}^{2}$ at time $=$ (1). The three curves correspond to models of plasma electron thermal ce.nducivity using (a) flux-limited diffusion with classical Spitzer conductivity, $(b)$ a fu $x$ himic reduced by ion acoustic turbulence, and (c) a global conductivity multiplier varied in time to reproduce the experimentally observed $x$-ray emission.

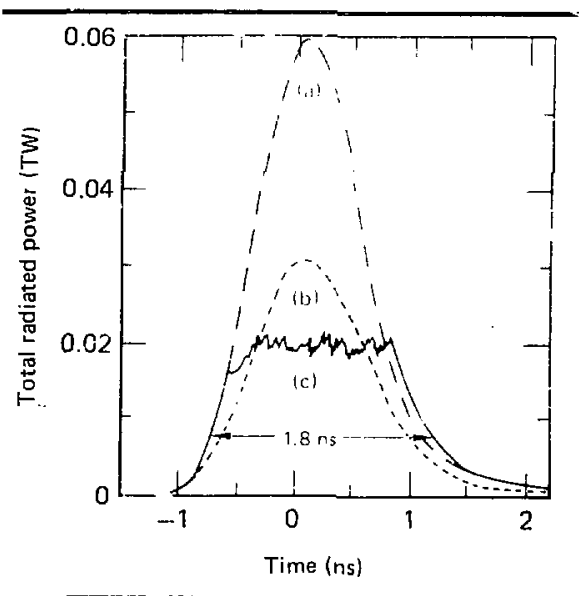


tensity of $5 \times 10^{14} \mathrm{~W} / \mathrm{cm}^{2}$. Abrupt temporal variations in the radiated power as the laser intensity rises have been interpreted as showing the onset of strongly inhibited electron thermal conduction in the laser-produced plasma. The laser intensity threshold for this effect is shown to increuse with the $\mathbf{Z}$ of the target. Consequently, for a fixed laser intensity of a few times $10^{14} \mathrm{~W} / \mathrm{cm}^{2}$, electron thermal conduction is more strongly inhibited for low- $Z$ targets than for high- $Z$ targets.

\section{Authors: P. H. Y. Lee and (;. E. Mcrlellan}

Major Contributors: K. C. Tirsell, E. M. Camphell, C. Caporaso, and M. D. Kosen

\section{References}

23. M. D. Rosen et al., Phis. Finids 22. 2020 (1979).

24. (i. B. Zimmerman. Nimerical Simalations of the High Densily approuch to laser fision, lawente livernore l.aboratory, livermore, (alif., UCRL.-7481I (1973).

25. R. (. Malone, R. I. Mecrory, and R. I. Morse. Phis. Rev. l.eit. 34. 721 (1975).

26. B. 11. Ripin at al. Phys. Rev. lett. 34. 1313 (1975).

27. J. S. Peirtiman and J. P. Anthes, 1ppri. Phys. L.ett. 27, 581 $1975)$.

28. B. Yaakobi and A. Ne. Phys. Rev. Lent. 36, 1077 (1976).

29. W'. C. Mead al al. Mhrs. Rev. Letr. 37, 489 (1976).

30. 1. (C. Young et al.. Apol. Phys. Lett. 30, 45 (1977).

3 B. Yatakobi and T. C. Brislow, Phls. Rev. Lett. 38. 350 (1977).

32. P. N. Camphell. R. R. Johnson. I. J. Mayer. l.. V. Powers. and D. C. Slater. Phys Rev. Lett. 39, 274 (1977).

33. R. Benantar, C. Popovies, R. Sigel, and J. Virntont. Ping.s. He'v: Letl. 42,766 (1979).

34. J. A. Stimper et al., Phys. Re'r: Lett. 26. 1012 (1971); for an exlensive review see J. A. Stamper. L'.S. Vaval Research J.ahoratory Report fo. 3872.1978 (unpublished).

35. D. W. Forslund. J. Coophys Res. 75, 17 (1970): R. Bickerlon. Vuct. Fusion 13. 457 (1973).

36. I. I. Valeo and I. R. Bernstein. Phys. Fluids 19. 1348 (1976).

37. H. W. Sinimons el al. Appl. Opt 17. 999 (1978).

38. K. (i. Tirsell, H. N. Kornblum, and V. W. Slivinsky. Bull. Am. Phys. Soc. 23, 807 (1978).

39. R. L. Kelly and L. J. Palumbo, L.S. Noval Research Laboratory Report No. 7599. (June 1973).

40. H. II. Condon and H. Odishaw. eds.. Handhook of Physics (Me(iraw Hill. New York, 1967). 2nd ed. p. 7-127.

41. A. H. Compton and S. K. Allison, X RAYS in Theory and Experiment (Van Nostrand Co.. New York, 1957), p. 89.

42. R. J. Faehl and W'. L. Kruer, Phys. Fluids 20. 55 (1977): W. M. Manheimer, D. G. Colombant. and B. H. Ripin. Phys. Rev. Letl. 38. 1139 (1977).

\section{High-Energy X-Ray \\ Measurements From Disks of \\ Different $\mathbf{Z}$}

We have traditionally measured high-energy $(20 \mathrm{keV} ₹ \mathrm{~h} \approx 100 \mathrm{keV}) \times$ rays to gather information about suprathermal electrons produced in the laser-plasma interaction. ${ }^{43}$ During the last year we have made such measurements at Argus, as part of the long-pulse $(t-0.9 \mathrm{~ns})$, variable- $Z$ disk experiments. The primary diagnostic was a 6-channel filter fluorescer (FFLL.X) spectrometer located in the plane of incidence and $14.5^{\circ}$ from the plane of polarization. ${ }^{44}$ The six energy channels were located at $20,29,50,54,70$, and $88 \mathrm{keV}$. Due to the low flux obtained from the majority of the targets, four of the six channeis employed only K-edge filters: in view of the rapidly falling spectra that were recorded, however, there was little difficulty in assigning aceurate energy bins to the different channels. The targets were rotated $30^{\circ}$ with respect to the laser axis with the beam incident near p-polarization. The filter fluorescer viewed the targets at an angle of $25^{\circ}$ to the disk normal. The geonietry of the experiment is shown in Fig. 6-28.

Samples of the data collected for plasmas with low (Be), intermediate (Ti), and high (U) $Z$ are shown in Fig. 6-29. The peak intensity incident on the targets ranged from 3 to $5 \times 10^{14} \mathrm{~W} / \mathrm{cm}^{2}$. The

Fig. 6-28. Experimental geometry for long-pulse, bighenergy $x$-ray measurements using a 6-channel filter fluorescer detector.

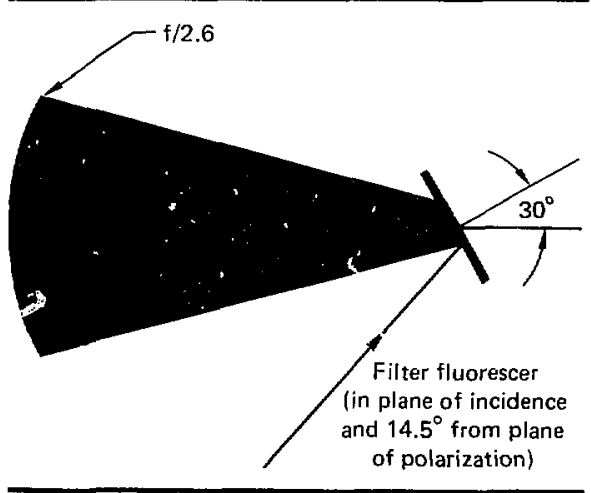


flux from these targets scales roughly linearly with nuclear charge $Z$, as we would expect for highenergy-electron, thick-target bremsstrahlung. Bremsstrahlung emission scales with $Z^{2}$ whereas stopping power scales with $Z .^{45}$ To illustrate this $Z$ dependent scaling, Fig. 6-29 gives the expected flux from $\mathrm{Ti}$ and $\mathrm{U}$ (dashed lines) based on the Be data (solid line), assuming identical electron spectra and absorption of light independent of target $Z$.

The data also suggest a hardening of the spectrum as the target $Z$ increases (e.g.. compare Be and U: This trend is in qualitative agreement with theoretical expectations based on recirculation of the hot electrons through the heating region at critical density, due to the target albedo increasing with $Z$ (with a predicted scaling of $Z^{1 / 4}$ ). ${ }^{46}$

The experimental results for these long-pulse irradiations also show the difficulty in describing the spectrum as a simple exponential with a slope defined as $\theta_{1-1}^{-1}$. This is most clearly evidenced by the titanium data, whose spectrum can be described as

Fig. 6-29. High-eaergy $x$-ray emission as a function of $Z ; Z$ scaling at flux normallzed to Be. Curves for $T i$ and $U$ (dashed lines) are expectec Paxes, based on menared Be data (solid line).

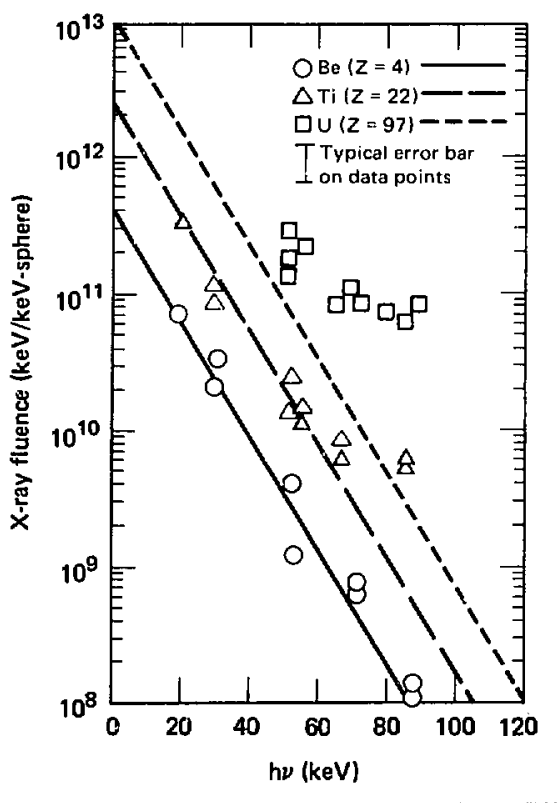

both is not tail and a superhot tail. The latter component could possibly arise from processes such as Raman scattering and $2 \omega_{\text {pe }}$ decay, occurring at onequarter critical density. ${ }^{47}$

To unfold from the measured $x$-ray spectrum both the electron spectrum and the energy absorbed via collective processes requires the use of any assumptions which cannot be as yet verifies $: x-$ perimentally. Interpretation of experimental results is affected, for example, by the shape of the electron distribution, by coronal losses, and by isotropy i the $x$-ray emission. Nonetheless, if we ignore coronal losses and assume both isotropy of $x$-ray emission and a Maxwellian electron distribution characterized by $\theta_{11}$. the measured flux levels suggest that for all the $Z$ materials only $₹ 1 \%$ of the incident energy appears in suprathermal electrons for these moderate-intensity, long-pulse irradiations. Calculations indicate that coronal losses may account for $80 \%$ of the suprathermal energy, however, and thus the hot-electron fraction suggested by the $x$-ray flux should be viewed only as a lower bound.

A full understanding of suprathermal electron generation under these irradiation conditions will require more sophisticated experimentation, to measure the electron spectrum, determine coronal losses. examıne the Raman and $2 \omega_{\mathrm{pe}}$ instabilities, and ascertain the isotrcpy of the $x$-ray emission.

Author: E. M. Campbell

Major Contributors: S. M. Lane, B. L. Pruett, and H. N. Kornblum

\section{References}

43. P. A. Hais, W. C. Mead, W. L. Kruer, D. W. Phillion, N. H. Kornblum. J. D. Lind!, D. MacQuigg. V. C. Rupert. and K. G. Tirsetl. Phys. Fluids 20. 332 (1977).

44. H. N. Kornblum, B. L. Pruett, K. G. Tirsell, and V. W. Slivinsky. "Filter Fluorescer Experiments on Argus Laser," presented to the Division of Plasma Physics at the Meeting of the American Physical Society. Colorado Springs. Colo. (1978).

45. J. D. Jackson. Classical Mechanics (New York: Wiley \& Sons, 1962). p. 513.

46. K. Estabrook. M. D. Rosen, Bull. Am. Phys. Soc. 24, 8 (1979).

47. J. R, Albritton, Bull. Am. Phys Soc. 24, 8 (1979). 


\section{Angular Distribution of Suprathermal X Rays}

The angular distribution of suprathermal $\checkmark$ rays enitled from laser-produced plasmas has gencrally been assumed $\omega$ be isotropic, except for thick largets: the total $x$-ray emission encrgy has wstaly been calculated simply by multiplying the ener $s$-inegraled spectrum by $4 \pi$. The angular distributions of low-energy $x$ rays and ions from such plasmass are anisolropic. ${ }^{48.49}$ however, and for some laser conditions the fast electrons produced in lasergas interactions are also directional, due to the reconaluce absorption effect. ${ }^{50}$ It is therefore both meresting and important to measure the angular diaribution of high-energy $x$ rays from laserproduced plasmias.

Some diclails of our measurement geometry are whown in 1.ig. 6-30. We placed eight x-ray detectors in Shival target chamber ports located at an arimuthal angle $\phi=126^{\circ}$ and at polar angles ranging irom d $=4510148^{\circ}$ : the larget is located al the

Fig. 6-30. Experimental geometry for measurements of highenergy $x$-ray angular distribution. Shiva laser beams are incident on the target (at the origin of the coordinate system) from above and below; orly one of elght detectors is shown.

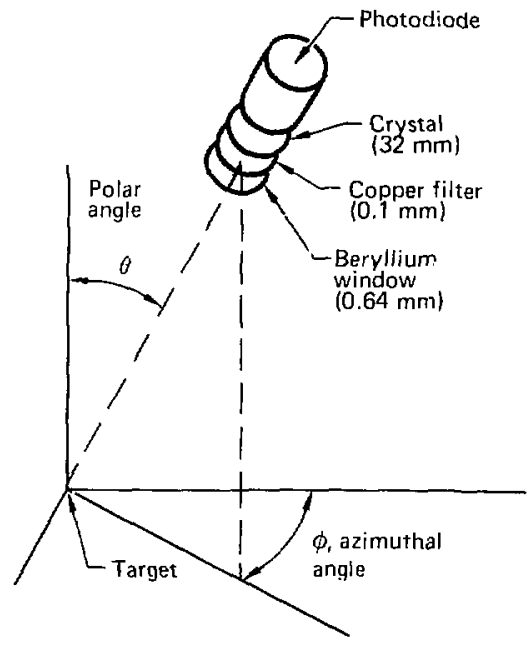

Fig. 6-31. Sample detector response to a fat x-ray spectrum.

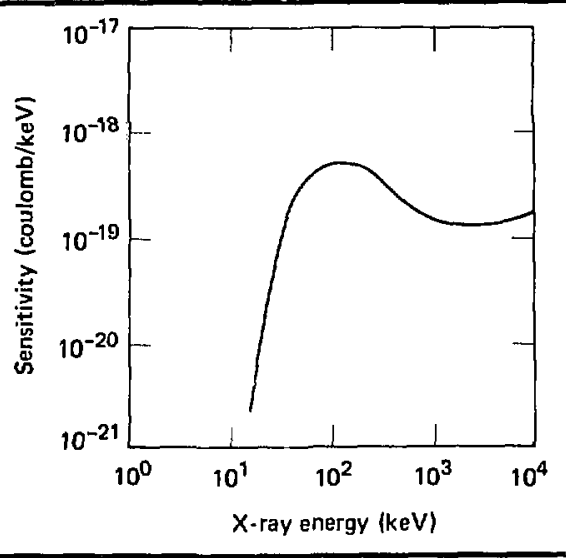

origin of the coordinate system in the figure. The detectors consist of a Be-windowed Nal(TI) erystal $50.8 \mathrm{~mm}$ in diameter and $32 \mathrm{~mm}$ thick, coupled to a photodiode with 5.20 response. Behind the 0.64$\mathrm{mm} \mathrm{Be}$ window on the target chamber is a $0.1-\mathrm{mm}$ Cu filter. followed by a Ta collimator $4.8 \mathrm{~mm}$ thick with an opening $36.1 \mathrm{~mm}$ in diameter (the collimeter is not shown in the figure). A typical response of the detector system is given for a flat $x$ ray spectrum in rig. 6-31. The cutoff of the lowenergy $x$ rays is determined by the $50 \%$ transmission of the Cu filter, at $37 \mathrm{keV}$. The calibrated sensitivities of the eight detectors varied up to $40 \%$ for $x$-raly energies between 8 and $97 \mathrm{keV}$.

Figure 6-32 shows the measured suprathermal $x$-ray angular distribution normalized at $90^{\circ}$. from gold disks $600 \mu \mathrm{m}$ in diameter and $15 \mu \mathrm{m}$ thick. and from a glass microspbere $149 \mu \mathrm{m}$ in diameter and $6.4 \mu \mathrm{m}$ thick filled with D-T gas. The angular distribution shows two strong peaks, one at abcut $60^{\circ}$ and the other at about $105^{\circ}$. That different targets guve similar angular distribution tends to suggest that the minimum at $90^{\circ}$ is not due to absorption of $x$ rays in the plane of the gold disks.

Any such unexpected result is initially suspect. however, and we tried many variations of the experiment to confirm the effect. We switched detectors. fitters, cables. scopes, etc., to check any possible systematic errors, and added $0.813-\mathrm{mm}$ Al filters benind the $\mathrm{Cu}$ filters to remove any possible $\mathrm{K}$-edge effect from the $\mathrm{Cu}$. Interposing $\mathrm{Pb}$ filters $0.5 \mathrm{~mm}$ 
Fig. 6-32. Angular distribution of suprathermal $x$ rays normalized at $90^{\circ}$, from gold disks and a D-T-filled glass microsphere. Note the uniform peak distribution at $60^{\circ}$ and $105^{\circ}$.

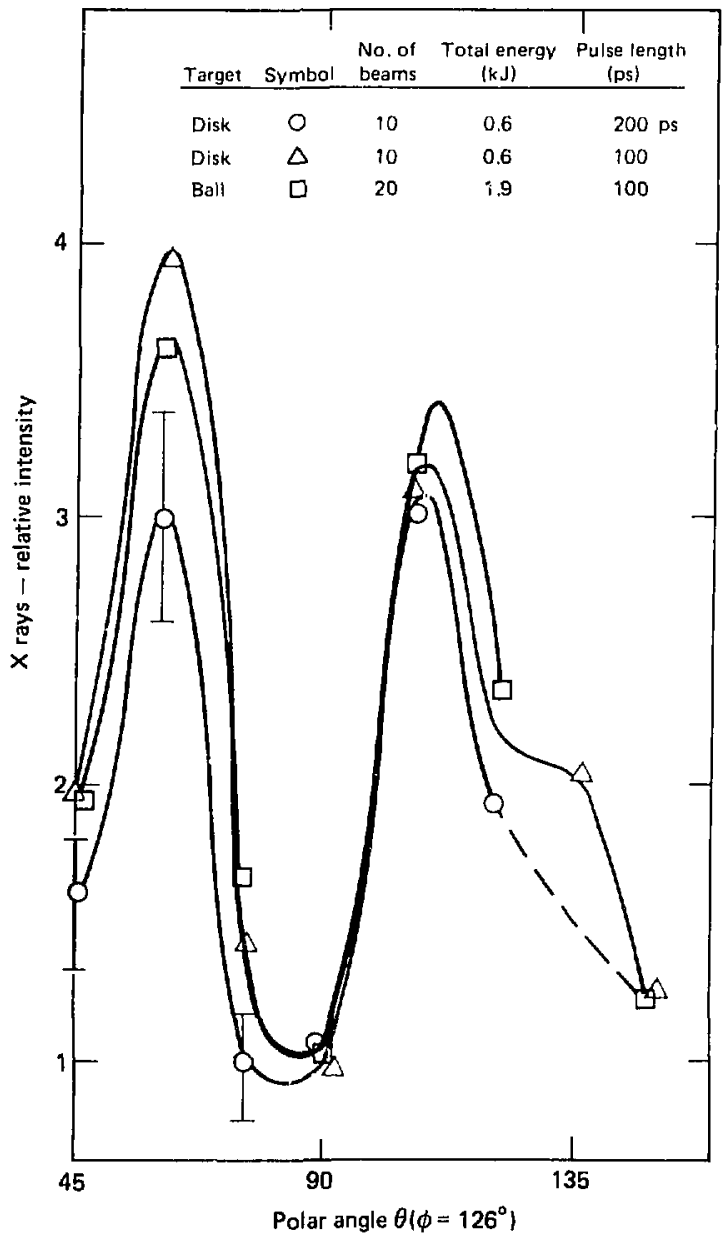

We next conducted a series of single-beam shoss to study the possible dependence of the peaks on the polarization or electric vector of the laser light; no such dependence was observed. This result is shown in Fig. 6-33, in which $J \phi^{\circ}$ and $\lambda \|^{\circ}$ (E) refer respectively to the change in the polariation angle and the change in the electric vecter direction of each be:am with respect to beam 13. The detecors are locialed in the plane of polarization of iseam 13. Generally the x-ray peak on the silme side of the incident beans is stronger than the peik at the onposite side of the gold disk: i.e., the peak at $60^{\circ}$ is stronger than at $105^{\circ}$ lor beams 13,11 and 17, which are incident from $/$ near $0^{\circ}$.

In order to see the angular distribution visually, a stack of 50 $m m-b y-50-m m x$-ray films behind a $0.1-\mathrm{mm}$ (u filter was placed 30 mra (at $\phi=162^{\circ}$. $\theta=105^{\circ}$ ) from a gold disk rligs. 6-34 and 6.351 . The orientation of the target and lilm pack is shown in lig. 6-35. The target was irradiated at $2.8 \times 10^{14} \mathrm{~W}^{\prime} \mathrm{cm}^{2}$ with the upper 10 Shiva heams: sample results are given in 1:ig. 6-34. which show ral the density distribution of the $1-r$. deposition on R-lype film. and (h) the density profile along a horiontal! line at the center of the film $1 \mathrm{~m}$ age. The profile was nol corrected for the vary ing distance and incident angle of $x$ rays on the film from the target.

thick reduced the signal more than tenfold, but the peaks remained. In some experiments we used all 20 Shiva beams, in others we ased only 10 . We also varied the pulse length and beam energy. Installing powerful magnets capable of removing $30-\mathrm{MeV}$ electrons from the paths to the $x$-ray detectors had no effect. indicating that the signal came from photons, not electrons.
In another shot. a slack of $18-\mathrm{mm}-$ by-92-mm film strips was placed $50 \mathrm{~mm}$ (at $\phi=i 98^{\circ} . \theta=90^{\circ}$ ) from a gold disk target. The film strips were curved so that their surfaces were at equal distances from the target and covered $110^{\circ}\left(A=35^{\circ}\right.$ to $\left.145^{\circ}\right)$. 


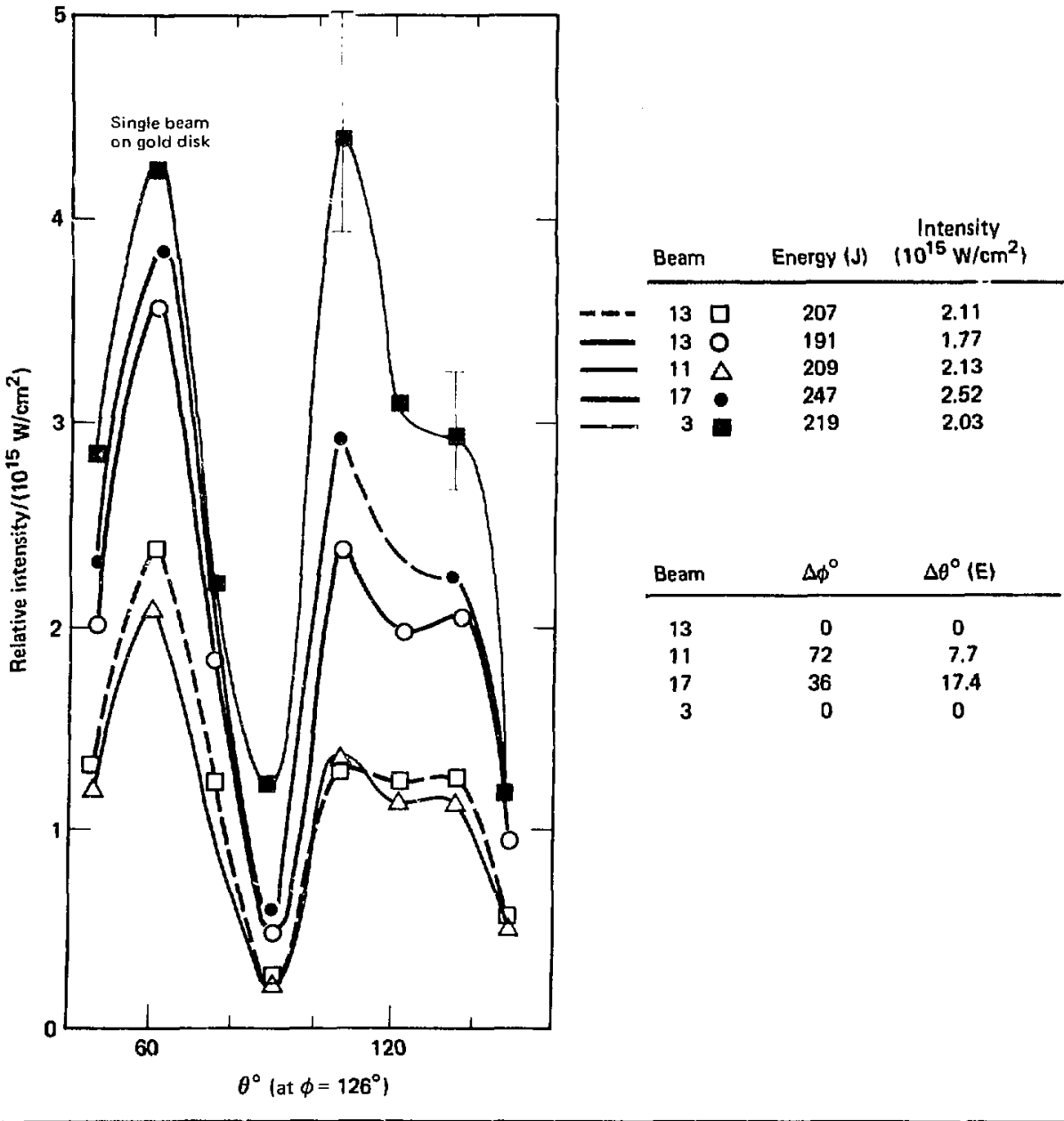

Target normal was pointing at $\theta=30^{\circ}$ and $\phi=90^{\circ}$, and the target was irradiated at $10^{16} \mathrm{~W} / \mathrm{cm}^{2}$ with the lower 10 Shiva beams. The geometry of this configuration is shown in Fig. 6-36; results are given in Fig. 6-37. ivote the strong peak at $\theta \approx 105^{\circ}$. The shallow dip in the intensity profile is due to absorption of $x$ rays in the target plane.

In conclusion, our film exposures indicate that there are definitely peak structures in the $x$-ray angular distribution, but exact correlation with the result of Nal detectors has yet to be established. It is not clear at present what mechanism may be responsible for generating the observed ans,ular dis- 
Fis. 6-34 (a) Density distritintion of the x-ray deposition on R-type fin, based on the target-file confizuration in Fis. 6-34 with incilent Inser intesedty of $2.8 \times 10^{14} \mathrm{~W} / \mathrm{cm}$ ? (b) Dewatty profile aloug a herizontal line at the center of the Tre imatex.
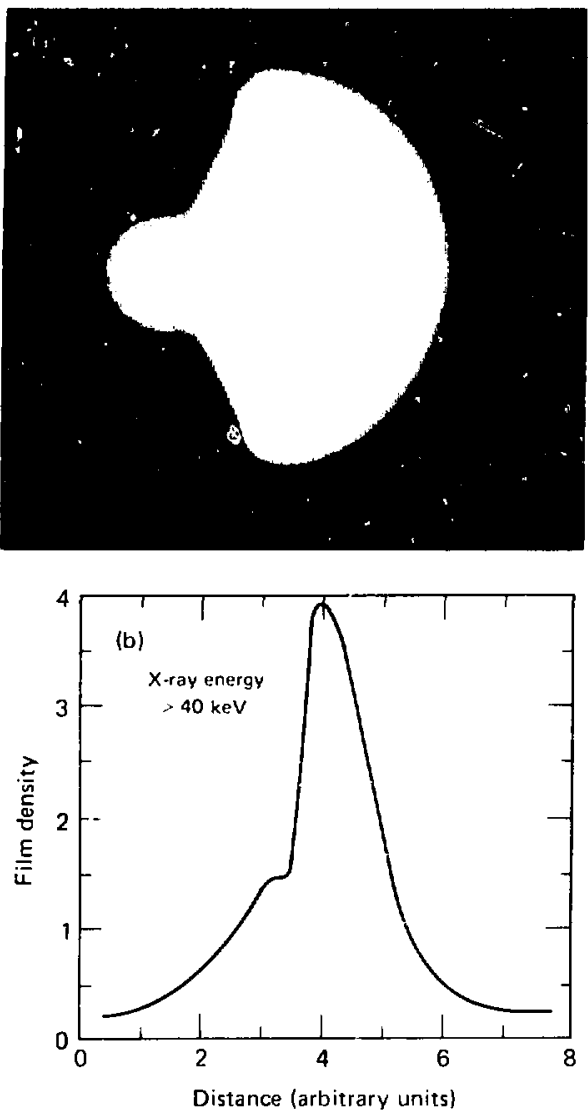

tribution. If our measurements are valid, however. then our previous estimate of the total energy in hot electrons (assuming isotropyl may be seriously in error. To explore this possibility we have nou fabricated a film cylinder $230 \mathrm{~mm}$ in diameter and $305 \mathrm{~mm}$ long. which will enable us to cover a large solid angle and thus conduct a more systematic $x$ ray film study of $x$-ray angular distribution.

Author: C. L. Wang

Major Contributors: H. N. Kornblum. G. R. Liepelt. K. D. Poor, and V. W. Slivinsky
Fig. 6-35. Experimental orientation of a gold dik target and $x-$ ray fillo paci.

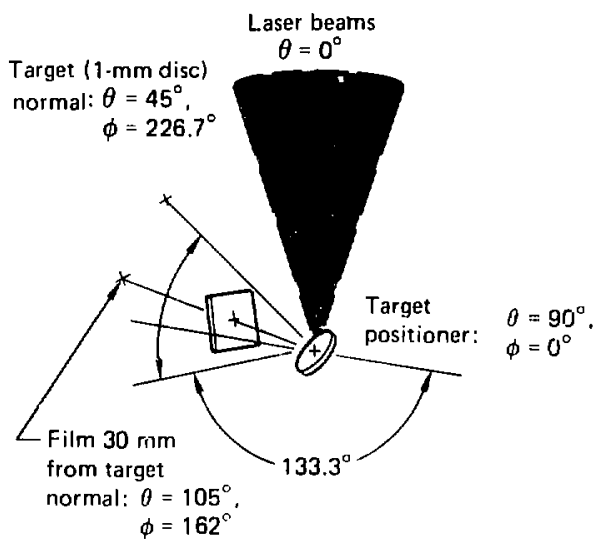

Fig. 6-36. Experimental orientation of a gold disk target and an x-ray film pack curved to expose the film surface at an:form distance from the target.

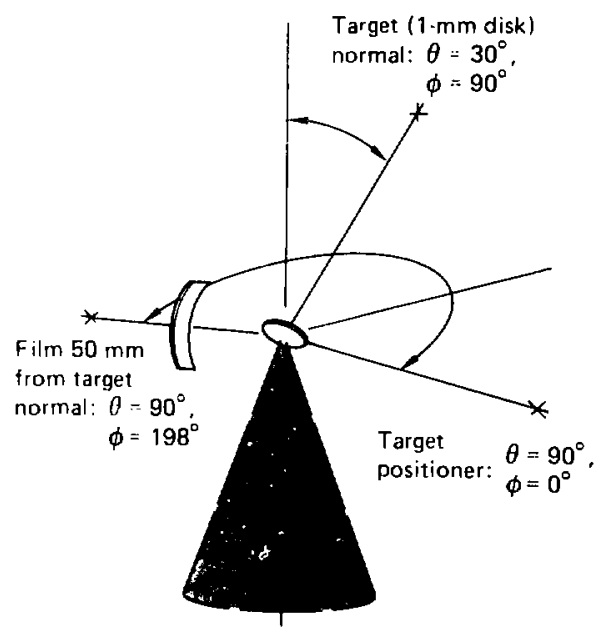

L.aser beams $\left(\theta=180^{\circ}\right)$ 
Fig. 6-37. (a) Density distribution of the x-ray deposition on R-type film, based on the target-film configuration in Fig. 6-36 with incident laser intensity of $10^{16} W / \mathrm{cm}^{2}$. (b) Density profile along a horizontal like at the center of the film image. Note the strong peak at $\theta \approx 105^{\circ}$.

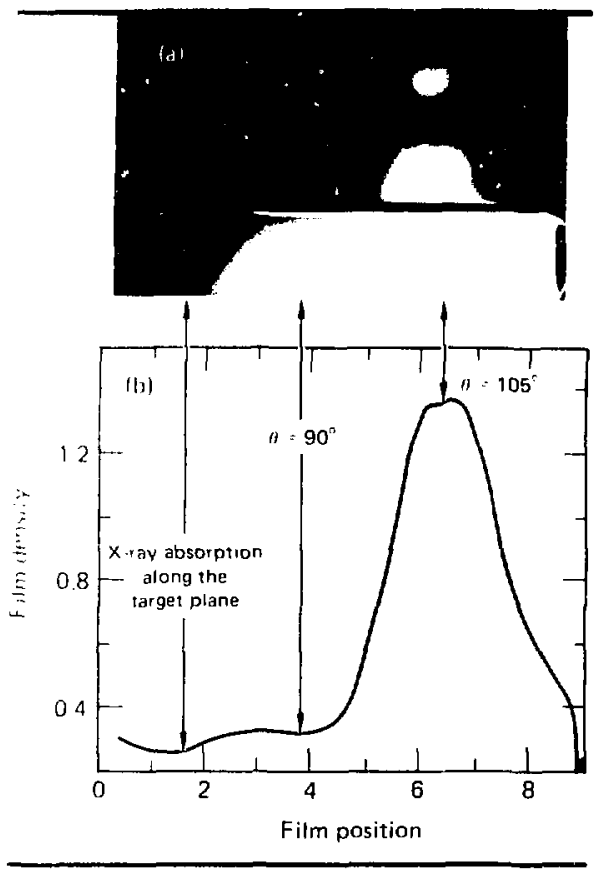

Refurences

I Rupert 1 I Hoyle. H. Y Kornblum, ind $\mathrm{k}$ (i

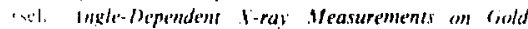

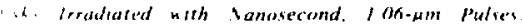

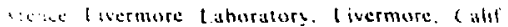
a. $\rightarrow+4 k 6,14978)$

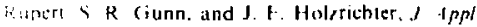
19121974,

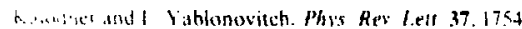
, i.

\section{Stimulated Raman Scattering Experiments}

For high-intensity $1.5 \times 10^{14} \mathrm{~W} / \mathrm{cm}^{2}$ at $\left.1 \mu \mathrm{m}\right)$. long-pulse $(\Sigma \mid \mathrm{ns}$ at $(\mu \mathrm{m})$ irradiation of laser fusion targets, stimulated Raman scattering (SRS) may scatter several percent of the incident beam into lower-intensity light. SRS is a parametric instability in which an incident photon decays into an electron-plasma wave (epw) and a lower-frequency photon. This process can occur only at electron densities below quarter-critical density $\left(0.25 \mathrm{n}_{\mathrm{c}}\right)$. The epw is usually collisionlessly damped by trapping. and creates suprathermal electrons which can preheat the pusher and D-T fuel. Even at modest intensities, laser bean filamentation in the plasma may create the high intensities needed for the Raman instahility to become important. Thus, experiments are needed to explare and quantify this process in long-pulse irradiation of large targets at both moderalt and high intensities.

We have made mealsurements which show that SRS is more significant at longer pulse lengths, and that at high intensities it occurs not only near 0.25 $\mathrm{n}_{\mathrm{c}}$ (where the light is seattered with frequency $\omega_{0} / 2$ ). but also at much lower densities. In this article we report the first observation of Raman scattering occurring in the very underdense plasma (at $\mathrm{n}_{\mathrm{e}} \approx 0.1$ $n_{\text {c) }}$ for a solid target.

The carliest ohservation of $\omega_{(0)} / 2$ light was reported by Bobin." who reported a line spectrum centered at $2 \lambda_{1}$ with a 60 .f width hetween halfintensity points. compared to $30-.4$ width for the incident laser light. This $\omega_{0} 2$ light can be altributed either to resonance Raman scattering or to linearmode conversion of the epu sproduced by the $2 \omega$ pe instahility into light waves. Watt has seen Raman scattering of ( $\mathrm{O}_{2}$ laser light irradiating a 200$)-\mathrm{mm}$ length of plasma formed in a solenoid. ${ }^{52}$ Many 1 I 2-dimensional particle code simulations of SRS have heen done by Fistabrook, "In one simulation. $1.06-\mu \mathrm{m}$ light at $3 \times 10^{15} \mathrm{~W} \cdot \mathrm{cm}^{2}$ was incident upon 10 -ke $v$ plasma with its initial density rising linearly from zero to $0.4 n_{c}$ in $105 \lambda_{0}$. Approximately $15 \%$ of the incident light was Raman-backscattered. Baidis ${ }^{54}$ has directly observed $\omega_{0 / 2} / 2$ epw's in a $\mathrm{CO}_{2}$ irradiated plasma using Thompson scattering

All our measurements were made (Fig. 6-38) with indium arsenide detectors cooled in Dewar flasks to liquid nitrogen temperature and reversebiased by about one volt. A $1-\mathrm{mm}$-diam quartz optical fiber brought the light collected in the target chamber to the indium arsenide detector outside the target chamber vacuum. The $1.064-\mu \mathrm{m}$ light and light of shorter wrvelength was blocked by filters both at the ligh' collector and at the detector; the $1.064-\mu \mathrm{m}$ light was attenuated by a factor on the or- 
Fig. 6-39. Experimental setup for measuring Raman-scattered light. The light callector is mounted at Argus on a theta arch which covers the angular range $25^{\circ}<\theta<155^{\circ}$. The visible-light-absorbing Coming $7-56$ filters have a transmittance below $0.75 \mu \mathrm{m}$ of $<10^{-4}$; the near-infrared-absorbing Corning 464 filters have a transmittance between $0.65 \mu \mathrm{m}$ and $1.1 \mu \mathrm{m} \mathrm{of}<10^{-3}$, and a9.9\% reffective coating at $1.064 \mathrm{\mu m}$. The band-pass interference filter has a nominal $800-f$ half-width and is blocked to better than $10^{-3}$.

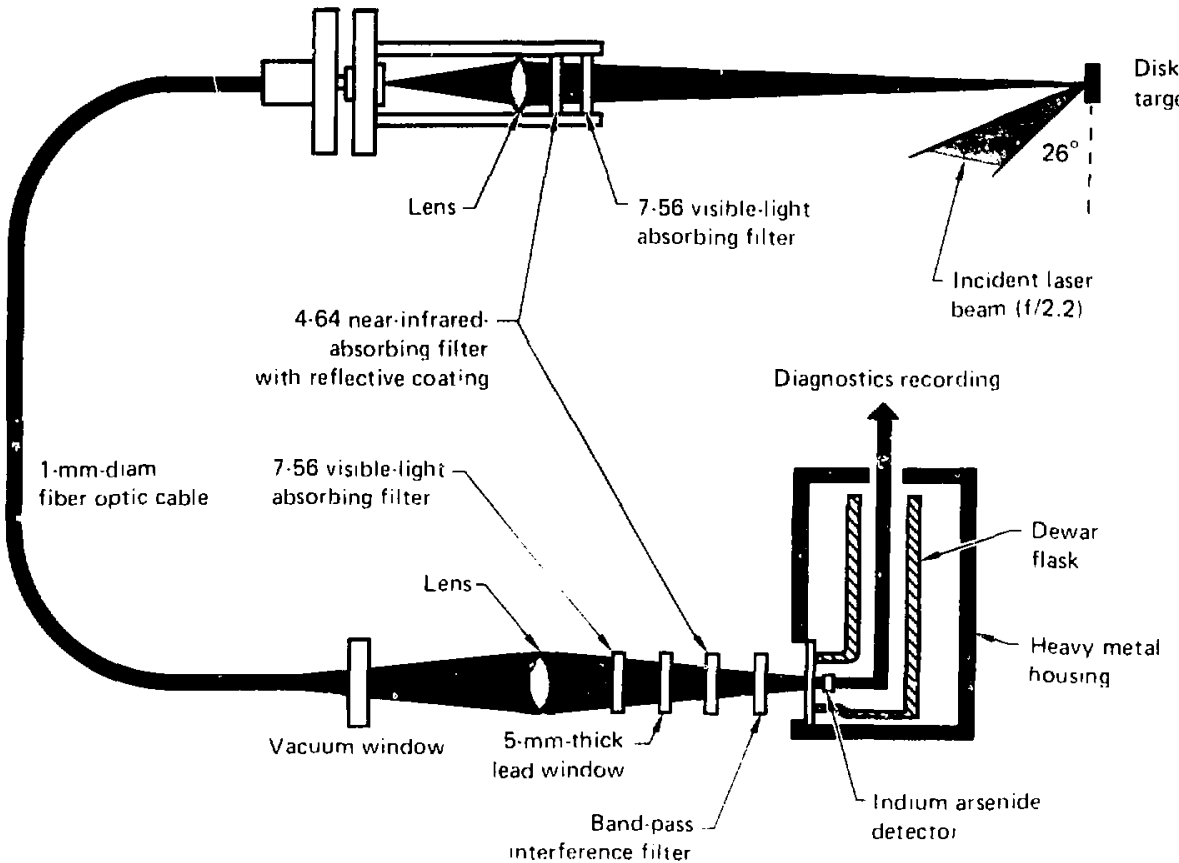

der of $10^{14}$. while shorter-1 avelength light sian a lotal attenuation of at leatst $10^{101}$. Corning $7-56$ visible-light-absorbing filters and corning $4-64$ near-infrared-absorbing filters with a dielectric coating highly reflective at $1.064 \mu \mathrm{m}$ were placed both in the light collector and in the filier holder. which were light-tight except for light transmitted through the filters. $X$-ray shielding was provided by the $\mathrm{W}$ - $\mathrm{Cu}$ housing and a lead-glass window in the filter holder. The light brought by the fiber optic cable from the target chamber was focused by a lens onto the 2-mm-diam indium arsenide detechor. Null experiments gave us confidence that we were actually looking at light from the target: when we blocked the light with a dark slide placed against the vacuum window, we observed no signal from a target shor. This elıminated other sources of the observed signal. such as electrical nouse. Mashlamp light. and high-energy $x$ ralys.

1ll the initial alignnent and focusing $u$ as done bs connectung one of the ends of the fiber optic cable to a visible light source. For most of the experiments. the focus of the collector was then moved a calculated distance from the best visible focus, so as $(0$ be in focus during the experiment at the wavelength of interest. If the light collector is in focus at a particular wavelength. all the light col- 
lected by the lens at that wavelength will be focused into the fiber optic. For some of the experiments, however, we deliberately defocused to reduce the light intensity at the fiber optic. The calibration factor is, of course. then multiplied by the fraction of the light making it into the fiber optic. No correction for dispersion was made in focusing the light onto the In-As detector; rather, we always focused the light to a spot just a little larger than the detector area. Dispersion will make the spot larger at longer wavelengths, but so long as the procedure is repeatable, so that the setup can be exactly duplicated when the calibration is done, it makes no difference.

We used two different calibration techniques. The most accurate calibration method employed a blackbody source whose temperature was set (and cliecked with a calibrated thermocouple) to be $1200 \mathrm{~K}( \pm 2 \mathrm{~K})$. and whose emissivity was known to be $0.99( \pm 0.01)$. Except for the neutral-density filter attenuation, the calibration setup duplicated the experimental setup. $\hat{A}$ separate calibration was performed for each band-pass filter. The precision aperture of the blackbody source was placed at the same distance from the light collector as the target was in the experiments. The $1.27-\mathrm{mm}$-diam aperture chosen was imaged by the light collector as a 0.5 mm-diam spot on the 1-mm-diam fiber optic: thus all the light collected was focused into the optical fiber. The absolute spectral intensity of the apertured blackbody source in $\mathrm{W} /(\mathrm{sr}-\mu \mathrm{m})$ is known to within $2 \%$. The current was measured by a Keithley nunovoltmeter shunted by $1 \mathrm{k} \Omega$. The internal impedance on the scales used ( $\mu V$ to $100 \mu V$ full scale) exceeds $100 \mathrm{k} \Omega$ and the In-As impedance at $77 \mathrm{~K}$ is also several hundred $\mathrm{k}$ ?, so the current can be accurately measured. The rather large $(\approx 100 \mathrm{nA})$ dc offset current was cancelled by sinking this current into an adjustable current source rather than the 1-k!? resistor. There was no problem in making measurements to $0.05-n A$ accuracy if the input soltage was nulled just prior to each measurement. To ensure repeatubility we made several measurements for each point.
The power per unit of emitting arex per unit of wavelength interval radiated by a blackbody source into $2 \pi$ steradians (one hemisphere) is given by

$$
\frac{W(\lambda, T)}{W_{\max }(T)}=\frac{0.2909}{(\lambda T)^{5}\left[\exp \left(\frac{\mathcal{C}_{2}}{\lambda T}\right)-1\right],}
$$

where $\lambda$ is in $\mu \mathrm{m}, T$ is in $K$, and $c_{2}=1.4388 \mathrm{~cm}-K$ is the second radiation constant. The maximum radiated power per unit wavelength for a fixed temperature is

$$
W_{\max }(T)=1.286 \times 10^{-15} \mathrm{~T}^{5} \quad w /\left(\mathrm{cm}^{2}-\mu \mathrm{m}\right) ;
$$

for $T=1200 \mathrm{~K}, \mathrm{~W}_{\max }(\mathrm{T})=3.21 \mathrm{~W} /\left(\mathrm{cm}^{2}-\mu \mathrm{m}\right)$.

The on-axis maximum spectral intensity from an apertured blackbody source is

$$
I_{\max }=\frac{\pi A}{\pi} w_{\max }(T) \text {, }
$$

where $\eta$ is the emissivity $(0.99)$ and $A$ is the aperture area, $0.0127 \mathrm{~cm}^{2}$. The units are power per unit solid angle per unit wavelength interval. Putting in the numbers, we find

$$
I_{\max }=0.013 W /(s-\mu m) .
$$

The spectral intensity of the blackbody source at any other wavelength is given by

$$
J(\lambda, T)=1_{\max }(T)\left(\frac{w(\lambda, T)}{W_{\max }(T)}\right) .
$$

A second, less accurate pulsed calibration method enabled us to calibrate the In-As detector at flux levels comparable to experimental ones, using a charge-integrating amplifier. The pulsed light source was a $Q$-switched Nd:Yag laser with $\lambda=$ $1.064 \mu \mathrm{m}$. The spectral transmission curves for the filters and fiber optic cable were combined with the spectral response curve for the In-As detector to obtain the overall spectral response.

Low-intensity experiments were carried out on

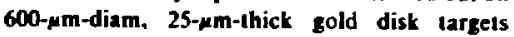
irradiated by one beam of the Argus laser facility. The disks were irradiated by $800 \mathrm{~J}$ in $950 \mathrm{ps}$. focused to a spot of about $425 \mathrm{~mm}$ minor diameter. $500 \mathrm{~mm}$ major diameter. An exception was a $\mathbf{8 a}$ $\left(\mathrm{NO}_{3}\right)_{2}$ disk larget which was irradiated with about 

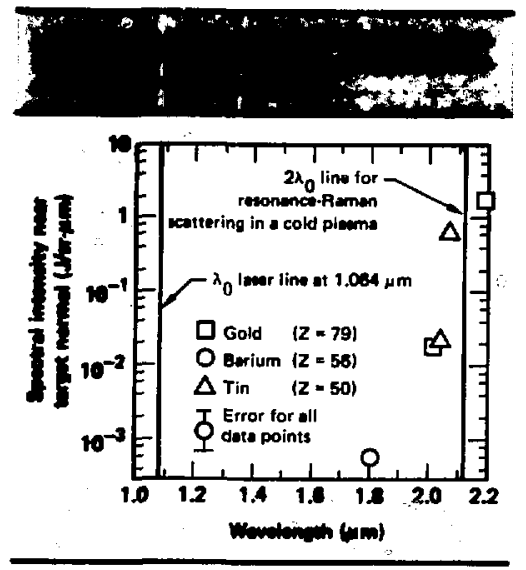

one third the energy focused to a correspondingly smaller spot size. The intensity was always about 5 $\times 10^{14} \mathrm{~W} / \mathrm{cm}^{2}$. The disks were tilted $30^{\circ}$ in the plane of polarization and the light collector always looked within $11^{\circ}$ of the target normal. This is necessary because the light at frequency $\omega_{0} / 2$ is generated very near its critical density and refraction will cause it to emerge at an angle very near to the normal regardless of its direction when first generated in the plasma.

Each point in Fig. 6-39 represents the result of one target experiment. The band-pass interference filter in front of the In-As detector was changed beIx:en shots, so we could get the spectral shape from a set of otherwise identical experiments. The width of each point is given by the FWHM band-pass of the interference filter. Target materials differed because our Raman light measurements were secondary diagnostics in an experimental series on varied- $Z$ disk targets designed to characterize $x$ radiography sources. Since all the target materials had at least a moderately high $Z$. however. we assumed that the $Z$ variation was unimportant for our measurements. The spectrum is highly peaked and extends to the red beyond $2 \lambda_{0}$. We did not make any measurement for wavelengths longer than $2.2 \mathrm{~mm}$. The signals at $1.8 \mathrm{\mu m}$ and $2.0 \mu \mathrm{m}$ could be caused by leakapes through the band-pass filters of the observed strong signal near $2 \lambda_{0}$. If one conservatively assumes a solid angle of about 0.25 sr and a spectral width of about $1000 \mathrm{~A}$, these measurements imply a conversion efficiency into $\omega_{0} / 2$ light of $5 \times$ $10^{-5}$. but this is only an order-of-magnitude estimate.

The $\omega_{0} / 2$ light spectrum extends to the red beyond $2.128 \mu \mathrm{m}\left(2 \lambda_{0}\right)$ due to the Bohm-Gross shift, as first explained by Kruer. ${ }^{55}$ If one solves the dispersion relations for the two light waves and the epw to find the density at which the Raman light wave is exactly at its critical density, one finds this density is below $0.25 n_{c}$ for a hot plasma and that the Raman light wave thus has a wavelength longer than $2 \lambda_{0}$. These dispersion relations are

$$
\omega_{0}^{2}=\omega_{p \mathrm{c}}^{2}+\mathrm{c}^{2} \mathrm{k}_{0}^{2}
$$

for the incident light wave,

$$
\omega^{* 2}=\omega_{p e^{2}+3 v}{ }^{2} k e^{2}
$$

for the electron-plasma wave, and

$$
\omega_{R}^{2}=\omega_{p e}^{2}+c^{2} k_{R}^{2}
$$

for a Raman light wave, where $\omega_{p e}$ is the electron plasma frequency, $v_{t c}=\sqrt{k T_{c} / m_{e}}$ is the electron thermal velocity, and $\omega^{*}$ and $k^{*}$ are the angular frequency and wave number of the epw, respectively. We set $\omega_{R}=\omega_{p c}$ and $\omega^{*}=\omega_{0}-\omega_{R}$ to find

$$
\left(\omega_{0}-\omega_{p e}\right)=\sqrt{\omega_{p e}^{2}+3 v_{t e}^{2} k^{22}} .
$$

Representing the square root by the first two terms in its binomial expansion, we get

$$
\frac{\lambda_{R}-2 \lambda_{0}}{2 \lambda_{0}}=\frac{\theta_{e}}{227 \mathrm{keV}} .
$$

where $\theta_{c}$ is the electron temperature in keV. This shift is about $0.5 \%$ per keV electron temperature. Figure 6-40 plots the wavelength of the Raman light for backscatter as a function of electron density for $T_{e}=0$ and $T_{e}=10 \mathrm{keV}$, again making the BohmGross approximation given by Eq. (11). 


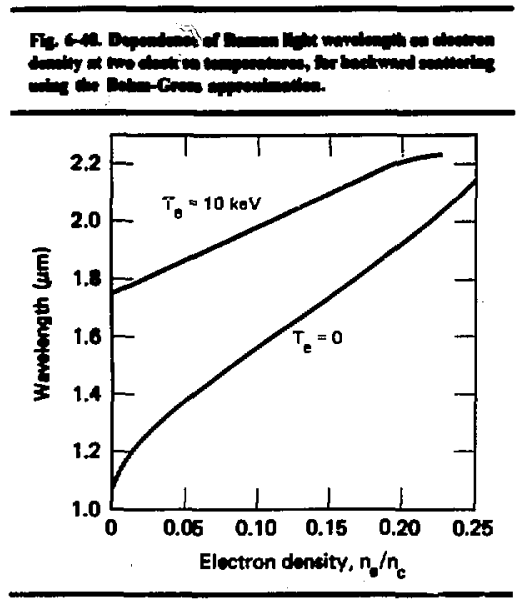

The threshold for SRS is expected to be lowest near quarter-critical density for two reasons:

- The group velocity of the Raman light wave is near zero at $0.25 n_{c}$ so the instability can become absolute rather than convective.

- For backward scattering the phase velocity of the Raman light wave is highest at $0.25 n_{c}$, resulting in the least Landau damping.

The phase velocity of the epw at $0.25 n_{c}$ is near $c / \sqrt{3}$ : an electron moving at this speed has a kinetic energy of $115 \mathrm{keV}$. Thus Raman light generated near its critical density produces electrons with energies on the order of $100 \mathrm{keV}$. In Fig. 6-41 the wave-breaking energy (the kinetic energy of an electron moving at the epw phase velocity) is plotted against electron density at various electron temperatures, assuming direct backscatter. For a given electron density. the phase velocity increases with electron temperature. As $n_{e}$ appronches zero. the phase velocity approaches $\sqrt{3}$ ve. but the BohmGross approximation is not valid if $k \lambda_{D_{k}} Z 1$. where $\lambda_{D e}=\mathrm{He} /$ the $_{\mathrm{he}}$ is the electron Debyc kngth. As electron temperature increases, the maximum electron density at which Raman scattering can occur decreases. This density is given by

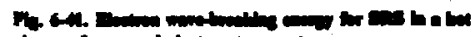

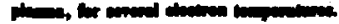

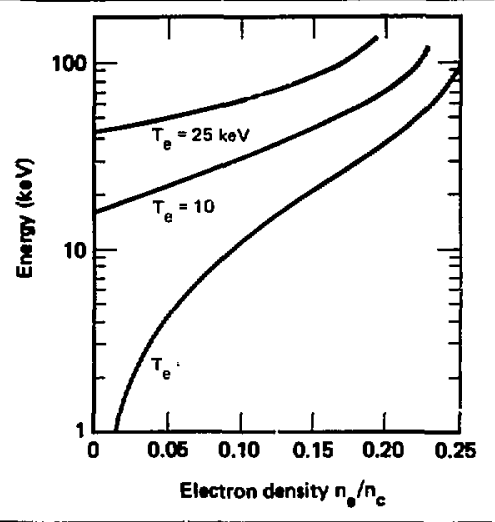

electron density at which Raman scattering can occur decreases. This density is given by

$$
\left(\frac{n_{e}}{n_{c}}\right)_{\max }=\left(\frac{1-\xi}{1+\sqrt{1-\xi+\xi^{2}}}\right)^{2} .
$$

where $\xi=3 \mathrm{v}_{\mathrm{te}}{ }^{2} / \mathrm{c}^{2}$ is a dimensionless parameter proportional to the electron temperature. In Fig. 6-42 the wave-breaking energy is plotted for sideand forward-scatter as well as backscatter.

C. S. Liu ${ }^{56}$ shows the condition for absolute instability in an inhomogeneous plasma to be

$$
\left(\frac{v_{0}}{c}\right)^{2}>\frac{4}{\left(k_{0} L\right)^{4 / 3}} \text {. }
$$

where $\mathbf{L}$ is the local density gradient defined by

$$
L^{-1}=\frac{d \ln n_{c}}{d x}
$$

Here $k_{0}$ is the wave number of the incident light in vacuum. and $v_{0}$ is the peak oscillatory velocity of the electron in the incident light field. The threshold condition given here actually agrees with Liu's (given in Eq. 1-31 on page 133 of Ref. 56), despite a 


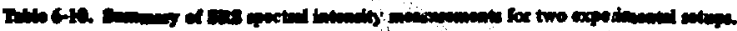

\begin{tabular}{|c|c|c|c|c|}
\hline \multirow{2}{*}{$\begin{array}{c}\text { Dheses } \\
(1+0)\end{array}$} & \multicolumn{2}{|c|}{ 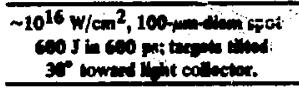 } & \multicolumn{2}{|c|}{ 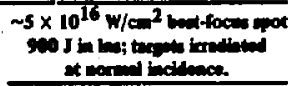 } \\
\hline & $J /(r-n)$, in plase & $\Delta 6$ & $J /(\mathrm{m}+\mathrm{m})$, i plate & $\Delta$ \\
\hline $\begin{array}{l}1.6 \\
1.8 \\
2.0 \\
2.13\end{array}$ & $\begin{array}{l}0.33 \\
>0.26 \\
\text { No wicenten } \\
0.4 \text { to } 1.1\end{array}$ & $\begin{array}{l}56^{\circ} \\
712 \\
112\end{array}$ & $\begin{array}{l}1.310 .1 .7 \\
0.24 \\
1.2 \\
\text { No meanrese }\end{array}$ & $\begin{array}{l}25^{\circ} \\
25^{\circ} \\
25^{\circ}\end{array}$ \\
\hline
\end{tabular}

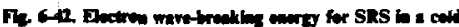

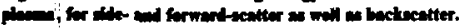

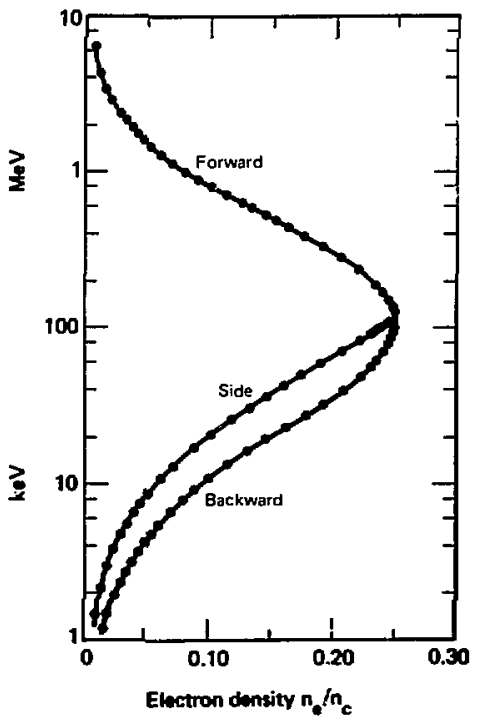

seeming discrepancy by a constant läctor. Liu's v $v_{0}$ is actually half the peak oscillatory velocity (see Eqs. $1-13$ and $1-19$ in Ref. 56), which for linearly polarized 1.064-km light is given in term of the intensity I by:

$$
\left(\frac{0}{c}\right)^{2}=\frac{1}{1.21 \times 10^{18} \mathrm{w} / \mathrm{cm}^{2}} \text {. }
$$

Simulations indicate $L \approx 150 \mu \mathrm{m}$, so that we were close to threshold for $1=5 \times 10^{14} \mathrm{~W} / \mathrm{cm}^{2}$. Of course, the peak intensity of the beam in hot spots may be higher than this average intensity by a factor of 2 or 3 .

At light intensities of $10^{16}$ and $10^{17} \mathrm{~W} / \mathrm{cm}^{2}$, the spectral intensity of the Raman-scattered light increased not only near the degeneracy frequency $\omega_{0} / 2$, but even more so at higher frequencies (Table 6-10). The Raman light intensity at $1.6 \mu \mathrm{m}$ and $1.8 \mu \mathrm{m}$ is comparable to that at $2.13 \mu \mathrm{m}$. All these measurements were made with the Raman light collector in the plane of polarization of the incident light. SRS, however, should have its maxilnum gain when the scattering plane is orthogonal to the plane of polurization; the electric vector of the Raman light would then be paralle! to the electric vector of the incident light. The growth rate of the Raman instability is proportional to the dot product of the two electric vectors, so out-of-planc scattering (which sve have not yet measured) is expected to occur preferentially.

For the experiments at $10^{16} \mathrm{~W} / \mathrm{cm}^{2}$, the gold disks were tilted $30^{\circ}$ in the plane of polarization and toward the Raman light collectors, so that they looked nearly normal to the target surface. However, the shots at $5 \times 10^{16} \mathrm{~W} / \mathrm{cm}^{2}$ were done at normal incidence, so the Raman light collectors were looking $25^{\circ}$ away from the incident beam and in the plane of polarization: at this intersity the fraction of the incident light energy appearing as Raman-scattered light is at least $0.05 \%$.

Raman-scattered light in the wavelength range from 1.6 to $1.8 \mu \mathrm{m}$ probably originates around 0.1 $\mathbf{n}_{G}$ although it is not possible to determine precisely at what density the Raman scaltering occurs without knowing more than just the wavelength of the Raman-shifted light: one must also know the 


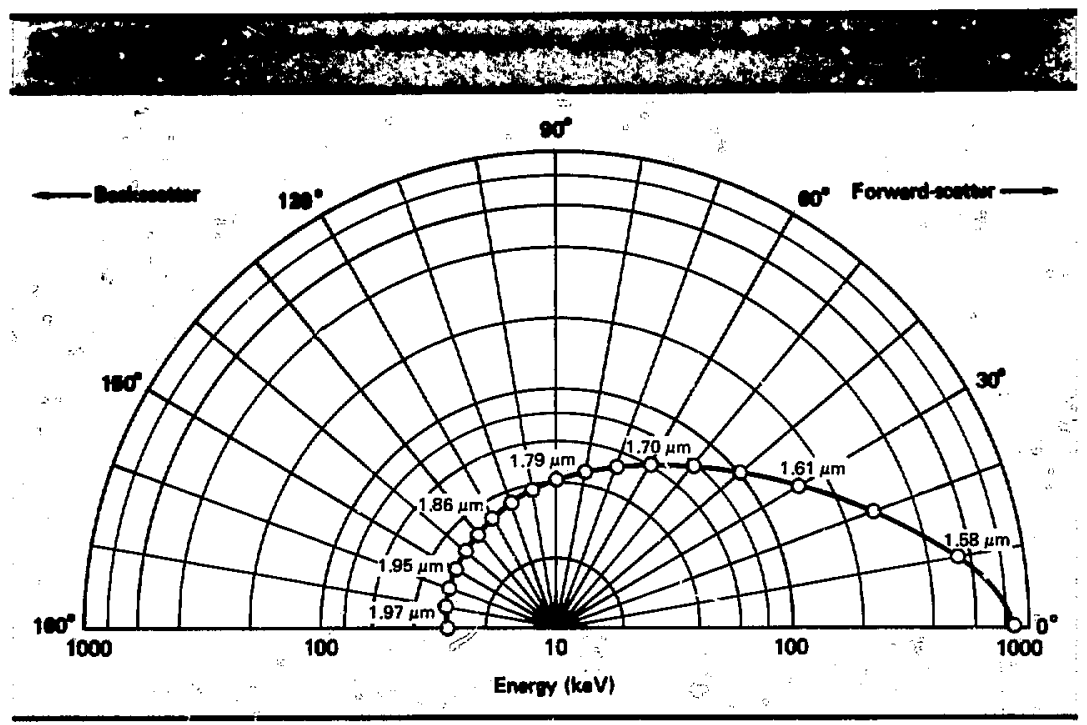

electron temperature and the scattering angle within the plasma. Backward Raman scattering has the highest growth rate, but also the highest Landau damping, since for backscatter the epw has the lowest phase velocity. The log-polar plot in Fig. $6-43$, of the wave-breaking energy at density $0.1 n_{c}$ and temperature $10 \mathrm{keV}$, shows that the epw for backscattering has a wave-breaking energy of only $31 \mathrm{keV}$ and thus will be highly Landau-damped. If the scattering direction is within 30 or $40^{\circ}$ of the forward direction, the Landau damping will be extremely small, though the growth rate will also be much reduced because of the smaller wave number of the epw.

An estimate of when SRS is important can be made on the basis of a simple steady state model in which the epw is assumed to be strongly damped and the plasma to be planar with a linear density gradient (Fig. 6-44). The incident light (not shown in Fig. 6-44) is assumed to be a monochromatic plane wave normally incident upon the plasma, and while the Raman-scattered light wave can have any frequency and be incident at any angle, it will not see significant growth enless the beat term in the ponderomotive force drives the epw nearly resonantly along part of the ray path. If there is a ray which will be amplified ten or more e-foldings in intensity, Raman scattering can be expected to occur.

How strongly damped the epw needs to be before a strongly damped approximation is valid depends upon the scale length. At some point in the plasma, let the epw be driven nearly resonantly at angular frequency $\omega^{*}$ and wave vector $k^{*}$. If a noninteracting electron-plasma wave packet with the same center frequency $\omega^{*}$ and propagation direction (but with the wave number required by the dispersion relation) damps before it can fall out of phase with the ponderomotive-force driving term, then the epw is considered to be stro:gly damped. We are then justified in neglecting propagation in the model and can assume the epw amplitude at any point is determined by the ponderomotive-force driving term at that same point. We also make the slowly varying approximation and assume $\delta \mathrm{n} / \mathbf{n}$ small.

If the friquency of the Raman-scattered light wave is fixed and its angle of incidence allowed to vary, we find that the ray path which gives maximum growth is the one for which the epw is driven nearly resonantly at its turning point. This is physically reasonable since the interaction length is 

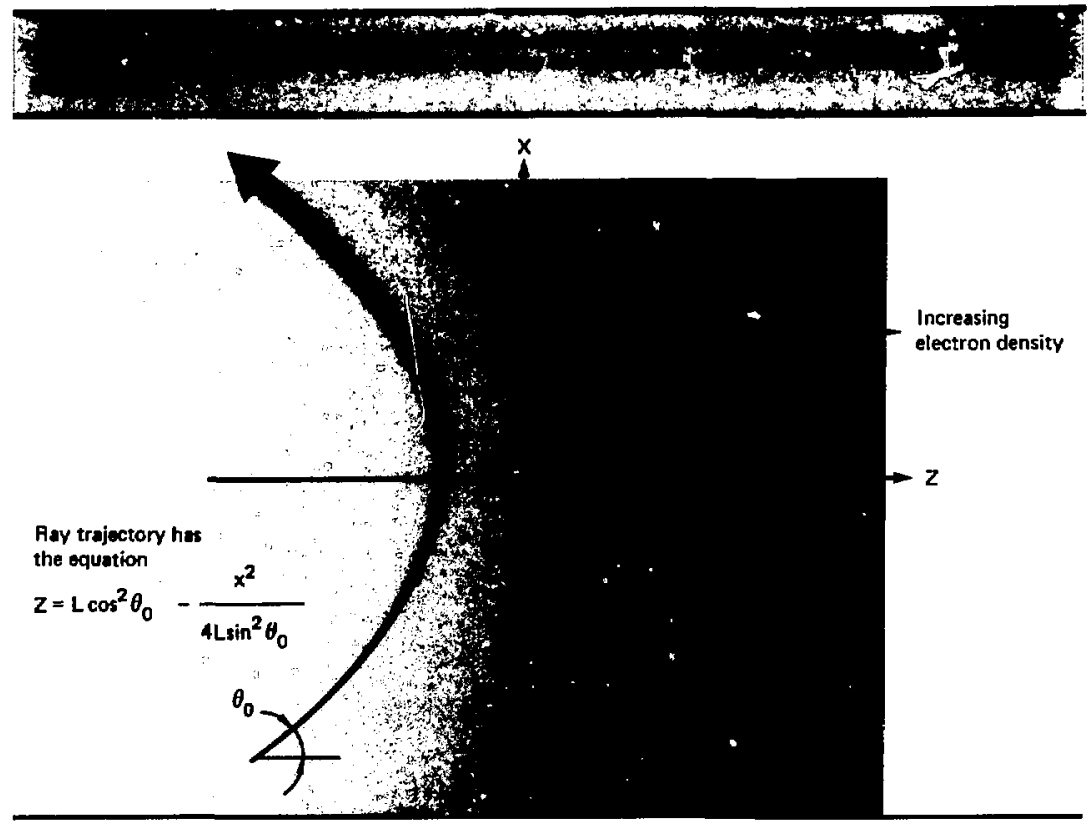

then maximum. Unrealistic results may be obtained for the growth seen by rays having turning points very far out in the underdense plasma, since in this model the interaction length increases without limit as the turning density goes to zero. The interaction length may be limited by the finite spot size or the curvature of the plasma density contours rather than by refraction.

The number of intensity e-foldings along the optimal path $\left(\lambda_{R}\right.$ fixed) is given by

$$
\begin{aligned}
k_{\max }= & \frac{3 \sqrt{3} \pi}{4}\left|{\overrightarrow{c_{R}}}_{\mathrm{e}_{0}}\right|^{2}\left(\frac{k^{* 2}}{k_{0}\left(\frac{\omega_{R}}{c}\right)}\right)\left(\frac{\omega^{*}}{\omega_{R}}\right) \\
& \times\left(\frac{u_{0}}{c}\right)^{2}\left(\frac{L}{\lambda_{0}}\right) \sqrt{\frac{\omega^{*}}{y_{L}}},
\end{aligned}
$$

where $\nu_{L}$ is the Landau damping rate, $\overrightarrow{e_{R}}$ and $\vec{b}$ are unit electric vectors, and $\mathbf{L}$ is the scale length, defined by

$$
n_{e}=n_{d}\left(\frac{x}{L}\right)
$$

The subscript $\mathbf{R}$ refers to the Raman-scattered light wave, the subscript $O$ to the incident light wave, and the superscript * to the epw.

Notice that as expected, $K_{\max }$ is proportional to the density scale length $L$ and to the incident laser intensity, but, surprisingly, is proportional only to $\nu_{\mathrm{L}}^{-1 / 2}$ rather than $\nu_{\mathrm{L}}^{-1}$, where $\nu_{\mathrm{L}}$ is the Landau damping rate. This dependence is explained when we observe that the interaction length increases with $\nu_{L^{1 / 2}}$ while the gruw th per unit distance varies with $\nu_{\mathrm{L}}^{-1}$. The Raman-shifted light wave will interact nearly resonantly with the other two waves only over a narrow electron-density interval whose width is proportional to $\nu_{\mathrm{L}}$. If the resonance point occurs near the turning point of the Raman-shifted light wave. the interaction length is then proportional to $\operatorname{Lv}_{\mathrm{L}}^{1 / 2}$.

We can now evaluate $K_{\max }$ for $n_{e}=0.1 n_{c}, T_{e}$ $=10 \mathrm{keV}$, to find

$$
K_{\max }=\frac{1}{22}\left(\frac{L}{\lambda_{0}}\right)\left(\frac{\omega^{0}}{V_{L}}\right)\left(\frac{1}{10^{16}}\right) .
$$


$A_{\text {gold disk simulution }} 35$ for $\mathrm{I}=10^{16} \mathrm{~W} / \mathrm{cm}^{2}$, spot size $=100 \mu \mathrm{m}$, and pulse length $=I$ ns shows $T_{c} \approx 12 \mathrm{keV}$ and $\mathrm{L} \approx 500 \mu \mathrm{m}$, where $\mathrm{L}^{-1}=$ $d\left(n_{c} / n_{c}\right) / d x$. Equation (2l) would give $K_{\max } \approx 25$. In this case it is the radius of curvature of the plusma rather than refraction which limit' the interaction length. Using a $250-\mu \mathrm{m}$ radius of curvature at $0.1 n_{0}$ we estimate $K_{\max } \approx 12$.

In conclusion. Raman scattering is an instability which must be seriously considered in designing laser-heated ICF targets, especially at high intensity $\left(1 \lambda^{2} \sim 10^{15} \mathrm{~W} / \mathrm{cm}^{2}-\mu \mathrm{m}^{2}\right)$.

Author: D. W. Phillion
Majer Centrimmers: b. I. Eamer, K. G. Estareak, and W. B. Laind

\section{Refereaces:}

S1. J. L. Bobin. M, Decroisett. B. Meyer, and Y. Vitel, Phys. Rev. Left. 30, 594 (1973).

52. R. G. Watt, R. D. Brooks, and Z. A. Pietrayk, Phys. Rev. Lett. 41, 271 (1978).

53. W. L. Kruter, K. Estabrook, B. F. Lasinsky, and A. B. Langdon, Phys. Fiulds (to be published).

54. H. A. Buldis, J. C. Samson, and P. B. Corkum, Phys. Rev. l.elc, 41, 1719 (1979).

55. Laser Program Anmual Repon-1978, Lawrence Livermore Luboratory, Livermore, Calif,, UCRL-50021-78 (1979), pp. $3-42$ to $3-45$.

56. Adwances In Plasma Physics, Albert Simon and W. B. Thompson, eds. (Wiley \& Sons, New York, 1976), vol. 6, p. 121.

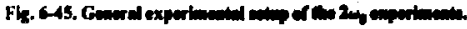

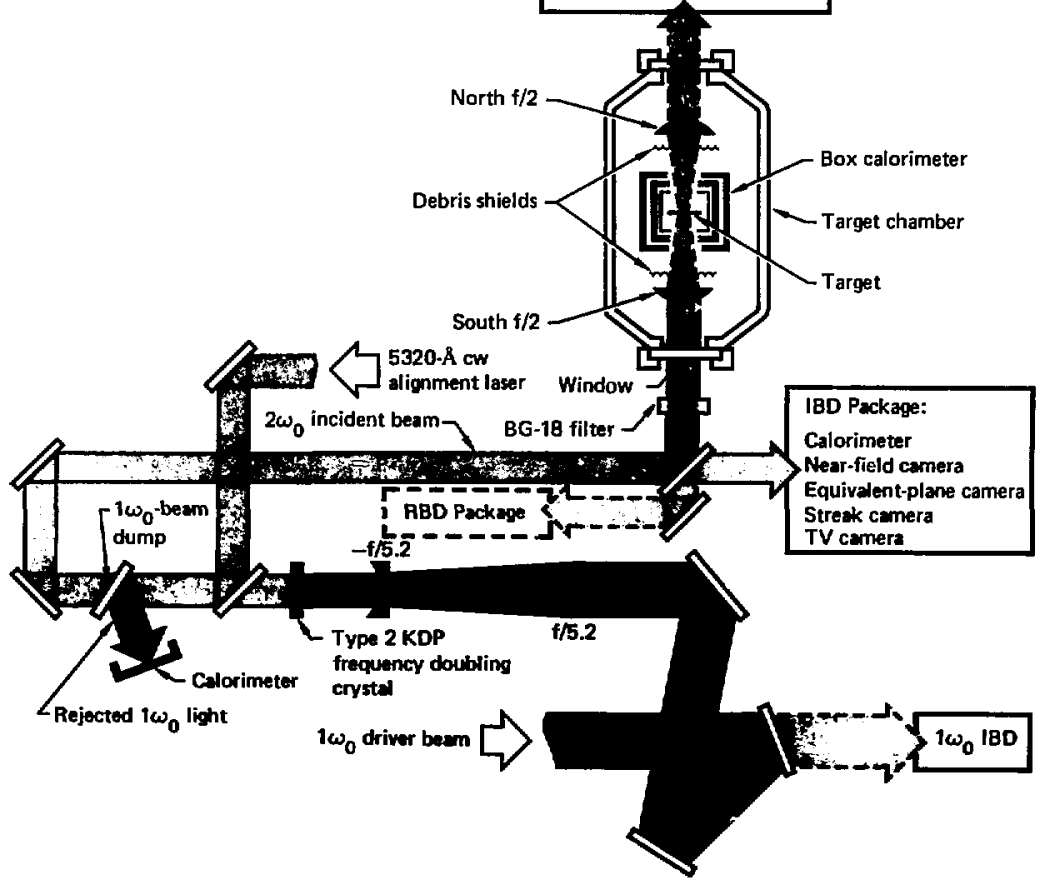

TBD Pack age:

MIC, calorimeter, TV camera 


\section{Experimeatal Comfizuration of the $2 \omega_{0}$ Experiments}

The experimental setup used at the Argus laser facility for target irradiation experiments at $0.532 \mu \mathrm{m}$ (frequency-doubled $1.06 \mu \mathrm{m}$ ) is shown schematically in Fig. 6-45: since the basic design principles and calibration techniques of the various instruments employed in the $2 \omega_{0}$ experiments are analogous to those described in detail in Refs. 57, 58, and 59, we will not repeat those descriptions here.

A system of three turning mirrors directs the $1.06-\mu \mathrm{m}$ driver beam onto a lens assembly that demagnifies and recollimates the beam from about $280 \mathrm{~mm}$ to approximately $80 \mathrm{~mm}$ in diameter. This is done to reconcile the beam size with the clear aperture $(90 \mathrm{~mm})$ of the frequency-doubling crystal (KDP type II, with a thickness $\approx 13 \mathrm{~mm}$ ). The unconverted $1.06-\mu \mathrm{m}\left(1 \omega_{0}\right)$ beam is eliminated from the frequency-doubled $\left(2 \omega_{0}\right)$ beam by reflection from a coated BK-7 glass slab (the "l $\omega_{0}$ beam dump" in Fig. 6-45); the $2 \omega_{0}$ beam is then directed into the target chamber using an $f / 2$ focusing lens ( $f / 2.2$ effective aperture). As discussed earlier in this report (see Section 2, "Argus Operations Summary"), frequency-doubling conversion efficiencies in excess of $55 \%$ were obtained at Argus at an incident-driver-beam radiation intensity of 1.5
$\mathrm{GW} / \mathrm{cm}^{2}$ or higher. In the experiments reported here, however, Fresnel reflection losses cut the actual $2 \omega_{0}$-beam energy incident on the target to approximately $30 \%$ of the $1 \omega_{0}$ driver-beam energy.

\section{Beam Diagnostics Packages}

Less than $3 \%$ of the incident $2 \omega_{0}$ beam is directed into an incident-beam diagnostics (IBD) package (Fig. 6.46), which includes a near-field camera, an equivalent-plane (target-plane) camera, a streak camera, ${ }^{57,58}$ a calorimeter, ${ }^{59}$ and a monitoring TV camera. A representative near-field image of the 200 beam is shown in Fig. 6-47, together with a corresponding near-field image of the driver lup beam. Comparison of the two pictures shows that the $2 \omega_{0}$-beam amplitude modulation exceeds that of the lap beam.

The equivalent-plane camera is used to obtain two-dimensional pictures of the incident $2 \omega_{0}$ beam near best focus; these pictures are in turn used to characterize the beam spatial intensity distribution there. Figuse 6-48 shows a representative equivalent-plane camera image taken near best focus. The presence of comatic aberrations made determination of the lens best-focus position very difficult.

We used rod shots to determine spot size and adjust the streak camera timing. Figure 6-49 shows

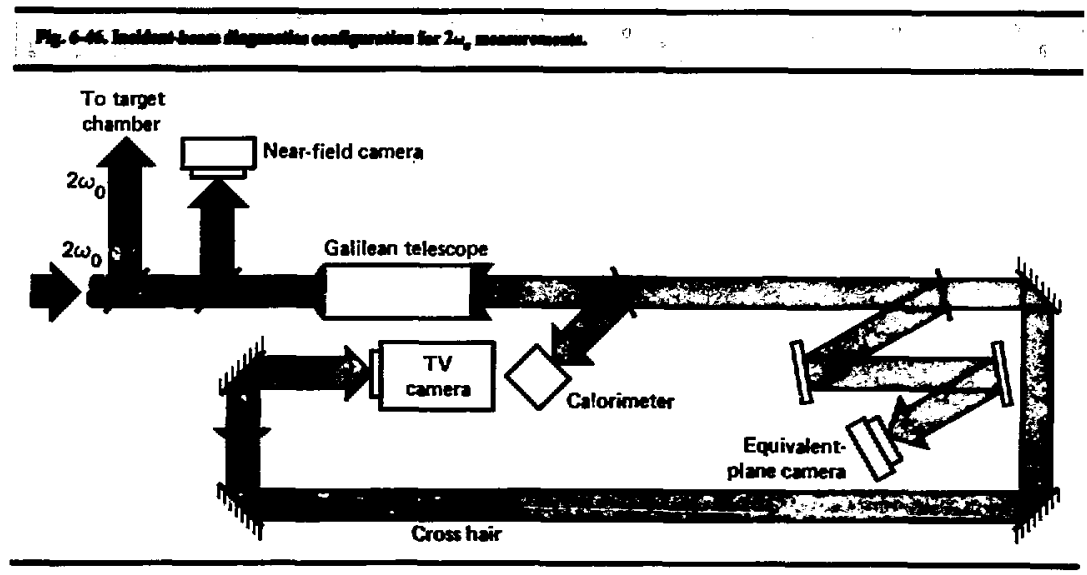




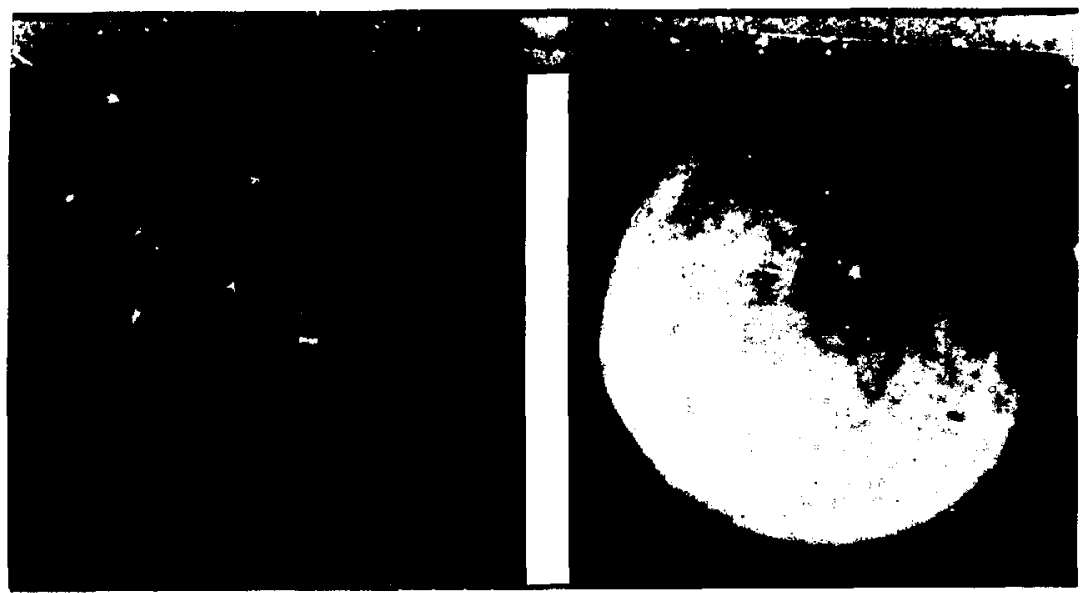

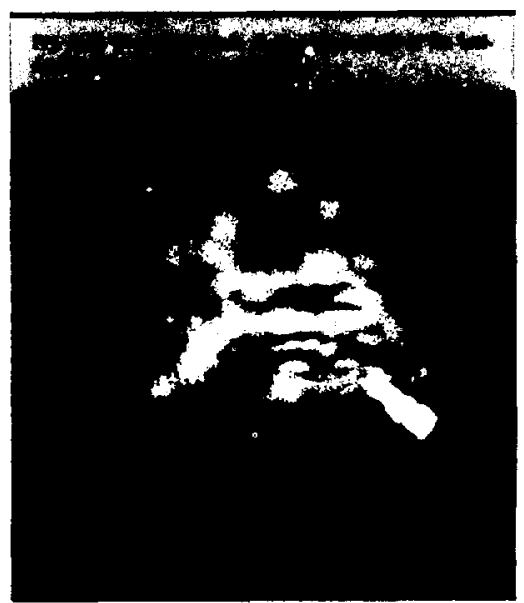

a sample $2 \omega_{0}$-beam streak picture, in which time is measured horizontally and radial distance measured vertically; the pulse duration was about $600 \mathrm{ps}$. Digitized pictures similar to the one shown here were instrumental in the determination of the tem-

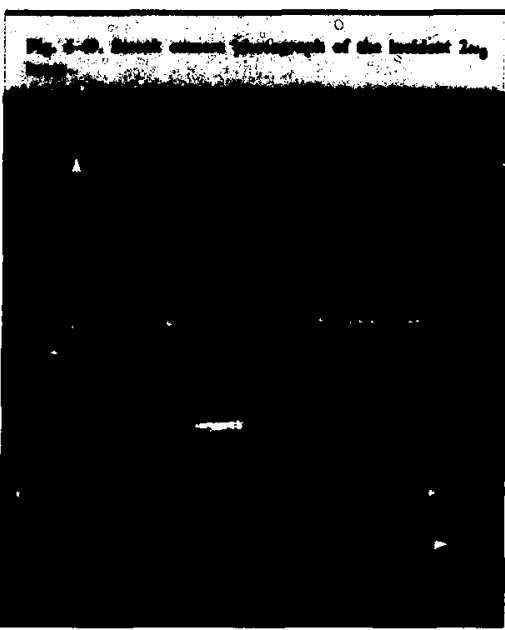

poral $2 \omega_{0}$ pulse widths discussed in Section 2 of this report.

We measured the incident $2 \omega_{0}$-beam energy with a calibrated calorimeter. We calibrated the calorimeter with calorimetric readings taken of the transmitted beam (without target), taking into account the chamber lens transmission coefficients; the chamber single-lens transmission, including the 


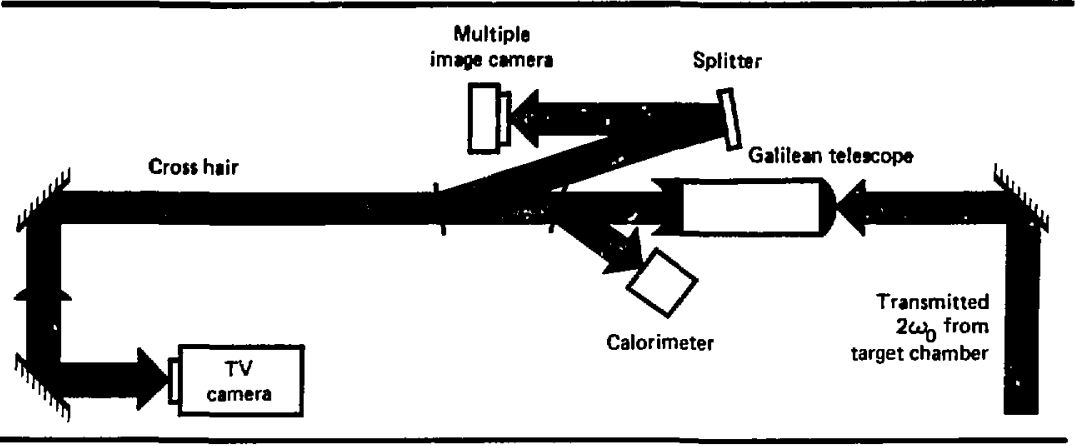

lens' protective debris shield, was found to be about 94\%. By positioning a BG-18 glass filter across the $2 \omega_{0}$ beam outside the target chamber, we determined that the level of residual $1.06-\mu \mathrm{m}$ light that leaked through the coated BK-7 glass (the $1 \omega_{0}$-beam dump) represented less than $1 \%$ of the total beam energy incident on the target.

The transmitted beam diagnostics (TBD) package (Fig. 6-50) included a calorimeter, a multiple image camera (MiC) similar to the one used for the equivalent-plane two-dimensional imaging of the incident beam, and a monitoring TV camera. Accurate irradiation spot sizes were determined from the MIC pictures of the transmitted beam. The monitoring TV camera was instrumental in target alignment and positioning.

\section{Target Aliznment and Spot Size Determination}

Three steps were required for $2 \omega_{0}$ beam alignment.

- The I $\omega_{0}$ beam was first made to properly point through the target chamber. This procedure involved replacing the $1.06-\mu \mathrm{m}$ beam dump with an uncoated BK-7 glass slab of the same thickness. With the chamber lenses temporarily removed, we aligned an attenuated $\mathrm{cw} 1.06-\mu \mathrm{m}$ beam through the chamber with the aid of reference crosshairs, turning mirrors, and infrared sensitive viewers.

- After replacing the simulated dump with the real one (but with the chamber lenses still out). we verified that the pulsed $2 \omega_{0}$ beam also properly

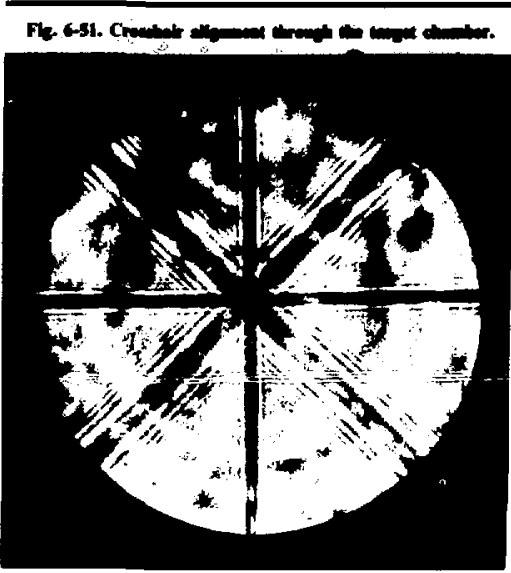

pointed through the target chamber. The final $2 \omega_{0}$ pulsed-beam alignment was achieved by fine tuning crosshair positions and verifying the alignment with photographs of the transmitted pulsed beam on rod shots. In Fig. 6-5I we show a sample transmittedbeam picture displaying two correctly matched crosshairs (one placed in front of the final turning mirror. one placed beyond the exit window of the target chamber).

- After remounting the two chamber lenses, we used an auxiliary cw $2 w_{0}$ alignment bser (whose optics were decoupled from those of the main beam: 


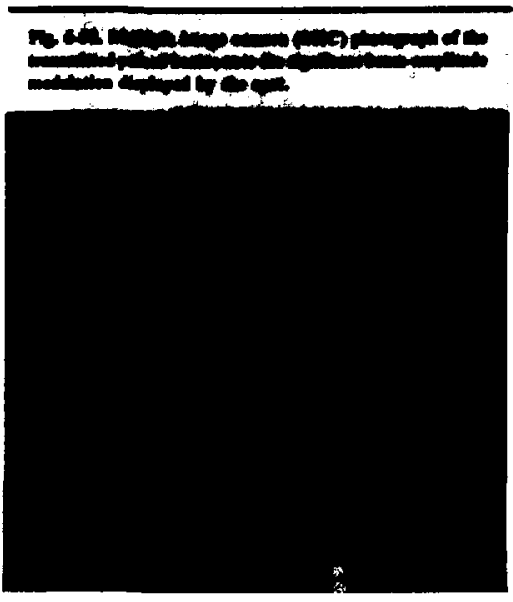

see Fig. 6-45) to check the orientation of the lenses. This was followed by another rod shot to recheck the $2 \omega_{0}$ pulsed-beam alignment. Finally, the auxiliary cw $2 \omega_{0}$ beam was used to monitor the alignment and to backlight the target after it had been aligned.

Before a target is inserted into the chamber, its orientation on the target holder is set on a specially built mounting table; in these experiments the table setup simulated the chamber geometrical configuration and was equipped with an angle-setting prealignment jig. The oriented target was then inserted into the chamber and exactly positioned at the center of the chamber, by adjusting its vertical position while viewing it through two perpendicular telescopic viewers that cross at the chamber center (T:O viewers). High-intensity white-light illuminators opposite the telescopes served to illuminate the target during this part of the procedure. Next, diffused $\mathrm{cw} 2 \omega_{0}$ light was used to project the target image on a TBD monitoring TV camera screen while the position of the cramber North lens was adjusted until the image appeared sharpest.

The chamber South lens was focused in a somewhat similar manner. The lens position was varied until the sharpest, smallest image was ob-

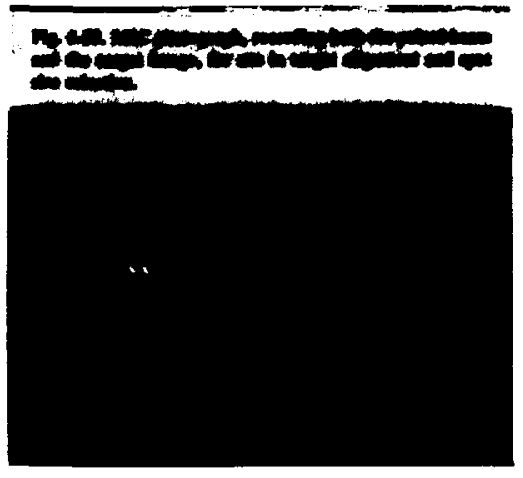

tained in photographs of the transmitted pulsed beam on a rod shot. A calibration curve was then used to deduce the position of the chamber South lens that would give the desired beam spot size at the target plane. The chamber South lens was moved toward or away from the target plane until the desired converging or diverging spot size was obtained on a two-dimensional MIC image of the transmitted pulsed beam. In the representative image in Fig. 6-52, it is evident that the spot displays significant beam-amplitude modulation. Photographs of the transmitted beam used for spot size selection were also digitized to provide necessary information about the beam's physical qualities.

Figure 6-53 illustrates the success of the methods just described for target aligliment and spol size selection. The film recorded both the pulsed beam and the image of the target, a plastic parylene disk oriented normal to the incident beam. The camera magnification was 38.2.

\section{Diagnostics Durime Target Shots}

The determination of energy balance during target shooting was made by calorimetric measurements. Target irradiation was done inside a box calorimeter that measured the scattered and refracted 2 an energy. The box calorimeter incorporated a plasma shicld (WG-280 glass) so that only scattered light could reach the energy-absorbing NG-1 glass pancls. Because of its vilal importance in the determination of light absorption by the target, the box calorimeter was designed for high sensitivities $\{>3$ 


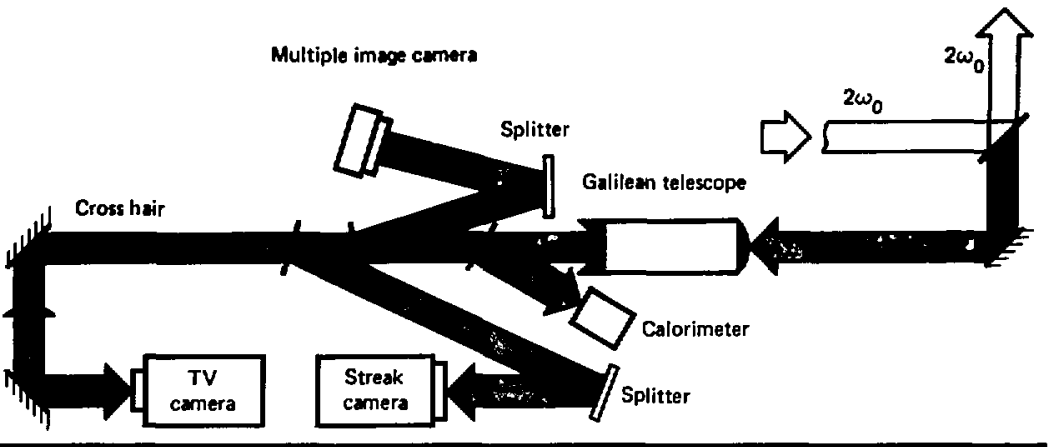

$\mu \mathrm{V} / \mathrm{J}$ for the North and South panels and $\sim 120$ $\mu \mathrm{V} / \mathrm{J}$ for the other four panels). The amplifier settings for the signals from the panels were closely monitored to prevent saturation. The plasma shield was replaced whenever there was evidence of excessive debris deposited on the WG-280 glass.

Corrections were made to account for losses through access and diagnostics holes in the box. The South and the North holes of the box subtended small solid angles of about $0.20 \mathrm{sr}$ each. Fortunately, however, the effective $\mathrm{f} / 2.2$ optics (for a beam diameter of $\sim 82 \mathrm{~mm}$ ) and the chamber South lens $(f f \approx 180 \mathrm{~mm})$ subtend a solid angle of about 0.18 steradian. Therefore, all the light scattered back through the chamber South hole was collected by the backscattered-light diagnostics instruments. Similarly, any forward-scattered light going through the North $1 / 2$ lens was registered in the TBD package. Preliminary results revealed that during target experiments the amount of forwardscattered light was negligible, so that no calorimetric measurements of the transmitted beam were recorded.

The reflected-beam diagnostics (RBD) package (Fig. 6-54) consisted of a calorimeter, a twodimensional MIC camera, two streak cameras (one for the spectrally analyzed light, the other for the nondispersed back-reflected light). an optical spectrometer, and a monitoring TV camers. An MIC image of back-reflected light taken during an experiment on a gold disk target is shown in Fiz. 6-55. Figure 6-56 shows two streak pictures from the

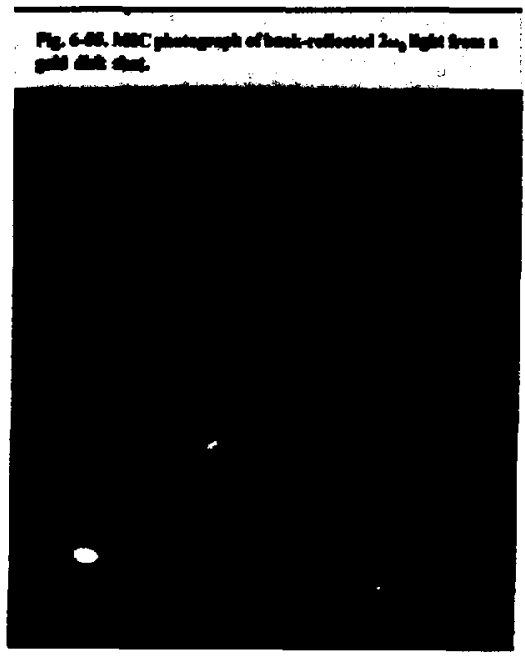

sume shot, one (a) for the temporal behavior of the spectrally analyzed backscattered light, the other (b) giving the time history and one spatial dimension of the scattered light. Analysis of the spectrally resolved light provided insight for our laser-plasma coupling studies (discussed in the following article). Spectral data was gathered via a 1-m-grating spectrograph coupled to an optical multichannel 


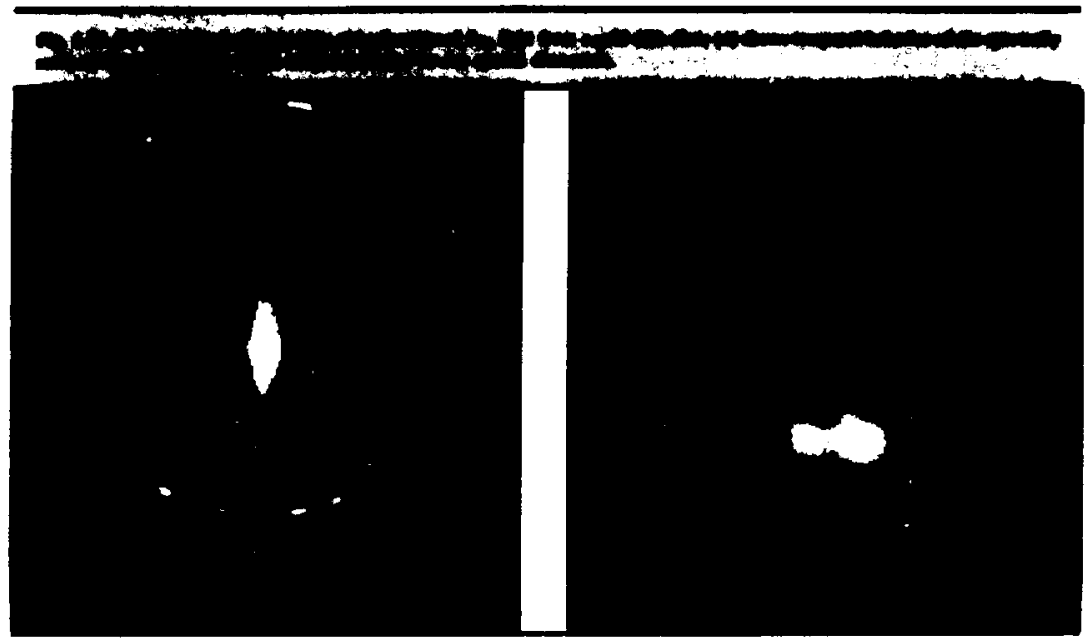

anilyzer (a 120SE detector head provided by Princeton Applied Research Laboratory). The spectrograph angular dispersion was $2.80 \times 10^{-4} \mathrm{rad} / A$, which gave a dispersion of $8.82 \times 10^{-2} A /$ channel on the optical multichannel analyzer.

Awhor: F. Ze

Major Contributors: E. M. Campheh, V. C. Rupert, J. E. Swain, and D. W. Phillion

\section{References}

57. D. R. MacQuigg and D. R. Speck, Beam Diagnostics on Agus, Lawrence Livermore Laboratory, Livermore, Calif.. UCRL-78+47 (1976).

58. Laser Program Amual Report-1975. Lawrence Livermore Laboratory, Livermore. Calif., UCRL-S002!-75 (1976). pp. 367 to 405 .

59. Laser Program Annual Report-S976. Liwrence Livermore Laboratory, Livermore. Calif., UCRL-5002I-76 (1977). pp. 3-55 to 3-60.

\section{Preliminary $2 \omega_{0}$ Results}

We have initiated a series of experimeris to study the wavelength scaling of physical prccesses such as absorption, stimulated scattering, energy transport, and suprathermal electron geseration. which are important in laser-driven inertial confinement fusion. Our variable-wavelength source will be provided by nonlinear frequency conversion of the fundamental 1.064- $\mu \mathrm{m}$ output of Nd lasers into the 2nd, 3rd and possibly 4 th harmonics $(0.532 \mu \mathrm{m}$, $0.355 \mu \mathrm{m}$, and $0.266 \mu \mathrm{m}$ respectively). As discussed in the previous article, we are presently utilizing a 90-mm-diam, 13-mm-thick KDP type II crystal to frequency-double one of the two Argus laser beams.

To date, we have used a box calorimeter ${ }^{60}$ to measure the absorption and scattering of light from both $\mathrm{Au}$ and $\mathrm{CH}$ disk targets irradiated with green light at intensities in the range of $10^{14}$ to $10^{15}$ $W / \mathrm{cm}^{2}$. Targets are typically $600 \mu \mathrm{m}$ in diameter and 14 to $25 \mu \mathrm{m}$ thick; pulse lengths $\left(2 \omega_{0}\right)$ are $600 \mathrm{ps}$ (FWHM). The laser spot (containing $90 \%$ of the energy) is elliptical, with a major axis ranging from 50 to $160 \mu \mathrm{m}$ and an eccentricity of 1.5 to 2 . Peakto-average intensity modulations within this spot are typically 3 or 4 to 1 .

In Fig. 6-57, the absorption of Au targets at intensities of 2 to $4 \times 10^{14} \mathrm{~W} / \mathrm{cm}^{2}$ is shown as a function of the angle of incidence between the disk normal and the laser propagation direction. The irradiations were done near p-polarization ( $E$ lies $14.5^{\circ}$ out of the plane of incidence). Al normal incidence, the laser-spot major axis was $110 \mu \mathrm{m}$; beam area was kept constant as the target was rotated through the various angles.

The angular variation was motivated by the following considerations: 


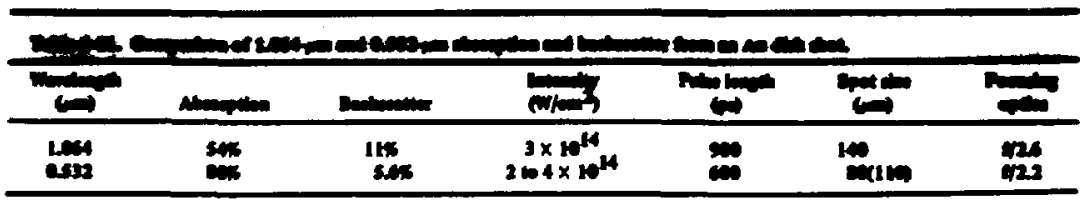

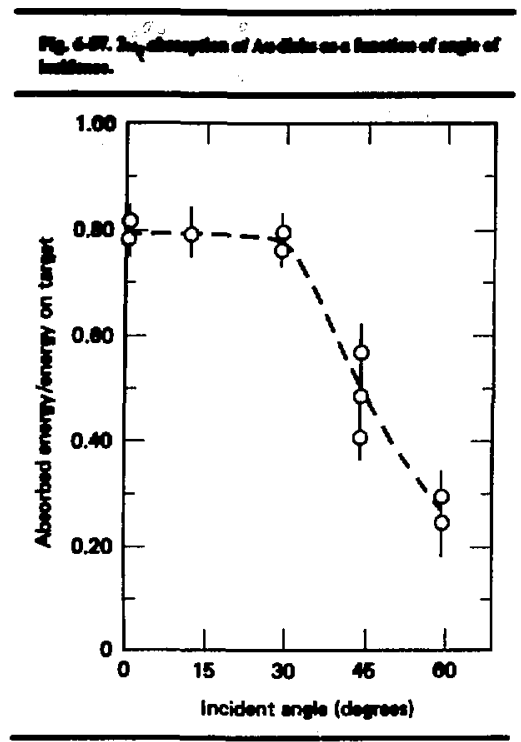

- Future measurements of low-ejergy (hy $\leqslant$ I keV) $x$ rays (which can provide information on thermal transport into the supercritical plasma) require a quantitative knowledge of the angular distribution of emission. ${ }^{\text {fI }}$ To map this out using immobile diagnostics (i.e.. our Dante systems) requires incidence angles up to $30^{\circ}$.

- Examination of the spectrum of the backseattered light at different incidence angles allows the potential unfolding of Doppler and Brillouin components.

- The specular and backscatter components of the scattered light can be determined with the six discrete calorimeter modules of the cubical box calorimeter as the targets are rotated through the various angles. Analysis of this data in terms of absorption mechanism or scale lenglh, however, is complicated by the fast optics (marginal rays are $12.8^{\circ}$ ) and possible two-dimensional expansion effects due to the relatively small laser spot and the long pulse length. This latter difficulty has arisen in the vast majority of long-pulse experiments performed to date. ${ }^{62}$

As shown in Fig. 6-57, the Au absorption is $80 \%$ at normal incidence and falls relatively slowly with angle of incidence. The weak dependence of absorption on the angle of incidence (roughly $\cos \theta^{l .4}$ ) sirnplines future $x$-ray conversion efficiency measuiements.

In Table 6-1] we compare the measured $A \mathbf{u}$ absorption and backscatter fraction obtained at normal incidence with $1.06-\mu \mathrm{m}$ and $0.532-\mu \mathrm{m}$ light under similar irradiation conditions. ${ }^{64}$ The large absorption obtained at the shorter wavelength is primarily due to more effective inverse bremsstrahlung absorption, although this may aiso be in part due to a reduction in stimulated Brillouin backscatter. ${ }^{63}$

Independent monitoring of signals from the six panels of the cubic box calorimeter and the measurement of the direct backscatter energy allows a crude determination of the angular dependence of the scattered light. In particular it is possible, by rotating the target through large angles $\left(45^{\circ}\right.$ and $\left.60^{\circ}\right)$ and using the responses of the individual pinels in the box calorimeter, to separote specular reflection from the backscatter. The basic idea behind this is indicated in Fig. 6-58, which shows a top view of the calorimeter and the orientation of a target at a $45^{\circ}$ incident angle. At incident angles of $45^{\circ}$ and $60^{\circ}$ (where target misalignment of $\pm 3^{\circ}$ does not influence the possible panel distribution), $36 \%$ and $55 \%$ (respectively) of the incident energy appears on the east panel (specular) of the box calorimeter, while only $6 \%$ and $10 \%$ (respectively) appear in tl. : backscatter direction. Here we define backscatter to include light collected by both the focusing lens and the south panel (i.e., angles up to $45^{\circ}$ from the laser axis).

In contrast, data obtained with Au disks oriented at $45^{\circ}$ (s-polarization) and irradiated at $3 \times$ 


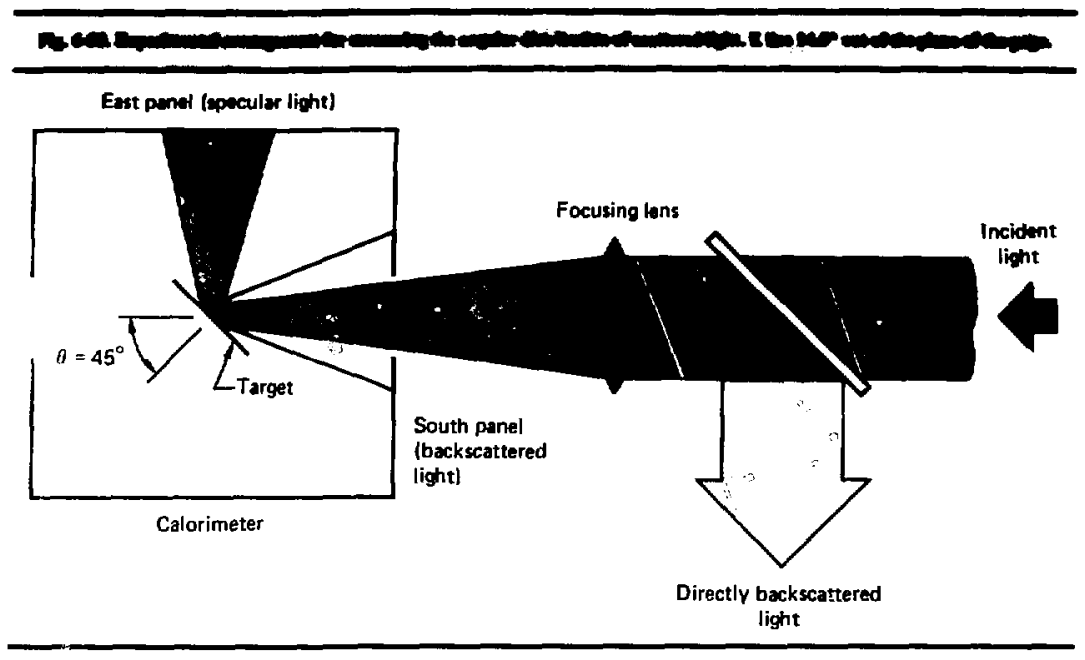

$10^{14} \mathrm{~W} / \mathrm{cm}^{2}$ with s-polarized $1.064-\mu \mathrm{m}$ light showed that $25 \%$ of the incident energy appeared in the backscatter direction.

For all of the target orientations, the amounts of energy collected by the top and bottom panels of the calorimeter (i.e., large-angle scattering $\left(n>45^{\circ}\right.$ ) out of tite plane of polarization] were negligible. This result is not surprising in view of the small laser spot (and hence the small number of growlh lengths for stimulated Brillouin side-scatter), and the tendency of refraction to direct the light along the density gradient. ${ }^{61.63}$ Though not conclusive and lacking quantitative data on refraction effects, our calorimeter results suggest that stimulated Brillouin scattering is reduced at the shorter wavelength (at least for densities less than $0.5 \mathbf{n}_{\mathbf{c}}$ ).

Another interesting feature of the scatteredlight distribution is found by plotting the energy directly backscattered through the $r / 2$ lens as a function of angle of incidence. The results (Fig6-59) show an unexpected increase in the backscattered energy as the incidence angle is increased to $60^{\circ}$. This phenomenon is not yet understood.

Evicience that Brillouin scattering is still operative at these irradiation conditions (although al a reduced kevel) can be found by examining the

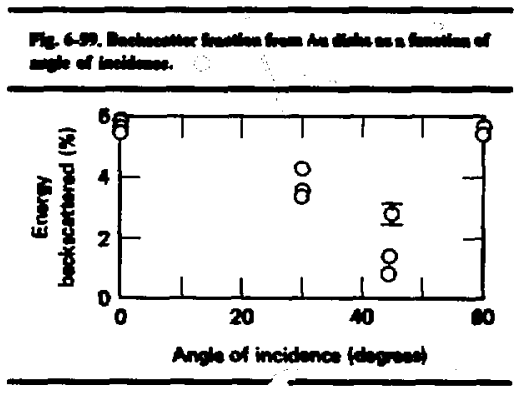

frequency spectrum of the backscattered light. One of the signatures of Brillouin scattering is the red shift of the scattered light. ${ }^{64}$ In an expanding plasma, however, the Brillouin shift is reduced by the blue Doppler shift. Since the plasma nominally blows off normal to the disk plane, rotating the target diminishes the Doppler shift by reducing the velocily prujection along the observation line of sight.

Figure 6-60(a) shows the time-integrated frequency spectra obrained in these experiments. As expected, the spectrum is increasingly red-shifted as the disk is rotaled. From these dala (surimarized in Table 6-12) and from the dispersion relation for ion 


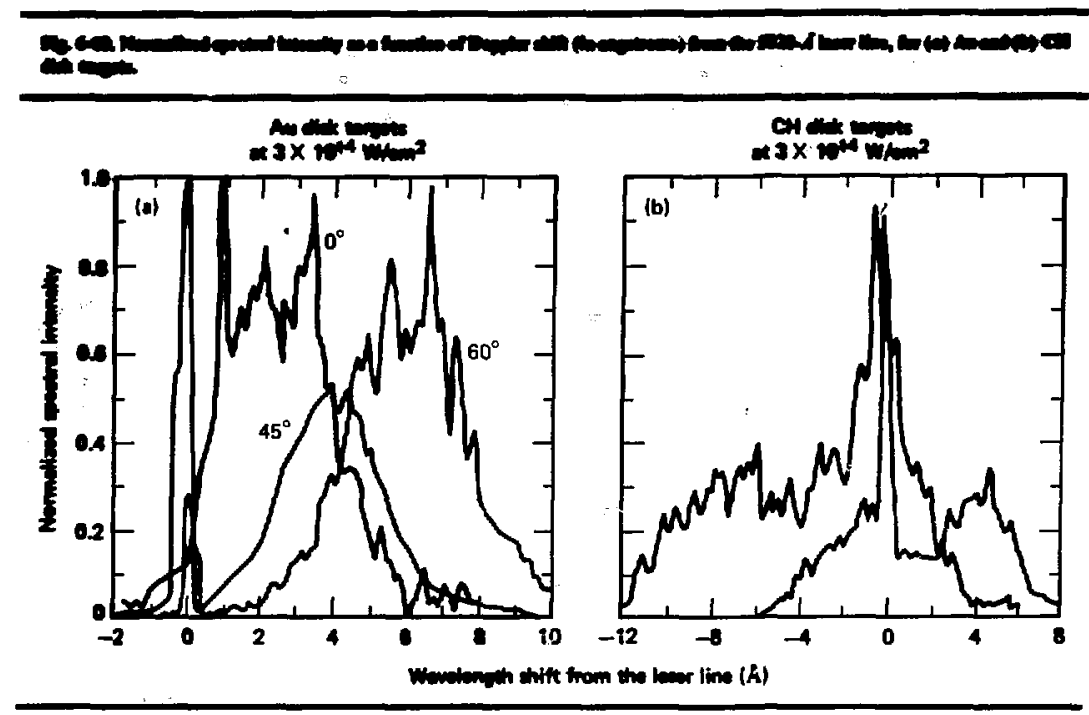

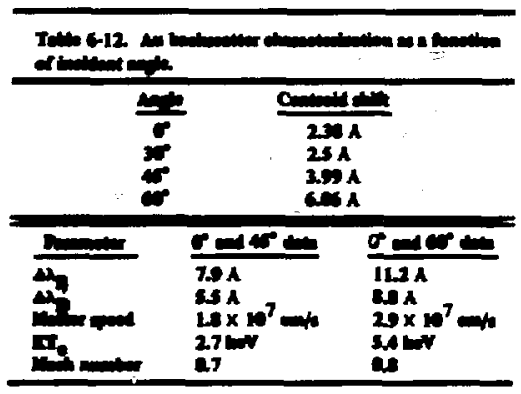

acoustic waves, we can obtain estimates of both the matter velocity and coronal ekctron temperature. To derive the electron temperature $T_{c}$ and matter velocity $U$, we have used the centroid of the spectrum and assumed

- A mean ionization state $\overline{\mathbf{Z}}$ of $\sim \mathbf{5 0}$.

- $2 T_{e}>3 T_{i}$.

- That the scattering takes place at $0.25 n_{c}$ (turning density at $60^{\circ}$ ).

- That no quantities chanee significantly as the disk is rotated.

\begin{tabular}{|c|c|c|c|}
\hline Mandald & $\bullet$ & Alomention (X) & 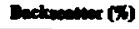 \\
\hline$\underset{c}{a}$ & $\underset{45}{6}$ & $\begin{array}{l}71 \pm 5 \\
1 \pm 5 \\
34 \pm 5 \\
49 \pm 1\end{array}$ & $\begin{array}{l}11 \pm 4 \\
5.4 \pm 0.5 \\
3 \pm 2 \\
1.1 \pm 1\end{array}$ \\
\hline
\end{tabular}

As can be seen from the inferred values, ahere is considerable scatter in the results, parcicularly in the electron temperature. This is not unexpected, since the electron temperature depends on the square of the Brillouin shift. Nonetheless it is interesting to note that the inferred quantities indicate that the flow is subsonic (i.e., Mach numbers $\sim 0.75$ ) where the scattering occurs,

In addition to the Au dises, several $\mathrm{CH}$ targets have been irradiated under similar conditions at both normal incidence and at $45^{\circ}$. The preliminary $\mathrm{CH}$ results are compared with the Au data in Tabl. 6-13. As expected, the lower-Z CH plasma absolb: kss efficiently than the Au plasma, although the difference is not as large as might be suspected with the $\boldsymbol{Z}$ scaling of classical absorption. 1 .e CH plasma 


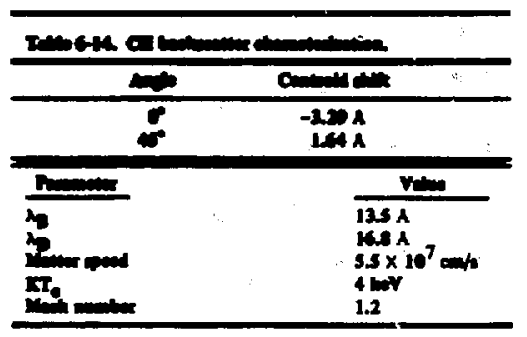

also backscattered more energy through the focusing lens than did the Au plasma. In previous longpulse $(0.9 \mathrm{~ns}) \quad 1.06-\mu \mathrm{m}$ experiments conducted at A rgus with similar intensity, the oppositc result was obtained-the backscalter fraction increased with $Z$ (Ref. 65).

The measured absorption of $\mathrm{CH}$ is consistent with the data obtained by Fabre, ${ }^{22}$ although the irradiation conditions are not identical. For example, at similar intensities, Fabre measured $63 \%$ absorption with 80-ps pulses. In addition, at lower intensities $\left(\sim 6 \times 10^{13} \mathrm{~W} / \mathrm{cm}^{2}\right)$ only a weak dependency on pulse width was observed (absorptions of $75 \%$ and $82 \%$ were observed at $80 . \mathrm{ps}$ and $2.5-\mathrm{ns}$ pulses). This weak dependence, however, may be due in part to the small laser spot $(50 \pm 15 \mu \mathrm{m}$ in diameter) used in the Fabre experiments.

In addition to calorimetry, we performed spectral analysis on the backscattered $\mathrm{CH}$ light [Fig. 6-60(b)]. As was observed with Au disks, the light is increasingly red-shifted as the target is rotated to $45^{\circ}$. In contrast to the Au results, however, the $\mathrm{CH}$ spectrum exhibits a net blue shift at normal incidence. Results of unfolding the Brillouin and Doppler shifts from the data are displayed in Table 6-14, which shows that the $\mathrm{CH}$ plasma expands more rapidly than the Au plasma. This is most likely due to both the lower $Z / A$ ( $A$ is the atomic weight) and increased radiation loss of the $A u$ plasma. Also, in contrast to the Au results, the $\mathrm{CH}$ plasma appears to be expanding supersonically (i.e.. Mach number $=1.2$ ).

\section{Anter: E. M. Compreli}

Meier Contrilumers: F. Ze, D. W. Preminend V. C. Repert

\section{References}

60. Later Program Annuol Report-19/5. Lawitence Livermore Laborator;, Livermore, Calif., L'SRL-50021-75 (1976). p. 404.

61. M. D. Rosen el al., Phys. Fluids 22, 10 (1979).

62. F. Amirinofr el al., presented to the Division of Plasma Physics at the 21st Meeling of the American Physical Socicty, Boston, MA (1979).

63. C. E. Mix and K. Estabrook, Lawrence Livermore Luboratory, Livermore, Calif. (submitted to Comments Plosmo Phys. Cont. Fusion).

64. D. W. Phillion, W. L. Kruer, and V. C. Rupert, Phy's. Rev. Let1. 39. 1529 (1974).

65. G. McClellan, P. H. Y. Lee, and G. Caporaso, Lawrence Livermore Lahoratory, Livermore. Calif., UCRL-83044 (submitted to Phys. Rev. Lett.).

\section{Summary of Disk Experiments}

In the past year we have continued our attempls to understand laser-plasma interaction phenomena occurring in large-yolume, long-scalelength plasmas. In particular we have extended our data base concerning pulse length, plasma composition, and wavelength, through a wide variety of disk-target irradiations at both the Shiva and Argus faeilities.

In studies at Shiva we have measured the intensity-dependence of the absorption and the $x$ ray emission from. $\mathrm{CH}$ and $\mathrm{Au}$ plasmas irradiated witb 2-11s (FWHM) 1.06- $\mu \mathrm{m}$ pulses. The absorption results are surprisingly insensitive to ${ }^{\prime}$ in a parameter regime where inverse bremsstrah..ng absorption should be operative. At low intensities $(5 \times$ $10^{13} \mathrm{~W} / \mathrm{cm}^{2}$ ) we have obtained absorptions as large as 70 to $80 \%$, although there is still evidence for low $(\sim 5 \%)$ levels of stimulated Brillouin backscatter. Low-energy (hy $₹ 1.5 \mathrm{keV}$ ) $x$-ray measurements from Au at low intensities also suggest that thermal transport inthibition may not be severe. At an intensity of $3 \times 10^{14} \mathrm{~W} / \mathrm{cm}^{2}$, the behavior of plasma interactions with $A u$ is very similar to that obtained with 1-ns irradiation, although we measured a slight increase in absorption. from $50 \%$ for I ns to $100 \%$ for $2 \mathrm{~ns}$. At the highest intensity examined $\left(3 \times 10^{15}\right.$ $W / \mathrm{cm}^{2}$ ), Au absorption was observed to drop from $35 \%$ to $25 \%$ as the pulce length was increased from 1 to $\mathbf{2} \mathbf{n s}$. Such results may illustrate the competition between inverse bremsstrahlung and stimulated Brillouin scattering. and show the potential problems of high-intensity operation with brgevolume plasmas. 
Interesting scattered-light angular distributions were measured with a box calorimeter on long-pulse (0.9-ns FWHM) large-focal-spot (350- $\mu \mathrm{m}$-diam) irradiations of Au disks. These experiments were conducted at Argus with $1.06-\mu \mathrm{m}$ light at peak intensities of $3 \times 10^{14} \mathrm{~W} / \mathrm{cm}^{2}$. We observed an increase in large-angle scattering $\left(\theta>45^{\circ}\right)$ out of the plane of polarization as the disk targets were isolated from $0^{\circ}$ to $30^{\circ}$ (p-polarization) with respect to the laser axis. At rormal incidence only $3 \%$ of the incident light suffered large-angle out-of-plane scattering, whereas up to $23 \%$ was observed at $30^{\circ}$. This scattered energy was comparable to the specular and backscatter components. We obtained similar results for intermediate- $Z$ (Ti) disk targets oriented at $30^{\circ}$. These data, though complicated by refraction and the poor angular resolution of the box calorimeter, are suggestive of Brillouin sidescatter.

A large fraction of our experimental time at Argus was allocated to interaction physics. We repeated and extended shori-pulse ( 0.1 ns), highintensity $\left(10^{15} \mathrm{~W} / \mathrm{cm}^{2}\right)$ energy-transport experiments with $\mathrm{CH}$-coated Al microdisks. Measurements of sub-kilovelt : rays and Al line radiation as a function of $\mathrm{CH}$ thickness indicated burn-lhrough depths of $\sim 0$. $1 \mu \mathrm{m}$ : this result is consistent with data obtained at other laboratories. Simulations of these experiments, though compromised by the lack of absorption data, suggest transport inhibition for both the thermal and suprathermal electrons. Modeling of the suprathermal electrons was also complicated, however, by the low $x$-ray fluxes between 10 and $50 \mathrm{keV}$ measured during these experiments (the levels were a factor of 4 to 10 times lower than $1.06-\mu \mathrm{m}$ experiments conducted at other ficilities). This result is not yet fully understood but may be due to better beam quality or a lower absorption level than we expecicd.

We also measured $x$-ray emission $(0.1 \mathrm{keV} \gtrless$ $h^{\prime}>\mathbf{8 0 ~ k e V ) ~ f r o m ~ d i s k s ~ o f ~ v a r i o u s ~} Z$ irradiatiated with 0.9-ns (FWHM) putses. The targets were Be. Ti, Au, and $U$ disks with atomic numbers of $4,22$. 79. and 92 respectively. The peak intensity was held constant at 3 to $5 \times 10^{14} \mathrm{~W} / \mathrm{cm}^{2}$. Several interesting features of the low-energy (h, $₹ 1.5 \mathrm{keV}$ ) x-ray emission were observed in these experiments:

- The total $x$-ray emission and spectrum were strongly dependent on $Z$.

- The cmitted energy initially increased rapidly with $Z$ and then leveled off duc to saturation of the plasma ionization level in the radiating reusion.

- The spectrum, measured with the broadband Dante system, displayed the presence of dominant line radiation on the intermediate- $Z$ (Ti) plasmas.

i,t a finad preak laser intensity the temporal profile of subkilovolt emission was found to depend on the $Z$. of the target matcrial. We have internreted this phenomenon as a signature of thermal tratisport inhibition with a Z-dependent intensity threshold.

- The flux of the high-energy (he $>10 \mathrm{keV}$ ) $x$ rays also was seen to scale with $Z$. It is difficult to assign an accurate value to the slope of the $x$-ray spectrum $\left(\theta_{\mathrm{H}}\right)$, but the data do show a hardening of the spectrum as target $Z$ increases. For example, the Be spectrum is best fit with a $\|_{H}$ of $\sim 10 \mathrm{keV}$ whereas the uranium data suggest $\|_{\mathrm{H}} \sim 20 \mathrm{keV}$. As we have found in our previous long-pulse (l-ns) experiments, the level of the hard-:-ray nux indicates that only a small fraction ( $₹ 5 \%$ ) of the incident energy appears in suprathermal electrons; this suprathermal level appears to be only weakly dependent on $\mathbf{Z}$.

We have also begun to examine the mechanisms for suprathermal-electron production in longpulse $(\tau \sim 1 \mathrm{~ns})$, high-intensity irradiations. We are particularly concerned with Raman and $2 \omega_{\mathrm{pe}}$ instabilities which are operative at densities $\leqslant 0.25 \mathrm{n}_{\mathrm{c}}$. M-zasurement of the scattered light from hign- $Z$ disk targets at wavelengths between 1.8 and $2.2 \mu \mathrm{m}$ has demonstrited that these processes occur not only at $0.25 n_{c}$ (resonant Raman scattering and $\left.2 \omega_{p c}\right)$ but at densities as low as $0.1 n_{c}$ [nonresonant Raman (Compton) scaltering]. The fraction of the light entergy appearing as scattered light between 1.8 and $2.2 \mu \mathrm{m}$ is estimated to be at least $5 \times 10^{-5}$ at intensities of $\sim 5 \times 10^{14} \mathrm{~W} / \mathrm{cm}^{2}$. When the peak intensity is increased to $\sim 10^{17} \mathrm{~W} / \mathrm{cm}^{2}$ we estimate a lower bound of $5 \times 10^{-4}$ for the conversion efficiency.

Recent experiments at Shiva have indicated an isotropy in the angular distribution of $x$ rays with energy of $>+0 \mathrm{keV}$. These $x$ rays are produced by suprathermal electrons. as they interact with the dense target materisl. Our measurements have been made with arrays of calibrated detectors and an extended film pack. The two-lobed distribution we 
have observed (see "Angular Distribution of Suprathermal X Rays" earlier in this section) is relatively insensitive to changes both in target unaterial (Au disks and glass microballoons) and irradiation conditions. Additional experiments are required to explore and quantify the source of these energetic $x$ rays.

Finally, recent modifications to the Argus facility have allowed us to begin to explore wivelength scaling of laser-plasma interaction pirysics. Experiments at $0.532 \mu \mathrm{m}$ have demonstrated high absorption efficiency of both $\mathrm{Au}$ and $\mathrm{CH}$ plusmas irradiated at peak intensities of $3 \times$ $10^{14} \mathrm{~W} / \mathrm{cm}^{2}$ with 600-ps (FWHM) pulses. As in our long-pulse (2 ns) $1.06-\mu \mathrm{m}$ experiments, we found only a weak $Z$-dependence for absorption, from $80 \%$ for $\mathrm{Au}$ to $70 \%$ for $\mathrm{CH}$. The angular dependence of the scatlered light and frequency analysis of the direct backscatter indicate that stimulated Brillouin scattering is still operative, although at a reduced level compared to $1.06 \mu \mathrm{m}$.

Author: E. M. Campbell

Major Contributors: D. W. Phillion, V. C. Rupert, D. L. Banner, P. H. Y. Lee, and C. L. Wang

\section{X Liquid Density Target Experiments}

The achievement of high-density, isentropic implosions is critically important for realizing high guin by means of laser-driven inertial confinement fusion. ${ }^{66}$ Most experimental experience to date, however, has been obtained with high-entropy, lowdensity exploding-pusher targets. ${ }^{67}$ While such targets have achieved high final D-T ion temperatures (2 to $8 \mathrm{keV}$ ), demonstrated thermonuclear burn, ${ }^{68}$ and produced high $\left(3 \times 10^{10}\right)$ neutron yields, ${ }^{69}$ they are not scalable to break-even conditions.

We have recently begun experiments on thickwalled capsules designed to achieve high-density implosions ( $\rho$ D.T $\sim 2 \mathrm{~g} / \mathrm{cm}^{3}$ ). Accurate diagnosis of targets used in such implosions is in an early stage, since the relatively cold, dense implosions preclude the use of many techniques that have been successfully employed in traditional exploding-pusher experiments. ${ }^{70}$ Thus, as experiments evolve toward a more direct study of ablatively driven targets, it is important to examine transitional targets which combine features of both simple exploding pushers and more advanced isentropic implosions. Such experiments serve as valuable checks on our simulation codes by allowing the use of several independent diagnostics to study the implosion. In addition, new experimental techniques designed to study high-density targets can be developed and compared with established, well-understood diagnostics.

For the above reasons, thick-walled polymercoated glass microspheres designed to reach D-T densities of $\sim 2 \mathrm{~g} / \mathrm{cm}^{3}$ (10X liquid density) were irradiated at Shiva. ${ }^{71}$ The basic $10 \times$ target capsule [Fig. 6-6l(a)] is a $140-\mu \mathrm{m}-\mathrm{i}$.d. silicate glass microsphere with a $5-\mu \mathrm{m}-1$ hick wall and $15-\mu \mathrm{m}$ coating of $\mathrm{CF}_{1.4}$. The targets are filled with 50 atnospheres of equimolar deuterium and tritium (10 $\mathrm{mg} / \mathrm{cm}^{3}$ ) and approximately 0.05 atmospheres of urgon (as will be discussed below, the trace amount of argon is used for diagnostic purposes and does not effect the implosion dynamics).

The targets were irradiated with $4 \mathrm{~kJ}$ in a 200 ps FWHM Gaussian pulse. The radially polarized Shiva beams, interacting with the plasma predominantly in p-polarization, were offset so that the marginal rays were tangential to the target [Fig. 6-61(b)]. Peak calculated intensities on the target were $\sim 4 \times 10^{15} \mathrm{~W} / \mathrm{cm}^{2}$ at the equator and the poles (intersecting the heam cluster axis). and $\sim 2 \times 10^{16}$ $W / \mathrm{cm}^{2}$ in two bands at intermediate latitude.

We fielded a large number of diagnostics during this experimental series:

- Target absorption was measured with arrays of both plasma and laser calorimeters ${ }^{72}$ and with scattered-light photodiodes. ${ }^{73}$

- Thermonuclear yields were measured with both lead and copper activation systems. ${ }^{74}$

- A filter fluorescer recorded the flux of 20 to-80-keV-bremsstrahlung produced by suprathermal electrons. ${ }^{75}$

- Time-integrated $x$-ray imaging of the target was accomplished by two Kilpatrick-Baez microscopes (giving polar and equatorial views) ${ }^{76}$ and a Fresnel zone plate camera (giving an equatorial view). ${ }^{77}$ Each microscope recorded images in four broad-band channels (derined by $K$ edge filters, the reflector material, and the angle of incidenoc) near $1.6,2,2.9$, and $3.5 \mathrm{keV}$. the zone 


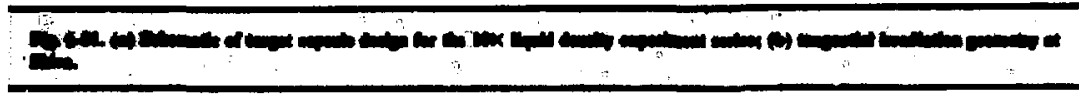

(a)
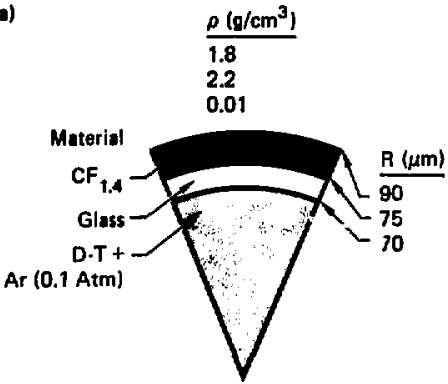

(b)

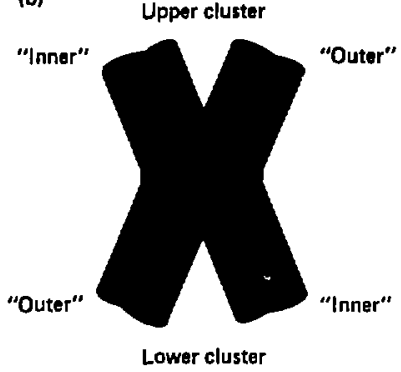

plate camera also had multi-image capability, through the use of 40- $\mu \mathrm{m}$-thick $A u$ zones and a film pack consisting of alternating layers of film and $x$ ray absorbers. ${ }^{77}$ Though dependent on the $x$-ray spectrum, the broad zone-plate image channels were nominally centered on $5.9,6.6,10,17$, and $21 \mathrm{keV}$. This high-energy ( $54-\mathrm{keV}$ ) imaging capability is particularly important for viewing intermediatedensity targets, as the large areal density of the stagnated pusher requires the imaging of multikilovolt $x$ rays in order to view the ptisher-fuel interface. ${ }^{70}$ In addition, the large collection solid angle of the zone plate camera allows high sensitivity while still maintaining a resolution of $8 \mu \mathrm{m}$.

- Compressed-core conditions were also diagnosed with one-dimensional imaging spectroscopy of the argon seed gas in the fuel. ${ }^{78} \mathrm{~A}$ lowdispersion PET crystal spectrograph, used in conjunction with a $20-\mu \mathrm{m}$ slit aligned for a pole-to-pole view, imaged the 3.14-keV Hac [1s $\left.2 \mathrm{p}\left({ }^{1} \mathrm{P}_{1}\right) \rightarrow 1 \mathrm{~s}^{2}\left({ }^{1} \mathrm{~S}_{0}\right)\right]$ argon line emanating from the compressed-fuel region. The small amounts of argon led to a low signal-to-noise ratio and precluded any Stark analysis of the line radiation.

- The areal density, $\rho \Delta R$, of the compressed glass pusher, integrated over the burn time, was measured by counting ${ }^{28} \mathrm{Al}$ created by the ${ }^{28} \mathrm{Si}(\mathrm{n}, \mathrm{p})$ ${ }^{28} \mathrm{Al}$ reaction. ${ }^{79}$ If we use the measured cross section for this reaction, the fractional concentration (25\%) of ${ }^{28} \mathrm{Si}$, and the measured 14.1-MeV neutron yield, $Y_{n}$, then measuring the number $N^{*}$ of activated ${ }^{28} A l$ atoms yields the pusher areal density (in $\mathrm{mg} / \mathrm{cm}^{2}$ ):

$$
(\rho \Delta R)_{\mathrm{eff}} \simeq 5 \times 10^{5}\left(\frac{\mathrm{N}^{*}}{\mathrm{Y}_{\mathrm{n}}}\right)
$$

It is possible to relate this measured quantity to the compressed-fuel conditions by both simple modeling and with detailed hydrodynamic simulations.

\section{Experimental Results}

We measured absorption on two shots for which the density-diagnostics instrumentation in the vicinity of the target was removed. Both the plasma calorimeters and Si PIN diodes indicated an absorption fraction of $23 \%$ ( $\pm 5 \%$ ). Previous experiments have shown that much of the light absorption at the high intensities and relatively short pulse lengths described above occurs via resonance absorption at the critical surface. Angle- and polarization-dependent absorption measurements have shown $\sim 40 \%$ absorption at 1 to $3 \times 10^{15}$ $w / \mathrm{cm}^{2}$ for p-polarized light at incidence angles of $30^{\circ}$ (Ref. 80). The reduction in absorption is most likely due both to irradiation geometry and to stimulated Brillouin scattering that watters the light before it reaches the critical surface. ${ }^{81}$

In Fig, 6-62 the measured $x$-ray fluence at 20, 50 , and $80 \mathrm{keV}$ is compared with a LASNEX simulation in which resonance absorption accounts for $\mathbf{9 0 \%}$ of the absorbed light. The effective slope of the $\mathrm{x}$-ray tail is calculated to be $\sim 21 \mathrm{keV}$. As shown 


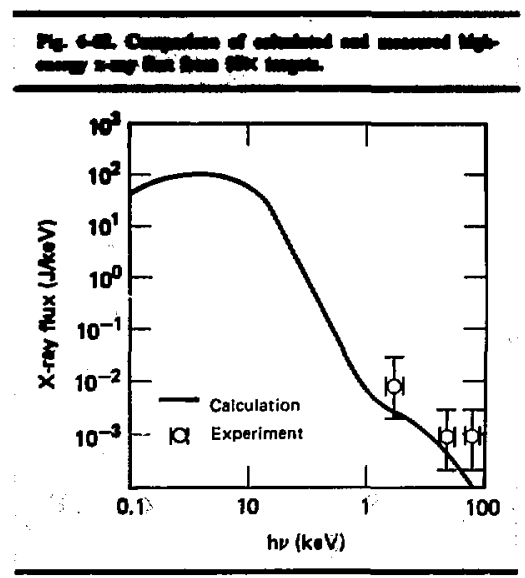

in the figure, the data agrees reasonably well with the simulation. Interpretation of the data is complicated, however, by recent experiments which suggest an apparent angular modulation of the high-energy (hu $\$ 50 \mathrm{keV}) \times$ rays $^{82}$; the origin of this modulation (and the spectral dependencies) are not presently understood and will be explored in future experiments.

Thermonuclear yields between $10^{8}$ and $10^{9}$ were obtained in the $10 \mathrm{X}$ experiments. For seven shots with an average incident energy of $3.8( \pm 0.4)$ $\mathrm{kJ}$ the measured neutron yield was $3.1( \pm 0.5) \times 10^{8}$. These yields are consistent to within a factor of 3 with onc-dimensional and two-dimensional LASNEX simulations, ${ }^{83}$ using the modeling generally consistent with that used for previous interaction and implosion experiments. The predicted yiclds, however, are not sensitive to thermalelectron transport inhibition, however, and thus the

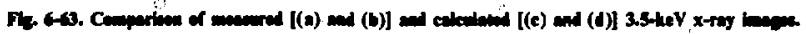

(a) Microscope images
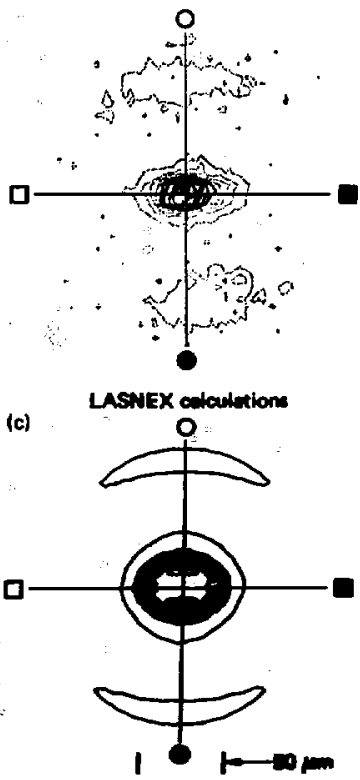

(b)

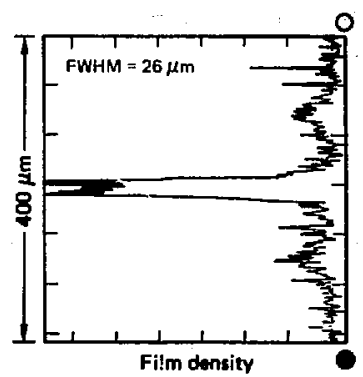

(d)

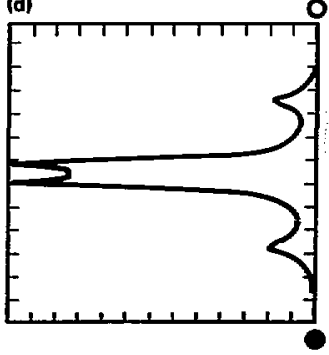

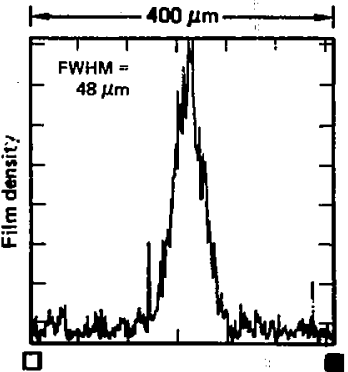

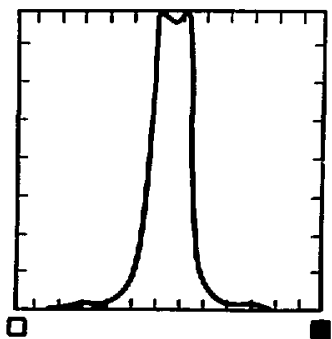


neutron measurement does not constrain this aspect of the modeling. Furthermore, neutron production is so strongly dependent on the fuel ion temperature and implosion dynamics of the target that additional details of the compression (such as symmetry and pusher fuel mixing) cannot be inferred from the TN-yield meas $\mu$ rement.

Characteristics of the heating and stagnation symmetry of the target were obtained using twodimensional continuum $x$-ray imaging. So many time-dependent factors are involved in producing the image, however, that deconvolution of quantitative physical information from the data, particularly in the imploded core, is impossible. The image is spatially integrated along a line of sight, spectrally integrated over broad channels, and temporally integrated over a complex, dynamic implosion. As implosions achieve higher final densities and correspondingly larger pusher areal densities, the additional complexily of $x$-ray transport arises. This results in the continuum images becoming increasingly determined by emission from the outer pusher material.

This problem is reduced by inaging $x$ rays of sufficiently high energy, for which the pusher is essentially transparent. The targets used in these experiments had measured and calculated $\mu \Delta R$ 's of $\sim 6 \mathrm{mg} / \mathrm{cm}^{2}$, requiring $x$ rays with energy $>5 \mathrm{keV}$ to see into the pusher-fuel interface. ${ }^{70}$ The atility to clearly see this interface is essential to ascertaining the fuel density, both for continuum and seed-gas imaging. This latter technique relies on information ahout symmetry to subsequently extrapolate from the one-dimensional image, obtained with a single slit-spectrograph combination, to a measurement of the $x$-ray emitting volume.

To illustrate these effects we show in Fig. 6-63(a) the image of the irradiated target recorded in the highest energy cbannel ( $3.5 \mathrm{keV})$ of the equatorial-viewing $x$-ray microscope. The asymmetry of the irradiation pattern is obvious from both the initial heating of the ablator and the oblate stagnated core. As can be seen from line scans through the digitized image along directions parallel and perpendicular to the laser axis [Fig. 6-63(b)], there is no clear delineation of the pusher-fuel interface. Thus we are unable to infer either the fuel density or the symmetry of the enclosed fuel volume from this experimental measurement.

Figures 6-63(c) and (d) show the corresponding $x$-ray image and line scans calculated in the two- dimensional modeling of the experiment using the appropriate filter functions of the microscope. In this simulation, in which we obtained a peak fuel density of $2 \mathrm{~g} / \mathrm{cm}^{3}$, the thermal electrons were inhibited by the 2-stream instability with an effective flux limiter of 0.03 (Ref. 77). The emission in this energy band is dominaled by free-hound radiation from silicon in the pusher, although the calculation suggests that there may be an additional contribution from the high- $Z$ argon fill mixed in with the fuel. The calculated images are in qualitative agreement with the overall shape of the measured emission region of the compressed core. The detuils of the modeling (and hence the calculated image), however, are extremely sensitive to iflumination symmetry, absorption, transport, the mix of the pusher and fuel, and (in the case of the high- $Z$ fill) to non-LTE ionization physics.

Figure 6-64(a) shows an $x$-ray image recorded in a broad energy bin near $6 \mathrm{keV}$, obtained with the multichannel zone plate, filter-film pack combination. A line scan along a direction colinear to the laser axis is shown in Fig. 6-64(b). Images recorded at this energy do not suffer from transport difficultits and are less afrected by temporal smearing; at the implosion temperatures $\left(T_{\mathrm{e}}<1 \mathrm{keV}\right)$ characteristic of these experiments, the emission time of the 6-keV $x$ rilys is only 10 to $20 \mathrm{ps.} \mathrm{Since} \mathrm{the} x$-ray emission is primarily free-hound radiation from silicon, however, the images are extremely sensitive to electron temperalure gradients.

As shown in Fig. 6-64(a), the core region is considerably smaller than that measured by the $x$ ray microscope; the core also exhibits considerable structure in the emission pattern. This latter feature may suggest the existence of a pusher-fuel interface, although the region of enhanced $x$-ray emission does not totally encompass the centrol region of the core. This lack of emission could easily result from temperature gradients along the interface. Both high-energy (hu $\Im 10 \mathrm{keV}$ ) images where suprathermal brerisstrahlung emission dominates and twodimensional simulations with thermal transport inhibition suggest that asymmetries in the high-energy core-ion emission can be correlated with regions of increased heating in a nonuniformly irradiated ablator.

The high-energy images did not reproduce from shot to shot during the experimental series, and in several of the experiments the presence of a 


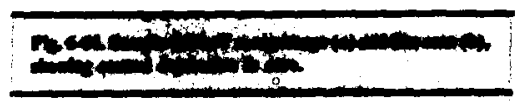

nomin

-
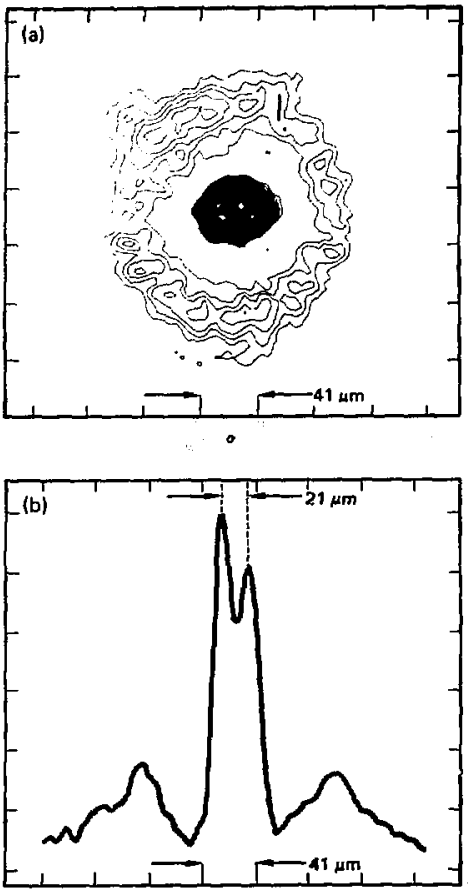

local minina is not obvious in the high-energy $x$-ray core emission. Nevertheless, despite a small data base, the performance of the capsules was nominally unchanged as measured by other experimental observables such as neutron yield, lowenergy (ho $₹ 3.5 \mathrm{keV}$ ) $x$-ray images, $\rho \Delta R$ at burn time, and argon line imaging. $A$ sample image and line scan are shown in Figs. 6-65(a) and (b); these should be compared with the corresponding zoneplate images in Fig. 6-64. It should be noted that dimensions of the core emission structure (i.e., two
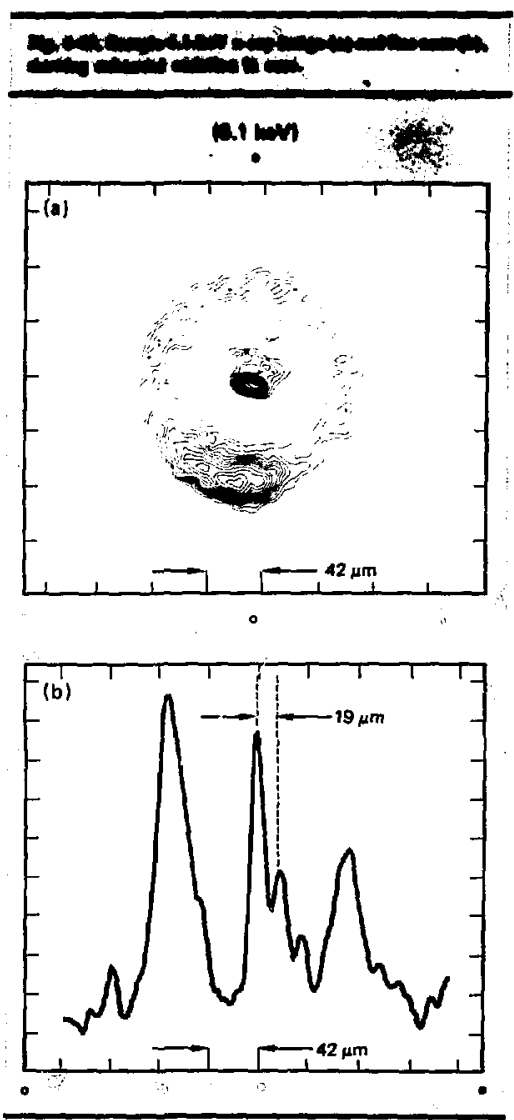

peaks separated by $21 \mu \mathrm{m}$ ) are similar in both images. The large difference in the asymmetries of the emission peaks may simply reflect the irreproducibility of the target irradiation.

Imaging of the line emission from a trace amount of argon mixed with the D-T $\left(\mathrm{n}_{\mathrm{Ar}} \sim 10^{-3}\right.$ nD.T) provides information about fuel compression that is not available with $x$-ray continumm imaging. The combination of low fills of argon (0.05 $₹ P_{A r} ₹$ 0.1 Atm), transport losses through the pusher (survival fraction $\sim 0.2$ ), and background emission from 


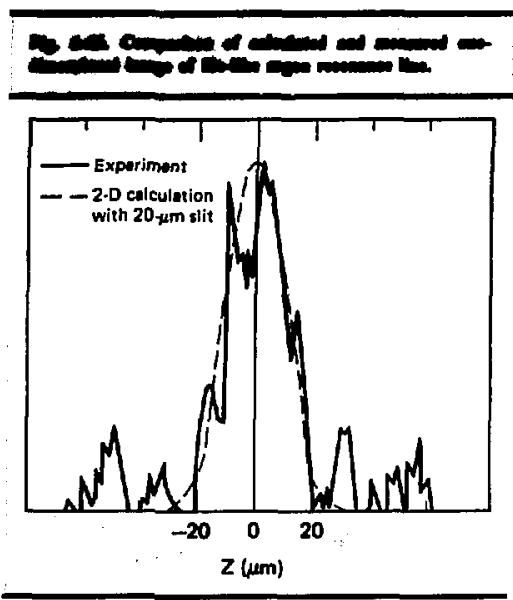

the silicon free-bound radiation from the pusher resulted in extremely poor signal-to-noise ratios ( 1.5:1 to 2:1). The data reduction is further compromised by the need to unfold the effects of the $20-\mu \mathrm{m}$ slit used in the experiment; this is particularly true when the slit is comparable to or larger than the source dimension to be imaged. To unfold the data we collapsed a uniformly emitting sphere or ellipsoid into one dimension, producing a parabolic radiance function. This profile is then convoluted with a slit response function and compared to the measured spatial profile.

Two experiments in which adequate data were obtained gave source dimensions of $\sim 25( \pm 10) \mu \mathrm{m}$. The slit was aligned to provide a pole-to-pole view (i.e., the minimum core dimension, as indicated by the continuum x-ray imaging). Rough estimates of the fuel density can be obtained from this data if we assume instantaneous emission and equality of the $x$-ray emission volume and fuel burn volume:

- For a spherical core one obtains a fuel density of $\sim 1.8 \mathrm{~g} / \mathrm{cm}^{3}$.

- For an oblate spheroid (with oblateness 2:1), as suggested by the iwo-dimensional modeling and continuum $x$-ray imaging, one obtains a density of $\sim 0.4$ to $0.5 \mathrm{~g} / \mathrm{cm}^{3}$.

In a more sophisticaled analysis we compared the measured argon spatial profile with the profile predicted by two-dimensional simulations which calculate the non-LTE evolution of the argon Helike emission during the implosion. " Figure 6-66 shows the calculated profile (including effects of the $20-\mu \mathrm{m}$ slit) and measured argon profiles. In this badly preheated implosion, mistiming of the argon emission relative to the peak compression results in a sampling of the fuel volume at about twice the minimum calculated value. Our observation is thus consistent with a two-dimensional simulation of the implosion which gives a D-T density of $\sim 1 \mathrm{~g} / \mathrm{cm}^{3}$; if the implosion continues until maximum stagnation the calculation of peak fuel density is $\sim 2 \mathrm{~g} / \mathrm{cm}^{3}$.

In the $10 \times$ series we measured effective pusher areal density $(\rho \Delta R)_{\text {eff, averaged over the } D-T \text { burn }}$ duration, with neutron activation of ${ }^{28} \mathrm{Si}$ (see Ref. 79 and Section 5, "Implosion Measurements with Neutron Activation Techniques"). Excellent data was obtained on 3 target shots, giving a mean value of $5.8( \pm 1) \mathrm{mg} / \mathrm{cm}^{2}$ (Ref. 84).

$A$ simple model serves to illustrate the connection between $\rho \Delta R$ and D-T density. The model

- Assumes the final state is an isobaric and isuthermal spherical core with instantaneous D-T burn.

- Incorporates the initial pellet geometry and a parameter $\epsilon$, representing the fraction of the initial pusher mass which is stagnated around the fuel at burn.

- Uses an ideal gas equation of state to relate the densities of the stagnated glass and D-T.

Solving the equations for conservation of fuel and pusher mass gives the final fuel density as $\rho_{\Gamma}=$ $\mathbf{G}(\rho\lrcorner \mathbf{R})_{\mathrm{e}}^{3 / 2}$, where $G$ is an algebraic expression containing the pellet parameters and $\epsilon$.

We can use this simple model to estimate the fuel density attained experimentally. Setting $\epsilon=0.5$. we obtain fuel densities of 1.5 to $2.5 \mathrm{~g} / \mathrm{cm}^{2}$ from the measured $(\rho \perp R)_{\text {eff }}$ (Ref. 71). If all the pusher is assembled at high density about the fuel (i.e., if $\epsilon=$ 1). and no mixing occurs, then we obtain a lower limit of 0.8 to $1.4 \mathrm{~g} / \mathrm{cm}^{3}$.

More detailed modeling of the activation dynamics is possible with LASNEX simulations which take into account both the finite length of the D-T burn and the imploded-pusher density profile. The plot of Fig. 6-67 gives the relationship between D-T density and pusher areal density at peak burn. ${ }^{71}$ Thus. the activation measurement implies 

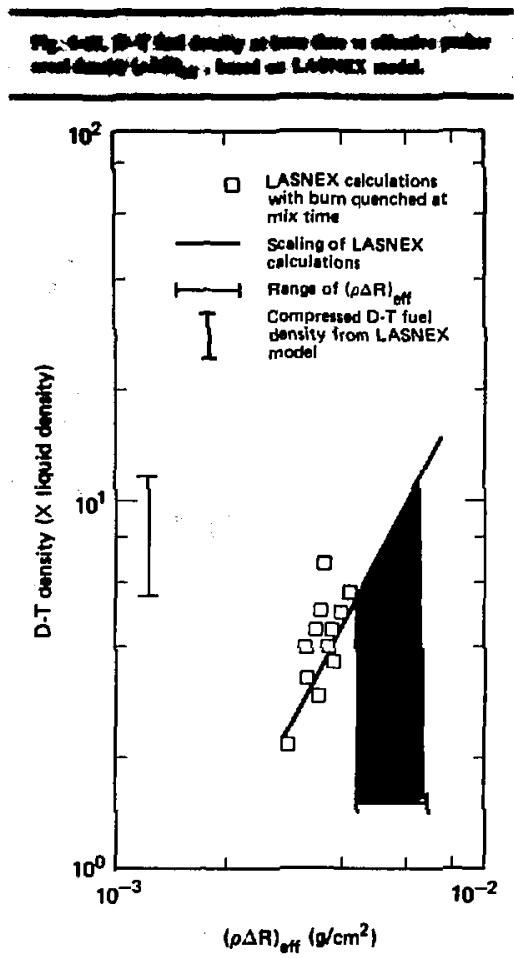

an average D-T density at burn time of $\sim 1$ to 2.4 $\mathrm{g} / \mathrm{cm}^{3}$ ( 5 to 12 times liquid density).

Two-dimensional effects (i.e., nonspherical implosions) modify the density that is attained but affeet the $p$ vs $(p-\lambda R)_{\text {efr }}$ relationship only in pathological cases. This is particularly true for the implosions described here, for which core asymmetries of $1.5: 1$ to $2: 1$ are suggested by both calculations and experiments.

Mistiming of the implosion (i.e., burn time occurring before maximum compression) also affects the interpretation of the activation measurement, in terms of maximum fuel density achieved in the implosion. Figure 6-68 gives the timing of the late stages of the implosion and shows that the fuel den- sity at the sumple time of the neutron activation measurement is approximately balf the peak calculuted value.

\section{Summary}

The $10 \times$ experiments are among the first to examine targets whose performance drviates from thut of the traditional thin-walled exploding pusher. Our measurements and calculations for this series indicate in particular the achievement of compression of the D-T fuel on the order of 5 to 12 times bquid density. Not all of our data is completely understood, but we have established the critical role of sophisticated two-dimensional simulations to describe asymmetric implosions.

A more quantiative understanding of the core conditions of high-density implosions requires an extension of our diagnostic capability:

- High-energy $x$-ray imaging where $x$-ray transport difficulties do not arise must be fully exploited in the future.

- The use of more than one imaging spectrometer will provide an accurate determination of the $x$-ray cmilting volume.

- Increased seed-gas fills, allowing the use of narrower slits, will reduce the present image unfold difficulty and allow Slark broadening analysis.

- The addition of $\sim 0.1$ atmosphere of Br into the fuel will make possible neutron activation metsurements of fuel areal density: simultaneous silicon activation measurements can be made in the pusher.

We have also derived several near-term improvements in design and diagnostics from the $10 \mathrm{X}$ experimental series. Several partially diagnosed experiments during this series showed more uniform ablator heating and increased core symmetry with a "ball in plate" target configuration (Fig. 6-69), thus making it possible to achieve more nearly spherical implosions. ${ }^{85}$ The neutron yield obtained on one shot was consistent with that measured on bare ball targets, indieating that thermal losses to the plate were nol excessive.

Besides providing more accurate data, these improvements and developments will constrain our simulation calculations and thus improve their credibility.

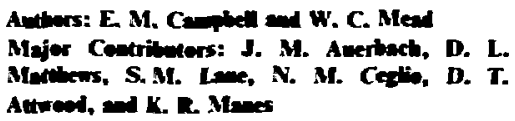




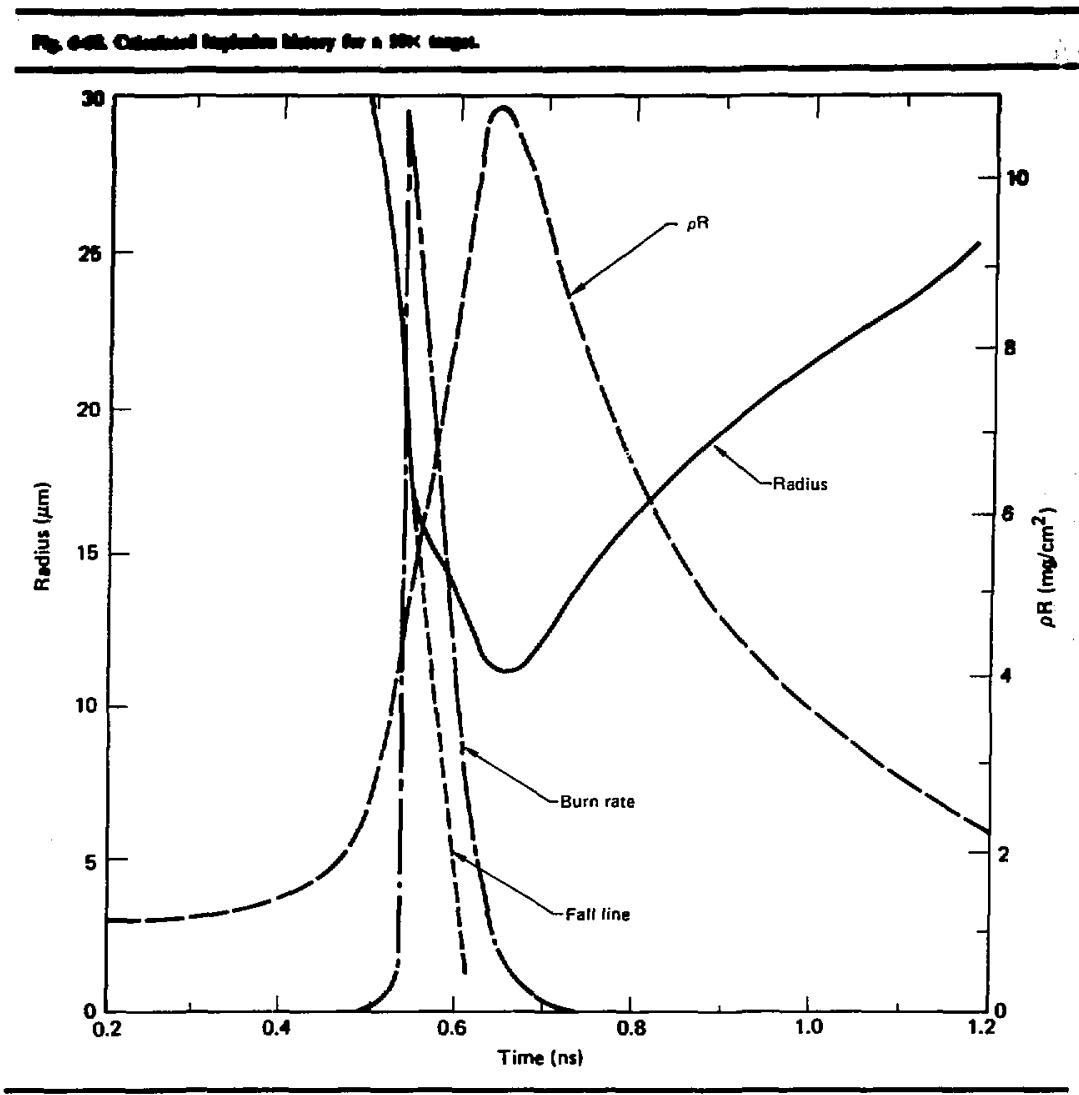

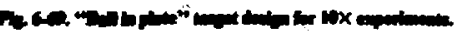

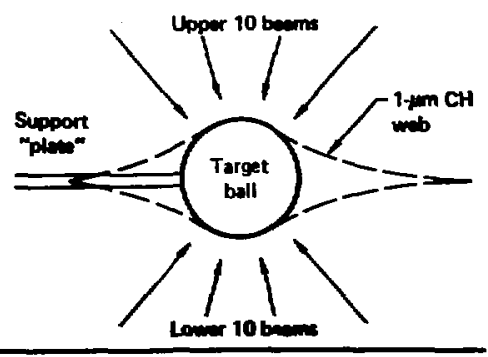

\section{References}

66. J. Nuckolls, L. Wood, A. Thiesson, and G. Zimmerman. Neture 23\%, 139 (1972).

67. E. K. Storm et al. Phýs. Rer. Lett. to 1570 (1978).

68. V. W. Slivinsky et al.. Phys. Rer. Letr. 35. I08j (1975).

69. D. R. Speck et at. Law rence Livermore Laboratory. Livermore. Calit.. LCRL-82117 (1979).

70. Y. L. Pan. and J. T. Larson. Lawrence Livermore Laborator. Livermore. Calif.. UCRL-79772 (1977): D. T. Attwood et al., Lawrence Livermore Laboratory. Livermore Calif.. UCRL-83541 (1979).

71. I. M. Auerbach. Lawrence Livermore Laboratorg. Livermore. Calif.. LCR1-89989 (1980) (submitted to Phys. Rer. (rni.). 
72. Laser Pnoprim Amund Rrport-1977. Lavrence Livermore Lahuratory. tivermore, Colif., UCRL.5002I-77 (1978), p. 3-37.

73. Laser Program A numb Aeporf-1975, Lawrence Livermore t.aboralory, I.ivermure, Calif., UCRL-S0021.76 (1977), p. 3.51.

74. Iaser I'rugram Annual Report/976. Lawrence Livermore I.aburatury, I.ivermore, Calif., UCRL.50021-76 (1977), ค. 3-105.

75. Lase" Program , 4nawal Report-1977. Luwrence Livermore I aburatory. I.ivermore. Calif., UCRL.50021-77 (1978), p) $3 \cdot(-4$.

-6. I. Seward el al., Rev. Sci. Instrum. 47, 464 (1976).

7? V. M1. Ceglio and J. T. Larson, thys. Rev. Lelt. 44, 9 (1980).

T. I. Y. Koppel et al. Lawrence Livermore Laboratory, 1 isermore, Calif., UCRL.-81477 (1979).

-. f: M. Cumpbell at al., Lawrence Livermore Laboratory. t ivernate. Calif. UCKL.83996 (1980) (to be nubtished in Mitr. Rev. L.elt.).

a! K. K. Manes al al. Phys Rev. Leff. 39, 281 (1977).

S1 13. I. Cohen and C. I. Mux, Phys. F/wids 22, 1115 (1979).

3. (C. Wang, H. Kornblum, and V. W. Slivinsky, Bull. Am. liny.s. Sinc. 24, 1106 (1979).

53 L.aser Progrum Anmual Report-1977. Lawfence Livetmore I.ahoratery. Livermore, Calif., UCRL-S0021-77 (1978). P. $4-31$.

H4. S. M. Lane. Li. M. Campbell, and C. K. Bennett. Lawrence liverinore laboratory, Livermone, Calif., UCRL.-B3882 (19k0) (10 be published in Appl. Phys. Lett.).

H5. W. C. Mead et al., Lawrence Livermore laboratory, Liverftore, Calif., UCRL-83163 (1979).

\section{D-'T Fuel Density Determination from Measurements of Pusher Areal Density $(\rho \Delta \mathbf{R})$}

For near-term investigation of D-T fuel compression in the intermediate-density ICF targets [linal fuel density $\rho_{f} \sim 2$ to $20 \mathrm{~g} / \mathrm{cm}^{3}$; neutron yield $\gamma \sim 10^{6}$ to $\left.00^{4}\right)$, we have chosen as our primary diagnostic the defermination of pusher areal density. $p \perp R$, by neutron activation. ${ }^{86}$ This technique has recently been used to estimate the final compressed state for thin-walled, single-shell exploding pusher targets as well as thiek-walled multilayered targets irradiated with pulses of 20010400 ps (Ref. 87 and the previous article).

With present-generation targets, the reutrons generated at peak D-T burn activate a measured fraction of ${ }^{28} \mathrm{Si}$ material in the glass pusher.

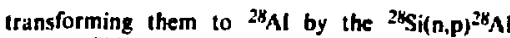
reaction. $* 6,84$ Assuming that the neutron yiekd $\alpha$ curs during a time period short compared with time scales of hydrodynamic motion, the number of activations are directly proportional to the product of the neutron yield $N$, and the pusher areal density $p \Delta R=\left[\int(R) d R\right]_{\text {pusher }}$ at the time of peak burn. The ${ }^{2 B}$ Al atoms decay by $\beta$-emission (with a half-life of 2.24 minutes) to excited ${ }^{2 k} \mathrm{Si}$, followed by a 1.78 . $\mathrm{MeV}$ gamma deexcitution to the ground state of $28 \mathrm{Si}$. By measuring the $\beta, \gamma$ coincidence decay rate from a known collected fraction of the pusher debris, ${ }^{\mathrm{Bk}}$ and taking the measured neutron yield, we can determine $\rho \perp R$ at peak burn (thit is, the neak neution production rate). ${ }^{89}$

An exact determination of $p د R$ from the number of pusher activations would require knowledge of the actual extended spatial distribution of neutron production, For spherically symmetric geometries, however, the issumption that the neutrons all originate at the center of the fuel produces the correct value for the determination of the number of activations (except for when the pusher and fuel are mixed at the time of peak burn). Consequently, except for analyzing the cases of pusher-fuel mix and nonspherical geometries, we will assume that the number of pusher activations is directly proportional to the product of the neutron yield and the pusher areal density. In this article we show how a simple. conservative estimate of the average fuel density at burn time, $\nu_{f}$ can be inferred from $\rho \perp R$.

\section{Target Geometry and Assumptions}

The typical multilayered, ablatively driven target shown in Fig. 6-70(a) is designed to acbieve 10 times the liquid density of D-T; although the figure shows the D-T fuel contained by a $\mathrm{SiO}_{2}$ puaher, our analysis can be performed for pushers of different materials as will as for pushers containing neutron-activation trace materials. Figure 670(b) shows a typical one-dimensional computer calculation of the density distribution at peak burn. The density jump at the pusher-fuel interface is the resulh of the requirement of local pressure balance and tbe different atomic weights of $\mathrm{SiO}_{2}$ and $\mathrm{CF}_{1.4}$ - 

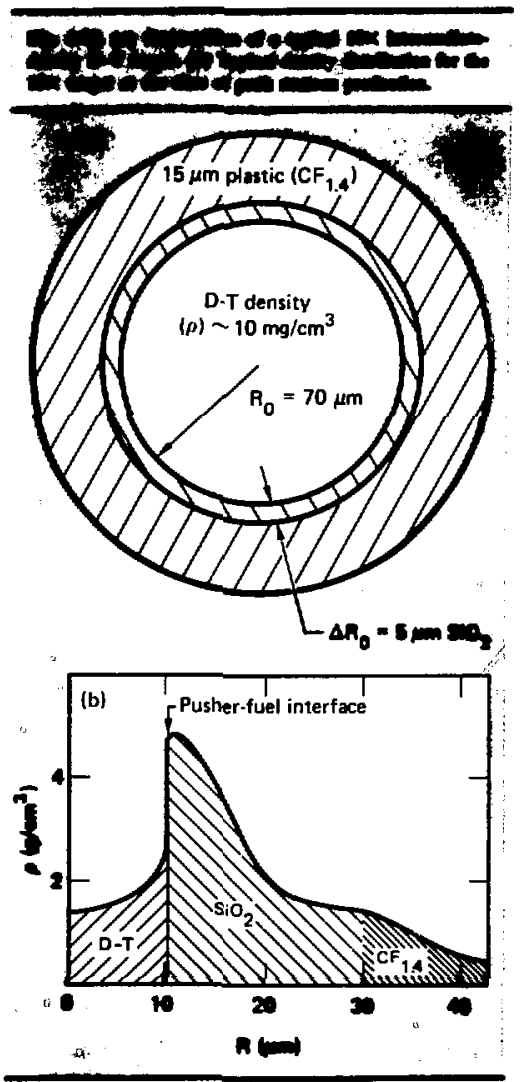

Computer code simulations show that the timing of peak compression and maximum neutron production in intermediate-density implosions depend on both the target design and irradiation conditions. For the targets discussed here. LASNEX calculations predict that peak burn occurs $\sim 150 \mathrm{ps}$ before maximum compression. ${ }^{89}$ Thus. provided the pusher and fuel are not severely mixed at the time of peak neutron production, fucl densilies determined from measurements of pusher areal density will in general be conservative (lower than the peak value).

To simplify the problem of determining the fuel density at peak burn. Our simpke model rests on four assumptions:
- Spherical symmetry.

- Conservation of both fuel and pusher mass.

- Uniform (though different) pusher and fuel densities.

- No mixing of pusher and fuel.

We will show later that relaxing the assumptions of mass conservation and uniform density distributions result in moderately higher calculated fuel densities for a given measured $\rho\lrcorner \mathrm{R}$, while the effects of mixing and asymmetry produce reductions by (at most) a factor of about 2 .

\section{Thin-Walled Targets}

Given the assumptions listed above it is straightforward to show that the statement of conservation of mass for the pusher and the fuel can be written

$$
\left(\frac{\rho_{\mathrm{f}}}{\rho_{\mathrm{f} 0}}\right)^{2 / 3}=\frac{\rho \Delta \mathrm{R}}{\rho_{0} \Delta \mathrm{R}_{0}} \frac{1+\frac{\Delta \mathrm{R}}{\mathrm{R}_{\mathrm{f}}}+\frac{1}{3}\left(\frac{\Delta \mathrm{R}}{\mathrm{R}_{\mathrm{f}}}\right)^{2}}{1+\frac{\Delta \mathrm{R}_{0}}{\mathrm{R}_{\mathrm{f0}}}+\frac{1}{3}\left(\frac{\Delta \mathrm{R}_{0}}{\mathrm{R}_{\mathrm{fo}}}\right)^{2}},
$$

where $\mu$ is pusher density, $\rho_{f}$ is fuel density, $R_{f}$ is fuel radius, and $\lambda R$ is pusher thickness; initial-state values are distinguished from final values by the subscript 0 . If in addition we are dealing with thinwalled targets and final experimental conditions such that

$$
\frac{\Delta R_{0}}{R_{f 0}}<1 \quad \frac{\Delta R}{R_{\mathrm{f}}}<1,
$$

then Eq. (23) immediately simplifies to

$$
\rho_{\mathrm{f}}=G_{0}(\rho \Delta R)^{3 / 2} .
$$

where $G_{0}=p_{f 0} /\left(\rho_{0} \Delta R_{g}\right)^{3 / 2}$ is a parameter composed of measured intial quantities. We observe that given our initial assumptions. a measurement of $\rho \mathbf{S R}$ at the time of peak D-T burn allows a direct and simple calculation of the average fuel density without independent knowledge of the pusher density or thickness.

\section{Thict-walled Targets in the Abeence of Mixins}

In most targets and final compressed-fuel conditions of interest. however. even if $\mathbf{S} \mathbf{R}_{0} / \mathbf{R}_{0} \ll 1$. $3 R_{1} / R_{\mathbf{r}}<k 1$ (and may indeed be $>1$ ) and Eq. (25) 
will significantly underestimate $\rho$. Yet it turns out that knowledge of the relationship between $p \rho$ and pusher density $p$ at the time of peak burn is sufficient to establish the more general relationship. $\mu_{f}=f(p \perp R)$, Still using the four assumptions outlined above, the conservation of pusher and fuel mass [Eq. (23)] can in this more general case be manipulated again to yield an expression between $\mu \Gamma$ and $p \triangle R$ of the form

$$
\rho_{f}=G\left(a, M_{p}, M_{f}\right)(\rho \Delta R)^{3 / 2} \text {, }
$$

where $M_{p}$ is the pusher mass. $M_{f}$ is the fuel mass, a $=\rho / \rho_{\mathrm{f}}$ (the ratio of pusher density to fuel density at the time of peak burn) and

$G\left(\alpha, M_{p}, M_{f}\right)=\left\{\alpha\left(\frac{3 M_{f}}{4 \pi}\right)^{1 / 3}\left[\left(1+\frac{M_{p}}{\alpha M_{f}}\right)^{1 / 3}-1\right]\right\}^{-3 / 2}$.

Thus, if $x$ is relatively insensitive to variations in largel parameters and performanee, $G$ will be (in the first approximation) a target-geometrydependent constant given by the initial conditions, and we find that even in the more general case we have a very simple relationship between of and $r \Delta R$.

By invoking continuity of pressure and temperature across the pusher-fuel interface at the time of peak D-T burn, then for silicale glass pushers and equimolar D-T fuel we find that at the interface

$$
p=\frac{16}{\langle Z\rangle+l} \rho_{f},
$$

where $Z$ is the average charge state of the pusher. Here we assume that

- The pusher and D-T are perfect gases.

- At the interface, the D-T ion temperature is equal to the electron temperature: this assumption is satisfied except for transiently in the target center.

- The pusher is in thermal equilibrium with the fuel electrons; for target sizes used in current experiments this assumption is also satisfied.

For our first estimate of the functional relationship between $\rho_{f}$ and $\rho \Delta R$, we will therefore assume that Eq. (28) is valid throughout the fuel and pusher, and use the D-T ion temperature at burn time. T, to evaluate $Z$. (We will later investigate the effect of fuel temperature equilibrium as well as nonuniform fuel and pusher density dis- tributions.) We choose $T$ to evaluate $Z$ be suse:

- $T$. the spatially and temporally averaged D-T ion temperature at hurn time, is the temperature most directly inferred from the target neutron yicld.

- Almost $x$ r: of the fuel mass lies beyond $R_{f} / 2$, where the temperature ratio approaches 1 .

- Even the transient ratio of electron to ion temperatures is much less than two.

Taking the specilic ealse of $\mathrm{SiO}_{2}$ pushers and using $T \sim 0.4 \mathrm{keV}$ als an approximate lower bound for the average D-T fuel temperature at peak burn. Eq. (28) becomes

$$
\rho=\alpha \rho_{f},
$$

where $1.5 \geq$ a $₹ 2.0$, and the upper and lower limits correspond to $Z \sim 7(\mathrm{~T} \sim 0.4 \mathrm{keV})$ and $Z=10$ for fully ionized $\mathrm{SiO}_{2}\left(T^{\top}>1 \mathrm{kt} V\right)$, respectively. The lower bound of $\mathrm{T} \sim 0.4 \mathrm{keV}$ was chosen becuuse temperatures much lower would result in neutron yiclds too low to allow determination of p p by neutron activation. Thus, for target performante of interest $(T>0.4 \mathrm{keV})$ the total vatriation in $\alpha$ is relatively small, and we can cnsure a conservative estimate of $p_{f}$ by assuming $\alpha=2$ throughout the experimental parameter range ( $T>0.4 \mathrm{keV}$ ). Curve 1 in Fig. 6-71(a) is a plot of Eq. (26), assuming o $\mathrm{c}=2$, for a $10 \times$ target of the type shown in Fig. 6-70.

If al more accurate value of $a$ is desired, however, we note that for $\mathrm{T}$ less than in few keV, neutron yield is a very sensitive function of the D.T ion temperature. ${ }^{.01 .91}$ Assuming that the D-T fuel conditions do not vary significantly during the burn, that burn time ( $10 \%$ to $90 \%$ ) is approximated by the final radius $R_{f}$ divided by the $D$ - $T$-ion sound speed. and given the D-T Maxwell-iveraged cross section. ${ }^{90}$ we can estimate the neutron yield as

$$
\mathrm{N}=2 \times 10^{27}\left(\rho_{10}{ }^{2} R_{\mathrm{TO}}{ }^{4}\right) c^{2 / 3} \mathrm{~T}_{\mathrm{exp}}^{-2 / 3}-\left(\frac{19}{\mathrm{~T}^{1 / 3}}\right)
$$

where $T$ is in keV, $R_{10}$ and $\rho_{50}$ are in cgs units, and $C$ is the fuel compression.

Figure 6-72 shows the result of solving Eq. (30) for the final D-T ion temperature as a function of neutron yield. for $10 \times$ targets as shown in Fig. 6-70(a): in experiments at Shiva (previous artick) a fuel density for these tarzets of up to 10 imes liquid D-T density was diagnosed using neutron activation. The curves show that for 2 given $\mathbf{N}$. the final temperature is not a strong function of the final 


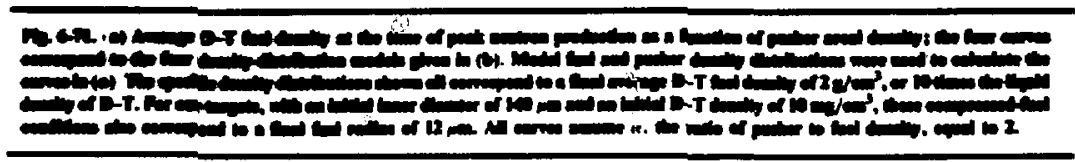

(a)

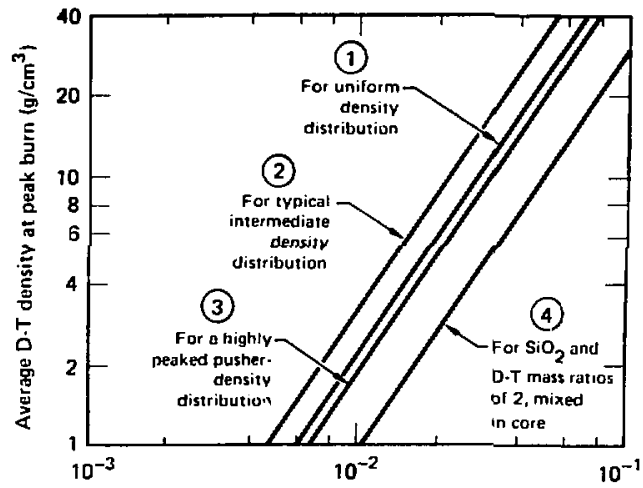

(b)

$\mathrm{SiO}_{2}$ pusher effective areal density $\rho \Delta \mathrm{R}\left(\mathrm{g} / \mathrm{cm}^{2}\right)$
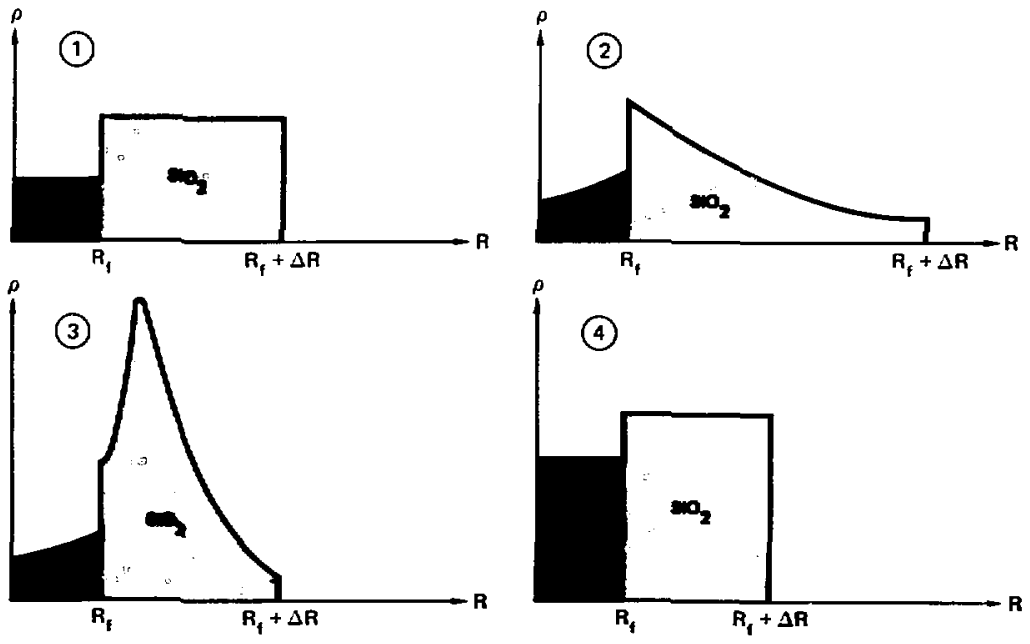


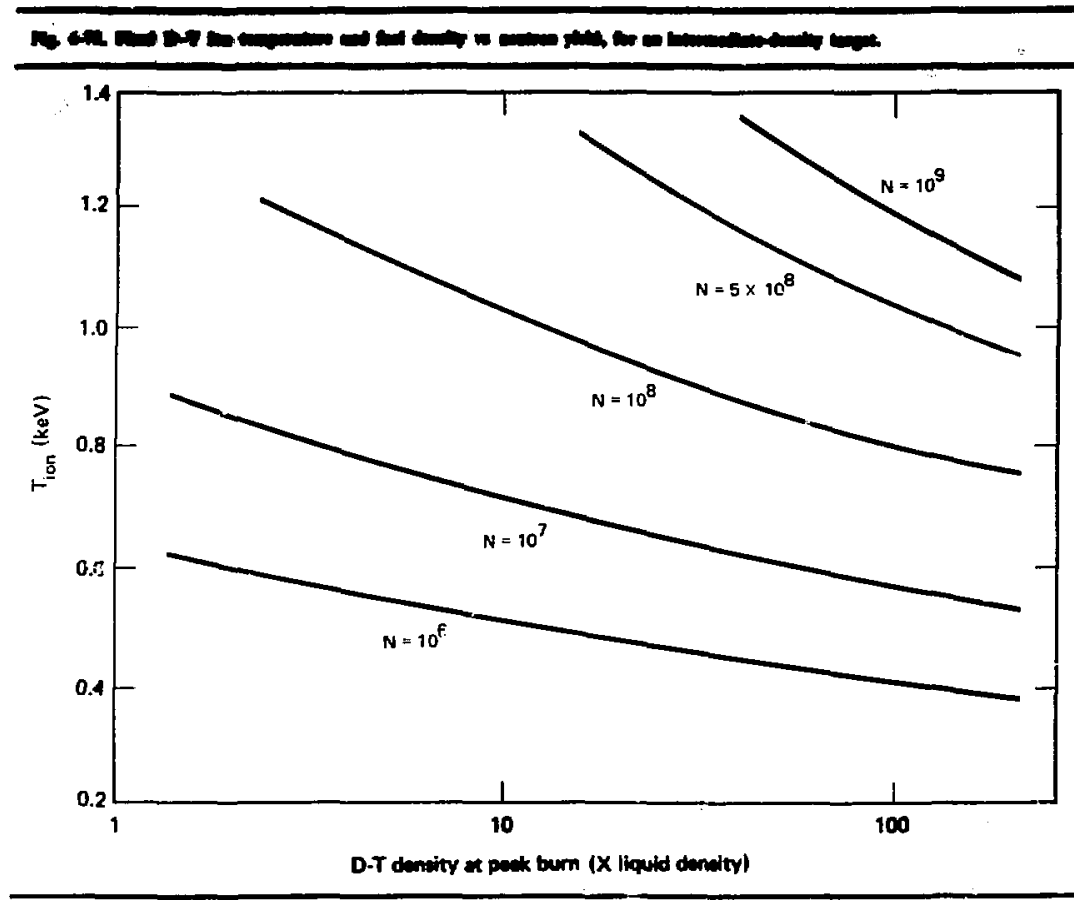

den sity. Thus a simple one-time iteration will suffice to give T sufficiently accurately to determine $\alpha$.

\section{Nonuniform Pusher Density Distributions}

We can now investigate the effects of relaxing some of our earlier assumptions. In the above inulysis we have assumed that whike fuel and pusher density have different values. they were each uniform with radius. As mentioned previously, a fuel density variation with radius will gon affect the activation of pusher material for ary given value of $\rho \mathbf{R}$, as long as the variation is spherically symmietric and no mixing has occurred. There are many reasonably possible pusher density distributions. however, that could greatly affect the pusher activation. If a portion of the pusher material is ablated during the implosion and thas significantly decompressed, then for a given average fuel density the pusher areal density will be less than in the case of uniform compression of all pusher material at burn time. In this case, curve 1 in Fig. 6-71(a) would underestimate the fuel density for a given (measured) $p \Delta R$ by approximately the amount of nonuniform compression: if $20 \%$ of the pusher mass of a $10 \times$ target is ablated away. of would be approximately $20 \%$ higher than is implied ty curve 1 .

While the effect of disequilibriurr on fuel temperalure conditions is a reduction in implied density. the restilt is relatively insensitive to the temperature natio. Even assuming a D-T ion temperature :hat is twice tbe electron temperature throughout the fuel region. the relationship of $\rho_{f}$ and $\rho د R$ will see oniy a $25 \%$ reduction. 


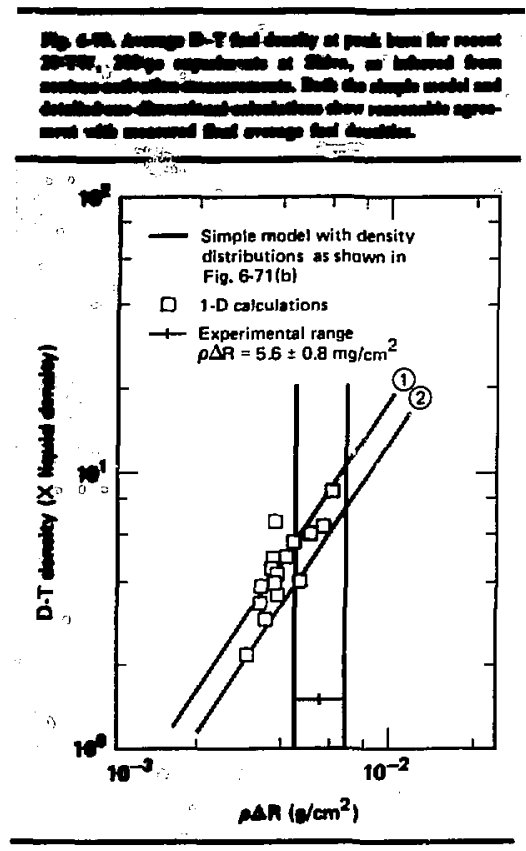

Nonuniform Radial Density

In investigating the effect of nonuniform radial density (and consequently pressure and temperature) distributions, we will still usc $T$ (the spatially and temporally averaged D-T fiel temperature) to evaluate $(Z$ ) and still consider Eq. (28) valid at the pusher-fuel interface. Simulations ${ }^{89}$ in. dicate that for our $10 \times$ target the pusher is decelerating near peak neutron production, so the most realistic configurations give density distributions that are peaked at or near the pusher-fuel interface. With the tools developed above, it is thus relatively easy to evaluate the effect of such nonuniform density distributions.

Curves 2 and 3 in Fig. 6-71(a) show the result of determining pr from the neutron-activationdetermined $\rho \perp R$, for two such peaked density distributions. The actual density-vs-radius distributions used in the computation are indicated (drawn to scale) in Fig. 6-73: rather than attempt to explore a variety of analytical expressions for $\rho(R)$, we have chosen these two generic distributions as examples. The numerical results quoted below are relativily insensitive to shape perturbations. For a given average fuel density, the effect of a nonuniform fuel distribution is to alter $\rho \Delta R$, from the value of the uniform density case.

We can now find $\rho \perp R$ by evaluating the act ual integral. $\rho(R) d R$. We note that the density distribution in model 2 in Fig. 6-71(b) (similar to that predicted by detailed computer simulations ${ }^{91}$ ) increases the implied averuge $\rho$ for a given $\rho \Delta R$. and that moving the peak in the pusher density distribution away from the interface, as shown in model 3 , brings the average $\nu_{i}$ vs $\rho \perp R$ back (approximately) lowards the isodensity result. A pusher density distribution could be generated that would reduce the avera ge $p_{f}$ by a factor of 2 or 3 from the isodensity curve; this, however, would require a pusher density distribution similar to model 3 as shown. but with a peak pusher density of $\sim 10$ times the average fuel density at the time of peak neutron production. Such high densities do not occur because the adjicent $\mathrm{SiO}_{2}$ glass and D-T are thermally coupled. and due to the finite time for the stagnation shock to propagate to the outer glass, the pressure on the outer glass is much less than the pressure on the D-T.

In the absence of mixing. the assumptions of conservation of mass and uniform density distribution in the pusher and fuel are thus wen to give a conservative cstimate of average peak fuel density $\nu_{f}$ as determined by $\rho \mathbf{R}$. In our discussion of the effect of mixing, we will therefore limit rurselves to the cuse of uniform density distributions.

\section{Effects of Mixing}

During the final stages of implosion. the dense pusher will be decelerated by the lower-density D-T fuel. In this situation. the pusher-fuel interface can suffer significant growth of spatial perturbations via Rayleigh-Taylor instabilities. This instability can result in significant mixing of the pusher into the D$T$ fuel. The mixing process is tinus dynamic rather than diffusive. driven by the density gradient at the interface. and can be viswalized as the final breakup 
of the wavy pusher-fuel interface and resultant intermixing of adjacent pusher and fuel "pockets." In addition, since adjacent layers of fuel and pusher mass are isobaric and isothermal, the ratio of pusher to fuel mass in the final mixed layer should be comparable to and probably no greater than the density ratio prior to mixing.

Simulations consider the upper unit of mix at burn time as when the pusher mix has affected approximately $2 / 3$ of the fuel mass. ${ }^{91}$ To ensure an even more conservative estimate on the effect of mixing, we will consider the situation when, at peak burn, the entire fuel region is involved in the mixing process; $\alpha$, the ratio of pusher to fuel density at the interface, will be used fer the ratio of pusher to fuel mass in the mix region. From the continuity of pressure and temperiture at the boundary of the pusher-fucl mixture and the pusher we thus have

$$
\rho=\frac{16}{\langle Z\rangle+1} \rho_{\mathrm{f}}+\rho_{\mathrm{C}}=\alpha \rho_{\mathrm{f}}+\rho_{\mathrm{C}}=2 \alpha \rho_{\mathrm{C}},
$$

where $\rho_{\mathrm{c}}$ is the pusher density in the core (i.e., in the pusher-fuel mixture), and $1.5 \gtrless \alpha \gtrless 2.0$ as before. Invoking conservation of fuel and pusher miss, together with the assumption of uniform density distributions, we can proceed as in the no-mix example, and it is again straightforward to show that [see Eq. (26)]

where

$$
\rho_{\mathrm{f}}=\mathrm{G}\left(\alpha_{1} \mathrm{M}_{\mathrm{p}}, \mathrm{M}_{\mathrm{f}}\right)(\rho \Delta \mathrm{R})_{\mathrm{eff}}^{3 / 2},
$$

$$
G\left(\alpha, M_{p}, M_{f}\right)=\sqrt{\frac{4 \pi}{3 M_{f}}}\left\{\frac{3}{4} \alpha+2 \alpha\left[\left(\frac{1}{2}+\frac{M_{p}}{2 \alpha M_{f}}\right)^{1 / 3}-1\right]\right\}^{-3 / 2} \text {. }
$$

Here $(\rho \Delta \mathrm{R})_{\mathrm{erf}}$ is the effective $\int \rho \mathrm{dR}$ as determined by the neutron activation diagnostic, and not simply jodR as in the unmixed case. The factor of $3 / 4$ in the expression for $G\left(\alpha, M_{p h} M_{f}\right)$ comes from the assumption lin computing the contribution to $(\rho \triangle \mathrm{R})_{\mathrm{eff}}$ that the neutrons are produced throughout the mixed core.

Equation (26) is plotted as curve 4 in Fig. 6-7l (a), again using the conservative value of $\alpha=2$ : the corresponding density-radius distribution (model 4) is shown in Fig. 6-71(b). We see that even with this severe degree of $\operatorname{mix}$ and with $\alpha=2$, the implied $\rho$ is reduced only by a factor of 2 over the unmixed case (curve and model I).
Since in the actual situation the outer layers of the pusher are cooler than the hot central fuel region, mixing of the pusher and fuel involving any significant amount of pusher mass would result in a reduction of the fuel ion temperature and consequently a reduction of the burn rate in the mix region. Let us therefore analyze a more realistic case for which an inner region of the fuel (say, $R<R_{f} / 2$ ) remains unnixed, and assume that the ion temperature in this region is $\sim 2$ times that of the mixed region, such that the neutrons are preferentially generated in this hot, unmixed region. In this case, the reduction in implied $\rho_{f}$ for a given $(\rho \Delta R)_{e f f}$ is only $35 \%$. Relaxing the assumption of no pushermass ablation, setting $\langle Z\rangle\rangle 7$, or relaxing the assumption of uniform density distributions will all again tend to increase the implied $\beta_{\mathrm{p}}$. Hence the net error should be less than twofold, and curve 4 in Fig. 6-71(a) is clearly a conservative lower bound of the effect of mix.

Although we do not show the calculations here, it is casy to show that only extreme deviations from spherical symmetry produce reductions greater than 10 to $20 \%$. Such large asymmetries would be readily observable in $x$-ray inicroscope images.

\section{Comparison of Model with Experimental Results and One-Dimensional Simulation}

We now use this simple model (based on the four assumptions) to predict the fuel densities obtained in the actual intermediate-density experiment series, and compare them with derailed onedimensional computer modeling. As mentioned earlier, a series of $140-\mu \mathrm{m}$-i.d., 5- $\mu \mathrm{m}$-thick $\mathrm{SiO}_{2}$ microshells coated with $15 \mu \mathrm{m}$ of $\mathrm{CF}_{1.4}$ and filled with $10 \mathrm{mg} / \mathrm{cc}$ of D-T were irradiated at Shiva with $20 \mathrm{TW}$ in a 200-ps (FWHM Gaussian) pulse. ${ }^{88}$ The target-wall thickness in these experiments offers greater pusher preheat protection aguinst suprathermal electrons than the thin-shelled explodingpusher targets. And, as a first step towards the targets ultimately required for high-density implosions, these multilayered targets were expected to operate in a more ablatively driven mode, ${ }^{97}$ and to reach final fuel densities of $\sim 2 \mathrm{~g} / \mathrm{cm}^{3}$ (10X liquid D-T density). The target and laser parameters and experimental results are summarized in Table 6-15. 


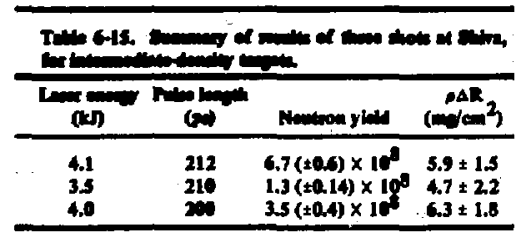

Using neutron activation, the average, elfective $\mathrm{SiO}_{2}$ pusher areal density at peak burn was determined in these experiments to be $5.6( \pm 0.8)$ $\mathrm{mg} / \mathrm{cm}^{2}$. Using Fig. 6-72, we observe that even taking our lowest experimental yalue for neutron yield $\left(1.3 \times 10^{8}\right)$ and a most optimistic value for the fuel density $\left(4 \mathrm{~g} / \mathrm{cm}^{3}\right.$, or 20 times the liquid D-T density) we find that the minimum possible D-T ion temperature for these experiments is $1 \mathrm{keV}$; consequently, $\alpha$ is found to be $\sim 1.5$. Using this value for $\alpha$ in the uniform density distribution model, our experimental $\rho \Delta R$ 's produce the range of $\rho$ 's plotted as curve 1 in Fig. 6-73, which also gives the onedimensional calculations, ${ }^{90}$ indicated by individual squares. Since the one-dimensional calculations imply that the actual density distribution is more like the one used for case 2 during the discussion of Fig. 6-71, however, we also show the fuel densities for this case (curve 2) in Fig. 6-73.

The one-dimensional calculations for this experiment show that peak neutron production occurs approximately $1.50 \mathrm{ps}$ before stagnation (or peak compression). Thus we would not expect any appreciable mix of the fuel and pusher at the time the neutron activation measurement is made, and for these experimental conditions the two curves 1 and 2 should provide a good lower and upper bound for the fuel density at tine time of peak burn. The relatively good agreement between the detailed computer calculations and the simple model gives us confidence that average fuel densities of 6 to 8 times liquid D-T densities were obtained at the time of peak burn. These results are also in good agreement with high-energy $x$-ray zone plate results and argon-imaging results from the same targets (reported in the preceding article).

The question of the final compressed density at stagnation is more complicated, as in this case the possibility of mix will have to be included. As we have no diagnostic measurement of $p \Delta R$ at this time. however. we can only use the densities found at peak burn as a lower. conservative estimate of the aclual peak densities. For diflerent targets and laser irradiation conditions under which peak burn and stagnation may occur simultaneously, although neutron activation will give us $\rho \perp R$ at the time of peak compression, we will have to seriously consider the effects of pusher-fuel mix in order to infer final fuel density.

\section{Conclusions}

With only a few physically justifiable assumptions we have shown that neutron activation measurements of pusher areal density can be used to infer final fuel density with uncertainties of less than a factor of 2. Our simple modeling is in good agreement with complex simulation codes which include neutron production as well as all of the relevant hydrodynamics. Using this model we have found that pusher areal densities of $5.6 \mathrm{mg} / \mathrm{cm}^{2}$, obtained in recent target experiments on the Shiva laser, imply a fuel density of 1 to $2 \mathrm{~g} / \mathrm{cm}^{3}$.

Authors: E. K. Storm, V. W. Slivinsky, and E. M. Camphell

\section{References}

86. H: M. Cilmphell. W. M. Ploeger. P. H. I.et, and S. M. Lane, L.awrence Livermore Laboratory, Livermore, Calif., UCKL-83096 (1979) (submitted to $\mathrm{A} / \mathrm{fl}$. Phy's. Left.).

67. J. M. Nuerhach, W. C. Metad, E. M. Camphell, D. 1. Matthews. D.S. Bailey, N. M. Ceglio. C. W. Hateher, L. N. Koppel. S. M. Lane. P. H. Lee. K. R. Manes, G. Mr(iellun. D. W. Phillion, R. L. Price, V. C. Rupert, V. W. Slivinsky. and C. D. Swift. Lawrence Livermore I.aborafory. I.jvermore, Calif., UCR I-83057: presented at the Mecting of the American Physical Society, Boston. Muss.. November 1979.

8K. IE. M. Cumphell, H. G. Hicks, W. C. Mead, L. W. Coltemun. C. W. Hulcher, 1. H. Dellis. M. J. Hoyle, J. T. Larson, and S, M. L.ane, L.awrence Livermore L.aboratory. livermort. Culir.. LCRL-k3072 (to be published in $J$. Appl. this.. Aprit 1980).

89. W. C. Stead. C. D. Orth, D. S. Hailey, G. McClellan, and k. Estabruok. Law rence livermare Laboratory, livermore, Calif. (ICRI_-\$3163 (1979).

90. S, L. Girein. Lau rence Livermore Laboratory: Livermore. Calif.. L (KL1.70522 1967)

91. E. K. Storm el al. Phys. Rev. Refr. 40. 1570 (197\$). 